For

UC-NRLF

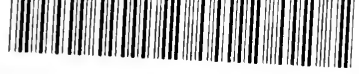

B 4 I $32 \quad 393$ 

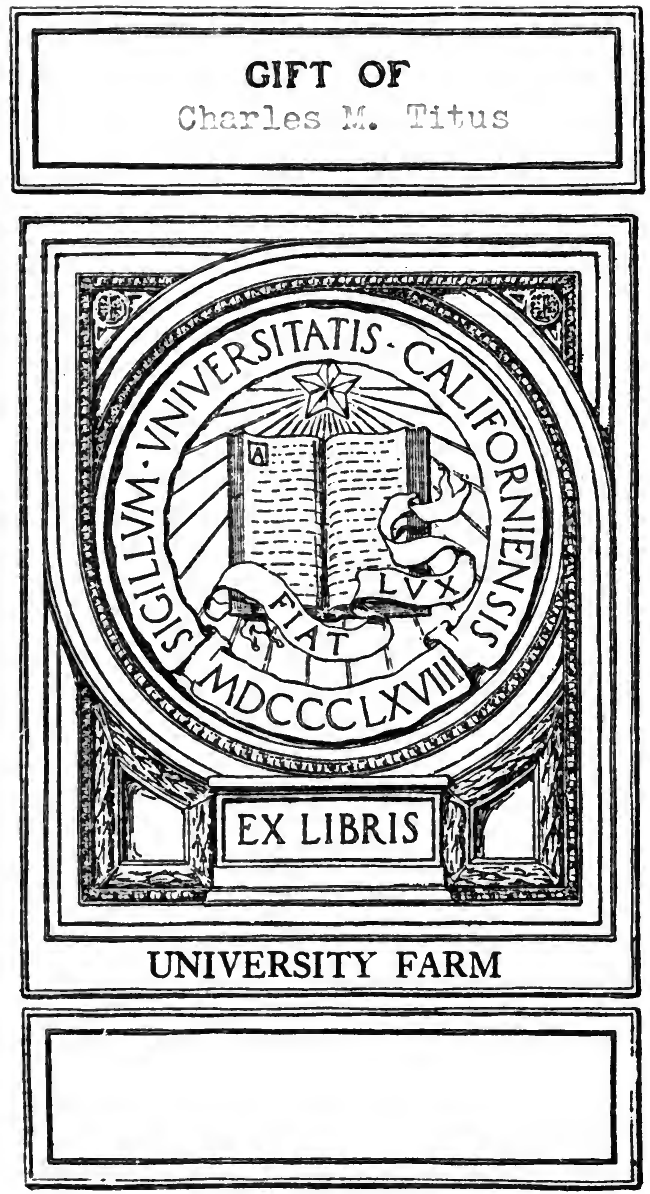

Digitized by the Internet Archive in 2007 with funding from Microsoft Corporation 


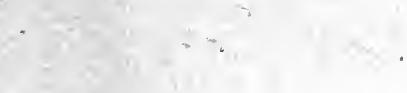

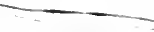





\section{ANALYTICAL MECHANICS}

\section{FOR ENGINEERS}

BY

FRED B. SEELY, M.S.

Professor of Theoretical and Applied Mechanics

AND

NEWTON E. ENSIGN, A.B., B.S.

Assistant Professor of Theoretical and Applied Mechanics

University of Illinois

TOTAL ISSUE, TEN THOUSAND

NEIV YORK

JOHN WILEY \& SONS, INC.

LoNdoN: CHAPMAN \& HALL, LIMITED

1921

UNIVERSITY OF CALIFORNIA 
Copyright, 1921,

By FRED B. SEELY

and

NEWTON E. ENSIGN 


\section{PREFACE}

THis book, as its name suggests, presents those principles of mechanies that are believed to be essential for the student of engineering.

Throughout the book the aim has been to make the principles of mechanics stand out clearly; to build them up as much as possible from common experience (the student's experience); to apply the principles to concrete problems of practical value; and to emphasize the physical rather than the mathematical interpretation of the principles. Important equations are printed in bold-faced type and the statements of the more important principles are italicized.

The book is divided into three parts; namely, Statics, Kinematics, and Kinetics. Statics is presented first because of its simplicity and its direct relation to the student's experience. However, in the first two chapters are developed certain concepts and elementary principles that are fully as important in kinetics as in statics, and the authors feel that it is essential to a satisfactory grasp of mechanics, as a whole, that sufficient time and care be taken to cause these elementary concepts and principles to crystallize in the student's mind before the more general principles and problems are studied. The equilibrium of the various types of force systems are treated both by the algebraic and by the graphical method. A large number of problems involving the equilibrium of the simpler structures and machines are given, and figures illustrating the structures and machines are used freely.

Although kinematics as herein developed is mainly a preliminary to kinetics, the authors' experience indicates that the kinematic properties of motion must be isolated and developed with care if they are to be used with success in the study of the kinetics of the motion. 
Both kinematics and kinetics have been developed with regard for the increasing importance of dynamics to engineers. The geometric and physical conceptions and interpretations of the quantities in kinematics have been emphasized rather than the mathematical conceptions. A treatment of acceleration is given which, it is hoped, will help to overcome some of the difficulties frequently found in the use of this quantity. The treatment of kinetics has been restricted to the more common types of motion found in engineering practice, but these motions have been treated more fully than is usual in elementary texts on mechanics. This is particularly true of plane motion. D'Alembert's principle (involving inertia forces) has been used for each type of motion as a second method of solution. The methods of procedure used in the analysis of kinetics problems are strongly emphasized both in the general discussions and in the solutions of illustrative problems.

Illustrative problems are given at the end of the more important articles and many problems are offered for solution. Great care has been exercised in selecting problems that are of practical interest and yet are easily comprehended and are free from unimportant details so that the principles used in their solution will stand out clearly. The answers to about one-half of the problems are given.

Graphical methods of representation and of solution have been used frequently in all three parts of the book. A knowledge of elementary calculus is assumed although little use is made of it in the first four chapters.

The discussion of centroids (Chapter V) is developed directly from the principle of moments-a principle given much emphasis throughout the book.

Several special topics are discussed in Section 3 of Chapter IX. They may be omitted without interfering with the continuity of the book, or any one of the topics may be studied alone without studying the whole section. Further, if it is desired to reduce the time given to kinetics, the second method of analysis of the motion of rotation or of plane motion (which employs D'Alembert's principle and inertia forces) in Section 2 of Chapter IX may be omitted. And, in general, the last part of the material in any section or chapter may be omitted without interfering with the student's progress in the first part of the next section or chapter. 
The authors wish to acknowledge their indebtedness to Professor A. N. Talbot for his interest in the book during its preparation and for his helpful suggestions on the treatment of certain important topies. The authors are also indebted to Professor G. A. Goodenough for valuable material in the treatment of Governors and to Professor O. A. Leutwiler for the data and figures for a number of valuable engineering problems.

F. B. S.

N. E. E.

URBANA, ILLINOIS,

December, 1920. 



\section{CONTENTS}

\section{PART I. STATICS}

\section{Chapter I. Fundamental Conceptions and Definitions}

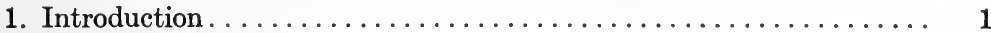

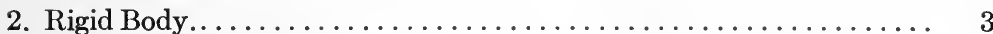

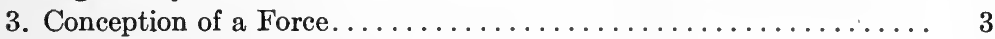

4. External Effects of a Force...................... 4

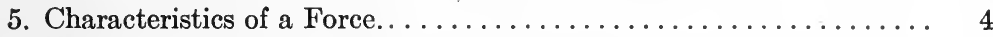

6. Measure of a Force. Units..................... 4

7. Scalar and Vector Quantities. Vector Representation of a Force... 6

8. Classification of Forces. Definitions................ 7

9. Principle of Transmissibility...................... 8

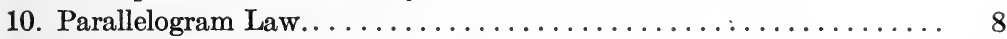

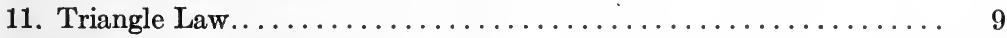

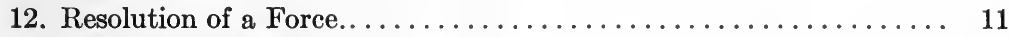

13. Moment of a Force.......................... 14

14. Principle of Moments. Varignon's Theorem................ 16

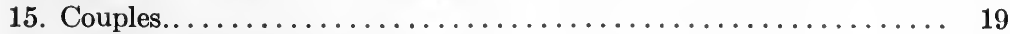

16. Characteristics of a Couple....................... 20

17. Vector Representation of a Couple................... 20

18. Resolution of a Force into a Force and a Couple............ 22

19. Methods of Solution of Problems.................... 24

Chapter II. Resultants of Force Systems

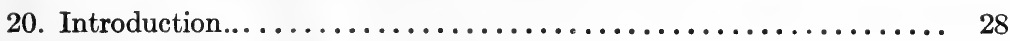

§ 1. Collinear Forces

21. Algebraic Method.............................. 28

§ 2. Concurrent Forces in a Plane

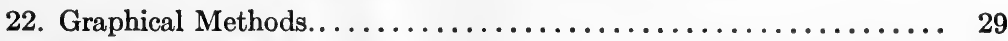

23. Algebraic Method........................... 31 
24. Graphical Methods............................ 34

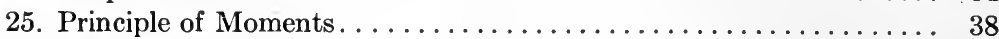

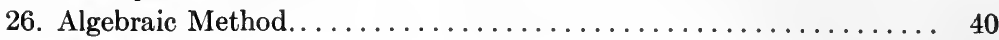

27. Couples................................. 44

§4. Non-concurrent, Non-parallel Forces in a Plane

28. Graphical Methods........................... 48

29. Principle of Moments. . . . . . . . . . . . . . . . . 49

30. Algebraic Method.......................... 50

31. Graphical Method......................... 53

32. Algebraic Method............................ 54

§6. Parallel Forces in Space

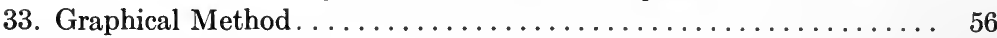

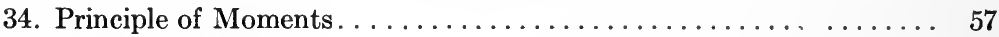

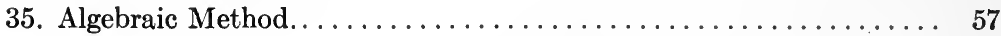

§ 7. Couples in Space

36. Resultant of a System of Couples..................... 60

37. Composition of Couples by Means of Vectors.............. 62

38. Resolution of Couples. ....................... 64

§8. Non-concurrent, Non-parallel Forces in Space

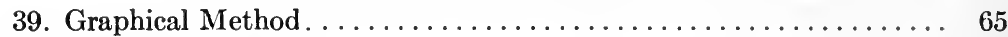

40. Principle of Moments. . . . . . . . . . . . . . . . . . . 65

41. Algebraic Method......................... 66

Chapter III. Equilibrium of Force Systems

§ 1. Introduction

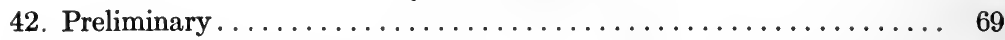

43. Graphical Conditions of Equilibrium ............... 70

44. Algebraic Conditions of Equilibrium................ 70

§2. Collinear Forces

45. Equations of Equilibrium...................... 72

§3. Concurrent Forces in a Plane

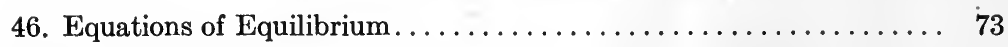

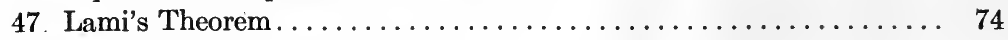

48 Tr ree Forces in Equilibrium. . . . . . . . . . . . 75

§4. Parallel Forces in a Plane

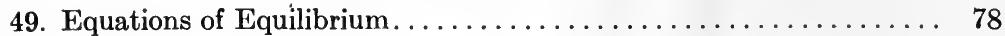


§ 5. Non-concurrent, Non-parallel Forces in a Plane

50. Equations of Equilibrium . . . . . . . . . 82

51. Graphical Solution of a Typical Problem................ 85

52. Choice of Moment-centers and of Directions of Resolution. . . . . . 87

§6. Equilibrium of Trusses, Cranes, and Cables

53. Stresses in Trusses. . . . . . . . . . . . . . 88

54. Method of Joints. . . . . . . . . . . . . . . . . . . . 89

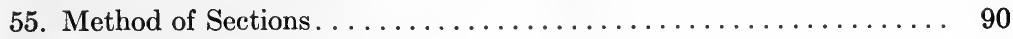

56. Graphical Analysis of Trusses. . . . . . . . . . . . $\ldots 5$

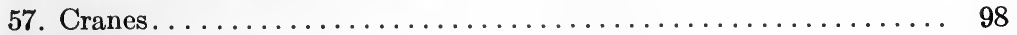

58. Stresses in Flexible Cables. . . . . . . . . . . . . . . . . . 102

59. The Parabolic Cable. . . . . . . . . . . . . . . . . . . . 102

60. The Catenary ............................... 106

§ 7. Concurrent Forces in Space

61. Equations of Equilibrium.

§ 8. Parallel Forces in Space

62. Equations of Equilibrium .

§ 9. Non-concurrent, Non-parallel Forces in Space

63. Equations of Equilibrium.

\section{Chapter IV. Friction}

64. Friction Defined.............................. 127

65. Coefficient of Friction. . . . . . . . . . . . . . . . . 128

66. Angle of Friction and the Friction Cone. . . . . . . . . . . . 129

67. Angle of Repose. . . . . . . . . . . . . . . . . . . 133

68. The Laws of Friction... . . . . . . . . . . . . . . . . . 134

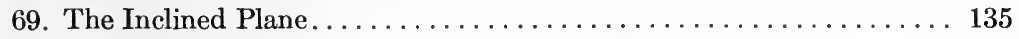

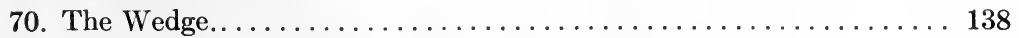

71. The Screw . . . . . . . . . . . . . . . . . . . . . 142

72. Journal Friction and the Friction Circle............... 145

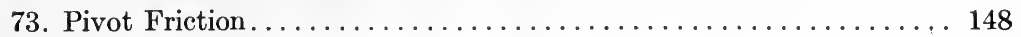

74. Solid Flat Pivot. . . . . . . . . . . . . . . . . . 149

75. Hollow Flat Pivot or Collar Bearing................. 150

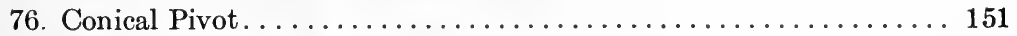

77. Rolling Resistance.......................... 153

78 Belt Friction ............................... 155

\section{Chapter V. First Moments and Centroids}

79. First Moments......................... 160

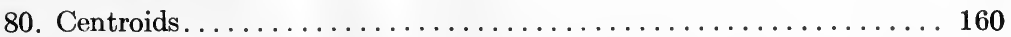

81. Planes and Lines of Symmetry . . . . . . . . . . . . . . 162

82. Centroids by Integration...................... 163 
83. Centroids of Composite Figures and Bodies. .............. PAGI

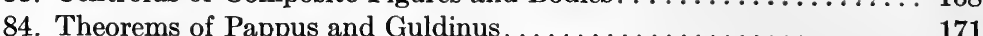

85. Center of Gravity and Mass-center.................. 173

86. Graphical Method of Determining Centroids of Areas. . . . . . . . 177

87. Determination of Center of Gravity by Experiment........... 178

\section{Chapter VI. Second Moment. Moment of Inertia}

\section{$\S 1$. Moments of Inertia of Areas}

88. Moment of Inertia of an Area Defined................. 180

89. Polar Moment of Inertia . . . . . . . . . . . . . . . . . . . . 181

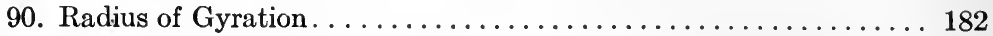

91. Parallel Axis Theorem for Areas..................... 183

92. Moments of Inertia by Integration.................. 184

93. Moments of Inertia of Composite Areas . . . . . . . . . . . . . . . . . 190

94. Product of Inertia Defined. . . . . . . . . . . . . . . . . . . 194

95. Axes of Symmetry . . . . . . . . . . . . . . . . . . . . 194

96. Parallel Axis Theorem for Products of Inertia . . . . . . . . . . . . 196

97. Moments of Inertia with Respect to Inclined Axes............ 198

98. Principal Axes. . . . . . . . . . . . . . . . . . . . . . . . . . . 199

99. Moments of Inertia of Areas by Graphical and Approximate Methods 202

\section{§2. Moments of Ineria of Bodies}

100. Moment of Inertia of Mass Defined................... 205

101. Radius of Gyration. . . . . . . . . . . . . . . . . . . . 207

102. Parallel Axis Theorem for Masses..................... 207

103. Moments of Inertia with Respect to Two Perpendicular Planes . . . . 208

104. Moments of Inertia of Simple Solids by Integration . . . . . . . . . . . 209

105. Moments of Inertia of Composite Bodies... . . . . . . . . . . . . . . 218

106. Moments of Inertia of Bodies by Experimental Methods. . . . . . . 220

\section{PART II. KINEMATICS}

107. Introduction . . . . . . . . . . . . . . . . . . . . 221

\section{Chapter VII. Motion of a Particle}

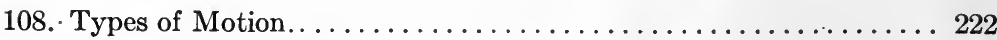

109. Linear Displacement. . . . . . . . . . . . . . . . . . . . . 222

110. Angular Displacement... . . . . . . . . . . . . . . . . . . . . 224

111. Relation between Linear and Angular Displacements. . . . . . . . 225

112. Linear Velocity and Speed. . . . . . . . . . . . . . . . . . . . 226

113. Distance-time Graph. . . . . . . . . . . . . . . . . . . . . 228

114. Angular Velocity................................ 229

115. Relation between Linear and Angular Velocities............ 230

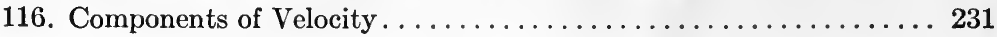


117. Linear Acceleration ........................... 235

118. Tangential and Normal Accelerations................... 236

119. Speed-time Graph.............................. 241

120. Angular Acceleration .......................... 242

121. Relation between Linear and Angular Accelerations........... 243

122. Axial Components of Acceleration.................... 245

123. Motion of a Projectile......................... 248

124. Uniformly Accelerated Rectilinear Motion............... 250

125. Uniformly Accelerated Circular Motion................ 251

126. Simple Harmonic Motion........................ 253

127. Non-uniformly Accelerated Motion................... 256

128. Relative Motion.............................. 262

129. Transverse and Radial Components of Acceleration........... 269

130. Coriolis' Law................................... 270

Chapter VIII. Motion of Rigid Bodies

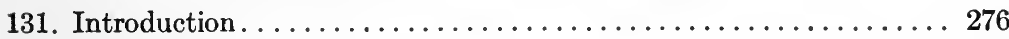

132. Translation............................... 276

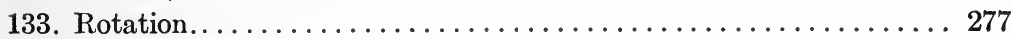

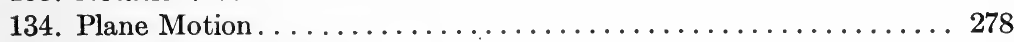

135. Instantaneous Center.......................... 284

\section{PART III. KINETICS}

Chapter IX.-Force, Mass, and Acceleration

\$1. Preliminary Considerations. Kinetics of a Particle

136. Introduction. .............................. 287

137. The General Kinetics Problem...................... 288

138. Characteristics of a Force System................... 288

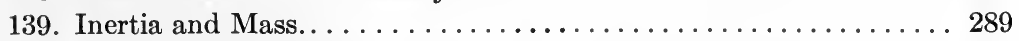

140. Newton's Laws............................... 293

141. Mathematical Statement of Newton's Second Law. Units....... 294

142. Other Systems of Units......................... 296

143. Equations of Motion for a Particle.................. 297

§2. Kinetics of Bodies

144. 'ntroduction. Methods of Analysis.................. 303

\section{Translation}

145. Kinetics of a Translating Rigid Body................. 305

\section{Rotation}

146. Kinetics of a Rotating Rigid Body ................... 313

147. Second Method of Analysis. Inertia Forces............... 325

148. Center of Percussion........................... 336 


\section{Plane Motion}

149. Kinetics of Plane Motion of a Rigid Body . . . . . . . . . . 336

150. Second Method of Analysis. Inertia Forces... . . . . . . . . . . 345

151. Limitations of the Moment Equation, $T=I \alpha \ldots \ldots \ldots \ldots \ldots \ldots 35$

152. Principle of the Motion of the Mass-center... . . . . . . . . . . 353

\section{§3. Special Topics in Kinetics}

153. Introduction . . . . . . . . . . . . . . . . . . . . . . . . 356

154. Hoop Tension in Flywheels. . . . . . . . . . . . . . . 356

155. Superelevation of Railway Track..................... 358

156. Simple Circular Pendulum. . . . . . . . . . . . . . . 360

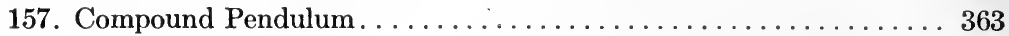

158. Center of Oscillation . . . . . . . . . . . . . . . . . . . . . . 364

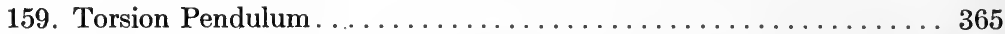

160. Experimental Determination of Moment of Inertia..... . . . . . . 367

\section{Balancing}

161. Need for Balancing......................... 369

162. Balancing of Rotating Masses. . . . . . . . . . . . . . . . . . . . 370

163. Several Masses in a Single Plane of Rotation.... . . . . . . . 372

164. Masses in Different Transverse Planes................. 373

\section{The Gyroscope}

165. The Problem Defined.............................. 378

166. Analysis of Forces in the Gyroscope.. . . . . . . . . . . . . . . 379

167. The Moment of the Gyroscopic Couple................ 382

Governors

168. The Action of Governors...................... 386

169. The Conical Pendulum. . . . . . . . . . . . . . . . . . . 387

170. The Loaded Governor. . . . . . . . . . . . . . . . . . 388

171. The Porter Governor.. . . . . . . . . . . . . . . . . . . . 389

172. The Centrifugal Shaft Governor................... 391

173. The Inertia Shaft Governor. . . . . . . . . . . . . . . . . 392

174. Comparison of the Two Types of Governors........ . . . . . 393

175. Analysis of Forces in the Rite's Inertia Governor . . . . . . . . . . . 393

\section{Chapter X.-Work and Energy}

176. Introduction . . . . . . . . . . 405

\section{§ 1. Work and Power}

177. Work Defined. . . . . . . . . . . . . . . . . . . . . . . . . . . 406

178. Algebraic Expressions for Work Done by a Force............. 406

179. Work Done by a Couple......................... 408

180. Work a Scalar Quantity. Sign and Units of Work........... 409

181. Graphical Representation and Calculation of Work........... 409 
182. Work Done on a Body by a Force System............ 414

183. Power Defined............................. 417

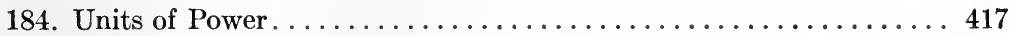

185. Special Equations for Power................... 418

\section{§ 2. Energy}

186. Energy Defined........................... 420

187. Potential Energy . . . . . . . . . . . . . . . . . . . . . . . . . . 421

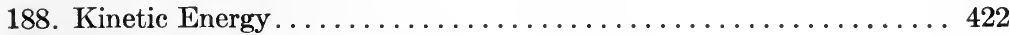

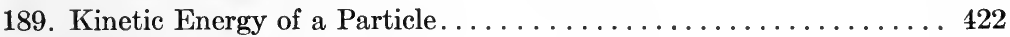

190. Kinetic Energy of a Body... . . . . . . . . . . . . . . . . 424

191. Non-mechanical Energy . . . . . . . . . . . . . . . . 428

\section{$\S 3$. Principles of Work and Energy}

192. Preliminary............................... 430

193. Principle of Work and Kinetic Energy... . . . . . . . . . 430

194. Application of Principle to Special Cases of Motion of Rigid Bodies. 432

195. Conservation of Energy........................ 439

196. Relation between Potential and Kinetic Energy for Conservative Systems. . . . . . . . . . . . . . . . . . . . 440

\section{§4. Efficiency. Dissipation of Energy}

197. Efficiency Defined. . . . . . . . . . . . . . . . . . . 442

198. Dissipation of Energy . . . . . . . . . . . . . . . . 443

199. Work Lost Due to Friction in Bearings................. 444

200. A Simple Dynamometer. Prony Brake................ 446

\section{Chapter XI.-Impulse and Momentum}

201. Preliminary.

\section{§1. Impulse}

202. Impulse and Impact Defined. Units. . . . . . . . . . . . . . . . 450

203. Components of Linear Impulse . . . . . . . . . . . . . . . . . . . . 451

204. Moment of Impulse. Angular Impulse . . . . . . . . . . . . . . . . 452

\section{§2. Momentum}

205. Momentum of a Particle Defined. Units.............. 453

206. Components of Momentum. Moment of Momentum......... 454

207. Linear Momentum of a Body. . . . . . . . . . . . . . . . 455

208. Angular Momentum of a Rotating Rigid Body ............. 456

209. Angular Momentum of a Rigid Body Having Plane Motion... . . . . 457

210. Preliminary ............................. 459

211. Principle of Linear Impulse and Linear Momentum. . . . . . . . . . 459

212. Principle of Angular Impulse and Angular Momentum......... 461 
213. Method of Analysis of the Motion of a Body by Means of Impulse

PAGE and Momentum. ........................... 462

214. Application of the Principles of Impulse and Momentum to Special

Types of Motion of Rigid Bodies................ 463

21.5. Conservation of Momentum.................... 467

216. Impact. . . . . . . . . . . . . . . . . . 471

217. Impact of Two Translating Bodies.................. 473

218. Impact of a Translating Body with a Rotating Body.......... 475

219. Loss of Kinetic Energy in Direct Central Impact. . . . . . . . . 476

220. Angular Momentum a Vector Quantity............... 477

221. The Gyroscopic Couple........................ 478 


\title{
ANALYTICAL MECHANICS FOR ENGINEERS
}

\author{
PART I. STATICS
}

\section{CHAPTER I}

\section{FUNDAMENTAL CONCEPTIONS AND DEFINITIONS}

1. Introduction.-The term Mechanics is used in a broad sense to denote the science which treats of the motion of bodies, rest being considered as a special case of motion. The science of mechanics constitutes a large part of our knowledge of the laws of the Universe, including the laws concerning gases and liquids as well as those of rigid bodies, and it takes a prominent place in the study of Astronomy and Physics as well as in the study of machines and structures which are involved in engineering practice.

The object of Analytical Mechanics as developed in this book is to determine the laws by which the motions (including the state of rest) of bodies (mainly rigid bodies) are governed and to apply these laws to conditions met in engineering practice.

In the development of the laws of mechanics certain concepts are assumed to be fundamental, that is, no one of them can be expressed in terms of the others or in simpler terms. Such concepts grow out of our experiences, and other ideas and laws are derived from these condensed experiences.

The fundamental concepts involved in the laws of mechanics of rigid bodies are: (1) force, which is made known to us through the tension and the compression of our muscles as a pull or a push, (2) bodies or inert material (matter) on which forces act and without which forces cannot exist, (3) space, and (4) time. A more definite understanding of force and inert bodies can be obtained after the laws of kinetics have been developed. To start with, 
however, it is necessary only to recognize the existence of these quantities.

In the process of the development of the laws of mechanics, considerable use is made of mathematics. It should be kept in mind, however, that mechanics is a physical science and that mathematics is used, mainly, as a tool to express and interpret physical laws.

For convenience, the study of mechanics is considered under three main divisions; namely, Statics, Kinematics, and Kinetics. Statics is that branch of mechanics which treats of bodies that are acted on by balanced forces and hence are at rest or are moving with uniform motion (without change of motion). Kinematics is that branch of mechanics which treats of the motion of bodies without considering the manner in which the influencing factors (force and matter) affect the motion. It deals with the fundamental concepts of space and time, and the quantities, velocity and acceleration, derived therefrom. It is, therefore, sometimes ealled the geometry of motion. Kinematics forms an important part of the study of mechanics, not only because it treats of a part of the general kinetics problem, but also because in many problems which involve mainly the relative motion of parts of a machine, the principles of kinematics, alone, are sufficient for the solution of the main problem. Such problems are discussed in treatises on Kinematics of Machinery, in which subject the motion of such machine elements as valve gears, quick-return mechanisms, etc., are considered. Kinetics is that branch of mechanics which treats of bodies which are acted on by unbalanced forces and, hence, have non-uniform or accelerated motions. In particular, it treats of the change of motion of bodies and the manner in which the change is related to the factors that affect it; namely, the actions of other bodies (forces), and the properties (inertia, etc.) of the bodies themselves. It will be noted that the study of the motion of a body that moves uniformly may be regarded either as a problem in statics or as a special case of a kinetics problem.

Since both statics and kinetics deal with the action of forces on bodies, that part of mechanics embraced in these two subdivisions of the subject is sometimes called Dynamics. Frequently, however, the term dynamics is used in technical literature to denote those subdivisions of mechanics with which the idea of motion is most closely associated, namely, kinematics and kinetics. 
For simplicity the kinematics and kinetics of a particle (material point) will be treated before extending the study to bodies. In many problems the body considered may be assumed, without serious error, to be a particle. This assumption may always be made when the dimensions of the body are negligible in comparison with the range of its motion. A body, however, may always be considered to be made up of particles.

2. Rigid Body.-As was stated in Art. 1, the bodies dealt with in this book are, in the main, considered to be rigid. A rigid body is defined as a definite portion of matter the parts (particles) of which do not move relative to each other. Actual solid bodies are never rigid. The relative motion (deformation) of their particles forms an important part of the study of Strength of Materials. But the theoretical laws which govern the motion of ideal rigid bodies may be used, usually with very small error or, with modifications if necessary, to determine the motion of actual solid bodies.

3. Conception of a Force.-It was stated in Art. 1 that force is one of the fundamental concepts on which the subject of mechanics of rigid bodies is built. A force is the action of one body on another body. The idea of force, then, implies the mutual actions of two bodies, since one body cannot exert a force on another body unless the other offers a resistance to the one. A force,therefore, never exists alone. Forces always occur in pairs; one force acting on one of two bodies and the other force on the other body. In fact, to every action there is an equal and opposite reaction. Our conception of force comes mainly from our experiences in which we have been one of the bodies between which mutual actions have occurred. The resistance which is offered by bodies to the action of other bodies arises out of (1) their ability to resist change of shape (rigidity) and (2) their ability to resist change of motion (inertia). If a body is acted on by one force, only, a change of motion of the body will always take place, but if the body is acted on by two or more forces, it may be held at rest.

Although a single force never exists, it is convenient in the study of the motion of a body to think of a single force and to consider only the actions of other bodies on the body in question without taking into account the reactions of the body in question on the other bodies. However. the fundamental nature of force should he kent in mind. 
4. External Effects of a Force. $\measuredangle$ When a force is applied to a rigid body the external effect on the body is either to change the motion of the body acted on or to develop resisting forces (reactions) between the body acted on and other bodies. Both of the foregoing effects, of course, may be produced simultaneously. For example, consider a body falling freely under the action of gravity. The sole external effect of the force acting on the body (its weight) is to produce an acceleration $g$ (32.2 ft. per sec. ${ }^{2}$ approximately). If the same body is placed on the floor of an elevator which is at rest, the sole external effect of the weight is to produce an upward reaction of the floor on the body. If now the elevator moves downward with an acceleration less than $g$, the effect of the weight is partly to cause an acceleration of the body (the same as that of the elevator) and partly to produce an upward pressure (reaction) of the floor on the body.

The internal effects of a force are to produce stress and deformation in the body on which the force acts. The internal effects of a force are discussed in books on Strength of Materials.

5. Characteristics of a Force.-From experience we learn that the external effects of a force depend on, (1) the magnitude of the force, (2) the position of the line of action of the force in the body, and (3) the sense of the force, that is, the direction along the line of action. $\rightarrow$ These three properties of a force are called its elements or characteristics. A change in any one of them causes a change in the external effect of the force. A discussion of the exact manner in which these characteristics influence the change of motion of a body forms an important part of the study of kinetics, and their influence on the reactions developed in holding a body at rest is considered in the study of statics.

6. Measure of a Force. Units.-Although we are conscious of forces of varying magnitudes, we are not able to compare the magnitudes with precision by means of our muscular sense. In order to express the magnitude of a force some standard force must be selected as a unit in terms of which other forces may be expressed. The unit of force commonly used by the engineer is the earth-pull (weight) on an arbitrarily chosen body, as found at a specified or standard position on the earth's surface. Examples of such units are the pound, ton, kilogram, etc. The earthpull on any body (its weight) varies slightly with its position (altitude and latitude) on the earth. For most engineering cal- 
culations, however, the variation in the weight of a body may be neglected. ${ }^{1}$

The unit of force as here defined is called a gravitational unit of force. (For a discussion of other units of force see Arts. 141 and 142.)

There are two common methods used by the engineer for measuring a force, that is, for finding the number of units in a force: (1) by use of the spring balance in its various forms such as steam gages, certain forms of dynamometers, testing machines, etc.; (2) by use of a beam or lever balance or system of levers such as platform scales, screw type of testing machines, etc.

(1) Due to the fact that many materials possess nearly perfect elasticity, within limits, the unit of force may be considered as the force required to produce a certain stretch or deflection of an elastic body, the specified stretch or deflection being that caused by the earth-pull on an arbitrarily chosen body at a standard locality. A properly graduated spring balance, therefore, furnishes one means of measuring any force in terms of the arbitrarily chosen unit of force.

(2) In the beam or lever balance, the force to be measured is applied at one end of a lever or system of levers and an arbitrarily selected body is placed at such a position on the other end of the lever that the earth-pull on the body balances the force. The arbitrarily selected body is a body on which the earth-pull at a standard location is the unit of force or some multiple thereof. The lever is so graduated that the number of units in the unknown force may be read off directly from the lever or beam.

Attention should here be called to the fact that it is impossible, fundamentally, to measure the earth-pull (weight) of a body by means of a beam or lever balance as stated under (2) above. The weight of the body, as already noted, varies with the position of the body on the earth's surface and this variation is not indicated by the beam balance. Hence if this variation. were large the beam balance could not be used for measuring a force. Its use, then, is permissible only because the quantity measured (not the earth-pull) is nearly equal (numerically) to the earth-pull or weight.

${ }^{1}$ The earth-pull on a body varies directly with $g$, the acceleration due to the earth-pull. The extreme variation in the value of $g$ corresponding to a change in the position of the body on the earth's surface from a high altitude at the equator to the pole is 0.6 per cent. Within the United States the maximum variation is about 0.3 per cent. 
Units of space (length), such as the foot, yard, mile, meter, etc., and units of time, such as the second, minute, hour, etc., are assumed to be familiar to the student.

7. Scalar and Vector Quantities. Vector Representation of a Force.-Quantities which possess magnitude only, as, for example, areas, volumes, etc., are called scalar quantities. Many quantities involved in the study of mechanics, however, have direction as well as magnitude. Any quantity which has direction as an inherent property as well as magnitude is called a vector quantity. Thus, as stated in the preceding article, the effect of a force depends on its direction as well as its magnitude, and hence force is a vector quantity. Other examples of vector quantities are velocity, acceleration, momentum, etc.

A vector quantity may be conveniently represented wholly or in part by means of a directed straight line. Any such line is called a vector. Thus, the direction of a force may be represented by a straight line drawn parallel to the action line of the force, the sense being represented by an arrow-head on the line, and the magnitude of the force may be represented by the length of the line according to some convenient scale. If the magnitude and direction, only, are to be represented, the vector may be drawn anywhere in the plane of the force. Such a vector is called a free vector. If, in addition, the action line of the force is to be represented, the vector must be laid off along the line of action. Such a vector is called a localized vector. Further, if it is desired to represent the point of application of the force, the point of application may be taken as the initial end of the vector, that is, the point from which the vector is drawn. It will be noted, however, that the point of application of a force which acts on a rigid body is not one of the essential characteristics of the force (Art. 5).

In dealing with the forces which act on a given body it is convenient frequently to represent the forces by free vectors. The diagram in which are represented the free vectors (that is, the vectors representing the magnitudes and directions of the forces) is called the vector diagram. $\rightarrow$ The diagram which represents the body and the action lines of the forces that act on the body is called the space diagram. Both diagrams as a rule play an important part in the complete solution of a problem and will be used frequently in the subsequent pages. $>$ In Fig. 1 is shown a wall bracket, the horizontal arm of which is acted on by three forces having points of 
applications at 1,2 , and 3 , the action lines being indicated by $\bar{u} b$, $b c$, and $c d$, as shown in the space diagram. $>$ The forces are represented in magnitude and direction in any convenient place by the vectors $A B, B C$, and $C D$, the lengths of the vectors representing the magnitudes of the forces according to a convenient scale. The direction of each vector is parallel to the action line of the force which it represents.

The notation used in the above illustration is known as Bow's notation and will be used frequently in the subsequent pages. According to this notation the action line of a force is denoted by two lower-case letters and the vector which represents the magnitude and direction of the force is denoted by the corresponding capital letters.

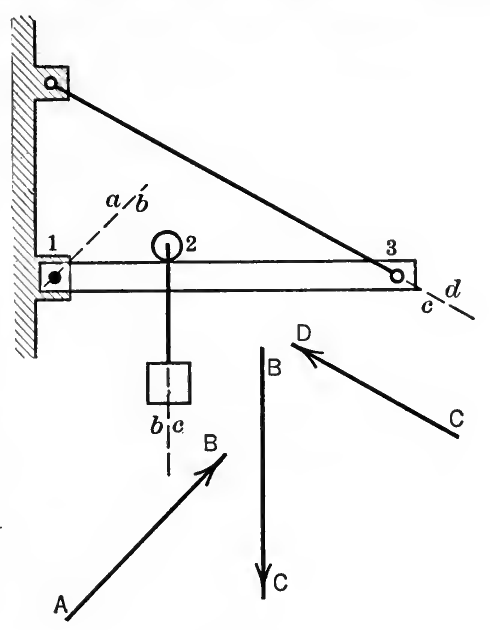

FIG. 1.

8. Classification of Forces. Definitions.-Forces may be classified as surface forces and body forces, sometimes called forces of contact and forces at a distance according as the action of one body on another is exerted over a portion of the surfaces of two bodies that are in contact or is distributed throughout the materials of the bodies. (The most important body force considered in mechanics is the earth-pull (weight). Magnetic forces are of the same class. A surface force becomes a concentrated force when the area over which the force is distributed is so small compared with the surface of the body acted on that it may be regarded as a point. This point is called the point of application of the force. The action line of a concentrated force is a line containing the point of application of the force and having the same direction as that of the force.

Any number of forces treated as a group constitute a force system. A force system is said to be concurrent if the action lines of all the forces intersect in a common point and non-concurrent if the action lines do not intersect at a point. A force system is said to be coplanar when all the forces lie in the same plane and non- 
coplanar when the forces do not lie in a common plane. A parallel force system is one in which the action lines of the forces are parallel, the senses of the forces not necessarily being the same, and a non-parallel system is one in which the action lines of the forces are not parallel. If the forces of a system have a common line of action the system is said to be collinear.

Two force systems are said to balance if, when applied simultaneously to a body, they produce no external effect on the body. The forces which hold a body at rest always balance. Forces or force systems which balance are said to be in equilibrium and the body or bodies on which they act are also said to be in equilibrium. If a body is acted on by a force system which is not in equilibrium there always is a change in the motion of the body. Such a force system is said to be unbalanced or to have a resultant. The resultant of a force system is the simplest equivalent system to which the given system will reduce, that is, the simplest system which will produce the same change of motion. The resultant of a force system is frequently a single force. For some force systems, however, the simplest equivalent system is composed of two equal, non-collinear, parallel forces of opposite sense, called a couple. And still other force systems reduce to a force and a couple as the simplest equivalent system. The process of reducing a force system to a simpler equivalent system is called composition. The process of expanding a force or a force system into a less simple equivalent system is called resolution. A component of a force is one of the two or more forces into which the given force may be resolved. The anti-resultant or equilibrant of a force system is the simplest force system which will balance the given system.

9. Principle of Transmissibility.-It was stated in Art. 7 that the external effect of a force on a rigid body does not depend on the point of application of the force. This very useful fact, the truth of which is found in our experience, is formally expressed in the principle of transmissibility. This principle states that the external effect of a force on a rigid body is the same for all points of application along its line of action. It will be noted that the external effect, only, remains unchanged. The internal effect of a force (stress and deformation) may be greatly influenced by a change in the point of application along the line of action.

10. Parallelogram Law.-The parallelogram law is the fundamental principle on which the composition and the resolution of 
forces is based. The law states that the resultant of two forees which act on a rigid body is represented in magnitude and in direction by the diagonal of a parallelogram, the sides of which represent the magnitudes and the directions of the two forces. In Fig. 2(a) $P$ and $Q$ are two of the forces acting on a rigid body (bell-crank), $Q$ being a pressure of $150 \mathrm{lb}$. at $B$ produced by a force, $P$, of $100 \mathrm{lb}$. at $A$. If $Q$ is laid off from a point $O$ (Fig. 2b) to some convenient scale in the proper direction and if, in like manner, $P$ is laid off from the same point then $R^{1}$ represents in magnitude and in direction the resultant of $P$ and $Q$. If the point $O$ is taken as the intersection of the action lines of $P$ and $Q$ as in Fig. $2 a$ then the diagonal represents the action line of the resultant as well as its magnitude and direction.

The parallelogram law is not susceptible of rigid proof. It is an assumption which expresses a relation between forces (and other directed quantities such
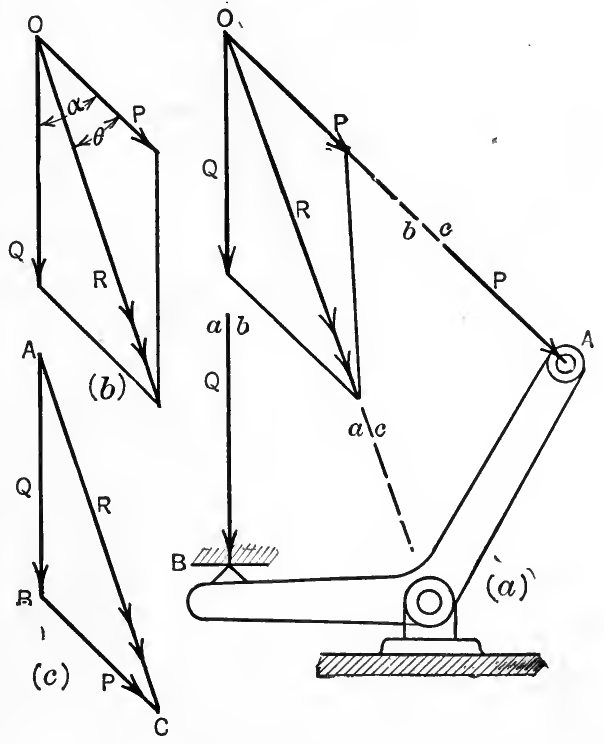

FIG. 2. as velocities, accelerations, etc.); an assumption, however, which appeals to experience as reasonable; which is used intuitively in interpreting and analyzing many of our common experiences; and which thereby becomes a fundamental or basic fact in mechanics.

11. Triangle Law.-The triangle law is a corollary of the parallelogram law. It states that the resultant of two concurrent forces is represented in magnitude and in direction by the third

${ }^{1}$ The fact that a force is the resultant of two or more forces is frequently indicated by two arrow-heads on the vector representing the force. This fact will also be indicated sometimes by drawing the force vector as a dashed or broken line. 
side of a triangie, the other two sides of which represent the two forces in magnitude and direction. Thus, in Fig. 2(c), the resultant $R$ of the two forces $P$ and $Q$ is represented in magnitude and in direction by the side $A C$ of the triangle $A B C$ in which $A B$ and $B C$ represent the magnitudes and the directions of $Q$ and $P$ respectively. The action line, $a c$, of the resultant is parallel to $A C$ and passes through the point of concurrence of the two forces. 'Although the triangle law is included in the parallelogram law, its extension to more than two forces, leading to the force polygon, makes its use more convenient than that of the parallelogram law.

Instead of determining the resultant of two forces graphically, from the parallelogram or the triangle of forces, it may be found algebraically. Thus, referring to Fig. 2(b), by use of trigonometry, the magnitude and the direction of the resultant may be expressed by the equations,

$$
\begin{aligned}
R & =\sqrt{P^{2}+Q^{2}+2 P Q \cos \alpha}, \\
\tan \theta & =\frac{Q \sin \alpha}{P+Q \cos \alpha},
\end{aligned}
$$

where $\alpha$ is the angle between the action lines of $Q$ and $P$, and $\theta$ is the angle between the action lines of $R$ and $P$.

Although it is not necessary to draw the parallelogram or triangle of forces to scale in determining the resultant of two concurrent forces by the algebraic method, the student should always make a free-hand sketch of the parallelogram or triangle of forces when using the above equations.

The expression for the resultant of two forces at right angles to each other $\left(\alpha=90^{\circ}\right)$ is of great importance. The resultant is completely determined by the above equations, which reduce for this special case to the following equations:

$$
\begin{aligned}
R & =\sqrt{P^{2}+Q^{2}}, \\
\tan \theta & =\frac{Q}{P} .
\end{aligned}
$$

It should be noted that two equations are needed to determine the magnitude and the direction of a resultant force, whereas one vector diagram is sufficient for the same purpose. 


\section{PROBLEMS}

1. Two forces having magnitudes of $7 \mathrm{lb}$. and $3 \mathrm{lb}$. have the same point of application on a body. The action line of the 3-lb. force is horizontal with its sense to the right, while that of the 7-lb. force makes an angle of $45^{\circ}$ with the horizontal with its sense upward to the left. Find the magnitude and the direction of the resultant. Solve graphically and check the result by the algebraic method.

Ans. Magnitude $=5.3 \mathrm{lb}$.

Direction, $111^{\circ} 28^{\prime}$ with the $3-1 \mathrm{lb}$. force.

2. A vertical force of $200 \mathrm{lb}$. which is applied at point $A$ of the bell-crank shown in Fig. 3 causes a horizontal pressure of $300 \mathrm{lb}$. on the vertical arm at point $B$. (a) Find the magnitudè and the direction of the resultant of the forces at $A$ and $B$ by the algebraic method. (b) Find the resultant completely by the graphical method.

12. Resolution of a Force. -In the two preceding articles it was assumed that a certain body was acted on by two other bodies, and the action of a third body was found which if allowed to replace

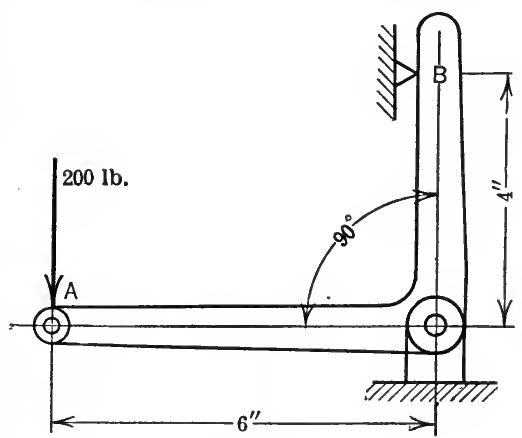

FIG. 3. the two would have the same external effect on the body in question. The reverse of this process, namely, the resolution of a force, is also of great importance in mechanics. The action of one body may be replaced by that of two bodies. The resolution of a force is acco nplished by means of the parallelogram and triangle laws and the components (resolved parts) may be found graphically or algebraically. For example, in Fig. $4(a), F_{1}$ represents the steam pressure on the crosshead, $D$, of a locomotive and $F_{2}$ represents the pressure on the crank-pin, $H$, of the driver. Let it be required to resolve $F_{1}$ into two components, one along the connecting rod $D H$, and the other parallel to the crank $O H$. The action lines, $a b$ and $b c$, of the two components must pass through $D$ as shown in the space diagram. The magnitudes and the directions of the components are represented graphically by $A B$ and $B C$ in the triangle of forces (Fig. 4b). This triangle was constructed by 
laying off $A C$ or $F_{1}$ parallel to $a c$ and by drawing from $A$ a line parallel to $a b$ and from $C$ another line parallel to $b c$, the two lines intersecting at $B$. Thus, the components of $F_{1}$ are represented in magnitude and in direction, but not in action line, by $A B$ and $B C$.

The resolution of a force into two rectangular components is of special importance. The particular value of resolving into rectangular components lies in the fact that these components may be found from very simple algebraic expressions. Thus, let the crankpin pressure $F_{2}$ (Fig. $4 a$ ) be resolved into two rectangular com-

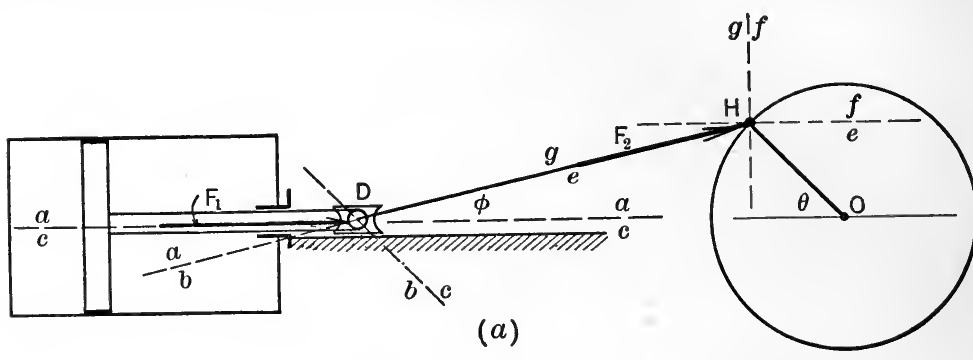

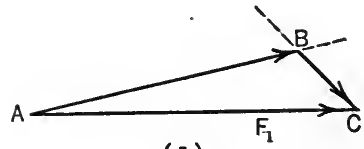

(b)

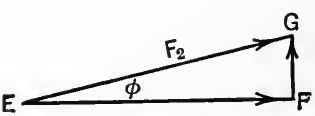

(c)

Fig. 4.

ponents (Fig. 4c). The action lines of the components must, of course, pass through the crank-pin, $H$. Thus, ef is the action line of the $x$-component and $f g$ is the action line of the $y$-component. From the triangle of forces the two components $E F$ and $F G$ may be found. Thus, if $\phi$ is the angle between the force and its $x$ component, then,

$$
E F=E G \cos \phi \quad \text { and } F G=E G \sin \phi .
$$

Or, in general, if $F$ denotes a force which makes an angle $\theta$ with the $x$-axis, the $x$ - and $y$-components of the force are,

$$
F_{x}=F \cos \theta \text { and } \quad F_{y}=F \sin \theta
$$

Hence, the component of a force in a given direction (the other component. being perpendicular thereto) equals the product of the 
magnitude of the force by the cosine of the acute angle which the force makes wiih the given direction.

A force can be resolved into two components in an indefinite number of ways by drawing lines from any point or pole to the ends of the force vector. Thus, in Fig. 5 three sets of components are shown for the force $A B$, the components being $A O$ and $O B$ for each position of the pole $O$.

It is frequently convenient to resolve a force into three rectangular components. This involves only a slight extension of the parallelogram law. Thus, the force $F$ (Fig. 6), represented by $O A$,

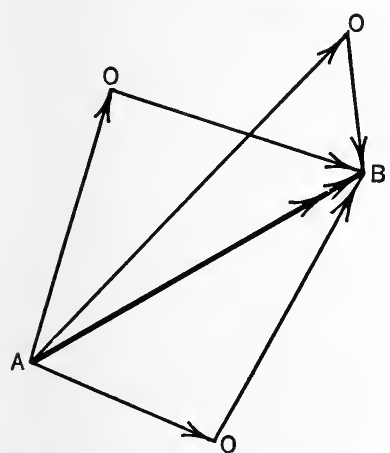

Fig. 5.

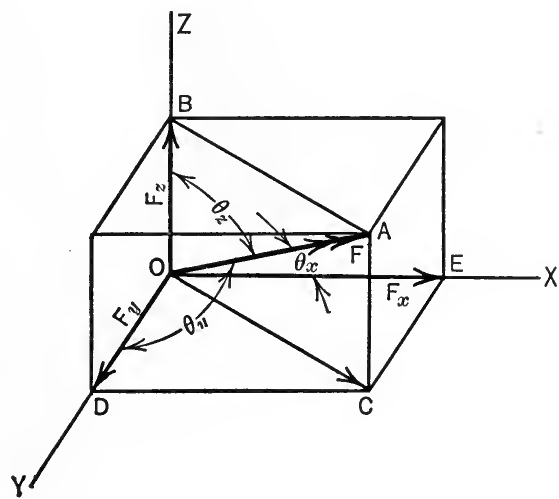

Fig. 6.

may be resolved into the two rectangular components $O B$ and $O C$, and the component $O C$ may be resolved further into two rectangular components $O D$ and $O E$. The magnitudes of the components of $F$ in the $x$-, $y$-, and $z$-directions, respectively, are

$$
F_{x}=F \cos \theta_{x} \quad F_{\nu}=F \cos \theta_{\nu} \quad F_{z}=F \cos \theta_{z},
$$

in which $\theta_{x}, \theta_{y}$, and $\theta_{z}$, are the angles which the force makes with the $x-, y$-, and $z$-directions respectively.

\section{PROBLEMS}

3. Given the three concurrent forces as shown in Fig. 7. Find the magnitude and the sense of the component of the system (sum of the components 
of the forces) along the line $A B$. Solve algebraically and check the result by the graphical method.

Ans. Magnitude $=24.2 \mathrm{lb}$.

Sense, Downward to the left.

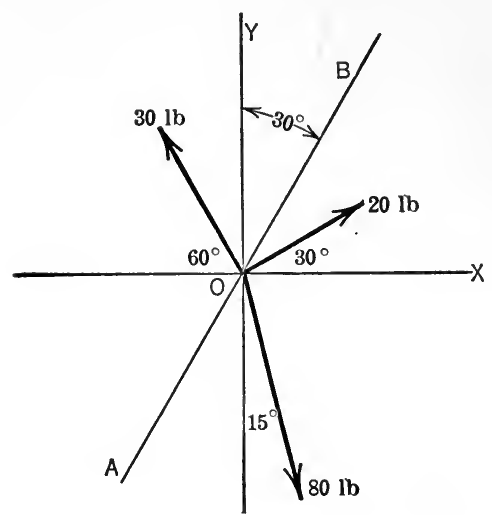

FIG. 7.

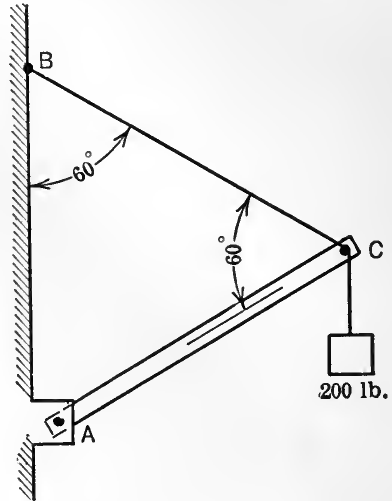

Fig. 8.

4. Resolve the weight of $200 \mathrm{lb}$. (Fig. 8) into (a) two rectangular components perpendicular and parallel respectively to $A C,(b)$ into two components parallel to $A C$ and $B C$ respectively.

5. Resolve the force $F$ (Fig. 9) into three rectangular components in the $x-, y$-, and $z$-directions.

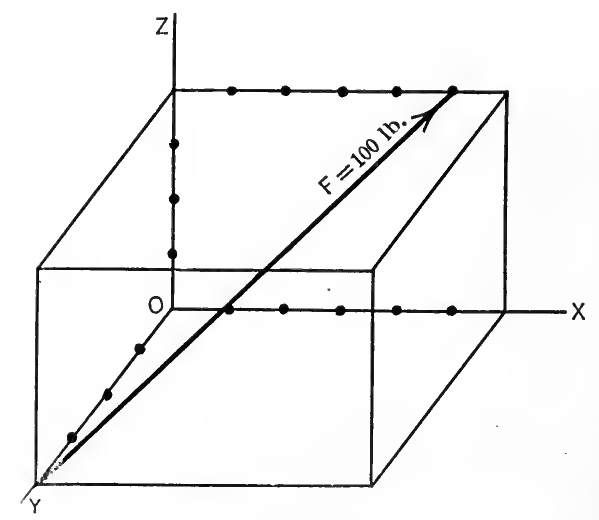

Fig. 9.

13. Moment of a Force.-The moment of a force about (with respect to) a point is the product of the magnitude of the force and the perpendicular distance from the point to the action line of the force. Thus, the moment of the force $F$ (Fig. 10) about the point $O$ is $F d$. The point about which the moment is taken is called $>$ the moment-center (or origin) and the distance $d$ is called the moment-arm. A unit moment is the product of a unit force and a unit distance such 
as a lb.-ft., lb.-in., ton-ft., etc. $>$ The moment of a force with respect to a point may also be regarded as the moment of the - force with respect to the line which passes through the point and is perpendicular to the plane determined by the point and

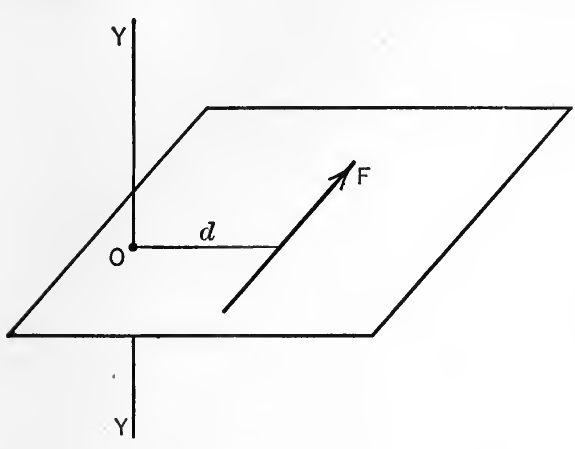

Fig. 10. the action line of the force. Thus, in Fig. 10, $F d$ also represents the moment of $F$ about the axis $Y Y$.

The physical significance of the moment of a force as defined above is built up intuitively from our experiences in which we have exerted moments on other bodies. It expresses a measure of the tendency of the force to turn the body on which it acts about a given axis.> Strictly speaking, the fact that the tendency of a force to turn a body about a given axis is directly proportional to the moment of the force, is an assumption which, when reinforced by experience and the analysis of observed facts, crystallizes into a fundamental or basic truth of mechanics.

7 In general, the moment of a force about an axis which is not perpendicular to the plane of the force is defined as the moment of the component of the force perpendicular to the given axis, the other

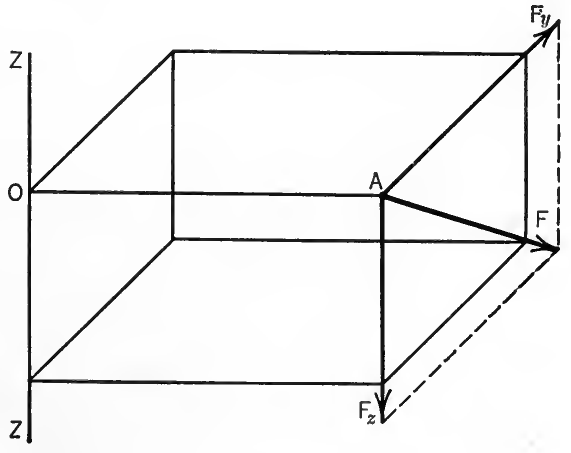

FIG. 11. component of the force being parallel to the axis. Thus, in Fig. 11 the moment of the force $F$ about the axis $Z Z$ is $F_{y} \cdot \overline{O A}$.

As a rule it will be convenient to select the moment axis as one of the coordinate axes. The moment of a force with respect 
to a coordinate axis will be considered as positive if the direction of rotation is counter-clockwise when viewed from the positive end of the axis.

\section{PROBLEMS}

6. A force of $20 \mathrm{lb}$. is exerted on the knob of a door as shown in Fig. 12. If the action line of the force lies in a plane perpendicular to the door, what is the moment of the force about the axis $Y Y$ ?

Ans. $42.4 \mathrm{lb} . \mathrm{ft}$.

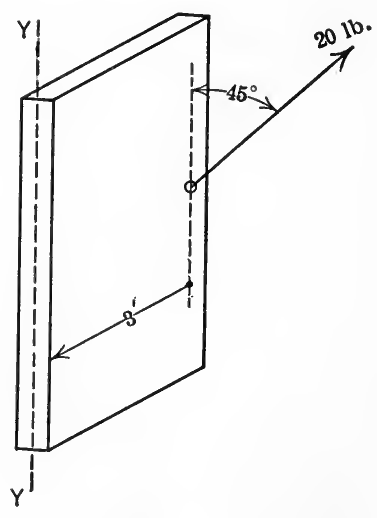

FIg. 12.

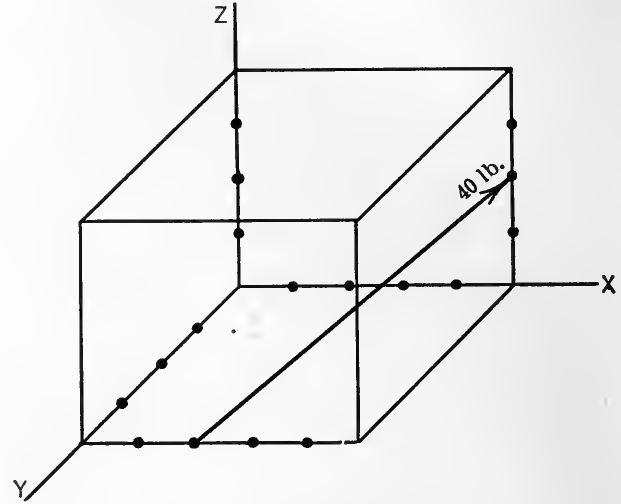

FIg. 13.

7. Find the moment of the 40-lb. force (Fig. 13) with respect to each of the coordinate axes; each division represents 1 in.

14. Principle of Moments. Varignon's Theorem.-The principle of moments is of great importance in mechanics. It applies to lines, areas, volumes, etc., as well as to forces. It will be considered, however, at this point only in connection with two concurrent forces. ${ }^{-}$The principle for this restricted case, which is known as Varignon's theorem, states that the algebraic sum of the moments of two concurrent forces about any point in their plane is equal to the moment of their resultant about the same point.

The principle of moments, like the parallelogram law, is an assumption; an assumption, however, which appeals to experience as reasonable; which is frequently and intuitively used in interpreting and analyzing observed facts, under various conditions; $>$ and which thereby becomes one of the fundamental facts or principles of mechanics. 
The fact that the principle of moments for forces is in agreement with the parallelogram law may be shown by deducing the principle from the parallelogram law as follows: In Fig. 14 let $P$ and $Q$ represent two forces concurrent at $A$, the resultant according to the parallelogram law being $R$. Let $O$ be any moment-center in the plane of the forces. It is required to prove that

$$
P p+Q q=R r,
$$

where $p, q$, and $r$ are the moment-arms of $P$, $Q$, and $R$, respectively. Let a set of rectangular

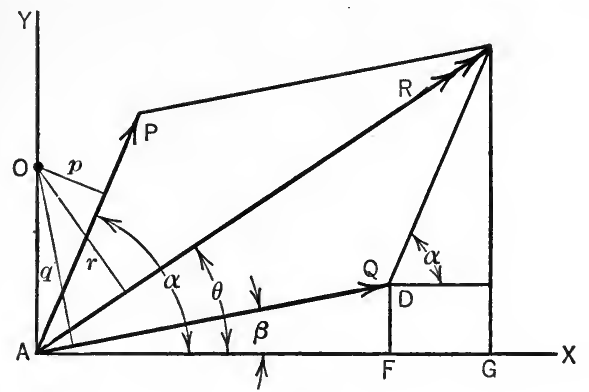

FIG. 14. coordinate axes $A X$ and $A Y$ be chosen as shown in the figure, $A Y$ passing through the moment-center $O$. Let $\alpha, \beta$, and $\theta$ denote the angles which the action lines of $P, Q$, and $R$, respectively, make with the $A X$ axis. From the figure it is seen that

that is,

$$
\overline{F G}+\overline{A F}=\overline{A G},
$$

$P \cos \alpha+Q \cos \beta=R \cos \theta$.

By multiplying both sides of this equation by $A O$, the following equation is obtained:

Hence,

$$
P \cdot \overline{A O} \cos \alpha+Q \cdot \overline{A O} \cos \beta=R \cdot \overline{A O} \cos \theta .
$$

$$
P p+Q q=R r \text {. }
$$

It will be noted that the definition of the moment of a force about a line as given in Art. 13 is in accordance with the principle of moments. It is often convenient to obtain the moment of a force about a point in its plane (or about an axis through the point perpendicular to the plane) by finding the sum of the moments of its rectangular components. In accordance with the principle of transmissibility a force may be resolved into components at any point along its line of action. Thus, in Fig. 15, $F_{x}$ and $F_{y}$ are one 
pair of rectangular components and ${F^{\prime}}_{x}$ and $F^{\prime}{ }_{y}$ are another pair of components of the force $F$. The moment of the force $F$ about the axis $Z Z$ is, by the principle of moments, equal to the sum of

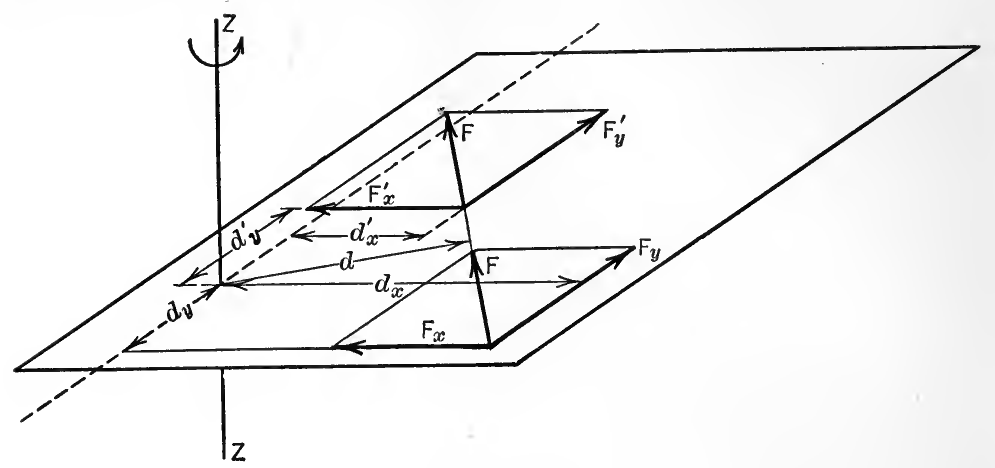

FIG. 15.

the moments of the forces of either pair of components. Hence, the moment of $F$ may be expressed as follows:

$$
F d=F_{y} \cdot d_{x}-F_{x} \cdot d_{y}=F^{\prime}{ }_{x} \cdot d^{\prime}{ }_{y}+F^{\prime}{ }_{y} \cdot d^{\prime}{ }_{x} .
$$

If the force $F$ is not in a plane perpendicular to the axis, as in

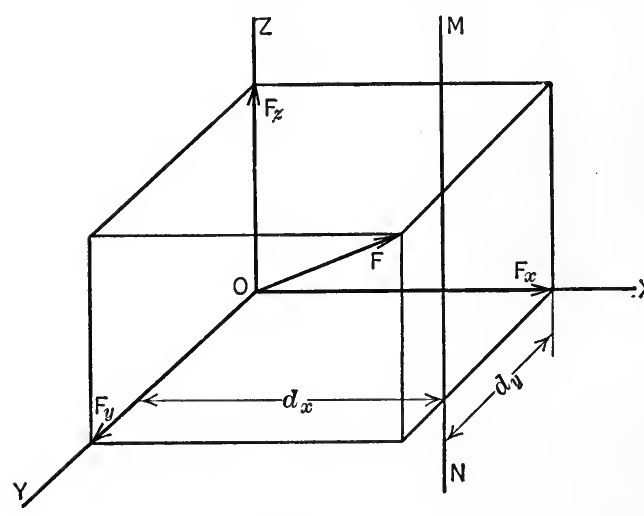

FIG. 16.

Fig. 16, the force may be resolved into three rectangular components, one being parallel to the axis and the other two in a plane which is perpendicular to the axis. The moment of the force is then the sum of the moments of the components which lie in this plane. Thus, in Fig. 16, the mo-

ment of $F$ about the axis $M N$ is,

$$
-F_{x} \cdot d_{y}+F_{y} \cdot d_{x}
$$

The component $F_{z}$, being parallel to the axis $M N$, has no moment about the line $M N$. 
15. Couples.-Two equal parallel forces which are opposite in sense and are not collinear are called a couple. A couple cannot be reduced to any simpler force system. The fact that the only effect of a couple is to produce or to prevent turning is obtained intuitively. The moment of a couple about any point in the plane of the couple (or any axis perpendicular to the plane of the couple) is defined as the algebraic sum of the moments of the forces of the couple about the point (or axis). From this definition it follows that the moment of a couple about any point in the plane of the couple (or axis perpendicular to its plane) is the product of the magnitude of either force of

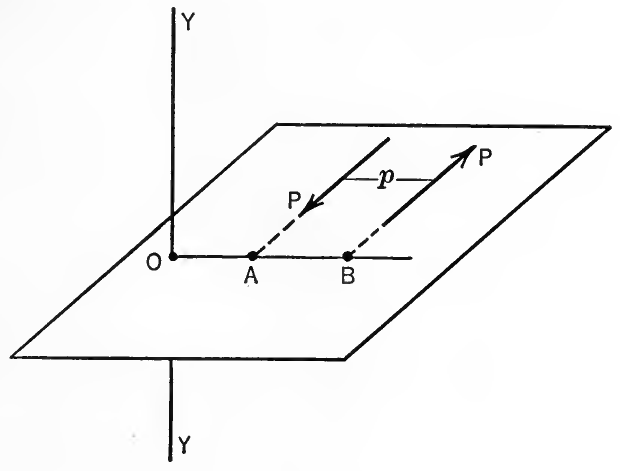

Fig. 17. the couple and the perpendicular distance (moment-arm) between the action lines of the two forces. This statement may be proved as follows: In Fig: 17 let $P, P$ be the two forces of a couple and $O$ any point in their plane or, $Y Y$ any axis perpendicular to their plane. The algebraic sum of the moments of the two forces about $O$ (or $Y Y$ ) is

$$
P \cdot \overline{O B}-P \cdot \overline{O A}
$$

which may be written thus,

$$
P(\overline{O B}-\overline{O A})=P \cdot \overline{A B}=P p .
$$

In like manner the moment of the couple may be shown to be the same about any other point in the plane (or any other axis parallel to $Y Y$ ). Since the moment of a couple depends only on the product of either force of the couple and the arm of the couple it follows that the turning effect of a couple on a rigid body about an axis in the body is the same for different magnitudes and lines of action of the forces, provided that the moment of the couple remains constant. For example, in Fig. 18, if cords are wrapped 
around two pulleys, of radii $r_{1}$ and $r_{2}$, which are keyed together and equal weights are attached to the other ends of the cords, the pulleys will rotate exactly the same as they would if forces $F, F$ were applied as shown by the dotted lines, provided that the moments of the two couples are the same, that is if $W\left(r_{2}-r_{1}\right)$ is equal to $F \cdot 2 r_{2}$. On the other

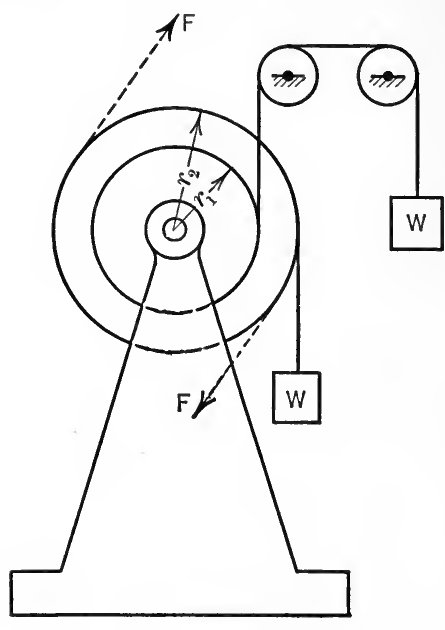

Fig. 18. hand if motion is to be prevented a couple having, a moment of $-\left(F \cdot 2 r_{2}\right)$ would be required.

16. Characteristics of a Couple.-The external effect of a couple when applied to a rigid body is either to cause a change in the rotational motion of the body or to develop a resisting couple due to the actions of other bodies on the body in question. Both of the foregoing effects may of course be produced simultaneously. From experience we learn that the external effect of a couple depends on (1) the magnitude of the moment of the couple, (2) the sense or direction of rotation of the couple, and (3) the aspect of the plane of the couple, that is, the direction or slope of the plane (not its location). These three properties of a couple are called its characteristics. Since parallel planes have the same aspect it follows from what has been stated above that two couples which have the same moment and sense are equivalent if their planes are parallel. The fact that the external effect of a couple is independent of the position of the plane of the couple and depends only on the direction of the plane is amply verified by experience.

7. Thus, in screwing a pipe into a joint by means of two pipe wrenches the forces applied at the end of the wrenches constitute a couple, and it is a matter of experience that the effort required is the same regardless of the position along the pipe at which the wrenches are applied (assuming perfect alignment between pipe and joint).

17. Vector Representation of a Couple.-In dealing with couples it is convenient to represent the couples by means of vectors. In order to represent a couple completely by a vector all of 
the characteristics of the couple must be represented. The moment of the couple may be represented to scale by the length of the vector. The aspect of the couple may be represented by drawing the vector perpendicular to the plane of the couple and hence parallel to the axis of the couple - that is, parallel to the axis about which the couple rotates or tends to rotate the body on which it acts. The sense of the couple may be represented by an arrow-head on the vector, the usual convention being to direct the arrowhead away from the plane of the couple in the direction from which the rotation appears counter-clockwise. This method of representing the sense of a couple involves the so-called convention of the right-handed screw, since a right-handed screw having an

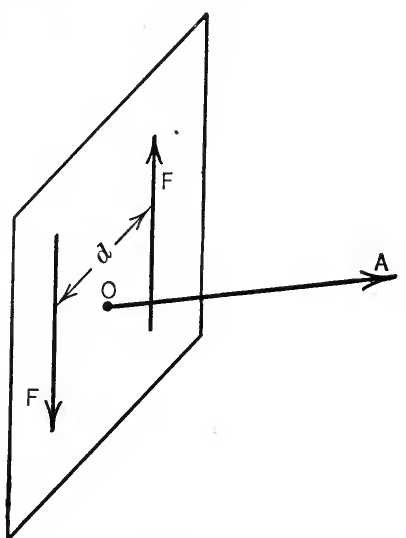

FIG. 19. axis perpendicular to the plane of the couple would move in the direction of the arrow if given a rotation which agrees in sense with that of the couple. Thus, in Fig. 19 the vector $O A$ completely represents the couple $F d$, provided that the length of $O A$ represents to scale the product $F \cdot d$.

\section{PROBLEMS}

8. The steering wheel of an automobile shown in Fig. 20 is 18 in. in diameter. Forces exerted by the hands on the wheel constitute a couple which is

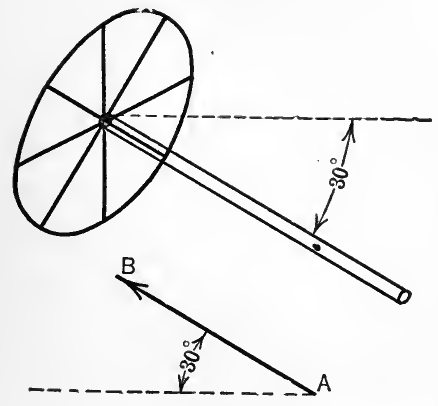

Fig. 20.

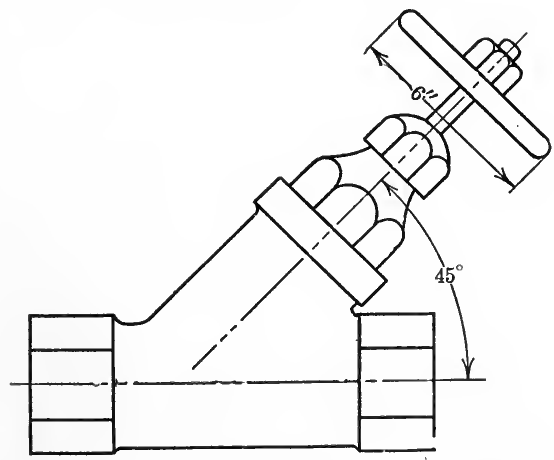

FIG. 21. 
represented by the vector $A B$. If the length of $A B$ is $1.5 \mathrm{in.}$ and the scale used is $1 \mathrm{in} .=60 \mathrm{lb}$.-in., represent the forces of the couple on the circumference of the wheel.

9. A couple having a moment of $60 \mathrm{lb}$.-in. is required to open the blow-off valve shown in Fig. 21. Represent the couple completely by a vector, using a scale of 1 in. $=24$ lb.-in.

18. Resolution of a Force into a Force and a Couple.-In many problems, both in staicics and kinetics, it is convenient to resolve a

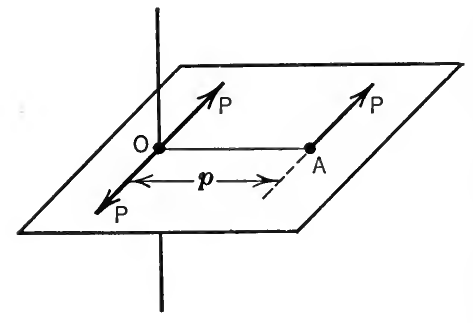

Fig. 22. force into a force parallel to the given force, and a couple in the plane of the force. Thus, in Fig. 22, let $P$ represent a force acting on a body at $A$. Let two equal, opposite, and collinear forces $P, P$ be introduced at any point $O$, each of the forces being parallel to the original force and of the same magnitude. The three forces are equivalent to the original one, since the two equal, opposite, and collinear forces have no effect on the body. The force system may now be considered to be a force $P$ acting at $O$ (parallel to the given force and of the same magnitude and sense), and a couple, the moment of which is the same as the moment of the original force about $O$.

The action lines of the forces of the couple, however, may be moved to any location in the plane of the forces and the magnitude of the forces of the couple may be changed to any value, provided that the forces remain equal and parallel to each other and also that the moment and sense of the couple are not changed. Couples will be discussed in greater detail in Art. 27.

Since a force may be resolved into a force and couple in the plane of the force it follows that a force and a couple in a plane may be combined into a resultant force in the same plane. Further, the resultant force has the same magnitude, direction, and sense as the given force and its action line is parallel to the action line of the given force. In other words, the sole effect of combining a couple with a force is to move the action line of the force into a parallel position, the force being unchanged in all other respects. 


\section{ILLUSTRATIVE PROBLEM}

10. In Fig. 23 is shown an arm mounted on an axle $O$. A vertical force $P=20 \mathrm{lb}$. acts on the arm at $C$. Resolve the force $P$ into a force acting through $O$ and a couple the forces of which act at $A$ and $B$.

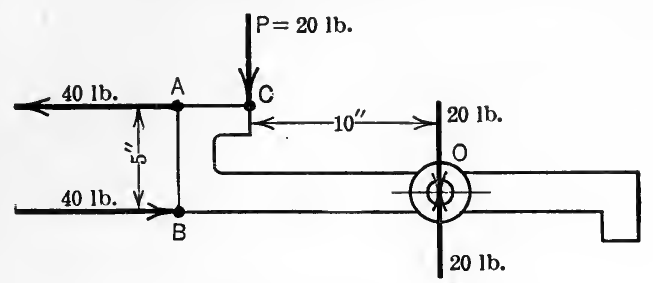

Fig. 23.

Solution.-By introducing two vertical $20-\mathrm{lb}$. forces at $O$ which are opposite in sense the force is resolved into a vertical downward force of $20 \mathrm{lb}$. at $O$ and a couple having a moment of $20 \times 10=200 \mathrm{lb}$.-in. This couple may be replaced by two horizontal forces, of $40 \mathrm{lb}$. each, acting at $A$ and $B$ as shown. The force at $O$ and the couple will produce exactly the same external effect on the arm as the original force at $C$.

\section{PROBLEMS}

11. A gusset plate $B$ (Fig. 24) is attached to another plate $A$ by means of four rivets as shown. A force of $10,000 \mathrm{lb}$. at the point $O(1.5 \mathrm{in}$. below dotted line) is transmitted to the member $A$. Resolve this force into a force, acting along the center line of the rivets, and a couple.

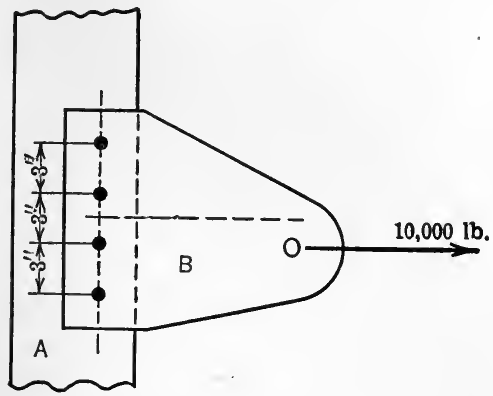

Fig. 24.

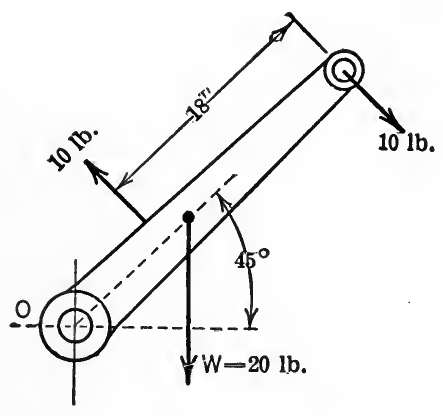

FIG. 25.

12. A body weighing $20 \mathrm{lb}$. is mounted on an axle $O$ (Fig. 25) and is acted on by a couple as shown in the figure, in addition to its weight and the axle reaction at $O$. Replace the weight and the couple by an equivalent single force. 
13. A load $P$ of $100 \mathrm{lb}$. acting on a wall bracket as shown in Fig. 26 causes the wall to exert a horizontal force of $150 \mathrm{lb}$. and a vertical force of $50 \mathrm{lb}$. at

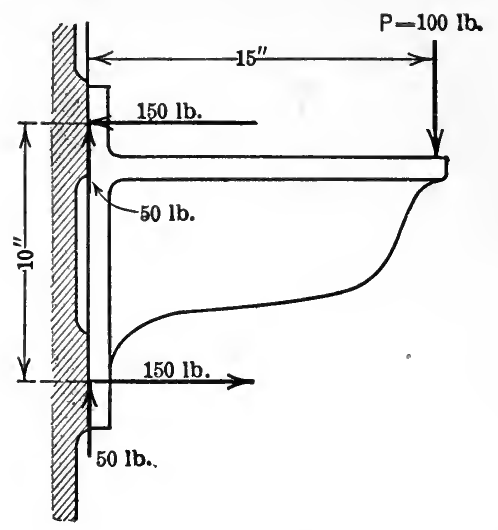

FIG. 26. each of the wall attachments. Consider the two 50-lb. forces to be equivalent to one $100-\mathrm{lb}$. force and replace this $100-\mathrm{lb}$ force and the two 150-lb. forces by an equivalent single force.

19. Methods of Solution of Problems. Algebraic and Graphical Methods.-In the analysis and solution of problems in mechanics two general methods may be used, namely, the algebraic and the graphical methods.

In the algebraic method of solution, quantities are represented by letters or by numbers which are dealt with according to the rules of mathematics. In the graphical method of solution, quantities are represented by lines which are dealt with by means of geometric constructions. Simple graphical methods have already been used in the preceding articles in connection with forces, couples, etc. In general, either of the two methods may be used in the solution of a problem. Some problems, however, may be solved much more easily by the algebraic method while other problems yield much more readily to the graphical method. The operations involved in the solution of a problem by the two methods are so radically different that one method of solution serves as an excellent check on the other method.

Approximate Methods.-In making computations it is important to keep in mind the degree of accuracy which should be obtained. The degree of accuracy desired will depend, in general, on two factors, namely:

(1) The degree of accuracy of the original data or quantities on which the computation is based, and

(2) The use which is to be made of the computed results.

The data on which many engineering computations are based are determined from experiments and hence are approximate values, the degree of approximation depending on the instruments 
and methods used, and on the care and skill of the observer. The computed results which are based on these values cannot have a greater degree of accuracy than that of the original data. In general a sum, difference, product, or quotient of two approximate values will not have a greater degree of accuracy than that of the least accurate of the two numbers. For example, if one numerical quantity is accurate to two significant figures and another quantity to three significant figures, the product of the two numbers will not be accurate to more than two significant figures, and hence more than two significant figures should not be retained in the result.

Dimensional Equations.--In algebraic equations in which the variables represent physical quantities, all of the terms of the equation must be of the same dimensions, or, to express the same idea in mathematical language, an algebraic equation which expresses a relation between physical quantities must be homogeneous. The use of this principle is of assistance in checking any equation for correctness and in determining the specific units in which a result is expressed when computed from a given equation. For each of these purposes the given algebraic equation is replaced by a dimensional equation.

The dimensional equation corresponding to any algebraic equation is formed by replacing each term of the given equation by a term which indicates the kinds of fundamental quantities in which the term is expressed and which also indicates the degree of the corresponding quantities in each term.

The fundamental quantities used in engineering are force, iength, and time $(F, L$, and $T)$. Hence, in an equation, a term which represents an area is replaced by $L^{2}$ in the dimensional equation since an area is the square of a length. A velocity is a length divided by time and hence is represented in the dimensional equation by $L T^{-1}$ and similarly for other quantities. It should be noted that in the dimensional equation only the kinds of fundamental quantities are indicated and not the specific units used in measuring these quantities and also that the number of such units is not indicated. Hence, numerical constants in the algebraic equation do not appear in the dimensional equation.

For example, consider the equation $a d^{2}+d^{3}=v$, in which $a$ represents an area, $d$ a length, and $v$ a volume. Since an area is the square of a length $\left(L^{2}\right)$ and a volume is the cube of a length 
$\left(L^{3}\right)$, the dimensional equation is $L^{4}+L^{3}=L^{3}$ and hence the given equation is incorrect.

Consider also the equation $P+k v=a s$ in which $P$ represents a force, $k$ a weight (force) per unic volume, $v$ a volume, $a$ an area, and $s$ a force per unit area. The dimensional equation then may be written,

$$
F+\frac{F}{L^{3}} \cdot L^{3}=L^{2} \cdot \frac{F}{L^{2}}
$$

That is,

$$
F+F=F,
$$

and hence the equation is dimensionally correct.

Further, consider the equation $E=\frac{P l}{a e}$, in which $P$ represents a force in pounds, $l$ a length in inches, $a$ an area in square inches, and $e$ a length in inches. Let it be required to determine the units in which $E$ is expressed. The dimensional equation is

$$
E=\frac{F \times L}{L^{2} \times L}=\frac{F}{L^{2}}
$$

Hence, in accordance with the units stated (pound and inch), $E$ is expressed in pounds per square inch (lb./sq. in. or lb./in. $\left.{ }^{2}\right)$.

\section{PROBLEMS}

14. The equation $Q=.622 \sqrt{2 g}(b-0.2 h) h^{3 / 2}$ is used to determine the quantity of water flowing per unit of time over a rectangular weir of width $b$ when the height of water above the weir is $h, g$ being the acceleration due to gravity $\left(32.2 \mathrm{ft} . /\right.$ sec. $\left.^{2}\right)$. (a) Write the dimensional equation. (b) What are the units of $Q$ if $b$ and $h$ are expressed in feet? (c) If $Q$ equals $24.4 \mathrm{cu}$. ft. per sec. and $b$ equals $3 \mathrm{ft}$. compute the value of $h$ to the same degree of accuracy as is used in expressing $Q$, that is, to three significant figures. Note: This formula is valid only when $.2 h$ is small compared with $b$. Hence the term $.2 h$ may be omitted and a trial value of $h$ may be found by solving the resulting equation. This value of $h$ may now be used in the term $.2 h$ and a second trial value of $h$ may be obtained by solving the resulting equation. This process may be repeated.

15. In finding the diameter of a pipe required to discharge a given quantity of water, the formula $d^{5}=A d+B$ is used, in which $A$ and $B$ are known quantities. If $A=15.5$ and $B=400$ compute the value of $d$ to three significant figures. If the given equation is dimensionally correct what can be said of the quantities $A$ and $B$ ? 
16. In the following equation, $T$ is the moment of a force, $S$ is a force per unit of area in pounds per square inch, $h, h_{o}, b$, and $b_{o}$, are lengths in inches. Is the equation dimensionally correct? What are the units of $T$ ?

$$
T=\frac{2}{9} S \frac{b^{3} h-b_{0}{ }^{3} h_{0}}{b}
$$




\section{CHAPTER II}

\section{RESULTANTS OF FORCE SYSTEMS}

20. Introduction.-The determination of the resultants of various force systems as discussed in this chapter is of importance, mainly, (1) in the study of the conditions which the forces of a system satisfy when they hold a body in equilibrium (Statics, Chapter III), and (2) in the study of the laws by which the motions of bodies are governed (Part III, Kinetics).

(1) The equations of equilibrium for a given type of force system are obtained by expressing the conditions which the forces must satisfy in order that the resultant of the system shall be zero. Therefore the resultant to which a given type of force system reduces must be known before the conditions which are required to make the resultant equal to zero can be established. Furthermcre, in dealing with forces in equilibrium it is frequently convenient to replace several of the forces of a balanced system by the resultant of the several forces and to deal with the resulting force system instead of the original system.

(2) The motions of bodies are influenced by the unbalanced part (resultant) of the forces which act on the bodies. In the study of the motions of physical bodies, therefore, a knowledge of the resultants of various force systems and of methods of expressing the characteristics of resultants in terms of the forces of the system must be understood.

\section{$\S 1$. Collinear Forces}

21. Algebraic Method.-The resultant will be found first for two collinear forces, $P$ and $Q$, having the same sense. It is a matter of experience that the two forces are equivalent to a single force which has a magnitude equal to $P+Q$ and a line of action and sense which are the same as the line of action and sense of the given forces. This proposition may be proved, however, by applying the parallelogram law. Thus, according to this law, the resultant of any two concurrent forces $P$ and $Q$, the lines of action of 
which make an angle $\alpha$ with each other, is a single force of magninitude, $R$, which is given by the equation $R=\sqrt{P^{2}+Q^{2}+2 P Q \cos \alpha}$ (Art. 11). In the special case of two collinear forces here considered, $\alpha$ equals zero and hence $R=\sqrt{P^{2}+Q^{2}+2 P Q}=P+Q$. Similarly, the resultant of two forces $P$ and $Q$ having opposite senses ( $P$ being the larger of the two forces) is a force the magnitude of which is given by the equation $R=P-Q$, the sense of $R$ being the same as the sense of the larger force $P$. Hence, the resultant of any two collinear forces is a single force having the same line of action as the given forces, the magnitude and sense being indicated by the algebraic sum of the forces. The extension of this method to any number of collinear forces may easily be made. Thus the resultant of two of the forces may be combined with a third force; the resultant thus obtained may then be combined with a fourth force, and so on, until the entire system is reduced to a single resultant force. Therefore the magnitude of the resultant of a collinear force system is given by the equation,

$$
R=\Sigma F
$$

\section{$\S 2$. Concurrent Forces in a Plane}

22. Graphical Methods. First Method.-The resultant of an unbalanced system of concurrent forces in a plane is a force which may be found by means of the parallelogram law. In Fig. 27 are shown three forces $F_{1}, F_{2}$, and $F_{3}$ which act on a body at the point $O$. The forces $F_{1}$ and $F_{2}$ may be combined into a single force $R_{1}$ by means of the parallelogram law. Similarly $R_{1}$ and $F_{3}$ may be combined into a single force $R_{2} . R_{2}$ then is the resultant of the given forces. By continuing this process any number of concurrent forces

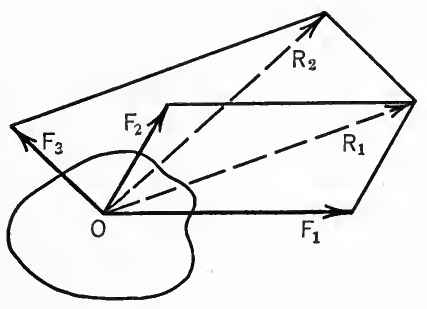

FIG. 27. may be combined into a single force. The order in which the forces are taken is immaterial. If the resultant force obtained by combining all except one of the forces of a concurrent system is equal to that one, collinear with it, and of opposite sense, the two 
forces cancel and hence the resultant of the given system is equal to zero.

Second Method.-Another graphical method of determining the resultant of a system of concurrent forces in a plane involves the application of the triangle law. Consider, for example, the three forces $F_{1}, F_{2}$, and $F_{3}$, which act on a body and concur at a point $O$ as shown in Fig. 28(a). In order to determine the resultant of the three forces, draw from any arbitrary point $A$ (Fig. 28b), a vector representing the magnitude and the direction of the force $F_{1}$; from the end of this vector draw another vector representing the
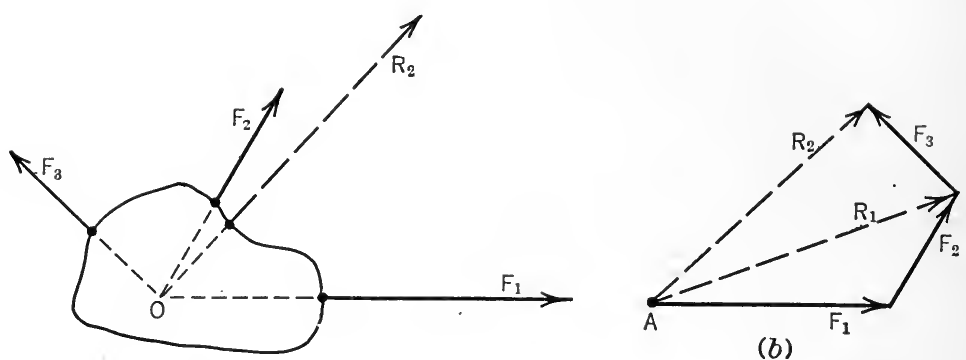

(a)

Fig. 28.

magnitude and the direction of the force $F_{2} . \quad R_{1}$, the resultant of $F_{1}$ and $F_{2}$, is represented in direction and magnitude by the vector drawn from $A$ to the end of $F_{2}$. To find the resultant of $R_{1}$ and $F_{3}$, and hence of the three given forces, draw from the end of $R_{1}$ (or $F_{2}$ ) a vector representing $F_{3}$ in magnitude and in direction. The resultant of $R_{1}$ and $F_{3}$ is then represented in magnitude and in direction by the vector $R_{2}$ drawn from $A$ to the end of $F_{3}$. It should be noted that this vector $R_{2}$ represents the magnitude and the direction, only, of the resultant of the given forces and not the action line of the resultant. The action line of the resultant must pass through the point $O$ in the body at which the forces are concurrent.

This method of determining the resultant may be stated formally as follows: In order to find the resultant of a system of concurrent forces in a plane, construct a polygon the sides of which are vectors representing the given forces in magnitude and in direction; a line drawn from the beginning of the first vector to 
the end of the last vector represents the magnitude and the direction of the resultant of the given system. If the force polygon closes, that is, if the end of the last vector coincides with the beginning of the first vector, the resultant of the given system is equal to zero.

23. Algebraic Method.-In Fig. 29(a) is represented a coplanar force system which is concurrent. Each of the four forces of the system may be resolved into two components, one lying along the $x$-axis and one along the $y$-axis. The $x$-components of the forces constitute a collinear system the resultant of which is a force along
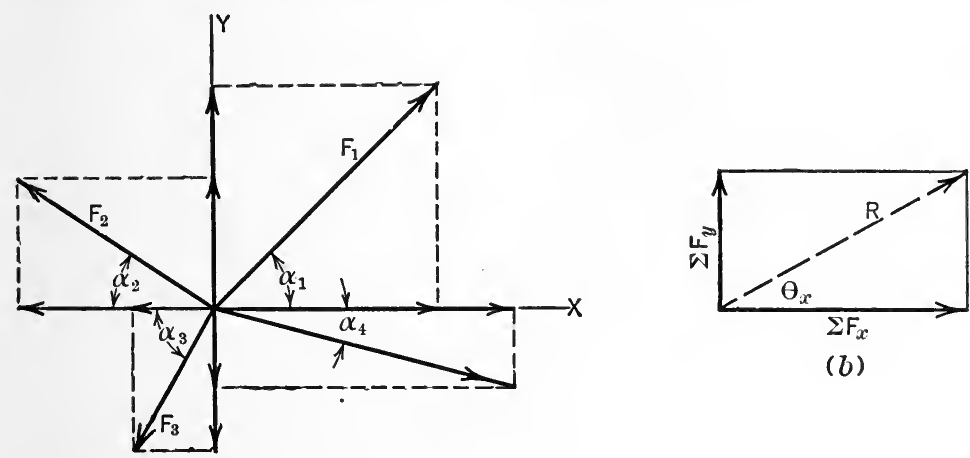

(b)

(a)

Fig. 29

the $x$-axis. The magnitude of this resultant is equal to $\Sigma F_{x}$. The $y$-components of the forces likewise constitute a collinear system the resultant of which is a force along the $y$-axis of magnitude $\Xi F_{y}$. Since the system, by this method, is reduced to two forces the resultant of the given system is the resultant of these two forces. If the magnitude of this resultant is denoted by $R$ and the direction which its action line makes with the $x$-axis by the angle $\theta_{x}$ as shown in Fig. $29(b)$, the resultant may be found from the equations:

$$
\begin{aligned}
R & =\sqrt{\left(\Sigma F_{x}\right)^{2}+\left(\overline{\left.\Sigma F_{y}\right)^{2}},\right.} \\
\tan \theta_{x} & =\frac{\boldsymbol{\Sigma} F_{y}}{\Sigma F_{x}} .
\end{aligned}
$$

The action line of the resultant must of course pass through the point of concurrence of th forces. 


\section{ILLUSTRATIVE PROBLEM}

17. Find the resultant of the system of concurrent forces shown in Fig $30(a)$.

Algebraic Solution:

$$
\begin{aligned}
\Sigma F_{x} & =20 \cos 30^{\circ}-30 \cos 60^{\circ}-10 \cos 45^{\circ}+25 \cos 45^{\circ} \\
& =17.32-15-7.07+17.67 \\
& =12.92 \mathrm{lb} . \\
\Sigma F_{y} & =20 \cos 60^{\circ}+30 \cos 30^{\circ}-10 \cos 45^{\circ}-25 \cos 45^{\circ} \\
& =10+25.98-7.07-17.67 \\
& =11.24 \mathrm{lb} .
\end{aligned}
$$

and

$\therefore R=\sqrt{(12.92)^{2}+(11.24)^{2}}=17.1 \mathrm{lb} . \quad$ (Fig. 30b)

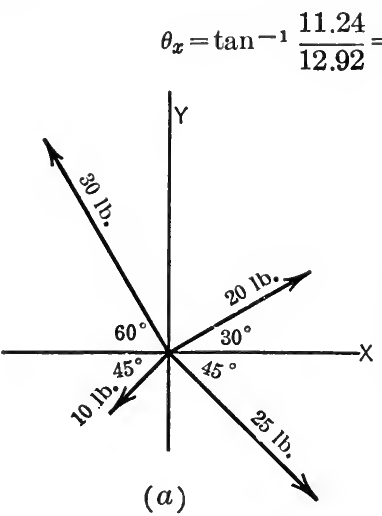

(a)

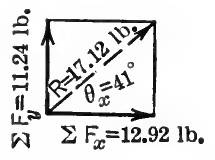

(b)

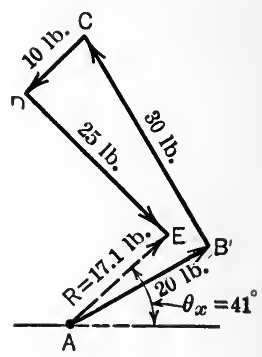

(c)

FIG. 30.

\section{Graphical Solution:}

To determine the resultant of the system graphically a force polygon is constructed as shown in Fig. $30(c)$. The resultant is represented by the vector $A E$. The magnitude of the resultant is found, by measuring the length of $A E$, to be $17.1 \mathrm{lb}$, and the angle which the resultant makes with the $x$-axis is found to be $41^{\circ}$.

\section{PROBLEMS}

In the following three problems the forces are concurrent at the origin. The values of $F$ are the magnitudes of the forces and the values of $\theta_{x}$ are the angles which the action lines of the forces make with the positive end of the $x$-axis, the angles being measured in a counter-clockwise direction. In each problem it is required to find the resultant of the forces specified 
18. $\begin{array}{ccccc}F & 100 \mathrm{lb} . & 60 \mathrm{lb} . & 40 \mathrm{lb} . & 50 \mathrm{lb} . \\ \theta_{x} & 30^{\circ} & 135^{\circ} & 240^{\circ} & 330^{\circ}\end{array}$ 19. $\begin{array}{ccccc}F & 20 \mathrm{lb} . & 10 \mathrm{lb} . & 15 \mathrm{lb} . & 5 \mathrm{lb} \\ \theta_{x} & 0^{\circ} & 45^{\circ} & 120^{\circ} & 270^{\circ}\end{array}$

Ans. $R=75$ lb., $\theta_{x}=25^{\circ} 55^{\prime}$.

20. $\begin{array}{ccccc}F & 25 \mathrm{lb} . & 10 \mathrm{lb} . & 30 \mathrm{lb} . & 40 \mathrm{lb} . \\ \theta_{x} & 0^{\circ} & 30^{\circ} & 135^{\circ} & 240^{\circ}\end{array}$

Ans. $R=24.7 \mathrm{lb}$., $\theta_{x}=37^{\circ} 35^{\prime}$.

21. Fig. 31 represents a body acted on by four forces. Determine completely the resultant of the four forces by the graphical method and check the results by the algebraic method. Ans. $R=186 \mathrm{lb}$; $\theta_{x}=164^{\circ} 45^{\prime}$.

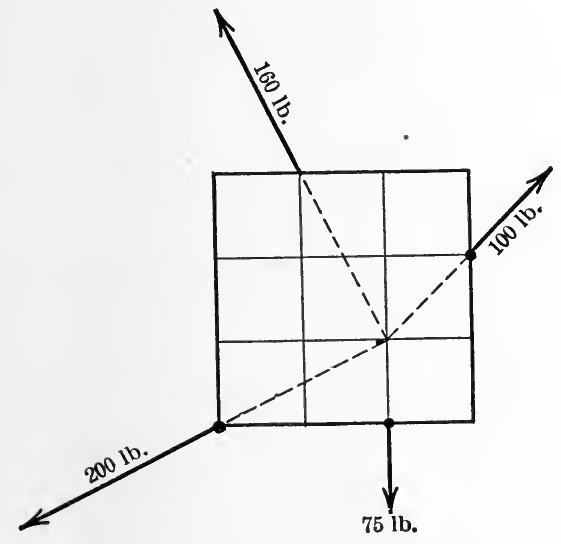

Fig. 31.

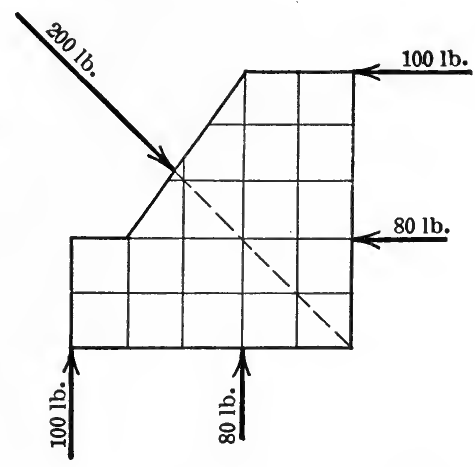

FIG. 32.

22. The body represented in Fig. 32 is acted on by five forces as shown. Replace the two 80-lb: forces by a single force and then find the resultant of this force and the remaining three forces.

23. Find by the graphical method the resultant of the five forces shown in Fig. 33.

24. Solve the preceding problem by the algebraic method.

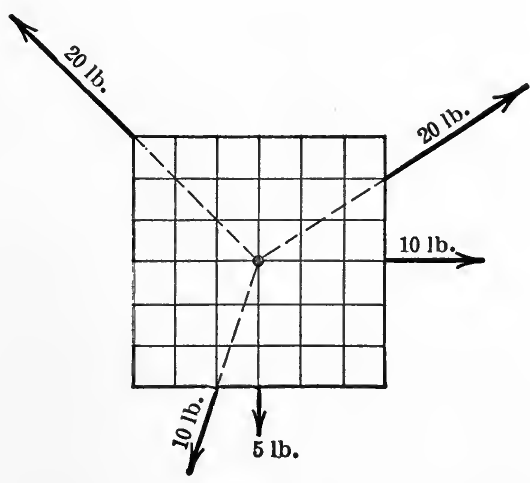

Fig. 33. 


\section{§3. Parallel Forces in a Plane}

24. Graphical Methods. First Method.-A system of two parallel forces having the same sense will first be considered. In Fig. 34 are shown two such forces, $P$ and $Q$, the points of application of which are $B$ and $A$ respectively. The resultant of these two forces cannot be found directly by the parallelogram law. However, by introducing at $B$ and $A$ any two equal, opposite, and collinear forces $F, F$, as shown in Fig. 34, the parallelogram law may be

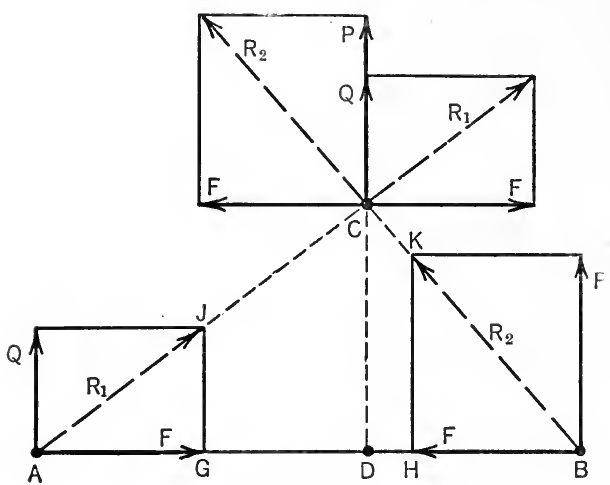

FIG. 34 used. Thus the forces $Q$ and $F$ may be replaced by their resultant $R_{1}$. Likewise $P$ and $F$ may be replaced by their resultant $R_{2}$. At $C$, the point of intersection of the lines of action of $R_{1}$ and $R_{2}$, let $R_{1}$ be resolved into components $Q$ and $F$, and $R_{2}$ into components $P$ and $F$. The two forces $F, F$ at $C$ cancel, thereby reducing the system to two collinear forces $P$ and $Q$ along the line $D C$. The resultant of these two forces, which is also the resultant of the original two forces, is a single force of magnitude $P+Q$ along the line $I C$. In order to determine the position of the action line $D C$ of the resultant, consider the two similar triangles $A J G$ and $A C D$. From these similar triangles there is obtained the relation,

$$
\frac{Q}{F}=\frac{\overline{C D}}{\overline{A D}}
$$

Similarly, from the triangles $B K H$ and $B C D$ the following relation is obtained,

$$
\frac{P}{F}=\frac{\overline{C D}}{\overline{B D}} \cdot \cdot \cdot . . . . . .
$$


By eliminating $F$ from equations (1) and (2) the resulting equation is

$$
\frac{P}{Q}=\frac{\overparen{A D}}{\overline{B D}}
$$

Hence the resultant of two parallel forces of like sense is a force having a line of action which is parallel to the lines of action of the two forces and which divides the distance between them internally into segments which are inversely proportional to the magnitudes of the forces. The magnitude of the resultant is the sum of the forces and its sense is the same as that of the forces.

If the forces $P$ and $Q$ are unlike in sense it can be shown in a manner similar to that used above that their resultant is a force of magnitude $P-Q$ ( $P$ being larger than $Q$ ), the sense of which is the same as that of $P$. The line of action of the resultant is parallel to the lines of action of the forces and divides the distance between them externally into segments which are inversely proportional to the magnitudes of the forces. The proof is left to the student.

It should be noted that the construction used in Fig. 34 fails if the two opposite, parallel forces are equal in magnitude since two such forces constitute a couple and cannot be replaced by a single force.

This method of finding the resultant of two parallel forces can be extended to any number of parallel forces. For, any two forces of such a system can be combined into a single resultant force; this resultant and a third force of the system can be combined in exactly the same manner, and so on, until the entire system has. been reduced to a single resultant force. It is evident that the magnitude of the resultant is equal to the algebraic sum of the given forces. If in combining the forces of a parallel system by the above method it is found that the resultant of all except one of the forces of the system is a force which is equal to the force omitted and opposite to it in sense, the two forces constitute a couple which is the resultant of the system. If, however, the lines of action of the two forces just mentioned are collinear, the forces balance and the resultant of the system is equal to zero. Hence if the resultant of a system of parallel forces in a plane is not equal to zero, the resultant is either a force or a couple.

The determination of the line of action of the resultant force by the method just discussed involves considerable work if there 
are many forces in the system. A second graphical method will now be considered. This method involves less construction than the method just considered and possesses other important advantages.

Second Method.-In Fig. 35(a) is shown a system of four parallel forces having the action lines $a b, b c, c d$, and $d e$. On a line parallel to the action lines of the forces are laid off vectors $A B, B C$ : $C D$, and $D E$ which represent the given forces in magnitude and direction (Fig. 35b). From any arbitrarily chosen point, $O$, lines are drawn to the points $A, B, C, D$, and $E$. The figure thus constructed is called a force polygon; the point $O$ is called the pole,

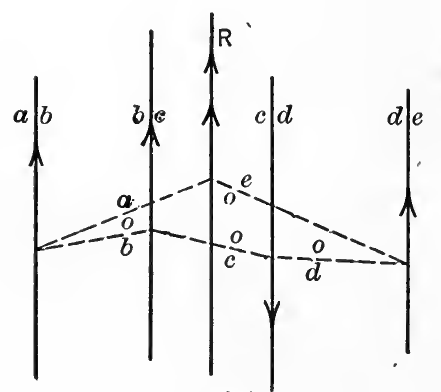

(a)

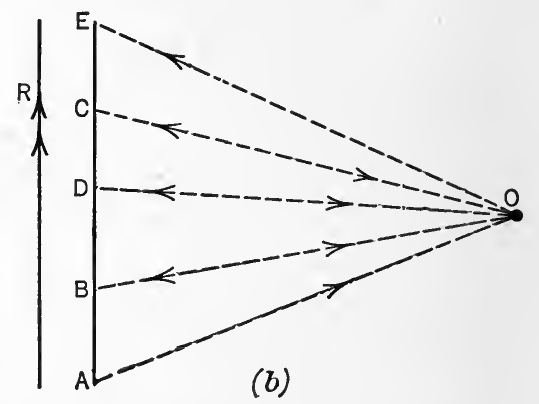

(b)

Fig. 35.

and the lines $O A, O B, O C, O D$, and $O E$ are called rays. From the triangle law it follows that by this construction the force $A B$ is resolved into two components represented in magnitude and in direction by the rays $A O$ and $O B . A O$ and $O B$, however, do not represent the lines of action of the two components, since the lines of action of the components of $A B$ must intersect on $a b$. Similarly, $B O$ and $O C$ represent the magnitudes and the directions of two components into which $B C$ is resolved, and so on for the remaining rays. The given system then may be replaced by another system of eight forces which are represented in direction and in magnitude by the rays $A O, O B, B O, O C, C O$, etc. The forces represented by the rays $O B$ and $B O$ ( $O C$ and $C O$, etc.), are equal in magnitude, opposite in sense, and their lines of action are parallel. 
If now the action lines of the pairs of parallel and equal forces $O B$ and $B O, O C$ and $C O, O D$ and $D O$, are made to coincide, each pair will cancel and there will remain only two forces, $A O$ and $O E$. The resultant of these two forces is the resultant of the given system and is represented in magnitude and in direction by the vector $A E$.

The action line of $A E$ will pass through the point of intersection of the action lines of its components $A O$ and $O E$. In order to locate the point of intersection of the action lines of these two forces it is necessary to construct another figure called the funicular polygon. This is a polygon the sides of which, called strings, are parallel to the rays of the force polygon. The funicular polygon is constructed as follows: From any point on $a b$, the action line of $A B$, strings $a o$ and $o b$ are drawn parallel to the rays $A O$ and $O B$. These strings represent the action lines of the forces $A O$ and $O B$ into which $A B$ was resolved. From the point of intersection of $o b$ and $b c$ the string $b o$ (which will coincide with the string $o b$ ) and the string $o c$ are drawn. From the point of intersection of $o c$ and $c d$ are drawn $c o$ and $o d$ and from the point of intersection of od and $d e$ are drawn $d o$ and $o e$. Since $o b$ and $b o$, the action lines of the equal and opposite forces $O B$ and $B O$, are collinear, the two forces $O B$ and $B O$ will cancel. Similarly for $O C$ and $C O$ and for $O D$ and $D O$. The system then is reduced to two forces represented in magnitude and in direction by the vectors $A O$ and $O E$, the action lines of the forces being $a o$ and $o e$. The resultant of these two forces, which is also the resultant of the given system, will pass through the point in which their action lines $a o$ and $o e$ intersect. The resultant then is completely determined by the force and funicular polygons; the former determines its magnitude and direction; the latter determines a point on its action line.

If the force polygon closes, that is, if $E$ coincides with $A$, the rays $A O$ and $O E$ represent two forces which are equal in magnitude and which have parallel action lines and opposite senses. The resultant in this case is a couple provided that the funicular polygon does not close. If, however, the funicular polygon also closes, that is if $a O$ and $o e$ coincide, the two forces $A O$ and $O E$ will balance and the resultant of the given system will be equal to zero.

In constructing the force and funicular polygons any point may be taken for the pole and the funicular polygon may be started 
at any point on the action line of any one of the forces. A change in the positions of these points has the effect, only, of locating a different point on the action line of the resultant if the resultant of the system is a force. If the resultant of the system is a couple, the effect is to change both the magnitude of the forces constituting the resultant couple and the length of its moment-arm. The couples will be equivalent, however; that is, they will have the same moment and sense.

\section{ILLUSTRATIVE PROBLEM}

25. Find by the graphical method the resultant of the four parallel forces shown in Fig. 36(a).

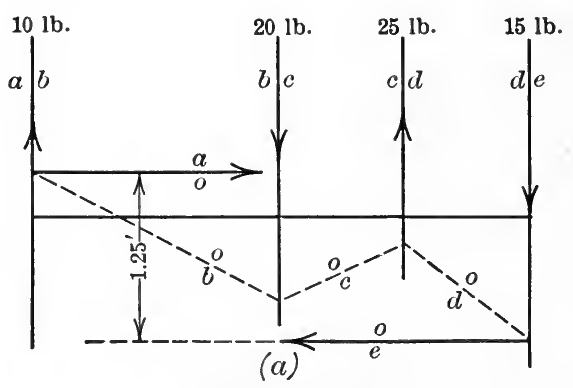

Scales: $1 \mathrm{in}=2 \mathrm{ft}$.

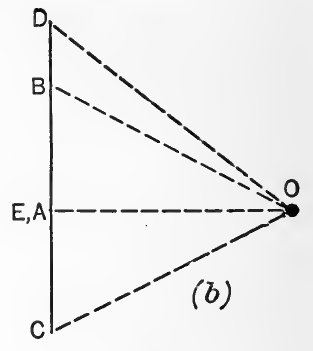

$1 . \mathrm{in} .=20 \mathrm{lb} . \checkmark$

FIG, 36.

Solution.- Since the force polygon closes the resultant is not a force and hence is a couple. In constructing the rays (Fig. $36 b$ ) it is convenient to take the pole $O$ so that the force represented by $O A$ has an integral value. $O A$ here represents to scale $20 \mathrm{lb}$. The funicular polygon in Fig. 36(a) is constructed according to the method described in Art. 24. The resultant is a couple consisting of the two 20-1b. forces $A O$ and $O E$. The arm as scaled from the funicular polygon is $1.25 \mathrm{ft}$. Hence the moment of the resultant couple is $-25 \mathrm{lb}$. $\mathrm{ft}$., the minus sign indicating a clockwise direction of rotation.

25. Principle of Moments.-The principle of moments as discussed in Art. 14 for the special case of two concurrent forces may be extended to all force systems. Briefly, the principle states that the moment of the resultant of a force system is equal to the algebraic sum of the moments of the forces of the system. The principle is of great importance in the determination, by the algebraic method, of $(a)$ the action line of the resultant of a system of forces 
when the resultant is a force, and (b) the moment and sense of the resultant of the force system when the resultant is a couple. A formal statement and proof of the principle of moments will not be given for each of the force systems considered since the method of proof is substantially the same for all the force systems. As applying to a system of parallel forces in a plane the principle may be stated formally as follows: The algebraic sum of the moments of the forces of a coplanar parallel system about any point in their plane is equal to the moment of the resultant of the system about the same point.

In demonstrating the truth of this statement use will be made of the diagram in Fig. 35 and of the methods of the preceding article. The given system of four forces (Fig. 35) was replaced by another system of eight forces which were represented in magnitude and in direction by the rays of the force polygon and in line of action by the strings of the funicular polygon. Six of these forces occur in pairs, the two forces of each pair being collinear, equal in magnitude, and opposite in sense. Obviously, the sum of the moments of the two forces of each pair with respect to any point in their plane is equal to zero. For any moment-center in the plane, by use of Varignon's theorem, the following relations may be written,

\begin{tabular}{|c|c|c|c|c|}
\hline "6 & $B C=$ & " & $\mathrm{BO}+$ & " \\
\hline “ & $C D=$ & "6 & $\mathrm{CO}+$ & “ \\
\hline " & $D E=$ & " & $D O+$ & " \\
\hline
\end{tabular}

If the two sides of the above equations are added the result obtained may be stated as follows:

The sum of the moments of the forces of the system

$$
=\text { moment of } A O+\text { moment of } O E \text {, }
$$

since the remaining terms on the right side of the equations cancel in pairs. But $A O$ and $O E$ are the components of the resultant force of the system and hence, by Varignon's theorem, the sum of the moments of $A O$ and $O E$ is equal to the moment of the resultant of the system. Hence, the proposition is proved for a parallel force system in which the resultant is a force.

If the resultant of the force system is a couple, that is, if the forces $A O$ and $O E$ are parallel, equal, and opposite as in Fig. 36, 
the proposition also holds, since the sum of the moments of $A O$ and $O E$ is equal to the moment of the resultant couple.

26. Algebraic Method.-A system of coplanar parallel forces is shown in Fig. 37, the forces being parallel to the $y$-axis. In the graphical determination of the resultant of such a system it was seen that the resultant was either a force or a couple. If the resultant is a force, $R$, its action line is parallel to the action lines of the forces of the system and its magnitude and sense are indicated by the algebraic sum of the forces, that is, $R=\Sigma F$. In

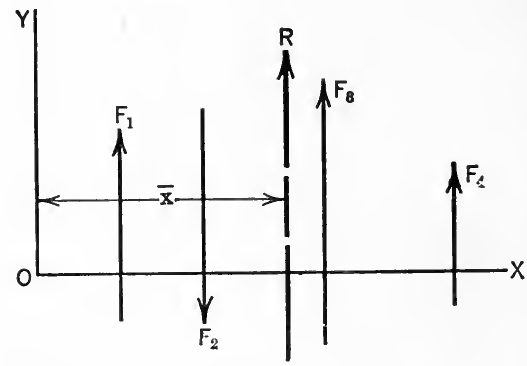

FIG. 37. order to locate the line of action of the resultant force the principle of moments will be applied. For convenience the origin, $O$, will be taken as the center of moments. The moment of $R$ then with respect to $O$ is equal to the algebraic sum of the moments of the forces with respect to the same point. If the distance from the action line of $R$ to the $y$-axis is denoted by $\bar{x}$, the moment of $R$ with respect to the origin is equal to $R \bar{x}$. Further, if the algebraic sum of the moments of the forces of the system with respect to the origin is denoted by $\Sigma(F x)$ or $\Sigma M_{0}$ the principle of moments may be expressed by the equation $R \bar{x}=\Sigma M_{0}$. The resultant, then, if a force, is parallel to the $y$-axis and is determined by the following equations,

$$
\begin{gathered}
R=\Sigma F \\
R \bar{x}=\Sigma M_{0} .
\end{gathered}
$$

It should be noted that the sign of $\bar{x}$ is not always indicated properly by the ratio of $\Sigma M_{0}$ to $R$. The sign of $\bar{x}$ may be determined by inspection, since the resultant force must lie on that side of the moment-center which will make the sense of its moment agree with that of the moment of the system.

If the resultant of all, except one, of the forces of the system is equal to that one and of opposite sense, $\Sigma F$ then equals zero and the resultant is a couple, the moment of which, according to the 
principle of moments, is equal to the algebraic sum of the moments of the forces of the system. Hence, if the resultant of a coplanar parallel force system is a couple the moment, $C$, of the couple may be determined from the expression,

$$
C=\Sigma M
$$

the aspect of the couple, of course, being the same as that of the plane of the forces.

If both $\Sigma F$ and $\Sigma M$ equal zero the resultant is equal to zero.

\section{ILLUSTRATIVE PROBLEMS}

26. Fig. 38 represents a beam resting on two supports and carrying four loads as indicated. Find the resultant of the loads.

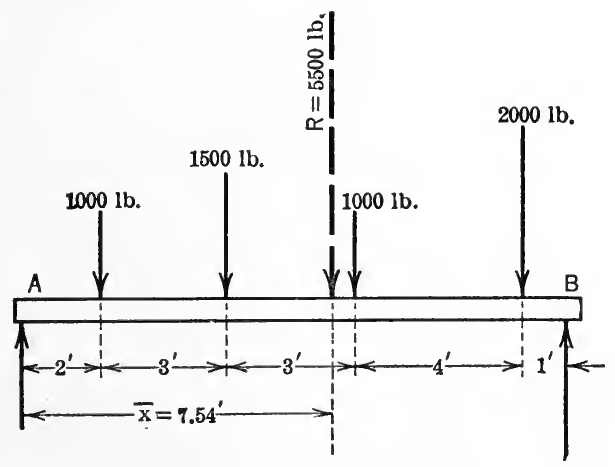

Fig. 38.

Solution. $-R=\Sigma F=-1000-1500-1000-2000=-5500 \mathrm{lb}$.

In order to locate the action line of the resultant, the point $A$ will be selected as the moment-center. Thus,

$$
R \bar{x}=\Sigma M_{A}=-1000 \times 2-1500 \times 5-1000 \times 8-2000 \times 12=-41,500 \quad \text { lb.ft. }
$$

Therefore,

$$
\bar{x}=\frac{41500}{5500}=7.54 \mathrm{ft} .
$$

Hence a single load of $5500 \mathrm{lb}$. at a point $7.54 \mathrm{ft}$. from $A$ will produce the same external effect (reactions at $A$ and $B$ ) as the four given forces; 
27. An arm mounted on an axle at $O$ (Fig. 39) is acted on by the four forces shown. Determine the resultant of the forces.

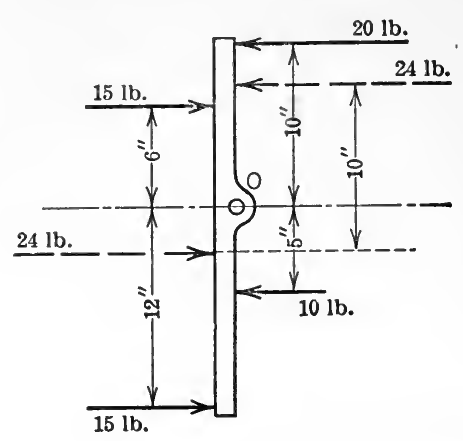

Fig. 39.

\section{Solution.-}

$$
R=\Sigma F=15+15-20-10=0 .
$$

Therefore the resultant is not a force, but is a couple, $C$, the moment of which may be found by taking moments about $O$. Thus,

$$
\begin{aligned}
C=\Sigma M_{0}=20 \times 10-15 \times 6-10 \times 5 \\
+15 \times 12=+240 \mathrm{lb} .- \text { in. }
\end{aligned}
$$

Hence any couple having a moment of $+240 \mathrm{lb}$.-in. will produce the same external effect on the arm (change in the rotational motion) as the four given forces. One resultant couple having forces equal to $24 \mathrm{lb}$. is shown in the figure.

\section{PROBLEMS}

28. Fig. 40 represents a beam $12 \mathrm{ft}$. long acted upon by five forces as shown. Find the anti-resultant of the five forces.

29. Five forces act on a body as shown in Fig. 41. Replace the two $60-\mathrm{lb}$. forces by a single force and then find the resultant of this force and the remaining three forces.

30. A force of $10 \mathrm{lb}$. acts along the $y$-axis, the sense of the force being positive. Resolve the force into components $P$ and $Q$ along the lines $x=1$ and $x=3$, respectively.

Ans. $P=15 \mathrm{lb} . \quad Q=-5 \mathrm{lb}$.

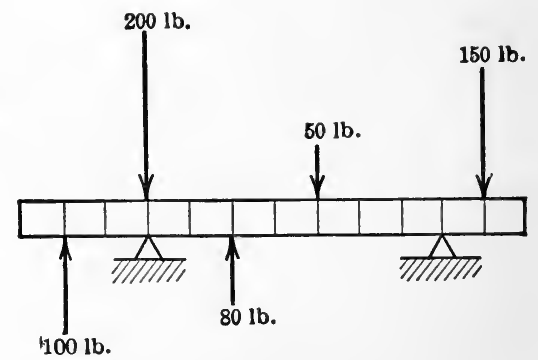

Fig. 40.

31. A bar $6 \mathrm{ft}$. long is hinged at $A$ and is acted on by five forces as shown in Fig. 42. Determine the resultant of the five forces.

Ans. $C=+90 \mathrm{lb} . \mathrm{ft}$.

32. A bar $10 \mathrm{ft}$. long is acted on by the forces shown in Fig. 43 . Find the equilibrant of the force system and represent it acting on the bar.

Ans. Equilibrant $=+15 \mathrm{lb}, 2 \frac{2}{3} \mathrm{ft}$. to the left of the $20-\mathrm{lb}$. force.

33. The 40-lb. force shown in Fig. 44 is the resultant of the 10-lb. force and a force $P$ not shown. Determine $P$ completely by means of a force and a funicular polygon and check the result by the algebraic method. 
In each of the following three problems find the resultant of the force system. The forces in each problem are parallel to the $y$-axis. The values

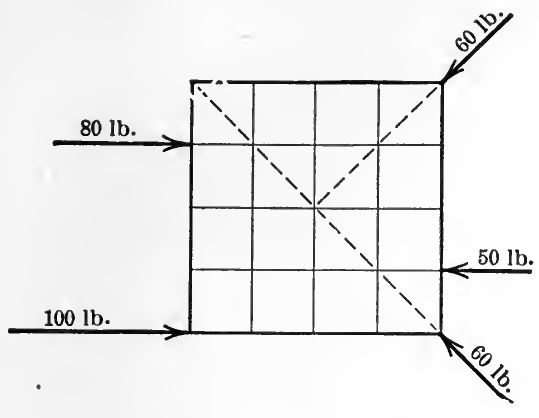

FIG. 41.

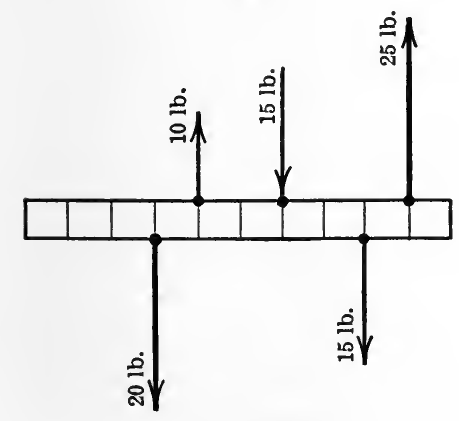

Fig. 43.

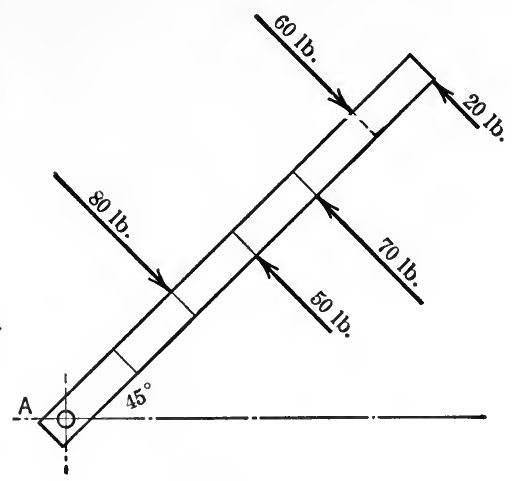

FIG. 42.

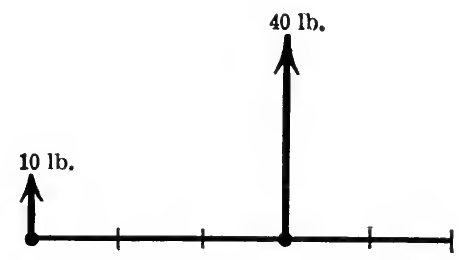

FIG. 44.

of $F$ given are the magnitudes of the forces (expressed in pounds) and the values of $x$ are the distances of the action lines of the forces from the $y$-axis (expressed in feet).

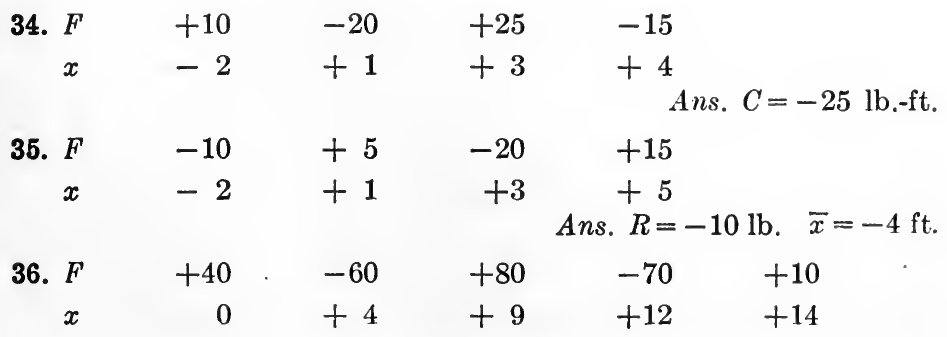


27. Couples.-In Art. 15 a couple is defined as two equal, parallel, non-collinear forces of opposite sense. The moment of a couple is defined as the algebraic sum of the moments of the two forces of the couple, and it is shown that the moment is the same for all moment-centers in the plane of the couple. Further, it is shown that the characteristics of a couple (moment, aspect, and sense) may be completely represented by means of a vector. Since couples play an important part in engineering problems it will be of advantage to consider certain additional propositions and transformations by means of which couples can easily be combined with other couples or with other forces.

Proposition I.-A couple may be translated into any parallel position in its own plane or into any parallel plane without changing its external effect on the body on which it acts.

Proof.-Consider the couple $P p$ having a moment-arm, $A B$, equal to $p$ as shown in Fig. 45. Construct a parallelogram having

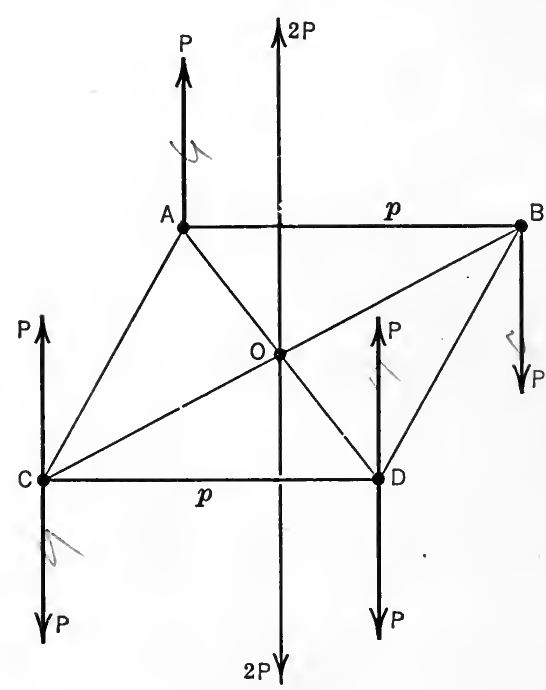

FIG. 45.

$A B$ as one side and $C D$ as the opposite side. This parallelogram may or may not be in the plane of the given couple. At each of the points $C$ and $D$ introduce two forces of opposite sense which are parallel to the forces of the given couple and equal to them in magnitude. The force $P$ at $A$ and the upward force $P$ at $D$ may be replaced by their resultant. This resultant is an upward force, $2 P$, the action line of which is parallel to the action lines of the forces of the given couple and is located half way between them.

Hence the action line passes through $O$, the point of intersection of the diagonals of the parallelogram. Similarly, the force $P$ at $B$ and the downward force $P$ at $C$ may be replaced by a downward force, $2 P$, acting through $O$ parallel to the forces of the given couple. The two forces $2 P, 2 P$ at $O$ cancel, thereby leaving only 
the upward force $P$ at $C$ and the downward force $P$ at $D$. Thesetwo forces constitute a couple which is equivalent to the given couple. Hence the proposition is established.

Proposition II. - A couple may be rotated through any angle in its plane without changing its external effect on the body on which ii acts.

Proof.-In Fig. 46 the two equal forces, $P_{1}$ and $P_{2}$, constitute a couple having a moment-arm, $A B$, equal to $p$. Through $O$, the midpoint of the arm, draw any line $C D$ making an angle $\theta$ with $A B$ and scale off $O D$ and $O C$ each equal to $\frac{1}{2} p$. At $C$ introduce two opposite forces, $P_{3}$ and $P_{4}$, which are perpendicular to $C D$, each of which is equal in magnitude to the forces $P_{1}$ and $P_{2}$ of the given couple. Similarly at $D$ introduce two forces, $P_{5}$ and $P_{6}$, perpendicular to $C D$, each being equal in magnitude to the forces of the given couple. Prolong the action line of $P_{1}$ until it intersects the action line of $P_{4}$ at $E$. The resultant

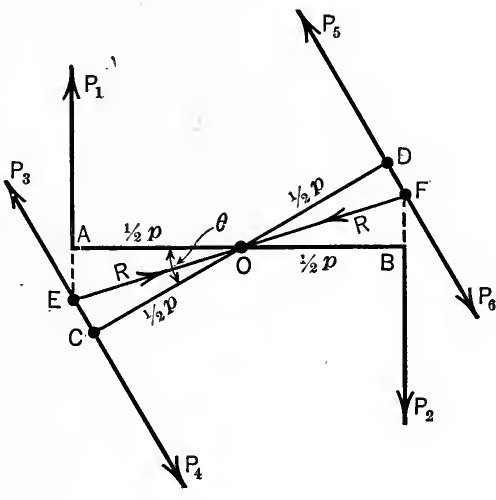

FIg. 46. of the two forces $P_{1}$ and $P_{4}$ is a force the action line of which bisects the angle $A E C$ and hence coincides with $E O$. Let $R$ denote the magnitude of this force. In the same way the resultant of $P_{5}$ and $P_{2}$ may be shown to be a force of magnitude $R$ the action line of which is $F O$. Since these two forces $R, R$ are equal, collinear, and opposite they cancel, thereby leaving the forces $P_{3}$ and $P_{6}$ which constitute a couple having a moment-arm $C D$ which, by construction, is equal to $p$. This equivalent couple, therefore, is merely the given couple rotated through an angle $\theta$.

Proposition III.-The magnitudes of the forces of a couple and the moment-arm of the couple may be changed to any values without changing the external effect of the couple, provided that the product of either force and the moment-arm remains constant.

Proof.-Given the couple $P p$ (Fig. 47) having a moment-arm, $A B$, equal to $p$. Let the arm be increased to $\overline{C D}=(p+2 x)$ as 
shown. At $C$ introduce two forces of opposite sense each equal to $\frac{P p}{p+2 x}$ and perpendicular to $C D$. Similarly, introduce two forces $\frac{P p}{p+2 x}$ at $D$. The force $P$ at $A$ and the upward force $\frac{P p}{p+2 x}$ at $D$ may be replaced by their resultant, which is an upward force of magnitude $\frac{2 P(p+x)}{p+2 x}$, the direction of the line of action being perpendicular to $C D$. The position of the line of action of this result-

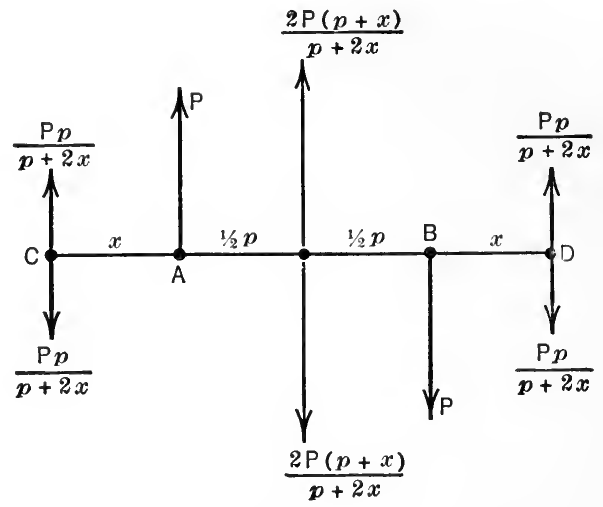

FIG. 47.

ant is found, by applying the principle of moments, to pass through the mid-point of $A B$. In a similar manner the resultant of the force $P$ at $B$ and the downward force $\frac{P p}{p+2 x}$ at $C$ is found to be a downward force of magnitude $\frac{2 P(p+x)}{p+2 x}$ perpendicular to $C D$, the action line of which also passes through the mid-point of $A B$. The two forces of magnitude $\frac{2 P(p+x)}{p+2 x}$ balance, thereby leaving two forces, $\frac{P p}{p+2 x}$ at $C$ and $\frac{P p}{p+2 x}$ at $D$, which constitute a couple the moment of which is equal to $P p$.

Proposition IV.-The resultant of any number of couples in a plane is a couple the moment of which is equal to the algebraic sum of the moments of the couples. 
This proposition can be proved by means of the three trans-formations discussed above. It is sufficient to prove the proposition for two couples, for, if two couples can be combined into a single resultant couple, evidently this couple can be combined with a third couple, and so on. Let the two couples $P p$ and $Q q$ (Fig. $48 a$ ) act on a body. The magnitude of the forces of the second couple may be changed from $Q$ to $P$ by changing the momentarm from $q$ to $\frac{Q q}{P}$ (Fig. 48b), as discussed in Proposition III. The second couple can then be rotated until the forces of the two couples are parallel (Fig. 48c). The second couple can now be translated until one of its forces is collinear with one of the forces of the first couple (Fig. 48d). This translation can always be made in such a way that these two equal, collinear forces are opposite in sense and hence will cancel, thereby leaving the two equal, parallel, and oppositeforces $P, P$ which constitute a couple. The arm of this resultant couple is $\left(p+\frac{Q q}{P_{i}}\right)$ and its moment is $P\left(p+\frac{Q q}{P}\right)=$ $P p+Q q$. Hence the moment of the resultant is equal to the sum of the moments of the

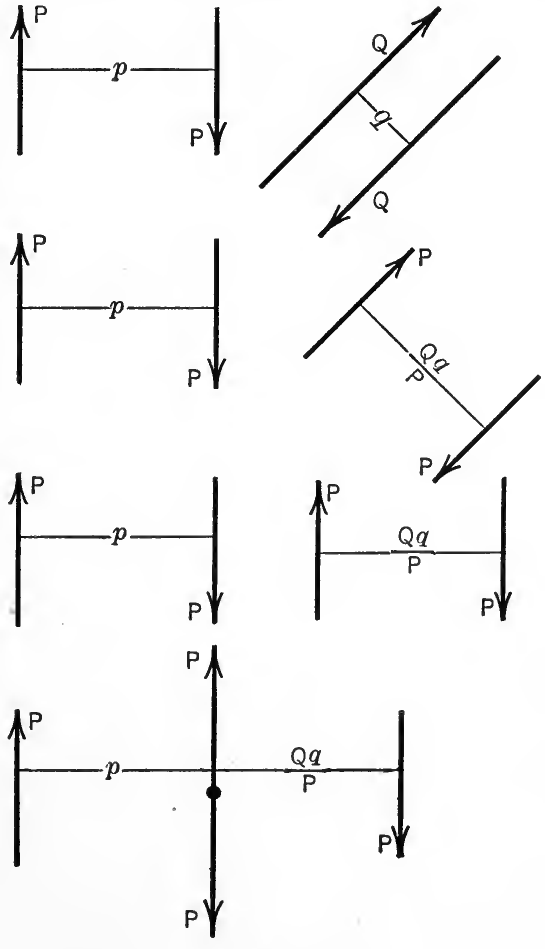

FIG. 48. given couples as stated in the proposition.

Proposition V.-Two coplanar couples which have the same moment and sense are equivalent.

This proposition follows from the three transformations pre- 
viously discussed, since, by means of them, one of the couples may be superimposed on the other.

\section{§4. Non-concurrent, Non-parallel Forces in a Plane}

28. Graphical Methods. First Method.-The resultant of a system of non-concurrent, non-parallel forces in a plane is either a force or a couple. If the resultant is a force it may be determined by use of the parallelogram law. In Fig. 49 are shown three non-concurrent, non-parallel forces $F_{1}, F_{2}$, and $F_{3}$ acting on a body. The forces $F_{1}$ and $F_{2}$ may be combined into a single resultant force $R_{1}$. $R_{1}$ and $F_{3}$ may be combined into a

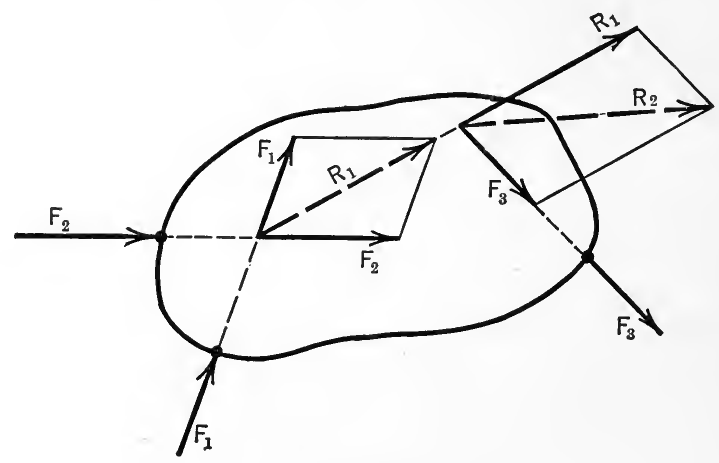

FIG. 49.

single force $R_{2}$ which is the resultant of the given system of forces.

If the resultant obtained by combining all, except one, of the forces of such a system is equal to that one and is parallel to it and of opposite sense, the two forces constitute a couple. Furthermore, if the action lines of these two forces are collinear the forces cancel and the resultant of the system is equal to zero.

Second Method.-The resultant of a sytem of non-concurrent, non-parallel forces in a plane may also be found by the construction of a force and a funicular polygon. This method is of great importance in engineering problems. The method of construction of these polygons is the same as that described in con- 
nection with a system of parallel forces (Art. 24) and will not here be discussed in detail for the non-parallel force system. The method will be illustrated, however, by the following problem.

\section{ILLUSTRATIVE PROBLEM}

37. A beam $9 \mathrm{ft}$. long is acted on by four forces as shown in Fig. 50 . Determine the resultant of the forces by use of the force and funicular polygons.

Solution.-The force polygon as shown in Fig. $50(b)$ is constructed by laying off the vectors $A B, B C, C D$, and $D E$ which represent the magnitudes and the directions of the given forces. The closing side $A E$ of the polygon represents the resultant force in magnitude and in direction. By measuring $A E$ to scale the magnitude of the resultant force is found

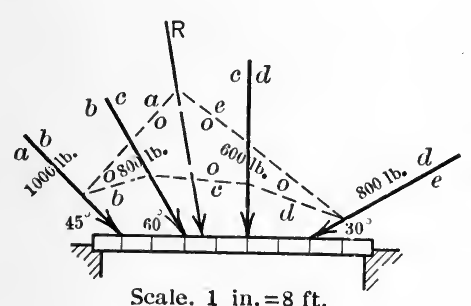

(a)

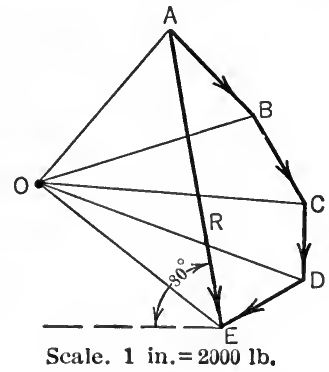

(b)

FIG. 50 .

to be $2450 \mathrm{lb}$. and the line $A E$ is found to make an angle of $80^{\circ}$ with the horizontal.

In Fig. $50(a)$ is shown the funicular polygon in which one point on the action line of the resultant is found, namely, the intersection of $o a$ and $o$. Therefore the action line of the resultant passes through this point and is parallel to $A E$. By measurement the action line is found to intersect the beam at a point $3.6 \mathrm{ft}$. from the left end of the beam. Hence, if the four forces were replaced by a single force, $R$, of $2450 \mathrm{lb}$. as shown in Fig. $50(a)$, the reactions at the ends of the beam (external effects) would be unchanged.

29. Principle of Moments.-The algebraic sum of the moments of the forces of a coplanar, non-concurrent, non-parallel force system about any point in the plane of the forces is equal to the 
moment of the resultant of the system about the same point. The proof is identical with that given for a system of parallel forces (Art. 25) and the principle will be used, in the algebraic method, for finding the action line of the resultant force or the moment of the resultant couple similar to the way it was used for parallel forces (Probs. 26 and 27).

30. Algebraic Method. - The resultant of a system of non-concurrent, non-parallel forces in a plane is either a force or a couple as explained in Art. 28. If the resultant is a force it may be determined as follows: In Fig. 51 $(a)$ is represented a body acted on by four forces $F^{\prime}, F^{\prime \prime}, F^{\prime \prime \prime}$, and $F^{\prime \prime \prime \prime}$. Let each force be replaced

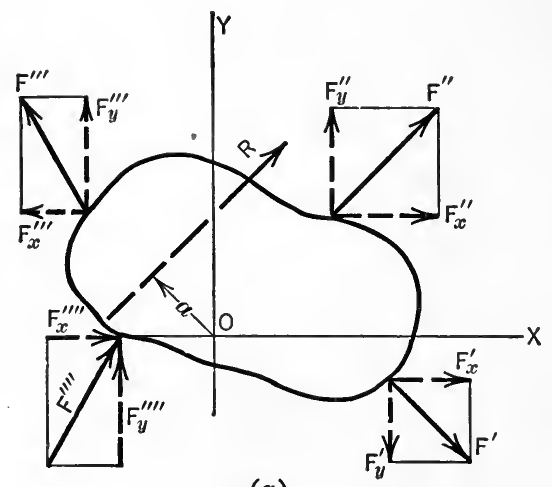

(a)

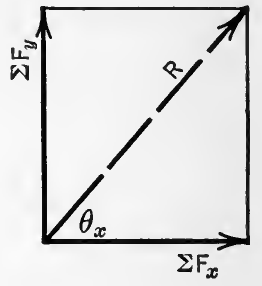

(b)

FIG. 51.

by its $x$ - and $y$-components. The original force system is now resolved into two systems of parallel forces, the resultant of each parallel system being a force parallel to the forces of the system. According to Art. 26 the resultant of the components in the $x$-direction is $\Sigma F_{x}$ and similarly, the resultant of the components in the $y$-direction is $\Sigma F_{y}$. Hence, the magnitude and the direction of the resultant of these two forces (which is also the resultant of the original forces) may be found from the equations $R=\sqrt{\left(\Sigma F_{x}\right)^{2}+\left(\Sigma F_{y}\right)^{2}}$ and $\tan \theta_{x}=\frac{\Sigma F_{y}}{\Sigma F_{x}}$ (Fig. 51b), in which $\theta_{x}$ is the angle which the action line of $R$ makes with the $x$-axis. In order to obtain the position of the line of action of the resultant force $R$ the principle of moments may be 
used. The principle is expressed by the equation $R a=\Sigma M_{0}$, in which $a$ (Fig. 51a) is the perpendicular distance from the moment-center, $O$, to the action line of the resultant, and $\Sigma M_{0}$ is the algebraic sum of the moments of the forces with respect to $O$.

Hence, if the resultant of a coplanar, non-concurrent, nonparallel system of forces is a force, it may be determined completely by the equations,

$$
\begin{aligned}
R & =\sqrt{\left(\Sigma F_{x}\right)^{2}+\left(\Sigma F_{y}\right)^{2}}, \\
\tan \theta_{x} & =\frac{\Sigma F_{y}}{\Sigma F_{x}}, \\
R a & =\Sigma M_{0} .
\end{aligned}
$$

If both $\Sigma F_{x}$ and $\Sigma F_{y}$ are equal to zero the resultant is not a force and hence is a couple the moment, $C$, of which, according to the principle of moments, is the algebraic sum of the moments of the forces of the system, that is,

$$
\boldsymbol{C}=\boldsymbol{\Sigma} M
$$

The center about which the moments of the forces are taken may be any point in the plane of the forces since the moment of a couple is the same about all points in the plane. The sense of rotation of the resultant couple is indicated by the sign of the algebraic summation and the aspect of the couple, of course, is the same as that of the plane of the forces.

If $\Sigma F_{x}$ and $\Sigma F_{y}$ are equal to zcro and $\Sigma M$ is also equal to zero, the resultant is equal to zero. 


\section{ILLUSTRATIVE PROBLEM}

38. Find the resultant of the system of four forces which act on the body represented in Fig. 52(a). Each space represents $1 \mathrm{ft}$.

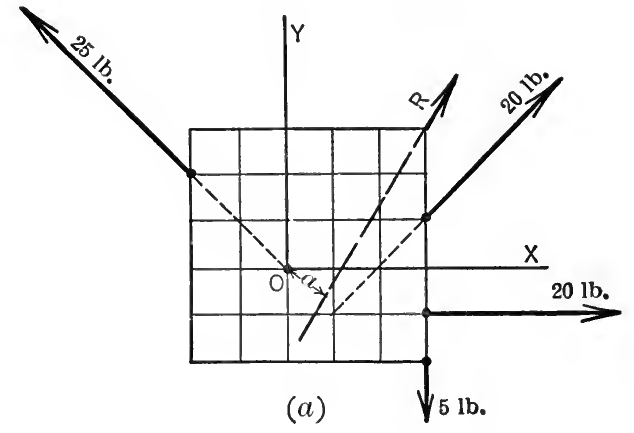

FIG. 52 .

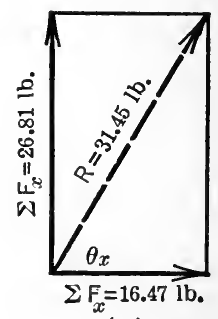

(b)

Solution.-The solution may be put in tabular form as follows:

$\begin{array}{rcccc}F & \theta_{x} & F_{x}=F \cos \theta_{x} & F_{y}=F \sin \theta_{x} & M_{0} \\ 20 & 45^{\circ} & 14.14 & 14.14 & 28.28 \\ 25 & 135^{\circ} & -17.67 & 17.67 & 0.00 \\ 5 & 270^{\circ} & 0.00 & -5.00 & -15.00 \\ 20 & 0^{\circ} & 20.00 & 0.00 & 20.00 \\ & & \Sigma F_{x}=16.47 \mathrm{lb} . & \Sigma F_{y}=26.81 \mathrm{lb} . & \Sigma M_{0}=33.28 \mathrm{lb} . \mathrm{ft} .\end{array}$

Therefore,

$$
\begin{gathered}
R=\sqrt{(16.47)^{2}+(26.81)^{2}}=31.45 \mathrm{lb}, \\
\theta_{x}=\tan ^{-1} \frac{26.81}{16.47}=58^{\circ} 25^{\prime} \\
a=\frac{33.28}{31.45}=1.06 \mathrm{ft} .
\end{gathered}
$$

\section{PROBLEMS}

39. Fig. 53 represents a board $3 \mathrm{ft}$. square acted upon by four forces as shown. Find completely the resultant of the forces. Specify the action line of the resultant by the perpendicular distance from the origin $O$.

Ans. $R=47.6 \mathrm{lb} . \theta_{x}=78^{\circ} 10^{\prime} . \quad a=1.98 \mathrm{ft}$. 
40. Determine completely the resultant of the five forces acting on the body shown in Fig. 54. Each space represents $1 \mathrm{ft}$.

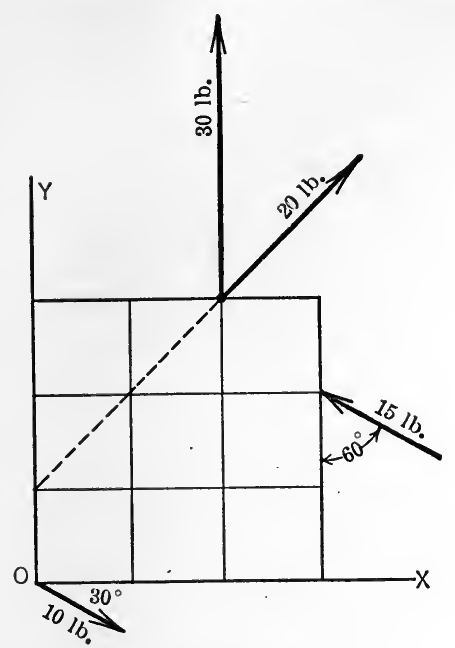

FIG. 53.

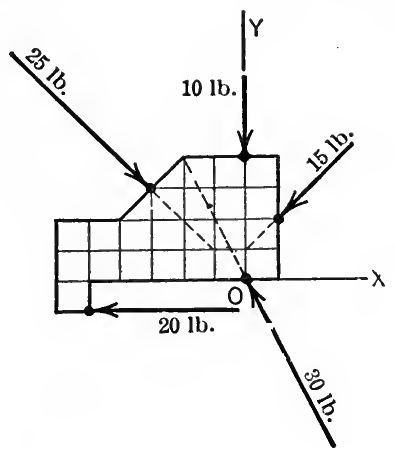

FIG. 54.

41. The forces of a coplanar system are specified below, the magnitudes of the forces being expressed in pounds and distances $x$ and $y$ in feet.

$\begin{array}{ccccc}F & 20 & 15 & 10 & 15 \\ x, y & 0,2 & 0,2 & 4,2 & 6,2 \\ \theta_{x} & 0^{\circ} & 45^{\circ} & 90^{\circ} & 135^{\circ}\end{array}$

Find the resultant of the system.

Ans. $R=37.1 \mathrm{lb} . \quad \theta_{x}=57^{\circ} 20^{\prime} \quad a=1.71 \mathrm{ft}$.

\section{$\S 5$. Concurrent Forces in Space}

31. Graphical Method.-The resultant of a system of noncoplanar, concurrent forces is a force which may be found by any of the graphical methods used in finding the resultant of a system of coplanar, concurrent forces as discussed in Art. 22. In applying any one of these methods, the forces are projected on two of the coordinate planes and the resultants of the two projected systems of forces are found. The resultant of the original force system will be a force the projections of which on the two planes are the resultant forces of the projected systems. If the force polygon 
for each of the projected systems closes the resultant of the given system vanishes. A graphical method of determining the resultant of any non-coplanar system of forces is less convenient than is the algebraic method of solution.

32. Algebraic Method.-Before discussing the method of determining the resultant of any number of non-coplanar, concurrent forces the special

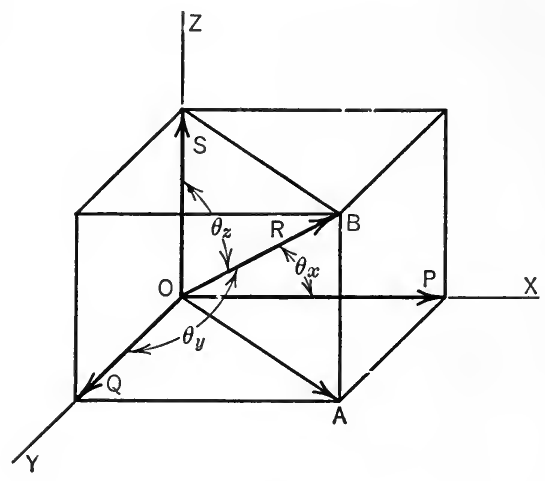

FIG. 55. case of three concurrent forces having action lines which are mutually perpendicular will be considered. In Fig. 55 are represented three such forces, $P, Q$, and $S$, the action lines of the forces being taken as the coordinate axes. The forces $P$ and $Q$ may be combined into a single force $O A$ the magnitude of which is $\sqrt{P^{2}+Q^{2}}$. The resultant of this force and the force $S$ is also the resultant of the given system of three forces and is represented by the vector $O B$, its magnitude being $\sqrt{P^{2}+Q^{2}+S^{2}}$. Hence the magnitude of the resultant, $R$, of the three forces and the angles $\theta_{x}, \theta_{y}$, and $\theta_{z}$, which the line of action of the resultant makes with the coordinate axes may be found from the equations,

$$
\begin{aligned}
R & =\sqrt{P^{2}+Q^{2}+S^{2}}, \\
\cos \theta_{x} & =\frac{P}{R}, \quad \cos \theta_{y}=\frac{Q}{R}, \quad \cos \theta_{z}=\frac{S}{R} .
\end{aligned}
$$

In finding the resultant of any number of non-coplanar, concurrent forces by the algebraic method it will be convenient to take the point of concurrence of the forces as the origin of a set of rectangular axes. Each force of the system may be resolved into components along the coordinate axes (Art. 12). The system is thus replaced by three collinear systems each of which may be replaced by a single force (Art. 21). Thus, the resultant of the components along the $x$-axis is a single force along the $x$-axis, the 
magnitude of which is expressed by $\Sigma F_{x}$. Similarly, the $y$-components may be replaced by a single force of magnitude $\Sigma F_{y}$ along the $y$-axis, etc. (Fig. 56). These three forces may be combined into a single force which is the resultant of the given system and which is completely defined by the following equations:

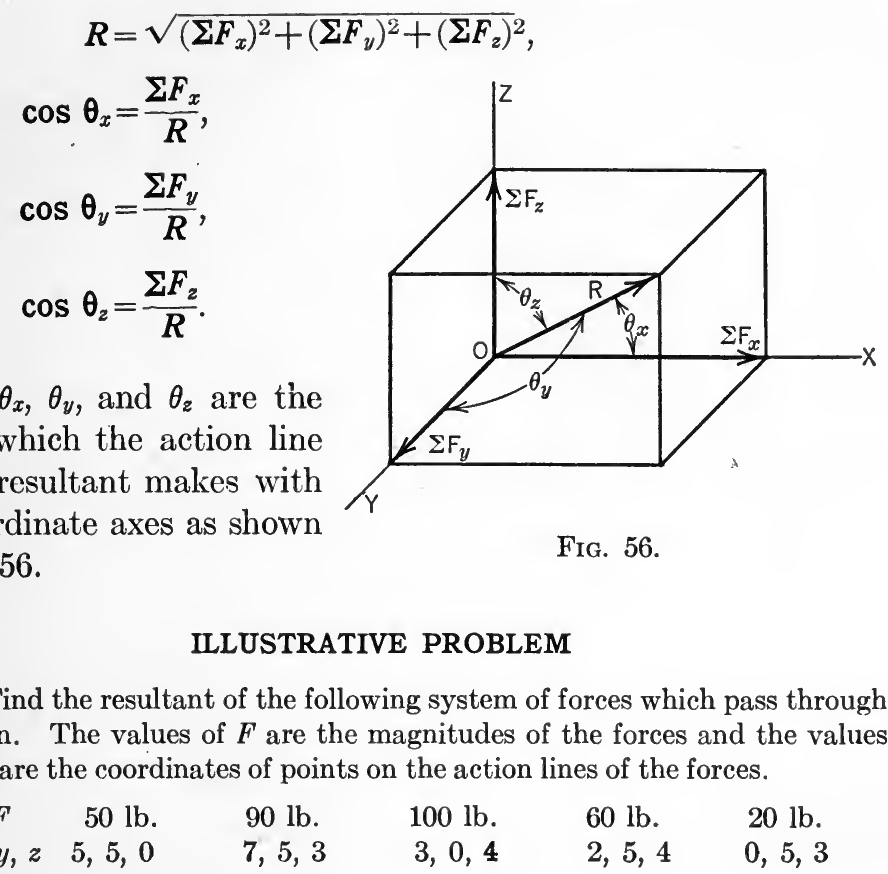

where $\theta_{x}, \theta_{y}$, and $\theta_{z}$ are the angles which the action line of the resultant makes with the coordinate axes as shown in Fig. 56.

Fig. 56 .

42. Find the resultant of the following system of forces which pass through the origin. The values of $F$ are the magnitudes of the forces and the values of $x, y, z$ are the coordinates of points on the action lines of the forces.
$x, z \quad 5,5,0$
$7,5,3$
$3,0,4$
$2,5,4$
$0,5,3$

Solution:

$$
\begin{aligned}
\Sigma F_{x} & =50 \cdot \frac{5}{\sqrt{50}}+90 \cdot \frac{7}{\sqrt{83}}+100 \cdot \frac{3}{5}+60 \cdot \frac{2}{\sqrt{45}}+20 \cdot \frac{0}{\sqrt{34}} \\
& =35.4+69.2+60+17.9+0 \\
& =182.5 \mathrm{lb} . \\
\Sigma F_{y} & =50 \cdot \frac{5}{\sqrt{50}}+90 \cdot \frac{5}{\sqrt{83}}+100 \cdot \frac{0}{5}+60 \cdot \frac{5}{\sqrt{45}}+20 \cdot \frac{5}{\sqrt{34}} \\
& =35.4+49.3+0+44.7+17.1 \\
& =146.5 \mathrm{lb} . \\
\Sigma F_{z} & =50 \cdot \frac{0}{\sqrt{50}}+90 \cdot \frac{3}{\sqrt{83}}+100 \cdot \frac{4}{5}+60 \cdot \frac{4}{\sqrt{45}}+20 \cdot \frac{3}{\sqrt{34}} \\
& =0+29.6+80+35.8+10.3 \\
& =155.7 \mathrm{lb} .
\end{aligned}
$$




$$
\begin{aligned}
& R=\sqrt{(182.5)^{2}+(146.5)^{2}+(155.7)^{2}}=281 \mathrm{lb} . \\
& \theta_{x}=\cos ^{-1} \frac{182.5}{281}=\cos ^{-1} .650=49^{\circ} 30^{\prime} . \\
& \theta_{y}=\cos ^{-1} \frac{146.5}{281}=\cos ^{-1} .521=58^{\circ} 35^{\prime} . \\
& \theta_{z}=\cos ^{-1} \frac{155.7}{281}=\cos ^{-1} .553=56^{\circ} 25^{\prime} .
\end{aligned}
$$

\section{PROBLEMS}

In the following problems the forces are concurrent at the origin. It is equired to find the resultants of the systems.
43. $\begin{aligned} & F \\ & x, y, z\end{aligned}$
$10 \mathrm{lb}$.
$20 \mathrm{lb}$.
$15 \mathrm{lb}$.
$1,2,1$
$2,2,3$
$3,1,2$
Ans, $R=43.1 \mathrm{lb} . \theta_{x}=53^{\circ} 10^{\prime} . \quad \theta_{y}=59^{\circ} 30^{\prime} . \theta_{z}=51^{\circ} 50^{\prime}$.
44. $F \quad 100 \mathrm{lb} . \quad 150 \mathrm{lb}$. $50 \mathrm{lb}$. $200 \mathrm{lb}$.
$x, y, z \quad 2,2,1 \quad 3,2,-2 \quad-4,-3,-5 \quad 3,-2,4$
Ans. $R=273 \mathrm{lb} . \theta_{x}=18^{\circ} 10^{\prime} . \quad \theta_{y}=80^{\circ} 45^{\prime} \quad \theta_{z}=74^{\circ} 20^{\prime}$.

$\begin{array}{llll}\text { 45. } & 10 \mathrm{lb} . & 30 \mathrm{lb} . & 20 \mathrm{lb} . \\ x, y, z & 1,2,1 & 1,4,2 & 2,3,3\end{array}$

\section{§6. Parallel Forces in Space}

33. Graphical Method.-The resultant of a system of noncoplanar, parallel forces (see Fig. 57) is either a force or a couple. If the resultant is a force the action line is parallel to the forces of the system, its magnitude and sense being given by the algebraic sum of the forces. The resultant force may be found graphically by a repeated use of the first method described in Art. 24 for finding the resultant of two parallel forces. Since this method of solution, however, involves constructions in various planes it is not convenient to use. The resultant force may also be found by the construction of a force and a funicular polygon as described in Art. 24. In using this method the forces are projected on two of the coordinate planes and the resultant of each of the two systems of projected forces is found. The resultant of the given force system is a force the projections of which on the two coordinate planes are the two resultants of the projected forces. 
34. Principle of Moments.-The algebraic sum of the moments of any number of non-coplanar, parallel forces about any line in space is equal to the moment of their resultant about the same line. The proof of this proposition is similar to the proof of the principle of moments given for a coplanar, parallel force system and will be left to the student. This principle will be used in the algebraic method of determining the resultant of a non-coplanar, parallel system in much the same way that it was used in Arts. 26 and 30 .

35. Algebraic Method.-In determining the resultant of a system of non-coplanar, parallel forces by the algebraic method it is convenient to select coordinate axes so that one axis is parallel to the forces. In Fig. 57 is shown a system of parallel forces referred to such a set of axes. The resultant, if a force, is parallel to the forces, its magnitude, $R$, being equal to the algebraic sum of the forces. The line of action of the resultant force is found by applying the principle of moments. Thus, if the algebraic sum of the moments of the forces with respect to the $x$-axis be denoted by $\Sigma M_{x}$ and the distance of the resultant from the $x$-axis be denoted by $\bar{y}$, then the principle of moments is expressed by the equation $R \bar{y}=\Sigma M_{x}$. In a similar manner, $R \bar{x}=\Sigma M_{y}$. The resultant, if a force, will then be completely defined by the following equations:

$$
\begin{aligned}
& R=\Sigma F, \\
& \bar{x}=\frac{\Sigma M_{y}}{R}, \\
& \bar{y}=\frac{\Sigma M_{x}}{R} .
\end{aligned}
$$

If the resultant of all, except one, of the forces of a parallel system is a force which is equal to that

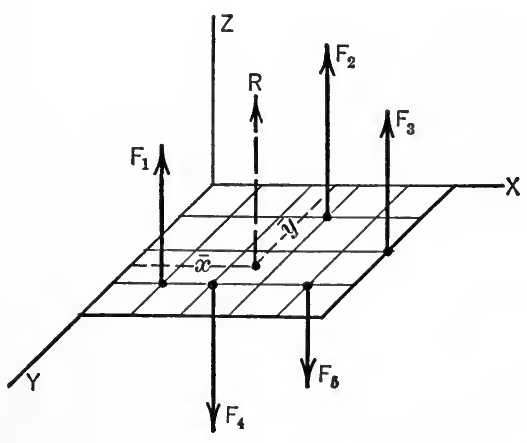

Fig. 57. one but of opposite sense, then $\Sigma F$ equals zero and hence the resultant is not a force and is therefore a couple. According to the principle of moments the moment, $C_{x}$, of the resultant couple with respect to the $x$-axis is 
equal to the algebraic sum of the moments of the forces with respect to the $x$-axis, that is,

Similarly,

$$
C_{x}=\Sigma M_{x}
$$

$$
C_{\nu}=\Sigma M_{\nu}
$$

Thus, the resultant is a couple which lies in a plane parallel to the $z$-axis, the moments of which, with respect to the $x$-and $y$-axes, are $C_{x}$ and $C_{y}$ as expressed above. Methods of expressing a resultant couple in terms of its component couples are discussed in Art. 36.

\section{ILLUSTRATIVE PROBLEM}

46. Find the resultant of the system of parallel forces as shown in Fig. 58. Each space in the figure represents $1 \mathrm{ft}$.

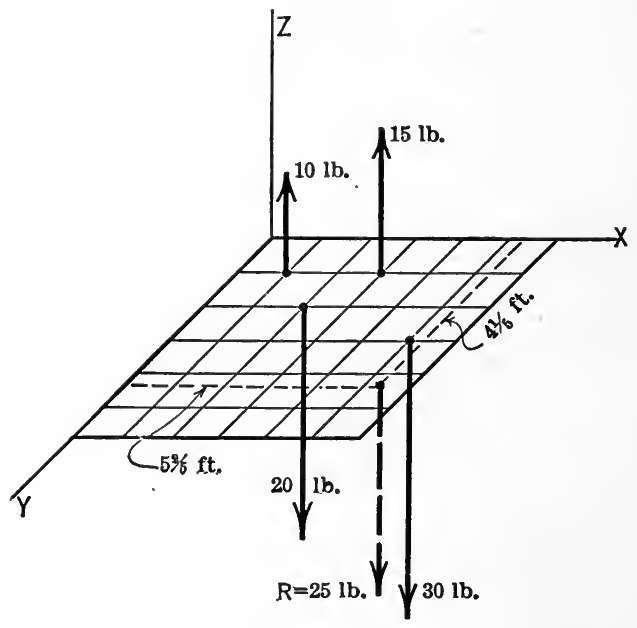

FIG. 58.

Solution:

$$
\begin{aligned}
R & =\Sigma F=10+15-20-30=-25 \mathrm{lb} . \\
\Sigma M_{x} & =20 \times 2+30 \times 3-10 \times 1-15 \times 1 . \\
& =105 \mathrm{lb} . \mathrm{ft} . \\
\Sigma M_{y} & =10 \times 1+15 \times 3-20 \times 2-30 \times 5 . \\
& =-135 \mathrm{lb} . \mathrm{ft} .
\end{aligned}
$$




$$
\therefore \bar{x}=\frac{135}{25}=5 \frac{2}{5} \mathrm{ft} \text {. }
$$

and

$$
\bar{y}=\frac{105}{25}=4 \frac{1}{5} \mathrm{ft} .
$$

Hence the resultant is a downward force of $25 \mathrm{lb}$. as shown in Fig. 58 .

Caution.-Care must be exercised in finding $\bar{x}$ and $\bar{y}$. For instance, in the above example if the value of $\Sigma M_{x}$ (+105 lb.-ft.) be divided by $R(-25 \mathrm{lb}$.) the quotient is $-4 \frac{1}{5} \mathrm{ft}$., which is not the value of $\bar{y}$, since a downward force of $25 \mathrm{lb}$. in this position would have a moment of $-105 \mathrm{lb} . \mathrm{ft}$. with respect to the $x$-axis. The magnitudes of $\bar{x}$ and $\bar{y}$ should be obtained by dividing the magnitudes of $\Sigma M_{y}$ and $\Sigma M_{x}$ by the magnitude of $R$ and their signs should be determined by inspection. The signs of $\bar{x}$ and $\bar{y}$, of course, must be such that the moment of the resultant will have the same sense of rotation as indicated by the algebraic sum of the moments of the forces of the system.

\section{PROBLEMS}

47. A board $6 \mathrm{ft}$. square is acted on by five forces as shown in Fig. 59 . Determine the resultant of the forces.

$$
\text { Ans. } R=+10 \mathrm{lb} . \quad \bar{x}=+2.5 \mathrm{ft} . \quad \bar{y}=+3.5 \mathrm{ft} .
$$

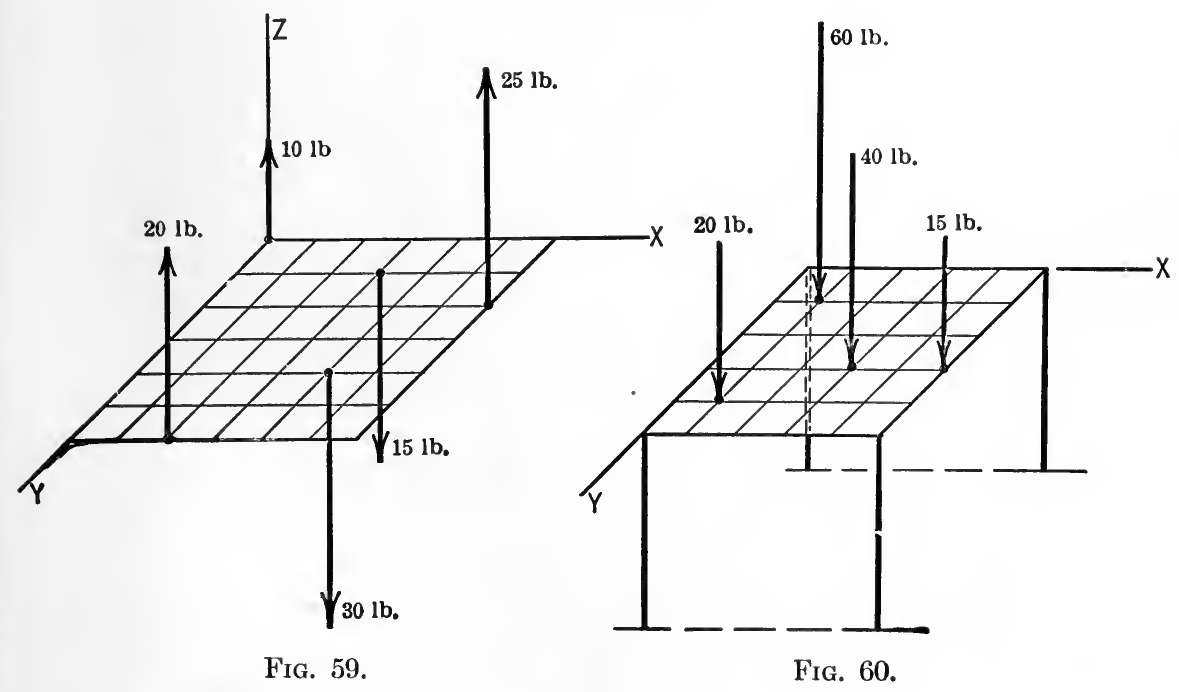

48. A table $5 \mathrm{ft}$. square carries four concentrated loads as shown in Fig. 60 . Find the resultant of the four loads.

Ans. $R=135 \mathrm{lb} . \quad \bar{x}=+2.04 \mathrm{ft} . \quad \bar{y}=+2.26 \mathrm{ft}$.

49. Find the resultant of the following system of forces which are parallel 
to the $z$-axis. The values of $x$ and $y$, expressed in feet, are the coordinates of the points where the action lines of the forces intersect the $x y$-plane.

$\begin{array}{cccccc}F & 20 \mathrm{lb} . & 10 \mathrm{lb} . & 25 \mathrm{lb} . & -15 \mathrm{lb} . & -10 \mathrm{lb} . \\ x, y & 1,3 & 3,2 & 6,6 & 2,5 & 6,4\end{array}$

\section{$\S 7$. Couples in Space}

36. Resultant of a System of Couples. Proposition.-The resultant of any number of couples is a couple.

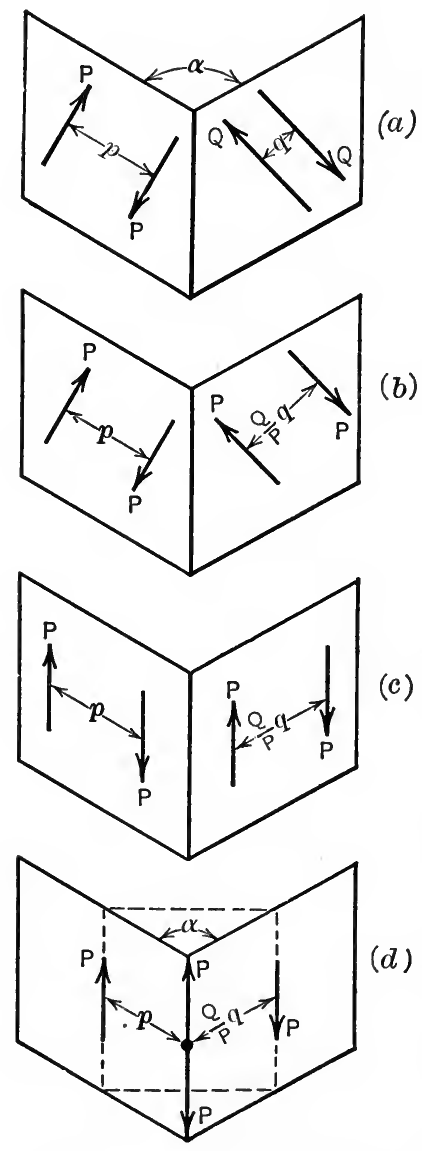

FIG. 61.

Proof.-It is sufficient to prove this proposition for two couples, only, since if two couples can be combined into a single resultant couple this couple can be combined with a third couple in exactly the same way and so on. Thus, consider the two couples $P p$ and $Q q$ in planes making an angle $\alpha$ with each other as shown in Fig. 61(a). The forces $Q, Q$ of

(b) the couple $Q q$ can be made equal to $P, P$ if the arm be changed to $\frac{Q q}{P}$ (Art. 27) as shown in Fig. 61(b). Each couple can then be rotated in its plane until the forces of the couples are parallel to the line of intersection of the two planes (Art. 27) as shown in Fig. 61(c). Now let the two couples be translated until one force of each couple lies in the line of intersection of the two planes. This translation can always be made so that the two forces in this line are opposite in sense and hence will cancel, thereby leaving a couple the forces of which are $P, P$ (Fig. 61d). The arm of this couple from trigonometry is,

$$
\sqrt{p^{2}+\frac{Q^{2} q^{2}}{P^{2}}-2 \frac{Q q p}{P} \cos \alpha} \text {. }
$$

Special Cases.-I. If the angle $\alpha$ equals $90^{\circ}$, that is, if the 
planes of the couples are perpendicular (Fig. 62), the moment, $C$, of the resultant couple is

$$
C=P \sqrt{p^{2}+\frac{Q^{2}}{P^{2}} q^{2}}=\sqrt{(P p)^{2}+(Q q)^{2}} .
$$

That is, the moment of the resultant couple is the square root of the sum of the squares of the moments of the two couples. The plane of the resultant couple makes an angle $\phi$ (Fig. 62) with the plane of the couple $P p$ such that

$$
\tan \phi=\frac{\frac{Q}{P} q}{p}=\frac{Q q}{P p},
$$

and the sense of rotation of the resultant couple is indicated in Fig. 62.

II. If $\alpha$ equals $180^{\circ}$, the couples are in the same or in parallel planes and hence have the same aspect. The moment of the resultant couple

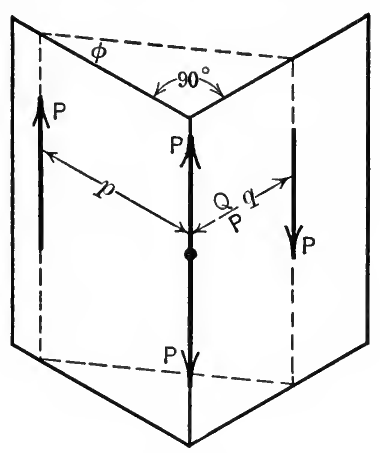

Fig. 62 . then is,

$$
C=P \sqrt{p^{2}+\frac{Q^{2}}{P^{2}} q^{2}+2 p \frac{Q}{P}} q=P\left(p+q \frac{Q}{P}\right)=P p+Q q .
$$

That is, the moment of the resultant couple is the algebraic sum of the moments of the two couples and its aspect, of course, is the same as that of each of the couples, the sense of rotation being indicated by the sign of the algebraic summation.

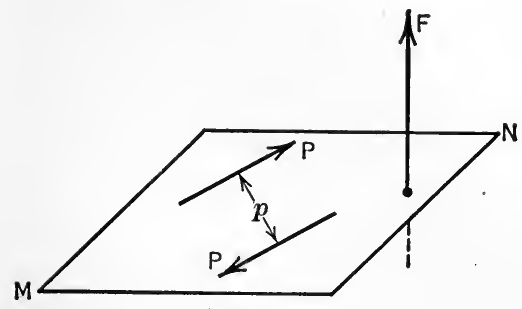

(a)

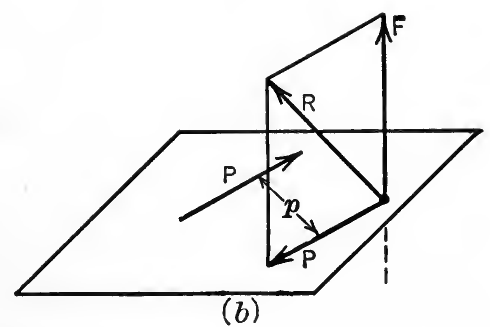

Fig. 63.

Resultant of a Couple and a Force.-A couple and a force not in the plane of the couple may be replaced by two non-coplanar forces. Thus, consider a couple $P p$ in the plane $M N$ and a force $F$ 
as shown in Fig. 63(a). The couple may be translated according to Art. 27 until one of the forces of the couple $P p$ and the force $F$ are concurrent (Fig. 63b). Therefore $P$ and $F$ now may be replaced by their resultant $R$ and hence the given system is replaced by a force $P$ in the plane of the original couple and a force $R$ not in the plane. Conversely, any two forces not in the same plane may be replaced by a force and a couple.

37. Composition of Couples by Means of Vectors. Proposition.-If any number of couples be represented by their vectors, the resultant of these vectors will represent completely the resultant couple.

It will be sufficient to prove the proposition for two couples. The extension of the proof to any number of couples is obvious.

Proof.-Given two couples $P_{1} p_{1}$ and $P_{2} p_{2}$ in planes which make an angle $\alpha$ with each other as shown in Fig. $64(a)$. It is
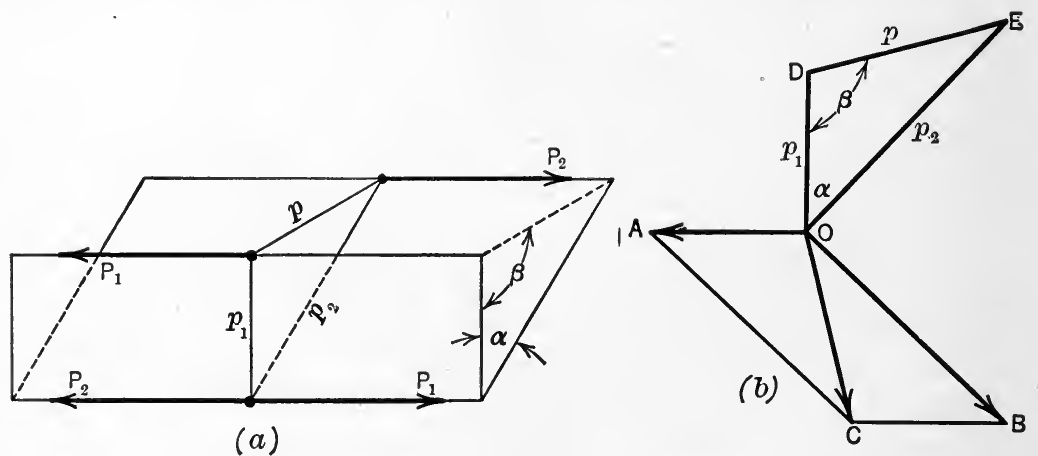

FIG. 64.

assumed that the couples have been transformed (see Art. 36) so that $P_{1}$ equals $P_{2}$. Let $D O E$ (Fig. 64b) represent a cross-section of the two planes shown in Fig. 64(a). The couple $P_{1} p_{1}$ can be represented by a vector $O A$ perpendicular to the plane of the couple, the length of $O A$ being proportional to $P_{1} p_{1}$. Likewise, the couple $P_{2} p_{2}$ can be represented by the vector $O B$ perpendicular to the plane of the couple, $O B$ being proportional to $P_{2} p_{2}$. Construct the parallelogram $O A C B$. In order to show that $O C$ represents completely the resultant couple it must be shown that $O C$ is perpendicular to the plane of the resultant couple (which is the same as showing that it is perpendicular to $D E$ ) and also that its 
length represents to scale the moment, $P p$, of the resultant couple. In the two triangles $O A C$ and $O D E$ the angle $O A C$ equals the angle $D O E$. The vector $O A$ is proportional to $P_{1} p_{1}$. This fact is expressed by the equation,

Similarly,

$$
O A=k P_{1} p_{1}
$$

Hence,

$$
O B=k P_{2} p_{2}=A C \text {. }
$$

$$
\frac{O A}{A C}=\frac{k P_{1} p_{1}}{k P_{2} p_{2}}=\frac{p_{1}}{p_{2}}=\frac{O D}{O E}
$$

Therefore, the triangles are similar and hence $O C$ is proportional to $D E$. That is,

$$
\frac{O C}{D E}=\frac{O A}{O D}=\frac{k P p_{1}}{p_{1}}=k P .
$$

Therefore,

$$
O C=D E \times k P=k P p .
$$

Hence $O C$ represents the moment of the resultant couple. Furthermore, $O C$ is perpendicular to $D E$, for, since the triangles $O A C$ and $O D E$ are similar and the corresponding sides $O A$ and $O D$ are perpendicular, it follows that the corresponding sides $O C$ and $D E$ are perpendicular. Hence, $O C$ represents the aspect as well as the magnitude of the resultant couple. It is plain from Fig. 64(b) that $O C$ also represents the sense of the resultant couple. Any number of couples then may be combined into a resultant couple by representing each couple by a vector and finding the resultant of the system of vectors. The resultant vector will represent completely the resultant couple.

- For an application of this method of combining couples see the discussion of the problem of balancing rotating masses, such as an engine crankshaft, in Chapter IX, Section III.

Three Couples in Mutually Perpendicular Planes.-Let the given planes be taken as the coordinate planes. The couple lying in the $y z$-plane may be represented completely by a vector along the $x$-axis. Let this vector be denoted by $C_{x}$. Similarly, the couples in the $x z$ - and $x y$-planes may be represented by vectors $C_{y}$ and $C_{z}$ along the $y$ - and $z$-axes respectively. The resultant of the three couples will be represented by the resultant of the three 
vectors $C_{x}, C_{y}, C_{\mathbf{z}}$. If the resultant of these three vectors be denoted by $C$ the resultant may be found from the equation,

$$
C=\sqrt{C_{x}^{2}+C_{y}^{\prime}+C_{z}^{2}} .
$$

Also, if $\phi_{x}, \phi_{y}$, and $\phi_{z}$ be the angles which the vector $C$ makes with the coordinate axes, these angles may be found from the equations,

$$
\phi_{x}=\cos ^{-1} \frac{C_{x}}{C^{\prime}}, \quad \phi_{y}=\cos ^{-1} \frac{C_{y}}{C^{\prime}}, \quad \phi_{z}=\cos ^{-1} \frac{C_{z}}{C^{\prime}} .
$$

That is, if the couples $C_{x}, C_{u}$, and $C_{z}$, which act on a given body, are replaced by the couple $C$ acting in the plane defined by the angles $\phi_{x}, \phi_{v}$, and $\phi_{z}$, the external effect on the body will be unchanged.

\section{ILLUSTRATIVE PROBLEM}

50. Determine the resultant of the three couples which act on the body (cube) as shown in Fig. 65. Each of the edges of the cube is $4 \mathrm{ft}$. in length.

Solution.-

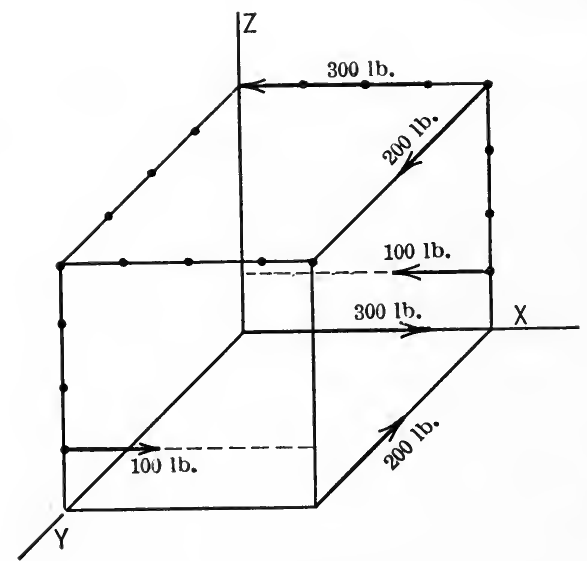

FIG. 65.

$$
\begin{aligned}
& C_{x}=200 \times 4=800 \mathrm{lb} . \mathrm{ft} . \\
& C_{y}=300 \times 4=1200 \mathrm{lb} . \mathrm{ft} . \\
& C_{z}=100 \times 4=400 \mathrm{lb} . \mathrm{ft} .
\end{aligned}
$$

Therefore,

$$
\begin{aligned}
C & =\sqrt{(800)^{2}+(1200)^{2}+(400)^{2} .} \\
& =1500 \mathrm{lb} . \mathrm{ft} . \\
\phi_{x} & =\cos ^{-1} \frac{800}{1500}=57^{\circ} 47^{\prime}, \\
\phi_{\nu} & =\cos ^{-1} \frac{1200}{1500}=36^{\circ} 50^{\prime}, \\
& \cdot \\
\phi_{z} & =\cos ^{-1} \frac{400}{1500}=74^{\circ} 30^{\prime} .
\end{aligned}
$$

Hence a single couple having a moment of $1500 \mathrm{lb} . \mathrm{ft}$. and located in a plane perpendicular to the line defined by the above angles will have the same external effect on the body as the three given couples.

38. Resolution of Couples.-In order to resolve a couple into two or more component couples, the vector representing the given couple may be resolved into component vectors each of which represents a component couple. 
Gne of the most important cases of the resolution of a couple is that in which the couple is resolved into three component couples lying in planes which are mutually perpendicular. Let the planes of the component couples be taken as the coordinate planes and let $\phi_{x}, \phi_{v}$, and $\phi_{z}$ be the angles which the vector representing the given couple, $C$, makes with the coordinate axes. If $C_{x}, C_{y}, C_{z}$, be the component couples lying in the $y z-, z x$-, and $x y$-planes, respectively, they may be found from the following equations:

$$
C_{x}=C \cos \phi_{x}, \quad C_{y}=C \cos \phi_{y}, \quad C_{z}=C \cos \phi_{z} .
$$

\section{§ 8. Non-concurrent, Non-parallel Forces in Space}

39. Graphical Method.-The resultant of a system of nonconcurrent, non-parallel forces in space may be a force and a couple, a single force, or a single couple. Further, since a force and a couple, not in the same plane, may be replaced by two noncoplanar forces (Art. 36), the resultant of such a system may also be regarded as two non-coplanar forces. If the graphical method is used in determining the resultant of such a system of forces, it is convenient to reduce the system to two forces rather than a force and a couple. The resultant may be found by selecting arbitrarily any plane which is not parallel to any of the action lines of the given forces and resolving each force, at the point where its action line pierces this plane, into two components, one perpendicular to this plane and one lying in the plane. The system consisting of the components which lie in the plane may be combined according to the method of Art. 28 and the components which are perwendicular to the plane may be combined according to the method of Art. 33. If the resultant of each of these systems is a force these two forces may be regarded as the resultant of the given system. The two forces may be replaced, however, by a force and a couple. Further, in special cases the force may vanish and thereby leave a single couple as the resultant of the given system. Again, the couple may vanish, in which case the resultant of the system is a force. If both the force and couple vanish the given force system has no resultant.

40. Principle of Moments.-The algebraic sum of the moments of the forces of any non-coplanar, non-concurrent, non-parallel system about any line is equal to the moment of the resultant 
of the system about the same line. Since the resultant in general consists of a force and a couple, the moment of the resultant about any line must be regarded as the algebraic sum of the moments of the force and the couple about the line.

41. Algebraic Method.-When the resultant of a system of non-concurrent, non-parallel forces in space is a force and a couple, the force may be made to pass through any arbitrary point, but, for different positions of this point, the moment of the couple will vary. In determining the resultant force and couple by the algebraic method it is convenient to select a rectangular set of coordinate axes so that the origin is the arbitrary point through which the resultant force passes. Each force of the system may be replaced by an equivalent parallel force through the origin and a couple as in Art. 18. Thus the system is reduced to a system of concurrent forces through the origin and a system of couples. The concurrent system at the origin may be combined into a resultant force, as in Art. 32, which is completely defined by the following equations:

$$
\begin{aligned}
& R=\sqrt{\left(\Sigma F_{x}\right)^{2}+\left(\Sigma F_{y}\right)^{2}+\left(\Sigma F_{z}\right)^{2}}, \\
& \theta_{x}=\cos ^{-1} \frac{\Sigma F_{x}}{R}, \\
& \theta_{y}=\cos ^{-1} \frac{\Sigma F_{y}}{R}, \\
& \theta_{z}=\cos ^{-1} \frac{\Sigma F_{z}}{R} .
\end{aligned}
$$

The system of couples may be replaced by a single couple as in Art. 37. For convenience in determining the resultant couple it will be considered to be resolved, as in Art. 38, into three component couples lying in the coordinate planes. Let the couple lying in the $z y$-plane be denoted by $C_{x}$, since it may be represented by a vector along the $x$-axis. The vector which represents this couple will also be denoted by $C_{x}$. Similarly, let the couples in the other two planes, as well as the vectors which represent them, be denoted by $C_{\nu}$ and $C_{z}$. The given system then is equivalent to the system shown in Fig. 66, namely, a force $R$ through the origin and the couples $C_{x}, C_{y}$, and $C_{z}$. Since the two systems are equivalent, the sum of the moments of the forces of the given system about any line is equal to the sum of the moments of the 
forces of the system shown in Fig. 66 about the same line. Thus, let moments be taken about the $x$-axis and let the moment of the given system about the $x$-axis be denoted by $\Sigma M_{x}$. The only part of the system shown in Fig. 66 which has a moment about the $x$-axis is the couple lying in the $y z$-plane, and the moment of this couple is $C_{x}$. Hence, $\Sigma M_{x}=C_{x}$. Similarly $\Sigma M_{y}=C_{y}$ and $\Sigma M_{z}=C_{z}$. The resultant of the three couples then may be determined as in Art. 36. Thus the resultant couple is defined by the following equations:

$$
\begin{aligned}
& C=\sqrt{\left(\Sigma M_{x}\right)^{2}+\left(\Sigma M_{y}\right)^{2}+\left(\Sigma M_{z}\right)^{2}}, \\
& \phi_{x}=\cos ^{-1} \frac{\Sigma M_{x}}{C} \\
& \phi_{y}=\cos ^{-1} \frac{\Sigma M_{y}}{C} \\
& \phi_{z}=\cos ^{-1} \frac{\Sigma M_{z}}{C} .
\end{aligned}
$$

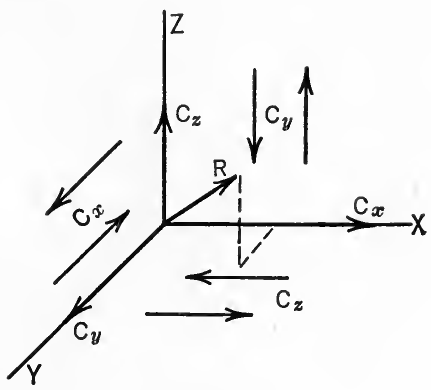

Fig. 66.

All of the force systems which have been discussed above may be considered as special cases of this system, and the six equations which define the resultant of this system will reduce to the same equations which were found necessary to define the resultants of the simpler systems.

\section{ILLUSTRATIVE PROBLEM}

51. Find the resultant of the system of four forces which act on the cube as shown in Fig. 67. Each side of the cube is $4 \mathrm{ft}$. long.

Solution: In resolving the 10-1b. force into its three rectangular components, $A$ will be taken as the point of application of the force and at this point the force may be resolved into two components, one along $A C$ and one along $A B$. The latter component may be resolved into components along $A E$ and $A F$. The quantities needed in the solution may be put in tabular form as follows, forces being expressed in pounds and moments in pound-feet.

\begin{tabular}{lrrrrrr}
$F$ & $F_{x}$ & $F_{y}$ & $F_{z}$ & $M_{x}$ & $M_{y}$ & $M_{z}$ \\
10 & -4.67 & -6.25 & -6.25 & 0.00 & -6.25 & 6.25 \\
15 & 0.00 & -10.60 & -10.60 & 0.00 & -42.42 & 42.42 \\
18 & 18.00 & 0.00 & 0.00 & 0.00 & -72.00 & 18.00 \\
40 & 0.00 & 40.00 & 0.00 & 40.00 & 0.00 & 0.00 \\
\hline
\end{tabular}

$\Sigma F_{x}=13.33 \quad \Sigma F_{y}=23.15 \quad \Sigma F_{z}=-16.85 \quad \Sigma M_{x}=40.0 \quad \Sigma M_{y}=-120.7 \quad \Sigma M_{z}=66.66$ 


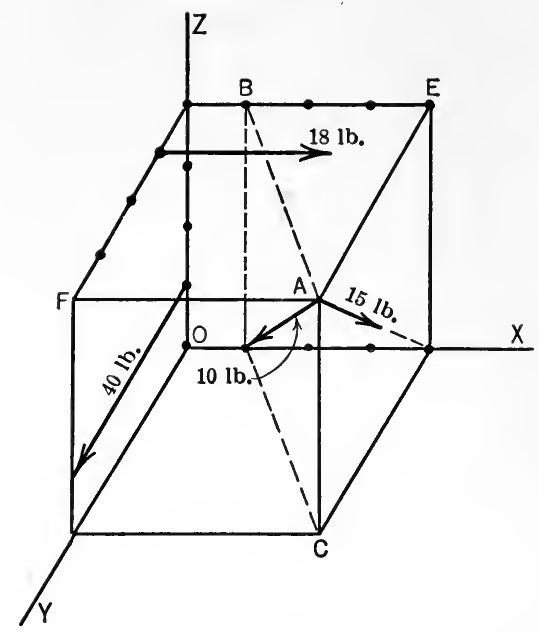

FIG. 67.

$$
\begin{aligned}
& R=\sqrt{(13.33)^{2}+(23.15)^{2}+(16.85)^{2}} \\
& =31.6 \mathrm{lb} \text {. } \\
& \theta_{x}=\cos ^{-1} \frac{13.33}{31.6}=65^{\circ} 0^{\prime} \\
& \theta_{y}=\cos ^{-1} \frac{23.15}{31.6}=42^{\circ} 50^{\prime} \\
& \theta_{z}=\cos ^{-1} \frac{-16.85}{31.6}=122^{\circ} 15^{\prime} \\
& C=\sqrt{(40)^{2}+(120.7)^{2}+(66.66)^{2}} \\
& =144 \text { lb.-ft. } \\
& \phi_{x}=\cos -\frac{40}{144}=73^{\circ} 50^{\prime} \\
& \phi_{y}=\cos ^{-1} \frac{-120.7}{144}=146^{\circ} 55^{\prime} \\
& \phi_{z}=\cos ^{-1} \frac{66.66}{144}=62^{\circ} 25^{\prime} \text {. }
\end{aligned}
$$

NoтE.--In specifying the angles which the vectors representing the resultant force and the resultant couple make with the coordinate axes, the smaller of the two angles which each vector makes with the positive end of the given axis is specified.

\section{PROBLEMS}

52. Find the resultant of the three forces which act on the cube as shown in Fig. 68. Each side of the cube is $4 \mathrm{ft}$. long.

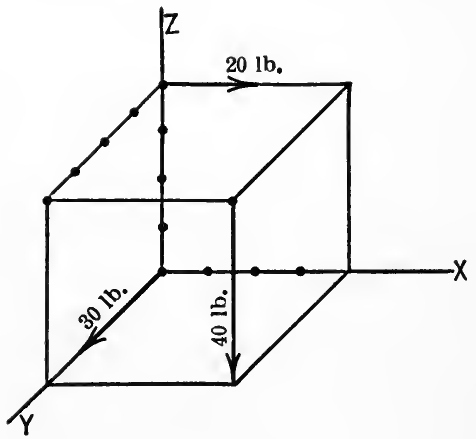

Fig. 68.

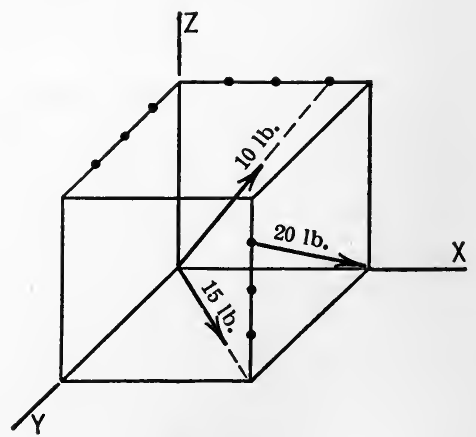

FIg. 69.

53. The sides of the cube shown in Fig. 69 are each $4 \mathrm{ft}$. long. Find the resultant of the three forces which act on the cube. 


\section{CHAPTER III}

\section{EQUILIBRIUM OF FORCE SYSTEMS}

\section{§ 1. InTRODUCTION}

42. Preliminary.-In the preceding chapter equations and graphical constructions were found by the use of which the resultants of the various force systems may be determined. In the present chapter are determined the algebraic and graphical conditions which the forces of the various force systems must satisfy in order that the resultants of the force systems shall be equal to zero; that is, in order that the force systems shall be in equilibrium. If a force system which is in equilibrium acts on a body, the body is either at rest or has a uniform motion.

The independent equations which must be satisfied by the forces which hold a body in equilibrium are called the equations of equilibrium, and the graphical constructions which the forces must satisfy are sometimes called equilibrium diagrams or equilibrium polygons.

Many problems in engineering practice involve bodies which are in equilibrium under the action of a system of forces as, for example, a bridge, roof-truss, crane, etc. In such problems there may be certain elements of the forces acting on the body which are unknown as, for example, the magnitude or the direction of one or more of the forces. These unknown elements or quantities may be found if their number is not greater than the number of equations of equilibrium for the force system involved. Such force systems are said to be statically determinate. If the number of unknown quantities in a force system is greater than the number of equations of equilibrium for that particular force system, the force system is said to be statically indeterminate, as, for example, the forces which act on a horizontal beam which rests on three or more supports and carries known vertical loads. The beam is in equilibrium under the action of a system of coplanar, parallel forces all of which are known except the three upward reactions of the supports. As is shown in Art. 49, there are only two independent 
equations of equilibrium for such a force system, and hence the three reactions cannot be found from the equations of equilibrium. The force system is therefore statically indeterminate.

43. Graphical Conditions of Equilibrium.-In the previous chapter it was shown that the resultant of an unbalanced force system in a plane is either a force or a couple. Further, it was shown that if the resultant is a force, it is represented in magnitude and in direction by the closing side of the force polygon, and that if the resultant is a couple, the two forces of the couple act along the first and last strings of the funicular polygon. Hence, if the force polygon closes, the resultant cannot be a force, but may be a couple. If, however, the funicular polygon also closes, that is, if the first and last strings along which the two forces of the couple act are collinear, the two forces cancel and hence the resultant couple vanishes. Hence there are two conditions which the forces of a coplanar force system must satisfy if they have no resultant, that is, if the forces are in equilibrium.

(1) The force polygon must close. If this condition is satisfied the resultant cannot be a force.

(2) The funicular polygon must close. If this condition is satisfied the resultant cannot be a couple.

The conditions of equilibrium for non-coplanar force systems may be stated in a similar manner. In order to determine the resultant of a non-coplanar system graphically, the forces of the system are projected on two of the coordinate planes and a force and a funicular polygon is drawn for each of the projected systems. The conditions of equilibrium for a non-coplanar system, then, are that the force and funicular polygons for each of the projected systems must close.

44. Algebraic Conditions of Equilibrium.-The two conditions which the graphical diagrams for a balanced force system must satisfy as stated in the preceding article may also be expressed algebraically. Thus, if the force polygon closes, the projections (components) of the forces on any line also form a closed polygon, as shown in Fig. 70, and since these components are collinear their vector sum is the same as their algebraic sum. Hence, the fict that the force polygon for the components closes may be expressed by stating that the algebraic sum of the components is equal to zero. Therefore, if the force polygon for the given system of 
forces closes, the algebraic sum of the components of the forces in any direction is equal to zero.

If the funicular polygon closes, the resultant cannot be a couple, since the first and last strings of the funicular polygon are collinear and hence the algebraic sum of the moments of the two equal and opposite forces which act along these strings is equal to zero. But the algebraic sum of the moments of these two forces is equal to the algebraic sum of the moments of the forces of the system. Therefore,

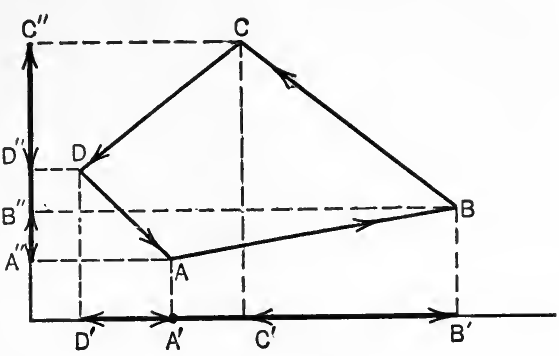

FIG. 70. the statement that the funicular polygon must close is equivalent to the statement that the algebraic sum of the moments of the forces of the system must equal zero. Hence the algebraic conditions of equilibrium are:

(1) The algebraic sum of the components of the forces in any direction must equal zero.

(2) The algebraic sum of the moments of the forces about any axis must equal zero.

An infinite number of equations could be written in accordance with these conditions by taking different directions of resolution and different moment axes, but all of the equations would not be independent. The number of independent equations is different for the various force systems, as will be discussed in the succeeding articles, but for any force system the independent equations of equilibrium are the equations which are necessary and sufficient to ensure that the resultant for that particular force system shall be equal to zero.

If a given body is in equilibrium under the influence of a system of forces some of which are unknown, wholly or in part, these unknown elements may be found by applying the equations of equilibrium which apply to that particular system of forces.

If the number of unknown elements in a system of forces which is in equilibrium is equal to the number of independent equations of equilibrium for that particular system, the determination of all of the unknown elements involves the use of all of the equations of 
equilibrium. Frequently, however, it is not required to determine all of the unknown elements in such a system, for, a single element only may be required, as for example, the magnitude of a certain force, the line of action of which is known. In such cases the unknown element may frequently be found by using only one of the equations of equilibrium. In applying the equilibrium equations the work may be materially simplified by properly selecting the directions of resolution and the axes of moments. Before applying the equations of equilibrium to any system of forces which holds a body in equilibrium it is important to have a clear idea of the forces which act on the body. For this purpose a free-body diagram is drawn. A free-body diagram is a diagram of a body showing the actions of all other bodies (forces) on the body considered. It does not show the actions of the given body on other bodies.

\section{§ 2. Collinear Forces}

45. Equations of Equilibrium.-A system of collinear forces is in equilibrium if the forces of the system satisfy either of the following equations:

or

$$
\Sigma F=0, \quad \cdot \quad \cdot \quad \cdot \quad \cdot \quad \cdot \quad \cdot
$$

where $A$ is any point not on the action line of the forces.

Proof.-As shown in Art. 21, if a collinear force system is not in equilibrium, the resultant of the force system is a force having the same action line as the forces and having a magnitude, $R$, which is given by the equation, $R=\Sigma F$. If, then, the equation $\Sigma F=0$, is satisfied the resultant is not a force and therefore the system is in equilibrium. The equation $\Sigma M_{A}=0$ is also sufficient to ensure equilibrium, for, in order to satisfy this equation, the resultant force must pass through the point $A$. But this is impossible, since the resultant force, if there be one, has the same line of action as the forces and hence cannot pass through $A$.

Therefore, if either one of the equations (1) and (2) is satisfied, the resultant is equal to zero and hence there is but one independent equation of equilibrium for a collinear force system. 


\section{ILLUSTRATIVE PROBLEM}

54. Two men pull on a rope with forces of $100 \mathrm{lb}$. each (Fig. 71a). What is the stress in the rope?

Solution.- Suppose the rope to be divided into two parts $A$ and $B$ as shown in Fig. 71(b). Consider as a free-body the part $A$. The forces acting

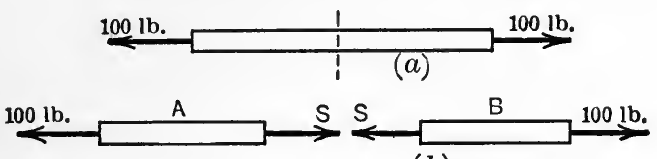

(b)

FIG. 71.

on $A$ are two in number, namely, the $100-\mathrm{lb}$. force and the force exerted by $B$ on $A$. The latter is the internal stress required. Let this stress be denoted by $S$. The equation of equilibrium then becomes,

Therefore,

$$
\Sigma F=S-100=0 .
$$

$$
S=100 \mathrm{lb} .
$$

Obviously, $B$ could have been taken as the free body and the same result would have been obtained.

\section{§ 3. Concurrent Forces in a Plane}

46. Equations of Equilibrium.-A system of coplanar, concurrent forces is in equilibrium if the forces of the system satisfy the following equations:

$$
\left.\begin{array}{l}
\Sigma F_{x}=0 \\
\Sigma F_{y}=0
\end{array}\right\} \cdot \quad \cdot \quad \cdot \quad \cdot \quad \cdot \quad \cdot
$$

where $x$ and $y$ denote any two non-parallel lines in the plane. It is convenient, however, to take as the two lines a set of rectangular axes with the point of concurrence of the forces as origin.

Proof.-In Art. 23 it was shown that if a concurrent system of forces in a plane is not in equilibrium the resultant is a force, the components of which are equal to $\Sigma F_{x}$ and $\Sigma F_{y}$. If, then, the forces of the system satisfy the equation $\Sigma F_{x}=0$ the resultant cannot have a component along the $x$-axis and if the equation $\Sigma F_{y}=0$ is satisfied the resultant cannot have a component along the $y$-axis. Therefore if both of these equations are satisfied, the resultant cannot be a force and hence the system must be in 
equilibrium. There are, then, only two independent equations of equilibrium for a coplanar, concurrent system of forces.

Another set of independent equations which, if satisfied by the forces of a coplanar, concurrent force system, are sufficient to ensure equilibrium may be expressed as follows:

$$
\left.\begin{array}{rl}
\Sigma F_{x} & =0 \\
\Sigma M_{A} & =0
\end{array}\right\} \cdot \ldots \cdot \ldots
$$

where $x$ denotes any line in the plane (taken for convenience as one of two rectangular axes through the point of concurrence of the forces) and $A$ is any point in the plane not on the $y$-axis.

Proof.-If the equation $\Sigma F_{x}=0$ is satisfied, the resultant cannot have a component along the $x$-axis, that is, the resultant, if there be one, must lie along the $y$-axis. If the equation $\Sigma M_{A}=0$ is satisfied, the resultant, if there be one, must pass through the point $A$. It is impossible for a force to satisfy the two equations simultaneously, and hence if both of the equations are satisfied the system is in equilibrium.

A third set of equations of equilibrium for a coplanar, concurrent force system is as follows:

$$
\left.\begin{array}{l}
\Sigma M_{A}=0 \\
\Sigma M_{B}=0
\end{array}\right\} \cdot \ldots . . . . .
$$

where $A$ and $B$ are any two points in the plane of the forces, provided that the line joining $A$ and $B$ does not pass through the point

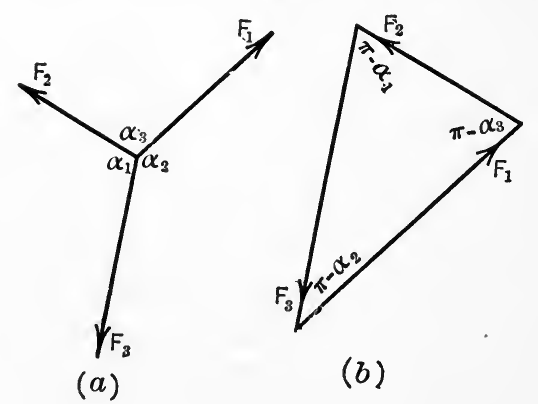

FIG. 72. at which the forces are concurrent. The proof that these equations are sufficient to insure equilibrium will be left to the student.

47. Lami's Theorem.When a coplanar, concurrent force system consists of only three forces, the equations of equilibrium may be expressed in a special form known as Lami's theorem. Let Fig. 72(a) represent three concurrent forces in equilibrium. The force polygon for the three forces is shown 
in Fig. $72(b)$. Since each side of a triangle is proportional to the sine of the opposite angle, the following equations are obtained from the force polygon:

That is,

$$
\frac{F_{1}}{\sin \left(\pi-\alpha_{1}\right)}=\frac{F_{2}}{\sin \left(\pi-\alpha_{2}\right)}=\frac{F_{3}}{\sin \left(\pi-\alpha_{3}\right)}
$$

$$
\frac{F_{1}}{\sin \alpha_{1}}=\frac{F_{2}}{\sin \alpha_{2}}=\frac{F_{3}}{\sin \alpha_{3}} \text {. }
$$

Hence, if three concurrent forces are in equilibrium, the magnitude of each force is proportional to the sine of the angle included between the action lines of the other two. This statement is known as Lami's theorem.

48. Three Forces in Equilibrium.-If three forces are in equilibrium the forces must be coplanar and either concurrent or parallel. In order that the three forces shall be in equilibrium, the resultant of any two of the forces must be a force which is collinear with the third force, of equal magnitude, and of opposite sense. Now, the resultant of the two forces will have the same line of action as the third force only if the two intersect on the action line of the third force, or if the two forces are parallel to the third force. Hence, the three forces must be either concurrent or parallel. This principle is of considerable importance, as it simplifies the solution of many problems. Consider, for example, the crane shown in Fig. 73(a). The forces acting on the crane are the reaction $R_{1}$ at the upper end (assumed to be horizontal), the load $W$, the weight of the crane (not shown), and the reaction $R_{2}$ at the lower end, the direction

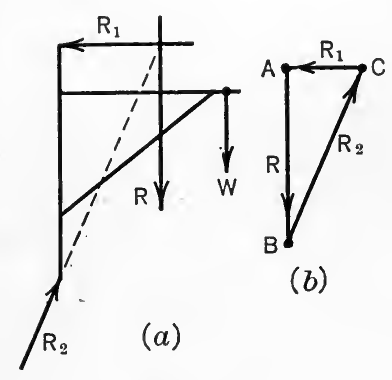

FIG. 73. of the latter force being unknown.

The load $W$ and the weight of the crane may be replaced by a single resultant force $R$, and the system will then consist of three forces $R_{1}, R_{2}$, and $R$. Since the three forces must be concurrent, $R_{2}$ must pass through the point of intersection of $R_{1}$ and $R$, and hence its action line is determined as indicated by the dotted line. The magnitudes of the reactions $R_{1}$ and $R_{2}$ may now be determined by drawing the force polygon (Fig. 73b). The force polygon 
is constructed by drawing $A B$ to represent the known force $R$ and by drawing from $A$ and $B$ lines parallel to $R_{1}$ and $R_{2}$, respectively, which intersect at $C$. The reaction $R_{1}$ is represented by $C A$ and $R_{2}$ is represented by $B C$.

\section{ILLUSTRATIVE PROBLEMS}

55. A body is held in equilibrium by a system of three concurrent forces as shown in Fig. 74. Find the values of $P$ and $\theta$.

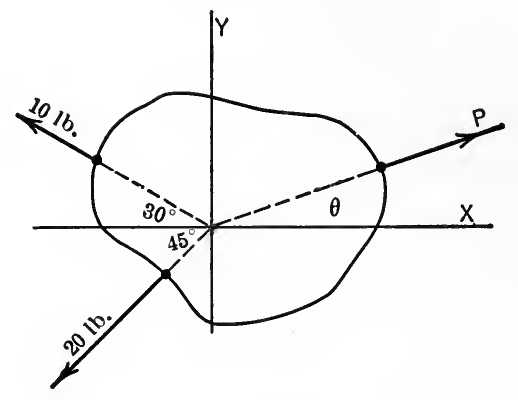

Fig. 74.

Solution.-The equations of equilibrium are,

$$
\Sigma F_{x}=P \cos \theta-10 \cos 30^{\circ}-20 \cos 45^{\circ}=0 \text {. }
$$

$\therefore \quad P \cos \theta=8.66+14.14=22.80 \mathrm{lb}$.

$$
\Sigma F_{\nu}=P \sin \theta+10 \sin 30^{\circ}-20 \sin 45^{\circ}=0 .
$$

$\therefore \quad P \sin \theta=14.14-5.00=9.14 \mathrm{lb}$.

By dividing (2) by (1), $\theta$ may be obtained. Thus,

$$
\begin{aligned}
& \tan \theta=\frac{9.14}{22.80}=.401 . \\
& \therefore \theta=21^{\circ} 50^{\prime} .
\end{aligned}
$$

By squaring and adding (1) and (2) $P$ may be obtained. Thus,

$$
\begin{aligned}
P^{2} & =(22.80)^{2}+(9.14)^{2}=603.5, \\
\therefore \quad P & =24.5 \mathrm{lb} .
\end{aligned}
$$


56. Fig. 75(a) represents a lower panel point of a pin-connected Pratt truss. The stresses in two of the members are $1000 \mathrm{lb}$. and $3000 \mathrm{lb}$. as shown. Find the stresses, $P$ and $Q$, in the other members.
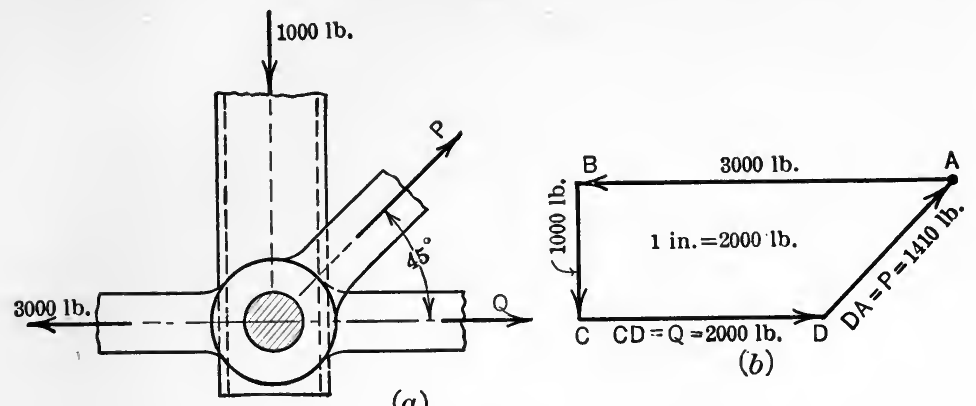

(a)

Fig. 75 .

Algebraic Solution.-

$$
\begin{aligned}
& \Sigma F_{x}=Q+P \cos 45^{\circ}-3000=0, \quad . \quad . \quad . \quad . \quad \\
& \Sigma F_{y}=P \sin 45^{\circ}-1000=0 .
\end{aligned}
$$

From (2),

$$
P=\frac{1000}{\sin 45^{\circ}}=1414 \mathrm{lb} \text {. }
$$

Substituting in (1),

$$
\begin{aligned}
Q & =3000-1414 \cos 45^{\circ} . \\
& =3000-1000=2000 \mathrm{lb} .
\end{aligned}
$$

Graphical Solution.-The problem may be solved graphically by constructing a closed force polygon as shown in Fig. 75(b). The polygon is constructed as follows: Vectors $A B$ and $B C$ are drawn to represent the $3000-1 \mathrm{~b}$. and 1000-lb. forces respectively. A line is then drawn from $C$ parallel to the direction of the force $Q$ and a line is drawn from $A$ parallel to the direction of the force $P$. These lines intersect at $D . \quad Q$ is then represented by $C D$ and $P$ by $D A$. The magnitudes of $Q$ and $P$ are found by measuring, according to the scale indicated, to be $2000 \mathrm{lb}$. and $1410 \mathrm{lb}$., respectively.

\section{PROBLEMS}

57. A sphere weighing $100 \mathrm{lb}$. rests between two smooth planes as indicated in Fig. 76. Find the reactions of the planes on the sphere.

Ans. $R_{1}=51.7 \mathrm{lb}$; $R_{2}=73.1 \mathrm{lb}$.

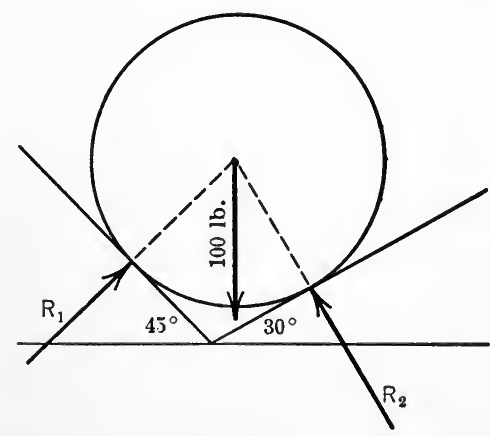

FIg. 76. 
58. A body weighing $80 \mathrm{lb}$. is supported by means of a number of cords as shown in Fig. 77. Find the tensions in the cords and the value of $\theta$.

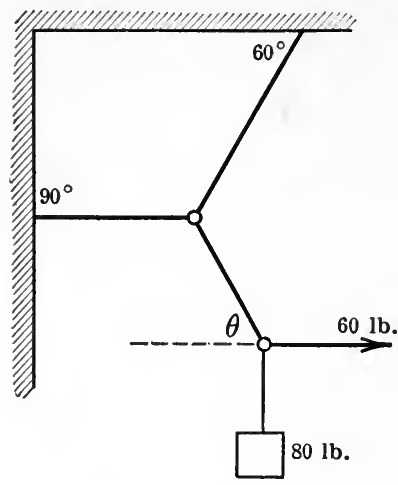

Fig. 77.

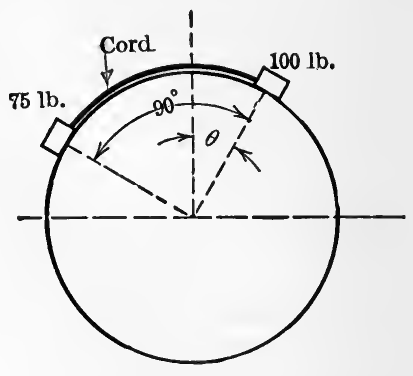

Fig. 78.

59. Two bodies weighing $75 \mathrm{lb}$. and $100 \mathrm{lb}$. rest on a smooth cylinder and are connected by a cord as shown in Fig. 78. Find the reactions of the cylinder on the bodies, the tension in the cord, and the value of $\theta$.

\section{\$4. Parallel Forces in a Plane}

49. Equations of Equilibrium.-A coplanar, parallel force system is in equilibrium if the forces of the system satisfy the equations,

$$
\left.\begin{array}{rl}
\Sigma F & =0 \\
\Sigma M_{A} & =0
\end{array}\right\} \cdot
$$

where $A$ is any point in the plane of the forces.

Proof.-According to Art. 26, the resultant of a coplanar, parallel force system which is not in equilibrium is either a force or a couple. If the resultant is a force the magnitude, $R$, is expressed by the equation $R=\Sigma F$, and if the resultant is a couple the moment, $C$, is expressed by the equation $C=\Sigma M$. If $\Sigma F=0$ the resultant is not a force and if $\Sigma M_{A}=0$ the resultant is not a couple. Hence, if both equations are satisfied the resultant of the force system can be neither a force nor a couple and therefore the system is in equilibrium. Two equations, then, are necessary and sufficient to ensure that the forces are in èquilibrium. In other words, there are only two indepen- 
dent equations of equilibrium for a system of parallel forces in a plane.

Another set of equations of equilibrium for a system of coplanar, parallel forces may be written as follows:

$$
\left.\begin{array}{l}
\Sigma M_{A}=0 \\
\Sigma M_{B}=0
\end{array}\right\}, \cdot \ldots . . . \cdot .
$$

where $A$ and $B$ are any two points in the plane, provided that the line connecting $A$ and $B$ is not parallel to the forces of the system. The proof that these equations are sufficient and necessary to ensure that the forces are in equilibrium will be left to the student.

An important problem in the equilibrium of coplanar, parallel forces is one in which the magnitudes of two forces are required, all of the other elements of the forces being known. The graphical solution of this problem is of particular interest. The graphical as well as the algebraic method of solution of such a problem is illustrated by Problem 62 .

\section{ILLUSTRATIVE PROBLEMS}

60. In the steelyard shown in Fig. 79 the distance $A B=5$ in. Find the force $R$ acting at $B$ and the distance $B C$ if the steelyard is balanced.

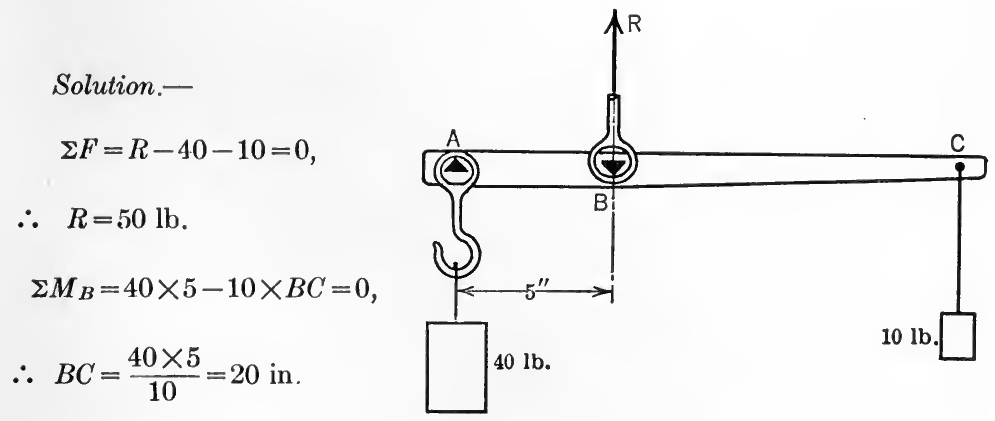

Fig. 79.

61. A load of $1200 \mathrm{lb}$. is applied to a beam $A B$ as shown in Fig. $80(a)$. The left end of the beam is carried by a second beam $C D$. Find the reactions on the second beam at $C$ and $D$. 
Solution.-The free-body diagrams for the two beams are shown in Fig. $80(b)$ and $80(c)$. The equation $\Sigma M_{A}=0$ becomes

$$
\begin{aligned}
& 1200 \times 9-R_{B} \times 12=0, \\
& \therefore R_{B}=900 \mathrm{lb} .
\end{aligned}
$$

There is, then, a load of $900 \mathrm{lb}$. acting on the beam $C D$ at $B$. The two reactions $R_{C}$ and $R_{D}$ may be found by applying either set of equilibrium equations. Thus, using the equations $\Sigma M_{C}=0$ and $\Sigma M_{D}=0$, the two reactions may be found as follows,

$$
\begin{aligned}
R_{D} \times 10-900 \times 6 & =0, \quad \therefore R_{D}=540 \mathrm{lb} . \\
-R_{C} \times 10+900 \times 4=0, & \therefore R_{C}=360 \mathrm{lb} .
\end{aligned}
$$

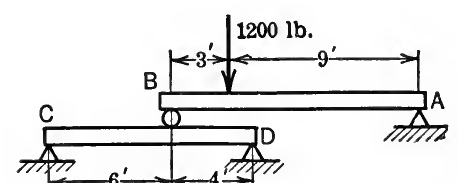

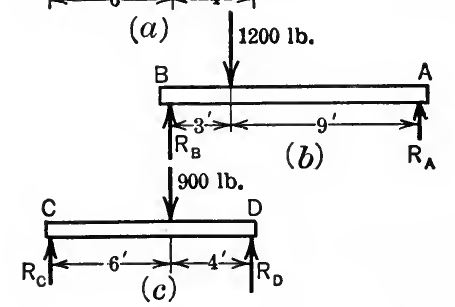

FIG. 80.

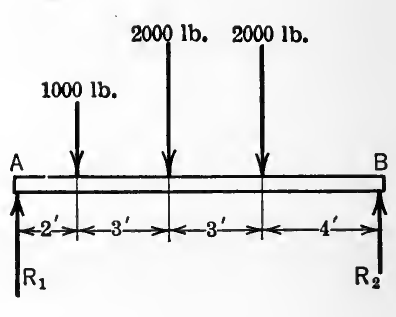

Fig. 81.

62. A beam $12 \mathrm{ft}$. long carries three loads as shown in Fig. 81 . Find the reactions at the ends of the beam.

$$
\begin{aligned}
& \text { Algebraic Solution.- } \\
& \begin{aligned}
\Sigma M_{B} & =-12 R_{1}+1000 \times 10+2000 \times 7+2000 \times 4=0, \\
12 R_{1} & =32000 \\
\therefore \quad R_{1} & =2666 \frac{2}{3} \mathrm{lb} . \\
\Sigma M_{A} & =12 R_{2}-1000 \times 2-2000 \times 5-2000 \times 8=0 \\
12 R_{2} & =28000, \\
\therefore R_{2} & =2333 \frac{1}{3} \mathrm{lb} .
\end{aligned}
\end{aligned}
$$

In order to check the results the equation $\Sigma F=0$ may be applied. Thus, $2666 \frac{2}{3}+2333 \frac{1}{3}=1000+2000+2000$. 
Graphical Solution.-In order to determine the reactions by the graphical method, a force and a funicular polygon are constructed as shown in Fig. 82. Since the forces are in equilibrium the two polygons must close. The three known forces are represented by $A B, B C$, and $C D$. The right reaction will be represented by $D E$, the position of the point $E$ being as yet unknown. Obvi-

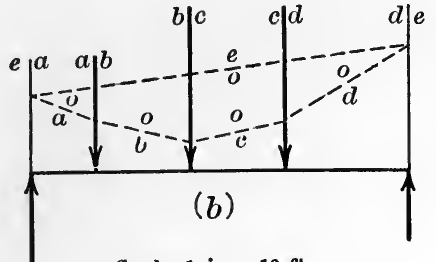

Scale. 1 in. $=10 \mathrm{ft}$.

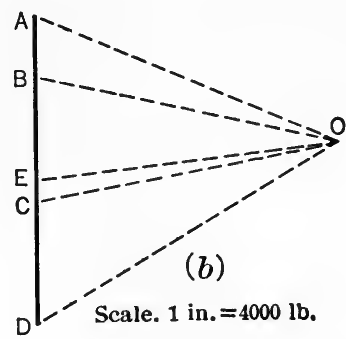

FIg. 82.

ously the left reaction will be represented by $E A$ since the force polygon must close. The strings $o a, o b, o c$, and $o d$ of the funicular polygon are drawn parallel to the corresponding rays. Since the string oe must intersect the string $o a$ on $e a$ and since $o e$ must also intersect the string $o d$ on $d e$, the position of the string $o e$ is determined. The direction of the ray $O E$ is now determined also, since it must be parallel to the string $o e$. Hence $E$ is the point where a line drawn through $O$ parallel to $o e$ intersects the line $A D$. The magnitudes of $D E$ and $E A$ are found, from the diagram, to be $2330 \mathrm{lb}$. and $2670 \mathrm{lb}$., which agree closely with the values found by the algebraic method of solution.

\section{PROBLEMS}

63. A beam $14 \mathrm{ft}$. long carries a concentrated load of $1000 \mathrm{lb}$. and a uniformly distributed load of $200 \mathrm{lb}$. per linear foot as shown in Fig. 83. If the weight of the beam is neglected find the reactions at the ends of the beam.

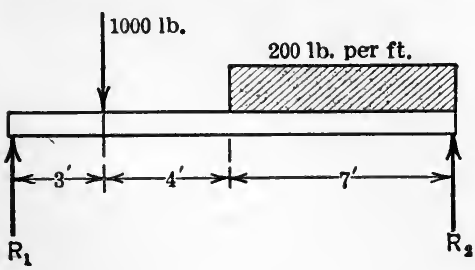

Fig. 83.

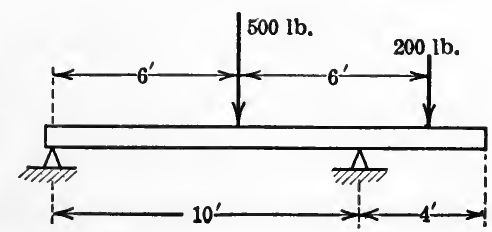

FIG. 84.

64. The beam shown in Fig. 84 weighs $10 \mathrm{lb}$. per linear foot. Find the reactions due to the weight of the beam and the loads shown.

Ans. $R_{1}=202 \mathrm{lb}$.; $R_{2}=638 \mathrm{lb}$. 
65. A bar $8 \mathrm{ft}$. long is held in equilibrium by the three forces shown in Fig. 85 and two forces acting along the lines $e f$ and $d e$. Find the magnitudes and senses of the two forces.

Ans. $D E=+560 \mathrm{lb} . \quad E F=-740 \mathrm{lb}$.

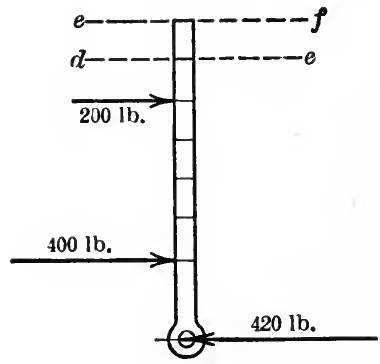

Frg. 85.

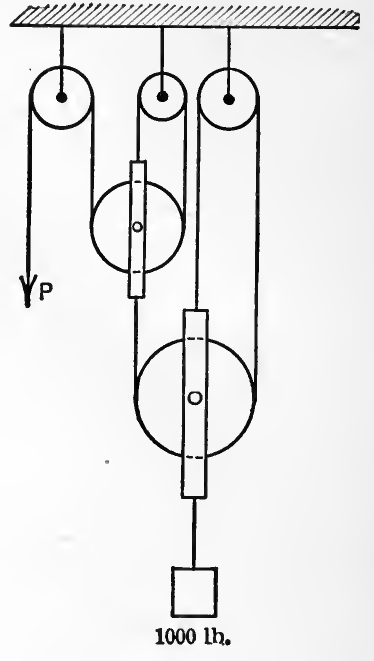

Fig. 86.

66. A load of $1000 \mathrm{lb}$. on the system of pulleys shown in Fig. 86 is held by a force $P$. Find the magnitude of $P$ and of the stresses in all cords, Neglect the weights of pulleys and axle friction.

\section{§5. Non-concurrent, Non-Parallel Forces in a Plane}

50. Equations of Equilibrium.-A system of coplanar, nonconcurrent, non-parallel forces is in equilibrium if the forces of the system satisfy the equations,

$$
\left.\begin{array}{rl}
\Sigma F_{x} & =0 \\
\Sigma F_{y} & =0 \\
\Sigma M_{A} & =0
\end{array}\right\} ; \quad \cdot \quad \cdot \quad \cdot \quad \cdot
$$

where $x$ and $y$ denote the coordinate axes and $A$ is any point in the plane of the forces.

Proof.-If a system of non-concurrent, non-parallel forces in a plane is not in equilibrium, the resultant of the system is either a force having components equal to $\Sigma F_{x}$ and $\Sigma F_{y}$ or a couple having a moment equal to $\Sigma M_{A}$ (Art. 30). If the equation $\Sigma F_{x}=0$ is 
saiisficd the resultant, if a force, must be parallel to the $y$-axis. If the equation $\Sigma F_{y}=0$ is satisfied, the resultant, if a force, must be parallel to the $x$-axis. A force cannot be parallel to both the $x$ - and $y$-axes and hence, if the first two equations are satisfied, the resultant of the system cannot be a force. If the equation $\Sigma M_{A}=0$ is satisfied, the resultant cannot be a couple. Therefore, if the forces of the system satisfy the three equations, the force system is in equilibrium.

Another set of independent equations of equilibrium for a non-concurrent, non-parallel system of forces in a plane may be written as follows:

$$
\left.\begin{array}{rl}
\Sigma F_{x} & =0 \\
\Sigma M_{A} & =0 \\
\Sigma M_{B} & =0
\end{array}\right\} \cdot
$$

where $x$ denotes any line or axis in the plane of the forces and $A$ and $B$ are any two points in the plane, provided that the line $A B$ is not perpendicular to the $x$-axis.

A third set of equilibrium equations for the force system here considered may be written as follows:

$$
\left.\begin{array}{l}
\Sigma M_{A}=0 \\
\Sigma M_{B}=0 \\
\Sigma M_{C}=0
\end{array}\right\} .
$$

where $A, B$, and $C$ are any three non-collinear points in the plane of the forces.

It will be left to the student to prove that either set of equations $(B)$ or $(C)$ are sufficient and necessary to ensure the equilibrium of a coplanar, non-concurrent, non-parallel system of forces.

Any one of the above sets of equations, therefore, may be used to determine the unknown elements of a coplanar, non-concurrent, non-parallel force system which is in equilibrium, provided there are not more than three such unknown elements or quantities. 


\section{ILLUSTRATIVE PROBLEMS}

67. A ladder rests with its upper end against a smooth vertical wall and its lower end on a smooth horizontal floor as shown in Fig. 87. Slipping is prevented by means of a stop at the lower end. If the ladder weighs $50 \mathrm{lb}$. and is of uniform cross-section, find the reactions of the wall, the floor, and the stop, on the ladder.

Solution.-A free-body diagram of the ladder is shown in Fig. 87. Since the wall and floor are smooth, the reactions at these surfaces are perpendicular to the surfaces and since the ladder has a uniform cross-section, its weight acts at the center of the ladder. Let the length of the ladder be denoted by $l$. The solution is effected by applying the three equations of equilibrium as follows:

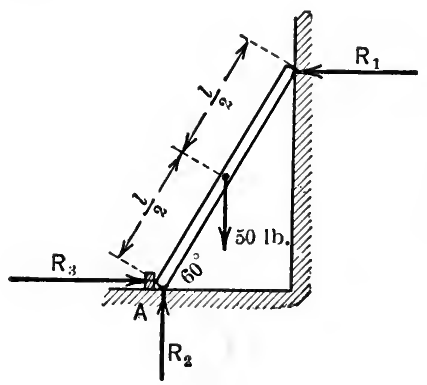

FIG. 87.

$$
\begin{aligned}
& \Sigma M_{A}=R_{1} \times l \sin 60^{\circ}-50 \times l / 2 \\
& \quad \cos 60^{\circ}=0, \\
& \therefore R_{1}=25 \cot 60^{\circ}=14.42 \mathrm{lb} . \\
& \Sigma F_{x}=R_{3}-R_{1}=0, \\
& \therefore R_{3}=R_{1}=14.42 \mathrm{lb} . \\
& \Sigma F_{y}=R_{2}-50=0, \\
& \therefore R_{2}=50 \mathrm{lb} .
\end{aligned}
$$

68. The wall bracket shown in Fig. 88(a) consists of a horizontal member $A B$, which is attached to the wall at $A$ by means of a smooth pin, and a rod $C B$, which is attached to the member $A B$ at $B$ and to the wall at $C$ by means
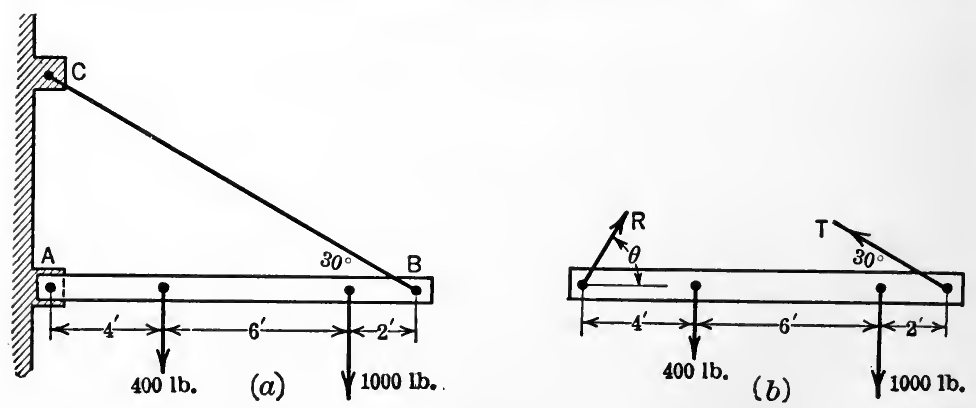

Fig. 88.

of smooth pins. Find the tension, $T$, in the rod and the pin reaction, $R$, at $A$ if the weights of the members are neglected.

Solution.-A free-body diagram of the member $A B$ is shown in Fig. $88(b)$ 
There are three unknown quantities in the force system, namely, $T, R$, and $\theta$. The three independent equations of equilibrium are,

$$
\begin{aligned}
\Sigma M_{A} & =T \times 12 \sin 30^{\circ}-1000 \times 10-400 \times 4=0, \quad . \quad . \quad . \quad . \\
\therefore T & =1930 \mathrm{lb} ., \\
\Sigma F_{x} & =R \cos \theta .-T \cos 30^{\circ}=0,
\end{aligned}
$$

$\therefore R \cos \theta=1930 \cos 30^{\circ}=1670$

$$
\Sigma F_{y}=R \sin \theta+T \sin 30^{\circ}-400-1000=0,
$$

$\therefore R \sin \theta=1400-1930 \sin 30^{\circ}=435$.

By solving equations (2) and (3) the following results are obtained,

$$
\begin{aligned}
R & =1730 \mathrm{lb} . \\
\theta & =14^{\circ} 35^{\prime} .
\end{aligned}
$$

\section{PROBLEMS}

69. A bar (Fig. 89) leans against a smooth vertical post and rests with its lower end on a smooth horizontal plane, slipping of the lower end being prevented by the cord as shown. If the weight of the bar is neglected, find the reactions of the plane at $A$ and of the post at $B$ and also find the tension in the cord.

$$
\text { Ans. } R_{A}=10.9 \mathrm{lb} . \quad R_{B}=51.1 \mathrm{lb} . \quad T=32.8 \mathrm{lb} .
$$

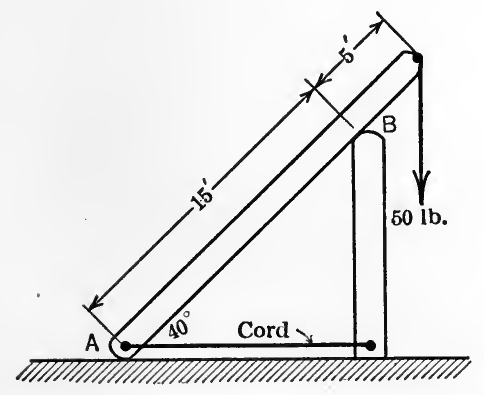

FIG. 89.

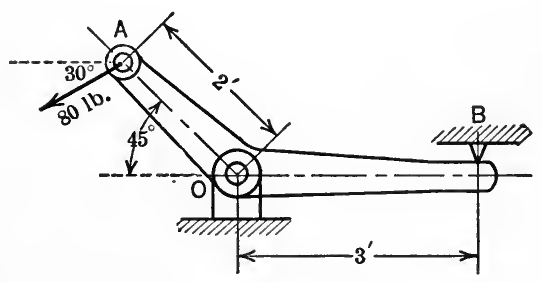

FIG. 90.

70. In Fig. 90 is shown a bell-crank mounted on a smooth pin at $O$ and subjected to a force of $80 \mathrm{lb}$. at $A$ as shown. Find the pin pressure at $O$ and the reaction at $B$. Solve algebraically and graphically.

51. Graphical Solution of a Typical Problem.-Any of the problems which involve a balanced non-concurrent, non-parallel force system in a plane in which there are not more than three unknown elements may be solved graphically as well as alge- 
braically. A graphical method of solution for one typical problem will here be discussed. In the force system considered all of the forces will be assumed to be known completely except two, the action line of one of these two being known and also one point on the action line of the other. The three unknown elements, then, are the magnitude of one of the two forces and the magnitude and the direction of the other.

As an example of such a force system, consider the forces acting on the beam shown in Fig. 91(a). The unknown elements are the magnitude of the vertical reaction at the left end of the beam and the magnitude and the direction of the reaction at the right end of the beam.
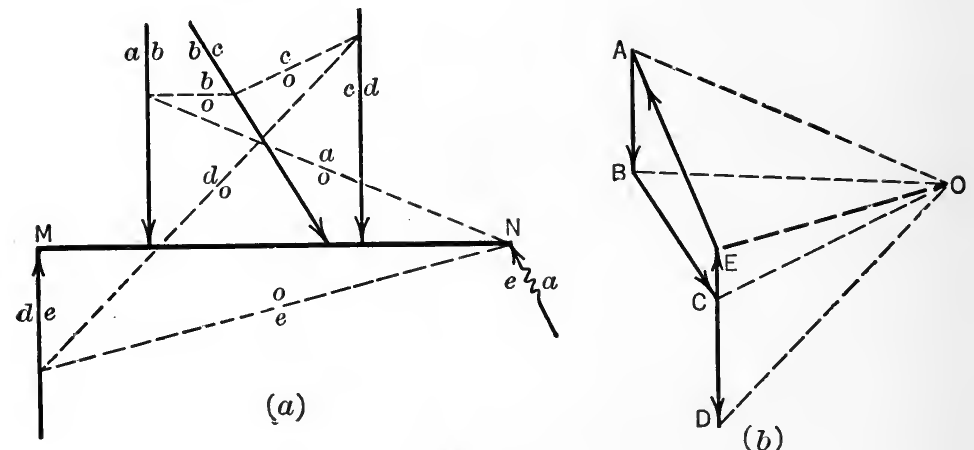

FIg. 91.

The force and funicular polygons are shown in Fig. 91. In constructing the force polygon, $A B, B C$, and $C D$ are first drawn to represent the three known forces (Fig. 91b). Since the magnitude of the force $D E$ is unknown the location of $E$ is not known, but it must lie in a vertical line through $D$. The rays are then drawn from $O$ after which the funicular polygon is constructed (Fig. 91a). Since the point $N$ is the only known point on the action line of the force $E A$, the funicular polygon must be started at this point. The strings $o a, o b, o c$, and $o d$ are drawn as shown. Since the string oe must intersect $o d$ on $d e$ and must also intersect $o a$ on $e a$ its position is determined. The ray $O E$ must be parallel to the string $o e$. Hence $E$ is the point of intersection of a vertical line through $D$ and a line through $O$ parallel to oe. $D E$ then represents the left reaction, and the right reaction is represented in magnitude and in direction by $E A$. 
52. Choice of Moment-centers and of Directions of Resolution.-In applying the three equations of equilibrium to a balanced force system, of the type here considered, in which there are not more than three unknown elements, the solution of the problem may frequently be simplified by selecting the moment-centers and the directions of resolution in a particular way. For example, in Fig. 92 is represented a portion of a roof truss which is held in equilibrium by the five forces shown, of which $P$ and $R$ are known completely and $F_{1}, F_{2}$, and $F_{3}$ are unknown in magnitude. By selecting $C$, the intersection of the two unknown forces $F_{2}$ and $F_{3}$, as a moment-center and applying the equilibrium equation $\Sigma M_{C}=0$, the force $F_{1}$ may be found from the one equation. Likewise by choosing $D$ as a moment-center and applying a second equation of equilibrium, $\Sigma M_{D}=0$, the force $F_{3}$ may be found directly. Similarly, $F_{2}$ may be found by selecting $A$ as the moment-center and applying the third equilibrium equation, $\Sigma M_{A}=0$. Thus by the proper selection of moment-centers each of the three equations involves one unknown quantity only.

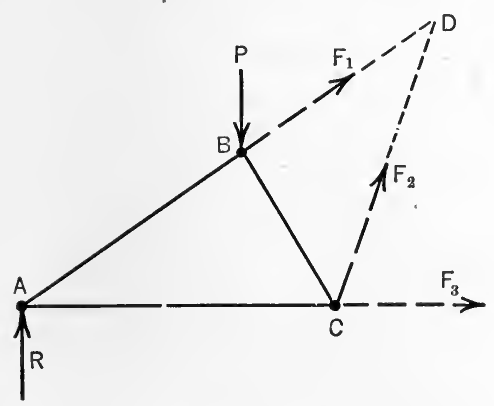

Fig. 92.

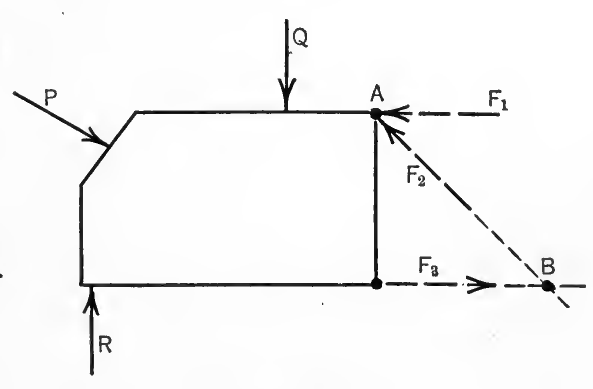

FIG. 93.

As another example, consider a body which is held in equilibrium by the six forces shown in Fig. 93, all of which are completely known except $F_{1}, F_{2}$, and $F_{3}$, which are unknown in magnitude. The forces $F_{1}$ and $F_{3}$ are parallel. By selecting $B$ as the moment-center, the force $F_{1}$ may be found from one equation, namely, $\Sigma M_{B}=0$. Likewise, $F_{3}$ may be found from the single equation $\Sigma M_{A}=0$, where $A$ is the intersection of the two forces $F_{1}$ and $F_{2}$. Further, $F_{2}$ may be found from the single equation $\Sigma F_{y}=0$ provided that the $y$-direction is chosen perpendicular to the forces $F_{1}$ and $F_{3}$. 


\section{§6. Equilibrium of Trusses, Cranes, and Cables}

53. Stresses in Trusses.-Important examples of balanced coplanar force systems are met in the analysis of the forces in trusses, cranes, and flexible cables. In determining the stresses in pin-connected trusses, only those trusses will here be considered for which the following assumptions may be made:

(1) The members of the truss lie in one plane and hence the forces acting on the truss and the stresses in the members form a coplanar force system.

(2) The members of the truss are connected at their ends by means of smooth pins.

(3) The loads on the truss act only at the pins, that is, at the ends of the members.

(4) The weights of the members are neglected, since the stresses due to the weights are small in comparison with the stresses due to other loads.

For practical purposes, in computing the stresses in many structures such as roof and bridge trusses, the structures may be considered to be in agreement with the above assumptions.

According to assumptions (2) and (3), the only forces acting on any member are the reactions of the smooth pins at the ends of the member, and since the member is in equilibrium under the action of these two forces, the two forces must be collinear, and hence the action line of each force must coincide with the axis of the member. Therefore the stress in each member is a direct or axial pull or push (tension or compression). Thus in the truss shown in Fig. 94(a), the member $A B$ is acted on by the pin pressures $F_{1}, F_{1}$ at the ends of the member which cause a compressive stress in the member (Fig. 94b), while the pin pressures $F_{2}, F_{2}$ which act at the ends of member $A C$ cause a tensile stress in the member (Fig. 94c). The stress $S$, then, in any member is numerically equal to the pressures of the pins at the ends of the member, as is indicated in the freebody diagrams of the two parts of $A C$ (Fig. 94d). The kind of stress (tension or compression) in a mem ber, therefore, is represented by arrows which show the directions of the pressures of the member on the pins. Thus, a compressive stress is represented by arrows along the member directed towards the ends of the member, etc. Thus, the stress in member $A C$ (Fig. 
$94 a$ ) is shown to be tension and the stress in member $A B$ is shown to be compression. Another convention commonly used to indicate the kind of stress in a member is to represent a tensile stress by a plus sign and a compressive stress by a negative sign. In the analysis of the stresses in a pin-ended structure two rather distinct methods may be used, namely, the method of joints and the method of sections.

54. Method of Joints. -By the method of joints the stresses in the members of a truss are found by the solution of the concurrent force systems which act at the pins, and hence not

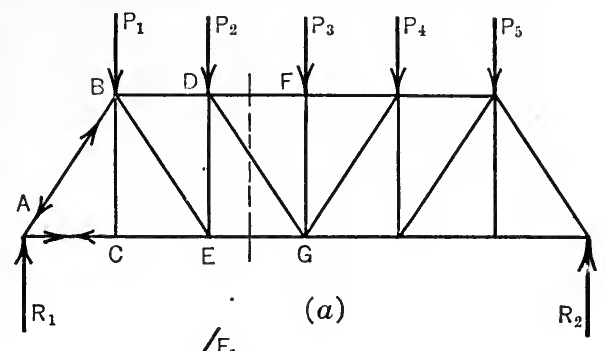
more than two unknown pin pressures at any joint or pin can be determined. For example, in the truss shown in Fig. 94(a) the pin at $A$ is in equilibrium under the influence of three forces (Fig. 95a), namely, the reaction $R_{1}$, which may be found if the dimensions of the truss and the loads carried by the truss are known, and the two unknown
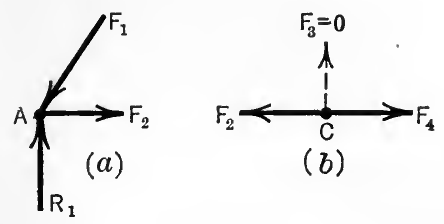

Fig. 95. forces (stresses) $F_{1}$ and $F_{2}$ which the members $A B$ and $A C$ exert on the pin. By using the two equations of equilibrium for a system of concurrent forces in a plane the two unknown forces may be found. The pin at $C$ may now be considered. This pin is in equilibrium under the influence of three forces (Fig. 95b) namely, the stress $F_{2}$ in the member $A C$ which has just been determined, and the two unknown stresses $F_{3}$ and $F_{4}$, in the members $C B$ and $C E$. These two unknown stresses may be determined from the two equations of equilibrium. The pin at $B$ may next be con- 
sidered, and so on until the stresses in all of the members have been determined. As already noted, in using this method the joints must be taken in such an order that there are not more than two unknown stresses at the joint considered, since there are only two equations of equilibrium for a concurrent force system and hence only two unknown stresses may be determined. In considering the equilibrium of the forces at any joint the senses of the two unknown forces are not always evident. When such is the case the forces may be assumed to be directed away from the joint, that is, the stresses may be assumed to be tensions. If, after applying the equations of equilibrium and solving for the two unknown forces, the sign of a force is found to be positive it is evident that the assumed sense of the force is correct, whereas a negative sign indicates that the sense of the force is opposite to that assumed. In most problems, however, the sense of each of the unknown forces is evident from inspection. The determination of the stresses in a truss by the method of joints often requires a large number of calculations with a corresponding chance for error. Thus, if the stress in a single member near the center of the truss is required it is necessary to start at the end of the truss and consider the equilibrium of the joints in order until a joint is reached which involves that particular member. By the method of sections (Art. 55) the stress in a single member may frequently be found by use of a single equation.

55. Method of Sections. - In determining the stresses in the members of a pin-connected truss by the method of sections a section is passed through the truss so that it cuts not more than three members in which the stresses are unknown. The part of the truss on either side of this section is then treated as a free body in equilibrium under the action of the known external forces which act on that part and the forces (stresses) which the members of the other part exert on the part considered. It will be noted that the method of sections involves a non-concurrent force system and hence there are three equations of equilibrium from which three unknown forces may be found. Consequently the section must not cut more than three members in which the stresses are unknown: As a rule any one of these three unknown forces (stresses) may be found by using one equilibrium equation as discussed in Art. 52. For example, consider the truss shown in Fig. 94. In order to determine the stress in the member $D G$ con- 
sider the section which cuts the members $D F, D G$, and $E G$. Thefree-body diagram of the portion of the truss to the left of this section is shown in Fig. 96(a). This part of the truss is in equilibrium under the action of six forces; namely, $R_{1}, P_{1}$, and $P_{2}$, which are assumed to be known, and the unknown stresses in the members $D F, D G$, and $E G$. The stress $\left(F_{6}\right)$ in the member $D G$ may be obtained from the equation $\Sigma F_{y}=0$, since neither of the other two unknowns will appear in this equation. Similarly the stress $\left(F_{5}\right)$ in the member $D F$ may be obtained from the single equation $\Sigma M_{G}=0$ and the stress $\left(F_{7}\right)$ in the member $E G$ may be obtained from the single equation $\Sigma M_{D}=0$.
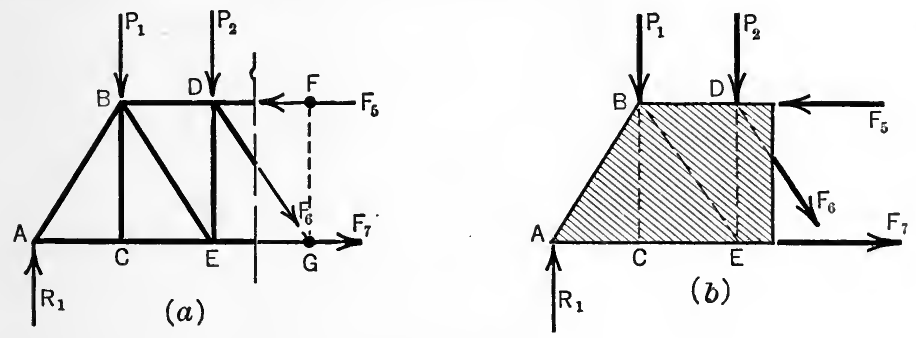

Frg. 96.

It is important to note that the forces $F_{5}, F_{6}$, and $F_{7}$ are regarded as internal forces (stresses) when the equilibrium of the truss as a whole is considered, but that they are regarded as external forces when the part of the truss on one side of the section is considered. In considering the equilibrium of the part of the truss on the left of the section (Fig. 96a) only the external forces which act on the left part are involved and hence the stresses in the members of the left part that are not cut by the section do not affect the values of $F_{5}, F_{6}$, and $F_{7}$. Thus the left part may be thought of as a solid block as indicated in Fig. 96(b) on which the forces $F_{5}, F_{6}$, and $F_{7}$ act together with $R_{1}, P_{1}$, and $P_{2}$, the six forces holding the block in equilibrium.

\section{ILLUSTRATIVE PROBLEM}

71. Determine the stresses in the members of a Howe truss when loaded as shown in Fig. 97(a).

Solution.-The stress in any member will be denoted by the same letters as the member itself. Thus, the stress in member $A B$ will be denoted by $A B$. By considering the equilibrium of the truss as a whole, the reartions 

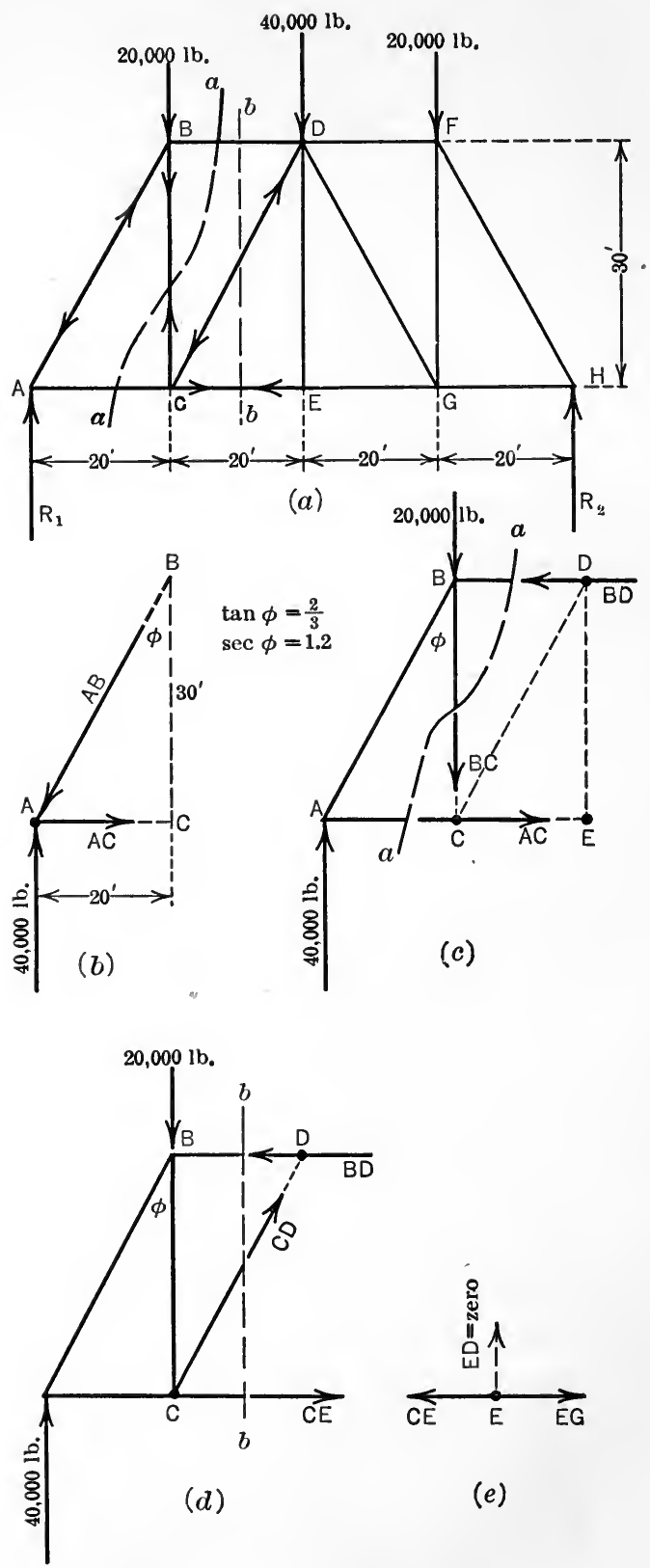

FIG. 97. 
$R_{1}$ and $R_{2}$ are found to be $40,000 \mathrm{lb}$. To find the stresses in members $A B$ and $A C$ the joint method is used. A free-body diagram of joint $A$ is shown in Fig. $97(b)$. By applying the equations of equilibrium for a concurrent force system, the stresses in the two members are found as follows:

$$
\begin{aligned}
\Sigma M_{B} & =-40,000 \times 20+A C \times 30=0 \\
\therefore A C & =\frac{40,000 \times 20}{30}=26,667 \mathrm{lb} . \text { (tension) } \\
\Sigma F_{y} & =-A B \cos \phi+40,000=0 . \\
\therefore A B & =\frac{40,000}{\cos \phi}=40,000 \mathrm{sec} \phi=48,000 \mathrm{lb} . \text { (compression) }
\end{aligned}
$$

To find the stresses in members $B C$ and $B D$ let a section $a a$ be passed (Fig. 97c). The forces acting on the part of the truss to the left of the section form a non-concurrent force system. But since the stress in member $A C$ is already known only two of the three equations of equilibrium are needed. Thus,

$$
\begin{aligned}
& \Sigma M_{C}=-40,000 \times 20+B D \times 30=0, \\
& \therefore B D=\frac{40,000 \times 20}{30}=26,667 \mathrm{lb} . \text { (compression) } \\
& \quad \Sigma F_{\nu^{\prime}}=40,000-20,000-B C=0, \\
& \therefore B C=20,000 \mathrm{lb} . \text { (tension) }
\end{aligned}
$$

In a similar manner the stresses in $C D$ and $C E$ may be found by the method of sections. By considering the part of the truss to the left of section $b b$ (Fig. 97d), the stresses in the two members are found as follows:

$$
\begin{aligned}
\Sigma M_{D} & =-40,000 \times 40+20,000 \times 20+C E \times 30=0, \\
\therefore \quad C E & =\frac{1,200,000}{30}=40,000 \mathrm{lb} . \text { (tension) } \\
\Sigma F_{y} & =40,000-20,000+C D \cos \phi=0, \\
\therefore C D & =-20,000 \mathrm{sec} \phi=-24,000 \mathrm{lb} \text {. (compression) }
\end{aligned}
$$

It will be noted that all of the stresses except $C D$ (Fig. $97 d$ ) are assumed to act in the correct directions, and hence are found to be positive whether they are tensile or compressive. The stress in $C D$ is assumed to be tension and hence the negative sign indicates that it is compression.

In considering the equilibrium of the forces which act on the pin $E$ (Fig. $97 e$ ), it is evident that the stress in $D E$ is zero, and that $E C$ equals $E G$. Further, since the truss is symmetrical with respect to the center line $D E$ and the loads are also symmetrical with respect to this line, it is obvious that the stresses in the members of the right half of the truss are equal to the stresses in the corresponding members of the left half. 


\section{PROBLEMS}

72. Find the stresses in the members of the Warren truss shown in Fig. 98. Ans. $A B=-4620 \mathrm{lb} . \quad D B=-3460 \mathrm{lb}$.

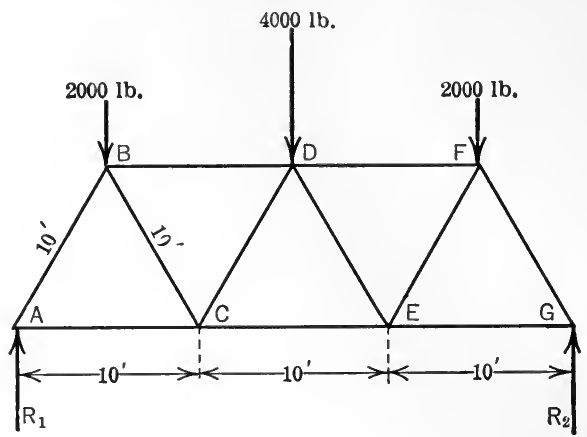

FIG. 98.

73. Find the stresses in the members of the Pratt truss shown in Fig. 99 in which the loads $P$ are each $10,000 \mathrm{lb}$.

Ans. $A B=-30,000 \mathrm{lb} . \quad B C=+10,000 \mathrm{lb} . \quad B D=-26,670 \mathrm{lb}$.

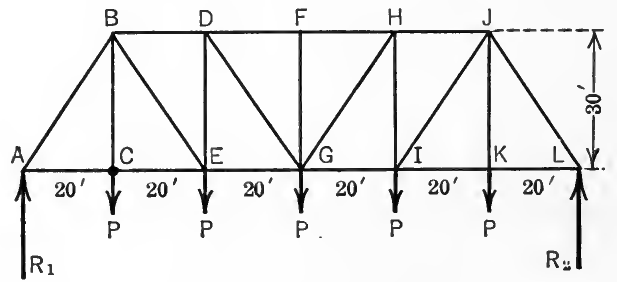

FIG. 99.

74. Find the stresses in the members of the Howe truss shown in Fig. 100. Ans. $A B=-12,700 \mathrm{lb} . \quad B C=-3000 \mathrm{lb} . \quad B D=-9000 \mathrm{lb}$.

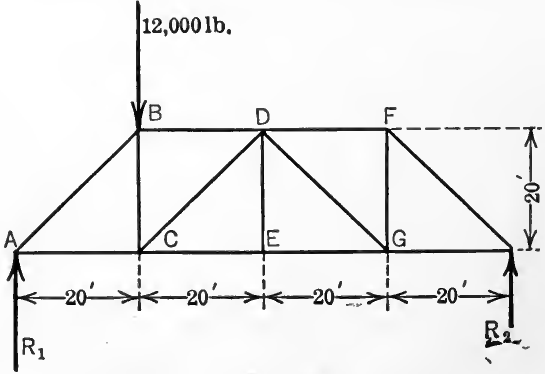

FIG. 100 . 
75. In the Fink truss shown in Fig. $101 B C$ is perpendicular to $A D$ and $B$ is the mid-point of $A D$. Find the stresses in the members.

$$
\text { Ans. } B C=-1730 \mathrm{lb} . \quad A B=-6000 \mathrm{lb} \text {. }
$$

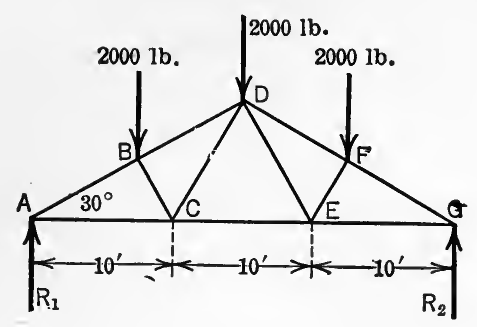

Frg. 101.

56. Graphical Analysis of Trusses.-The graphical method of analysis of framed structures is sometimes simpler than the algebraic method. This statement is true particularly when the form of the structure is such that a considerable amount of calculation is necessary to determine the directions and moment-arms of the forces involved. The graphical method consists essentially in constructing the force polygons for the concurrent forces at the joints and superimposing these polygons. The method will be explained in detail with reference to the Pratt truss shown in Fig. 102(a). It will be assumed that the loads carried at the upper panel points are equal. It is convenient to use the Bow system of notation (Art. 7). In this system the regions on either side of the action line of a force (either external or internal) are denoted by numbers or letters as shown in Fig. 102(a) and the force is denoted by the two numbers or letters adjacent to the action line of the force. For example, the left reaction is denoted by $X_{1}-Y$; the load at the upper panel point at the left end of the truss by $X_{1}-X_{2}$; the stress in the diagonal in the second panel by 2-3; the stress in the lower cord at the left end of the truss by $Y-1$; etc. The order of the numbers or letters denoting an external force, or an internal stress in any member, may be reversed. Thus the stress in the diagonal in the second panel may also be denoted by 3-2. The force polygon shown in Fig. 102(b) is constructed as follows: The load line $X_{1} X_{2} X_{3} X_{4} X_{5} X_{6}$ is first laid off. Since the loads are symmetrical, the reactions will be equal, and hence $Y$ will be midway between the points $X_{1}$ and $X_{6}$. 
Consider now the three concurrent forces at the lower left-hand panel point. Of these three forces the left reaction, $Y-X_{1}$, is completely known and the directions of the other two, $X_{1}-1$ and $1-Y$, are known. Since these three forces are in equilibrium, their force polygon must close. Hence, to determine the magnitudes of the last two forces a line is drawn in Fig. 102(b) through $X_{1}$ parallel to $X_{1}-1$, and a line is drawn through $Y$ parallel to $1-Y$. These lines intersect in the point 1 , and the magnitudes of the stresses are indicated by the lengths of the lines $X_{1}-1$ and $1-Y$ in the force polygon. It will be noted that the pressure of the member $X_{1}-1$ on the pin at the lower end is downward to the left and hence the member is in compression.
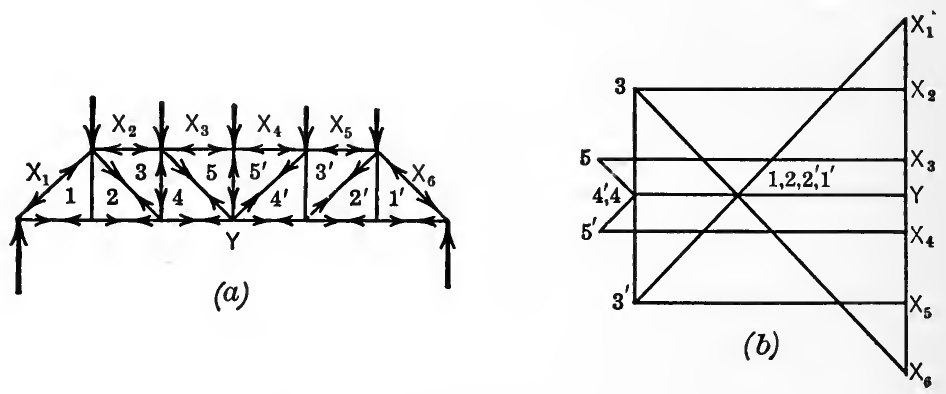

FIG. 102.

As soon as the stresses in the various members are found arrow-heads should be placed on the members indicating whether the stresses are tensions or compressions. The second lower panel point should next be taken. The stress in the member $Y-1$ is now known and in order that the polygon for this joint shall close it is evident that the point 2 must coincide with 1 . Therefore the stress in 1-2 is zero and the stress in $2-Y$ is the same as the stress in $Y-1$. The first upper panel point can now be taken and the stresses in $X_{2}-3$ and 3-2 may be found. The forces at the remaining joints may be found in a similar manner. The student should follow through the construction (Fig. 102b) and build up the complete polygon. 


\section{ILLUSTRATIVE PROBLEM}

76. Find the stresses in the members of the truss shown in Fig. 103(a). The members $X_{1}-1$ and 2-3 are parallel and the members $X_{1}-2$ and $Y-1$ are parallel.
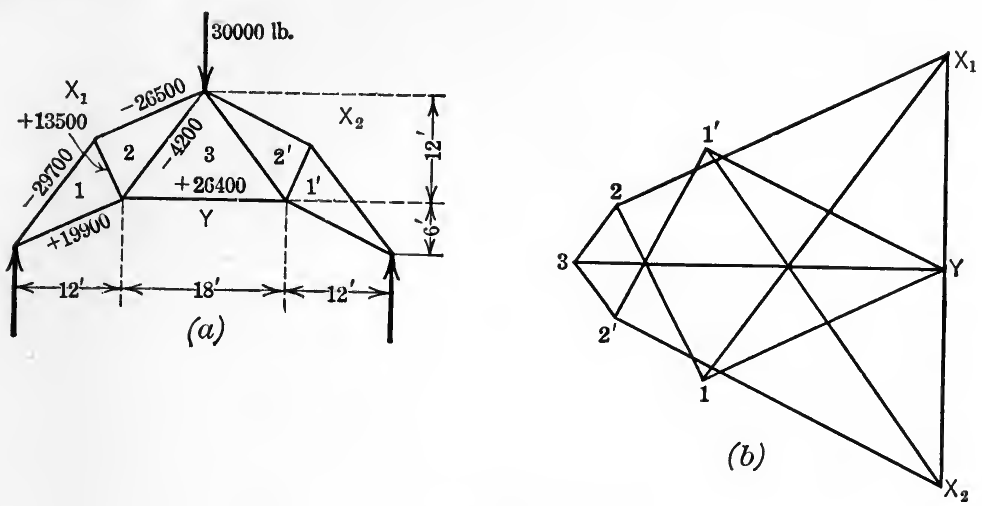

FIG. 103.

Solution.-The stresses are determined from the force polygon shown in Fig. 103(b). The magnitudes of the stresses are shown on the members in Fig. 103(a), the plus or minus sign indicating whether the stress in the member is tension or compression.

\section{PROBLEMS}

77. The Fink truss shown in Fig. 104 is subjected to wind loads as indicated, the loads being perpendicular to the upper chord $A D$. The truss

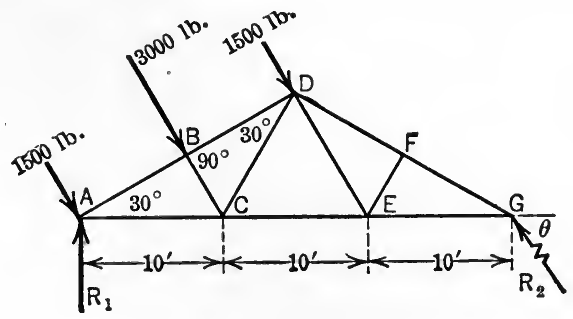

Fig. 104.

rests on a smooth plate at the left end and hence the reaction at that end is vertical. Find the magnitude and direction of the reaction at the right end of the truss and the stresses in the members. Use the graphical method. 
78. Find, by the graphical method, the stresses in the members of the truss shown in Fig. 105, the value of $P$ being 10,000 lb.

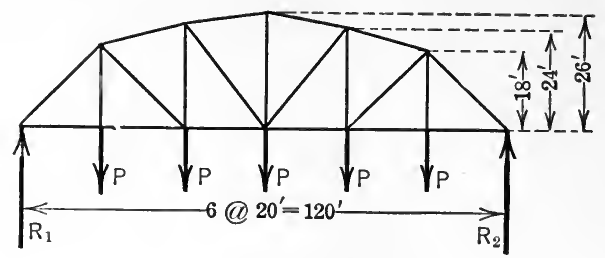

Fig. 105.

57. Cranes.--It was stated in Art. 53 that in the analysis of the forces which act on the members of a crane balanced coplanar force systems are involved. The loads on cranes, unlike those on trusses, are not applied at the pins only. That is, assumptions (2), (3) and (4) of Art. 53 may not always be made in the analysis of the forces acting on cranes. The force system acting on a member of a crane, therefore, includes forces other than the two pin pressures at the ends of the member, and hence the stress in a crane member, in general, is not a direct axial tension or compression as is the case in a truss. In order to calculate the stress in a crane member a knowledge of the subject of Strength of Materials is needed, and consequently the stresses in crane members will not, in general, here be found. The main problem here considered in connection with cranes, therefore, is that of determining only the forces acting on the various members of the crane. Since the stresses in the members of a crane are not single forces directed along the axes of the members, the method of sections as used in the preceding articles cannot be employed. The general method of procedure in the analysis of the forces acting on crane members will be first to consider the equilibrium of the structure as a whole and determine the external reactions, and then to consider the equilibrium of the various members separately and determine the forces (pin pressures, etc.) which act on the separate members. 


\section{ILLUSTRATIVE PROBLEM}

79. In the crane shown in Fig. 106 the post $A D$ weighs $1200 \mathrm{lb}$. and the boom $B E$ weighs $1000 \mathrm{lb}$. The member $C E$ is a cable the weight of which will be neglected. The reaction at $D$ will be assumed to be horizontal. Find the reactions at $A$ and $D$, the pin reaction at $B$, and the tension in the cable $C E$.

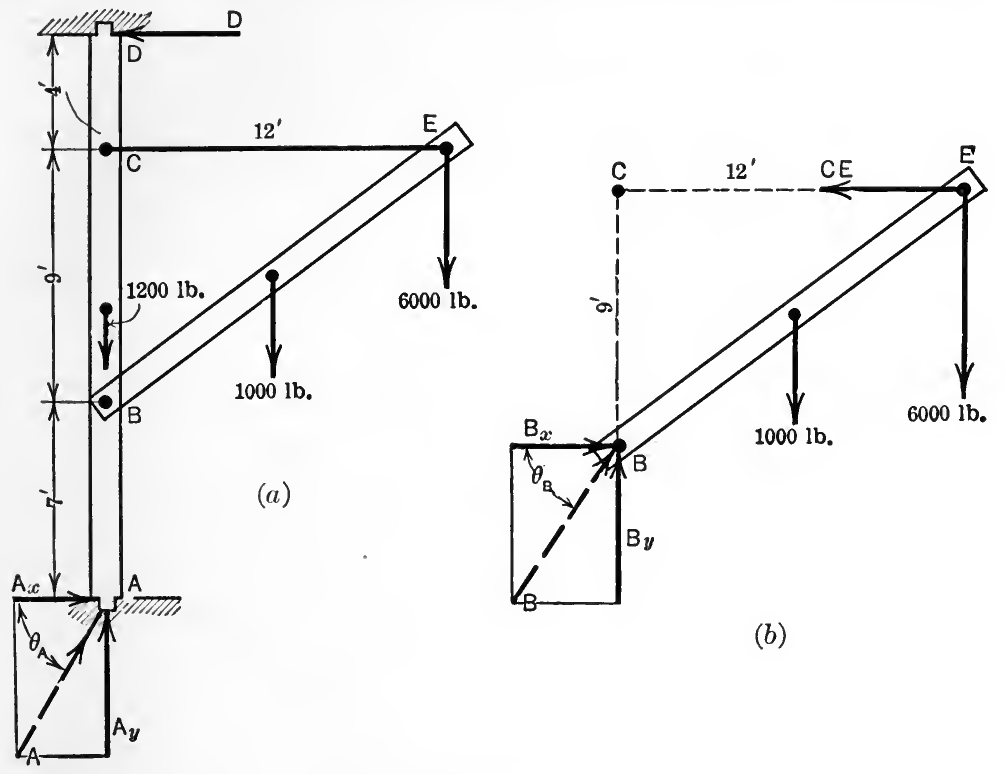

FIG. 106.

Solution.-First consider the equilibrium of the entire crane, a free-body diagram of which is shown in Fig. 106(a). The reaction at $D$ will be denoted by $D$. The reaction at the pin $A$ will be denoted by $A$ and, for convenience, will be resolved into its horizontal and vertical components which will be denoted by $A_{x}$ and $A_{y}$. The unknown reactions are obtained by applying the equations of equilibrium as follows:

$$
\begin{aligned}
\Sigma M_{A} & =20 D-6 \times 1000-12 \times 6000=0, \\
\therefore D & =\frac{78,000}{20}=3900 \mathrm{lb} . \\
\Sigma F_{x} & =A_{x}-D=0 \\
\therefore D & =A_{x}=3900 \mathrm{lb} . \\
\Sigma F_{y} & =A_{y}-1200-1000-6000=0 \\
\therefore A_{y} & =8200 \mathrm{lb} .
\end{aligned}
$$


Whence,

and,

$$
A=\sqrt{A x^{2}+A y^{2}}=\sqrt{(3900)^{2}+(8200)^{2}}=9075 \mathrm{lb} .
$$

$$
\theta_{A}=\tan ^{-1} \frac{8200}{3900}=64^{\circ} 35^{\prime} \text {. }
$$

Next consider the equilibrium of the member $B E$. The forces acting on this member are four in number; namely, the tension in $C E$ which will be denoted by $C E$, the load of $6000 \mathrm{lb}$., the weight of the member, and the pressure of the pin at $B$ on the member, as shown in the free-body diagram (Fig. 106b). The last force is resolved into its horizontal and vertical components as shown in the free-body diagram. By applying the equations of equilibrium the unknown quantities may be found as follows:

$$
\begin{aligned}
\Sigma M_{B} & =9 \times C E-6 \times 1000-12 \times 6000=0, \\
\therefore C E & =\frac{78,000}{9}=8667 \mathrm{lb} . \\
\Sigma F_{x} & =B_{x}-C E=0, \\
\therefore B_{x} & =C E=8667 \mathrm{lb} . \\
\Sigma F_{y} & =B_{y}-1000-6000=0 .
\end{aligned}
$$

Whence,

$$
\therefore B_{y}=7000 \mathrm{lb} \text {. }
$$

and,

$$
B=\sqrt{B x^{2}+B_{y}^{2}}=\sqrt{(8667)^{2}+(7000)^{2}}=11,100 \mathrm{lb} .
$$

$$
\theta_{B}=\tan ^{-1} \frac{7000}{8667}=38^{\circ} 55^{\prime} \text {. }
$$

80. Determine the forces acting on the members of the crane shown in Fig. 107 $(a)$. Neglect the weights of the members.
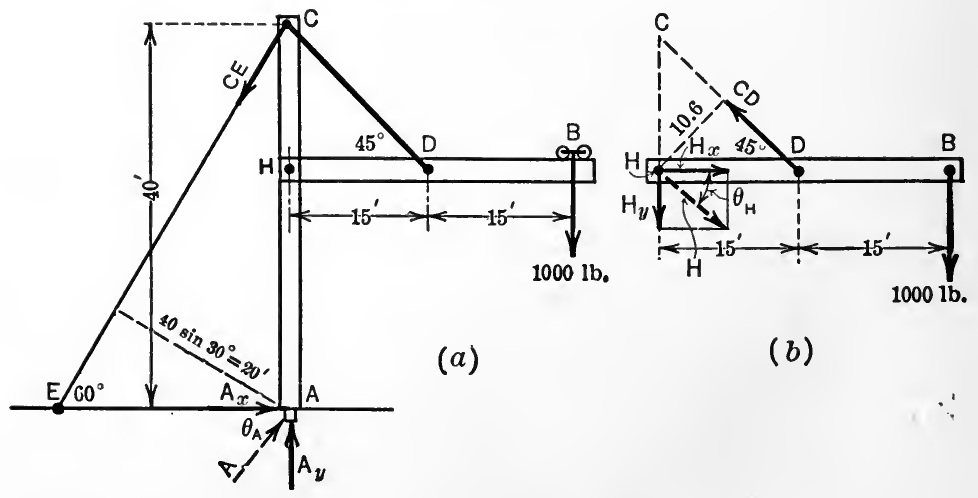

Fig. 107. 
Solution.-Consider first the equilibrium of the entire crane, a free-body diagram of which is shown in Fig. 107(a). By applying the three equations of equilibrium, the unknown quantities may be found. Thus,

$$
\Sigma M_{A}=20 \times C E-30 \times 1000=0 .
$$

$\therefore C E=\frac{30,000}{20}=1500 \mathrm{lb}$.

$$
\Sigma F_{x}=A_{x}-C E \sin 30^{\circ}=0,
$$

$\therefore A_{x}=C E \sin 30^{\circ}=750 \mathrm{lb}$.

$$
\Sigma F_{\nu}=A_{\nu}-1000-C E \cos 30^{\circ}=0 \text {, }
$$

Whence,

$$
\therefore A_{\nu}=1000+1300=2300 \mathrm{lb} \text {. }
$$

and,

$$
A=\sqrt{(750)^{2}+(2300)^{2}}=2420 \mathrm{lb} .
$$

$$
\theta_{A}=\tan ^{-1} \frac{2300}{750}=71^{\circ} 55^{\prime} \text {. }
$$

Consider next the equilibrium of the member $B H$. The forces acting on $B H$ are shown in Fig. 107(b). From the equations of equilibrium the unknown quantities may be found as follows:

Whence,

$$
\begin{aligned}
\Sigma M_{H} & =10.6 \times C D-30 \times 1000=0, \\
\therefore C D & =\frac{30,000}{10.6}=2830 \mathrm{lb} . \\
\Sigma F_{x} & =H_{x}-C D \cos 45^{\circ}=0, \\
\therefore H_{x} & =2830 \times .707=2000 \mathrm{lb} . \\
\Sigma F_{\nu} & =-H_{\nu}+C D \cos 45^{\circ}-1000=0, \\
\therefore H_{y} & =-1000+2830 \times .707=1000 \mathrm{lb} .
\end{aligned}
$$

and,

$$
H=\sqrt{(2000)^{2}+(1000)^{2}}=2240 \mathrm{lb} .
$$

$$
\theta_{H}=\tan ^{-1} \frac{1000}{2000}=26^{\circ} 35^{\prime} \text {. }
$$

\section{PROBLEMS}

81. In the crane shown in Fig. 108 the post $A E$ weighs $1600 \mathrm{lb}$. and the member $\mathrm{CH}$ weighs $1200 \mathrm{lb}$. The remaining members are cables the weights of which may be neglected. The member $C H$ passes through a slot in the post $A E$. Determine the external reactions, and the tensile stresses in the members $B C, C D$, and $D H$. Assume the reaction at $E$ to be horizontal and that there is no reaction between $C H$ and $A E$. 
82. The crane in Fig. 109 is in equilibrium under the action of the two loads shown and the reactions at $B$ and $A$, the reaction at $B$ being horizontal. Find the reactions at $A$ and $B$ and the tension in the member $D F$. Find also the pressure of the pin at $C$ on the member $C F$.

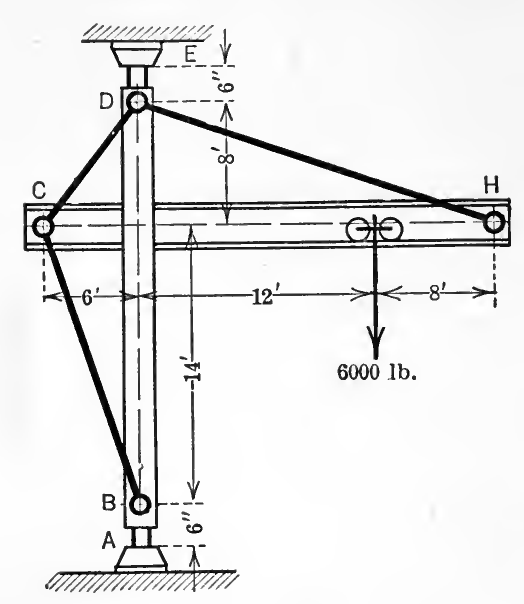

FIG. 108.

$$
\text { Ans. } B=5200 \mathrm{lb} . \quad D F=15400 \mathrm{lb} \text {. }
$$

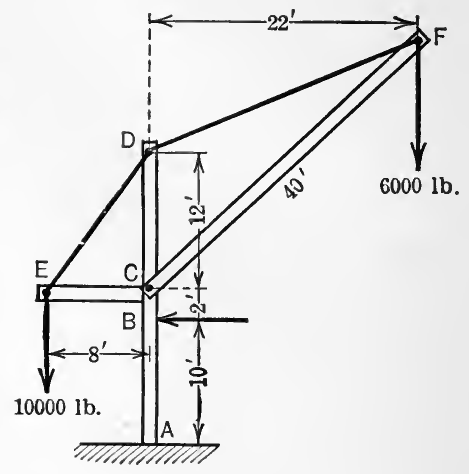

FIG. 109.

58. Flexible Cables.-In the following two articles the equilibrium of flexible cables or cords will be discussed. A cable is said to be perfectly flexible when it can offer no resistance to bending. A flexible cable, then, can transmit a stress only along its axis, that is, the stress at any point of a flexible cable is tangent to the curve assumed by the cable. Although physical cables and cords are not perfectly flexible the resistance they offer to bending is generally so small that it can be neglected without serious error. In the discussion of cables it will be assumed that the cables are perfectly flexible and inextensible.

59. The Parabolic Cable.-If a flexible cable is suspended from two points and carries a load that is distributed uniformly horizontally (Fig. 110a), the curve assumed by the cable is a parabola, as will presently be shown. In the present discussion the points from which the cable is suspended will be assumed to be in the same horizontal plane. An example of a cable carrying a load which closely approximates that above indicated is the cable of a suspension bridge, since the weight of the roadway is uniformly distributed horizontally and the weights of the cable and hangers are small in comparison with the weight of the roadway and 
therefore may be neglected. Another example is that of a tightly stretched cable (that is, one in which the sag is small as compared with the span) which carries no load except its own weight, as for example, the cable of an electric transmission line, a telegraph wire, etc. In this case the load carried by the cable (its weight) is distributed uniformly along the curve assumed by the cable, but since the sag is small the horizontal projection of an arc of the curve is approximately equal to the length of the arc, and hence the load is distributed approximately uniformly in the horizontal direction.

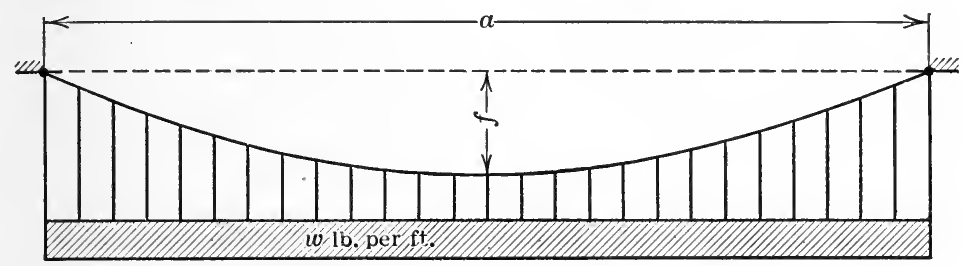

(a)

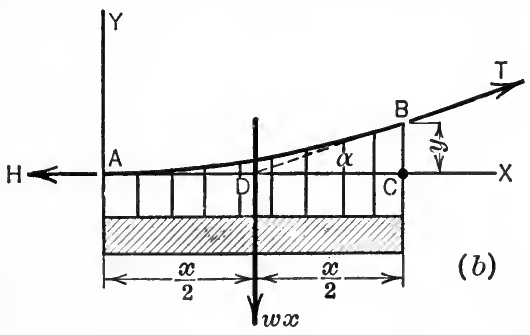

FIG. 110.

In the solution of problems involving the parabolic cable, use is made of the equation of the curve assumed by the cable (parabola) and of equations which express relations between the span, sag, length of the cable, tension, etc. In order to determine the equation of the parabola a portion $A B$ of the cable will be considered as a free body (Fig. 110b). $A$, the lowest point of the cable, will be taken as the origin of coordinates and the tension at this point will be denoted by $H$. The tension at any point, $B$, will be denoted by $T$. The portion of cable $A B$, then, is in equilibrium under the action of the three forces $H, T$, and the vertical load $w x$ which acts through the point $D$ midway between $A$ and $C$. Since these three forces are in equilibrium they must be concurrent, 
and hence the action line of $T$ passes through $D$. The equations of equilibrium are:

$$
\begin{aligned}
& \Sigma F_{x}=T \cos \alpha-H=0, \quad . \quad . \quad . \quad . \quad . \\
& \Sigma F_{\nu}=T \sin \alpha-w x=0 . \quad . \quad . \quad . \quad .
\end{aligned}
$$

By dividing (2) by (1), the equation obtained is,

But,

$$
\tan \alpha=\frac{w x}{H} \text {. }
$$

Hence,

$$
\tan \alpha=\frac{2 y}{x} \text {. }
$$

That is,

$$
\frac{2 y}{x}=\frac{w x}{H}
$$

$$
y=\frac{w x^{2}}{2 H} \text {. }
$$

The curve, then, is a parabola with its vertex at $A$ and its axis vertical. By squaring and adding (1) and (2) and extracting the square root of each side of the resulting equation, the following expression for $T$ is found,

$$
T=\sqrt{H^{2}+w^{2} x^{2}} . \quad . \quad . \quad . \quad . \quad .
$$

In applying the above equations we are concerned with the tension at the point of support since at this point the tension is a maximum. Hence, if the span be denoted by $a$ and the maximum value of $y$ (that is, the sag) by $f$, equations (3) and (4) reduce to

and

$$
f=\frac{w a^{2}}{8 \bar{H}}, \quad \text {. . . . . . . . . . }
$$

$$
T=\frac{1}{2} w a \sqrt{1+\frac{a^{2}}{16 f^{2}}}, \quad \text {. . . . . }
$$

in which $T$ represents the tension at the points of support.

The length of the cable will now be determined in terms of the span and sag. In any curve the length of an arc is obtained from the equation

From equation (3), $\frac{d y}{d x}=\frac{w x}{H}$.

$$
s=\int \sqrt{1+\left(\frac{d y}{d x}\right)^{2}} d x
$$


Hence, if the length of the cable be denoted by $l$, we have

$$
l=2 \int_{0}^{\frac{a}{2}} \sqrt{1+\frac{w^{2} x^{2}}{H^{2}}} d x .
$$

Substituting for $H$ from equation (5), this equation becomes

$$
l=2 \int_{0}^{\frac{a}{2}} \sqrt{1+\frac{64 f^{2} x^{2}}{a^{4}}} d x .
$$

The exact expression for $l$, obtained from this integral, involves a logarithmic function and is difficult to apply. A simpler expression for $l$ may be obtained by expanding the expression under the integral into a series and integrating the series term by term. This method leads to the following equation,

$$
l=a\left[1+\frac{8}{3}\left(\frac{f}{a}\right)^{2}-\frac{32}{5}\left(\frac{f}{a}\right)^{4}+\ldots\right] . . . .
$$

Since the sag ratio $f / a$ is generally small, the series converges rapidly and it is sufficient in most practical computations to use only the first two or three terms of the series to obtain a close approximation to the value of $l$.

\section{ILLUSTRATIVE PROBLEM}

83. The horizontal load carried by each cable of a suspension bridge is $1000 \mathrm{lb}$. per ft. The span of the bridge is $800 \mathrm{ft}$. and the sag is $50 \mathrm{ft}$. Determine the tensions at the ends and at the middle of the cable and also find the length of the cable.

Solution.-From equations (5) and (6),

and,

$$
H=\frac{1000 \times(800)^{2}}{8 \times 50}=1,600,000 \mathrm{lb} .
$$

$$
T=\frac{1}{2} \times 1000 \times 800 \sqrt{1+\frac{(800)^{2}}{16 \times(50)^{2}}}=1,650,000 \mathrm{lb} .
$$

The length of the cable may be determined by using equation (7). Thus,

$$
l=800\left[1+\frac{8}{3}\left(\frac{50}{800}\right)^{2}-\frac{32}{5}\left(\frac{50}{800}\right)^{4}\right]=808.24 \mathrm{ft} .
$$




\section{PROBLEMS}

84. A telegraph wire weighing $0.1 \mathrm{lb}$. per foot is stretched between two poles $150 \mathrm{ft}$. apart. 'The tension in the wire at the insulators (which are in the same horizontal plane) is $500 \mathrm{lb}$. Find the sag, assuming that the weight of the wire is uniformly distributed horizontally. Find also the length of the wire.

$$
\text { Ans. } f=0.562 \mathrm{ft} . \quad l=150.005 \mathrm{ft} .
$$

85. Each cable of a suspension bridge carries a load of $1200 \mathrm{lb}$. per foot uniformly distributed along the horizontal. The span is $1000 \mathrm{ft}$. and the sag is $50 \mathrm{ft}$. Find the maximum stress in the cable and the length of the cable.

86. A cable $100 \mathrm{ft}$. in length is suspended from two points in a horizontal plane which are $99 \mathrm{ft}$. apart. If the cable carries a load that is uniformly distributed along the horizontal what is the sag of the cable?

$$
\text { Ans. } f=6.10 \mathrm{ft} \text {. }
$$

60. The Catenary.-The curve assumed by a flexible cable of uniform cross-section which is suspended from two points, and which carries no load except its own weight (Fig. $111 \mathrm{a}$ ), is called a

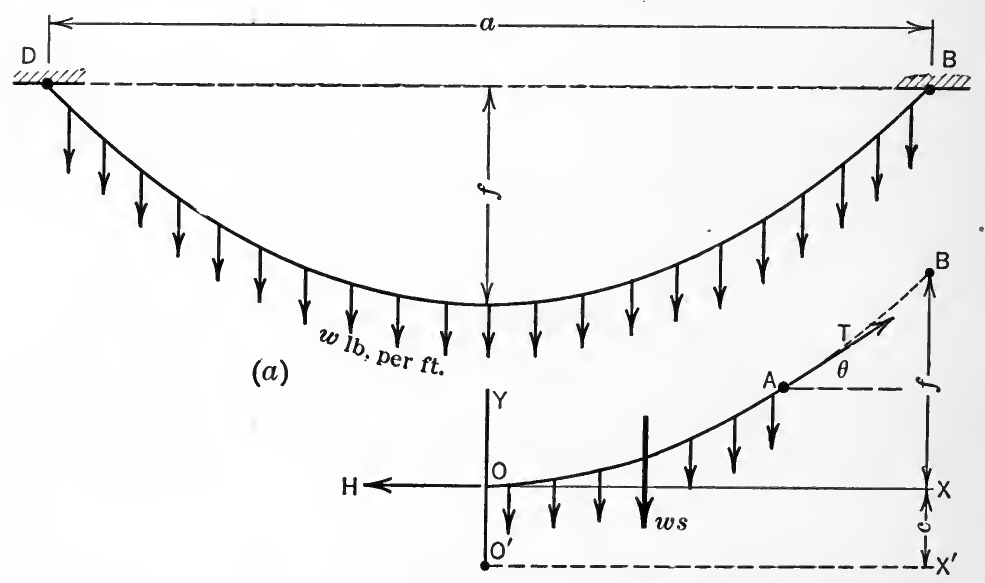

(b)

Fig. 111.

catenary. The load which causes a cable to assume the form of a catenary, then, differs from that which causes the form of a parabola in that the load is distributed uniformly along the cable in the former case, whereas in the latter case the load is distributed uniformly horizontally.

The discussion of the catenary is of practical importance only 
for cables in which the sag ratio is large, since for a small sag ratio the curve assumed by a cable may be regarded with small error as being a parabola, as discussed in the preceding article.

In order to determine the equation of the catenary and also to derive certain important relations between such quantities as the sag, span, length of cable, tension, etc., the equilibrium of a portion, $O A$, of the cable (Fig. 111b) will be considered, $O$ being the lowest point of the cable and $A$ any other point. The point $O$ will be taken as the origin of coordinates, the weight of the cable per unit of length will be denoted by $w$, and the length of the arc $O A$ will be denoted by $s$. The portion, $O A$, of the cable is in equilibrium under the influence of three forces, namely, the tension $H$ at the point $O$, the tension $T$ at the point $A$, and the weight ws. The angle which $T$ makes with the horizontal will be denoted by $\theta$. The equations of equilibrium for the concurrent force system are,

$$
\begin{aligned}
& \Sigma F_{x}=T \cos \theta-H=0, \quad \therefore T \cos \theta=H . \quad . \quad . \quad . \\
& \Sigma F_{y}=T \sin \theta-w s=0, \quad \therefore T \sin \theta=w s . \quad . \quad . \quad .
\end{aligned}
$$

From (1) and (2) we have,

$$
\tan \theta=\frac{w s}{H}=\frac{s}{c} \text { where } \frac{H}{w}=c \text { (a constant). }
$$

Hence,

$$
s=c \tan \theta \quad \text { or } \quad s=c \frac{d y}{d x} . \quad . \quad . \quad . \quad .
$$

This equation is the intrinsic equation of the catenary. The cartesian equation will now be found. In any curve,

$$
\frac{d s}{d y}=\sqrt{1+\left(\frac{d x}{d y}\right)^{2}} \text {. }
$$

Hence, from (3), the following equation is obtained,

Therefore,

$$
\frac{d s}{d y}=\sqrt{1+\frac{c^{2}}{s^{2}}}=\frac{\sqrt{s^{2}+c^{2}}}{s} .
$$

Integrating,

$$
d y=\frac{s d s}{\sqrt{s^{2}+c^{2}}} .
$$

$$
y+A=\sqrt{s^{2}+c^{2}} .
$$


If now the origin is transferred to $O^{\prime}$, where $O O^{\prime}=c$, then $y=c$ when $s=0$ and hence $A=0$. The last equation, therefore, becomes,

$$
y=\sqrt{s^{2}+c^{2}}
$$

Eliminating $y$ from (3) and (4),

Integrating this equation,

$$
d x=\frac{c d s}{\sqrt{c^{2}+s^{2}}}
$$

$$
x+B=c \log _{c}\left(s+\sqrt{s^{2}+c^{2}}\right) .
$$

Since $s=0$ when $x=0, B=c \log _{e} c$ and hence the last equation bècomes,

$$
x=c \log _{e} \frac{s+\sqrt{s^{2}+c^{2}}}{c}=c \log _{e} \frac{y+s}{c} .
$$

Equation (5) can also be written in the form,

$$
\sqrt{s^{2}+c^{2}}+s=c e^{\frac{x}{c}} . \quad \text {. . . . . . . . }
$$

By inverting each side of (6). and rationalizing the denominator of the left side, the following equation is obtained,

$$
\sqrt{s^{2}+c^{2}}-s=c e^{-\frac{x}{c}} \text {. . . . . . . . . }
$$

Adding (6) and (7) and using (4),

$$
y=\frac{c}{2}\left(e^{\frac{x}{c}}-e^{-\frac{x}{c}}\right)=c \cosh \frac{x}{c} . \quad . \quad . \quad . \quad . \quad .
$$

This is the cartesian equation of the catenary. Subtracting (7) from (6) we have,

$$
s=\frac{c}{2}\left(e^{\frac{x}{c}}-e^{-\frac{x}{c}}\right)=c \sinh \frac{x}{c} . . \quad . \quad . \quad . \quad . \quad .
$$

Squaring and adding (1) and (2) we have,

Hence;

$$
T^{2}=H^{2}+w^{2} s^{2}=w^{2} c^{2}+w^{2} s^{2}=w^{2} y^{2} .
$$$$
T=w y . \quad \cdot \quad \cdot \quad \cdot \quad \cdot \quad \cdot \quad \cdot \quad \cdot \quad \cdot \quad \cdot \quad \cdot
$$ 
Summarizing, then, the following important properties of the catenary may be stated:

(1) The horizontal component of the stress at any point is constant and equal to $w c$.

(2) The vertical component of the stress at any point is equal to $w s$.

(3) The total stress $T$ at any point is equal to $w y$.

In engineering problems which involve the catenary we are concerned particularly with the tension at the points of support, since at these points the tension is a maximum. Hence, in the above formula, $T$ will be regarded as the tension at the points of support and the values of $x, y$, and $s$ will be regarded as the values of the variables at these points. Therefore, if the length of the cable be denoted by $l$, the span by $a$, and the sag by $f$, then the values of $x, y$, and $s$ in the above equations become $\frac{a}{2}, f+c$, and $\frac{l}{2}$, respectively.

It may be noted that when the sag of the catenary is small the curve very closely approximates a parabola, since the load is approximately uniform horizontally. The formulas of Art. 59 are generally used when the sag is small, since they are much easier to apply and the results obtained are sufficiently accurate for practical purposes. When the sag is large as compared with the span, however, the above formulas should be used.

Since the relations between the quantities as expressed by the above equations are complicated, many of the problems which involve the catenary can be solved only by trial.

\section{ILLUSTRATIVE PROBLEM}

87. A cable weighing $4 \mathrm{lb}$. per foot is stretched between two points in the same horizontal plane. The length of the cable is $600 \mathrm{ft}$. and the tension at the points of support is $2000 \mathrm{lb}$. Find the sag and also the distance between the points of support.

Solution.-From equation (10),

$$
y=\frac{T}{w}=\frac{2000}{4}=500 \mathrm{ft} .
$$

From equation (4),

$$
c=\sqrt{y^{2}-s^{2}}=\sqrt{(500)^{2}-(300)^{2}}=400 \mathrm{ft} .
$$

Hence, $\quad f=y-c=500-400=100 \mathrm{ft}$. 
From equation (5),

Hence,

$$
\begin{aligned}
x & =c \log _{e} \frac{\sqrt{s^{2}+c^{2}}+s}{c}=400 \log _{e} \frac{\sqrt{(300)^{2}+(400)^{2}}+300}{400} \\
& =277.2 \mathrm{ft} .
\end{aligned}
$$$$
a=2 x=554.4 \mathrm{ft} \text {. }
$$

\section{PROBLEMS}

88. A cable $100 \mathrm{ft}$. long is suspended between two points which are in the same horizontal plane and $80 \mathrm{ft}$. apart. What is the sag at the mid-point of the cable?

$$
\text { Ans. } f=26.54 \mathrm{ft} \text {. }
$$

89. A cable weighing $2 \mathrm{lb}$. per foot is stretched between two points in the same horizontal plane which are $150 \mathrm{ft}$. apart. If the sag is $5 \mathrm{ft}$. what is the length of the cable and the tension at the points of support.

Ans. $l=150.44 \mathrm{ft} . \quad T=1130 \mathrm{lb}$.

\section{§ 7. Concurrent Forces in Space}

61. Equations of Equilibrium.-A non-coplanar, concurrent system of forces is in equilibrium if the algebraic sums of the components of the forces along any three non-coplanar lines through the point of concurrence of the forces are equal to zero. As a matter of convenience the three lines will be taken as a set of rectangular axes through the point of concurrence, in which case the equations of equilibrium may be written:

$$
\begin{aligned}
& \Sigma F_{x}=0 \\
& \Sigma F_{y}=0 \\
& \Sigma F_{z}=0
\end{aligned}
$$

Proof.-The resultant of a concurrent system of forces in space, if not in equilibrium, is a force (Art. 32). In order to satisfy the equation $\Sigma F_{x}=0$, the resultant, if there be one, must lie in the $y z$-plane. In order to satisfy the equation $\Sigma F_{y}=0$, the resultant must lie in the $x z$-plane, and in order to satisfy the equation $\Sigma F_{z}=0$, the resultant must lie in the $x y$-plane. It is impossible for a force to lie in the three planes simultaneously and hence, if the forces of the system satisfy the above equations, the resultant cannot be a force and, therefore, the system must be in equilibrium. 


\section{ILLUSTRATIVE PROBLEM}

90. The wall bracket (Fig. 112a) is composed of two flexible cables, $A C$ and $B C$, and a stiff rod, $D C$, which is pin-connected at $D$ and $C$. The points $A, B$, and $C$ lie in a horizontal plane and $A, B$, and $D$ lie in a vertical plane, $D$ being vertically beneath $E$, the mid-point of $A B$. Find the stresses in the three members due to the $100-\mathrm{lb}$. load shown.

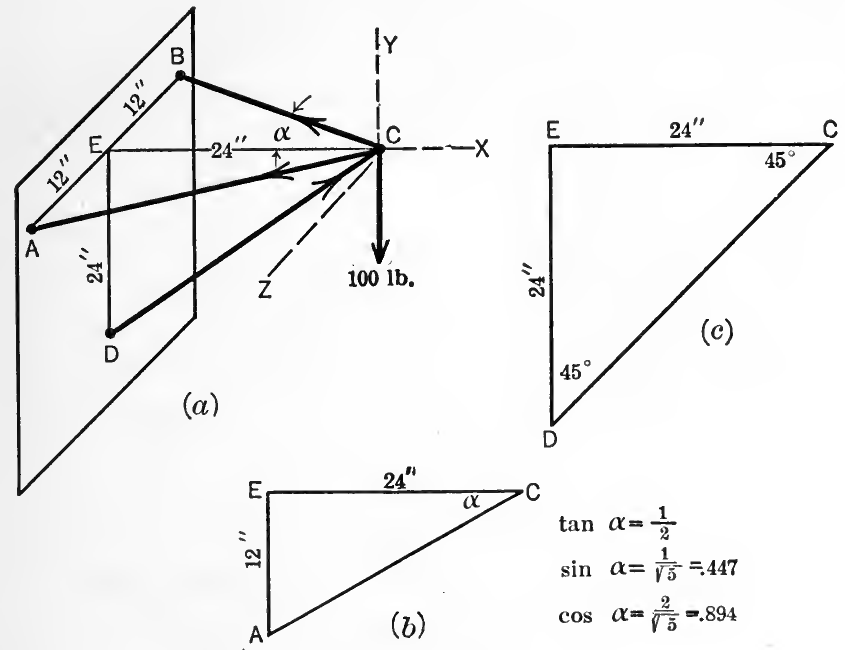

Fig. 112 .

Solution.-The pin $C$ is in equilibrium under the action of the 100-lb. load and the reactions of the three members, these reactions being equal to the stresses in the corresponding members. The free-body diagram of the pin $C$ is shown in Fig. 112(a). By selecting axes as indicated and applying the equations of equilibrium, the following equations are obtained:

$$
\begin{aligned}
\Sigma F_{y} & =D C \cos 45^{\circ}-100=0, \\
\therefore \quad D C & =\frac{100}{\cos 45^{\circ}}=141.4 \mathrm{lb} . \\
\Sigma F_{z} & =A C \sin \alpha-B C \sin \alpha=0, \\
\therefore \quad A C & =B C . \\
\Sigma F_{x} & =D C \cos 45^{\circ}-A C \cos \alpha-B C \cos \alpha=0
\end{aligned}
$$

$D C \cos 45^{\circ}=2 A C \cos \alpha$,

$$
\therefore A C=\frac{D C \cos 45}{2 \cos \alpha}=\frac{141.4 \times .707}{2 \times .894}=55.9 \mathrm{lb} \text {. }
$$

Hence there is a compressive stress of $141.4 \mathrm{lb}$. in the rod $D C$ and a tensile stress of $55.9 \mathrm{lb}$. in each of the cables $B C$ and $A C$. 


\section{PROBLEMS}

91. A weight of $150 \mathrm{lb}$. is suspended from hooks at points $A, B, C$, of a ceiling as shown in Fig. 113. $A D, B D$, and $C D$ are cords each $10 \mathrm{ft}$. in length.

Find the stresses in the cords.

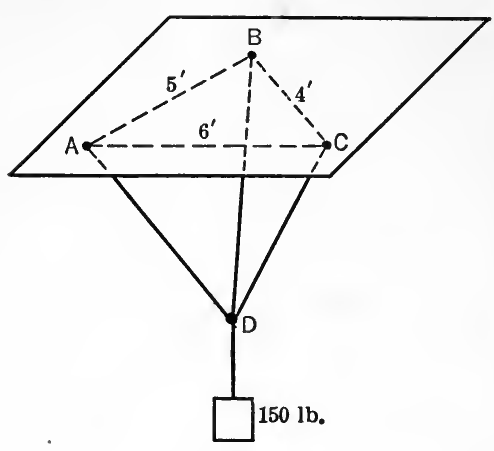

FIG. 113.

$$
\begin{aligned}
A n s . & A D=71.68 \mathrm{lb} . \quad B D \\
= & 18.08 \mathrm{lb} . \quad C D=67.24 \mathrm{lb} .
\end{aligned}
$$

92. Fig. 114 represents a stiffleg derrick. The member $A C$ lies in the $x y$-plane and the members $B D$ and $B E$ lie in vertical planes making angles of $45^{\circ}$ with the $x y$-plane. The weight carried is such as to produce a tensile stress of $5000 \mathrm{lb}$. in the member $B C$. Find the stresses in the members $A C, B D$, and $B E$.

Ans. $A C=5670 \mathrm{lb}$.

$$
B E=B D=4910 \mathrm{lb} \text {. }
$$

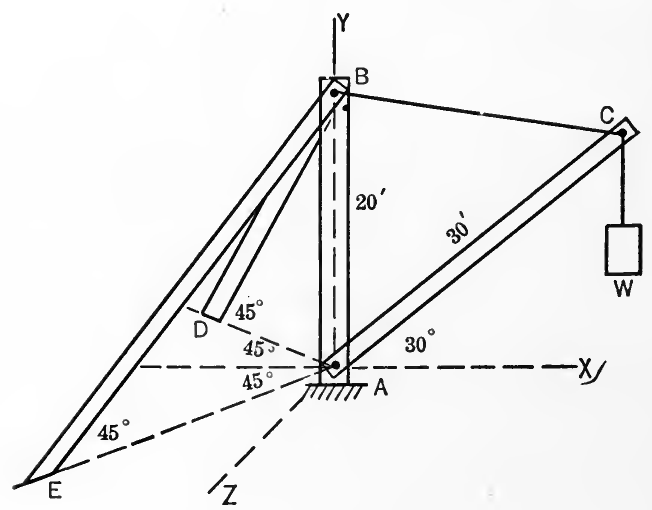

FIG. 114.

\section{§ 8. Parallel Forces in Space}

62. Equations of Equilibrium.-A system of parallel forces in space is in equilibrium if the algebraic sum of the forces is zero, and if the algebraic sum of the moments of the forces with respect to each of two non-parallel lines is equal to zero, provided that neither one of the lines is parallel to the forces of the system. It will be convenient to select a set of rectangular axes so that one of the 
axes (the $z$-axis, say) is parallel to the forces. If the axes are so selected the equations of equilibrium may be written:

$$
\begin{aligned}
\Sigma F & =0, \\
\Sigma M_{x} & =0, \\
\Sigma M_{y} & =0 .
\end{aligned}
$$

Proof.-The resultant of a system of parallel forces in space which is not in equilibrium is either a force or a couple (Art. 35). If $\Sigma F=0$, the resultant cannot be a force. If the resultant is a couple it must lie in a plane parallel to the $x z$-plane in order to satisfy the equation $\Sigma M_{x}=0$, and in order to satisfy the equation $\Sigma M_{y}=0$ it must lie in a plane parallel to the $y z$-plane.

A plane, however, cannot be parallel to both the $x z$ - and $y z$ planes, and hence, if the two moment equations are satisfied the resultant cannot be a couple. Therefore if the forces of the system satisfy the three above equations, the force system is in equilibrium.

\section{ILLUSTRATIVE PROBLEM}

93. In Fig. 115, $A B C$ represents a triangular plate, the sides of which are each $2 \mathrm{ft}$. in length. It is held in a horizontal position by vertical cords at the three vertices. A weight of $200 \mathrm{lb}$ is suspended from the point $E$ which lies on the median $A D$, the distance $D E$ being 6 in. Find the stresses in the cords neglecting the weight of the plate. follows:

Solution-The stresses may be found from the equilibrium equations as

$$
\begin{aligned}
\Sigma M_{x} & =T_{3} \times 2 \sin 60^{\circ}-200 \times \frac{1}{2}=0, \\
\therefore T_{3} & =\frac{100}{2 \sin 60^{\circ}}=57.7 \mathrm{lb} . \\
\Sigma M_{2} & =T_{2} \times 1-T_{1} \times 1=0, \\
\therefore T_{2} & =T_{1} . \\
\Sigma F & =T_{1}+T_{2}+T_{3}-200=0, \\
T_{1}+T_{2} & =200-T_{3}, \\
2 T_{1} & =142.3,
\end{aligned}
$$$$
\therefore T_{1}=T_{2}=71.15 \mathrm{lb} \text {. }
$$

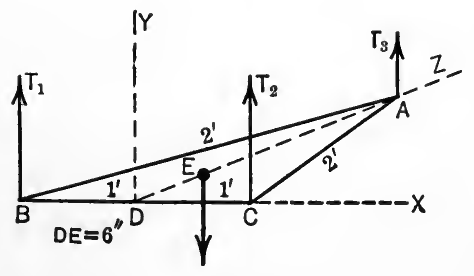

FIG. 115. 


\section{PROBLEMS}

94. A square table weighing $50 \mathrm{lb}$. stands on four legs at the mid-points of the sides. Find the greatest weight that can be placed on one corner of the table without causing it to overturn.

95. A uniform circular plate weighing $200 \mathrm{lb}$. is supported in a horizontal position at three points on its circumference. Find the reactions at the supports if the points divide the circumference into arcs of $90^{\circ}, 135^{\circ}$, and $135^{\circ}$.

\section{§ 9. Non-concurrent, Non-parallel Forces in Space}

63. Equations of Equilibrium.-A system of non-coplanar, non-concurrent, non-parallel forces is in equilibrium if the algebraic sum of the components of the forces in each of three directions is equal to zero and if the algebraic sum of the moments of the forces with respect to each of three axes is equal to zero, provided that the directions of resolution are so chosen that lines drawn through any arbitrary point in these three directions are not coplanar, and that the moment axes do not lie in a plane, and that no two of them are parallel. It will be convenient to take the coordinate axes for the axes of resolution and for the moment axes, in which case the equations of equilibrium may be written as follows:

$$
\begin{array}{ll}
\Sigma F_{x}=0, & \Sigma M_{x}=0, \\
\Sigma F_{y}=0, & \Sigma M_{y}=0, \\
\Sigma F_{z}=0, & \Sigma M_{z}=0 .
\end{array}
$$

Proof.-The resultant of a non-concurrent, non-parallel system of forces in space is, in general, a force and a couple (Art. 41). If the first three equations are satisfied the resultant force must vanish and if the last three equations are satisfied the resultant couple must vanish. If, therefore, the forces of the system satisfy the six equations the force system is in equilibrium.

\section{ILLUSTRATIVE PROBLEM}

96. Fig. 116(a) represents a windlass used in lifting heavy weights. The end bearings will be regarded as smooth and the force $P$ applied to the crank will be assumed to be perpendicular to the axis of the cylinder and also perpendicular to the crank. Find the value of $P$ required to hold the $450-1 \mathrm{~b}$. 
weight and also find the reactions at the bearings, assuming that the crank is inclined $30^{\circ}$ to the vertical.
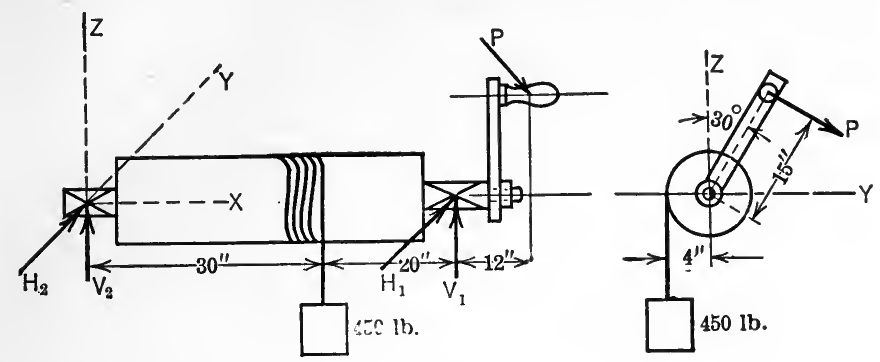

FIG. 116.

Solution.-The coordinate axes will be taken as shown in the figure. There are four forces acting on the windlass, namely, the weight of $450 \mathrm{lb}$., the force $P$, and the reactions at the bearings. Since the bearing reactions are unknown in direction as well as in magnitude it will be convenient to resolve them into horizontal and vertical components, $H, V_{1}$ and $H_{2}, V_{2}$, as indicated in the figure. Applying the equations of equilibrium to the system of forces acting on the windlass we have,

$$
\begin{aligned}
& \Sigma F_{y}=P \cos 30^{\circ}+H_{1}+H_{2}=0 . \\
& \Sigma F_{2}=V_{1}+V_{2}-P \sin 30^{\circ}-450=0 . \\
& \Sigma M_{x}=15 P-450 \times 4=0 . \\
& \Sigma M_{y}=P \sin 30^{\circ} \times 62+450 \times 30-50 V_{1}=0 . \quad . \quad . \quad . \\
& \Sigma M_{z}=50 H_{1}+P \cos 30^{\circ} \times 62=0 .
\end{aligned}
$$

The solution of these equations gives the following values:

$$
\begin{aligned}
& P=120 \mathrm{lb} . \\
& H_{1}=-128.8 \mathrm{lb} . \\
& V_{1}=344.4 \mathrm{lb} . \\
& H_{2}=24.92 \mathrm{lb} . \\
& V_{2}=165.6 \mathrm{lb} .
\end{aligned}
$$




\section{GENERAL PROBLEMS}

97. The pressure between the rubbing surfaces of the friction clutch shown in Fig. 117 is $15 \mathrm{lb}$. per square inch normal to the surfaces. What force $P$ on the bell crank is required to produce this pressure?

$$
\text { Ans. } P=196 \mathrm{lb} \text {. }
$$

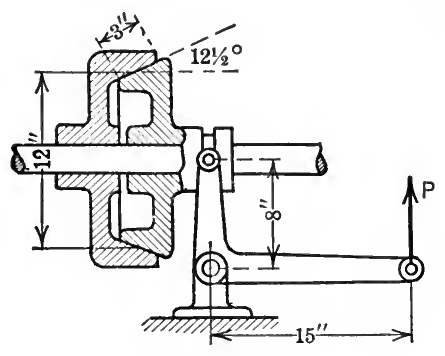

Fig. 117.

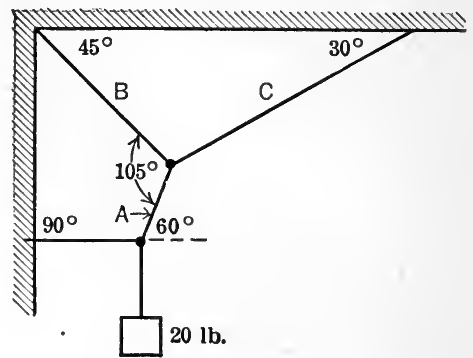

Fig. 118.

98. A body weighing $20 \mathrm{lb}$. is held in equilibrium by four cords as shown in Fig. 118. What are the stresses in cords $A, B$, and $C$ ?

$$
\text { Ans. } A=23.1 \mathrm{lb} . \quad B=1195 \mathrm{lb} . \quad C=231 \mathrm{lb} \text {. }
$$

99. A sphere which weighs $40 \mathrm{lb}$. is held on a smooth inclined plane by means of a cord which is attached to a ceiling as shown in Fig. 119. Determine the pressure of the plane against the sphere and the tension in the cord.

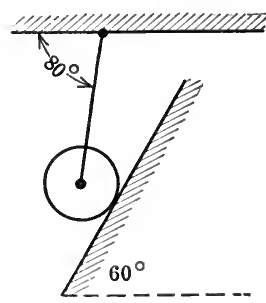

FIG. 119.

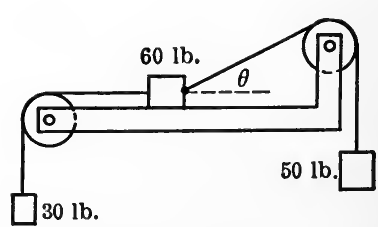

Fig. 120.

100. A body weighing $60 \mathrm{lb}$. is held in equilibrium on a smooth surface by two cords which pass over frictionless pulleys and carry suspended weights of $30 \mathrm{lb}$. and $50 \mathrm{lb}$. as shown in Fig. 120. Find the reaction of the surface on the body and the angle $\theta$.

Ans. $R=20 \mathrm{lb} . \quad \theta=53^{\circ} 8^{\prime}$.

101. The pillar crane shown in Fig. 121 is bolted to the floor by six bolts as shown. The pressure (or the pull) between the base of the crane and the floor is assumed to be concentrated along the axes of the bolts. Find the ten- 
sion in the two bolts to the left of the $Y Y$ axis and the pressure between the base and the floor at the corresponding bolts to the right of the axis, caused by the load of $2000 \mathrm{lb}$. shown.

Ans. 2170 (tension); 2830 (compression).

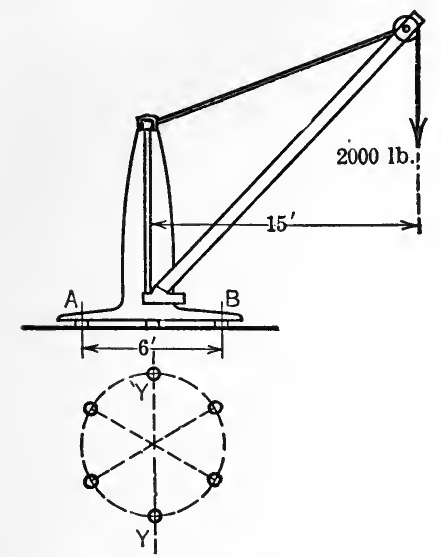

Fig. 121.

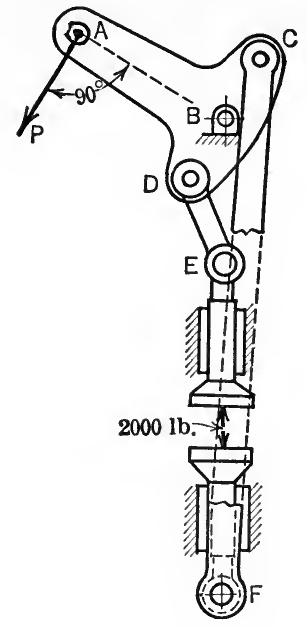

FIG. 122.

102. Find $R$, the maximum unbalanced pressure (recoil pressure) that can exist on the breech of a 3-in. gun (Fig. 123), when firing, without causing the wheels to leave the ground, assuming the earth pressures and the weight of the gun to be as shown in Fig. 123.

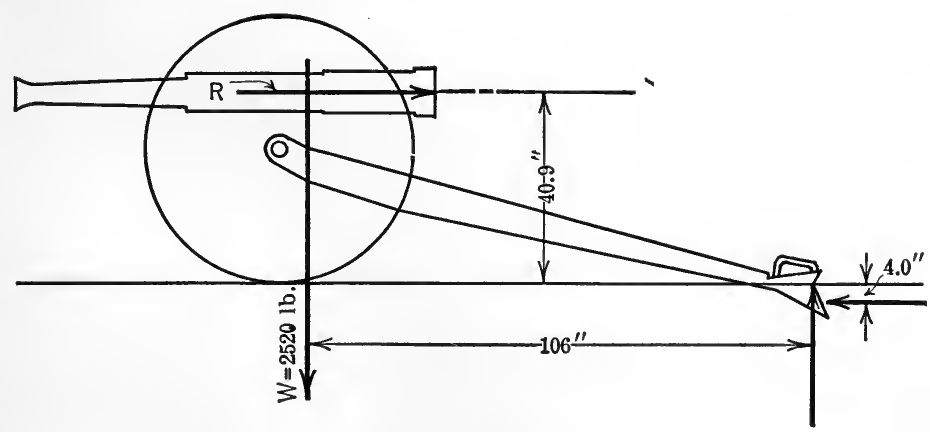

FIG. 123.

103. A uniform beam weighing $W \mathrm{lb}$. rests with its ends on two smooth planes which are inclined at angles of $60^{\circ}$ and $30^{\circ}$ with the horizontal. Find the inclination of the beam with the horizontal.

Ans. $\theta=30^{\circ}$. 
104. The two bodies shown in Fig. 124 are held in equilibrium on two smooth rods by a connecting cord. If the bodies weigh $200 \mathrm{lb}$. and $50 \mathrm{lb}$., find the reactions of the rods, the tension in the cord, and the angle $\theta$.

105. The power press shown in Fig. 122 has the following dimensions: $A B=2.5 \mathrm{ft} . \quad B C=B D=1 \mathrm{ft} . \quad D E=1.5 \mathrm{ft} . \quad F C=8 \mathrm{ft} . \quad F B=7.1 \mathrm{ft}$. The points $B, E$, and $F$ are on the same vertical line. What force $P$ is required to cause a pressure of $2000 \mathrm{lb}$. between the jaws of the press?

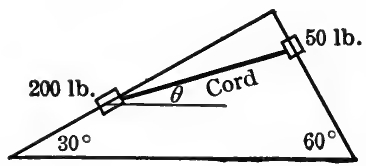

FIG. 124:

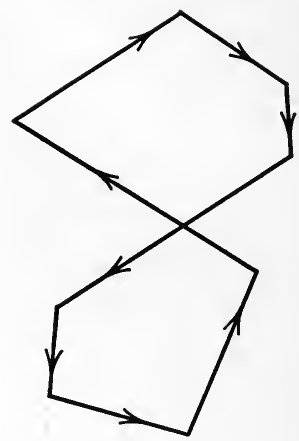

FIG. 125.

106. Forces act along the sides of the polygon shown in Fig. 125. The forces are proportional to the lengths of the corresponding sides. If the areas of the two loops are equal, show that the system of forces is in equilibrium.

107. A body weighing $80 \mathrm{lb}$. is suspended by two strings of lengths $5 \mathrm{ft}$. and $12 \mathrm{ft}$., the upper ends of which are attached to a horizontal plane, the distance between the two points of attachment being $13 \mathrm{ft}$. Find the tensions in the strings. Ans. $30.8 \mathrm{lb} ; 73.8 \mathrm{lb}$.

108. A beam $8 \mathrm{ft}$. long rests on two horizontal supports and is loaded as shown in Fig. 126. If the weights of the beams are neglected, find the reactions $R_{1}$ and $R_{2}$ at the points of support.

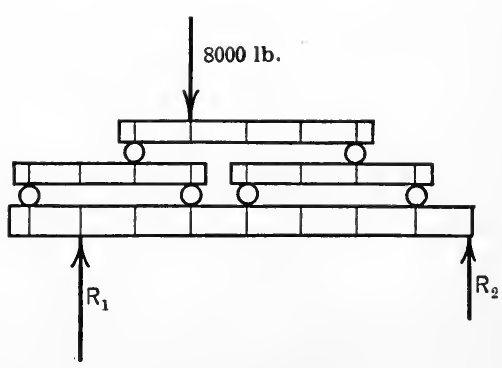

FIG. 126.

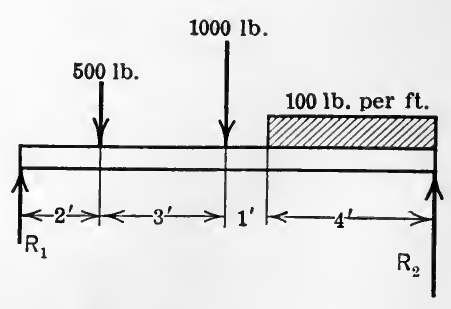

FIG. 127. 
109. A horizontal beam $10 \mathrm{ft}$. long is supported at its ends and loaded with two concentrated loads and a uniformly distributed load of $100 \mathrm{lb}$. per ft. over a length of $4 \mathrm{ft}$. as shown in Fig. 127. Find the reactions $R_{1}$ and $R_{2}$ at the points of supports.

Ans. $R_{1}=980 \mathrm{lb} . \quad R_{2}=920 \mathrm{lb}$.

110. A camp stool loaded as shown in Fig. 128 rests on a smooth floor. Find the reactions of the floor on the stool.

111. Find the magnitudes of the forces which must act along lines I and II (Fig. 130) in order to hold in equilibrium the three forces shown. Solve by the graphical method.

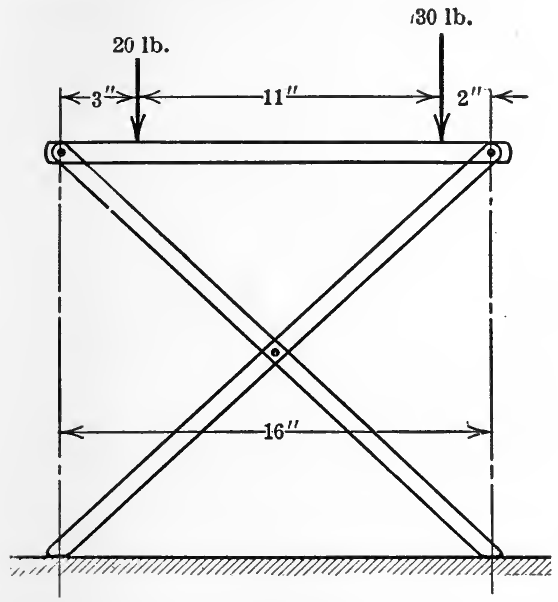

Fig. 128.

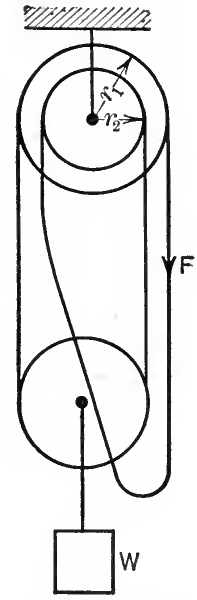

FIg. 129.

112. In Fig. 132 is shown one form of a dynamometer. The pressure on the scale beam at $D$ is balanced by the poise weights $A$ and $B$. The weight of $A$ and $B$ together is $150 \mathrm{lb}$. and that of $B$ alone is $3.5 \mathrm{lb}$. The divisions on the large scale are $1 \mathrm{in}$. apart and those on the small scale are $0.4 \mathrm{in}$. apart.

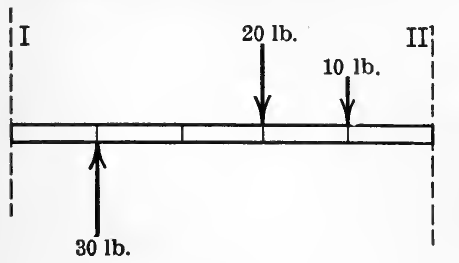

Fig. 130.

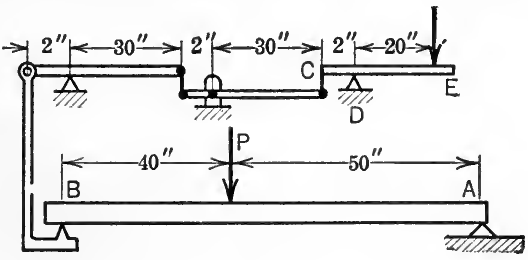

FIG. 131.

When both $A$ and $B$ are set at zero on the scales they are just balanced by the weight $C$. If, in order to maintain balance, $A$ is set at the 2 nd division and $B$ 
is set at the 15th division, what is the pressure of the dynamometer on the scale beam at $D$ ?

Ans. $R=26.75 \mathrm{lb}$.

113. In Fig. 131 is shown a diagrammatic sketch of an apparatus for measuring a load on a beam. If a force of $5 \mathrm{lb}$. at $E$ is required to balance the scale beam $C D E$ when the beam $A B$ carries a load $P$ in the position shown, find the magnitude of the load.

114. In Fig. 129 is represented a differential chain hoist. Two sheaves of radii $r_{1}$ and $r_{2}$ are fastened together and a continuous chain passes around the small sheave, then around a movable pulley of diameter $r_{1}+r_{2}$, and then around the larger sheave. Neglecting the resistance due to friction, find the relation between the applied force $F$ and the load $W$ which it will hold.

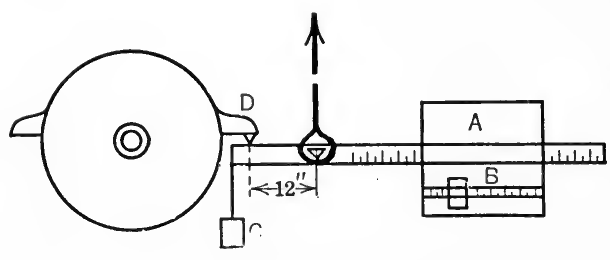

FIG. 132.

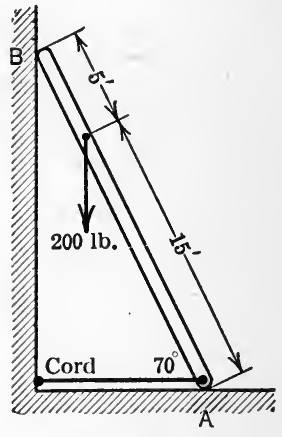

FIg. 133.

115. The bar shown in Fig. 134 is connected to a fixed support by a smooth pin at $A$ and rests on a smooth surface at $B$. Find the reaction at $B$ and the magnitude and direction of the pin pressure at $A$.

$$
\text { Ans. } A=25.4 \mathrm{lb} \text {. }
$$

116. A bar rests against two smooth surfaces as shown in Fig. 133 and is prevented from slipping by means of a cord attached to the lower end. If the weight of the bar is neglected, find the reaction of each surface and the tension in the cord.

Ans. $R_{A}=200 \mathrm{lb} . \quad R_{B}=54.6 \mathrm{lb} . \quad T=54.6 \mathrm{lb}$.

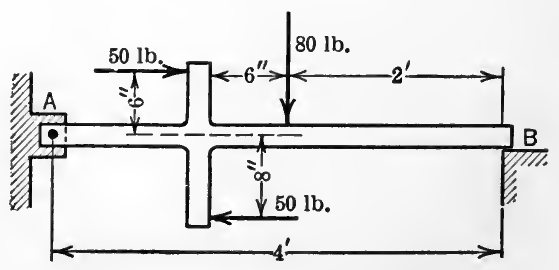

Fig. 134. 
117. In the crane represented in Fig. 135 the weight of the post $A B$ is 1 ton, the weight of the boom $C E$ is 0.8 ton and that of the brace $D F$ is 1 ton. The reaction at $B$ is horizontal. Find the reaction at $B$ and the horizontal and vertical components of the reaction at $A$. Also find the pin reaction on the brace at $F$ and at $I$. Ans. $B=5.84$ tons; $D=11.2$ tons; $F=11.9$ tons.

118. In the crane represented in Fig. 136, the weight of the post $A B$ is $1600 \mathrm{lb}$, the weight of the boom $D E$ is $1200 \mathrm{lb}$., and the weight of the rod $C E$ may be neglected. Find the tension in the rod and the reaction of the pin at $D$ on the post.

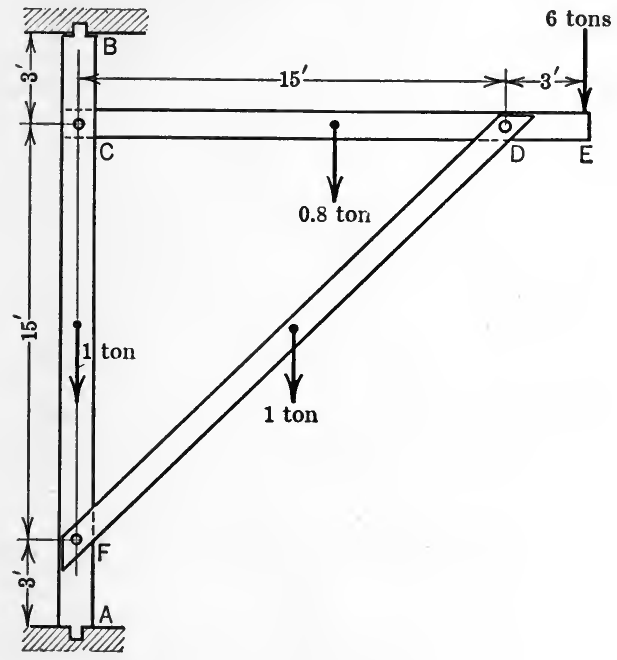

FIG. 135.

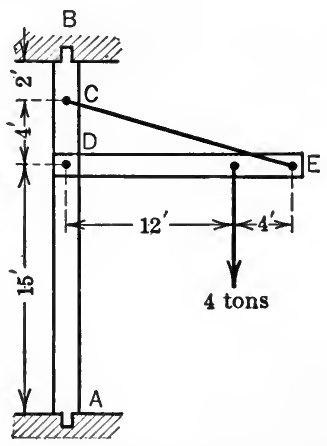

FIG. 136.

119. Two members $A B$ and $B C$ (Fig. 137) are connected by a smooth pin at $B$ and their lower ends rest on a smooth horizontal surface, slipping on the surface being prevented by a cord which connects the ends $A$ and $C$. The weight of $A B$ is $120 \mathrm{lb}$. and the weight of $B C$ is $180 \mathrm{lb}$. The member $B C$

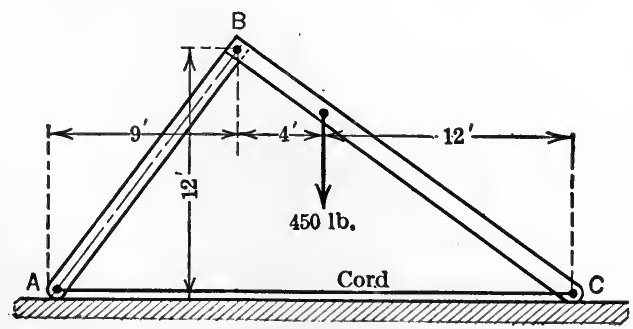

Fig. 137. 
also carries a concentrated load of $450 \mathrm{lb}$. as shown. Find the reactions of the surface, the tension in the cord, and the pin reaction at $B$.

Ans. $R_{A}=372 \mathrm{lb} . \quad R_{C}=378 \mathrm{lb} . \quad T=234 \mathrm{lb} . \quad R_{B}=344 \mathrm{lb}$.

120. In Fig. 139 is shown a shear for cutting steel bars in a repair yard. What force $P$ is required to give a pressure of $70,000 \mathrm{lb}$. on the anvil when the $3 \mathrm{ft} .-6 \mathrm{in}$. arm is vertical. Solve graphically.

121. Two bars $A C$ and $B D$ (Fig. 138) are connected to each other by a smooth pin at $C$ and to the floor by smooth pins at $A$ and $B . \quad A C=C B=4 \mathrm{ft}$. $C D=3 \mathrm{ft}$. Find the pin pressures at $A, B$, and $C$.

$$
\text { Ans. } A=140 \mathrm{lb} . \quad B=109.8 \mathrm{lb} . \quad C=140 \mathrm{lb} \text {. }
$$

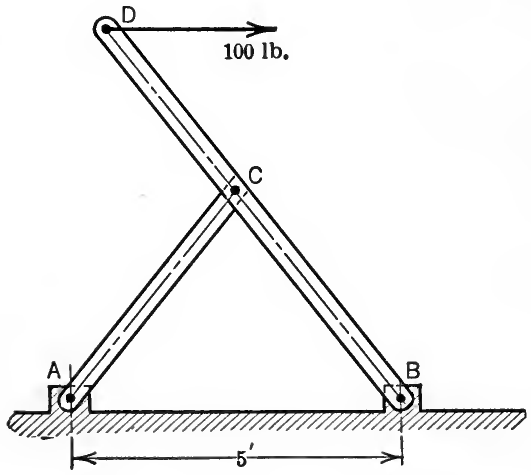

FIG. 138.

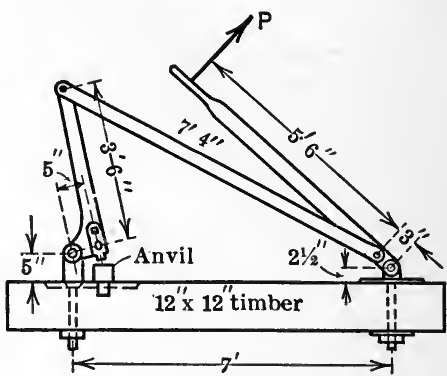

Frg. 139.

122. In Fig. 140, when $r$ is zero, the tension in the spring, $S$, is $50 \mathrm{lb}$. The modulus of the spring is 58 lb. per inch. Find the tension in the spring in pounds when $r=3$ in. and also find the pulls $P, P$ required to cause this spring tension. What is the total stretch of the spring?

Ans. $S=94.1 \mathrm{lb} . \quad P=24.3 \mathrm{lb}$. Stretch $=1.62$ in.

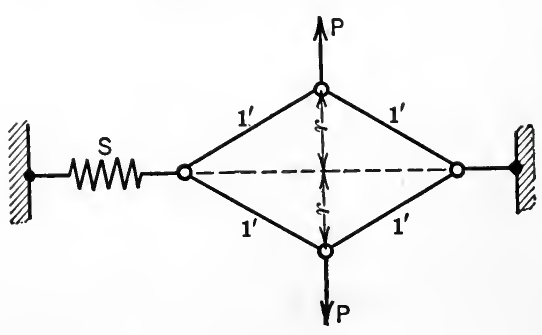

Fig. 140.

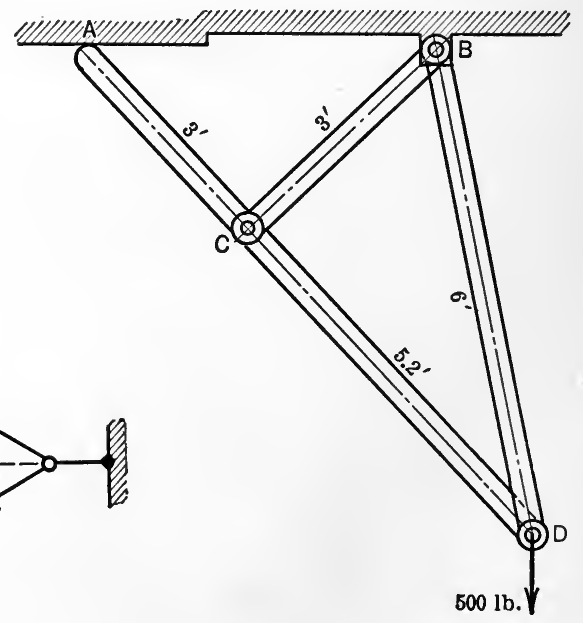

Fig. 141. 
123. The weights of the members $A D, B C$, and $B D$ of the frame showu in Fig. 141 are $100 \mathrm{lb}$., $50 \mathrm{lb}$., and $75 \mathrm{lb}$, respectively. Find the pin pressure at $C$ and the pressure at $A$, the surface at $A$ being smooth.

$$
\text { Ans. } A=152 \mathrm{lb} . \quad C=225 \mathrm{lb} \text {. }
$$

124. Find by the method of sections, the stresses in the members $C E, D E$, and $D F$ of the Howe truss shown in Fig. 142.

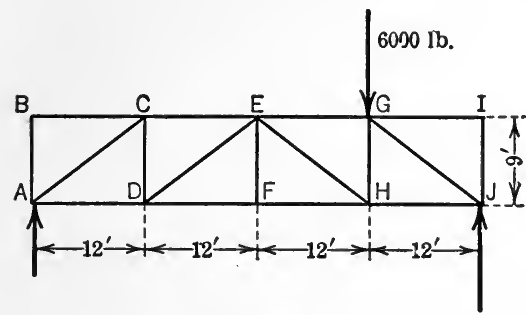

FIG. 142.

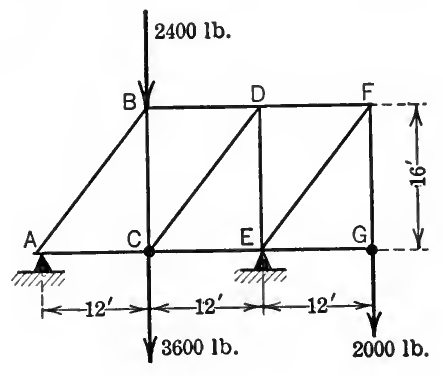

Fig. 143.

125. The truss shown in Fig. 143 is supported at $A$ and $E$. Find the stresses in the members $B D, C D$, and $C E$.

126. Find the stresses in the members $C E, D E$, and $D F$ of the truss shown in Fig 144. Ans. $C E=+440 \mathrm{lb}$; $D E=+500 \mathrm{lb}$; $D F=-550 \mathrm{lb}$.

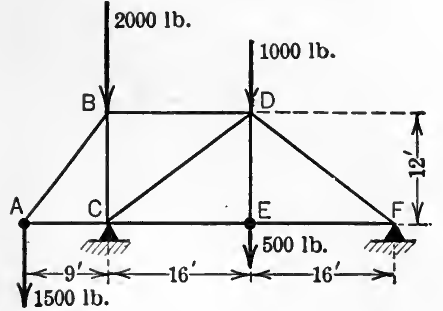

Fig. 144.

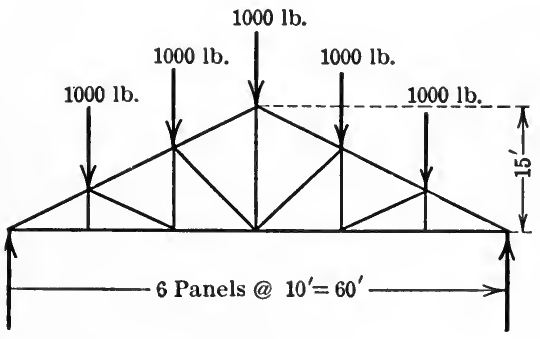

Fig. 145

127. Determine graphically the stresses in the members of the Pratt truss shown in Fig. 145.

128. The members of the crane shown in Fig. 146 are connected by smooth pins. Neglecting the weights of the members, find by the graphical method, the stresses in the members due to the 4-ton load. 
129. The framework shown in Fig. 147 is connected at the points. $A, D$, $C$, and $E$ by smooth pins and is supported by a smooth surface at $B$. Find

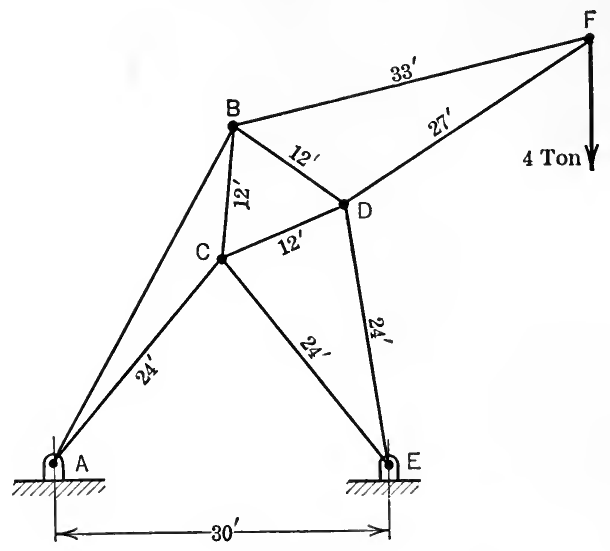

FIG. 146.

the pin reactions at $A, D, C$, and $E$, and the reaction at $B$. Neglect the weights of the members.

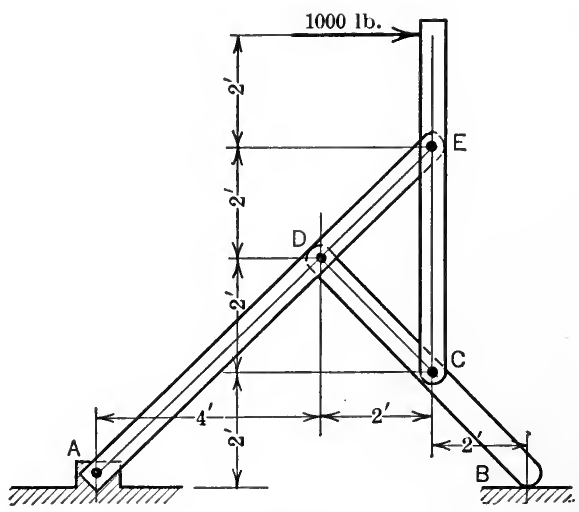

FIG. 147.

130. In the hydraulic crane shown in Fig. 148 the weight of the boom $A B C$ is $500 \mathrm{lb}$, as shown in the figure, and the weights of the members $A D$ and $B D$ may be neglected. In raising the boom the hydraulic pressure from the cylinder transmits a pressure $P$ to the pin at $D$. Neglecting the frictional resistance find the value of $P$ required to raise the boom when loaded as shown. 
Find also the stresses in $B D$ and $A D$ and the pressures of the rolls at $A$ and at $D$ against the vertical post.

Ans. $P=2500 \mathrm{lb}$.; $D=A=5540 \mathrm{lb}$. $D B=+6550 \mathrm{lb}$; $A D=-760 \mathrm{lb}$.

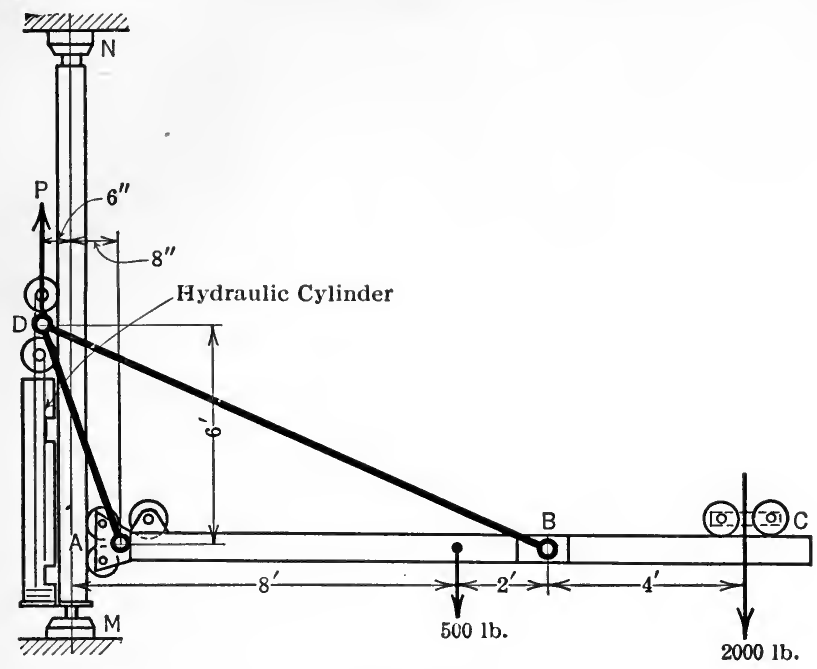

FIG. 148.

131. The crank-pin pressures, $P_{1}$ and $P_{\mathrm{a}}$, on the crank shaft shown in Fig. 150 are $6000 \mathrm{lb}$. and $4800 \mathrm{lb}$. respectively. Find the bearing reactions $R_{1}$

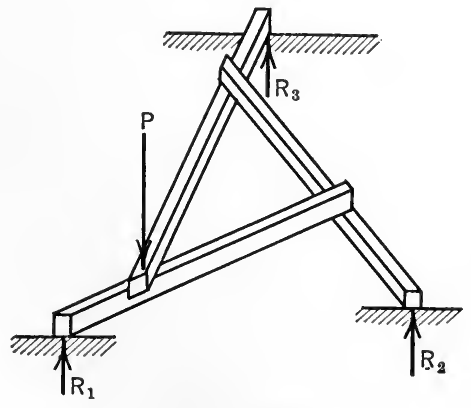

FIG. 149.

and $R_{2}$ (Fig. 150b) and the resisting moment $Q q$ required for equilibrium of the shaft.

Ans. $R_{1}=3120 \mathrm{lb} . \quad R_{2}=1920 \mathrm{lb} . \quad Q q=64,800 \mathrm{lb}$.-in.

132. Three rods each of length $l$ rest on each other (Fig. 149) in such a 
way as to form a central equilateral triangle each side of which is $\frac{2}{3} l$. A load $P$ is applied at one corner as shown. Find the reactions $R_{1}, R_{2}$, and $R_{3}$ in terms of $P$.

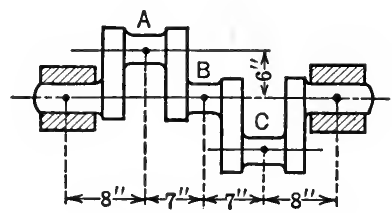

(a)

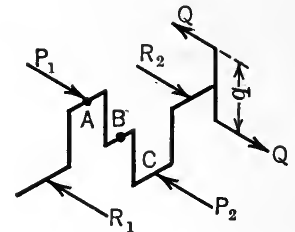

(b)

FIG. 150. 


\section{CHAPTER IV}

\section{FRICTION}

64. Friction Defined.-Friction is defined as the resistance which one body offers to the motion of a second body when the second body slides or tends to slide over the former. The frictional force is tangent to the surfaces of contact of the two bodies and always opposes motion.

Friction is of great importance in engineering practice. Since it always opposes motion, it is an undesirable and expensive factor in the operation of many machines or machine elements, and in such cases is reduced as much as practicable by means of lubricants. In other machines it becomes a very desirable and useful element, as in various forms of brakes, friction drives, etc. In fact, many of our normal physical activities, such as walking, would be impossible without the aid of friction.

If the resistance between two bodies prevents motion of one body relative to the other, the resistance is called static friction, while the frictional resistance between two bodies which move relative to each other is called kinetic friction. If the friction is static, the amount of friction developed is just sufficient to maintain equilibrium with the other forces acting on the body. That is, static friction is an adjustable force the magnitude of which is determined from

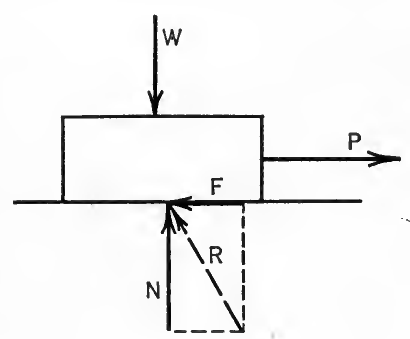

FIG. 151. the equations of equilibrium for the forces which act on the body. Thus, let Fig. 151 represent a body in equilibrium on a rough horizontal plane under the action of a horizontal force $P$, which tends to move the body, the reaction, $R$, of the plane, and the weight, $W$, of the body. Let the reaction $R$ be resolved into two components $F$ and $N$ parallel and perpendicular, 
respectively, to the plane. The component $F$ along, or tangent to, the plane is the frictional force as defined above. The component $N$ is called the normal pressure and $R$ is called the total reaction. Since the body is in equilibrium, the equations of equilibrium must be satisfied, and hence $F$ is equal to $P$. If the force $P$ is gradually increased, $F$ must increase in the same ratio in order to maintain a condition of equilibrium. There is a definite limit, however, to the amount of frictional resistance that can be developed, and when the value of $P$ exceeds this limiting value motion will ensue. The limiting or maximum value of the frictional force is called limiting friction and is denoted by $F^{\prime}$. Its value depends on the normal pressure and on the roughness of the surfaces of contact.

65. Coefficient of Friction.-In order to compare the frictional resisting properties of various pairs of materials or of the same pair of materials under varying conditions of their surfaces of contact, and in order to calculate the maximum frictional force corresponding to any normal pressure, a certain experimental constant, called the coefficient of friction, is used.

The coefficient of static friction for any two surfaces is defined as the ratio of the limiting friction to the corresponding normal pressure. Thus, if the coefficient of static friction is denoted by $\mu$, it may be expressed as follows:

$$
\mu=\frac{F^{\prime}}{N} \quad \text { or } \quad F^{\prime}=\mu N
$$

It is important to note that $F^{\prime}$ in the above equation is the maximum friction which the surfaces can develop, that is, the friction corresponding to impending motion. Thus the maximum frictional force which any two surfaces can develop is equal to $\mu N$.

The value of $\mu$ must be determined experimentally and, as stated above, it is a constant for any two materials for a definite condition of the surfaces of contact. It varies considerably, however, for different conditions of the surfaces, and it varies widely for different pairs of materials, as is shown in the following table which gives the values of the coefficient of friction for dry surfaces as determined by Morin and others. 


\section{Coefficient of Static Friction}

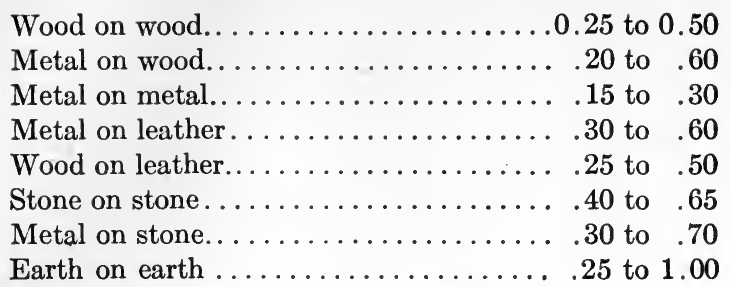

If two surfaces move relative to each other, the ratio of the friction developed to the corresponding normal pressure is defined as the coefficient of kinetic friction. The value of the coefficient of kinetic friction for two surfaces is influenced by more factors than is the value of the coefficient of static friction. A brief discussion of the influencing factors is given in Art. 68. For values of the coefficient of kinetic friction for various conditions of rubbing surfaces the reader is referred to Goodman's "Mechanics Applied to Engineering."

66. Angle of Friction and the Friction Cone.-The angle of static friction for two surfaces is defined as the angle between the directions of the total reaction and the normal pressure when motion is impending. Thus in Fig. 152 , if the force $P$ is just large enough

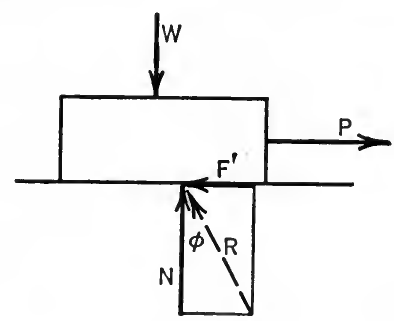

Fig. 152. to develop the limiting friction, the angle which $R$, the reaction of the plane on the body, makes with the normal pressure, $N$, is the angle of static friction and is denoted by $\phi$.

Since the components of $R$, parallel and perpendicular respectively to the plane, are $F^{\prime}$ and $N$, it is evident from the figure that $\tan \phi=\frac{F^{\prime}}{N}$. But since the ratio $\frac{F^{\prime}}{N}$ is defined as the coefficient of static friction, $\mu$, the following important relation may be written:

$$
\mu=\tan \phi,
$$

that is, the coefficient of static friction is equal to the tangent of the angle of static friction.

If the two surfaces move relative to each other then the angle between the total reaction and the normal pressure is called the 
angle of kinetic friction. Its value is somewhat less than the angle of static friction, since the frictional force after motion ensues becomes less than the limiting friction. The relation $\mu=\tan \phi$ also holds for kinetic friction, the value of $\mu$ for kinetic friction being somewhat less than for static friction. The angle of friction (for both static and kinetic friction) is convenient to use particularly in the solution of problems by graphical methods.

If a body rests on a rough plane and certain forces are applied

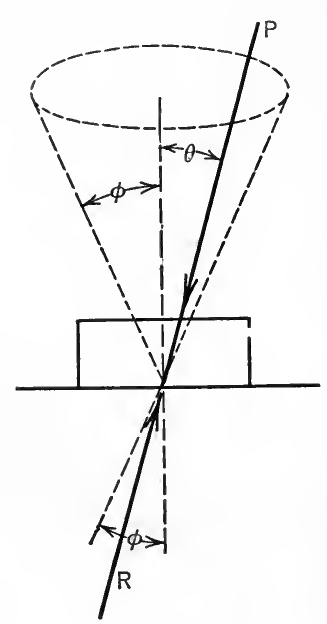

FIG. 153. to the body, a convenient graphical method of determining whether the body will slide or not makes use of the cone of friction. The cone of friction for two plane surfaces is the cone generated by revolving, about the normal to the plane, a line making with the normal an angle equal to the angle of friction as shown in Fig. 153. If the resultant of all of the forces acting on the body, except the reaction of the plane (total reaction), falls within the cone of friction, the body will not slide; if the resultant falls outside the cone of friction, the body will slide. Thus in Fig. 153 let $P$ represent the resultant of all of the forces acting on the body except $R$, the reaction of the plane. If the angle which $P$ makes with the normal to the plane is called $\theta$, the force tending to move the body is $P \sin \theta$, and the normal pressure is $P \cos \theta$. The body will not slide if

$$
P \sin \theta<\mu P \cos \theta,
$$

that is, if $\tan \theta<\mu$ or if $\tan \theta<\tan \phi$, or if

$$
\theta<\phi .
$$

If $\theta>\phi$, that is, if $P$ falls outside of the cone of friction the body will slide, and if $\theta=\phi$ motion is impending.

\section{ILLUSTRATIVE PROBLEM}

133. A lift shown in Fig. 154 slides on a vertical shaft having a square cross-section 2 in. on a side. Find the greatest distance, $x$, that a load $W$ can be placed from the edge of the shaft and still cause the lift to slide on the shaft. Neglect the weight of the lift and use 0.2 for the coefficient of friction. 
Graphical Solution.-Since motion impends, the angle between the reaction $R_{1}$ and the normal pressure $N_{1}$ of the shaft at $A$ is equal to the angle of friction; that is, the tangent of the angle is equal to 0.2 . Hence, by laying off ten spaces along the normal and two spaces perpendicular to the normal, as shown in Fig. 154, the action line of $R_{1}$ is determined. In a similar manner the action line of $R_{2}$ is found. Now the three forces $R_{1}, R_{2}$, and $W$ must be concurrent in order to be in equilibrium (Art. 48) and hence, $W$ must pass through the intersection of $R_{1}$ and $R_{2}$. Now the intersection of these two forces can never be nearer to the shaft than the point $D$, since the angle $\phi$

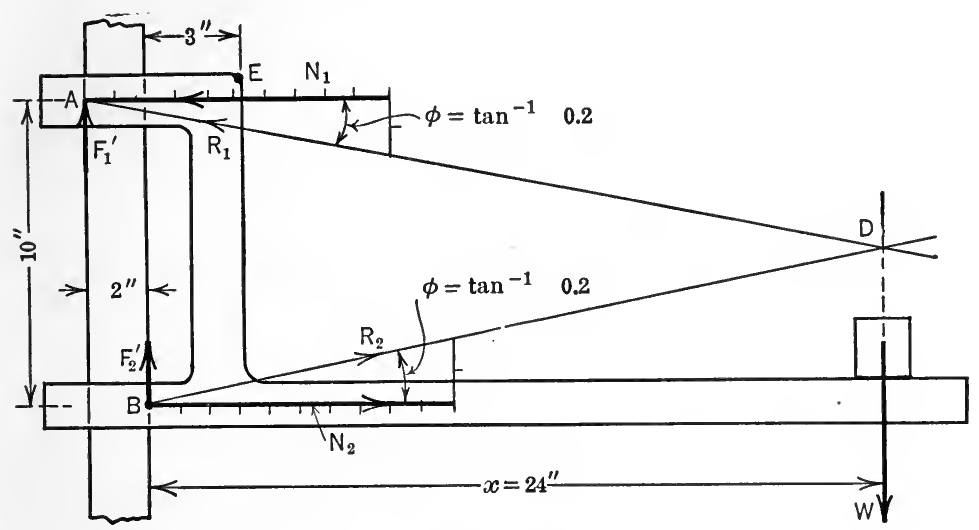

FIG. 154.

cannot be greater than $\tan ^{-1} \cdot 2$. The distance, $x$, of $D$ from the shaft is found by measurement to be 24 in.

Algebraic Solution.-The five forces, $F^{\prime}{ }_{1}, N_{1}, F^{\prime}{ }_{2}, N_{2}$, and $W$ which hold the lift in equilibrium as shown in the free-body diagram (Fig. 154) form a coplanar non-concurrent force system and hence there are three equations of equilibrium as follows:

in which

$$
\begin{gathered}
\Sigma F_{x}=N_{2}-N_{1}=0 . \quad . \quad . \quad . \quad . \quad . \quad . \quad . \quad . \\
\Sigma F_{y}=-W+0.2 N_{1}+0.2 N_{2}=0 . \\
\Sigma M_{B}=-W x+10 N_{1}-2 \times 0.2 N_{1}=0, \quad . \quad . \quad .
\end{gathered}
$$$$
0.2 N_{1}=F_{1}^{\prime} \text { and } 0.2 N_{2}=F_{2}^{\prime} \text {. }
$$

From (1) and (2) we have,

$$
W=0.4 N_{1}=0.4 N_{2} \text {. }
$$

By substituting this value of $W$ in (3) the equation obtained is,

whence,

$$
-0.4 N_{1} \cdot x+10 N_{1}-0.4 N_{1}=0,
$$

$$
x=24 \text { in. }
$$




\section{PROBLEMS}

134. If the weight $W$ in the preceding problem is $500 \mathrm{lb}$. what vertical force $P$ applied at $E$ will be just sufficient to start the lift up?

135. The coefficient of friction for the 100-1b. body and the table (Fig. 155) is 0.4. What weight, $W_{2}$, will cause motion of the three bodies to impend? The pulleys are assumed to be frictionless and the cords to be flexible.

Ans. $W_{2}=47 \mathrm{lb}$.

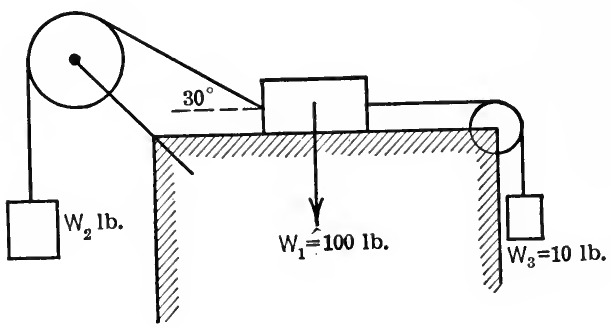

FIg. 155.

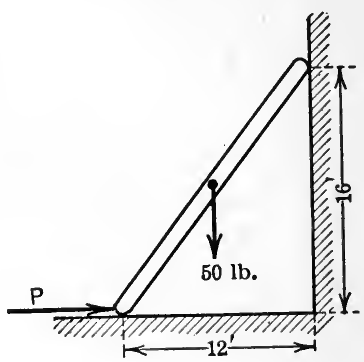

Fig. 156.

136. The ladder shown in Fig. 156 weighs $50 \mathrm{lb}$. The coefficient of friction for the ladder and vertical wall is 0.25 , and for the floor and ladder 0.5. Find the horizontal force $P$ which will cause motion to impend to the right.

Ans. $P=51 \mathrm{lb}$.

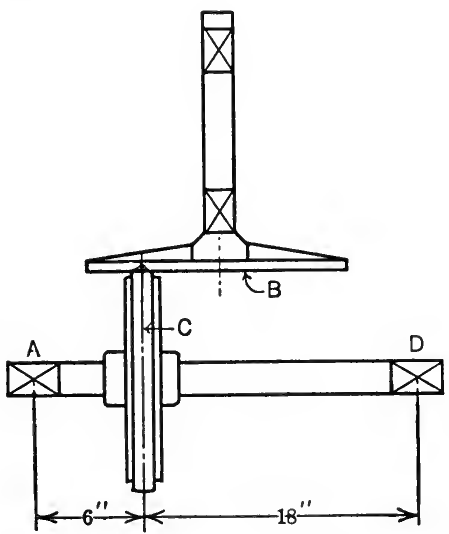

Fig. 157.

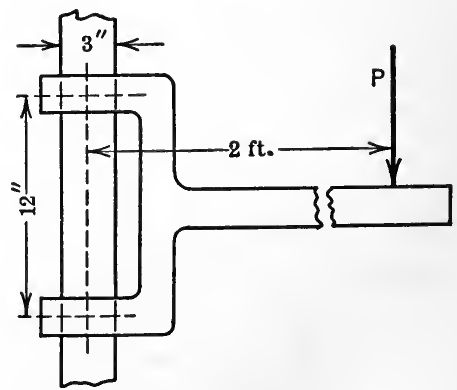

Fig. 158.

137. A rectangular block of wood $10 \mathrm{in.} \mathrm{by} 10 \mathrm{in.} \mathrm{by} 20 \mathrm{in}$. stands on end on a horizontal floor. The block is acted on by a horizontal force applied through the center of the top face and perpendicular to one side. The block 
weighs $40 \mathrm{lb}$. and the coefficient of friction is 0.20 . If the force is gradually increased until motion ensues will the block slide or tip? What is the magnitude of the force when the block starts to move?

138. A crown friction drive as indicated in Fig. 157 is used on screw power presses, motor trucks, etc. The cast iron disk $B$ rotates at 1000 r.p.m. and drives the crown wheel $C$, which is faced with leather-fiber. The diameter of $C$ is 20 in. and the value of $\mu$ is 0.35 . If a turning moment of $100 \mathrm{ft}$.-lb. is transmitted to the crown wheel shaft when slipping impends, what is the normal pressure between the disk and the crown wheel? What is the pressure on the bearings at $A$ and $D$ ?

139. A load, $P$, of $80 \mathrm{lb}$. is applied to the arm (Fig. 158) at a distance of 2 ft. from the axis of the shaft. If $\mu=0.15$ will the arm slide on the shaft?

140. A cone clutch as shown in Fig. 159 is used on automobiles to connect the motor with the transmission. If the normal pressure between the two

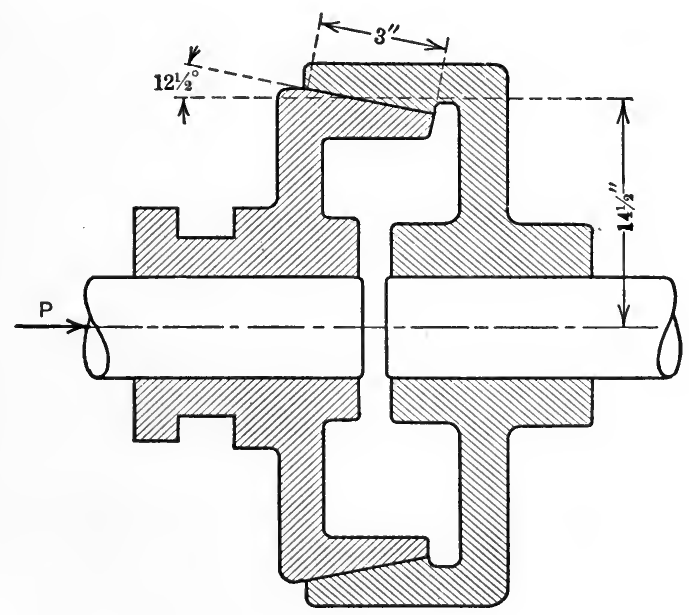

FIG. 159.

surfaces of contact, as maintained by a spring, is $15 \mathrm{lb}$. per square inch and the coefficient of friction is 0.25 , what is the maximum torque the clutch can transmit? Assume that the frictional force has a mean arm of $14 \frac{1}{2}$ in. What spring pressure, $P$, is required to produce the normal pressure of $15 \mathrm{lb}$. per square inch?

Ans. $T=1235$ lb.-ft.; $P=887 \mathrm{lb}$.

67. Angle of Repose.-If a body rests on an inclined plane, as shown in Fig. 160, and is acted on by no forces except its weight and the reaction of the plane, and if $\alpha$, the angle of inclination of the plane to the horizontal, is such that motion of the body 
impends down the plane, the angle $\alpha$ is defined as the angle of repose.

Since the body is in equilibrium under the action of the two forces $R$, the reaction of the plane, and $W$, its weight, these forces

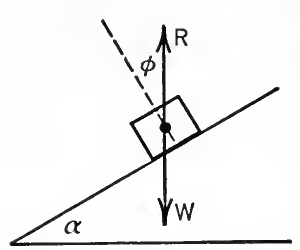

FIG. 160. must be equal, opposite, and collinear. Hence the reaction $R$ is vertical. Furthermore, the angle which $R$ makes with the normal to the plane is $\phi$, the angle of friction. It is evident from the figure that the angles $\alpha$ and $\phi$ are equal. The angle of repose for two surfaces can be found easily by experiment, after which the coefficient friction for the surfaces may be found from the relation $\mu=\tan \phi=\tan \alpha$.

68. The Laws of Friction.-One of the earliest contributions to our knowledge of the laws of friction was made by Coulomb, who published, in 1781, the results of experiments on the friction of plane dry surfaces. Later experiments by Morin confirmed, in the main, the results obtained by Coulomb. The results of the experiments of Morin on dry surfaces, published in 1831, may be stated as follows:

1. The friction between two bodies when motion is impending (limiting friction) is proportional to the normal pressure; that is, the coefficient of friction is independent of the normal pressure.

2. The coefficient of static friction is independent of the area of contact.

3. The coefficient of kinetic friction is less than the coefficient of static friction and is independent of the relative velocity of the rubbing surfaces.

Although these laws are probably correct for the conditions under which the tests were made, they must be modified in order to apply to friction which is developed under conditions quite different from those found in the experiments. The pressures used in the experiments of Morin varied from $\frac{3}{4} \mathrm{lb}$. per square inch to $100 \mathrm{lb}$. per square inch. It has been found in later experiments that for pressures less than $\frac{3}{4} \mathrm{lb}$. per square inch the value of the cocfficient of static friction increases somewhat. For very great pressures the coefficient also increases. The highest velocity used in Morin's experiments was $10 \mathrm{ft}$. per sec. For greater velocities than this it has been found in later experiments that 
the coefficient of kinetic friction decreases with the velocity. The experiments of Jenkin show that for extremely low velocities (the lowest velocity measured was $.0002 \mathrm{ft}$. per sec.) there is an increase in the coefficient of kinetic friction. These experiments indicate that the value of the coefficient of kinetic friction gradually increases as the velocity decreases and passes without discontinuity into that of static friction.

From experiments made by Tower, Goodman, Thurston, and others, on lubricated surfaces, it has been found that the laws of friction for lubricated surfaces are almost the reverse of those stated for dry surfaces. For example, it is found that the friction of two surfaces is almost independent of the nature of the surfaces and of the normal pressure so long as there is a film of lubricant between the surfaces. Again, for lubricated surfaces, it is found that the friction is materially affected by the temperature, which is not true in the case of dry surfaces.

69. The Inclined Plane.-Applications of the inclined plane are found in various machines such as the jack-screw, the screw type of testing machine, wedges, etc. Three variations of the problem which arise in connection with the use of the inclined plane will now be considered.

Case I. To Determine the Force Required to Start a Body up the Plane.-Let Fig. 161 represent a body resting on a plane, the angle of inclination of which is $\alpha$. It is required to find the value of a force, $P$, which will make motion of the body impend up the plane when the action line of $P$ makes an angle of $\theta$ with the plane. Although motion of the body

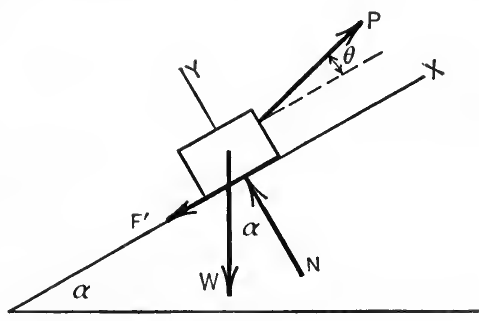

FIG. 161. impends, it will not occur unless the value of $P$ is increased slightly beyond the critical value here considered. And since the body is still in equilibrium the equations of equilibrium may be applied to the system of forces acting on it. In addition to the required force $P$, the forces acting on the body are the weight, $W$, of the body, and the reaction of the plane. The latter force will be resolved into components $N$, perpendicular to the plane, and $F^{\prime}$ (equal to $\mu N$ ), parallel to the plane, as shown in the 
free-body diagram. If the $x$-axis be taken parallel to the plane and the $y$-axis perpendicular to the plane, the equations of equilibrium may be written as follows:

$$
\begin{aligned}
& \Sigma F_{x}=P \cos \theta-\mu N-W \sin \alpha=0, \\
& \Sigma F_{y}=N+P \sin \theta-W \cos \alpha=0 .
\end{aligned}
$$

Eliminating $N$ from the two equations we have:

$$
P=\frac{W(\sin \alpha+\mu \cos \alpha)}{\cos \theta+\mu \sin \theta} .
$$

If $\tan \phi$ be substituted for $\mu$ this may be written,

$$
P=W \frac{\sin (\alpha+\phi)}{\cos (\theta-\phi)} \text {. }
$$

If the values of $W, \alpha$, and $\phi$ are specified, $P$ may be regarded as a function of $\theta$. The value of $P$ is a minimum when $\theta$ is equal to $\phi$, since this value of $\theta$ makes $\cos (\theta-\phi)$ a maximum; the minimum value of $P$, then, is $W \sin (\alpha+\phi)$. If the force $P$ is applied parallel to the plane its value becomes $W \frac{\sin (\alpha+\phi)}{\cos \phi}$.

Case II. To Determine the Force Required to Prevent Motion Down the Plane when $\alpha>\phi$.- In this case the forces acting on the body are the same as shown in Fig. 161 except that the frictional force is directed up the plane. By writing the equations of equilibrium as before and solving, the equation obtained is,

$$
P=\frac{W \sin (\alpha-\phi)}{\cos (\theta+\phi)} \text {. }
$$

For this case $P$ is a minimum when $\theta=-\phi$, and the minimum value of $P$ is $W \sin (\alpha-\phi)$. If the force $P$ is applied parallel to the plane the value of $P$ is $\frac{W \sin (\alpha-\phi)}{\cos \phi}$.

Case III. To Determine the Force Required to Start the Body Down the Plane when $\alpha<\phi$. - In this case the forces which act on the body are as shown in Fig. 161, except that $P$ and $\mu N$ are reversed. By writing the equilibrium equations as before and solving, the equation obtained is,

$$
P=\frac{W \sin (\phi-\alpha)}{\cos (\theta+\phi)} \text {. }
$$


For this case $P$ is a minimum when $\theta=-\phi$ and the minimum value of $P$ is $W \sin (\phi-\alpha)$. If $P$ is applied parallel to the plane, the value of $P$ becomes $\frac{W \sin (\phi-\alpha)}{\cos \phi}$.

\section{ILLUSTRATIVE PROBLEM}

141. A small body weighing $50 \mathrm{lb}$. is placed on a rough plane which is inclined $30^{\circ}$ with the horizontal (Fig. 162). The body is acted on by a force $P$, the action line of which lies in the plane and makes an angle of $30^{\circ}$ with the line of greatest slope in the plane. If the coefficient of friction is $\frac{1}{3}$, find the value of $P$ that will just start the block in motion, and find the direction in which the block will begin to move.

Solution.-There are three forces acting on the body, namely, the force $P$, the earth pull of $50 \mathrm{lb}$., and the reaction of the plane. The $50-1 \mathrm{~b}$. force may be resolved into two components; $50 \mathrm{sin} 30^{\circ}$ in the plane (Fig. 162) and $50 \mathrm{cos}$ $30^{\circ}$ (not shown) perpendicular to the plane. The reaction of the plane may be resolved into two components, $N$ (not shown) perpendicular to the plane and $\frac{1}{3} N$ lying in the plane. The frictional force $\frac{1}{3} N$ acts opposite to the direction in which the body will begin to move. Let $\theta$ be the angle which the frictional force makes with the line of greatest slope. The two forces which are perpendicular to the plane are in equilibrium and hence are equal. Thus,

$$
N=50 \cos 30^{\circ} \text {. }
$$

The three forces in the plane are in equilibrium; hence, by Lami's theorem (Art. 47),

$$
\begin{array}{r}
\frac{50 \sin 30^{\circ}}{\sin \left(\theta+30^{\circ}\right)}=\frac{P}{\sin \theta}= \\
\sin \left(\theta+30^{\circ}\right)=\frac{3 \sin ^{2} 30^{\circ}}{\cos 30^{\circ}}=\frac{\sqrt{3}}{2}
\end{array}
$$

Hence,

By eliminating $N$ from (1) and (2) the equation obtained is,

Therefore,

$$
\begin{aligned}
\theta+30^{\circ} & =60^{\circ} . \\
\theta & =30^{\circ} .
\end{aligned}
$$

Substituting $\theta$ in (2) we have,

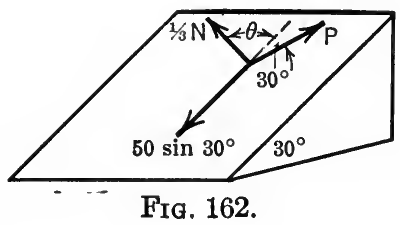

$$
P=\frac{50}{2 \sqrt{3}}=14.4 \mathrm{lb} \text {. }
$$

\section{PROBLEMS}

142. A body weighing $W \mathrm{lb}$. rests on a rough plane inclined at an angle $\theta$ to the horizontal. What horizontal force must be applied in order to start the body up the plane if the angle of friction is $\phi$ ? Express the force in terms $\phi$ and also in terms of $\mu$.

$$
\text { Ans. } P=W \tan (\theta+\phi)=W \frac{\sin \theta+\mu \cos \theta}{\cos \theta-\mu \sin \theta}
$$


143. A homogeneous rectangular prism, the dimensions of which are $1 \mathrm{ft}$. by $1 \mathrm{ft}$. by $2 \mathrm{ft}$., stands on end on a flat square

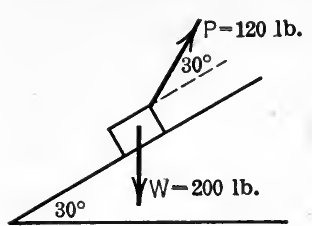

Fig. 163. board, the edges of the base of the prism being parallel to the edges of the board. The coefficient of friction for the board and prism is 0.2 . If one side of the board is gradually raised will the prism slide or tip?

144. A body weighing $100 \mathrm{lb}$. rests on a rough plane inclined $45^{\circ}$ to the horizontal. A horizontal force of $50 \mathrm{lb}$. is just sufficient to prevent the body

from moving down the plane. Find the coefficient of friction for the body and plane. Ans. $\mu=\frac{1}{3}$.

145. Two bodies weighing $50 \mathrm{lb}$. and $100 \mathrm{lb}$. rest on an inclined plane and are connected by a cord which is parallel to the line of greatest slope. The body weighing $50 \mathrm{lb}$. is below the one weighing $100 \mathrm{lb}$., and the coefficient of friction for the 50-lb. body is $\frac{1}{5}$ and that for the $100-1 b$. body is $\frac{1}{4}$. Find the inclination of the plane to the horizontal and the tension in the cord, when motion impends.

146. A body weighing $200 \mathrm{lb}$. rests on a plane inclined $30^{\circ}$ to the horizontal and is acted on by a force of $120 \mathrm{lb}$. as shown in Fig. 163. The coefficient of friction for the body and plane is 0.3 . Find the friction between the body and plane. Ans, $3.92 \mathrm{lb}$.

70. The Wedge.-Wedges are used for lifting-devices, cotterpins, keys, etc. In the wedge shown in Fig. 164(a), it is required to find the value of the force $P$ which must be applied to the wedge to overcome the forces $W, W$, applied to blocks $A$ and $B$. It will be assumed that the angle of friction for all rubbing surfaces is the same. A free-body diagram of the block $A$ and of the wedge

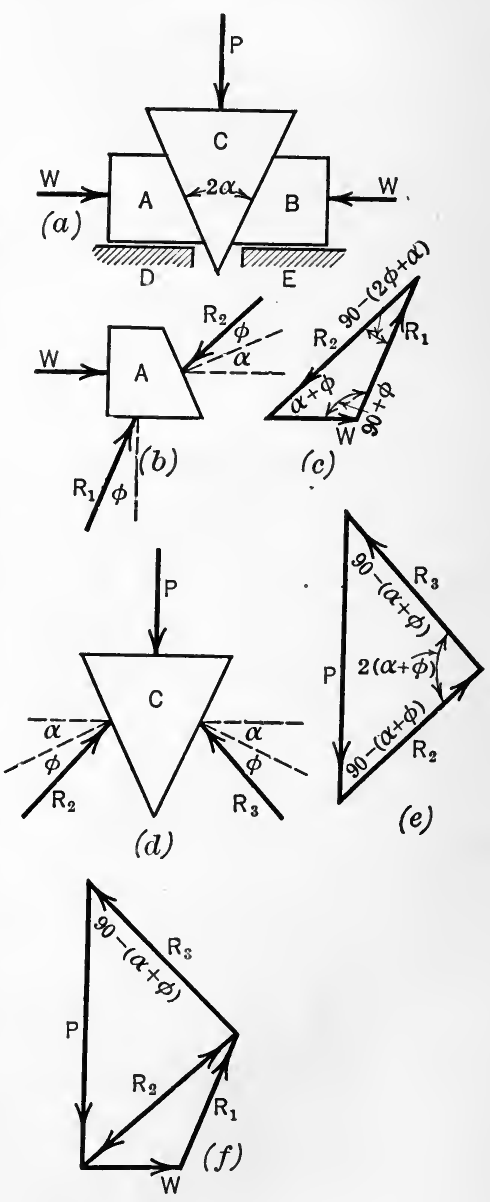

Fig. 164. 
$C$ is shown in Fig. 164(b) and $164(d)$, respectively. The force $P$ may be found graphically by constructing the force polygons for the forces acting on $A$ and $C$. Since the force $W$ is known completely and since the directions of the reactions $\left(R_{1}\right.$ and $\left.R_{2}\right)$ of $D$ and $C$ on $A$ are known in direction, the force polygon for $A$ can be drawn as shown in Fig. 164(c). Having found the reaction $R_{2}$, it is now possible to complete the force polygon for the forces acting on the wedge $C$. The reaction of $A$ on $C$ is equal and opposite to the reaction of $C$ on $A$, that is, it is equal to the force $R_{2}$ just found, but reversed in sense. The force polygon for $C$ is shown in Fig. 164(e) from which $P$ may be found. Fig. 164(f) shows the two force polygons superimposed. From the force polygons it is easy, by means of trigonometry, to derive the expression for $P$ in terms of $W, \phi$, and $\alpha$. It will be of interest, however, to find the value of $P$ by use of the equations of equilibrium. Thus, the equations of equilibrium for the forces acting on $A$ and $C$, respectively, are,

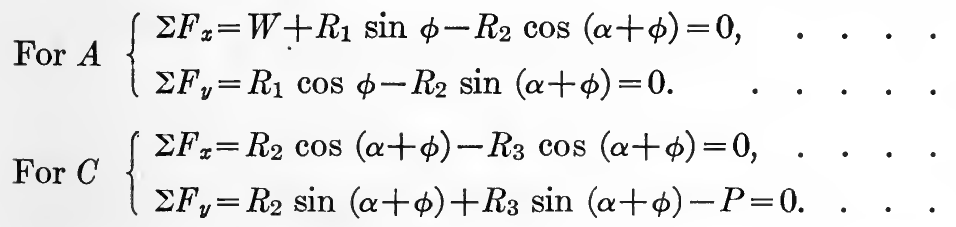

By eliminating $R_{1}$ from (1) and (2) the equation obtained is,

$$
R_{2}=\frac{W \cos \phi}{\cos (\alpha+2 \phi)}
$$

By eliminating $R_{3}$ from (3) and (4) the equation obtained is,

$$
P=2 R_{2} \sin (\alpha+\phi) . \quad . \quad . \quad . \quad . \quad .
$$

By eliminating $R_{2}$ from (5) and (6) the final equation is,

$$
P=\frac{2 W \cos \phi \sin (\alpha+\phi)}{\cos (\alpha+2 \phi)} \text {. }
$$

Another problem which arises in connection with the wedge is the determination of the least force, $P$, which will prevent the wedge from being forced upward by the loads $W, W$. The procedure in the solution of this problem is similar to that used in the problem just discussed. The frictional forces, however, will be reversed and hence the reactions will make angles $\phi$ on the other 
sides of the normals to the surfaces. The value of $P$ for this case is given by the equation,

$$
P=\frac{2 W \cos \phi \sin (\alpha-\phi)}{\cos (\alpha-2 \phi)} \text {. }
$$

When $\alpha$ is less than $\phi$, it is evident that the value of $P$ is negative and hence the wedge is self-locking, that is, it would stay in place even if the force $P$ were reduced to zero.

A third problem which arises in connection with the wedge is the determination of the least pull, $P$, which is required to withdraw a self-locking wedge, as for example, in lowering a heavy body by means of the wedge. By writing the equations of equilibrium and solving, the value of the pull is found to be

$$
P=\frac{2 W \cos \phi \sin (\phi-\alpha)}{\cos \alpha} .
$$

\section{ILLUSTRATIVE PROBLEM}

147. Fig. 165(a) represents a cotter joint. The angle $\alpha$ equals $15^{\circ}$ and the

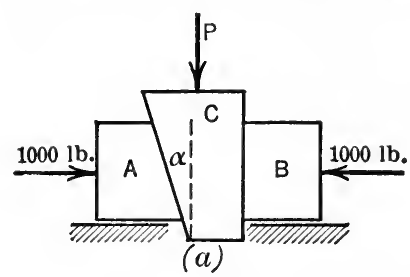
angle of friction for all rubbing surfaces is $12^{\circ}$. What is the value of the force $P$ required to overcome the 1000 -lb. forces applied on parts $A$ and $B$ ?

Solution.-Free-body diagrams of the block $A$ and the cotter pin $C$ are shown in Fig. $165(b)$ and $165(c)$ respectively. The equations of equilibrium for the two blocks may be written as follows:

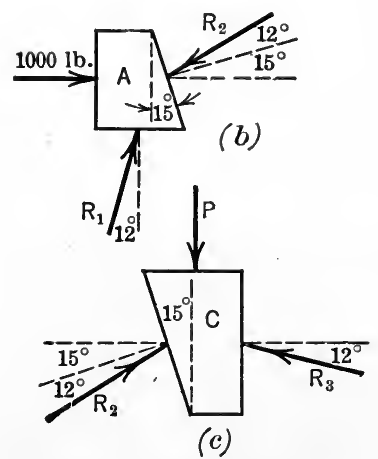

$$
\begin{aligned}
& \text { For } A \\
& \left\{\begin{array}{r}
\Sigma F_{x}=1000+R_{1} \sin 12^{\circ} \\
-R_{2} \cos 27^{\circ}=0, \\
\Sigma F_{y}=R_{1} \cos 12^{\circ}-R_{2} \sin 27^{\circ}=0 .
\end{array}\right.
\end{aligned}
$$

For $C$

$\left\{\begin{array}{l}\Sigma F_{x}=R_{2} \cos 27^{\circ}-R_{3} \cos 12^{\circ}=0, \\ \Sigma F_{y}=R_{2} \sin 27^{\circ}+R_{3} \sin 12^{\circ}-P=0 .\end{array}\right.$

By eliminating $R_{1}$ from (1) and (2) the equation obtained is,

$$
R_{2}=\frac{1000 \cos 12^{\circ}}{\cos 39^{\circ}} .
$$


By eliminating $R_{3}$ from (3) and (4) the following equation is obtained:

$$
R_{2}=\frac{P \cos 12^{\circ}}{\sin 39^{\circ}}
$$

By equating values of $R_{2}$ in (5) and (6) the value of $P$ may be found. Thus,

Therefore,

$$
\frac{P \cos 12^{\circ}}{\sin 39^{\circ}}=\frac{1000 \cos 12^{\circ}}{\cos 39^{\circ}} \text {. }
$$

$$
P=1000 \tan 39^{\circ}=810 \mathrm{lb} \text {. }
$$

\section{PROBLEMS}

148. The load of $100,000 \mathrm{lb}$. (Fig. 166) is raised by applying forces $P, P$ to the wedges. What is the required value of $P$ if the coefficient of friction is 0.2 for all surfaces of contact?

Ans. $P=26,667 \mathrm{lb}$.

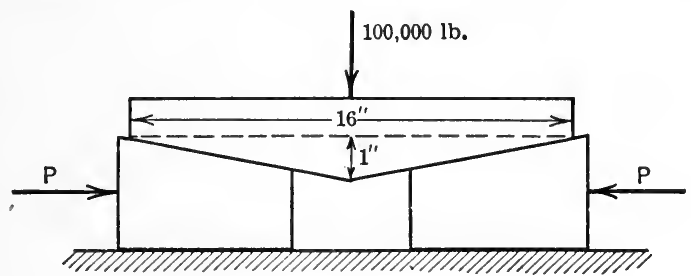

Frg. 166.

149. Find the value of the force $P$ which mist be applied to the wedge $A$ (Fig. 167) in order to raise a weight $W$ carried by the block $B$, assuming that the coefficients of friction for all rubbing surfaces are equal.

$$
\text { Ans. } P=W \tan (2 \phi+\alpha) \text {. }
$$

150. Find the expression asked for in Problem 149 if friction is neglected at all surfaces except that between the block $B$ and the wedge $A$.

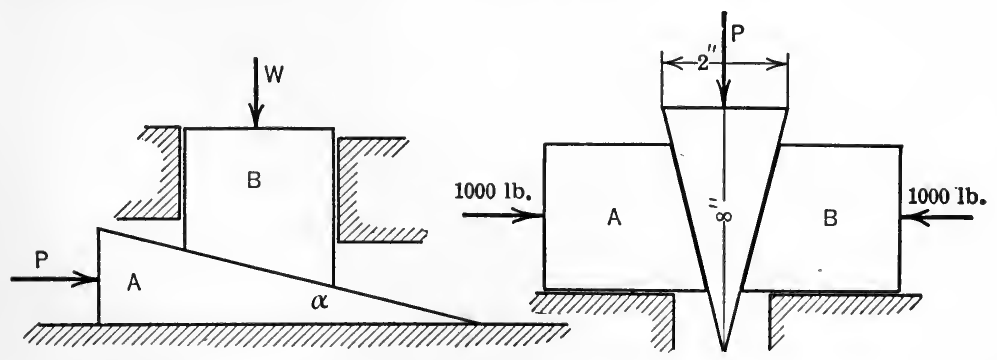

Fig. 167.

Fig. 168.

151. Find the force $P$ which must be applied to the wedge shown in Fig. 168 in order to overcome the 1000-lb. forces applied to blocks $A$ and $B$ as 
shown. Assume the coefficient of friction for all rubbing surfaces to be 0.1 . Ans. $467 \mathrm{lb}$.

152. What is the value of $P$ in Problem 151 if the horizontal surfaces, on which blocks $A$ and $B$ rest, are smooth?

71. The Screw.-A screw is, in effect, an inclined plane wound around a cylinder. Screws are made with square threads and
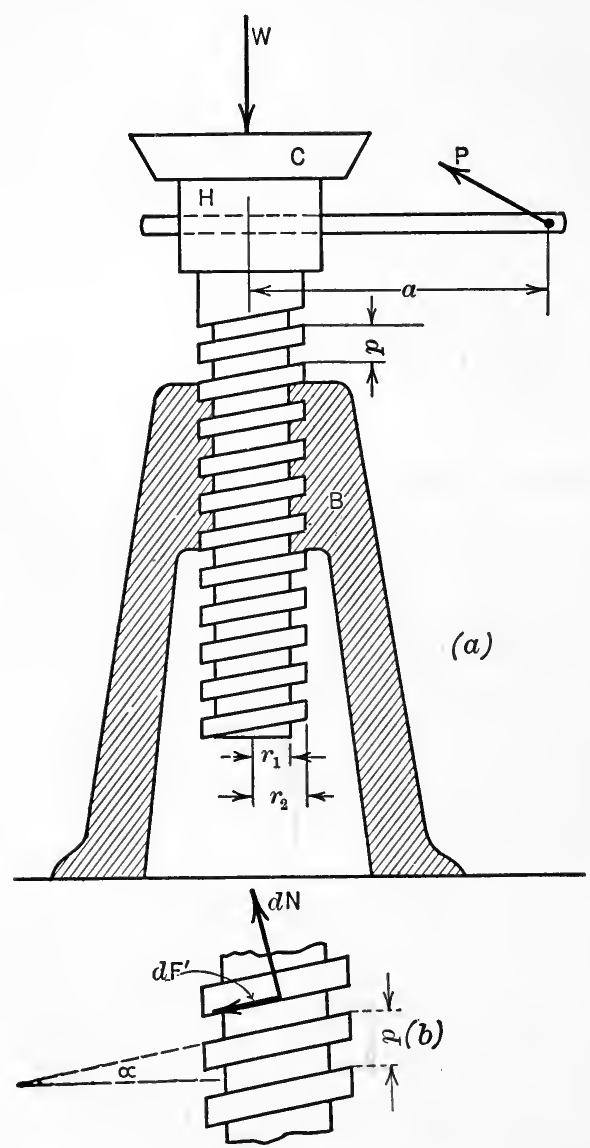

Fig. 169. with triangular threads, but square - threaded screws, only, will be considered here. Fig. 169 $(a)$ shows a jack-screw with square threads which is used in raising or lowering heavy loads. The radius of the base of the thread is denoted by $r_{1}$ and the outer radius by $r_{2} ; \alpha$ is called the pitch angle and $p$ is called the pitch of the screw. Let it be required to find the force, $P$, which, when applied at the end of a lever of length $a$, is just sufficient to raise the load $W$. The forces which hold the screw in equilibrium are: the force $P$; the pressure of the cap, $C$, on the head, $H$; and the reaction of the nut, $B$, on the screw. The latter is distributed over the area of the threads in contact with the nut. If the friction between the cap and the head of the screw is neglected, the pressure of the cap on the head of the screw will be a vertical force equal to $W$. The problem will first be solved on this assumption. Two of the equilibrium equations which apply to this type of force 
system will be sufficient for the solution of the problem, namely, $\Sigma F_{z}=0$ and $\Sigma M_{z}=0$ where $z$ is taken as the axis of the screw. The reaction between the nut and the thread of the screw on an element of area $d A$ will be denoted by $d R$. This force may be resolved into components $d N$ normal to the thread and $d F^{\prime}$ parallel to the thread as shown in Fig. 169(b). In taking moments about the axis of the thread it will be sufficiently accurate to consider the moment-arm of $d F^{\prime}$ to be equal to the mean radius of the thread, $\frac{1}{2}\left(r_{1}+r_{2}\right)$, which will be denoted by $r$. The equilibrium equations stated above, then, become:

$$
\begin{gathered}
\Sigma F_{z}=\Sigma d N \cos \alpha-\Sigma d F^{\prime} \sin \alpha-W=0, \\
\Sigma M_{z}=P a-\Sigma r d N \sin \alpha-\Sigma r d F^{\prime} \cos \alpha=0 .
\end{gathered}
$$

Since $d F^{\prime}=\mu d N$, these equations may be written:

$$
\begin{aligned}
& \cos \alpha \Sigma d N-\mu \sin \alpha \Sigma d N-W=0, \quad \text {. . . . . } \\
& P a-r \sin \alpha \Sigma d N-\mu r \cos \alpha \Sigma d N=0 . \quad \text {. . . . }
\end{aligned}
$$

By eliminating $\Sigma d N$ from (1) and (2) the equation obtained is,

$$
P a=W r \frac{\sin \alpha+\mu \cos \alpha}{\cos \alpha-\mu \sin \alpha} .
$$

By substituting $\tan \phi$ for $\mu$ this equation may be written in the form,

$$
P a=W r \tan (\phi+\alpha) .
$$

If the pitch angle $\alpha$ is large and the angle of friction is small, the load $W$ will cause the screw to run down unless a force is applied to prevent it. The force $P$ required to hold the load is found by a method of analysis similar to that used above, the only difference being that the sense of the frictional force is reversed. The least value of $P$ required to prevent the screw from running down is given by the equation,

$$
P a=W r \tan (\alpha-\phi) .
$$

If $\alpha=\phi$ in the above equation, the force $P$ reduces to zero, that is, the load will be held by friction alone. If $\alpha<\phi$, a force 
is required (the sense of which is opposite to that in "the two cases considered) to lower the load. The value of the force required to lower the load is given by the equation,

$$
P a=W r \tan (\phi-\alpha) \text {. }
$$

In practice, jack-screws are always made self-locking, that is, $\alpha$ is made less than $\phi$. The values of $P$ for the three cases just considered will be changed somewhat if friction between the cap and the head of the screw is considered. If the area of contact between the cap and the head is a full circle the effective arm of the frictional resistance is two-thirds the radius of the circle as will be shown in Art. 74. In practice, however, the area of contact is usually a hollow circle, and it will be sufficiently accurate to take the mean radius as the effective arm of the frictional resistance. If the mean radius is denoted by $r^{\prime}$ and the coefficient of friction between the cap and the head of screw by $\mu$, the values of $P$ are given by the following equations:

To raise the load, $P a=W r \tan (\phi+\alpha)+\mu W r^{\prime}$.

To hold the load, $P a=W r \tan (\alpha-\phi)-\mu W r^{\prime}$,

To lower the load, $P a=W r \tan (\phi-\alpha)+\mu W r^{\prime}$.

\section{PROBLEMS}

153. The mean diameter of the screw of a square-threaded jack-screw is $1.8 \mathrm{in}$. The pitch of the thread is

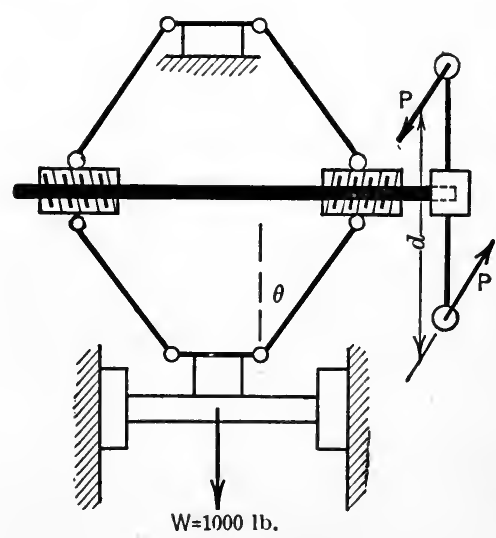

FIG. 170. $0.4 \mathrm{in}$. and the coefficient of friction for the screw and nut is 0.12 . What force must be applied at the end of a lever $18 \mathrm{in}$. long to raise a weight of $5000 \mathrm{lb}$.? What force is required to lower the weight?

$$
\text { Ans. } 48.1 \mathrm{lb} .12 .3 \mathrm{lb} \text {. }
$$

154. A weight of $1000 \mathrm{lb}$. is lifted by applying a couple, $P d$, to the hand wheel of the apparatus shown in Fig. 170. The diameter, $d$, of the hand wheel is 20 in.; the mean diameter of the screw is 1.5 in.; the pitch of the thread is $\frac{1}{3}$ in.; and the coefficient of friction is 0.15 . Find the values of the forces of the couple when the value of $\theta$ is $15^{\circ}$. 
155. The shaft-straightening hand press shown in Fig. 171 is used for bending or straightening $3 \frac{1}{2} \mathrm{in}$. steel shafts. What force, $P$, applied at the end of a 36-in. lever is required to produce a pressure, $Q$, of $24,000 \mathrm{lb}$. on the shaft? The threads have a mean diameter of 2 in. and there are four threads per inch. Consider friction between the screw and nut only, and use a value of 0.2 for the coefficient of friction.

Ans. $P=161 \mathrm{lb}$.

72. Journal Friction and the Friction Circle.-In Fig. 172 is shown an axle or journal in a bearing, the diameter of which is slightly greater than the diameter of the axle. The difference in the diameters is exaggerated in the figure for the sake of clearness.

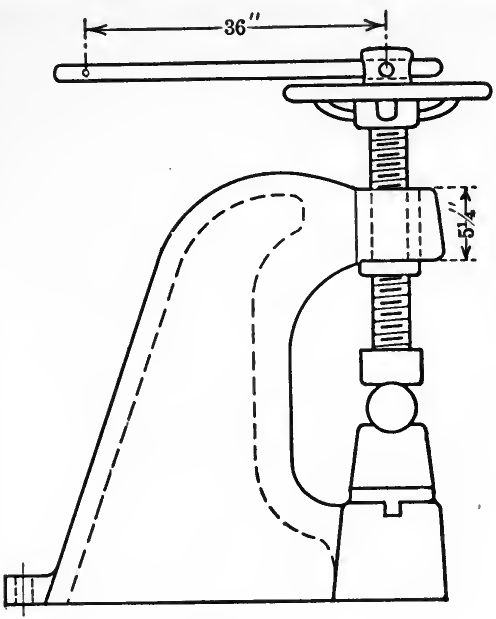

FIG. 171. Since the contact between the axle and the bearing is along a line, the reaction of the bearing on the axle is distributed along the line of contact and may be replaced by a resultant force, $R$, at

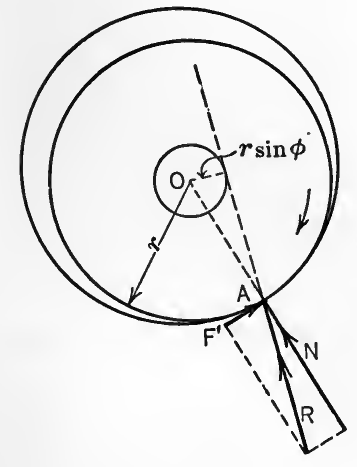

Fig. 172. $A$, the midpoint of the line of contact. If the axle rotates in the bearing or, if motion impends, the angle between the action line of the reaction, $R$, and the normal to the surfaces at $A$ is the angle of friction $\phi$. In the former case the angle is the angle of kinetic friction and in the latter case it is the angle of static friction. If the radius of the axle is denoted by $r$, a circle having a center which coincides with the center, $O$, of the axle and which has a radius equal to $r \sin \phi$ will be tangent to the action line of $R$. This circle is called the friction circle. It will be noted that when the angle $\phi$ is small, $\sin \phi$ is approximately equal to $\tan \phi$ and hence also approximately equal to $\mu$. Therefore, for small values of $\mu$ the radius of the friction circle may be taken as $\mu r$ without serious error. 
The friction circle is of importance in locating the line of action of the reaction between an axle and its bearing since this line is tangent to the friction circle when motion occurs or is impending. For example, in Fig. 172 the line of action of the reaction is one of the two tangents that can be drawn from $A$ to the friction circle. The direction of motion (or impending motion) will determine which one of the two tangents should be used. Thus, if the axle rotates, or is about to rotate, in a clockwise direction the action line of the reaction is as indicated in Fig. 172. In general the position of the line of contact between the axle and bearing is not

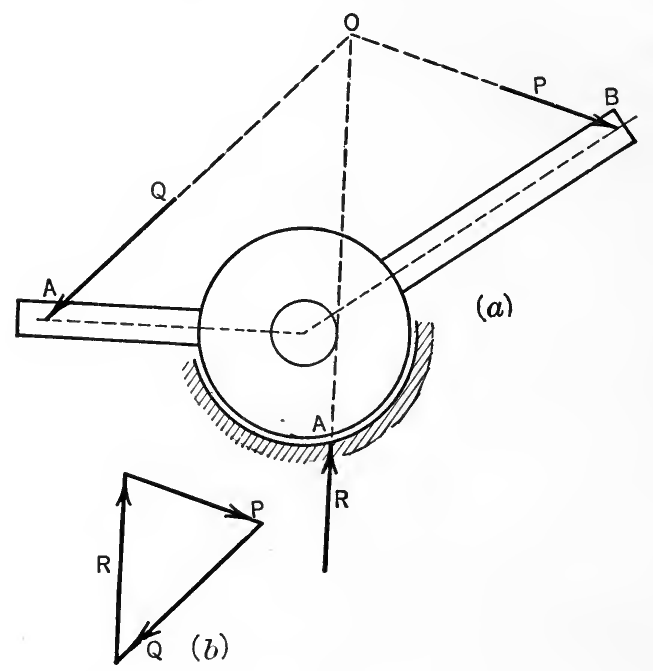

FIG. 173.

initially known. This position can usually be determined, however, by means of the friction circle. For example, consider the bell-crank, shown in Fig. 173(a), which is acted on by a known force $Q$. Let it be required to determine the magnitude of the force, $P$, which must be applied along the line $O B$ in order to cause motion to impend in a clockwise direction. The forces acting on the bell-crank are $Q, P$, and the reaction, $R$, of the bearing. Since the three forces are in equilibrium they must be concurrent. Hence if $P$ and $Q$ intersect at $O, R$ must also pass through $O$. Furthermore, $R$ must be tangent to the friction circle. There are 
only two tangents that can be drawn from $O$ to the friction circle. The one to be used is indicated in the figure. Therefore, the location of the line of contact between the axle and the bearing is at $A$. Since the action line of $R$ is now known, the magnitudes of $P$ and $R$ can easily be found by drawing the force polygon (Fig. 173b) for the three forces. If motion impends in the opposite direction and it is required to find the least value of the force $P$ which will maintain equilibrium the other tangent would be used.

\section{ILLUSTRATIVE PROBLEM}

156. Fig. 174 represents a pulley, having a diameter of $2 \mathrm{ft}$., mounted on a 2-in. axle. The coefficient of friction between the axle and the bearing in

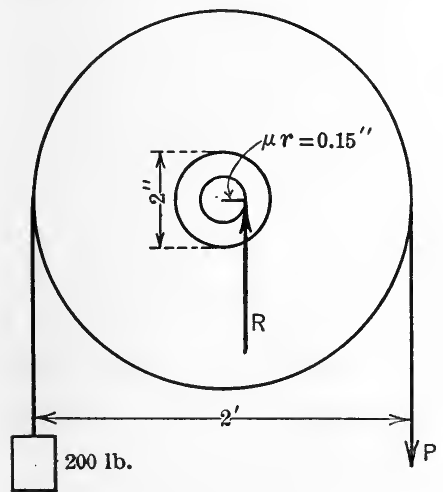

Frg. 174. which it rests is 0.15 . What is the least value of the force $P$ which will raise the 200-lb. weight, assuming that the friction between the rope and pulley is sufficient to prevent slipping? Neglect the weight of the pulley.

Solution.-There are three forces acting on the pulley: the 200-lb. force, $P$, and $R$, the reaction of the bearing. Since these three forces are in equilibrium they must be parallel (Art. 48). Hence $R$ must be vertical and, since it must also be tangent to the friction circle, its line of action is determined. The radius of the friction circle is $\mu r$ or $0.15 \mathrm{in}$. The equations of equilibrium are:

$$
\begin{aligned}
& \Sigma F_{y}=R-P-200=0 . \\
& \Sigma M_{0}=200 \times 12+0.15 R-12 P=0 .
\end{aligned}
$$

Eliminating $R$ from these equations we have,

$$
P=205 \mathrm{lb} \text {. }
$$

If the force $R$ acted through the center of the axle, that is, if the axle were smooth, the value of $P$ would be $200 \mathrm{lb}$. 


\section{PROBLEM}

157. A load of $1000 \mathrm{lb}$. is raised by a wooden wheel and axle as shown in Fig. 175. What is the least value of the force $P$ which will raise the load if the coefficient of friction between the axle and the bearing is 0.4 ?

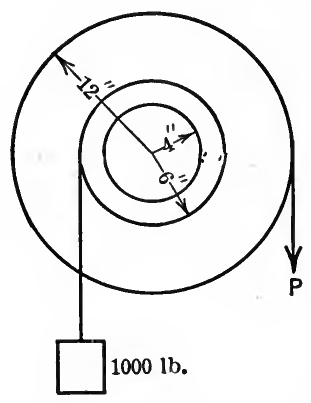

FIG. 175.

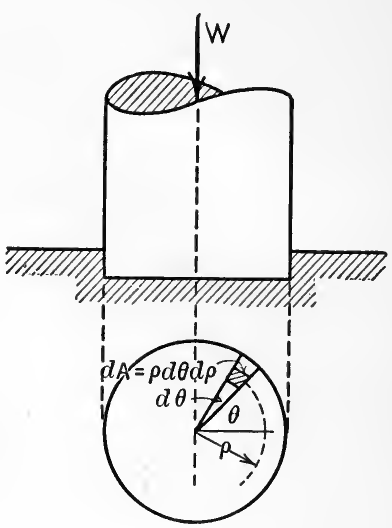

FIg. 176.

73. Pivot Friction.-In Fig. 176 is shown a pivot in a step bearing. The pivot will be assumed to turn in its bearing and hence kinetic friction, only, is involved. In order to determine the resisting moment due to the frictional forces at the end of a pivot, certain assumptions will be made as follows:

1. The coefficient of friction is constant over the end of the pivot.

2. The pressure between the pivot and the bearing at any point

(a) is constant, or

(b) varies in such a manner that the wear of the pivot in the direction of its axis (axial wear) is uniform over the area of the pivot. Or, as sometimes stated, the wear, at any point, normal to the rubbing surfaces (normal wear) is proportional to the work of friction.

Since the value of the coefficient of kinetic friction varies somewhat with the velocity of the rubbing surfaces, it is evident that the coefficient is not constant over the end of the pivot, for the velocity of any point varies as its distance from the axis of the pivot. The assumption made in (1), however, will not cause 
serious error if a mean value of the coefficient is used. For a new pivot the assumption $2(a)$ seems reasonable, although the pressure, probably, will not remain constant after wear occurs. If the wear normal to the pivot (normal wear) at any point of the pivot is proportional to the work done by the frictional force at that point, that is, to the product of the frictional force, $F^{\prime}$, and the distance traveled by the point, the normal wear will also be proportional to the normal pressure, since $F^{\prime}$ is equal to $\mu N$. If, then, the normal pressure is constant, it is evident that, for a time, the greater wear will occur near the circumference of the pivot. The effect of this greater wear at the edge of the pivot will be to increase the intensity of pressure near the center and to decrease it near the edge of the pivot. The increased pressure near the center will in turn result in increasing the wear near the center and in decreasing it near the edge. This process will continue until a uniform condition has been established, after which, the wear will be uniform over the end of the pivot. The assumption made in $2(b)$, therefore, seems reasonable for pivots that have been in use for some time. The frictional moment will now be determined for several pivots.

74. Solid Flat Pivot. Uniform Pressure.-In the flat pivot shown in Fig. 176 the axial load will be denoted by $W$, the radius of the pivot by $r$, and the area of the pivot by $A$. The frictional moment may be found as follows:

Pressure per unit of area $\quad=\frac{\mathrm{W}}{\pi r^{2}}$.

Pressure on element of area $\quad=\frac{W}{\pi r^{2}} d A=\frac{W}{\pi r^{2}} \rho d \rho d \theta$.

Frictional force on element $d A=\frac{\mu W}{\pi r^{2}} \rho d \rho d \theta$.

Moment of frictional force on $d A=\frac{\mu W}{\pi r^{2}} \rho^{2} d \rho d \theta$.

Total frictional moment

$$
\begin{aligned}
& =\frac{\mu W}{\pi r^{2}} \int_{0}^{2 \pi} \int_{0}^{r} \rho^{2} d \rho d \theta \\
& =\frac{2}{3} \mu W r .
\end{aligned}
$$

Uniform Wear.- Since the wear at any point is proportional to the distance traveled by that point, it is proportional to the dis- 
tance, $\rho$, of the point from the axis of the pivot. The wear, however, is also proportional to the friction force and hence, to the pressure, $p$. That is, $p \rho$ is equal to some constant, $C$. The frictional moment, then, may be found as follows:

$$
\begin{aligned}
\text { Frictional moment } & =\int_{0}^{2 \pi} \int_{0}^{r} \mu p \dot{\rho}^{2} d \rho d \theta \\
& =\mu C \int_{0}^{2 \pi} \int_{0}^{r} \rho d \rho d \theta \\
& =\mu C \pi r^{2} . \quad . \quad .
\end{aligned}
$$

But, the total pressure must be equal to $W$. Hence, another equation containing $C$ may be found. Thus,

$$
\begin{aligned}
W & =\int_{0}^{2 \pi} \int_{0}^{r} p \rho d \rho d \theta \\
=C \int_{0}^{2 \pi} \int_{0}^{r} d \rho d \theta & \\
& =2 \pi C r .
\end{aligned}
$$

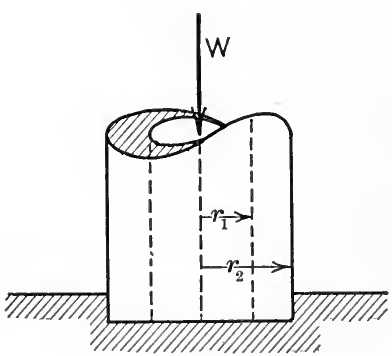

(a)

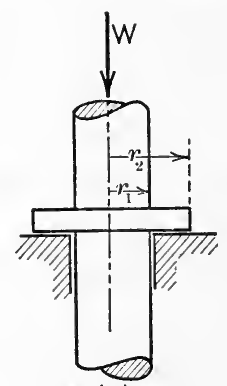

(b)

FIG. 177.

By eliminating $C$ from equations (1) and (2) the resulting expression is,

Frictional moment $=\frac{1}{2} \mu W r$.

75. Hollow Flat Pivot or Collar Bearing.-The determination of the frictional moment for a hollow flat pivot (Fig. 177a), or collar bearing (Fig. 177b), involves the same steps as were used in finding 
the frictional moment for a solid flat pivot and, hence, will not be given in detail. The frictional moment is found to be:

For uniform pressure, $\frac{2}{3} \frac{\mu W\left(r_{2}{ }^{3}-r_{1}^{3}\right)}{r_{2}^{2}-r_{1}^{2}}$.

For uniform wear, $\quad \frac{1}{2} \mu W\left(r_{1}+r_{2}\right)$.

76. Conical Pivot. Uniform Pressure.-In finding the frictional moment for a conical pivot (Fig. 178) it will first be assumed that the intensity of pressure, $p$, normal to the surface is constant. The frictional force at any point of course is horizontal. Referring to Fig. 178, we find the frictional moment as follows:

Frictional force on an element of area $d A=\mu p d A$;

Moment of this force

$$
=\mu p \rho d A ;
$$

Total frictional moment

$$
\begin{aligned}
& =\int \mu p \rho d A \\
& =\int_{0}^{r} \mu p \rho 2 \pi \rho \frac{d \rho}{\sin \alpha} \\
& =\frac{2 \pi \mu p}{\sin \alpha} \frac{r^{3}}{3} . \quad . \quad
\end{aligned}
$$

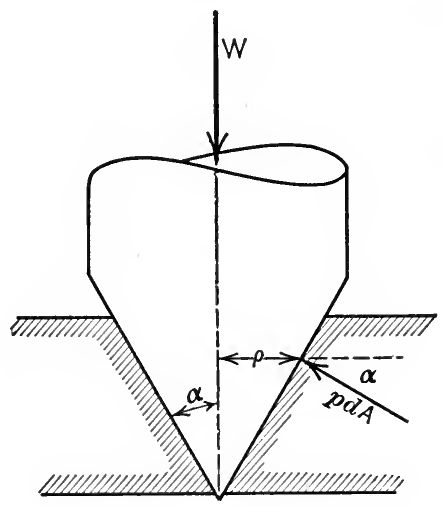

Fig. 178.

The value of the constant pressure $p$ in (1) may be expressed in terms of $W$ as follows: Since the sum of the vertical components of the pressures on the elements of area must be equal to the axial load $W$, we may write,

$$
W=\int p d A \sin \alpha=p \sin \alpha \int d A=p A \sin \alpha,
$$

where $A$ is the area of contact between the pivot and bearing. And, since $A \times \sin \alpha=\pi r^{2}$ the above equation may be written,

$$
W=p \pi r^{2} \text {. }
$$

By eliminating $p$ from equations (1) and (2) the final expression is,

$$
\text { Frictional Moment }=\frac{2}{3} \frac{\mu W r}{\sin \alpha} .
$$


Uniform Wear.--If the wear in the direction of the axis of the pivot is constant it can be shown that,

$$
\text { The frictional moment }=\frac{1}{2} \frac{\mu W r}{\sin \alpha} .
$$

The proof is left to the student.

\section{PROBLEMS}

158. The weight of the vertical shaft and the rotating parts of a turbine is $100,000 \mathrm{lb}$. and the diameter of the shaft is $10 \mathrm{in}$. Assuming the coefficient of friction to be 0.015 and the bearing to be a flat-ended pivot, find the frictional moment (1) when the pressure is uniform and (2) when the wear is uniform.

159. Find the moment of the friction on a collar bearing, when subjected to a pressure of $6000 \mathrm{lb}$., if the radii of the collar are $3.5 \mathrm{in}$. and $4.5 \mathrm{in}$. and the coefficient of friction is 0.025 . Assume the pressure to be uniform.

160. Derive an expression for the frictional moment for the spherical pivot shown in Fig. 179 for each of the following assumptions: (1) uniform normal pressure; (2) uniform wear in the axial direction.

$$
\text { Ans. (1) } W \mu r\left(\frac{\alpha-\sin \alpha \cos \alpha}{\sin ^{2} \alpha}\right) ; \text { (2) } \frac{W \mu r \sin ^{2} \alpha}{\alpha+\sin \alpha \cos \alpha} \text {. }
$$

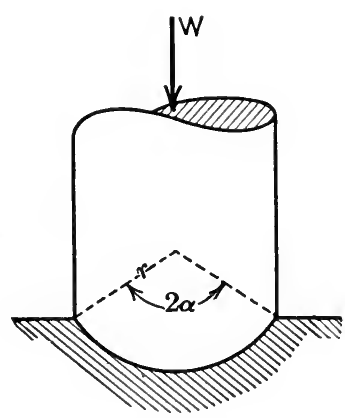

Fig. 179.

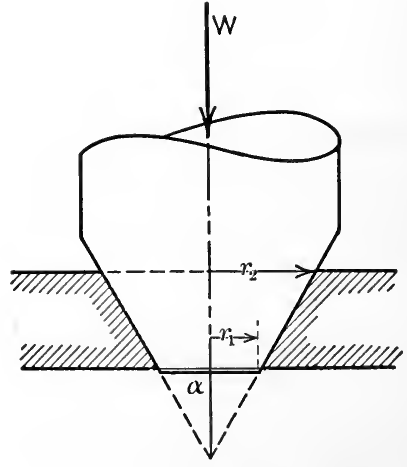

Fig. 180.

161. Determine the frictional moment for the conical pivot shown in Fig. 180 for each of the following assumptions: (1) uniform normal pressure; (2) uniform axial wear.
Ans. (1) $\frac{2 W \mu}{3 \sin \alpha} \cdot \frac{r_{2}{ }^{3}-r_{1}{ }^{3}}{r_{2}{ }^{2}-r_{1}{ }^{2}}$.
(2) $W \mu \frac{r_{2}+r_{1}}{2 \sin \alpha}$. 
77. Rolling Resistance.-If a rigid wheel or roller which carries a vertical load rests on a rigid horizontal surface, a horizontal force, however small, will cause the wheel or roller to roll on the surface. If a wheel rolls over a yielding surface, however, a resistance to the motion is encountered due to the fact that the surface immediately in front of the wheel is being deformed.

In Fig. 181 is shown a wheel carrying a vertical load $W$. Let $P$ be a horizontal force which causes the center of the wheel to move with a constant velocity. Since the surface on which the wheel rolls deforms under the wheel, the pressure between the wheel and the surface is distributed over the area of contact. The resultant pressure or reaction of the surface on the wheel, then, passes through some point, $B$, in the area of contact as shown in the figure. Since the velocity of the wheel is constant,

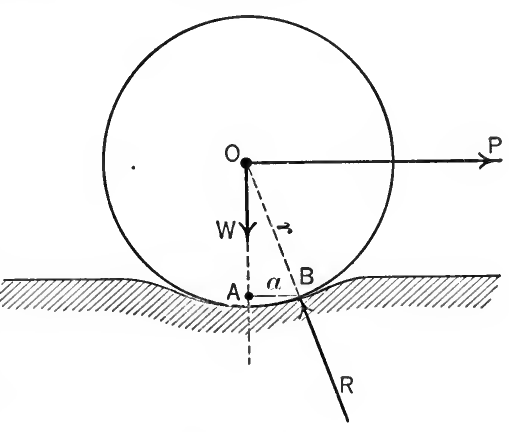

FIG. 181. the three forces acting on it are in equilibrium and hence the reaction, $R$, of the surface on the wheel must pass through $O$, the center of the wheel. Taking moments about $B$ we have,

$$
\Sigma M_{B}=W \times A B-P \times O A=0 .
$$

Since the depression is usually small, $O A$ is approximately equal to $r$, the radius of the wheel. By using this approximation and denoting $A B$ by $a$, the value of $P$ is found to be,

$$
P=\frac{W a}{r} \text {. }
$$

The force $P$ is equal to the horizontal component of the reaction $R$ and is called the rolling friction or rolling resistance; the distance $a$ is sometimes called the coefficient of rolling resistance. However, since $a$ is a linear quantity and not a pure number it is not a true coefficient. The value of $a$ is generally expressed in inches. The laws of rolling resistance are not well known, and there is need of further investigation on the subject. It was assumed by Coulomb that the coefficient of rolling resistance is independent of the radius 
of the wheel. Tests by Dupuit indicate that the coefficient varies as the square root of the diameter. Whether the conclusion of the latter is correct or not, it seems reasonable to assume that the value of the coefficient depends on the diameter of the wheel. The values of the coefficient of rolling resistance given by various investigators are not in close agreement and should be used with caution.

\section{Coefficients of Rolling Resistance}

(Due to Coulomb and Goodman)

$$
a \text { (inches) }
$$

Lignum vitæ on oak. . . . . . . . . 0.0195

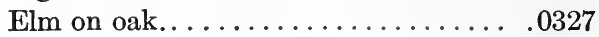

Steel on steel.................. 007 to 0.015

Steel on wood................06 to .10

Steel on macadam road........... $05 \quad .20$

Steel on soft ground. . ........... 3.0 to 5.0

Pneumatic tires on good road.......0.02 to 0.022

Pneumatic tires on mud road ...... .04 to .06

Solid rubber tire on good road....... . . 04

Solid rubber tire on mud road....... .09 to .11

The resistance due to rolling may be regarded as equivalent to a couple. In order to show that this statement is true, consider the wheel represented in Fig. 182. A flexible cord passing

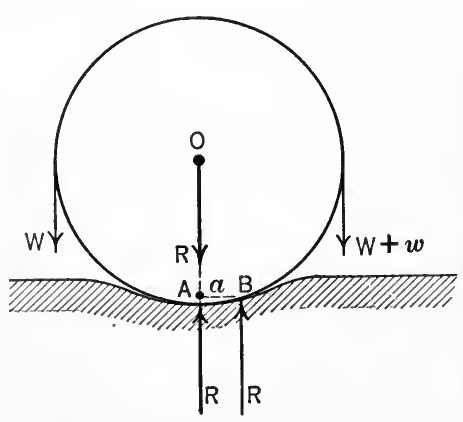

FIG. 182. over the wheel carries at its ends weights $W$ and $W+w$. Assume $w$ to be just great enough to cause the wheel to move with a constant velocity. Since the wheel is in equilibrium under the action of the two vertical forces, $W$ and $W+w$, and the reaction, $R$, of the surface at $B$, it is evident that the latter force must be vertical. The reaction $R$ at $B$ may be replaced by an equal parallel force through $A$ and a couple having a moment equal to $R a$. Since the force, $R$, through $A$ also passes through the center of the wheel it will not effect the rotation. Therefore the rolling resistance is equivalent to the couple $R a$. The moment $w r$, then, which causes the wheel to roll is equal to $R a$. 
For moving heavy weights, such as machines, houses, etc., two or more rollers are frequently used as shown in Fig. 183. If the coefficient of rolling resistance is the same at the top and bottom of the rollers it can be shown that the force $P$ required to overcome friction is given by the expression,

$$
P=\frac{W a}{r} .
$$

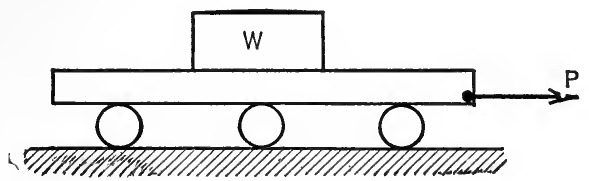

FIG. 183.

\section{PROBLEMS}

162. The rolling resistance for the wheels of a freight car is $3 \mathrm{ib}$. per ton. If the diameter of the car wheels is 33 in., what is the coefficient of rolling resistance? Ans. 0025 in.

163. An oak beam which carries a load of $5000 \mathrm{lb}$. rests on elm rollers the diameters of which are 6 in. The rollers rest on a horizontal oak track. What horizontal force is required to move the load if the weight of the beam is neglected?

164. What is the rolling resistance of a wagon wheel on a macadam road if the diameter of the wheel is $4 \mathrm{ft} .6$ in.? Assume the coefficient of rolling resistance to be 0.2 in.

Ans. $14.8 \mathrm{lb}$. per ton.

78. Belt Friction.-Belt friction is important in the transmission of power by belt and rope drives and in resisting large loads by means of band brakes, capstans, etc. If a belt, rope, or steel band passes over a smooth cylinder or pulley the tensions in the belt, rope, or band on the two sides of the pulley are equal. If the cylinder or pulley is rough, however, the tensions will not, in general, be equal. In the present article the relation between the tensions in the belt, etc., on the two sides of a rough pulley, when the belt is about to slip, will be determined. It is evident that the greater tension must be just large enough to overcome the smaller tension in addition to the friction between the belt and the pulley. In Fig. 184(a) is represented a belt on a pulley, the angle of contact being $\alpha$ and the belt tensions being $T_{1}$ and $T_{2}$. Let 
$T_{1}$ be the greater tension and let it be assumed that the belt is about to slip on the pulley. The normal pressure between the belt and the pulley per unit length of belt, that is, the intensity of pressure at any point, will be denoted by $p$, and the tension in the belt at the same point will be denoted by $T$. Fig. $184(b)$ is a freebody diagram of an element of belt of length $d s$. The forces acting on this element are the tensions $T$ and $T+d T$ at the ends, and the reaction of the pulley. The latter force may be resolved into a component, $d N=p d s$, normal to the face of the
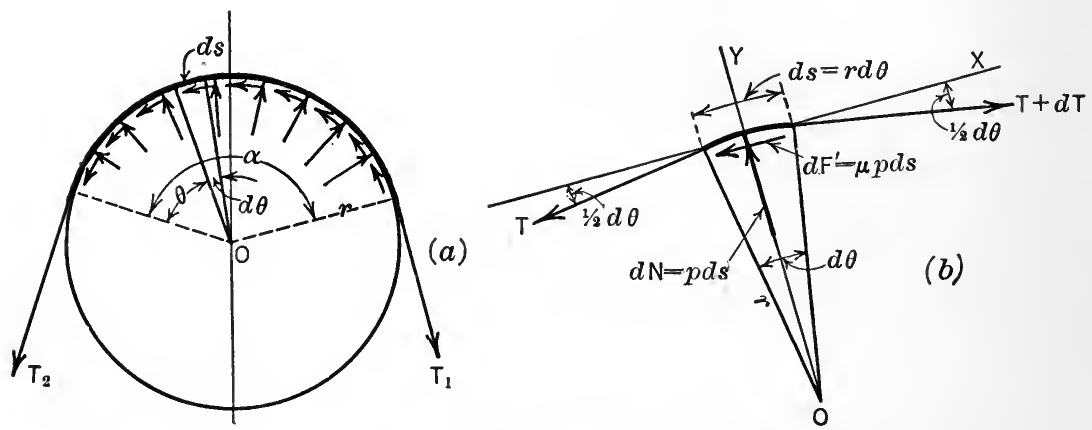

FIG. 184.

pulley and a frictional component, $d F^{\prime}=\mu p d s$, tangent to the face of the pulley. The equations of equilibrium may be applied as follows:

$$
\begin{aligned}
& \Sigma F_{x}=(T+d T) \cos \frac{d \theta}{2}-T \cos \frac{d \theta}{2}-\mu p d s=0 . \\
& \Sigma F_{y}=p d s-(T+d T) \sin \frac{d \theta}{2}-T \sin \frac{d \theta}{2}=0 . \quad .
\end{aligned}
$$

Since $\frac{d \theta}{2}$ is small, $\cos \frac{d \theta}{2}$ is approximately equal to unity and $\sin \frac{d \theta}{2}$ is approximately equal to $\frac{d \theta}{2}$. The term $d T \sin \frac{d \theta}{2} \mathrm{i}$ is a small quantity of the second order and may be neglected. By using these approximations, equations (1) and (2) become,

$$
\begin{array}{llllllll}
d T-\mu p d s=0, & . & . & . & . & . & . & . \\
p d s-T d \theta=0, & . & . & . & . & . & . & .
\end{array}
$$


Eliminating $p d s$ from equations (3) and (4) we have,

$$
\frac{d T}{T}=\mu d \theta . \quad \text {. . . . . . . . }
$$

By integrating equation (5) the relation between $T_{1}$ and $T_{2}$ may be found as follows:

$$
\begin{aligned}
& \int_{T_{2}}^{T_{1}} \frac{d T}{T}=\int_{0}^{\alpha} \mu d \theta \\
& \log _{e} \frac{T_{1}}{T_{2}}=\mu \alpha
\end{aligned}
$$

That is,

$$
\frac{T_{1}}{T_{2}}=e^{\mu \alpha}
$$

or,

$$
T_{1}=T_{2} e^{\mu \alpha}
$$

where $e$ is the base of natural logarithms and $\alpha$ is measured in radians. It should be noted that in the derivation of equation (6) the belt is assumed to be perfectly flexible.

\section{ILLUSTRATIVE PROBLEM}

165. In the band brake shown in Fig. 185 the force $P$ is $100 \mathrm{lb}$., the angle of contact, $\alpha$, is $270^{\circ}\left(\frac{3}{2} \pi\right.$ radians), and the coefficient of friction, $\mu$, for the band and the brake wheel is 0.2 . If the brake wheel rotates in a counter-clockwise direction find the tensions in the band and the frictional moment developed.

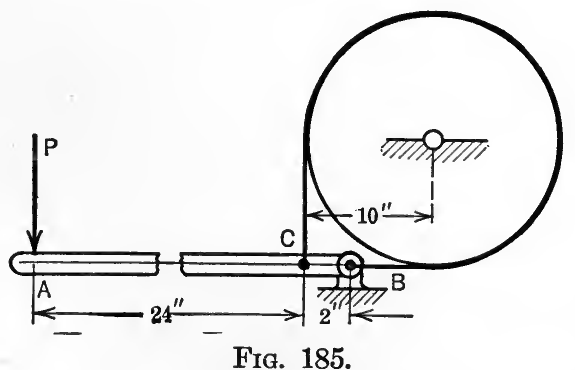

Solution.- Since the operating lever $A C B$ is in equilibrium the equation $\Sigma M_{B}=0$ may be applied, from which the band pull at $C$, that is, the tension $T_{2}$, is found. Thus,

$$
\begin{aligned}
\Sigma M_{B} & =100 \times 26-T_{2} \times 2=0, \\
\therefore T_{2} & =1300 \mathrm{lb} .
\end{aligned}
$$


Since $T_{2}$ is now known, the tension $T_{1}$ may be found from the belt-friction formula. Thus,

$$
\begin{aligned}
T_{1} & =T_{2} \times e^{\mu \alpha} \\
& =1300 \times(2.718)^{0.2 \times \frac{3}{2} \pi,}
\end{aligned}
$$

$\log T_{1}=\log 1300+0.3 \pi \log 2.718$

$=3.114+.942 \times .434$

$=3.522$.

$\therefore T_{1}=3330 \mathrm{lb}$

Frictional moment $=\left(T_{1}-T_{2}\right) \times 10$

$$
=2030 \times 10=20,300 \mathrm{lb} \text {.-in. }
$$

\section{PROBLEMS}

166. A body weighing $2000 \mathrm{lb}$. is suspended by means of a rope wound $1 \frac{1}{2}$ turns around a drum. If the coefficient of friction is 0.3 what force must be exerted at the other end of the rope to hold the body?

167. A boat is brought to rest by means of a rope which is wound around a capstan. If a force of $4000 \mathrm{lb}$. is exerted by the

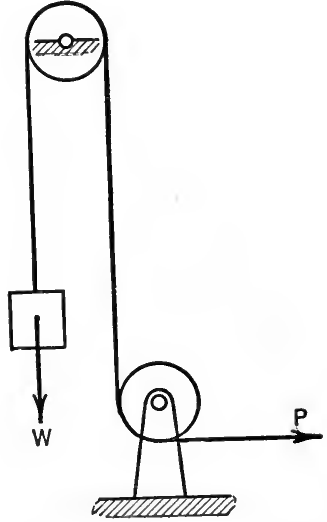

FIG. 186. boat and a pull of $100 \mathrm{lb}$. is exerted on the other end of the rope, find the number of turns the rope makes around the capstan, assuming the value of $\mu$ to be 0.25 .

Ans. 2.36 turns.

168. A body weighing $500 \mathrm{lb}$. is raised by means of a rope which passes over a round beam, the angle of contact being $180^{\circ}$. If the coefficient of friction is 0.4 , what is the least force which will raise the body? What is the least force which will hold the body?

169. A rope is wound twice around a post. If a pull of $50 \mathrm{lb}$. at one end of the rope will just support a force of $6000 \mathrm{lb}$. at the other end, what is the coefficient of friction?

170. A body having a weight, $W$, of 1 ton is suspended by means of a wire rope which passes over two fixed drums, as shown in Fig. 186. If the coefficient of friction for the rope and drums is 0.3 , what force, $P$, will be required $(a)$ to hold the body; (b) to start the body upwards?

Ans. (a) $P=488 \mathrm{lb}$. 
171. In Fig. 187 is represented a band brake, the angle of contact of the

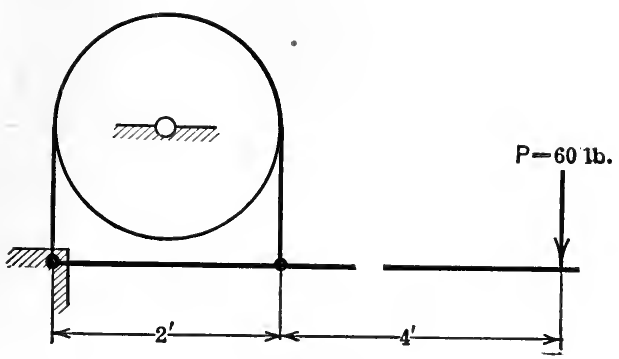

FIG. 187.

band on the brake wheel being $180^{\circ}$. If the coefficient of friction is 0.2 , find the frictional moment developed $(a)$ when the brake wheel rotates clockwise; $(b)$ when the brake wheel rotates counter-clockwise

Ans. (a) 157 lb.-ft. 


\section{CHAP'TER V}

\section{FIRST MOMENTS AND CENTROIDS}

79. First Moments.-In the preceding chapters moments of forces with respect to points and lines have frequently been used. In the analysis of many problems in engineering, however, expressions are frequently met which represent moments of volumes, masses, areas, and lines. The moment of a volume, mass, ${ }^{1}$ area, or line with respect to an axis or plane is the algebraic sum of the moments of the elementary parts of the volume, mass, area, or line, the moment of an elementary part being the product of the elementary part (volume, mass, area, or line) and its distance from the moment axis or plane. This moment of a volume, mass, etc., is called the first moment when it is desired to distinguish it from the moment of inertia (or second moment) of the volume, area, etc. (see Chapter VI), since the coordinate distances of the parts of the volume, mass, area, or line enter into the expression for the first moment to the first power and into the expression for the second moment to the second power.

80. Centroids. - In dealing with a system of parallel forces in a plane it was found from the principle of moments that the algebraic sum of the moments of the forces about a point or axis is equal to the moment of the resultant of the forces with respect to the same point or axis. That is, $\Sigma(F \cdot x)=\Sigma F \cdot \bar{x}=R \bar{x}$ (Art. 26). This equation was frequently used in Chapter II to locate the position of the resultant, that is, to determine $\bar{x}$ after first finding the sum of the moments of the forces, $\Sigma(F \cdot x)$. When the position of the resultant is known, however, the moment of the system of forces is most easily found by determining the moment $(R \bar{x})$ of the resultant.

1 The term mass cannot be defined completely nor its physical significance discussed until the laws of motion of physical bodies are treated. (See Chapter IX.) As here used it is sufficient to think of mass as the inert material or matter of which bodies are composed, the quantitative expression of which is the volume of the body times a density factor. 
In a similar way the moment of a volume, mass, area, or line with respect to an axis or plane (made up of the sum of the moments of the several parts or elements of the volume, mass, area, or line) may be expressed as the product of the whole volume, mass, area, or line, and a distance, $\bar{x}$ (or $\bar{y}$, etc.), from the axis or plane such that this product equals the algebraic sum of the moments of the elementary parts of the volume, mass, area, or line.

This relation is sometimes called the principle of moments for areas, volumes, etc., and it leads to an equation of the same form as does the same principle in the case of forces. Thus, for an area $A$, an element of which is denoted by $a$ or $d A$, the equation is,

$$
\Sigma(a x)=\Sigma a \cdot \bar{x}=A \bar{x},
$$

or

$$
\int(d A \cdot x)=\int d A \cdot \bar{x}=A \bar{x}
$$

The resulting distance ( $\bar{x}$ or $\bar{y}$, etc.), is called the centroidal distance, and the point located by the centroidal distances is called the centroid ${ }^{1}$ of the volume, mass, area, or line. The centroid of a volume, mass, area, or line, then, is that point at which the whole volume, mass, area, or line may be conceived to be concentrated and have the same moment with respect to an axis or plane as has the volume, mass, area, or line when distributed in its natural way.

Hence the coordinates $(\bar{x}$ and $\bar{y})$ of the centroid of an area, $A$, and of a line, $L$, are expressed by the following equations, in 'which $A=\Sigma a=\int d A$ and $L=\Sigma l=\int d L$.

$$
\bar{x}=\frac{\Sigma(a x)}{A}=\frac{\int x d A}{A}, \quad \bar{x}=\frac{\Sigma(l x)}{L}=\frac{\int x d L}{L},
$$

${ }^{1}$ The term center of gravity is sometimes used in technical literature to denote not only the point in a body through which the resultant earth-pull acts, but also to denote what is here defined as the centroid of a line, area, volume, or mass. Thus, the phrase "center of gravity" of an area, or volume, etc., is used instead of the phrase " centroid" of the area, or volume, etc. Further, the term centroid is sometimes used in a restricted sense as applying only to geometrical figures (lines, areas, and volumes), in which case, the term mass-center or center of mass is used instead of centroid of mass in connection with physical bodies. 


$$
\bar{y}=\frac{\Sigma(a y)}{A}=\frac{\int y d A}{A} . \quad \bar{y}=\frac{\Sigma(l y)}{L}=\frac{\int y d L}{L} .
$$

Likewise, the coordinates $(\bar{x}, \bar{y}$, and $\bar{z})$ of the centroid of a volume, $V$, and of a mass, $M$, may be expressed by similar equations, in which $V=\Sigma v=\int d V$ and $M=\Sigma m=\int d M$. Thus,

$$
\begin{array}{ll}
\bar{x}=\frac{\Sigma(v x)}{V}=\frac{\int x d V}{V}, & \bar{x}=\frac{\Sigma(m x)}{M}=\frac{\int x d M}{M}, \\
\bar{y}=\frac{\Sigma(v y)}{V}=\frac{\int y d V}{V}, & \bar{y}=\frac{\Sigma(m y)}{M}=\frac{\int y d M}{M}, \\
\bar{z}=\frac{\Sigma(v z)}{V}=\frac{\int z d V}{V}, & \bar{z}=\frac{\Sigma(m z)}{M}=\frac{\int z d M}{M} .
\end{array}
$$

81. Planes and Lines of Symmetry.-If a geometrical figure (volume, area, or line) is symmetrical with respect to a plane or line, the centroid of the figure lies in the given plane or line. This statement is evident from the fact that the moments of the parts of the figure on the opposite sides of the plane or line are numerically equal but of opposite sign. If a figure is symmetrical with respect to each of two planes or lines, the centroid of the figure lies in the line of intersection of the two planes or at the point of intersection of the two lines. If the figure has three planes of symmetry, the centroid coincides with the point of intersection of the three planes. The foregoing statements apply also to the centroids of the masses of homogeneous physical solids which are symmetrical with respect to one or more planes, since the centroid of a volume coincides with the centroid of the mass of a homogeneous body which is congruent with the volume. The centroids of many simple figures may be partially or completely determined from symmetry. Thus, the centroids of the volumes or of the surface areas of the following solids are as indicated below:

(1) Sphere or ellipsoid; the center of the sphere or ellipsoid.

(2) Right prism or cylinder; the mid-point of the axis.

(3) Hemisphere; on the radius perpendicular to its base.

(4) Right cone; on its geometrical axis. 
And the centroids of the following areas, and of their bounding lines, are as indicated below:

(5) Circle or ellipse; the center of the circle or ellipse.

(6) Isosceles triangle; on the median bisecting the angle between the equal sides.

(7) Semicircle; on the radius perpendicular to the base.

82. Centroids by Integration.--In determining the centroid of a volume, mass, area, or line by the method of integration, from the equations of Art. $80\left(V \bar{x}=\int x d V, A \bar{x}=\int x d A\right.$, etc.), it is possible to select the element of volume, area, etc., in various ways and to express the element in terms of either cartesian or polar coordinates. The resulting integral may be a single, a double, or a triple integral, depending on the way the element is selected. The integral, of course, is a definite integral, the limits of integration depending on the boundary curve or surface of the figure or body. In any case the element of volume, mass, area, or line must be taken so that,

1. All points of the element are the same distance from the line or plane about which moments are taken; otherwise, the distance from the line or plane to the element will be indefinite. Or, so that,

2. The centroid of the element is known, in which case the moment of the element about the moment axis or plane is the product of the element and the distance of its centroid from the axis or plane.

The centroids of some of the common figures (lines, areas, and volumes) will be found in the following illustrative problems.

\section{ILLUSTRATIVE PROBLEMS}

Find, by the method of integration, the centroids of the following figures with respect to the axes indicated.

172. Arc of a Circle.-The radius which bisects the arc will be taken as the $x$-axis (Fig. 188). By symmetry the centroid lies on this axis. Hence $\bar{y}=0$. If $r$ denotes the radius of the arc and $2 \alpha$ the subtended angle, then, in terms of polar coordinates, the element of arc, $d L$, and its distance $x$ from the $y$-axis are $d L=r d \theta$ and $x=r \cos \theta$. Thus, the element of arc is selected in accordance with the first of the above rules and $\bar{x}$ may be found as follows:

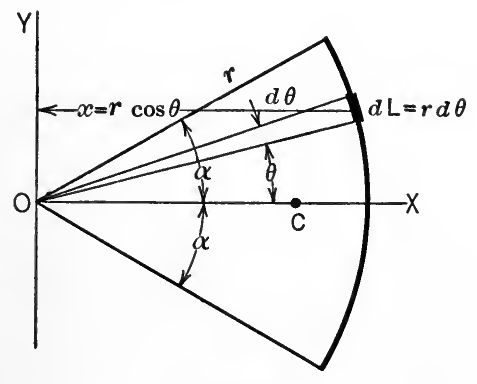

FIG. 188. 


$$
\begin{aligned}
L \bar{x} & =\int x d L \\
& =\int_{-\alpha}^{+\alpha} r \cos \theta \cdot r d \theta \\
& =r^{2} \int_{-\alpha}^{+\alpha} \cos \theta d \theta=2 r^{2} \sin \alpha .
\end{aligned}
$$

Therefore,

$$
\bar{x}=\frac{2 r^{2} \sin \alpha}{L}=\frac{2 r^{2} \sin \alpha}{2 r \alpha}=\frac{r \sin \alpha}{\alpha} .
$$

If the arc is a semicircle, that is, if $\alpha=90^{\circ}=\frac{\pi}{2}$ radians, then, $\bar{x}=\frac{2 r}{\pi}$. That is, the distance of the centroid of a semicircular arc from the center of the circle is slightly less than two-thirds of the radius of the circle.

173. Area of a Triangle.-In accordance with the first of the above rules the elements of area will be taken as strips parallel to the base of the triangle (Fig. 189). Since each element is bisected by the median drawn from the vertex opposite the base, the centroid of each element, and hence of the entire area, lies on this median. If $x$ denotes the width of the strip, the area of the strip is $d A=x d y$. Thus,

$$
A \bar{y}=\int x y d y .
$$

From similar triangles, the relation between $x$ and $y$ is,

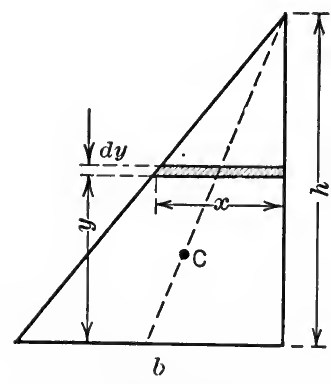

FIG. 189.

$$
\begin{aligned}
\frac{x}{h-y} & =\frac{b}{h} \quad \text { or } \quad x=\frac{b}{h}(h-y) . \\
A \bar{y} & =\frac{b}{h} \int_{0}^{h}(h-y) y d y=\frac{1}{6} b h^{2} .
\end{aligned}
$$

Therefore,

$$
\bar{y}=\frac{\frac{1}{6} b h^{2}}{\frac{1}{2} b h}=\frac{1}{3} h .
$$

The centroid of a triangular area, then, is at a distance of one-third of the altitude from the base.

174. Sector of a Circle. First Method.-The element of area wiil be selected in accordance with the first of the above rules as indicated in Fig. 190. Since the area is symmetrical with respect to the $x$-axis, the centroid lies on this axis and hence $\bar{y}=0$. The value of $x$ may then be found from the equation, 
Therefore,

$$
\begin{aligned}
A \bar{x} & =\int x d A \\
& =\int_{0}^{r} \int_{-\alpha}^{+\alpha} \rho \cos \theta \cdot \rho d \rho d \theta=\frac{2}{3} r^{3} \sin \alpha . \\
\bar{x} & =\frac{\frac{2}{3} r^{3} \sin \alpha}{A}=\frac{\frac{2}{3} r^{3} \sin \alpha}{r^{2} \alpha}=\frac{2}{3} \frac{r \sin \alpha}{\alpha} .
\end{aligned}
$$

If $\alpha=90^{\circ}=\frac{\pi}{2}$ radians, that is, if the sector is a semicircular area,

$$
\bar{x}=\frac{4 r}{3 \pi} .
$$

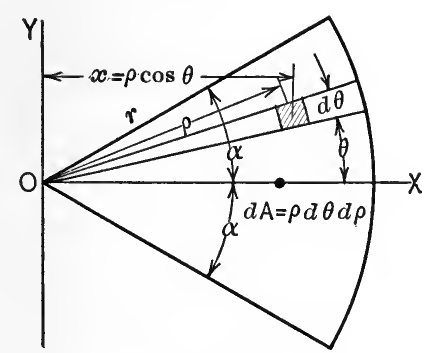

FIG. 190.

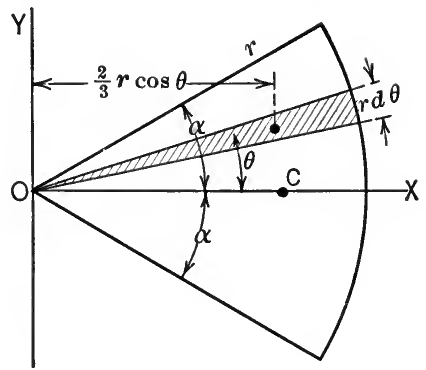

Fig. 191.

Second Method.-In accordance with the second of the above rules, the element of area will be selected as a triangle, as indicated in Fig. 191. The area of the triangle is $\frac{1}{2} r^{2} d \theta$ and the distance of its centroid from the $y$-axis is $\frac{2}{3} r \cos \theta$. Hence, the moment of the triangle with respect to the $y$-axis is $\frac{1}{3} r^{3} \cos \theta d \theta$ and $\bar{x}$ is obtained from the equation,

Therefore,

$$
\begin{aligned}
A \bar{x} & =\int_{-\alpha}^{+\alpha} \frac{1}{3} r^{3} \cos \theta d \theta \\
& =\frac{2}{3} r^{3} \sin \alpha .
\end{aligned}
$$

$$
\bar{x}=\frac{\frac{2}{3} r^{3} \sin \alpha}{r^{2} \alpha}=\frac{2}{3} \frac{r \sin \alpha}{\alpha} .
$$

175. Volume of a Right Circular Cone.-The axis of the cone will be taken as the $x$-axis (Fig. 192). By symmetry, $\bar{y}=0$ and $\bar{z}=0$. In finding $\bar{x}$, a thin lamina parallel to the base of the cone will be selected as the element of volume, the centroid of the lamina being at its center at the distance $x$ from the $y z$-plane. Hence, $\bar{x}$ may be found as follows:

$$
\begin{aligned}
V \bar{x} & =\int x d V \\
& =\int_{0}^{h} x \pi z^{2} d x .
\end{aligned}
$$


From similar triangles, the relation between $x$ and $z$ is,

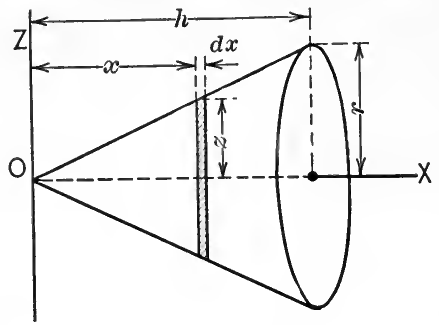

$$
\frac{z}{x}=\frac{r}{h} \quad \text { or } \quad z=\frac{r}{h} x .
$$

Hence,

FIG. 192.

$$
V \bar{x}=\frac{\pi r^{2}}{h^{2}} \int_{0}^{h} x^{3} d x=\frac{1}{4} \pi r^{2} h^{2} .
$$

Therefore,

$$
\bar{x}=\frac{\frac{1}{4} \pi r^{2} h^{2}}{\frac{1}{3} \pi r^{2} h}=\frac{3}{4} h .
$$

176. Volume of a Hemisphere.-The axis of symmetry will be taken as the $z$-axis (Fig. 193). By symmetry $\bar{x}=0$ and $\bar{y}=0$. A thin lamina parallel to the base will be selected as the element of volume, the centroid of which is at the distance $z$ from the $x y$-plane. Hence, the $z$-coordinate of the centroid of the volume of the hemisphere may be found from the equation,

$$
\begin{aligned}
V \bar{z} & =\int z d V, \\
& =\int_{0}^{r} z \pi x^{2} d z=\pi \int_{0}^{r} z\left(r^{2}-z^{2}\right) d z=\frac{1}{4} \pi r^{4} .
\end{aligned}
$$

Therefore,

$$
\bar{z}=\frac{\frac{1}{4} \pi r^{4}}{V}=\frac{\frac{1}{4} \pi r^{4}}{\frac{2}{3} \pi r^{3}}=\frac{3}{8} r .
$$

177. Parabolic Segment.-Let the segment be bounded by the $x$-axis, the line

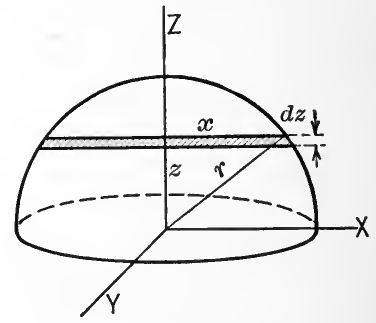

FIG. 193. $x=a$, and the parabola $y^{2}=\frac{b^{2} x}{a}$ as shown in Fig. 194. A strip parallel to the $y$-axis will be selected as the element of area, the area of the strip being expressed by $y d x$. The area of the segment, then, is,

$$
\begin{aligned}
A & =\int d A=\int_{0}^{a} y d x=\frac{b}{\sqrt{a}} \int_{0}^{a} \sqrt{x} d x \\
& =\frac{b}{\sqrt{ } a}\left[\frac{2}{3} x^{3 / 2}\right]_{o}^{a}=\frac{2}{3} a b .
\end{aligned}
$$

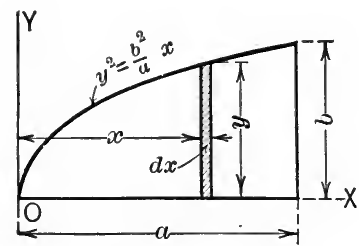

Fig. 194.

To find $\bar{x}$;

$$
\begin{aligned}
A \bar{x} & =\int x d A \\
& =\int_{0}^{a} x y d x=\frac{b}{\sqrt{a}} \int_{0}^{a} x^{3 / 2} d x=\frac{2}{5} b a^{2} .
\end{aligned}
$$

Therefore, $\quad \bar{x}=\frac{\frac{2}{5} b a^{2}}{A}=\frac{\frac{2}{5} b a^{2}}{\frac{2}{3} a b}=\frac{3}{5} a$. 
To find $\bar{y}$, the same elementary strip will be selected, but since each point of the element is not the same distance from the $x$-axis, its moment must be expressed as the product of the area of the strip and its centroidal distance, $\frac{y}{2}$, from the $x$-axis. Thus,

Therefore,

$$
A \bar{y}=\int_{0}^{a} \frac{1}{2} y \cdot y d x=\frac{1}{2} \frac{b^{2}}{a} \int_{0}^{a} x d x=\frac{1}{4} b^{2} a .
$$

$$
\bar{y}=\frac{\frac{1}{4} b^{2} a}{A}=\frac{\frac{1}{4} b^{2} a}{\frac{2}{3} a b}=\frac{3}{8} b \text {. }
$$

178. Area of Quadrant of an Ellipse.-The semi-axes of the ellipse will be denoted by $a$ and $b$ (Fig. 195) and hence the equation of the ellipse is

$$
\frac{x^{2}}{a^{2}}+\frac{y^{2}}{b^{2}}=1 \text {. }
$$

A strip parallel to the $y$-axis will be selected as the element of area. From the equation of the ellipse $y$ may be expressed in terms of $x$ by the equation,

Hence,

$$
y=\frac{b}{a} \sqrt{a^{2}-x^{2}}
$$

$$
A=\int_{0}^{a} y d x=\frac{b}{a} \int_{0}^{a} \sqrt{a^{2}-x^{2}} d x=\frac{1}{4} \pi a b .
$$

To find $\bar{x}$;

$$
\begin{aligned}
\frac{1}{4} \pi a b \cdot \bar{x} & =\int x d A \\
& =\int_{0}^{a} x \cdot y d x=\frac{b}{a} \int_{0}^{a} x \sqrt{a^{2}-x^{2}} d x=\frac{1}{3} a^{2} b .
\end{aligned}
$$

Therefore,

$$
\bar{x}=\frac{\frac{1}{3} a^{2} b}{\frac{1}{4} \pi a b}=\frac{4 a}{3 \pi} .
$$

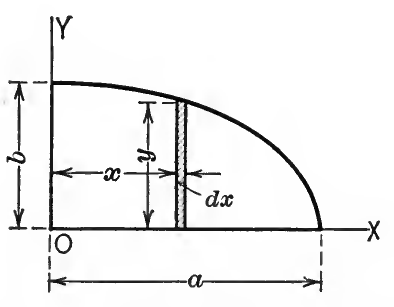

Fig. 195.

To find $\bar{y}$, the same strip will be used for the element of area, its centroid being at the distance $\frac{1}{2} y$ from the $x$-axis. Thus,

Therefore,

$$
\frac{1}{4} \pi a b \cdot \bar{y}=\int_{0}^{a} \frac{1}{2} y \cdot y d x=\frac{b^{2}}{2 a^{2}} \int_{0}^{a}\left(a^{2}-x^{2}\right) d x=\frac{1}{3} a b^{2} .
$$

$$
\bar{y}=\frac{\frac{1}{3} a b^{2}}{\frac{1}{4} \pi a b}=\frac{4 b}{3 \pi} .
$$

\section{PROBLEMS}

179. A pulley having a thin rim is $2 \mathrm{ft}$. in diameter. How far from the center of the pulley is the centroid of each half of the rim?

180. Find the centroid of a semicircular area, the radius of which is 9 in. 
181. Show that the centroid of the surface of a right circular cone is on the axis of the cone at a distance of $\frac{2}{3} h$ from the apex, where $h$ is the altitude of the cone.

182. The radius of the base of a right circular cone is 5 in. and its height, is $2 \mathrm{ft}$. Locate the centroid of $(a)$ the volume of the cone; $(b)$ the curved surface area of the cone.

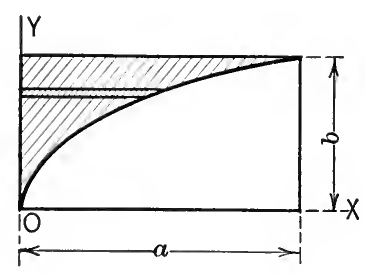

FIg. 196.

183. Show that the distance of the centroid of the surface of a hemisphere from the base is $\frac{1}{2} r$ where $r$ is the radius of the hemisphere.

184. Locate the centroid of the area included between the $y$-axis, the line $y=b$, and the parabola $y^{2}=\frac{b^{2} x}{a}$ as shown in Fig. 196 . Select the element of area as shown in the figure. Ans. $\bar{x}=\frac{3}{10} a, \bar{y}=\frac{3}{4} b$.

185. A paraboloid is generated by rotating the parabola $y^{2}=p x$ about the $x$-axis. Locate the centroid of the volume included between the paraboloid and the plane $x=a$.

$$
\text { Ans. } \bar{x}=\frac{2}{3} a \text {. }
$$

83. Centroids of Composite Figures and Bodies.-As noted in Art. 80, if the centroid of a line, area, volume, or mass is known, the moment with respect to an axis or plane is most easily found by multiplying the line, area, volume, or mass by the distance of the centroid from the axis or plane. Thus, if a given line, area, volume, or mass can be divided into parts, the centrojds of which are known, the moment of the whole line, area, etc., may be found without integrating by obtaining the algebraic sum of the moments of the parts into which the line, area, volume, or mass is divided, the moment of each part being the product of that part and the distance of its centroid from the line or plane. Thus, for example, in the case of a composite area, if $a_{1}, a_{2}, a_{3}$, etc., denote the parts into which the area $A$ is divided and $x^{\prime}{ }_{o}, x_{o}{ }^{\prime \prime}, x_{o}{ }^{\prime \prime}$, etc., denote the $x$-coordinates of the centroids of the respective parts, then,

$$
\left(a_{1}+a_{2}+a_{3}+\ldots\right) \bar{x}=a_{1} x_{0}{ }^{\prime}+a_{2} x_{0}{ }^{\prime \prime}+a_{3} x_{0}^{\prime \prime \prime}+\ldots,
$$

or

$$
\Sigma a \cdot \bar{x}=\Sigma\left(a x_{0}\right)
$$

or,

Similarly,

$$
A \bar{x}=\Sigma\left(a x_{0}\right) .
$$

$$
A \bar{y}=\Sigma\left(a y_{0}\right) \text {. }
$$


From similar equations the centroid of a composite line, volume, or mass may be found. Thus,

$$
\begin{array}{lll}
L \bar{x}=\Sigma\left(l x_{0}\right), & V \bar{x}=\Sigma\left(v x_{0}\right), & M \bar{x}=\Sigma\left(m x_{0}\right), \\
L \bar{y}=\Sigma\left(l y_{0}\right), & V \bar{y}=\Sigma\left(v y_{0}\right), & M \bar{y}=\Sigma\left(m y_{0}\right), \\
& V \bar{z}=\Sigma\left(v z_{0}\right), & M \bar{z}=\Sigma\left(m z_{0}\right) .
\end{array}
$$

\section{ILLUSTRATIVE PROBLEMS}

186. Locate the centroid of the T-section shown in Fig. 197.

Solution.-If axes be selected as indicated it is evident from symmetry that $x=0$. By dividing the given area into areas $a_{1}$ and $a_{2}$ and by taking moments about the bottom edge of the area, $\bar{y}$ may be found as follows:

$$
\begin{aligned}
A \bar{y} & =\Sigma\left(a y_{0}\right), \\
\bar{y} & =\frac{12 \times 1+12 \times 5}{6 \times 2+6 \times 2}=3 \mathrm{in} .
\end{aligned}
$$

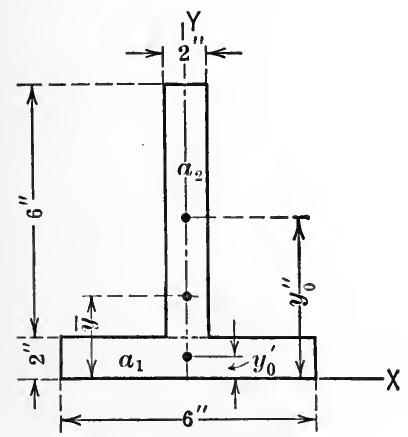

FIG. 197.

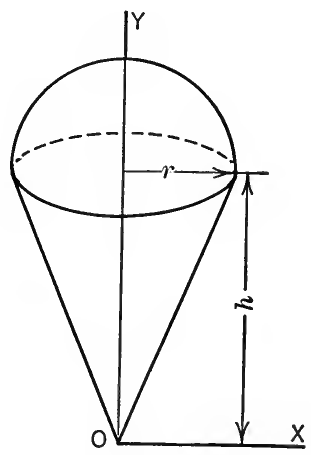

Fig. 198.

187. Locate the centroid of the volume of the cone and hemisphere shown in Fig. 198, the values of $r$ and $h$ being 6 in. and 18 in. respectively.

Solution.-The axis of symmetry will be taken as the $y$-axis. From symmetry then $x=0$. By taking the $x$-axis through the apex of the cone as shown the equation $V \bar{y}=\Sigma\left(v y_{0}\right)$ becomes,

That is,

$$
\left(\frac{1}{3} \pi r^{2} h+\frac{2}{3} \pi r^{3}\right) \bar{y}=\frac{1}{3} \pi r^{2} h \times \frac{3}{4} h+\frac{2}{3} \pi r^{3}\left(h+\frac{3}{8} r\right) .
$$

Therefore,

$$
\frac{1}{3} \pi r^{2}(h+2 r) \bar{y}=\frac{1}{3} \pi r^{2}\left(\frac{3}{4} h^{2}+2 r h+\frac{3}{4} r^{2}\right) \text {. }
$$

$$
\begin{aligned}
\bar{y} & =\frac{\frac{3}{4} h^{2}+2 r h+\frac{3}{4} r^{2}}{h+2 r} \\
& =\frac{\frac{3}{4} \times(18)^{2}+2 \times 6 \times 18+\frac{3}{4} \times(6)^{2}}{18+2 \times 6} \\
& =16.2 \mathrm{in} .
\end{aligned}
$$




\section{PROBLEMS}

188. Locate the centroid of the channel section shown in Fig. 199.

Ans. $\bar{x}=0.79$ in.

189. The radii of the upper and lower bases of the frustum of a right circular cone are $r_{1}$ and $r_{2}$ and the altitude is $a$. Find the distance of the centroid above the lower base.

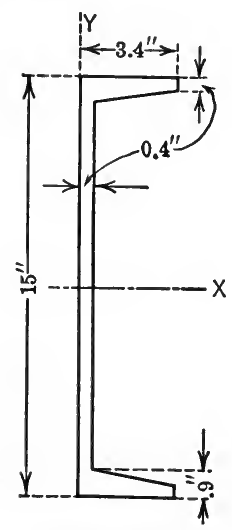

FIg. 199.

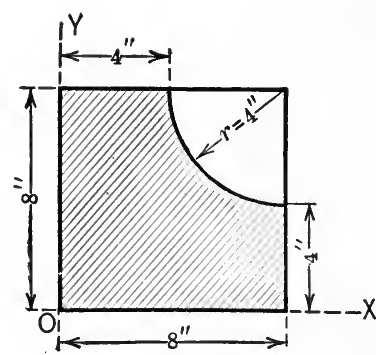

FIG. 200.
Ans. $\bar{y}=\frac{a}{4} \cdot \frac{r_{2}^{2}+2 r_{1} r_{2}+3 r_{1}^{2}}{r_{2}^{2}+r_{1} r_{2}+r_{1}^{2}}$

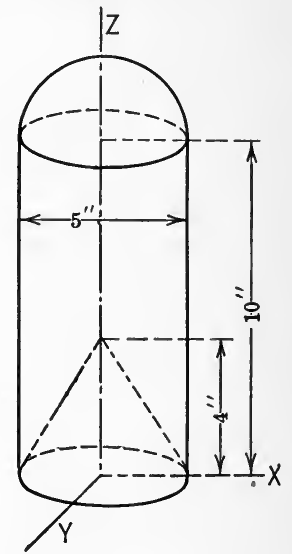

Fig. 201.

190. Locate the centroid of the shaded area shown in Fig. 200.

191. In Fig. 201 is represented a homogeneous solid which consists of a hemisphere and a right circular cylinder from which a cone is removed. Locate the centroid of the solid with respect to the axes indicated.

Ans. $\bar{z}=6.45$ in.

192. Locate the centroid of the segment of a circle as shown in Fig. 202. In the expression for $\bar{x}$ make $\alpha=\frac{\pi}{2}$, and see if the result agrees with the result found in Prob. 174 for a semicircle.

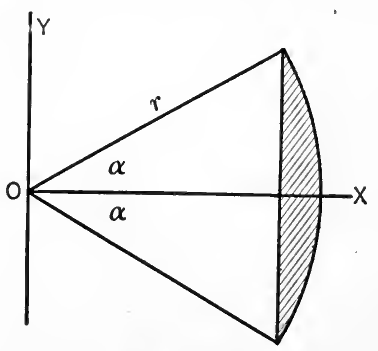

FİG. 202 .

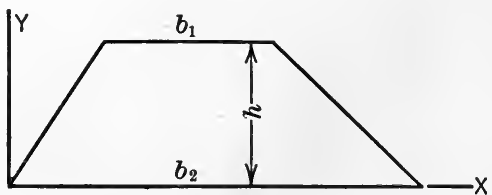

Fig. 203, 
193. Find the distance from the larger base of the centroid of the area of the trapezoid shown in Fig. 203. Ans. $y=\frac{h}{3} \cdot \frac{2 b_{1}+b_{2}}{b_{1}+b_{2}}$.

194. A slender steel rod is bent in the form shown in Fig. 204. Locate the centroid of the rod with respect to the axes shown.

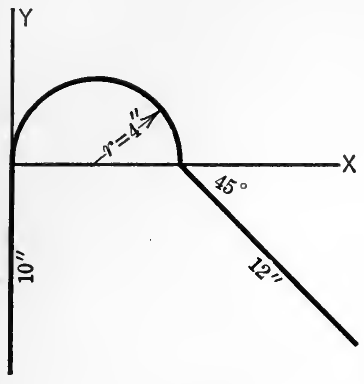

Fig. 204.

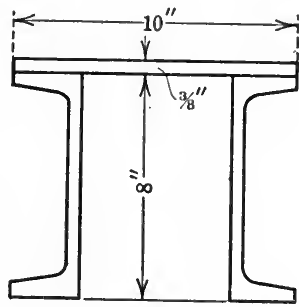

FIG. 205.

195. Fig. 205 represents the cross-section of the end post of a bridge. The area of each channel section is $4.78 \mathrm{sq}$. in. Find the distance from the top of the cover plate to the centroid of the section.

84. Theorems of Pappus and Guldinus.-I. The area of a surface of revolution generated by revolving a plane curve about any non-intersecting axis in its plane is equal to the product of the length of the curve and the length of the path described by the centroid of the curve.

Proof.-Let the curve $A B$ (Fig. 206) be revolved about $O X$. The area of the surface generated is given by the equation,

$$
A=\int 2 \pi y \cdot d L=2 \pi \int y d L=2 \pi \bar{y} \cdot L,
$$

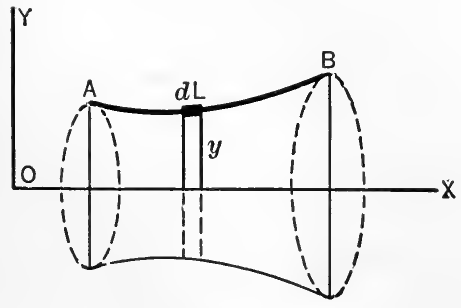

Frg. 206.

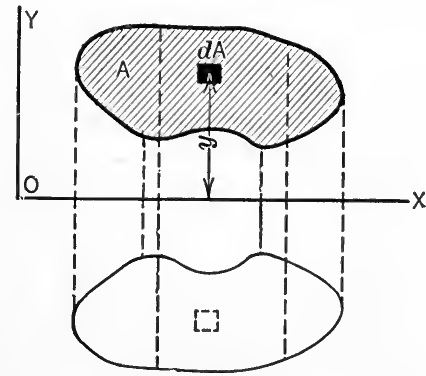

FIG. 207. 
where $\bar{y}$ is the distance of the centroid of the curve from $O X$ and $L$ is the length of the curve.

II. The volume of the solid generated by revolving any plane area about any non-intersecting line in its plane is the product of the area and the length of the path described by the centroid of the area.

Proof.-Let the plane area $A$ (Fig. 207) be rotated about the axis $O X$. Each elementary area $d A$ will generate a circular ring the volume of which is $2 \pi y d A$ and hence the entire volume generated is given by the equation,

$$
V=\int 2 \pi y d A=2 \pi \int y d A=2 \pi \bar{y} \cdot A,
$$

where $\bar{y}$ is the distance of the centroid of the area from $O X$.

\section{ILLUSTRATIVE PROBLEMS}

196. Find the surface area and the volume of the solid generated by rotating a circle of radius $r$ about a line in the plane of the circle at a distance $a$ from its center, $a$ being greater than $r$.

Solution:

$$
\begin{aligned}
& A=2 \pi a \times 2 \pi r=4 \pi^{2} a r, \\
& V=2 \pi a \times \pi r^{2}=2 \pi^{2} a r^{2} .
\end{aligned}
$$

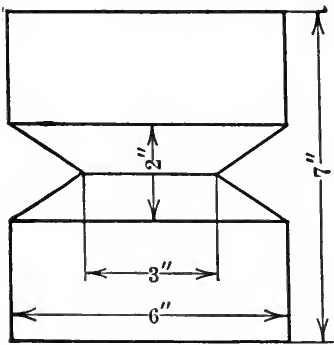

FIG. 208.

197. A V-shaped groove is turned out of a cylinder as indicated by Fig. 208. Find the volume of the material removed.

Solution.-The distance of the centroid of the triangle, which generates the volume, from the axis of the cylinder is 2.5 in. and the area of the triangle is $1.5 \mathrm{sq}$. in. Hence,

$$
\begin{aligned}
V & =2 \pi \times 2.5 \times 1.5 \\
& =23.5 .5 \mathrm{cu} . \mathrm{in} .
\end{aligned}
$$

\section{PROBLEMS}

Solve the following problems by the theorems of Pappus and Guldinus: 198. Find the surface area and the volume of a sphere. 199. Find the volume of a cone. 
200. Find the $y$-coordinate of the centroid of the trapezoid shown in Fig. 209. Determine the volume of the frustum of a cone generated by revolving the trapezoid about the $x$-axis.

201. Find the volume of the ellipsoid generated by revolving the right half of the ellipse, $\frac{x^{2}}{a^{2}}+\frac{y^{2}}{b^{2}}=1$, about the $y$-axis.

\section{Center of Gravity and Mass-} Center. Center of Gravity.-As noted in Art. 80, if the distance of a certain point (centroid) in an area, volume,

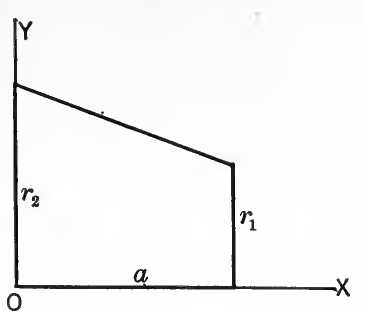

FIG. 209. etc., from a given line or plane is known, the moment of the area, volume, etc., with respect to the line or plane may be found by multiplying the whole area, volume, etc., by this (centroidal) distance. Similarly, the moment of the resultant earth-pull on a body (weight of the body) with respect to an axis may be found by multiplying the weight of the body by the distance of a certain point in the body from the axis. This point is called the center of gravity of the body. The center of gravity of a body, then, is a point in the body (or its extension) such that if the body were conceived to be concentrated at this point the moment of the weight of the body with respect to any axis would be the same as the algebraic sum of the moments of the weights of the particles of the body with respect to the same axis.

The weights of the particles of a body constitute a system of parallel forces having points of application which are fixed in the body and it can be shown ${ }^{1}$ that the resultant of such a force

${ }^{1}$ Center of a System of Parallel Forces. If a system of parallel forces have fixed points of application in a rigid body the resultant of the system will pass through a fixed point in the body regardless of the direction of the parallel force

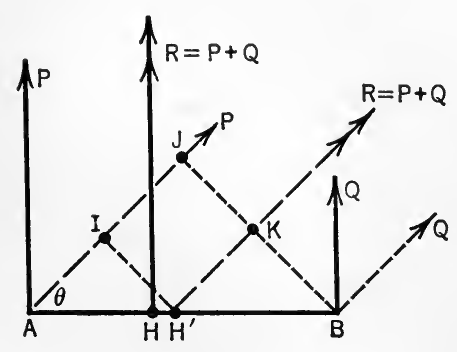

FIG. 210. system. This point is called the center of the system of forces.

In order to show that the resultant of such a force system doespass through a definite point in the body for all directions of the parallel force system, it is sufficient to prove the proposition for two such forces. In Fig. 210 are shown two parallel forces $P$ and $Q$ which act at points $A$ and $B$ of a rigid body (not shown). Let it be assumed first that the forces act in the direction indicated 
system passes through a definite point in the body regardless of the way in which the body may be turned. Hence the center of gravity of a body is a definite point in the body through which the weight of the body (earth-pull on the body) acts regardless of the position of the body.

If, then, $W$ denotes the weight of a body and $w$ or $d W$ denotes the weight of any particle of the body the coordinates of which are $x, y$, and $z$, the coordinates $\bar{x}, \bar{y}$, and $\bar{z}$ of the center of gravity of the body may be found from the equations:

$$
\begin{aligned}
& W \bar{x}=\Sigma w x=\int x d W, \\
& W \bar{y}=\Sigma w y=\int y d W, \\
& W \bar{z}=\Sigma w z=\int z d W,
\end{aligned}
$$

by the full lines. If the point in which the resultant of $P$ and $Q$ intersects the line $A B$ be denoted by $H$ it can be shown (Art. 24) that $A H=\frac{Q}{P+Q} \times A B$. Let it be assumed next that the action lines of $P$ and $Q$ are rotated through an angle $90^{\circ}-\theta$ as indicated by the dotted lines, and that the action line of the resultant of the two forces in this position intersects the line $A B$ in the point $H^{\prime}$. Let $B J$ and $H^{\prime} I$ be drawn perpendicular to the action lines of $P$ and $Q$ and let the point where the resultant of $P$ and $Q$ intersects $B J$ be denoted by $K$. It can be shown as before that,

Hence in the triangle $A I H^{\prime}$,

$$
J K=I H^{\prime}=\frac{Q}{P+Q} B J=\frac{Q}{P+Q} A B \sin \theta .
$$

$$
A H^{\prime}=\frac{I H^{\prime}}{\sin \theta}=\frac{Q}{P+Q} \cdot A B .
$$

Since $A H=A H^{\prime}$ it is evident that $H^{\prime}$ coincides with $H$, and hence the resultant of $P$ and $Q$ passes through a definite point $H$ on the line $A B$ regardless of the direction of the two parallel forces.

It is obvious that the proof may be extended to any number of forces, for, the resultant of $P$ and $Q$, just found, may be combined with a third force, $S$, which has a fixed point of application $C$ (say) in the body, and in the same manner it can be shown that the resultant of these two forces will pass through a fixed point on the line $H C$ and so on.

So far as the relations of the forces to the body are concerned it is obviously immaterial whether the body remains fixed and the forces be rotated relative to the body as discussed above, or whether the forces remain fixed in direction and the body be rotated. Hence the center of a system of parallel forces which have fixed directions and which have fixed points of application in a rigid body may also be defined as the point in the body through which the resultant of the forces will pass regardless of the way in which the body is turned. 
Mass-center.-As defined in Art. 82, the centroid of the mass of a body is the point in the body (or its extension) such that if the mass were conceived to be concentrated at this point, its moment with respect to any line or plane would be equal to the algebraic sum of the moments of the masses of the particles of the body with respect to the line or plane. In discussions (particularly in Kinetics) in which the relation between mass and weight are involved, the term mass-center (or center of mass) is frequently used instead of centroid of mass.

It will be shown in Part III (Kinetics) that the mass of a body is proportional to its weight $\left(M=\frac{W}{g}\right)$. It can be shown, therefore, that the center of mass of a body may, for all practical purposes, be regarded as the same point as the center of gravity of the body. Thus the $x$-coordinate of the mass-center is

$$
\bar{x}=\frac{\int x d M}{M}=\frac{\int x g d W}{g W} .
$$

Now, if $g$ be regarded as constant (and for all practical purposes it may be so regarded), the last term in the above equation becomes $\frac{\int x d W}{W}$, and this is the expression for the $x$-coordinate of the center of gravity of the body.

\section{ILLUSTRATIVE PROBLEM}

202. Gravel is piled on a floor so that the pressure on the beams which support the floor increases in the direction of the beams as shown in Fig. 211. The intensity of pressure varies from zero at the left end of the beam to a maximum of $p_{m}=800 \mathrm{lb}$. per foot at the right end. What is the weight of the gravel and how far from the left end of the beam does the resultant weight act?

Solution.-Let the intensity of pressure at any distance $x$ from the left end

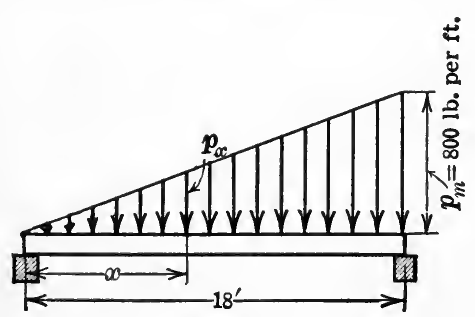

FIG. 211. of the beam be $p_{x} \mathrm{lb}$. per foot. This pressure may be assumed to be constant 
over a length $d x$ and hence the weight of gravel over this length is $d W=p_{x} d x$. But $p_{x}=\frac{x}{l} \cdot p_{m}$. Therefore the total pressure on the beam (weight of gravel) is,

$$
\begin{aligned}
W & =\int p_{x} d x \\
& =\int_{0}^{l} \frac{x}{l} p_{m} d x=\frac{p_{m}}{2} \times l=7200 \mathrm{lb} .
\end{aligned}
$$

Hence, the total pressure of the gravel (its weight) is the same in magnitude as it would be if the gravel were spread uniformly to a depth equal to one-half that of the maximum depth. The total weight, however, would then act at the center of the beam, whereas, according to the above distribution it must act at a distance $\ddot{x}$ such that,

Therefore,

$$
\begin{aligned}
W \bar{x} & =\int d W x \\
\left(\frac{p_{m}}{2} \times l\right) \bar{x} & =\int_{0}^{l} \frac{x}{l} p_{m} d x \cdot x=\frac{p_{m} l^{2}}{3} .
\end{aligned}
$$

$$
\bar{x}=\frac{2}{3} l .
$$

This result might have been obtained, without taking the above detailed steps, from the fact that the gravel may be conceived to be concentrated in a plane (of triangular shape), the position of the center of gravity of the gravel then being the same as the centroid of the triangular area (Prob. 173).

\section{PROBLEMS}

203. Two hornogeneous spheres $A$ and $B$, connected by a rod, are mounted on a vertical axis as shown in Fig.

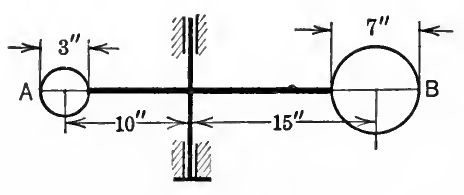

FIG. 212. 212. The weights of the spheres are $20 \mathrm{lb}$. and $60 \mathrm{lb}$., respectively, and the weight of the rod is $10 \mathrm{lb}$. How far from the axis is the center of gravity of the three bodies? Ans. 7.94 in.

204. Four bodies $A, B, C$, and $D$ are carried by a rotating shaft as shown in Fig. 213. The weights of the bodies are $20,15,10$, and $8 \mathrm{lb}$., respectively, and the distances of their centers of gravity from the axis of the shaft are $12,6,5$, and $10 \mathrm{in}$., respectively. Find the center of gravity of the four bodies when in the positions shown. 
205. The density at any point of a slender rod varies directly as the square of the distance of the point from the end of the rod. Show that the center of mass of the rod is $\frac{3}{4} l$ from the given end.
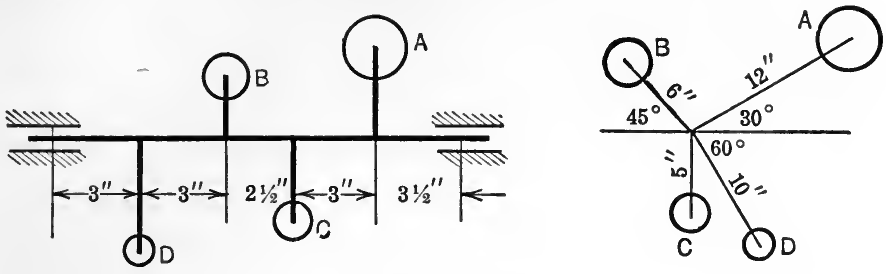

Fig. 213.

206. A beam is loaded with brick piled so as to produce the distribution of pressure as indicated in Fig. 214. Find the center of gravity of the hrick.

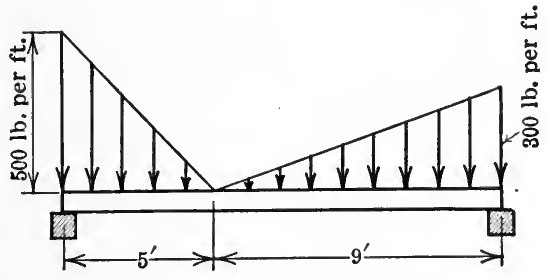

FIG. 214.

207. Three particles of equal mass are placed at the vertices of a triangle. Show that the mass-center of the particles coincides with the centroid of the area of the triangle.

86. Graphical Method of Determining Centroids of Areas.If the boundary of an area is an irregular curve which cannot be represented by an equation, the centroid cannot be determined by the method of integration. In such cases, however, the centroid may be determined by a graphical method which will now be described. Let $B B^{\prime} B^{\prime} B$ (Fig. 215) be an area, the centroid of which is to be found.

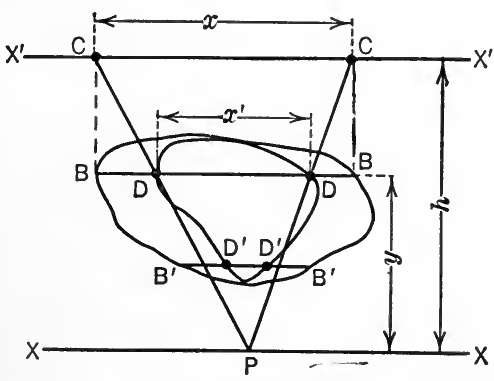

Fig. 215. Draw two parallel lines $X X$ and $X^{\prime} X^{\prime}$ any convenient distance 
$h$ apart. Draw a line $B B$ parallel to $X X$ and project the points $B, B$ on the line $X^{\prime} X^{\prime}$, thereby obtaining the points $C, C$. Connect the points $C, C$ to any point $P$ on $X X$. Let the points where the lines $C P$ intersect $B B$ be denoted by $D, D$. Repeat this process for other lines $B^{\prime} B^{\prime}, B^{\prime \prime} B^{\prime \prime}$, etc., and locate similarly, points $D^{\prime}, D^{\prime} ; D^{\prime \prime}, D^{\prime \prime}$; etc. Connect the points $D, D ; D^{\prime}, D^{\prime} ; D^{\prime \prime}, D^{\prime \prime}$; etc., by a smooth curve. Let the area enclosed by this curve be denoted by $A^{\prime}$. Then, if the given area be denoted by $A$, the distance of the centroid of the area from the line $X X$ is given by the following equation,

$$
\bar{y}=\frac{A^{\prime}}{A} h \text {. }
$$

Proof.-If $D D$ and $C C$ are denoted by $x^{\prime}$ and $x$ respectively, then from the similar triangles $P D D$ and $P C C$ the following relation is obtained,

$$
\frac{x}{h}=\frac{x^{\prime}}{y} \quad \text { or } \quad x y=h x^{\prime}
$$

The distance, $\bar{y}$, of the centroid of the given area from $X X$, then, may be found a follows:

$$
\bar{y}=\frac{\int y \cdot x d y}{A}=\frac{h \int^{\circ} x^{\prime} d y}{A}=h \frac{A^{\prime}}{A} .
$$

The areas $A$ and $A^{\prime}$ may be determined by the use of a planimeter or any other convenient method and the value of $\bar{y}$ is then determined from the above equation. In a similar manner, by taking any two lines $Y Y$ and $Y^{\prime} Y^{\prime}$ and proceeding as above, the distance, $\bar{x}$, of the centroid from $Y Y$ may be determined.

87. Determination of Center of Gravity by Experiment.When a body is irregular in shape, the center of gravity cannot be determined by the method of integration since the limits of the integral cannot be determined. The center of gravity of such a body, however, may be determined by the following experimental methods.

Method of Suspension.-If a body be suspended by a cord the center of gravity is on the (vertical) line coinciding with the axis of the cord. This statement follows from the fact that the two 
forces which hold the body in equilibrium (the upward tension in the cord and the downward earth-pull) must be equal, opposite, and collinear, and the earth-pull or weight of the body of course acts through the center of gravity of the body. Hence if a body be suspended from each of two points, the center of gravity will be located in each of two lines in the body and hence is at the point of intersection of the two lines.

Method of Balancing.-If a body be balanced on a knife-edge the center of gravity of the body will be in a vertical plane through the knife-edge. Hence if the body be balanced on a knife-edge in three different positions, three such planes in the body will be located, and the center of gravity of the body is the point in which the three planes intersect. A slightly different method involving the same principle, applicable particularly to large bodies of known weights, consists in resting one end of the body on a line of support (knife-edge) and the other end on platform scales. By weighing the reaction (pressure) on the scales and by measuring the distance between the knife-edge and the point of support on the platform scales the action line of the weight of the body may be found, since it lies in a plane which divides the distance between the knife-edge and the point of support into segments inversely proportional to the magnitudes of the reactions at the supports. By repeating the process for two other positions of the body, three such planes may be found as in the above method, and hence the center of gravity may be located. 


\section{CHAPTER VI}

\section{SECOND MOMENT. MOMENT OF INERTIA}

\section{§1. Moments of Inertia of Areas}

88. Moment of Inertia of an Area Defined.-In the analysis of many engineering problems as, for example, in determining the stresses in a beam or column, expressions of the form $\int x^{2} d A$ are frequently met, in which $d A$ represents an element of an area $A$, and $x$ is the distance of the element from some axis in, or perpendicular to, the plane of the area, the limits of integration being such that each element of the area is included in the integration. An expression of this form is called the second moment of the area or the moment of inertia of the area with respect to the given axis. The moment of inertia of an area with respect to an axis in, or perpendicular to, the plane of the area may, then, be defined as the sum of the products obtained by multiplying each element of the area by the square of its distance from the given axis.

The term moment of inertia is somewhat misleading, since inertia is a property of physical bodies, only, and hence an area does not possess inertia. For this reason the term, second moment of an area, is to be preferred, particularly when contrasting the expressions of the form here discussed with expressions which were defined as first moments of areas in Chapter V. It may be noted that each term $x^{2} d A$ in the summation which represents the moment of inertia of an area can be written in the form $x(x d A)$, and hence represents the mornent of the moment of an element of area, that is, the second moment of the element. The term moment of inertia, however, is very widely used, due to the fact that the expression is of the same form as an expression to be discussed later (Art. 100) which is defined as the moment of inertia of a body and which does have a physical significance.

The moment of inertia of an area with respect to an axis will be denoted by $I$ for an axis in the plane of the area and by $J$ for an axis perpendicular to the plane of the area. The particular 
axis (or direction of the axis) about which the moment of inertia is taken will be denoted by subscripts. Thus, the moments of inertia of the area $A$ (Fig. 216) with respect to the $x$ - and $y$-axes are expressed as follows:

and

$$
I_{x}=\int y^{2} d A,
$$

$$
I_{y}=\int x^{2} d A .
$$

Units and Sign.-Since the moment of inertia of an area is the sum of a number of terms each of which is the product of an area and the square of a distance, the moment of inertia of an area

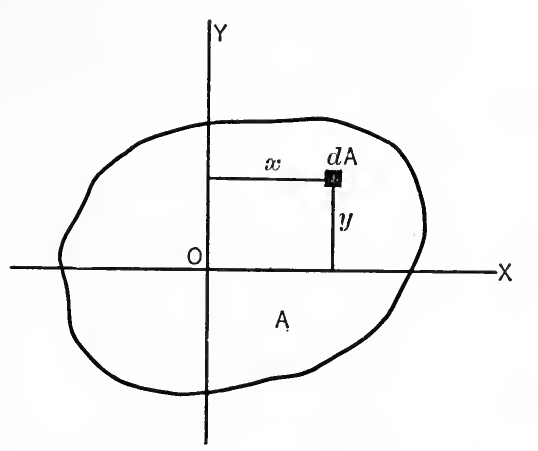

Fig. 216. is expressed as a length to the fourth power. If, then, the inch (or foot) be taken as the unit of length, the moment of inertia will be expressed as inches (or feet) to the fourth power (written in. ${ }^{4}$ or $\left.\mathrm{ft.}^{4}\right)$. Further, the sign of each of the products $x^{2} d A$ is always positive since $x^{2}$ is always positive, whether $x$ is positive or negative, and $d A$ is essentially positive. Therefore the moment of inertia, or second moment, of an area is always positive. In this respect it differs from the first moment of an area, which may be positive, negative, or zero, depending on the position of the moment axis.

89. Polar Moment of Inertia.-The moment of inertia of an

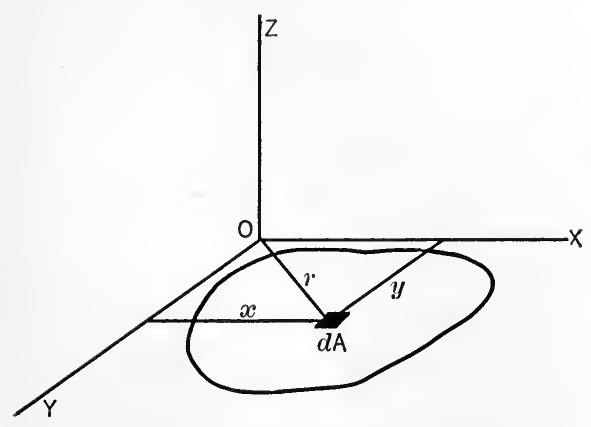

FIG. 217. area with respect to a line perpendicular to the plane of the area is called the polar moment of inertia of the area and, as noted in Art. 88, will be denoted by $J$. Thus the polar moment of inertia, with respect to the $z$-axis, of an area in the $x y$-plane (Fig. 217) may be expressed as follows: 
Therefore,

$$
\begin{aligned}
J_{z} & =\int r^{2} d A \\
& =\int\left(x^{2}+y^{2}\right) d A \\
& =\int x^{2} d A+\int y^{2} d A .
\end{aligned}
$$

$$
J_{z}=I_{y}+I_{x}
$$

Hence the following proposition may be stated:

The polar moment of inertia of an area with respect to any axis is equal to the sum of the moments of inertia of the area with respect to any two rectangular axes in the plane of the area which intersect on the given, polar axis.

90. Radius of Gyration.- Since the moment of inertia of an area $\left(\int x^{2} d A\right.$ or $\int r^{2} d A$, etc., $)$ is four dimensions of length, it may be expressed as the product of the total area, $A$, and the square of a distance, $k$. Thus,

$$
I_{x}=\int y^{2} d A=A k_{x}^{2},
$$

or

$$
J_{z}=\int r^{2} d A=A k_{z}^{2}
$$

The distance $k$ is called the radius of gyration of the area with respect to the given axis, the subscript denoting the axis with respect to which the moment of inertia is taken. The radius of gyration of an area with respect to a line, then, may be defined as a distance such that, if the area were conceived to be concentrated at this distance from the given line, the moment of inertia would be the same as the moment of inertia of the actual or distributed area with respect to the same line.

From the equation $I_{y}=\int x^{2} d A=A k_{y}{ }^{2}$, it will be noted that $k_{y}{ }^{2}$, the square of the radius of gyration with respect to the $y$-axis, is the mean of the squares of the distances, from the $y$-axis, of the equal elements of area into which the given area may 
be divided, and that it is not the square of the mean of these distances. The mean distance $(\bar{x})$ of the elements of area from the $y$-axis is the centroidal distance as discussed in the preceding chapter. Hence $A \bar{x}^{2}$ does not represent the moment of inertia of an area with respect to the $y$-axis.

91. Parallel Axis Theorem for Areas.-If the moment of inertia of an area with respect to a centroidal axis in the plane of the area is known, the moment of inertia with respect to any parallel axis in the plane may be determined, without integrating, by means of a proposition which may be established as follows: In Fig. 218 let $Y Y$ be any axis through the centroid, $C$, of an area and let $Y^{\prime} Y^{\prime}$ be any axis parallel to $Y Y$

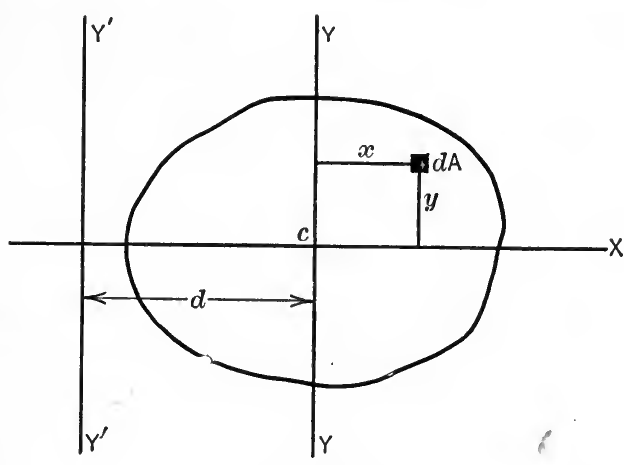

FIG. 218. and at a distance $d$ therefrom. Further, let the moment of inertia of the area with respect to the axis $Y Y$ be denoted by $\bar{I}$ and the moment of inertia with respect to $Y^{\prime} Y^{\prime}$ by $I$. By definition then,

$$
\begin{aligned}
I & =\int(x+d)^{2} d A \\
& =\int x^{2} d A+2 d \int x d A+d^{2} \int d A .
\end{aligned}
$$

Therefore,

$$
I=\bar{I}+A d^{2} \text { since } \int x d A=A \bar{x}=0 .
$$

Hence the following proposition may be stated:

The moment of inertia of an area with respect to any axis in the plane of the area is equal to the moment of inertia of the area with respect to a parallel centroidal axis plus the product of the area and the square of the distance between the two axes. This proposition is called the parallel axis theorem.

A corresponding relation exists between the radii of gyration of the area with respect to two parallel axes, one of which passes 
through the centroid of the area. For, by replacing $I$ by $A k^{2}$ and $\bar{I}$ by $A \bar{k}^{2}$ the above equation becomes,

$$
A k^{2}=A \bar{k}^{2}+A d^{2} \text {. }
$$

Whence,

$$
k^{2}=\bar{k}^{2}+d^{2},
$$

where $k$ denotes the radius of gyration of the area with respect to any axis in the plane of the area and $\bar{k}$ denotes the radius of gyration of the area with respect to a parallel centroidal axis.

Similarly, for polar moments of inertia and radii of gyration, it can be shown that,

and,

$$
J=\bar{J}+A d^{2}
$$

$$
k^{2}=\bar{k}^{2}+d^{2},
$$

where $\bar{J}$ and $\bar{k}$ denote the polar moment of inertia and radius of gyration, respectively, of the area with respect to the centroidal axis and $J$ and $k$ denote the polar moment of inertia and radius of gyration, respectively, of the area with respect to an axis parallel to the centroidal axis and at a distance $d$ therefrom.

92. Moments of Inertia by Integration.-In determining the moment of inertia of a plane area with respect to a line, it is possible to select the element of area in various ways and to express the area of the element in terms of either cartesian or polar coordinates. Further, the integral may be either a single or double integral, depending on the way in which the element of area is selected; the limits of integration are determined, of course, from the boundary curve of the area. In any case, however, the elementary area must be taken so that:

(1) All points in the element are equally distant from the axis with respect to which the moment of inertia is to be found, otherwise the distance $x$ in the expression $x^{2} d A$ would be indefinite. Or, so that,

(2) The moment of inertia of the element, with respect to the axis about which the moment of inertia of the whole area is to be found, is known, the moment of inertia of the area then being found by summing up the moments of inertia of the elements. Or, so that,

(3) The centroid of the element is known and also the moment of inertia of the element with respect to an axis which passes 
through the centroid of the element and is parallel to the given axis; the moment of inertia of the element may then be expressed by means of the parallel axis theorem.

The moments of inertia of some of the simple areas will now be found in the following illustrative problems.

\section{ILLUSTRATIVE PROBLEMS}

208. Determine the moment of inertia of a rectangle, in terms of its base $b$ and altitude $h$, with respect to $(a)$ a centroidal axis parallel to the base; (b) an axis coinciding with the base.

Solution.-(a) Centroidal Axis.-The element of area will be selected in accordance with rule (1) above, as indicated in Fig. 219. The moment of inertia of the rectangular area with respect to the centroidal axis, then, is,

$$
\begin{aligned}
\bar{I}_{x} & =\int y^{2} d A=\int_{-\frac{h}{2}}^{+\frac{h}{2}} y^{2} b d y \\
& =\frac{1}{12} b h^{3} .
\end{aligned}
$$

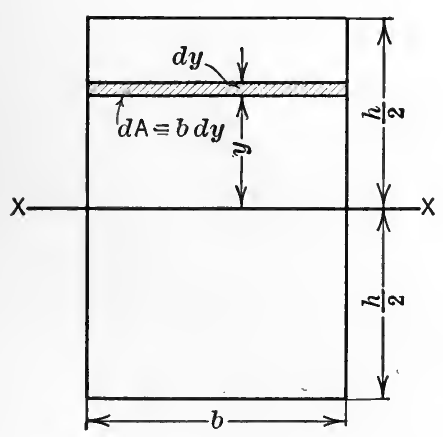

Frg. 219.

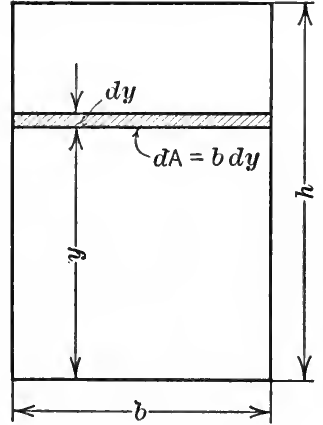

FIg. 220.

(b) Axis Coinciding with the Base. First Method.-The element of area will be selected as indicated in Fig. 220. The moment of inertia of the rectangle with respect to the base, then, is,

$$
\begin{aligned}
I_{b} & =\int y^{2} d A=\int_{0}^{h} y^{2} b d y \\
& =\frac{1}{3} b h^{3} .
\end{aligned}
$$


Second Method.- Since the moment of inertia of the rectangle with respect to a centroidal axis is $\frac{1}{12} b h^{3}$, the moment of inertia with respect to the base may be found from the parallel axis theorem (Art. 91). Thus,

$$
\begin{aligned}
I_{b} & =\bar{I}_{x}+A\left(\frac{h}{2}\right)^{2} \\
& =\frac{1}{12} b h^{3}+b h \times \frac{h^{2}}{4} \\
& =\frac{1}{3} b h^{3} .
\end{aligned}
$$

209. Determine the moment of inertia of a triangle, in terms of its base $b$ and altitude $h$, with respect to $(a)$ an axis coinciding with its base; (b) a centroidal axis parallel to the base.

Solution.-(a) Axis Coinciding with the Base.-The elementary area will be selected as shown in Fig. 221. The moment of inertia of the area of the triangle with respect to the base, then, is,

$$
I_{b}=\int y^{2} d A=\int y^{2} x d y \text {. }
$$

But, from similar triangles,

Hence,

$$
\frac{x}{b}=\frac{h-y}{h} .
$$

Therefore,

$$
x=\frac{b}{h}(h-y) \text {. }
$$

$$
\begin{aligned}
I_{b} & =\frac{b}{h} \int_{0}^{h} y^{2}(h-y) d y \\
& =\frac{1}{12} b h^{3} .
\end{aligned}
$$

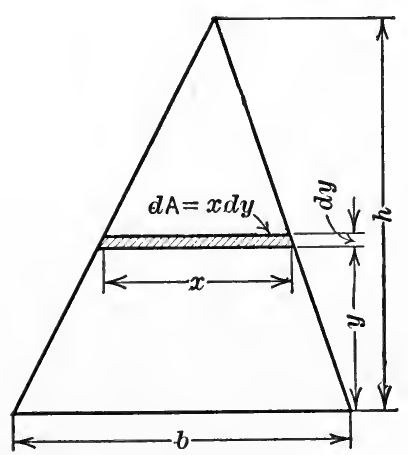

FIG. 221.

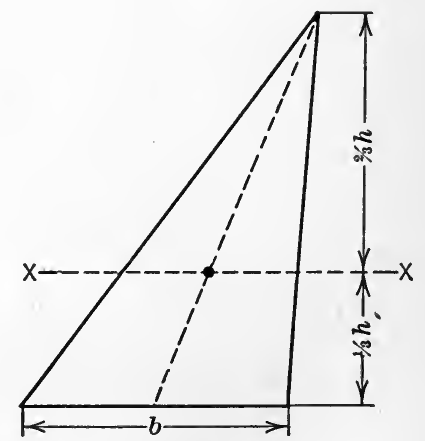

FIG. 222

(b) Centroidal Axis Parallel to the Base.-The centroidal axis parallel to the base (axis $X X$ ) is shown in Fig. 222 (see Prob. 173). Using the parallel 
axis thoerem, the moment of inertia of the triangular area with respect to the centroidal axis is,

$$
\begin{aligned}
\bar{I}_{x} & =I_{b}-A\left(\frac{1}{3} h\right)^{2} \\
& =\frac{1}{12} b h^{3}-\frac{1}{2} b h \times \frac{1}{9} h^{2} \\
& =\frac{1}{36} b h^{3} .
\end{aligned}
$$

210. Determine the moment of inertia of the area of a circle, in terms of its radius $r$, with respect to an axis coinciding with the diameter; $(a)$ using cartesian coordinates; (b) using polar coordinates.

Solution.-(a) Cartesian Coordinates.-The element of area will be selected as shown in Fig. 223. The moment of inertia of the circular area with respect to the diameter, then, is,

$$
\begin{aligned}
\bar{I}_{x} & =\int y^{2} d A=\int y^{2} 2 x d y \\
& =2 \int_{-r}^{+r} y^{2} \sqrt{r^{2}-y^{2}} d y \\
& =\frac{1}{4} \pi r^{4} .
\end{aligned}
$$

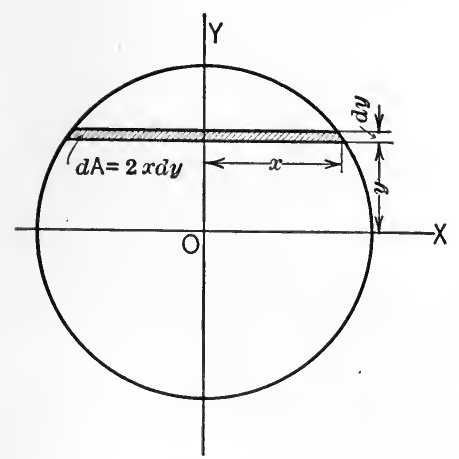

FIG. 223.

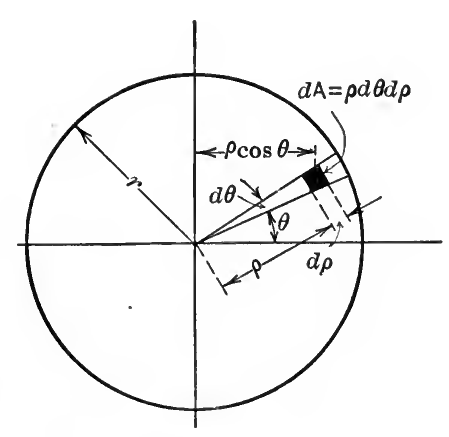

FIG. 224.

(b) Polar Coordinates.-The element of area will be selected as shown in Fig. 224. Hence,

$$
\begin{aligned}
\bar{I}_{x} & =\int y^{2} d A \\
& =\int_{0}^{r} \int_{0}^{2 \pi}(\rho \sin \theta)^{2} \rho d \rho d \theta
\end{aligned}
$$




$$
\begin{aligned}
& =\int_{0}^{r} \int_{0}^{2 \pi} \rho^{3} \sin ^{2} \theta d \rho d \theta \\
& =\frac{r^{4}}{4} \int_{0}^{2 \pi} \sin ^{2} \theta d \theta \\
& =\frac{r^{4}}{4} \times \pi=\frac{1}{4} \pi r^{4}
\end{aligned}
$$

211. Determine the polar moment of inertia of the area of a circle of radius $r$ with respect to a centroidal axis: $(a)$ by integration; $(b)$ by use of the theorem of Art. 89.

Solution.-(a) By Integration.-By selecting the element of area as indicated in Fig. 225, the polar moment of inertia of the circular area is,

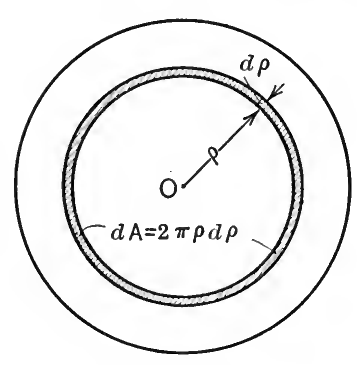

Fig. 225.

$$
\begin{aligned}
J_{z} & =\int \rho^{2} d A \\
& =\int_{0}^{r} \rho^{22} 2 \pi \rho d \rho \\
& =\frac{1}{2} \pi r^{4} .
\end{aligned}
$$

(b) By Use of Theorem of Art. 89.Since $I_{x}$ and $I_{y}$ are each equal to $\frac{1}{4} \pi r^{4}$ (Prob. 210), the polar moment of inertia of the area of the circle is,

$$
\begin{aligned}
J_{z} & =I_{x}+I_{y^{4}} \\
& =\frac{1}{4} \pi r^{4}+\frac{1}{4} \pi r^{4} \\
& =\frac{1}{2} \pi r^{4} .
\end{aligned}
$$

212. Find the moment of inertia, with respect to the $x$-axis, of the area bounded by the parabola $y^{2}=2 x$, and the line $x=8$ in.

Solution. First Method.-The element of area will be selected in accordance with rule (1) of Art. 92 as indicated in Fig. 226. The moment of inertia of the given area with respect to the $x$-axis, then, is,

$$
\begin{aligned}
1_{x} & =\int y^{2} d A \\
& =2 \int y^{2}(8-x) d y=2 \int_{0}^{4} y^{2}\left(8-\frac{y^{2}}{2}\right) d y \\
& =2\left[\frac{8 y^{3}}{3}-\frac{y^{5}}{10}\right]_{0}^{4} \\
& =136.5 \text { in. }{ }^{4}
\end{aligned}
$$


Second Method.-The elementary area will be selected in accordance with rule (2) of Art. 92 as indicated in Fig. 227. Since each elementary area is a

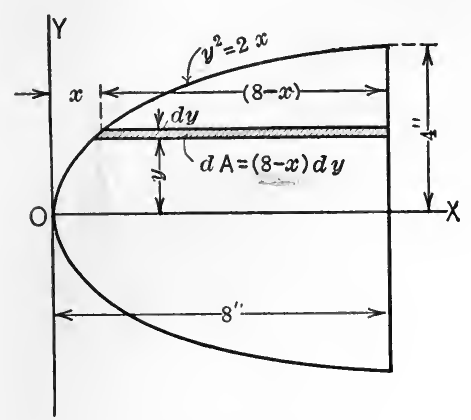

Fig. 226.

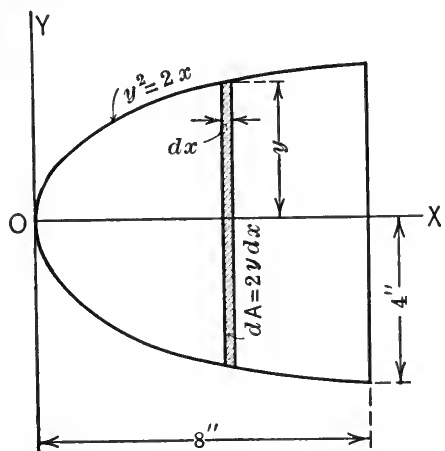

Fig. 227.

rectangle of width $d x$ and height $2 y$, the moment of inertia of the element with respect to the $x$-axis is $\frac{1}{12} d x(2 y)^{3}=\frac{2}{3} y^{3} d x$ (see Prob. 208). Hence, the moment of inertia of the given area is

$$
\begin{aligned}
I_{x} & =\frac{2}{3} \int_{0}^{8} y^{3} d x=\frac{2}{3} \int_{0}^{8}(2 x)^{3 / 2} d x \\
& =\frac{4 \sqrt{2}}{3} \int_{0}^{8} x^{3 / 2} d x \\
& =\frac{4 \sqrt{2}}{3}\left[\frac{2}{5} x^{5 / 2}\right]_{0}^{8} \\
& =136.5 \mathrm{in.}^{4}
\end{aligned}
$$

\section{PROBLEMS}

213. Determine the moment of inertia of the area of a circle, with respect to an axis tangent to the circle, in terms of, $r$, the radius of the circle.

214. Determine the polar moment of inertia of the area of a rectangle of base $b$ and altitude $d$ with respect to the centroidal axis.

$$
\text { Ans. } \bar{J}=\frac{1}{12} b d\left(b^{2}+d^{2}\right) .
$$

215. Find the moment of inertia and radius of gyration of a circular area, 16 in. in diameter, with respect to a diameter.

216. Determine the moments of inertia of the area of an ellipse, the principal axes of which are $2 a$ and $2 b$, with respect to the principal axes.

$$
\text { Ans. } I_{a}=\frac{1}{4} \pi a b^{3} . \quad I_{b}=\frac{1}{4} \pi b a^{3} .
$$


217. The base of a triangle is $8 \mathrm{in}$. and its altitude is $10 \mathrm{in.} \mathrm{Find} \mathrm{the}$ moment of inertia and radius of gyration of the area of the triangle with respect to the base.

218. Find the polar moment of inertia and radius of gyration of the area of a square, each side of which is 15 in., with respect to an axis through one corner of the square.

219. Find the polar moment of inertia, with respect to a centroidal axis, of the area of an isosceles triangle having a base $b$ and altitude $h$.

$$
\text { Ans. } J=\frac{1}{1} \frac{1}{2} b h\left(\frac{1}{4} b^{2}+\frac{1}{3} h^{2}\right) \text {. }
$$

93. Moments of Inertia of Composite Areas.-When a composite area can be divided into a number of simple areas, such as triangles, rectangles, and circles, for which the moments of inertia are known, the moment of inertia of the entire area may be obtained by taking the sum of the moments of inertia of the several areas. Likewise, the moment of inertia of the part of an area that remains after one or more simple areas are removed may be found by subtracting, from the moment of inertia of the total area, the sum of the moments of inertia of the several parts removed.

\section{ILLUSTRATIVE PROBLEMS}

220. Locate the horizontal centroidal axis, $X X$, of the T-section shown in Fig. 228 and find the moment of inertia of the area with respect to this centroidal axis.

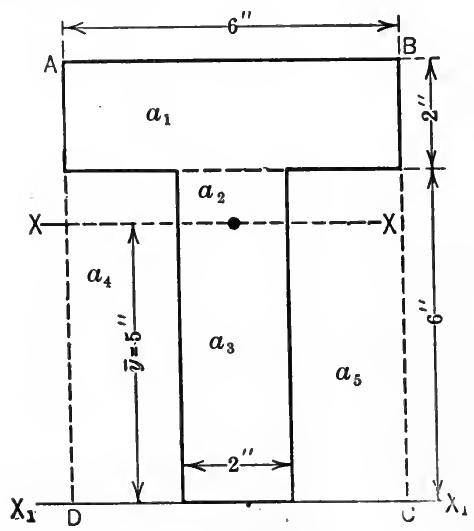

Fig. 228.

Solution. First Method.-The distance, $\bar{y}$, of the centroid of the area from the axis $X_{1} X_{1}$ may be found from the equation,

Thus,

$$
A \bar{y}=\Sigma\left(a y_{0}\right) \text {. }
$$

$$
\begin{aligned}
\bar{y} & =\frac{12 \times 7+12 \times 3}{12+12} \\
& =5 \mathrm{in} .
\end{aligned}
$$

The moment of inertia with respect to the $X X$ axis is the sum of the moments of inertia of the three parts $a_{1}, a_{2}$, and $a_{3}$, with respect to that axis. Thus,

$$
\begin{aligned}
I_{x} & =\frac{1}{12} \times 6 \times(2)^{3}+12 \times(2)^{2}+\frac{1}{3} \times 2 \times(1)^{3}+\frac{1}{3} \times 2 \times(5)^{3} \\
& =4+48+.67+83.33 \\
& =136 \text { in. }{ }^{4}
\end{aligned}
$$


Second Method.-The moment of inertia of the T-section may also be determined as follows: First find the moment of inertia of the T-section with respect to the axis $X_{1} X_{1}$ by subtracting the moments of inertia of the parts $a_{4}$ and $a_{5}$ from the moment of inertia of the rectangular area $A B C D$ and then find $\vec{I}_{x}$ for the T-section by use of the parallel axis theorem. Thus, the moment of inertia, $I_{x}$, of the T-section with respect to the $X_{1} X_{1}$ axis is,

and

$$
I_{x}=\frac{1}{3} \times 6 \times(8)^{3}-2 \times \frac{1}{3} \times 2 \times(6)^{3}=736 \text { in. }{ }^{4},
$$

$$
\bar{I}_{x}=I_{x}-A d^{2}=736-24 \times(5)^{2}=136 \text { in. }{ }^{4}
$$

221. Find the moment of inertia of the channel section shown in Fig. 229, with respect to the line $X X$. Find also the moment of inertia with respect to the parallel centroidal axis.

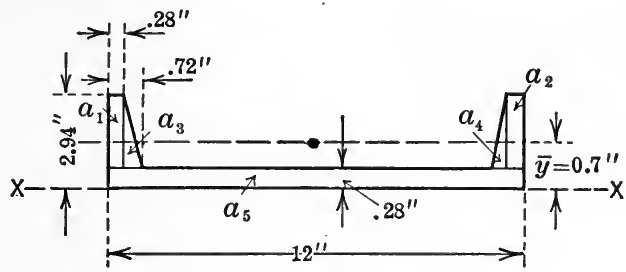

Fig. 229.

Solution.-The area may be divided into triangles and rectangles as shown in the figure. The values used in the solution may be put in tabular form as shown below, where $a$ denotes the area of any part, $y_{0}$ the distance of the centroid of the part from the line $X X, I_{0}$ the moment of inertia of the part with respect to its own centroidal axis parallel to $X X$, and $I^{\prime}{ }_{x}$ the moment of inertia of the part with respect to the axis $X X$.

\begin{tabular}{c|c|c|c|c|c|c}
\hline Part. & $a$. & $y_{0}$ & $a y_{0}$ & $I_{0 .}$ & $a y_{0}{ }^{2}$. & $I^{\prime}{ }_{x}=I_{0}+a y_{0}{ }^{2}$ \\
\hline$a_{1}$ & .745 & 1.61 & 1.20 & .44 & 1.93 & 2.37 \\
$a_{2}$ & .745 & 1.61 & 1.20 & .44 & 1.93 & 2.37 \\
$a_{\mathrm{s}}$ & .585 & 1.17 & .68 & .23 & .80 & 1.03 \\
$a_{4}$ & .585 & 1.17 & .68 & .23 & .80 & 1.03 \\
$a_{5}$ & 3.360 & .14 & .47 & .02 & .07 & .09 \\
\cline { 2 - 5 } & 6.02 in. $^{2}$ & & 4.23 in. & & & 6.89 in.4 \\
\hline
\end{tabular}

Thus the moment of inertia $I_{x}$ of the area with respect to the $X X$ axis is,

$$
I_{x}=\Sigma I^{\prime}{ }_{x}=6.89 \text { in. }{ }^{4}
$$

Further, the total area is $A=\Sigma a=6.02$ in. $^{2}$ and the moment of the area with respect to the $X X$ axis is $\Sigma\left(a y_{0}\right)=4.23$ in. $^{3}$ Hence, the distance, $\bar{y}$, of the centroid of the area from the $X X$ axis is,

$$
\bar{y}=\frac{\Sigma\left(a y_{0}\right)}{A}=\frac{4.23}{6.02}=0.70 \text { in }
$$


Therefore, the moment of inertia with respect to a line through the centroid and parallel to $X X$ is given by the equation,

$$
\begin{aligned}
\bar{I}_{x} & =I_{x}-A d^{2} \\
& =6.89-6.02 \times(.70)^{2} \\
& =6.89-2.95 \\
& =3.94 \mathrm{in} .^{4}
\end{aligned}
$$

\section{PROBLEMS}

222. A wooden column is built up of four 2 -in. by 8 -in. planks as shown in Fig. 230. Find the moment of inertia of the cross-section with respect to the centroidal axis $X X$. Ans. $I_{x}=981$ in. ${ }^{4}$

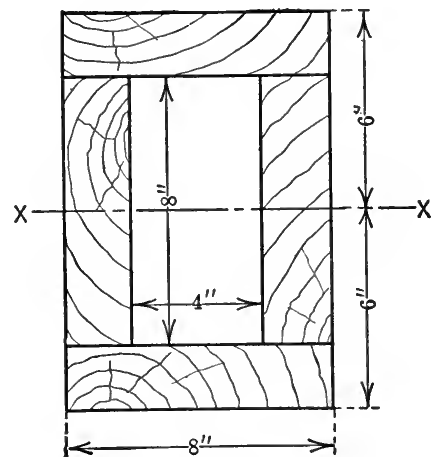

Fig. 230 .

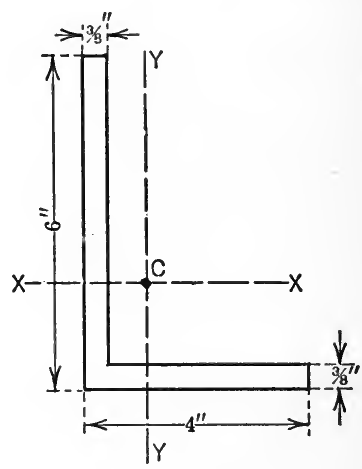

FIG. 231.

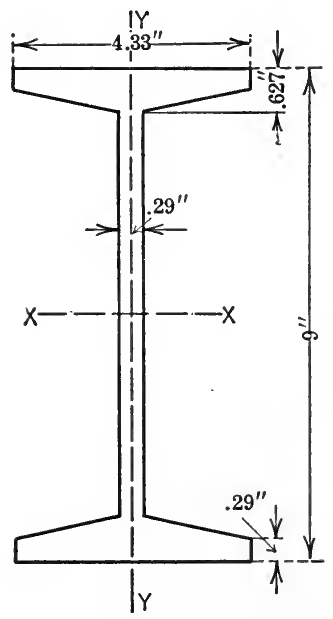

Fig. 232.

223. Find the moment of inertia of the angle section (Fig. 231) with respect to each of the centroidal axes parallel to the two legs of the angle.

224. In Fig. 232 is shown the crnss-section of a standard 9-in. 21-lb. I-beam (fillets are neglected). Find the moments of inertia of the section with respect to the centroidal axes, $X X$ and $Y Y$. Ans. $I_{x}=84.9$ in..$^{4} ; I_{y}=5.16$ in. ${ }^{4}$

225. In Fig. 233 is shown the cross-section of a standard $3 \frac{1}{4}$-in. by 5 -in. standard Z-bar (fillets are neglected). Find the moments of inertia of the section with respect to the centroidal axes $X X$ and $Y Y$.

226. In Fig. 234 is shown a built-up section made of a $\frac{1}{2}$-in. by 20 -in. plate and 
four angles. Find the moment of inertia of the section with respect to the $X X$ axis.

Ans. $I_{x}=1850$ in. $^{4}$

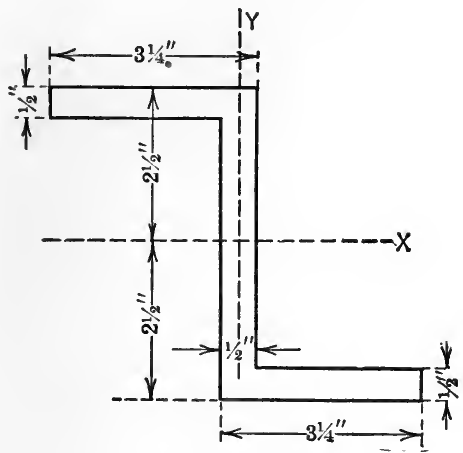

FIG. 233.

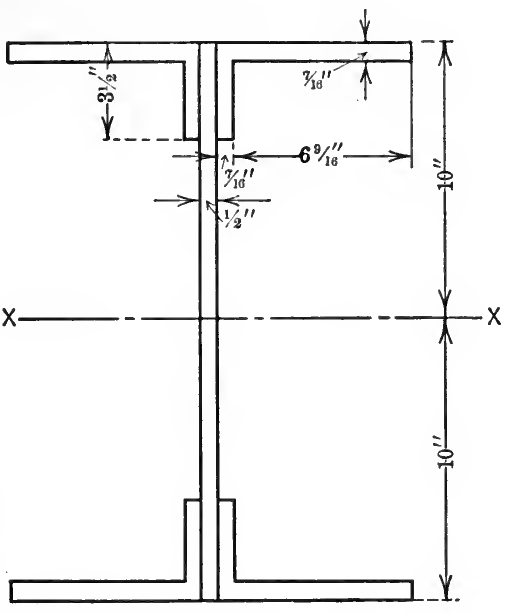

Fig. 234 .

227. In Fig. 235 is represented a 16 -in. circular plate in which there are drilled five 2 -in. holes and one 1 -in. hole as shown. Find the moment of inertia of the area of the holes with respect to the $X X$ axis and also with respect to the $Y Y$ axis.

Ans. $I_{x}=252$ in. ${ }^{4}$

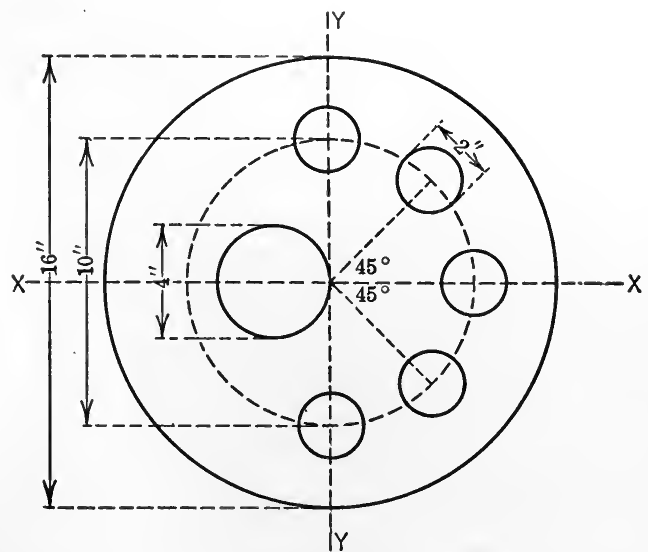

Fig. 235. 
228. Find the moment of inertia of a trapezoid (Fig. 236), in terms of its bases and altitude, with respect to $(a)$ an axis coinciding with its larger base; (b) a centroidal axis parallel to the bases.

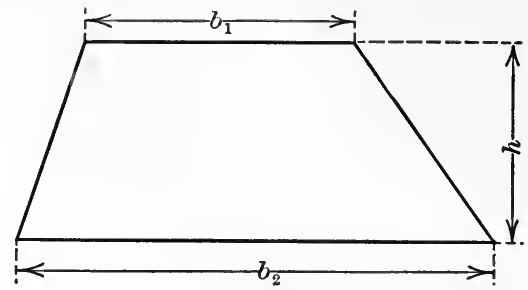

FIG. 236.

94. Product of Inertia Defined.-If the moments of inertia of an area with respect to any two rectangular axes are known, the moment of inertia with respect to any other axis through the point of intersection of the two axes may frequently be obtained most easily in terms of the moments of inertia of the area with respect to the two rectangular axes and an expression of the form $\int x y d A$ in which $d A$ is an element of the given area and $x$ and $y$ are the coordinates of the element with respect to the two rectangular axes. This expression is called the product of inertia of the area with respect to the axes and is denoted by $P_{x y}$. Hence, the product of inertia of an area with respect to any two rectangular axes may be defined as the sum of the products obtained by multiplying each element of area by the product of the two coordinates of the element with respect to the two rectangular axes. That is,

$$
P_{x y}=\int x y d A \text {. }
$$

The product of inertia of an area, like the moment of inertia of an area, is of four dimensions in length and is therefore expressed as inches (or feet, etc.) to the fourth power (in. ${ }^{4}, \mathrm{ft}^{4}{ }^{4}$, etc.). Unlike moment of inertia, however, the product of inertia of an area is not always positive, but may be negative or may be zero, since either $x$ or $y$ may be negative and hence their product may be negative or equal to zero.

95. Axes of Symmetry.-The product of inertia of an area with respect to two rectangular axes is zero if either one of the axes is an axis of symmetry. This follows from the fact that for each 
product $x y d A$ for an element on one side of the axis of symmetry there is an equal product of opposite sign for the corresponding element on the other side of the axis and hence, the expression $\int x y d A$ equals zero.

\section{ILLUSTRATIVE PROBLEM}

229. Find the product of inertia of the area of the triangle, shown in Fig. 237 , with respect to the $x$ - and $y$-axes.

Solution.-The relation between $x$ and $y$ is given by the equation $y=\frac{2}{3} x$. Hence,

$$
\begin{aligned}
P_{x y} & =\int x y d A=\int_{0}^{6} \int_{0}^{3 / 3 x} x y d x d y \\
& =\frac{2}{9} \int_{0}^{6} x^{3} d x=72 \text { in. } .^{4}
\end{aligned}
$$

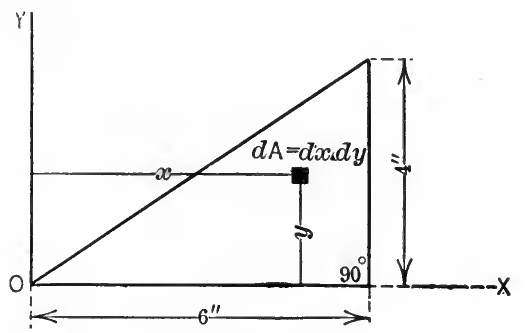

Fig. 237.

\section{PROBLEMS}

230. Find the product of inertia of the area of a rectangle, having a base $b$ and an altitude $h$, with respect to two adjacent sides.

Ans. $P_{x y}= \pm \frac{1}{4} b^{2} h^{2}$.

231. Find the product of inertia of the quadrant of a circular area, shown in Fig. 238, with respect to the $x$ - and $y$-axes, in terms of its radius $r$.

Ans. $P_{x y}=\frac{1}{8} r^{4}$.

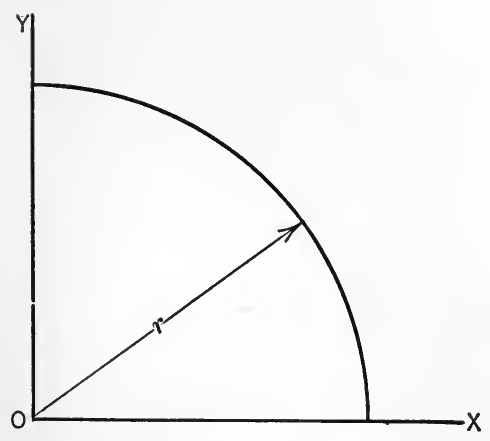

FIG. 238.

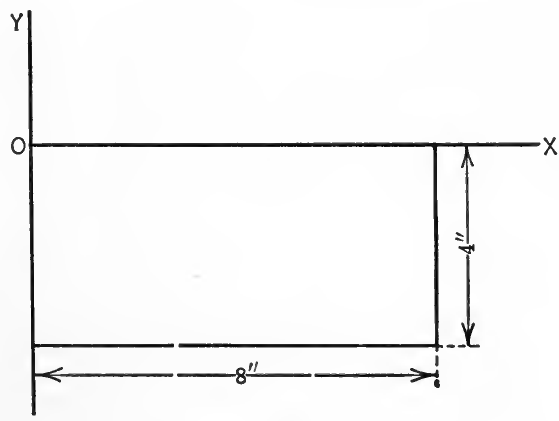

FIG. 239.

232. Find the product of inertia of the rectangular area with respect to the $x$ - and $y$-axes as shown in Fig. 239. 


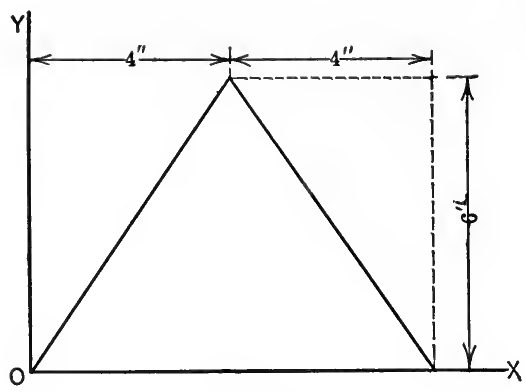

FIG. 240.

233. Find the product of inertia of the triangular area with respect to the $x$-and $y$-axes as shown in Fig. 240.

$$
\text { Ans. } P_{x y}=192 \text { in. }{ }^{4}
$$

234. Find the product of inertia, with respect to the coordinate axes, of the area bounded by the parabola $y^{2}=a x$, the line $x=b$, and the $x$-axis.

$$
\text { Ans. } P_{x y}=\frac{1}{6} a b^{3} \text {. }
$$

96. Parallel Axis Theorem for Products of Inertia.-When the product of inertia of an area is known for any pair of rectangular axes passing through the centroid of the area, the product of inertia of the area with respect to any parallel set of axes may be determined without integrating. Thus, in Fig. 241, $X^{\prime} X^{\prime}$ and $Y^{\prime} Y^{\prime}$ are axes which pass through the centroid, $C$, of the area; $X X$ and $Y Y$ are parallel axes passing through the point $O$. The coordinates of $C$ with respect to $X X$ and $Y Y$ are denoted by $\bar{x}$ and $\bar{y}$. If the product

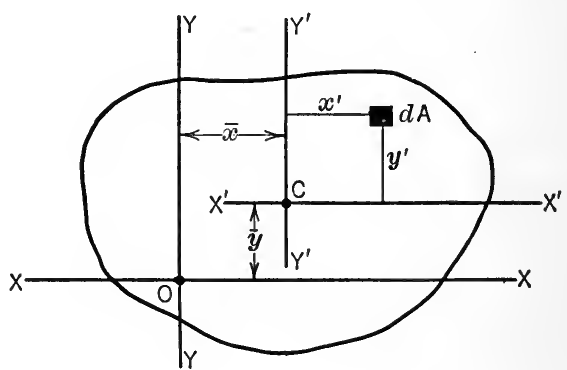

Fig. 241. of inertia of the area with respect to $X X$ and $Y Y$ be denoted by $P_{x y}$ and the product of inertia with respect to $X^{\prime} X^{\prime}$ and $Y^{\prime} Y^{\prime}$ be denoted by $\bar{P}_{x y}$, then, by definition,

$$
\begin{aligned}
P_{x y} & =\int\left(x^{\prime}+\bar{x}\right)\left(y^{\prime}+\bar{y}\right) d A \\
& =\int x^{\prime} y^{\prime} d A+\bar{x} \bar{y} \int d A+\bar{y} \int x^{\prime} d A+\bar{x} \int y^{\prime} d A .
\end{aligned}
$$

Since each of the last two integrals is the first moment of the area with respect to a centroidal axis, each integral is equal to zero. The equation then becomes,

$$
P_{x y}=\bar{P}_{x y}+A \bar{x} \bar{y} .
$$


That is, the product of inertia of any area with respect to any pair of rectangular axes in its plane is equal to the product of inertia of the area with respect to a pair of parallel centroidal axes plus the product of the area and the coordinates of the centroid of the area with respect to the given pair of axes.

\section{ILLUSTRATIVE PROBLEM}

235. Find the product of inertia of the area shown in Fig. 242 with respect to the $x$ - and $y$-axes.

Solution. - The area may be divided into rectangles $a_{1}$ and $a_{2}$ as shown. Using the formula $P_{x y}=\bar{P}_{x y}+A \bar{x} \bar{y}$ we have, for the area $a_{1}$,

$$
P_{x y}=0+12 \times 1 \times 3=36 \text { in. }{ }^{4},
$$

and for area $a_{2}$,

$$
P_{x y}=0+4 \times 3 \times 1=12 \text { in. } 4
$$

Hence, for the entire area,

$$
P_{x y}=36+12=48 \text { in. } .^{4}
$$

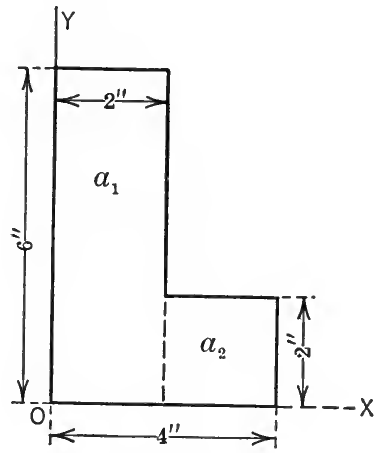

Fig. 242.

\section{PROBLEMS}

236. Find the product of inertia of the area shown in Fig. 243 with respect to the $x$ - and $y$-axes.

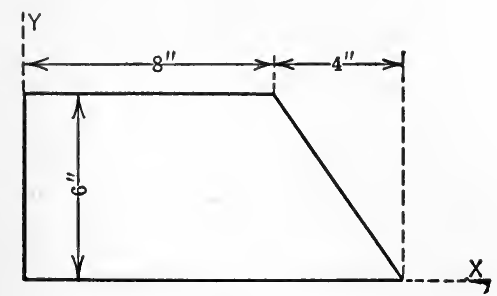

Fig. 243.

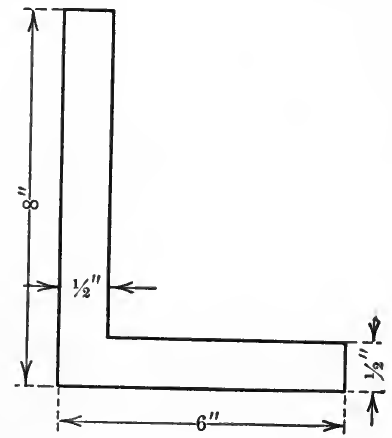

FIG. 244.

237. Locate the centroid of the angle section shown in Fig. 244 and determine the product of inertia with respect to centroidal axes parallel to the two legs of the angle.

Ans. $P=-18.3$ in. ${ }^{4}$ 
97. Moments of Inertia with Respect to Inclined Axes.-The moments of inertia of an area with respect to different lines (in the plane of the area) which pass through a given point are in general unequal. The determination of the moment of inertia of an area by the method of

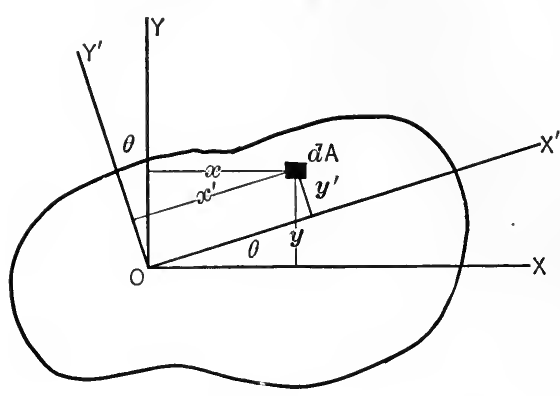

FIG. 245 integration is comparatively simple for certain lines but rather difficult for other lines. Equations (1) and (2) below make it possible to determine the moment of inertia with respect to any line passing through a given point in the area in terms of the moments of inertia and product of inertia of the area with respect to two rectangular axes passing through the point. The equations may be derived as follows: The moment of inertia of the area shown in Fig. 245 with respect to $O X^{\prime}$ is expressed by the equation,

$$
\begin{aligned}
I_{x^{\prime}} & =\int y^{\prime 2} d A=\int(y \cos \theta-x \sin \theta)^{2} d A \\
& =\cos ^{2} \theta \int y^{2} d A+\sin ^{2} \theta \int x^{2} d A-2 \sin \theta \cos \theta \int x y d A \\
& =I_{x} \cos ^{2} \theta+I_{y} \sin ^{2} \theta-2 P_{x y} \sin \theta \cos \theta . \quad \text {. . . . . . }
\end{aligned}
$$

In a similar manner the following equation may be derived,

$$
I_{y^{\prime}}=I_{x} \sin ^{2} \theta+I_{y} \cos ^{2} \theta+2 P_{x y} \sin \theta \cos \theta .
$$

Thus, from these equations, the moment of inertia of an area with respect to an axis inclined at an angle $\theta$ with one of a given pair of rectangular axes may be found, without integrating, if the moments of inertia and the product of inertia of the area with respect to the given rectangular axes are known.

By adding equations (1) and (2) the following important equation is obtained,

$$
I_{x^{\prime}}+\dot{I}_{y^{\prime}}=I_{x}+I_{y}
$$


That is, the sum of the moments of inertia of an area with respect to all pairs of rectangular axes, having a common point of intersection, is constant. It should be noted also that each side of equation (3) is equal to the polar moment of inertia of the area with respect to an axis passing through the point (Art. 89).

Axes for Which the Product of Inertia is Zero.-It may be shown that through any point in an area there is one set of rectangular axes for which the product of inertia is zero. Thus from Fig. 245.

$$
\begin{aligned}
P_{x^{\prime} y^{\prime}} & =\int x^{\prime} y^{\prime} d A=\int(x \cos \theta+y \sin \theta)(y \cos \theta-x \sin \theta) d A \\
& =\left(\cos ^{2} \theta-\sin ^{2} \theta\right) \int x y d A+\cos \theta \sin \theta \int\left(y^{2}-x^{2}\right) d A \\
& =P_{x y} \cos 2 \theta+\frac{1}{2}\left(I_{x}-I_{y}\right) \sin 2 \theta
\end{aligned}
$$

Hence $P_{x^{\prime} y^{\prime}}=0$ when $\tan 2 \theta=\frac{2 P_{x y}}{I_{y}-\frac{I_{x}}{I_{x}}}$.

98. Principal Axes.-In the analysis of many engineering problems the moment of inertia of an area must be found with respect to a certain axis called a principal axis. A principal axis of inertia of an area, for a given point in the area, is an axis about which the moment of inertia of the area is either greater or less than for any other axis passing through the given point. It can be proved that through any point in an area two rectangular axes can be drawn for which the moments of inertia of the area are greater and less, respectively, than for any other axes through the point. There are then two principal axes of inertia of an area for any point in the area. Further, it can be shown that axes for which the product of inertia is zero are principal axes. And, since the product of inertia of an area is zero for symmetrical axes, it follows that axes of symmetry are principal axes. The above statements may be demonstrated as follows:

The direction of the principal axes may be determined from equation (1) of Art. 97 which may be written in the form,

$$
\begin{aligned}
I_{x^{\prime}}= & I_{x} \frac{1+\cos 2 \theta}{2}+I_{y} \frac{1-\cos 2 \theta}{2}-P_{x y} \sin 2 \theta \\
& =\frac{I_{x}+I_{y}}{2}+\frac{I_{x}-I_{y}}{2} \cos 2 \theta-P_{x y} \sin 2 \theta .
\end{aligned}
$$


The value of $\theta$ which will make $I_{x^{\prime}}$ have a maximum or a minimum value may be found by equating the first derivative of $I_{x^{\prime}}$ with respect to $\theta$ to zero. Thus,

$$
\frac{d I_{x^{\prime}}}{d \theta}=\sin 2 \theta\left(I_{y}-I_{x}\right)-2 P_{x y} \cos 2 \theta=0
$$

whence,

$$
\tan 2 \theta=\frac{2 P_{x y}}{I_{y}-I_{x}}
$$

From this equation two values of $2 \theta$ are obtained which differ by $180^{\circ}$, the corresponding values of $\theta$ differing by $90^{\circ}$. For one value of $\theta$ the value of $I_{x^{\prime}}$ will be a maximum and for the other, a minimum. If $P_{x y}=0$ (which will always be the case if either the $x$ - or $y$-axis is an axis of symmetry) the value of $\theta$ is zero, and hence axes of symmetry are principal axes.

\section{ILLUSTRATIVE PROBLEM}

238. Find the moments of inertia of the angle section, shown in Fig. 246, with respect to principal axes passing through the centroid.

Solution.-The steps in the solution will be made as follows:

(1) The centroid of the area will be located, that, is $\bar{x}$ and $\bar{y}$ will be found.

(2) The moments of inertia and the product of inertia $\left(\bar{I}_{x}, \bar{I}_{y}\right.$, and $\left.\bar{P}_{x y}\right)$ with respect to the centroidal $x$ - and $y$-axes will then be found by the methods discussed in Arts. 93 and 96.

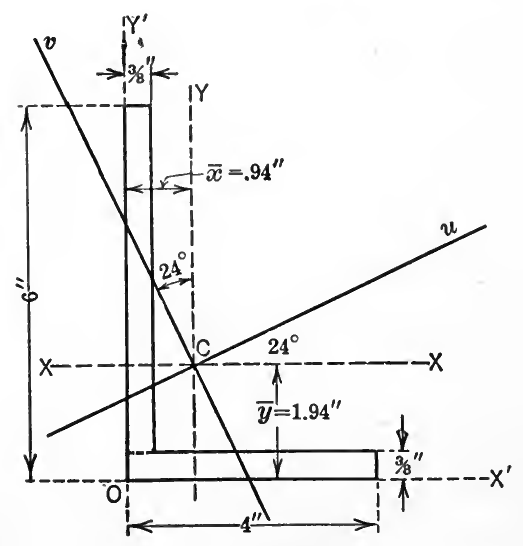

FIG. 246.

(3) The directions of the principal axes will then be found by use of the equations of Art. 98.

(4) The moment of inertia with respect to each of the principal axes, $u$ and $v$, will then be found by means of equations (1) and (2) of Art. 97.

$$
\begin{aligned}
\bar{x} & =\frac{4 \times \frac{3}{8} \times 2+5 \frac{5}{8} \times \frac{3}{8} \times \frac{3}{16}}{4 \times \frac{3}{8}+5 \frac{5}{8} \times \frac{3}{8}} \\
& =\frac{3.396}{3.61}=0.94 \mathrm{in} . \\
\bar{y} & =\frac{4 \times \frac{3}{8} \times \frac{3}{16}+5 \frac{3}{8} \times \frac{3}{8} \times 3 \frac{3}{16}}{4 \times \frac{3}{8}+5 \frac{5}{8} \times \frac{3}{8}} \\
& =\frac{7.01}{3.61}=1.94 \mathrm{in} .
\end{aligned}
$$




$$
\begin{aligned}
\bar{I}_{x}= & \frac{1}{12} \times \frac{3}{8} \times\left(5 \frac{5}{8}\right)^{3}+5 \frac{5}{8} \times \frac{3}{8} \times\left(1 . \frac{1}{4}\right)^{2} \\
& +\frac{1}{12} \times 4 \times\left(\frac{3}{8}\right)^{3}+4 \times \frac{3}{8} \times\left(1 \frac{3}{4}\right)^{2} \\
= & 5.57+3.30+0.02+4.59 \\
= & 13.48 \mathrm{in} .^{4} \\
\bar{I}_{y}= & \frac{1}{12} \times 5 \frac{5}{8} \times\left(\frac{3}{8}\right)^{3}+5 \frac{5}{8} \times \frac{3}{8} \times\left(\frac{3}{4}\right)^{2} \\
& +\frac{1}{12} \times \frac{3}{8} \times 4^{3}+4 \times \frac{3}{8} \times\left(1 \frac{1}{16}\right)^{2} \\
= & 0.02+1.19+2.00+1.69 \\
= & 4.90 \mathrm{in.} .^{4}
\end{aligned}
$$

To determine $\bar{P}_{x y}$, the value of $P_{x^{\prime} y^{\prime}}$ will first be found and then the value of $\bar{P}_{x y}$ may be found by means of the formula in Art. 96 . Thus,

$$
\begin{aligned}
P_{x^{\prime} y^{\prime}} & =\int_{3 / 8}^{6} \int_{0}^{3 / 8} x y d x d y+\int_{0}^{3 / 8} \int_{0}^{4} x y d x d y \\
& =1.26+0.56 \\
& =1.82 \mathrm{in.} .
\end{aligned}
$$

Using the formula of Art. 96 we have

$$
\begin{aligned}
\bar{P}_{x y} & =P_{x^{\prime} v^{\prime}}-A \bar{x} \bar{y} \\
& =1.82-3.61 \times(-0.94) \times(-1.94) \\
& =-4.76 \text { in. }{ }^{4}
\end{aligned}
$$

The directions of the principal axes are found from the formula of Art. 98 . Thus,

$$
\begin{aligned}
\tan 2 \theta & =\frac{2 \times(-4.76)}{4.90-13.48}=1.11, \\
\therefore 2 \theta & =48^{\circ} \text { or } 228^{\circ}, \\
\theta & =24^{\circ} \text { or } 114^{\circ} .
\end{aligned}
$$

From the formula of Art. 97, the moment of inertia with respect to the axis making an angle of $24^{\circ}$ with $C X$ (denoted by $u$ ) is,

$$
\begin{aligned}
I_{u} & =13.48 \cos ^{2} 24^{\circ}+4.90 \sin ^{2} 24^{\circ}-2(-4.76) \sin 24^{\circ} \cos 24^{\circ} \\
& =11.23+0.81+3.53 \\
& =15.59 \text { in. }^{4}
\end{aligned}
$$

Using $\theta=114^{\circ}$ and denoting the corresponding axis by $v$, we have,

$$
I_{0}=13.48 \cos ^{2} 114^{\circ}+4.90 \sin ^{2} 114^{\circ}-2(-4.76) \sin 114^{\circ} \cos 114^{\circ}
$$


$=2.23+4.08-3.53$

$=2.78$ in $^{4}$

Hence, the principal moments of inertia are 15.59 in. ${ }^{4}$ and 2.78 in. ${ }^{4}$ The corresponding radii of gyration are $2.08 \mathrm{in}$. and $0.88 \mathrm{in}$.

\section{PROBLEMS}

239. In the Z-section shown in Fig. $247, \bar{I}_{x}=25.32$ in. ${ }^{4}$ and $\bar{I}_{y}=9.11$ in. ${ }^{4}$ Find the principal moments of inertia.

Ans. 31.2 in. ${ }^{4} ; 3.20$ in. ${ }^{4}$

240. Show that the moment of inertia of the area of a square is constant for all axes in the plane of the area which pass through the center.

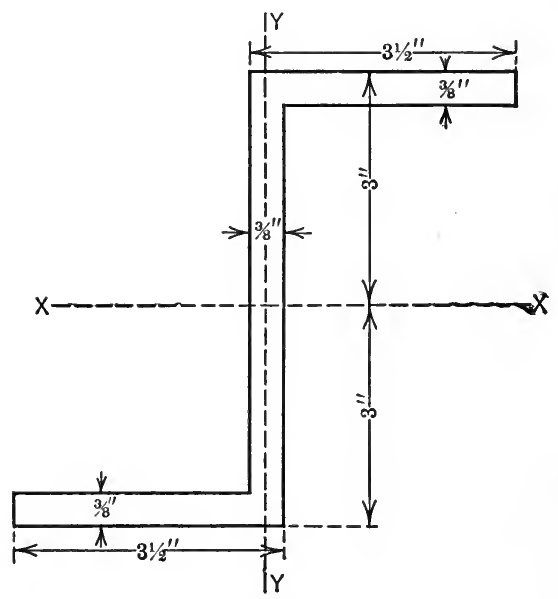

Frg. 247.

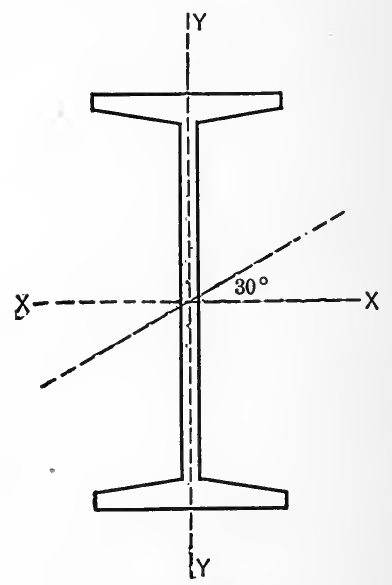

FIG. 248.

241. Fig. 248 represents the cross-section of a standard 10-in. 25-lb. I-beam. $\bar{I}_{x}=122.1$ in. ${ }^{4}, \bar{I}_{y}=6.89$ in. ${ }^{4}$, and $A=7.37$ in. ${ }^{2}$ Find the moment of inertia and radius of gyration of the section with respect to a line making an angle of $30^{\circ}$ with the $x$-axis. ${ }^{4}$

Ans. $I=93.3$ in. ${ }^{4}$

\section{Moments of Inertia of Areas by Graphical and Approximate} Methods.-It is sometimes necessary to determine the moment of inertia of an area having a bounding curve which cannot be defined by a mathematical equation. The moment of inertia of such an area may be determined by a graphical method or by an approximate method. 
Graphical Method.-Let the area within the outer curve in Fig. 249 be denoted by $A$ and let the line with respect to which the moment of inertia of $A$ is to be determined be denoted by $X X$. Draw any line, such as $X^{\prime} X^{\prime}$, parallel to and at the distance $h$ from $X X$. Draw any chord, such as $B B$, and project the points $B, B$ on $X^{\prime} X^{\prime}$ thereby obtaining the points $C, C$. Connect the points $C, C$ to any point, $P$, on $X X$ by lines intersecting $B B$ at points $D, D$. Project the points $D, D$, on $X^{\prime} X^{\prime}$ thereby obtaining the points $E, E$. Connect the points $E, E$, to $P$ by lines cutting the line

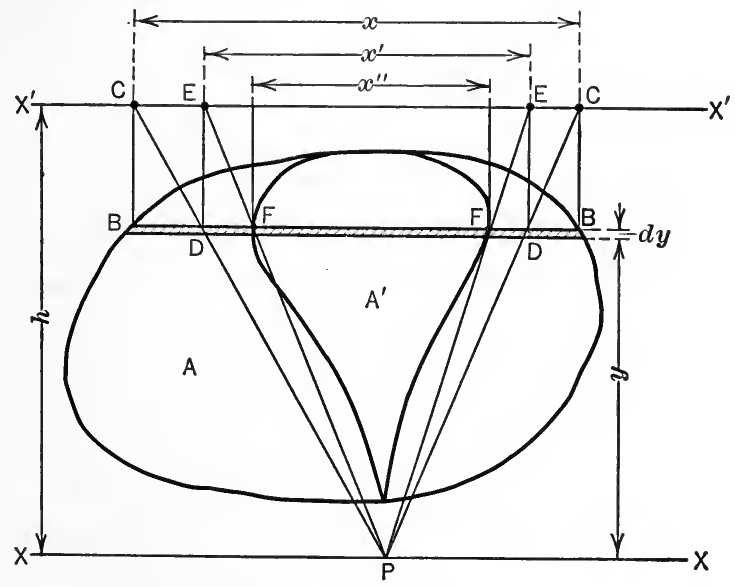

Fig. 249.

$B B$ in points $F, F$. This construction may be repeated for a number of chords parallel to $B B$, thus obtaining a number of points similar to $F, F$. All such points may be connected by a smooth curve, the area within which will be denoted by $A^{\prime}$. The moment of inertia of $A$ with respect to the axis $X X$ is given by the product of the area of $A^{\prime}$ and $h^{2}\left(A^{\prime} h^{2}\right)$. This fact may be proved as follows: Denoting $B B$ by $x, D D$ by $x^{\prime}$, and $F F$ by $x^{\prime \prime}$ we have, from the similar triangles $P F F$ and $P E E$,

$$
\frac{x^{\prime \prime}}{y}=\frac{x^{\prime}}{h}, \quad \text {. . . . . . . . . }
$$

and, from the similar triangles $P D D$ and $P C C$,

$$
\frac{x^{\prime}}{y}=\frac{x}{h} \text {. . . . . . . . . . }
$$


From (1) and (2), the following equation is obtained,

$$
x y^{2}=h^{2} x^{\prime \prime} \text {. }
$$

The moment of inertia of the area $A$ with respect to $X X$, then, is given by the equation,

$$
\begin{aligned}
I & =\int y^{2} d A=\int y^{2} x d y=h^{2} \int x^{\prime \prime} d y \\
& =h^{2} A^{\prime} .
\end{aligned}
$$

The area $A^{\prime}$ may be obtained by means of a planimeter or by approximate methods.

Approximate Method.-Although the method described above is of importance, the amount of work involved in any numerical problem is considerable. An approximate method will now be described which can easily be applied to any area. For convenience, however, a simple area will be selected so that the approximate value of the moment of inertia as determined by this method may be compared with the exact value. Thus, let the moment of inertia of the area of a rectangle, with respect to an axis coinciding with its base, be found. The area may be divided into any convenient number of equal narrow strips parallel to the base, as shown in Fig. 250. (The narrower

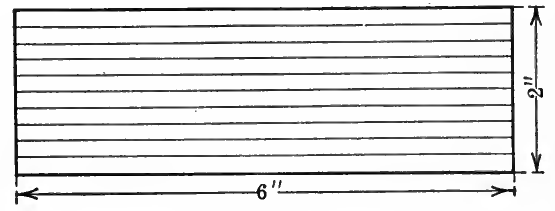

FIG. 250. the strips the more closely will the result agree with the exact result.) Let the area be divided into ten such strips each 0.2 in. in width. The moment of inertia of the rectangle is equal to the sum of the moments of inertia of the strips. The moment of inertia of any particular strip with respect to the base of the rectangle is

$$
\frac{1}{12} \times 6 \times\left(\frac{1}{5}\right)^{3}+6 \times \frac{1}{5} \times y^{2},
$$

where $y$ is the distance of the centroid of the particular strip from the base. The first term is small and may be omitted without serious error. The moment of inertia of each strip then is approx- 
imately equal to the product of the area of the strip and the square of the distance of its centroid from the base. Hence, the moment of inertia of the rectangle is,

$$
\begin{aligned}
I & =\frac{6}{5}\left(.1^{2}+.3^{2}+.5^{2}+.7^{2}+.9^{2}+{\overline{1.1^{2}}}^{2} \overline{1.3}^{2}+{\overline{1.5^{2}}}^{2} \overline{1.7^{2}}+\overline{1.9}^{2}\right) \\
& =\frac{6}{5} \times 13.3 \\
& =15.96 \text { in. }{ }^{4}
\end{aligned}
$$

According to Art. 92 the exact value is,

$$
I=\frac{1}{3} b h^{3}=\frac{1}{3} \times 6 \times 2^{3}=16 \text { in. }{ }^{4}
$$

\section{§2. Moments of Inertia of Bodies}

100. Moment of Inertia of Mass Defined.-In the analysis of the motion of a body, the body is frequently regarded as a system of particles and expressions are frequently met in the analysis which involve the mass of a particle and its distance from a line or plane. The product of the mass of a particle and the first power of its distance from a line or plane is called the first moment of the particle as discussed in Art. 79 while the product of the mass of the particle and the square of its distance from the line or plane is called the second moment of the mass of the particle or more frequently the moment of inertia of the mass of the particle (or briefly the moment of inertia of the particle) with respect to the line or plane. The moment of inertia of a system of particles (mass-system or body) with respect to a line or plane is the sum of the moments of inertia of the particles with respect to the given line or plane. Thus, if the masses of the particles of a system are denoted by $m_{1}, m_{2}, m_{3}, \ldots$ and the distances of the particles from a given line are denoted by $r_{1}, r_{2}, r_{3} \ldots$, the moment of inertia of the system may be expressed as follows:

$$
\begin{aligned}
I & =m_{1} r_{1}^{2}+m_{2} r_{2}^{2}+m_{3} r_{3}^{2}+\ldots \\
& =\Sigma m r^{2} .
\end{aligned}
$$

If the mass system constitutes a continuous body the summation in the above equation may be replaced by a definite integral, and the expression for the moment of inertia of the body then becomes,

$$
I=\int r^{2} d M
$$


where $d M$ represents an element of mass of the body and $r$ is the distance of the element from the given line or plane. The limits of the integral must, of course, be so chosen that each element of mass of the body is included in the integration. Therefore, the moment of inertia of a body with respect to a line or plane may be defined as the sum of the products obtained by multiplying each elementary mass of the body by the square of its distance from the given line or plane. The moment of inertia of the mass of a body (or more briefly the moment of inertia of a body) has a physical significance, since common experience teaches that if a body is free to rotate about an axis, the farther from the axis the material is placed, that is, the greater the moment of inertia of the body becomes, the greater is the moment of the forces required to give the body a prescribed rotation in a specified time. Thus, if a rod is free to rotate about a vertical axis and carries two spheres which may be moved along the rod, experience shows that the farther from the axis the spheres are placed the greater is the torque required to produce a definite rotation in a given time.

Units.-No special one-term name has been given to the unit of moment of inertia of a body, hence, the units of mass and the unit of length used are specified. Thus, if the mass of a body is expressed in pounds and the dimensions of the body are expressed in feet, the moment of inertia of the body is expressed in poundfoot $^{2}$ units (written lb.-ft. ${ }^{2}$ ). In engineering problems, however, the pound is generally used as the unit of force, in which case mass is equal to force divided by acceleration $\left(M=\frac{W}{g}\right)$, as will be discussed in Arts. 139 and 141, and the unit of mass is, therefore, a derived unit being expressed in terms of the units of force (pound), length (foot), and time (second). Thus, the dimensional equation for mass is $\frac{W}{g}=F \div \frac{L}{T^{2}}=\frac{F T^{2}}{L}$. And since the unit of moment of inertia involves the units of mass and of length squared, then in engineering problems the unit of moment of inertia is also a derived unit, the dimensions of which are $M L^{2}=\frac{F T^{2}}{L} \times L^{2}=F T^{2} L$, that is, lb.-sec ${ }^{2}$-ft. The name geepound or slug is sometimes used for the engineer's unit of mass, the first because the unit of mass is the mass of a body that weighs $g$ (32.2 approximately) pounds, and the second because mass is a measure of the sluggishness (inertia) 
of the body. Thus the moment of inertia of a body is sometimes expressed in geepound-foot ${ }^{2}$ or slug-foot ${ }^{2}$ units (geepound$\mathrm{ft}^{2}$ or slug-ft. ${ }^{2}$ ). It is important, however, to keep in mind that the unit of moment of inertia is a derived unit, being expressed in terms of the fundamental units of force, length, and time.

101. Radius of Gyration.-It is frequently convenient to express the moment of inertia of a body in terms of factors, one of which is the mass of the whole body. Since each term in the expression for moment of inertia as defined above is one dimension in mass and two dimensions in length, the moment of inertia of a body may be expressed as the product of the mass, $M$, of the whole body and the square of a length. This length is defined as the radius of gyration of the body and will be denoted by $k$. Thus, the moment of inertia, $I$, of a body with respect to a given line or plane may be expressed by the product $M k^{2}$, and hence,

$$
I=\int r^{2} d M=M k^{2} \quad \text { or } \quad k=\sqrt{\frac{I}{M}} .
$$

The radius of gyration of a body with respect to any axis, then, may be regarded as the distance from the axis at which the mass may be conceived to be concentrated and have the same moment of inertia with respect to the axis as does the actual (or distributed) mass.

Viewed differently, the radius of gyration of a body with respect to an axis is a distance such that the square of this distance is the mean of the squares of the distances from the axis of the (equal) elements of mass into which the given body may be divided (not the square of the mean of the distances).

102. Parallel Axis Theorem for Masses.-If the moment of inertia of a body with respect to an axis passing through its centroid (center of gravity or mass-center) is known, the moment of inertia of the body with respect to any parallel axis may be found, without integrating, by use of the following proposition.

The moment of inertia of a body with respect to any axis is equal to the moment of inertia of the body with respect to a parallel axis through the mass-center of the body plus the product of the mass of the body and the square of the distance between the two axes.

This proposition may be stated in equational form as follows:

$$
I=\bar{I}+M d^{2}
$$


where $\bar{I}$ denotes the moment of inertia of the body with respect to an axis through the mass-center and $I$ denotes the moment of

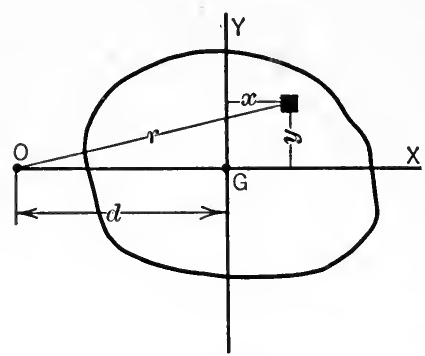

FIG. 251. inertia with respect to a parallel axis which is at a distance $d$ from the axis through the mass-center.

Proof.-Let Fig. 251 represent the cross-section of a body containing the mass-center, $G$. Further, let the moment of inertia of the body with respect to an axis through $G$ and perpendicular to this section be denoted by $\bar{I}$ and let the moment of inertia with respect to a parallel axis through the point $O$ be denoted by $I$. The expression for $I$, then, is,

$$
\begin{aligned}
I & =\int r^{2} d M \\
& =\int\left[(x+d)^{2}+y^{2}\right] d M \\
& =\int\left(x^{2}+y^{2}\right) d M+d^{2} \int d M+2 d \int x d M .
\end{aligned}
$$

Therefore,

$$
I=\bar{I}+M d^{2}
$$

since

$$
\int x d M=M \bar{x}=0 \text {. }
$$

This theorem is frequently called the parallel axis theorem for masses. A similar relation may be found between the radii of gyration with respect to the two axes. Thus, if the radii of gyration with respect to the two parallel axes be denoted by $k$ and $\vec{k}$, the above equation may be written,

Hence,

$$
M k^{2}=M \bar{k}^{2}+M d^{2},
$$

$$
k^{2}=\bar{k}^{2}+d^{2} \text {. }
$$

103. Moments of Inertia with Respect to Two Perpendicular Planes.-The determination of the moment of inertia of a body 
with respect to a line is frequently simplified by making use of the following theorem: The sum of the moments of inertia of a body with respect to two perpendicular planes is equal to the moment of inertia of the body with respect to the line of intersection of the two planes.

Proof.-If the moment of inertia of the body (Fig. 252) with respect to $x y$ - and $x z$-planes be denoted by $I_{x y}$ and $I_{x z}$, respectively, the expressions for the moments of inertia are,

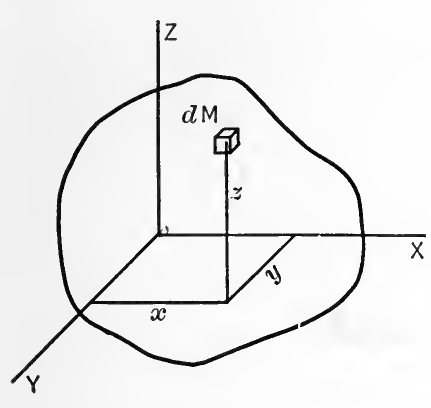

FIg. 252.

and

$$
I_{x y}=\int z^{2} d M
$$

$$
I_{x z}=\int y^{2} d M
$$

By adding these two equations the resulting equation is,

$$
I_{x y}+I_{x_{2}}=\int\left(z^{2}+y^{2}\right) d M
$$

$$
=I_{x} \text {. }
$$

104. Moments of Inertia of Simple Solids by Integration.- - In determining the moment of inertia of a body with respect to an axis by the method of integration, the mass of the body may be divided into elements in various ways, and either cartesian or polar coordinates may be used, leading to a single, double, or triple integration, depending on the way the element is chosen. The elements of mass should always be selected, however, so that,

(1) All points in the element are equally distant from the axis (or plane) with respect to which the moment of inertia is to be found, otherwise the distance from the axis to the element would be indefinite. Or, so that,

(2) The moment of inertia of the element with respect to the axis about which the moment of inertia of the body is to be found is known; the moment of inertia of the body is then found by summing up the moments of inertia of the elements. Or, so that,

(3) The mass-center of the element is known and the moment of inertia of the element with respect to an axis through its masscenter and parallel to the given axis is known, in which case, the moment of inertia of the element may be expressed by use of the parallel axis theorem (Art. 102). 
The moment of inertia of some of the simpler solids are found in the following problems.

Note: The symbol $\delta$ will be used in the following pages to denote the density of a body.

\section{ILLUSTRATIVE PROBLEMS}

242. Determine the moment of inertia of a homogeneous right circular cylinder with respect to its geometrical axis.

Solution.-In accordance with the first of the above rules, the element of mass may be selected as indicated in Fig. 253. The volume of this element is $h \rho d \rho d \theta$, and if the density be denoted by $\delta$, the mass of the element is $\delta h \rho d \rho d \theta$. Hence, the expression for the moment of inertia becomes,

$$
\begin{aligned}
\bar{I} & =\int \rho^{2} d M=\int_{0}^{r} \int_{0}^{2 \pi} \rho^{2} \delta h \rho d \rho d \theta \\
& =\frac{1}{2} \pi \delta h r^{4} .
\end{aligned}
$$

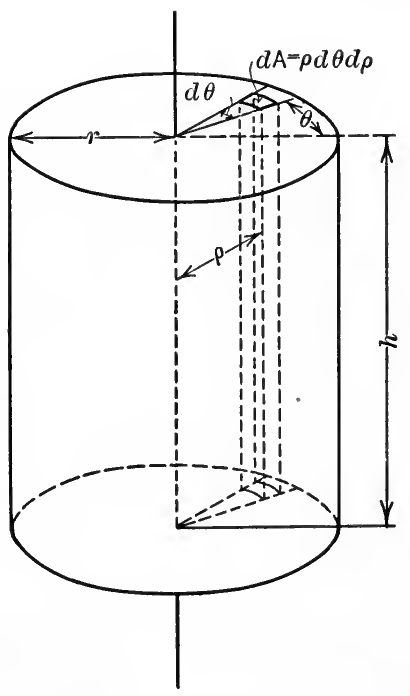

FIG. 253.

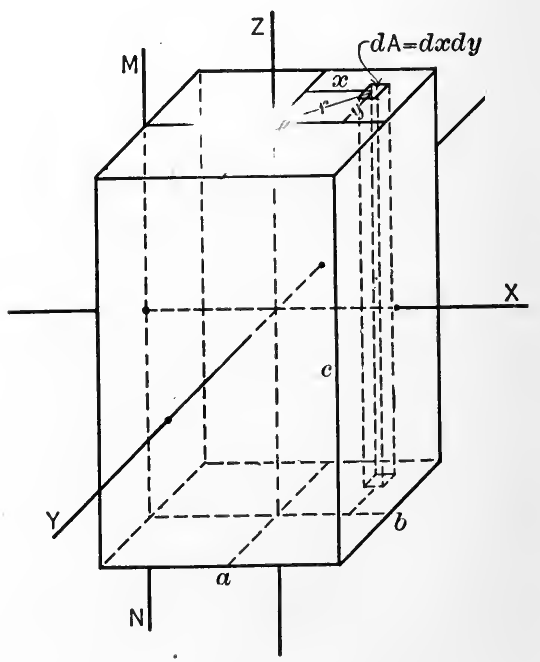

Fig. 254.

Since the mass, $M$, of the cylinder is $\delta \pi r^{2} h$, the above equation may be written,

$$
\vec{I}=\frac{1}{2} M r^{2} .
$$

243. Determine the moment of inertia of a homogeneous rectangular 
parallelopiped with respect to (a) a central axis parallel to an edge; (b) a median line of one face.

Solution. (a) Central Axis Parallel to an Edge.-Let the lengths of the edges be denoted by $a, b$, and $c$, and let the $z$-axis, parallel to the edge $c$, be the central axis about which the moment of inertia is to be found, as shown in Fig. 254. 'The parallelopiped may be divided into elementary volumes as indicated, the mass of each element being $\delta c d x d y$. The moment of inertia with respect to the $z$-axis, then, is,

$$
\begin{aligned}
I_{z} & =\int r^{2} d M=\int_{-\frac{a}{2}}^{+\frac{a}{2}} \int_{-\frac{b}{2}}^{+\frac{b}{2}} \delta c\left(x^{2}+y^{2}\right) d x d y \\
& =\delta c \int_{-\frac{b}{2}}^{+\frac{b}{2}}\left(\frac{a^{3}}{12}+y^{2} a\right) d y \\
& =\delta c\left(\frac{a^{3} b}{12}+\frac{a b^{3}}{12}\right) \\
& =\frac{1}{12} \delta a b c\left(a^{2}+b^{2}\right) \\
& =\frac{1}{12} M\left(a^{2}+b^{2}\right) .
\end{aligned}
$$

In a similar way it can be shown that the moments of inertia with respect to central axes parallel to the edges $a$ and $b$ are

and

$$
I_{x}=\frac{1}{12} M\left(b^{2}+c^{2}\right)
$$

$$
I_{\nu}=\frac{1}{12} M\left(a^{2}+c^{2}\right) .
$$

Thus, the moment of inertia of a homogeneous rectangular prism with respect to a central axis parallel to any one of the three edges is expressed in terms of the mass and the lengths of the other two edges.

(b) A Median Line of One Face.-In order to determine the moment of inertia with respect to a median line of one face the formula of Art. 102 may be used. Thus, if the line $M N$ (Fig. 254) be taken as the axis, the moment of inertia with respect to $M N$ is

$$
\begin{aligned}
I_{M N} & =\bar{I}_{z}+M\left(\frac{a}{2}\right)^{2} \\
& =\frac{1}{12} M\left(a^{2}+b^{2}\right)+\frac{1}{4} M a^{2}=\frac{1}{12} M\left(4 a^{2}+b^{2}\right) .
\end{aligned}
$$

244. Determine the moment of inertia of a homogeneous sphere with respect to a diameter. 
Solution.-The cross-section of the sphere in the $x y$-plane is shown in Fig. 25j. In accordance with the second rule above, the element of volume may be taken as a thin circular lamina included between two planes parallel to the $x z$-plane, as shown in cross-section. This element, then, may be regarded as a circular cylinder of radius $x$ and altitude $d y$. The mass of the elemental cylinder is $\delta \pi x^{2} d y$ and its moment of inertia with respect to the $y$-axis is $\frac{1}{2} \delta \pi x^{4} d y$ (Prob. 242). Hence, the moment of inertia of the entire sphere with respect to the $y$-axis is,

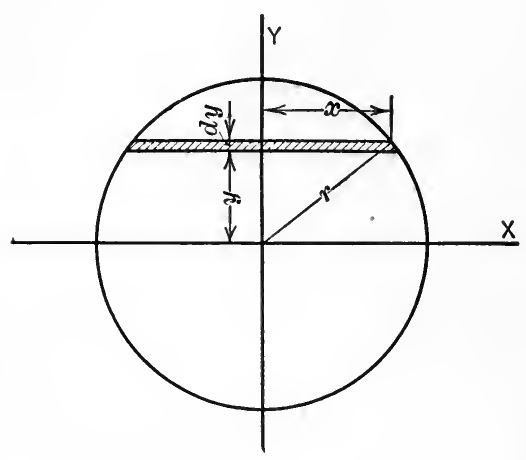

FIG. 255.

$$
\begin{aligned}
\bar{I} & =\frac{1}{2} \delta \pi \int_{-r}^{+r} x^{4} d y \\
& =\frac{1}{2} \delta \pi \int_{-r}^{+r}\left(r^{2}-y^{2}\right)^{2} d y \\
& =\frac{8}{15} \delta \pi r^{5} \\
& =\frac{2}{5}\left(\frac{4}{3} \delta \pi r^{3}\right) r^{2} \\
& =\frac{2}{5} M r^{2} .
\end{aligned}
$$

245. Determine the moment of inertia of a homogeneous right circular cone about its geometrical axis.

Solution.-A cross-section of the cone in the $x y$-plane is shown in Fig. 256, the $y$-axis being taken as the geometric axis. In accordance with the second rule above, the element of volume is taken as a circular lamina included between two planes parallel to the base, the height of the lamina being $d y$. The mass of this elemental cylinder is $\delta \pi x^{2} d y$ and its moment of inertia with respect to the $y$-axis is $\frac{1}{2} \delta \pi x^{4} d y$. The moment of inertia of the cone with respect to the $y$-axis, then, is,

$$
\bar{I}_{y}=\frac{1}{2} \delta \pi \int_{0}^{h} x^{4} d y .
$$

The relation between $x$ and $y$ may be found from similar triangles. Thus,

$$
\frac{h}{r}=\frac{h-y}{x} .
$$

That is,

$$
x=\frac{r}{h}(h-y) .
$$

Hence,

$$
\overline{I_{\nu}}=\frac{1}{2} \delta \pi \overline{r^{4}} \int_{0}^{h}(h-y)^{4} d y
$$

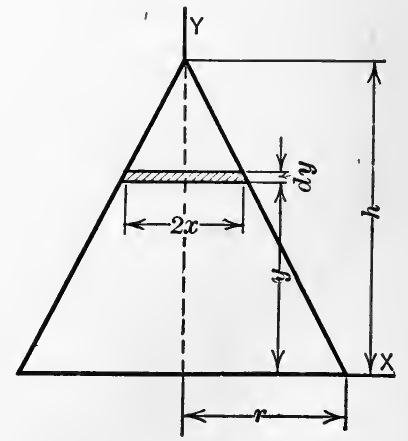

Fig. 256. 


$$
\begin{aligned}
& =\frac{1}{10} \delta \pi h r^{4} \\
& =\frac{3}{10}\left(\frac{1}{3} \delta \pi h r^{2}\right) r^{2} \\
& =\frac{3}{10} M r^{2} .
\end{aligned}
$$

Second Method.-If the element of volume be taken in accordance with the first rule above, as indicated in Fig. 257, the element of mass then is $\delta z \rho d \rho d \theta$ and hence the moment of inertia of the cone with respect to the $z$-axis is,

$$
\bar{I}_{z}=\int_{0}^{r} \int_{0}^{2 \pi} \delta z \rho^{3} d \rho d \theta .
$$

The relation between $z$ and $p$ may be found from similar triangles. Thus,

or,

$$
\frac{z}{r-\rho}=\frac{h}{r}
$$

Hence,

$$
z=\frac{h}{r}(r-\rho) .
$$

$$
\begin{aligned}
\overline{I_{2}} & =\frac{\delta h}{r} \int_{0}^{r} \int_{0}^{2 \pi} \rho^{3}(r-\rho) d \rho d \theta \\
& =\frac{1}{10} \delta \pi h r^{4} \\
& =\frac{3}{10} M r^{2} .
\end{aligned}
$$

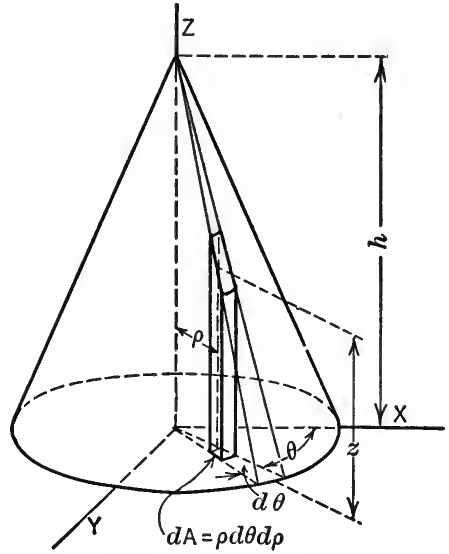

FIG. 257.

246. Show that the moment of inertia of a homogeneous right prism, having a cross-section of any shape, with respect to a plane coinciding with one of the bases of the prism, is $I=\frac{1}{3} M l^{2}$ in which $M$ is the mass of the rod and $l$ is the length of the rod. Further, show that for a slender rod the above expression represents, with slight error, the moment of inertia of the rod with respect to an axis passing through one end of the rod and perpendicular to the rod.

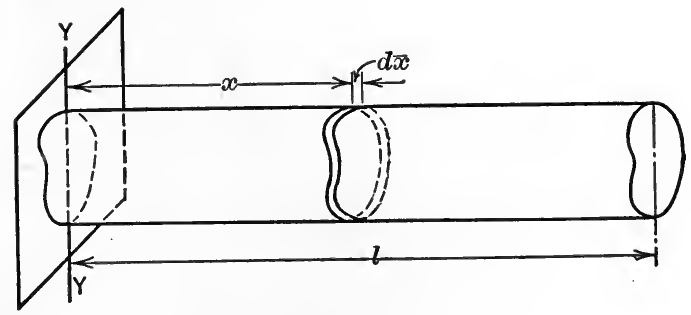

Frg. 258.

Solution.-In accordance with the first of the above rules, the element of mass may be selected as a thin lamina parallel to the end plane as shown in 
Fig. 258. The volume of the element is $A d x$ and its mass is $\delta A d x$, where $A$ is the cross-section of the prism. Hence,

$$
\begin{aligned}
I & =\delta A \int_{0}^{l} x^{2} d x \\
& =\frac{\delta A l^{3}}{3}=\frac{1}{3}(\delta A l) l^{2}=\frac{1}{3} M l^{2} .
\end{aligned}
$$

If the moment of inertia is taken with respect to an end axis $(Y Y)$, then, $x$ (Fig. 258) is not the distance of all points in the lamina from the axis; the greater part of the lamina is farther from the axis than the distance $x$. Therefore the product $d M \cdot x^{2}=(\delta A d x) x^{2}$ is less than the real moment of inertia of the lamina, and hence $\frac{1}{3} M l^{2}$ is less than the true moment of inertia of the rod with respect to the end axis $Y Y$. But, the products $d M x^{2}$ for the laminas which are greatly in error (for the laminas near the axis $Y Y$ ) have littleinfluence in determining the value of the summation, $\int d M x^{2}$, in comparison with the laminas far removed from the axis and for which the products $d M x^{2}$ are only slightly in error. Hence, the expression $\frac{1}{3} M l^{2}$ is a close approximation to the moment of inertia of a slender rod, as for example, a flywheel spoke or connecting rod, etc., with respect to an axis through an end. Thus, the error in the expression $\frac{1}{3} M l^{2}$ is only 1 per cent for a circular cylinder when the length of the cylinder is only 4.3 times its diameter.

247. Show that the moment of inertia of a homogeneous thin circular lamina with respect to an axis through the mass-center, parallel to the bases of the lamina, is approximately $\frac{1}{4} M r^{2}$, in which $M$ is the mass of the lamina and $r$ is the radius.

Solution.-A top view and an end view of the lamina are shown in Fig. 259. The element of volume will be taken as a prism of altitude $t$ and cross-section $\rho d \rho d \theta$, as indicated in the figure. The mass of the element is $\delta t \rho d \rho d \theta$. Now if the thickness $t$ is relatively small, all points in any elementary prism are approximately at the same distance $(y=\rho \sin \theta)$ from the $x$-axis except for those prisms which are near the axis, and these prisms

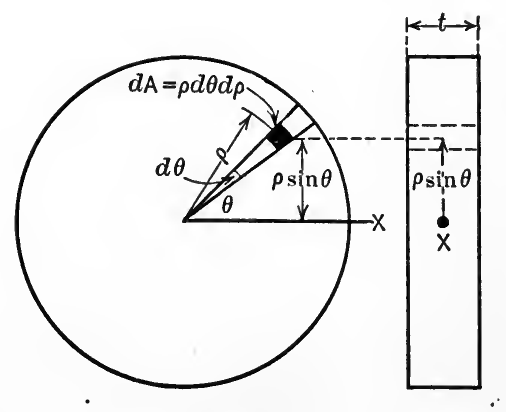

FIG. 259. contribute little to the moment of inertia of the lamina with respect to the $x$-axis. Thus, the moment of inertia of the lamina with respect to an axis through its mass-center parallel to the bases of the lamina is approximately,

$$
\begin{aligned}
I_{x} & =\int_{0}^{r} \int_{0}^{2 \pi} \delta t \rho^{3} \sin ^{2} \theta d \rho d \theta \\
& =\frac{1}{4} \delta l \pi r^{4}=\frac{1}{4}\left(\delta t \pi r^{2}\right) r^{2} \\
& =\frac{1}{4} M r^{2} .
\end{aligned}
$$


It should be noted that the smaller the value of $t$ becomes, the closer is the approximation. The above expression is also a close approximation to the moment of inertia of the lamina with respect to a diameter of either base of the lamina.

248. Determine the moment of inertia of a homogeneous right circular cylinder with respect to a diameter of one of the bases.

Solution. First Method.-In accordance with the third of the above rules, the element of volume may be taken as a thin circular lamina parallel to the base as indicated in Fig. 260. The mass of the element is $\delta \pi r^{2} d z$ and the moment of inertia of the element with respect to a centroidal axis parallel to the $x$-axis, as found in the preceding problem, is $\frac{1}{4} \delta \pi r^{4} d z$. The moment of inertia of the element with respect to the $x$-axis, then, as found by the parallel axis theorem (Art. 102), is

$$
{ }^{\frac{1}{4}} \delta \pi r^{4} d z+\delta \pi r^{2} z^{2} d z .
$$

Hence, the moment of inertia of the entire cylinder with respect to the $x$-axis is,

$$
\begin{aligned}
I & =\int_{0}^{h}{ }_{1}^{\frac{1}{4}} \delta \pi r^{4} d z+\int_{0}^{h} \delta \pi r^{2} z^{2} d z \\
& =\frac{1}{4} \delta \pi r^{4} h+\frac{1}{3} \delta \pi r^{2} h^{3} \\
& =\frac{1}{12} \delta \pi r^{2} h\left(3 r^{2}+4 h^{2}\right) \\
& =\frac{1}{12} M\left(3 r^{2}+4 h^{2}\right) .
\end{aligned}
$$

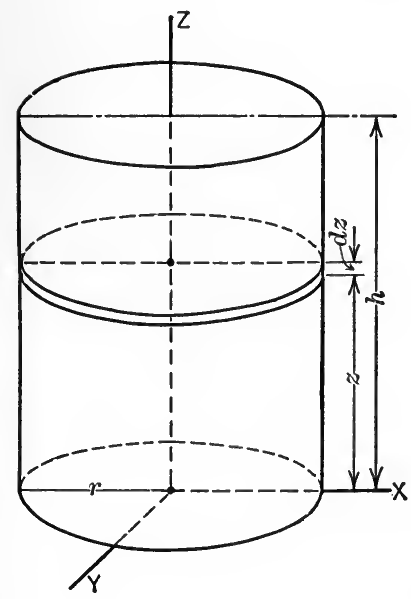

FIG. 260.

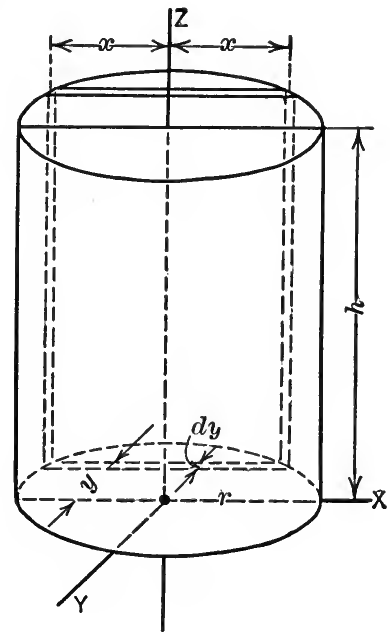

FIG. 261.

Second Method.-The moment of inertia with respect to the $x$-axis may also be found by adding the moments of inertia with respect to the $x y$ - and $x z$-planes (Art. 103). Thus, in Fig. 261,

$$
I_{x}=I_{x y}+I_{x z} .
$$


From Problem 246 the moment of inertia with respect to the end $(x y)$ plane is

$$
I_{x y}=\frac{1}{3} M h^{2} .
$$

To find the moment of inertia with respect to the $x z$-plane an element of mass may be selected as indicated in Fig. 261. Thus,

Therefore,

$$
\begin{aligned}
I_{x z} & =\int y^{2} d M=\int_{-r}^{+r} y^{2}(\delta h 2 x d y) \\
& =2 \delta h \int_{-r}^{+r} y^{2} \sqrt{r^{2}-y^{2}} d y \\
& =\frac{1}{4} \delta h \pi r^{4}=\frac{1}{4} M r^{2} . \\
I_{x} & =\frac{1}{3} M h^{2}+\frac{1}{4} M r^{2} \\
& =\frac{1}{12} M\left(3 r^{2}+4 h^{2}\right) .
\end{aligned}
$$

249. Determine the moment of inertia of a homogeneous right circular cone with respect to a diameter of the base.

Solution.-In accordance with the third of the above rules the element of volume may be taken as a thin cylindrical lamina parallel to the base, as indicated in Fig. 262. The mass of this element is $\delta \pi x^{2} d z$ and its moment of inertia with respect to its centroidal axis parallel to the $x$-axis is $\frac{1}{4} \delta \pi x^{4} d z$ (see Problem 247). The moment of inertia with respect to the $x$-axis, according to the parallel axis theorem (Art. 102), is $\frac{1}{4} \delta \pi x^{4} d z+\delta \pi x^{2} z^{2} d z$. Hence, the moment of inertia of the entire cone with respect to the $x$-axis is,

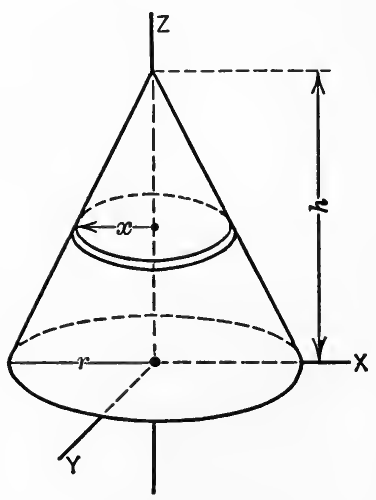

FIg. 262.

$$
I_{x}=\int_{0}^{n} \frac{1}{4} \delta \pi x^{4} d z+\int_{0}^{n} \delta \pi x^{2} z^{2} d z
$$

From similar triangles,

$$
x=\frac{r}{h}(h-z) \text {. }
$$

Hence,

$$
\begin{aligned}
I_{x} & =\frac{1}{4} \delta \pi \frac{r^{4}}{h^{4}} \int_{0}^{h}(h-z)^{4} d z+\delta \pi \frac{r^{2}}{h^{2}} \int_{0}^{h} z^{2}(h-z)^{2} d z \\
& =\frac{1}{20} \delta \pi h r^{4}+\frac{1}{30} \delta \pi r^{2} h^{3} \\
& =\frac{1}{3} \delta \pi r^{2} h\left(\frac{3}{20} r^{2}+\frac{1}{10} h^{2}\right) \\
& =M\left(\frac{3}{20} r^{2}+\frac{1}{10} h^{2}\right) \\
& =\frac{1}{20} M\left(3 r^{2}+2 h^{2}\right) .
\end{aligned}
$$

250. Determine the moment of inertia of a sphere with respect to a central axis if the density at any point varies directly as the distance of the point from a central plane perpendicular to the axis. 
Solution.-The moment axis will be taken as the $y$-axis of coordinates (Fig. 263). The element of volume will be taken as a thin lamina parallel to the $x z$-plane. The density at all points in this lamina is proportional to $y$ and hence may be denoted by $k y$. Hence, the element of mass is $k y \pi x^{2} d y$ and the mass of the sphere is

$$
M=k \pi \int_{-r}^{+r} y x^{2} d y=k \pi \int_{-r}^{+r}\left(r^{2}-y^{2}\right) y d y=\frac{1}{2} \pi k r^{4}
$$

The moment of inertia of the cylindrical lamina is $\frac{1}{2} k y \pi x^{4} d y$ (Prob. 242) and the moment of inertia of the entire sphere is the sum of the moments of inertia of the elementary cylinders. Thus,

$$
\begin{aligned}
\bar{I} & =\frac{1}{2} k \pi \int_{-r}^{+r} y x^{4} d y \\
& =\frac{1}{2} k \pi \int_{-r}^{+r} y\left(r^{2}-y^{2}\right)^{2} d y \\
& =\frac{1}{6} k \pi r^{6} \\
& =\frac{1}{3}\left(\frac{1}{2} k \pi r^{4}\right) r^{2} \\
& =\frac{1}{3} M r^{2} .
\end{aligned}
$$

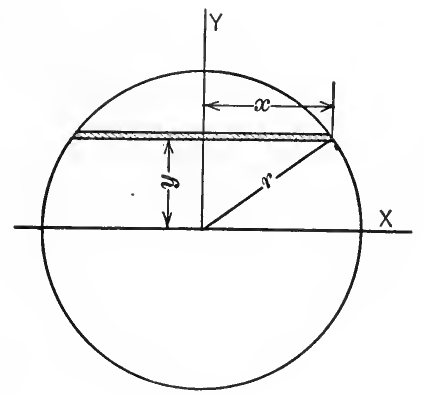

FIg. 263.

251. Find the moment of inertia, with respect to a central axis, of a castiron sphere $10 \mathrm{in}$. in diameter. Assume the weight of cast iron to be $450 \mathrm{lb}$. per cubic foot.

Solution.-The mass of the sphere is,

$$
\begin{aligned}
M & =\frac{4}{3} \pi\left(\frac{5}{12}\right)^{3} \times \frac{450}{32.2}=4.27 \frac{\text { lb.-sec. }}{\mathrm{ft} .} \text { or slugs. } \\
I & =\frac{2}{5} M r^{2} \\
& =\frac{2}{5} \times 4.27 \times\left(\frac{5}{12}\right)^{2} \\
& =.296 \mathrm{lb} . \text {-sec. }^{2} \text {-ft. (or slug-ft. }{ }^{2} \text { ). }
\end{aligned}
$$

\section{PROBLEMS}

252. Determine the moment of inertia of a homogeneous right circular cone about an axis through the center of gravity perpendicular to the geometrical axis.

Ans. $\frac{3}{20} M\left(r^{2}+\frac{1}{4} h^{2}\right)$. 
253. A homogeneous cylindrical rod is $2 \mathrm{ft}$. long and the radius is 2 in. If the moment of inertia is found with respect to a line through one end of the rod perpendicular to its axis by using the approximate formula of Problem 246 , what is the error in the result, in per cent?

254. Determine the moment of inertia of a steel cylinder 6 in. in diameter and $12 \mathrm{in}$. high, about its geometric axis. Assume the weight of steel to be $490 \mathrm{lb}$. per cubic foot.

255. Show that the moment of inertia of a homogeneous slender rod with respect to an axis through the mid-point of the rod and perpendicular to the rod is approximately $\frac{1}{12} M l^{2}$.

256. Determine the moment of inertia of a homogeneous ellipsoid, the principal axes of which are $2 a, 2 b$, and $2 c$, about the axis $2 a$.

$$
\text { Ans. } I=\frac{1}{5} M\left(b^{2}+c^{2}\right) .
$$

257. Determine the moment of inertia of a homogeneous elliptic cylinder, in which the principal axes of the cross-section are $2 a$ and $2 b$, with respect to (1) the geometrical axis, and (2) an axis through the center of gravity coincident with the axis $2 a$ of the cross-section.

$$
\text { Ans. (1) } I=\frac{1}{4} M\left(a^{2}+b^{2}\right) \text {; (2) } I=\frac{1}{12} M\left(h^{2}+3 b^{2}\right) \text {. }
$$

105. Moments of Inertia of Composite Bodies. - If a body can be divided into several finite parts, the moment of inertia of each of which is known, the moment of inertia of the given body may be obtained by adding the moments of inertia of the several parts. In like manner if parts of a body are removed, the moment of inertia of the remainder may be obtained by subtracting from the moment of inertia of the original body the sum of the moments of inertia of the parts removed.

\section{ILI.USTRATIVE PROBLEMS}

258. Determine the moment of inertia of a homogeneous, hollow, circular cylinder with respect to its geometric axis, in terms of its mass $M$ and its inner and outer radii, $r_{1}$ and $r_{2}$.

Solution.-Let $I_{2}$ and $M_{2}$ denote the moment of inertia and the mass of a solid cylinder of radius $r_{2}$ and let $I_{1}$ and $M_{1}$ have similar meanings for the cylinder of radius $r_{1}$ which is removed. Then,

$$
\begin{aligned}
I & =I_{2}-I_{1}=\frac{1}{2} M_{2} r_{2}{ }^{2}-\frac{1}{2} M_{1} r_{1}^{2} \\
& =\frac{1}{2} \delta \pi r_{2}^{4} h-\frac{1}{2} \delta \pi r_{1}^{4} h=\frac{1}{2} \delta \pi h\left(r_{2}^{4}-r_{1}^{4}\right) \\
& =\frac{1}{2} \delta \pi h\left(r_{2}{ }^{2}-r_{1}{ }^{2}\right)\left(r_{2}{ }^{2}+r_{1}{ }^{2}\right)=\frac{1}{2} M\left(r_{1}{ }^{2}+r_{2}{ }^{2}\right) .
\end{aligned}
$$


259. Determine the moment of inertia of the cast-iron flywheel shown in Fig. 264 with respect to the axis of rotation. Assume the weight of cast iron to be $450 \mathrm{lb}$. per cubic foot.

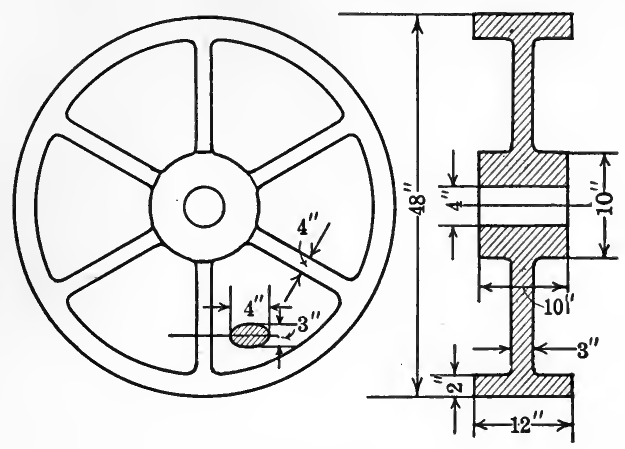

Fig. 264.

Solution.-The rim and hub are hollow cylinders and the spokes may be regarded as slender rods.

The weight of the rim $=\pi\left[\left(\frac{24}{12}\right)^{2}-\left(\frac{22}{12}\right)^{2}\right] \times \frac{12}{12} \times 450=903 \mathrm{lb}$.

The weight of the hub $=\pi\left[\left(\frac{5}{12}\right)^{2}-\left(\frac{2}{12}\right)^{2}\right] \times \frac{10}{12} \times 450=172 \mathrm{lb}$.

The weight of each spoke $=\pi \times \frac{1.5}{12} \times \frac{2}{12} \times \frac{17}{12} \times 450=41.7 \mathrm{lb}$.

For the rim,

$$
I=\frac{1}{2} \frac{903}{32.2}\left[\left(\frac{24}{12}\right)^{2}+\left(\frac{22}{12}\right)^{2}\right]=102.3 \text { slug-ft. }{ }^{2}
$$

For the hub,

$$
I=\frac{1}{2} \times \frac{172}{32.2}\left[\left(\frac{5^{\circ}}{12}\right)^{2}+\left(\frac{2}{12}\right)^{2}\right]=0.54 \text { slug-ft. }{ }^{2}
$$

For the spokes, $I=6 \times\left[\frac{1}{12} \times \frac{41.7}{32.2} \times\left(\frac{17}{12}\right)^{2}+\frac{41.7}{32.2} \times\left(\frac{13.5}{12}\right)^{2}\right]=11.1$ slug-ft. ${ }^{2}$

Hence the moment of inertia of the flywheel is,

$$
I=102.3+0.54+11.1=113.9 \text { slug-ft. }{ }^{2}
$$

\section{PROBLEMS}

260. Determine the moment of inertia of the frustum of a homogeneous right circular cone with respect to the geometrical axis, the radii of the bases being $r_{2}$ and $r_{1}$. Ans. $I=\frac{3}{10} M \frac{r_{2}^{5}-r_{1}^{5}}{r_{2}{ }^{3}-r_{1}^{3}}$. 
261. The head of the mallet shown in Fig. 265 is a rectangular parallelopiped and the handle is a right circular cylinder. If the weight of the material is $\frac{1}{4} \mathrm{lb}$. per cubic inch, find the moment of inertia of the mallet with respect to the line $Y Y$. Ans. $I=3.83$ slug-ft. 2

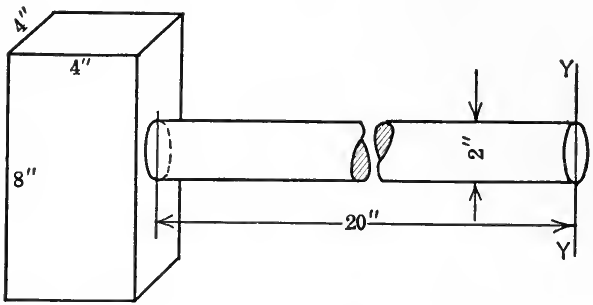

FIG. 265.

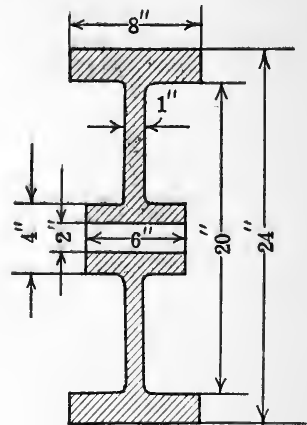

FIG. 266.

262. The wheel shown in Fig. 266 is made of cast iron and has a solid web. Determine the moment of inertia of the wheel with respect to the axis of rotation. Assume the weight of cast iron to be $450 \mathrm{lb}$. per cu. $\mathrm{ft}$.

263. From a round steel disk which is 20 in. in diameter and 4 in. thick, are bored four holes, each $4 \mathrm{in}$. in diameter. The axes of the holes are parallel to the geometric axis of the disk and 5 in. therefrom. Find the moment of inertia of the remainder of the disk with respect to its geometric axis, assuming the weight of steel to be $490 \mathrm{lb}$. per cu. $\mathrm{ft}$.

Ans. $I=3.51$ slug-ft. ${ }^{2}$

264. Two spheres are connected by a horizontal rod and are free to rotate about a vertical axis midway between the spheres. The diameter of each sphere is $9 \mathrm{in}$., the distance between their centers is $2 \mathrm{ft}$., and the diameter of the rod is $2 \mathrm{in}$. Find the moment of inertia of the rod and spheres with respect to the axis of rotation. Assume that the rod and spheres are made of cast iron which weighs $450 \mathrm{lb}$. per cu. $\mathrm{ft}$.

106. Moments of Inertia of Bodies by Experimental Methods. -If a body is irregular in shape, the moment of inertia cannot be found by methods of integration, since it is impossible to determine the limits of the integral. The moments of inertia of such bodies may be determined experimentally, however, by methods which make use of the laws of motion of a pendulum. These methods of determining the moment of inertia are discussed under the subject of Pendulums (Chapter IX). 


\section{PART II}

\section{KINEMATICS}

107. Introduction.-Kinematics treats of the motion of bodies without considering the manner in which the motion is influenced, either by the forces acting on the bodies or by the character of the bodies themselves. That is, the bodies are treated as geometric solids and not physical bodies. When the geometric solids are endowed with physical properties, we are led to a study of force, energy, momentum, etc., that is, to a study of Kinetics (Part III).

Kinematics deals with the relation between distance, time, velocity, and acceleration. In order to build up the fundamental conceptions which are involved in the study of the motion of bodies, the kinematics of a particle (material point) will be treated first. A particle is a body, or part of a body, the dimensions of which are negligible compared with its range of motion. Bodies are made up of particles, and the study of the motion of bodies is largely a study of the motion of their particles.

In treating of the motion of bodies (Chapter VIII), only rigid bodies will be considered, and the motion of the rigid bodies will be restricted to translation, rotation, and plane motion. 


\section{CHAPTER VII}

\section{MOTION OF A PARTICLE}

108. Types of Motion.-The motion of a particle along a straight line path is called rectilinear motion. The motion of a particle along a curved path is called curvilinear motion. If the moving particle describes equal distances in equal periods of time, however small, the motion is said to be uniform. If unequal distances are described by the moving point in equal periods of time, the motion is said to be non-uniform or variable.

Thus, if the crank shaft of a steam engine revolves at a constant

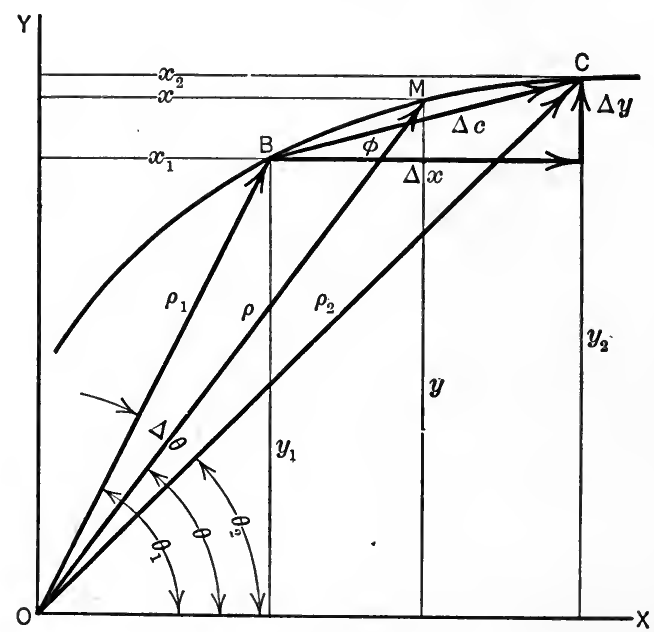

FIG. 267.

number of revolutions per minute, the crosshead of the engine has a non-uniform rectilinear motion, the crank pin has a uniform curvilinear motion, and any intermediate point on the connecting rod has a non-uniform, curvilinear motion.

109. Linear Displacement.-The displacement of a moving point is its change of position. The position of a moving point, at 
any instant, may be specified in a number of ways, as, for example, by stating the rectangular coordinates or the polar coordinates of the point. Thus, in Fig. 267 the position, at any instant, of the point $M$ as it travels along the curve from $B$ to $C$ may be specified by the coordinates $(x, y)$ or by $(\rho, \theta), \rho$ being the radius vector and $\theta$ its direction angle. The displacement $\Delta c$ of the point as it moves from the position $\left(x_{1}, y_{1}\right)$ or $\left(\rho_{1}, \theta_{1}\right)$ to the position $\left(x_{2}, y_{2}\right)$ or $\left(\rho_{2}, \theta_{2}\right)$ is the straight line $B C$, that is, the vector drawn from $B$ to $C$. This displacement may be expressed as the vector sum $^{1}$ of its $x$ - and $y$-components. Thus,

in which,

$$
\Delta c=\Delta x+\Delta y,
$$

$\Delta x=x_{2}-x_{1}$ is the displacement in the $x$-direction, and

$\Delta y=y_{2}-y_{1}$ is the displacement in the $y$-direction.

The magnitude of $\Delta c$ may be expressed algebraically by the equation,

$$
\Delta c=\sqrt{(\Delta x)^{2}+(\Delta y)^{2}},
$$

and its direction with the $x$-axis may be expressed by the equation,

$$
\tan \phi=\frac{\Delta y}{\Delta x}
$$

The displacement $\Delta c$ may be expressed also as the vector difference of the radius vectors to the two positions of the moving point. Thus,

$$
\Delta c=\rho_{2} \rightarrow \rho_{1},
$$

that is, $\Delta c$ is the directed distance which must be added to $\rho_{1}$ to give $\rho_{2}$. Or, in other words, $\rho_{2}$ is the vector sum of $\rho_{1}$ and $\Delta c$.

${ }^{1}$ The symbol $\rightarrow$ will be used in this and succeeding chapters, when dealing with vector quantities, to denote the fact that the addition of the quantities is a geometric or vector addition. Thus, if $R$ is the resultant of the forces $P$ and $Q$, the triangle law may be expressed by the equation $R=P \rightarrow Q$. And, if $u$ and $w$ are components of the velocity $v$, this fact may be expressed by the equation $v=u \nrightarrow w$. Similarly, the symbol $\rightarrow$ will be used to denote vector subtraction. Thus, $v_{2} \rightarrow v_{1}$ denotes the geometric or vector difference of two velocities and not their scalar difference, that is, the difference of their magnitudes, only. 
Expressed algebraically, by the law of cosines, the magnitude of $\Delta c$ may be written,

$$
\Delta c=\sqrt{\rho_{1}^{2}+\rho_{2}^{2}-2 \rho_{1} \rho_{2} \cos \Delta \theta} .
$$

The unit of displacement is any convenient unit of length, such as the inch, foot, mile, etc. It will be noted, however, that displacement is a directed distance or length, that is, a vector quantity. Displacements, therefore, may be combined and resolved according to the parallelogram (or triangle) law like forces and other vector quantities. It is important to note that by one of the above equations the displacement is expressed as the vector sum of two directed distances, whereas by the other equation it is expressed as the vector difference of two directed distances. Further, one vector equation is sufficient to express both the magnitude and the direction of the displacement, whereas two algebraic equations are required for the same purpose.

If the displacement of the particle is decreased indefinitely, the point $C$ (Fig. 267) will approach the point $B$ and, in the limit, the chord $\Delta c$ becomes coincident with the tangent to the path at $B$. Therefore, the direction of motion of the particle at any point on its path is tangent to the path at that point.

110. Angular Displacement.-The angular displacement of a moving point is the change in the angle which the radius vector to the point makes with some fixed reference line. Thus, in Fig. 267, the angular displacement, $\Delta \theta$, corresponding to the linear displacement, $\Delta c$, is,

$$
\Delta \theta=\theta_{1}-\theta_{2}
$$

The unit of angular displacement may be any convenient angular measure, such as the degree, revolution, radian, etc. It is important to note that the angular displacement of a point depends upon the reference point or pole about which the radius vector is assumed to revolve. If the point moves on a circular arc, the radius vector is usually taken as the radius of the circle and the pole is the center of the circle. 


\section{PROBLEMS}

265. An automobile starts from the position $A$, as specified with reference to the axes shown in Fig. 268, and travels to position $B$ along the road indicated by the curved path. Find $(a)$ the magnitude and direction of the linear displacement of the automobile; (b) the angular displacement.

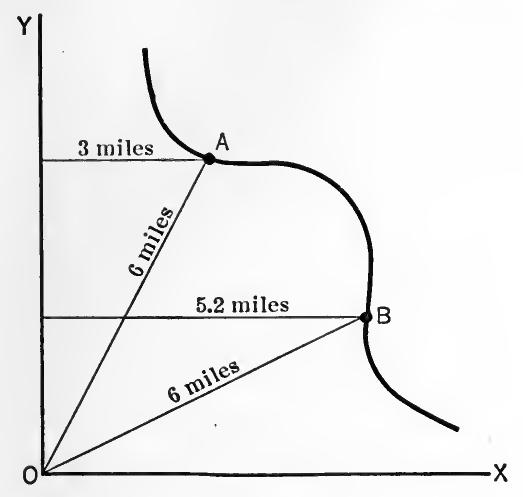

Fig. 268.

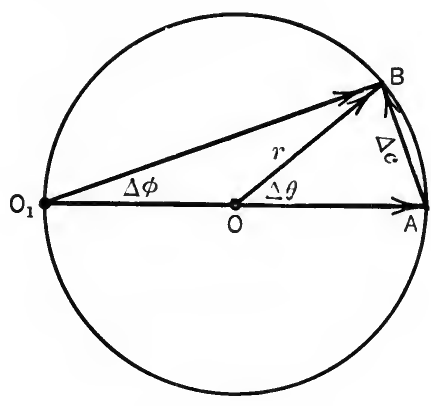

FIG. 269.

266. A point moves on a circular path of radius $r$ from $A$ to $B$ (Fig. 269), the angular displacement, with reference to the center, $O$, of the circle, being denoted by $\Delta \theta$ and, with reference to the pole $O_{1}$, by $\Delta \phi$. Show that the magnitude of $\Delta c$, the linear displacement of the point, is expressed by the equation,

$$
\Delta c=2 r \sin \frac{\Delta \theta}{2}
$$

If $\Delta \theta=\frac{\pi}{4}$ radians $\left(45^{\circ}\right)$, what is angular displacement, $\Delta \phi$, with respect to $O_{1}$ ?

\section{Relation between Linear} and Angular Displacements.-If a point moves along a circular path, the linear displacement, $d c$ (Fig. 270), corresponding to an indefinitely small angular displacement $d \theta$ may be considered to be coincident with the arc $d s$, which is subtended by the angle $d \theta$. Since the arc of a circle is the product of the radius and the central angle, when the angle is measured in radians, the rela-

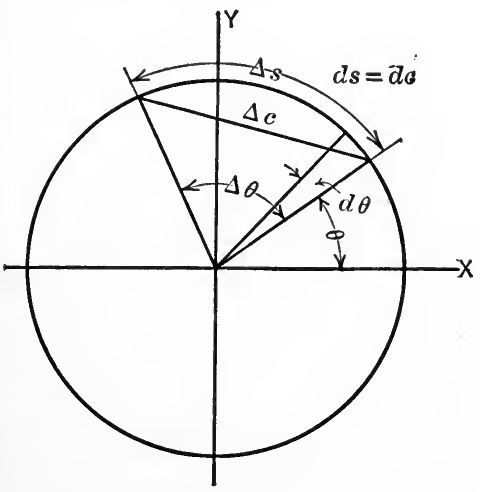

FIG. 270. 
tion between the linear and angular displacements may be expressed by the equation,

$$
d s=r d \theta .
$$

For a large angular displacement $\Delta \theta$, the corresponding linear

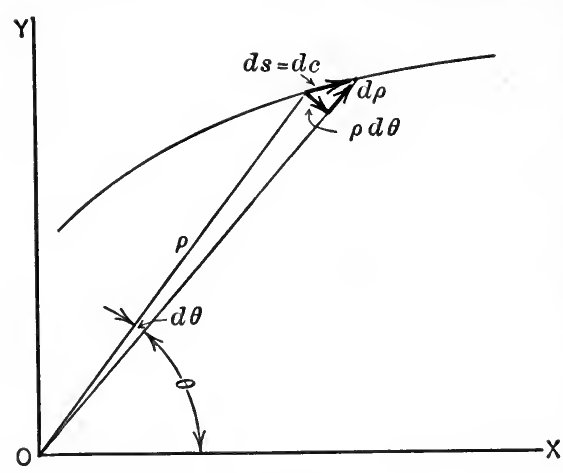

FIG. 271. displacement $\Delta c$ is not equal to $r \Delta \theta$. The distance $\Delta s$ along the arc, however, is expressed by $r \Delta \theta$.

If the moving point does not travel on a circular path, that is, if the path does not have a constant radius of curvature, the equation $d s=r d \theta$ may be used, provided that $r$ is the radius of curvature of the path at the given position of the point and that $d \theta$ is measured with respect to the center of curvature as the pole.

If, however, the pole is not chosen as the center of curvature of the path (Fig. 271), the displacement may then be expressed in terms of its two components as follows:

$$
d s=\rho d \theta+d \rho,
$$

in which $\rho$ is the radius vector to the point and not the radius of curvature of the path at that point.

112. Linear Velocity and Speed.-The linear velocity of a moving particle is the time rate at which the particle is changing position, or, more briefly, the time rate of linear displacement. The direction of the velocity of the moving particle at a given point on its path is tangent to the path at that point (Art. 109). Hence velocity, like displacement, possesses both magnitude and direction. The magnitude of the velocity of a point is called the speed of the point. Speed, therefore, is a scalar quantity. It may be defined as the time rate of describing distance (not the time rate of displacement). Although the terms velocity and speed are frequently used interchangeably, it is important to associate with the word velocity the two properties which it possesses, for, a change in 
the direciion of a velocity is fully as important in the laws of motion of physical bodies as is a change in the speed.

If a point has a uniform motion along any path, the speed of the point is the ratio of any distance, $\Delta s$, described by the point, to the corresponding interval of time, $\Delta t$. Thus,

$$
v=\frac{\Delta s}{\Delta t} \text {. }
$$

It will be noted that if a particle has uniform motion, whether rectilinear or curvilinear, the speed of the particle is constant. The velocity, however, is constant only in the case of uniform rectilinear motion, since in any curvilinear motion the velocity of the particle continually changes direction.

If the motion of the point is non-uniform, the above equation does not give the speed of the point at each instant in the interval, but gives only the average speed for the time interval, $\Delta t$. The instantaneous speed is the average speed over an indefinitely small period of time including the instant, or, expressed in mathematical form, the speed at any instant is,

$$
v=\underset{\Delta t \doteq 0}{\operatorname{Limit}} \frac{\Delta s}{\Delta t}=\frac{d s}{d t} . \quad . \quad . \quad . \quad .
$$

The direction of $v$, as already noted, is tangent to the path at the point on the path where the moving particle is located at the instant.

The unit of velocity may be any convenient unit of length per unit of time; such as, foot per second (ft./sec.), mile per hour (mi./hr.), centimeter per second (cm./sec.), etc.

In order to find the value of $v$ by differentiation, as indicated in equation (1), $s$ must be expressed algebraically in terms of $t$.

\section{ILLUSTRATIVE PROBLEM}

267. A point moves along a straight line path according to the law, $s=6 t^{2}+4$. What is the velocity of the point at the end of 5 sec.?

$$
\text { Solution.- } \quad v=\frac{d s}{d t}=\frac{d}{d t}\left(6 t^{2}+4\right)=12 t .
$$

Hence, when $t=5$ sec., the velocity of the point is,

$$
v=12 \times 5=60 \mathrm{ft} . / \mathrm{sec} \text {. }
$$




\section{PROBLEMS}

268. A point moves along a path according to the law $s=3 t^{2}+2 t+t^{-1}$, $s$ and $t$ being in feet and seconds, respectively. What is the speed of the point when $t=2$ sec.? Ans. $v=13.75 \mathrm{ft} . / \mathrm{sec}$.

269. A point moves along a straight line according to the law $v=t^{3}+4 t^{2}+2$. If $s=4 \mathrm{ft}$. when $t=2 \mathrm{sec}$., what is the value of $s$ when $t=3$ sec.?

Ans. $s=47.58 \mathrm{ft}$.

113. Distance-time Graph. - If a point moves in such a way that the distance $s$, in equation (1) of Art. 112, cannot be expressed algebraically in terms of $t$, or, if the relation between $s$ and $t$ leads to a complex equation, the speed may often be found conveniently, by a graphical process, from the distance-time $(s-t)$ curve.

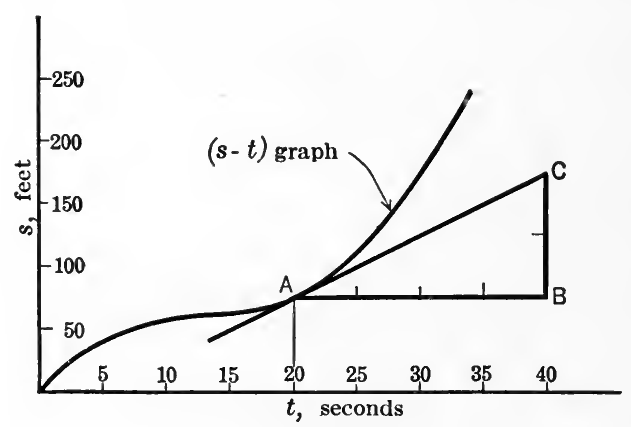

FIG. 272.

Thus, instead of expressing the relation between $s$ and $t$ algebraically, it is shown graphically by plotting a series of points, the rectangular coordinates of each point being simultaneously values of $s$ and $t$. One such graph is shown in Fig. 272.

Since the slope to the $(s-t)$ curve is represented by $\frac{d s}{d t}$ and since $v=\frac{d s}{d t}$ (Art. 112), then the slope at any point of the $(s-t)$ graph represents, to some scale, the speed at the corresponding instant. 


\section{ILLUSTRATIVE PROBLEM}

270. If the curve in Fig. 272 is the $s-t$ graph for a point moving on a straight-line path, what is the velocity of the point at the end of 20 sec.?

Solution:

$$
\text { Slope at } A=\frac{B C}{A B}=\frac{2 \text { units vertically }}{4 \text { units horizontally }}=\frac{1}{2} \text {. }
$$

But 1 unit vertically represents $50 \mathrm{ft}$. and 1 unit horizontally represents 5 sec. Hence,

$$
\text { Unit slope }=\frac{50 \mathrm{ft} .}{5 \mathrm{sec} .}=10 \mathrm{ft} . / \mathrm{sec} \text {. }
$$

Therefore velocity at end of 20 sec. $=\frac{1}{2} \times 10$

$$
=5 \mathrm{ft} . / \mathrm{sec} \text {. }
$$

114. Angular Velocity.-The angular velocity of a moving particle is defined as the time rate of angular displacement of the particle.

If equal angular displacements occur in equal time intervals, however small, the motion is said to be uniform, and the angular velocity, $\omega$, is expressed as the ratio of any angular displacement, $\Delta \theta$, to the time interval, $\Delta t$, during which the displacement occurs. Thus,

$$
\omega=\frac{\Delta \theta}{\Delta t}
$$

If unequal angular displacements occur in equal time intervals, the motion is said to be non-uniform or variable. For such motion the above equation gives the average angular velocity during the time interval $\Delta t$. When the angular velocity varies during the interval, its value at any instant is the average velocity over an indefinitely small time interval including the instant. Or, expressed mathematically, the instantaneous angular velocity is,

$$
\omega=\underset{\Delta t \doteq 0}{\operatorname{Limit}} \frac{\Delta \theta}{\Delta t}=\frac{d \theta}{d t}
$$

The unit of angular velocity is any cosrenient unit of angular displacement per unit of time; such as, degree per second (deg./sec.), revolution per minute (r.p.m.), radian per second (rad./sec.), etc. 
In order to determine the angular velocity from the above equation, $\theta$ must be expressed algebraically in terms of $t$, a requirement similar to that met in using the equation $v=\frac{d s}{d t}$ in Art. 112. The angular velocity may also be found from an angular displacement-time $(\theta-t)$ graph by a graphical method similar to that used in determining the linear velocity in Art. 113.

115. Relation between Linear and Angular Velocities.-In many problems in kinematics it is convenient to express the linear velocity of a point in terms of its angular velocity. The relation between the two velocities may be found as follows: Let a point $M$ move on a circular path of radius $r$ (Fig. 273a);
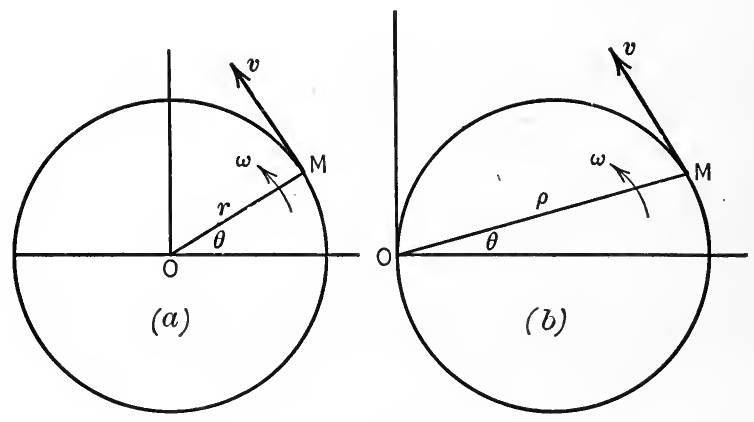

FIG. 273.

let $v$ be the linear velocity of the point at any instant and let $\omega$ be the angular velocity of the point at the same instant. From definition,

$$
v=\frac{d s}{d t} \text { and } \omega=\frac{d \theta}{d t}
$$

But the distance $d s$ traversed in the time $d t$ may be expressed in terms of the corresponding angular displacement $d \theta$, by the equation $d s=r d \theta$ (Art. 111). Therefore,

$$
v=\frac{r d \theta}{d t}=r \omega
$$

Hence, at any instant, the linear velocity of a point moving on a 
circular path is the product of its angular velocity ${ }^{1}$ (with respect to the center of the path) and the radius of the path.

If the particle does not move on a circular path, the equation $v=\omega r$ is also true if $r$ is the radius of curvature of the path at the given position of the particle, and if $\omega$ is the angular velocity of the particle with respect to the center of curvature as the pole.

Further, if the point moves on a curve of any form and the center of curvature is not taken as the pole (Fig. 273b), then the term $\rho \omega$, where $\rho$ denotes the radius vector to the point, gives one component only of the linear velocity, as is shown in the next article.

\section{PROBLEMS}

271. A flywheel $6 \mathrm{ft}$. in diameter rotates at 120 r.p.m. Find the linear velocity, in feet per second, of a point on its circumference.

272. A rod $4 \mathrm{ft}$. long rotates in a horizontal plane about a vertical axis through one end of the rod, so that the linear velocity of its mid-point is $60 \mathrm{ft}$. per sec. Find the angular velocity of the rod, in r.p.m.

273. An automobile is traveling at 30 miles per hour on a straight road. An observer is stationed at $O$ (Fig. 274). Find the angular velocity of the automobile with respect to the observer $(a)$ when the automobile is at $A ;(b)$ when at $B$.

116. Components of Velocity.-It is frequently convenient to find the velocity of a moving point by determining its components, or to deal

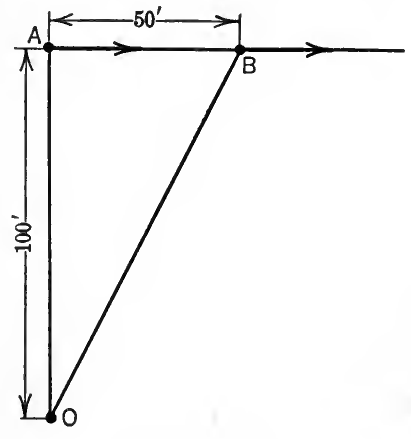

Fig. 274. with the components of the velocity instead of the total velocity. The assumption that velocity may be resolved into components

${ }^{1}$ When angular velocity is involved in the same expression with linear velocity, the angular velocity is expressed in radians per unit of time. Since the number of radians turned through is a length along a circular arc divided by the length of the radius and, hence, is a ratio of length to length, it has no dimensions. Thus the dimensional equation for $v=\omega r$ is $\frac{L}{T}=\left(\frac{L}{L} \times \frac{1}{T}\right) \times L$, and hence the equation is dimensionally correct (Art. 19). Likewise, angular displacement and angular acceleration are expressed in radians and radians per second ${ }^{2}$, respectively, under similar conditions. 
follows from the fact that velocity is a vector quantity. Its truth, however, is abundantly confirmed by the agreement of results, deduced by the use of velocity components, with observed facts.

Two sets of components only are here determined; namely, the

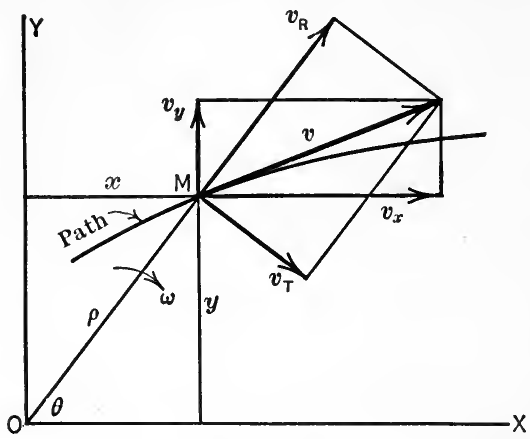

Fig. 275. axial components $\left(v_{x}\right.$ and $v_{y}$ ), parallel, respectively, to the $x$ - and- $y$ axes, and the radial and transverse components $\left(v_{R}\right.$ and $\left.v_{T}\right)$, parallel and perpendicular, respectively, to the radius vector (Fig. 275).

Thus, since the linear velocity of a point in any direction is the rate at which the position of the point is changing in the given direction, the axial components of the velocity $v$ of the moving point $M$ (Fig. 275) are given by the expressions, ${ }^{b}$

$$
v_{x}=\frac{d x}{d t} \quad \text { and } \quad v_{y}=\frac{d y}{d t}
$$

or, if the coordinates, $x$ and $y$, of the particle change uniformly, then,

$$
v_{x}=\frac{\Delta x}{\Delta t}=\frac{x_{2}-x_{1}}{t_{2}-t_{1}} \quad \text { and } \quad v_{\nu}=\frac{\Delta y}{\Delta t}=\frac{y_{2}-y_{1}}{t_{2}-t_{1}} .
$$

Likewise, at any instant, the transverse and radial components, $v_{T}$ and $v_{R}$, are expressed as follows:

$$
v_{T}=\frac{\rho d \theta}{d t} \quad \text { and } \quad v_{R}=\frac{d \rho}{d t},
$$

1 These equations follow from the definition of velocity provided that the assumption is made that the components of a displacement may be treated, in all respects, like the total displacement itself and that the vector sum of the rates of change of the components is the same as the rate of change of the total displacement. The equations may be derived through an algebraic series of steps beginning with the definition of a velocity component, $v_{x}=v \cos \alpha$ and substituting therein the values of $v$ and $\cos \alpha$; namely, $v=\frac{d s}{d t}$ and $\cos \alpha$ $=\frac{d x}{d s}$. This method gives an apparent definiteness to the conclusion but is lacking somewhat in its emphasis on the fundamental characteristics of the quantities involved. 
in which $\rho d \theta$ and $d \rho$ are the component displacements in the transverse and radial directions during the time interval $d t$, as shown in the preceding article, and $\omega$ is the angular velocity of the particle with reference to the pole $O$. It will be noted that when $O$ is chosen as the center of curvature of the path the transverse component of the velocity becomes the total velocity, tangent to the path, $v_{R}$ then being equal to zero.

Since the pole $O$ is arbitrarily chosen, the components $v_{T^{\prime}}$ and $v_{R}$ are different for different positions of the pole or origin, whereas $v_{x}$ and $v_{y}$ are independent of the origin but depend on the directions of the coordinate axes. Further, if the path of the point is circular and the pole $O$ is at the center or, in general, if $O$ is the center of curvature of the path, at the instant, then $d \rho$ and $\frac{d \rho}{d t}$ are zero and the expressions for $v_{T}$ and $v$ are the same.

\section{ILLUSTRATIVE PROBLEM}

274. In the quick-return mechanism shown in Fig. $276, O O_{1}=18$ in. and the crank $O A=8 \mathrm{in}$. If the angular velocity of the crank is 40 r.p.m., what is the velocity of the block $A$ ? Find graphically the component of the velocity of $A$ perpendicular to the rocker arm $O_{1} M$ (the transverse component). Find also the angular velocity of $O_{1} M$.

Solution.-The block moves on the circular path of radius $r=8$ in. Let $\omega$ denote the angular velocity of $A$ with reference to the pole $O$ and $\omega_{1}$ the angular velocity of $A$ with reference to the pole $O_{1}$ (that is, let $\omega_{1}$ denote the angular velocity of $O_{1} A$, or $\rho$ ). The direction of the velocity of $A$ is tangent to the circle and its magnitude is,

$$
v=\omega r=\frac{40 \times 2 \pi}{60} \times \frac{8}{12}=2.79 \mathrm{ft} . / \mathrm{sec} .
$$

By resolving $v$, graphically, into its transverse

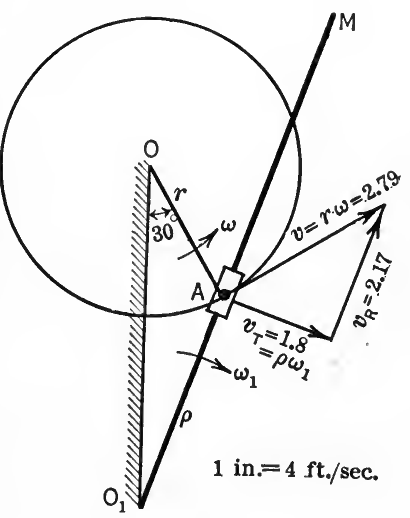

FIG. 276. and radial components as shown in the figure, the following values are found.

But,

$$
v_{T}=1.8 \mathrm{ft} . / \mathrm{sec} \text {. and } v_{R}=2.17 \mathrm{ft} . / \mathrm{sec} .
$$

$$
v_{T}=\omega_{1} \rho=\omega_{1} \times O_{1} A .
$$

By measuring, $O_{1} A$ is found to be 11.7 in. 
Therefore,

$$
\omega_{1}=\frac{1.8 \times 12}{11.7}=1.84 \mathrm{rad} . / \mathrm{sec} .=17.6 \text { r.p.m. }
$$

\section{PROBLEMS}

275. A rigid body composed of two disks of different diameters (Fig. 277) turns about the center $O$ so that a point on the circumference of each disk

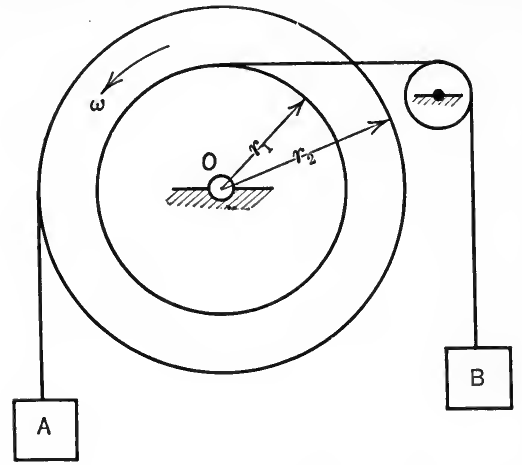

FIG. 277.

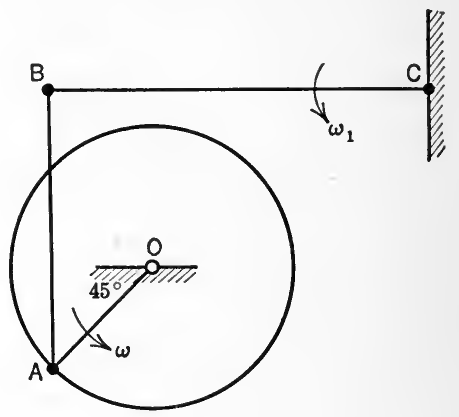

FIG. 278.

moves with a constant angular velocity of 80 r.p.m. If $r_{1}=2 \mathrm{ft}$. and $r_{2}=3 \mathrm{ft}$., what is the velocity of body $A$ and of body $B$ ? $A$ unwinds from the large disk as $B$ winds up on the small disk. How far will $A$ and $B$ travel in 6 sec.?

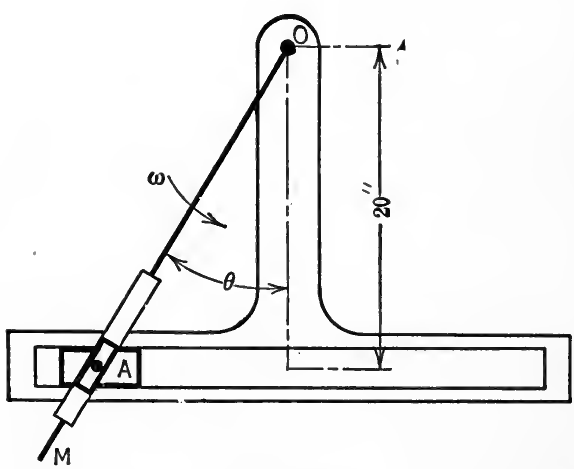

Fig. 279.

276. The link $B C$ (Fig. 278) is $2 \mathrm{ft}$. long and has an angular velocity, $\omega_{1}$, of 50 r.p.m. If the link $B A$ is perpendicular to $B C$, at the instant considered, what is the angular velocity of the crank $O A$ if $O A$ is 9 in. long?

277. If the angular velocity of the oscillating arm $O M$ (Fig. 279 ) is 40 r.p.m., in the position shown $\left(\theta=30^{\circ}\right)$, find the transverse component of the velocity of the block $A$, referred to $O$ as the pole. Also find the total velocity of $A$.

$$
\text { Ans. } v_{A}=9.3 \mathrm{ft} . / \mathrm{sec} \text {. }
$$

278. The cam shown in Fig. 280 revolves about the axis $O$, causing the roller $A$ to change its $x$-coordinate at the rate of 4 in. per second when $\theta=30^{\circ}$. Find the angular velocity of the bell-crank $A O_{1} B$ if $O_{1} A$ is 18 in. 


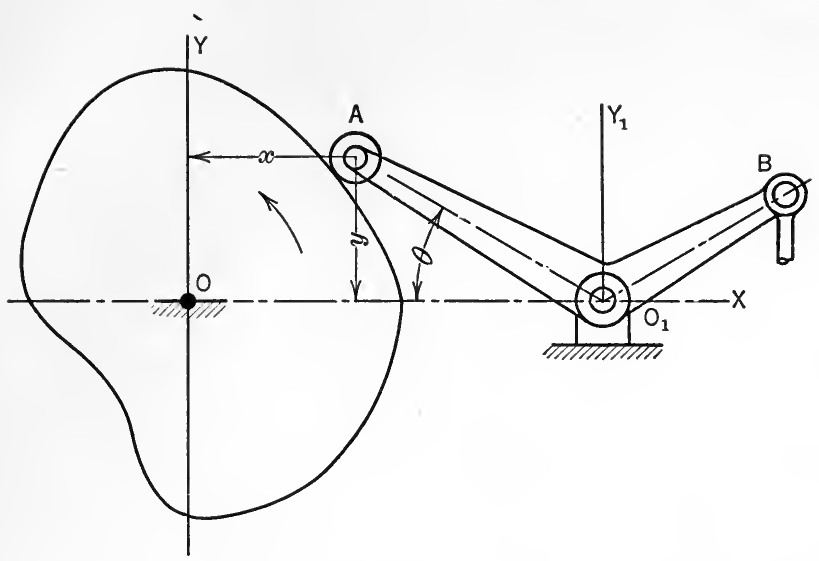

Frg. 280.

117. Linear Acceleration.-The acceleration of a moving point, at any instant, is the time rate at which its velocity is changing at the instant. The velocity of the point has, at any instant, a definite magnitude (speed) and a definite direction. A change in the velocity occurs if either its magnitude or its direction changes. Hence the acceleration of the point may be the rate of change of the velocity due to a change in the magnitude of the velocity, or it may be the rate of change of the velocity due to a change in the direction of the velocity. These statements are not mere arbitrary statements; they are in accord with our experience, for experience teaches that a particle tends to maintain the direction of its velocity as persistently as it tends to maintain the magnitude of its velocity. The two properties of velocity are inherent, independent properties. Hence,

Whenever the velocity of a particle changes in magnitude the particle is accelerated.

Whenever the velocity of a particle changes in direction the particle is accelerated.

If the direction and magnitude of the velocity change simultaneously, the acceleration of the particle is then the vector sum of the two accelerations which arise as the result of the separate changes.

In rectilinear motion the velocity of a particle can change in magnitude only, whereas in curvilinear motion the velocity of the 
particle may change in magnitude but must change in direction. Therefore a particle cannot have a curvilinear motion without being accelerated.

\section{PROBLEMS}

279. A quarter-mile track is made up of two straight parallel sides con-

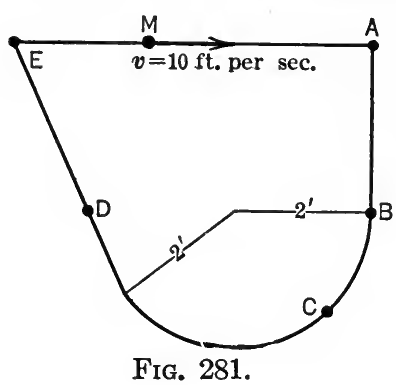
nected at the ends by semicircles. A boy runs the quarter mile in 50 seconds at uniform speed. Does the boy have an acceleration while on the straight sides? While on the curved ends? Why?

280. A particle moves with a constant speed of $10 \mathrm{ft}$. per sec. on the path shown in Fig. 281. Does the particle have an acceleration when at $A$ ? at $B$ ? at $C$ ? at $D$ ? What is the value of the acceleration of the particle when at $A$, and at $D$ ? .

118. Tangential and Normal Acceleration. Tangential Acceleration. - The facts stated in the preceding article make it possible to determine, for a given motion, whether or not a particle is being accelerated. The exact magnitude and direction of the acceleration produced in each of the two ways mentioned in the preceding article will now be found.

In Fig. 282 let a point move so that its velocity changes in

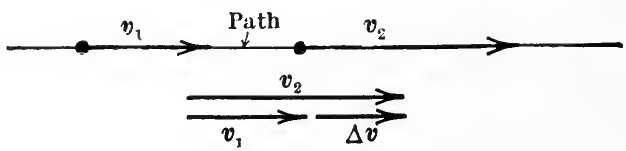

FIG. 282.

magnitude only. That is, let the point move on a straight line path. Let $v_{1}$ be the velocity at one instant and let $v_{2}$ be the velocity after the time interval $\Delta t$. If the velocity changes uniformly, the magnitude of the acceleration is the ratio of any change in the velocity, $\Delta v$, to the time interval, $\Delta t$, during which the change $\Delta v$ occurs. Since $v_{2}$ and $v_{1}$ have the same direction, $\Delta v$ is the algebraic difference of $v_{2}$ and $v_{1}$ as well as the vector difference. Hence, the magnitude of the acceleration for uniformly accelerated, rectilinear motion is,

$$
a-\frac{\Delta v}{\Delta t}=\frac{v_{2}-v_{1}}{t_{2}-t_{1}} \text {. . . . . . . . }
$$


If the velocity of the moving point in Fig. 282 does not change uniformly, then equation (1) gives only the average acceleration during the period $\Delta t$. When the acceleration varies from instant to instant its value at any instant is the average acceleration during a very small time interval including the instant. Or, expressed mathematically, the instantaneous acceleration for rectilinear motion is,

$$
a=\underset{\Delta t \doteq 0}{\operatorname{Limit}} \frac{\Delta v}{\Delta t}=\frac{d v}{d t}
$$

$v$ being the velocity of the particle at the instant. In order to find $a$ from the above equation, $v$ must be expressed algebraically in terms of $t$.

The direction of the acceleration of the particle is the same as that of the change of velocity, $\Delta v$, but $\Delta v$ is parallel to $v$, that is, along (or tangent to) the path. If $v_{2}$ is smaller than $v_{1}$, the sense of $\Delta v$ is negative, that is, opposite to that of $v$, and hence the acceleration then is negative.

If the particle moves on a circular path so that, as the velocity changes from $v_{1}$ to $v_{2}$, its direction as well as its magnitude changes, then $\frac{d v}{d t}$ expresses only that part of the acceleration which is due to the change of the magnitude of the velocity. And the direction of $\frac{d v}{d t}$ at any instant, as already noted, is parallel to the velocity, or tangent to the path at the point where the particle is located at the instant. Therefore, $\frac{d v}{d t}$ is called the tangential acceleration and is written,

$$
a_{t}=\frac{d v}{d t}
$$

Hence, $\frac{d v}{d t}$ is the total acceleration of a moving point, only, when the point has a rectilinear motion.

Normal Acceleration.-The acceleration produced by a change in the direction of the velocity of a point will now be considered. In Fig. 283 let a particle move on a circular path with constant speed. That is, let the velocity change in direction only. At one instant the velocity is $v_{1}$, and during a time interval $\Delta t$ the velocity changes to $v_{2}$. The change in the velocity during the time interval, that is, the vector difference between $v_{2}$ and $v_{1}$, is 
$\Delta v$, as shown in Fig. 283, since $\Delta v$ is the velocity that must be added to $v_{1}$ to give $v_{2}$, or, $v_{2}$ is the resultant of $v_{1}$ and $\Delta v$. For

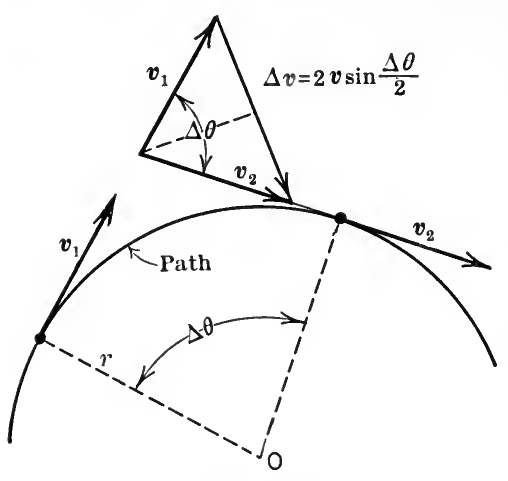

FIG. 283. motion in which the direction of the velocity does not change (rectilinear motion), the vector difference of the velocities of the point and the scalar (or algebraic) difference are the same, but in curvilinear motion (Fig. 283) the algebraic difference is zero, whereas the vector difference, or change, of the velocities, is $\Delta v$, the magnitude of which is,

$$
\begin{aligned}
\Delta v & =v_{2} \rightarrow v_{1} \\
& =2 v_{1} \sin \frac{\Delta \theta}{2}=2 v_{2} \sin \frac{\Delta \theta}{2} \\
& =2 v \sin \frac{\Delta \theta}{2},
\end{aligned}
$$

in which $v$ is the magnitude of the velocity at any instant, since $v_{2}=v_{1}=v$. Hence, since the acceleration at any instant is the average acceleration during an indefinitely small time interval including the instant, the magnitude of the instantaneous acceleration due to the change of direction of the velocity is,

$$
a=\underset{\Delta t \doteq 0}{\operatorname{Limit}} \frac{2 v \sin \frac{\Delta \theta}{2}}{\Delta t} .
$$

Now, as $\Delta t$ approaches zero, $\sin \frac{\Delta \theta}{2}$ approaches $\frac{\Delta \theta}{2}$ and $\frac{\Delta \theta}{\Delta t}$ approaches $\frac{d \theta}{d t}$. Hence, in the limit,

$$
a=v \frac{d \theta}{d t} .
$$

But $\frac{d \theta}{d t}$ is the angular velocity of the moving point which corre- 
sponds, at any instant, to the linear velocity $v$. Further, $v=\omega r$ (Art. 115). Therefore,

$$
a=v \omega=\omega^{2} r=\frac{v^{2}}{r} .
$$

The particular form most convenient for use depends upon the particular problem under consideration.

The direction of this acceleration is the limiting direction of $\Delta v$ as $\Delta t$ approaches zero, and since $\Delta \theta$ approaches zero as $\Delta t$ approaches zero, it will be seen from Fig. 283 that the limiting direction of $\Delta v$ is perpendicular to $v_{1}$ and, hence, is toward the center about which the point is turning, that is, normal to the path. This acceleration is, therefore, called the normal acceleration and is written,

$$
a_{n}=v \omega=\omega^{2} r=\frac{v^{2}}{r} .
$$

If the path on which the particle moves is not a circular path, the above expression also holds provided that $r$ is the radius of curvature of the path at the point where the particle is located at the instant, and that $\omega$ is the angular velocity with respect to the center of curvature as a pole. Summarizing, the two following important theorems may be stated:

I. When the velocity, $v$, of a particle changes in magnitude, an acceleration is produced the value of which is $\frac{d v}{d t}$; its direction at any instant is parallel to that of the velocity, that is, tangent to the path at the point at which the particle is located at the instant. Thus,

$$
a_{t}=\frac{d v}{d t}
$$

II. When the velocity, $v$, of a particle changes in direction, an acceleration is produced the value of which is $v \omega=\omega^{2} r=\frac{v^{2}}{r} ;$ its direction, at any instant, is perpendicular to the velocity, towards the center of curvature of the path, at the point where the particle is located at the instant. Thus,

$$
a_{n}=v \omega=\omega^{2} r=\frac{v^{2}}{r}
$$


The unit of acceleration is any convenient unit of velocity per unit of time; such ás, foot per second per second (ft./sec. ${ }^{2}$ ), mile per hour per second (mi./hr./sec.), etc.

\section{ILLUSTRATIVE PROBLEM}

281. Two pulleys are connected so that they turn together about the center $O$ (Fig. 284), causing the weight $A$ to unwind and the weight $B$ to wind up. If the angular velocity of the points $M$ and $P$ change uniformly from 10 r.p.m. to 60 r.p.m. during a period of 2 sec., find: (a) the tangential acceleration of each of the two points at any instant during the 2 sec.; $(b)$ the acceleration of $A$ and of $B ;(c)$ the total acceleration of $M$ at the beginning and of $P$ at the end of the two-second period.

Solution.-(a) The velocity of the point $M$ changes in magnitude from $v_{1}=\omega_{1} r_{1}=\frac{10 \times 2 \pi}{60} \times \frac{3}{4}=\frac{\pi}{4} \mathrm{ft}$./sec. to $v_{2}=\omega_{2} r_{1}=\frac{3}{2} \pi \mathrm{ft}$./sec. in 2 sec. Hence the tangential acceleration of $M$ is,

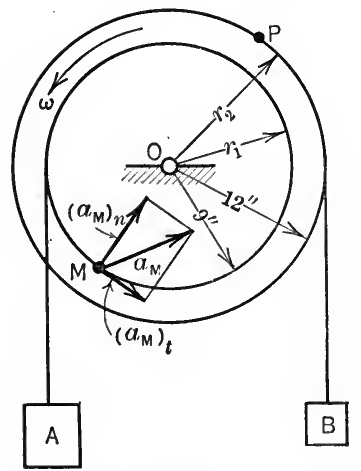

FIG. 284.

$$
\left(a_{M}\right)_{t}=\frac{v_{2}-v_{1}}{\Delta t}=\frac{\frac{8}{2} \pi-\frac{1}{4} \pi}{2}=\frac{5}{4} \pi=1.96 \mathrm{ft} . / \text { sec. }^{2} .
$$

The velocity of $P$ changes from $\frac{\pi}{3} \mathrm{ft}$./sec. to $2 \pi \mathrm{ft}$./sec. in 2 sec. Hence,

$$
\left(a_{P}\right)_{t}=\frac{v_{2}-v_{1}}{\Delta t}=\frac{2 \pi-\frac{1}{3} \pi}{2}=\frac{5}{6} \pi=2.62 \mathrm{ft} . / \mathrm{sec}^{2}
$$

(b) The magnitude of the velocity of any point on the circumference of the small pulley changes the same amount as does the velocity of $A$. Therefore, the tangential acceleration of $M$ is the same in magnitude as the acceleration of $A$. Hence,

Likewise,

$$
a_{A}=\left(a_{M}\right)_{t}=1.96 \mathrm{ft} . / \mathrm{sec}^{2}
$$

$$
a_{B}=\left(a_{P}\right)_{t}=2.62 \mathrm{ft} . / \mathrm{sec}^{2} \text {. }
$$

(c) The normal acceleration of $M$ at the beginning of the 2 -see. period is,

$$
\left(a_{M}\right)_{n}=\omega_{1}^{2} r_{1}=\left(\frac{10 \times 2 \pi}{60}\right)^{2} \times \frac{9}{12}=0.82 \mathrm{ft} . / \text { sec. }^{2}
$$

and the normal acceleration of $P$ at the end of 2 scc. is,

$$
\left(a_{P}\right)_{n}=\omega_{2}^{2} r_{2}=\left(\frac{60 \times 2 \pi}{60}\right)^{2} \times \frac{12}{12}=39.4 \mathrm{ft} . / \text { sec. }^{2} .
$$

Therefore the total acceleration of $M$ is,

$$
a_{M}=\sqrt{(1.96)^{2}+(.82)^{2}}=2.12 \mathrm{ft} . / \text { sec. }^{2}
$$


And the total acceleration of $P$ is,

$$
a_{P}=\sqrt{(2.62)^{2}+(39.4)^{2}}=39.5 \mathrm{ft} . / \text { sec. }^{2} .
$$

The acceleration of $M$, only, is shown in Fig. 284 .

\section{PROBLEM}

282. A train, while rounding a curve of $\frac{1}{4}$ mile radius, changes its speed uniformly from $20 \mathrm{mi}$./ hr. to $30 \mathrm{mi}$./ hr. in $20 \mathrm{sec}$. What is the total acceleration of the train at the beginning and at the end of the 20 -sec. period?

119. Speed-time Graph.-In finding the total acceleration of a point from the equation $a=\frac{d v}{d t}$ (in rectilinear motion), or, in finding the tangential acceleration from the equation $a_{t}=\frac{d v}{d t}$ (in curvilinear motion), by the calculus method, $v$ must be expressed algebraically in terms of $t$. The relation between $v$ and $t$ may sometimes be shown more conveniently, graphically, by plotting a speed-time $(v-t)$ graph the coordinates of any point on which are simultaneous values of the speed and time of the moving point (similar to the distance-time graph in Art. 113). One such graph is shown in Fig. 285. Since the slope of the speed-time curve is represented by $\frac{d v}{d t}$, the slope of the $v-t$ graph at any point represents, to some scale, the acceleration of the moving point at the corresponding instant. It is important to note that this method gives only that acceleration which is due to the change in the magnitude of the velocity. It is applicable, therefore, only to the total acceleration for rectilinear motion or to the tangential acceleration for curvilinear motion.

\section{ILLUSTRATIVE PROBLEM}

283. In Fig. 285 is represented the speed-time graph for a point which moves on a circular path. What is the magnitude of the tangential acceleration at the end of 15 sec.?

Solution. $-a_{t}$ at the end of 15 sec.

$$
\text { =slope at } A
$$$$
=\frac{2 \times 20}{4 \times 5}=2 \mathrm{ft} . / \mathrm{sec}^{2}
$$

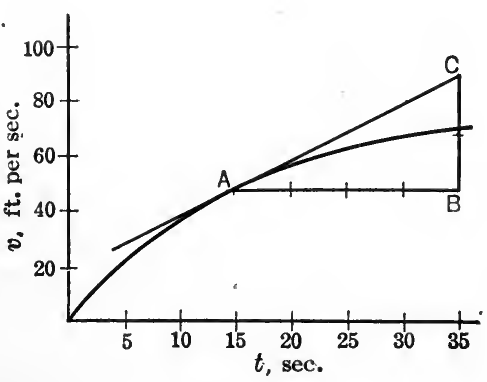

FIG. 285. 


\section{PROBLEMS FOR ARTICLES 118 AND 119}

284. A train running on a straight track has a speed of $30 \mathrm{mi}$. per hr. when passing a certain mile post and a speed of $50 \mathrm{mi}$. per hr. when passing the next mile post. If the rate of change of speed is uniform and the time required to travel the mile is $1.5 \mathrm{~min}$, , what is the acceleration of the train?

285. A point moves on a circular path having a radius of $2 \mathrm{ft}$. If its speed changes uniformly from $100 \mathrm{ft}$. per min. to $240 \mathrm{ft}$. per min. during a period of 3 sec., what are the tangential and normal accelerations $(a)$ at the beginning of the period; $(b)$ at the end of the period?

286. A flywheel $8 \mathrm{ft}$. in diameter turns so that the angular velocity of a point on its rim changes from 100 r.p.m. to 40 r.p.m. at a uniform rate during a period of $4 \mathrm{sec}$. Find the tangential acceleration of a point on the rim during the $4-\mathrm{sec}$. period. Find the total acceleration of a point on the rim at the end of the period. Ans. $a_{t}=6.28 \mathrm{ft} . / \mathrm{sec}^{2} ; a=70.5 \mathrm{ft} . / \mathrm{sec}^{2}{ }^{2}$

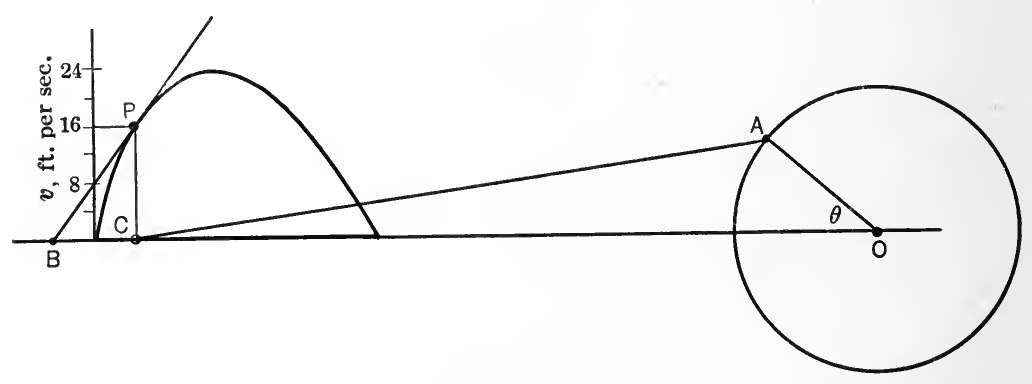

FIG. 286.

287. In Fig. 286 is shown the speed-displacement graph for the crosshead, $C$, of a steam engine. The crank $O A$ revolves at a constant speed of 233 r.p.m. Show that the slope to the speed-displacement curve represents the ratio of the acceleration of $C$ at any instant to its speed at the same instant. If the scale of abscissas is $1 \mathrm{in} .=1.75 \mathrm{ft}$. and if $B C$ measures $0.33 \mathrm{in}$., what is the acceleration of the crosshead in the position shown? How could the position of $C$ be found for which $C$ has zero acceleration?

120. Angular Acceleration.- The angular acceleration of a moving point is the time rate of change of the angular velocity of the point. If the angular velocity, $\omega$, of the point changes uniformly, the angular acceleration, $\alpha$, is expressed by the ratio of any change, $\Delta \omega$, in the angular velocity to the corresponding time interval, $\Delta t$. Thus,

$$
\alpha=\frac{\Delta \omega}{\Delta t}=\frac{\omega_{2}-\omega_{1}}{t_{2}-t_{1}}
$$


If the angular velocity of the point does not change uniformly, the above equation gives only the average acceleration during the time interval $\Delta t$. When the acceleration varies from instant to instant its value at any instant is the average acceleration during an indefinitely small time interval including the instant. Or, expressed mathematically, the instantaneous angular acceleration is,

$$
\alpha=\underset{\Delta t \doteq 0}{\operatorname{Limit}} \frac{\Delta \omega}{\Delta t}=\frac{d \omega}{d t}
$$

And since $\omega=\frac{d \theta}{d t}, \alpha$ may also be expressed by the equation,

$$
\alpha=\frac{d}{d t}\left(\frac{d \theta}{d t}\right)=\frac{d^{2} \theta}{d t^{2}} .
$$

In order to find $\alpha$ from the above equations, $\omega$ and $\theta$ must be expressed in terms of $t$.

The unit of angular acceleration is any convenient unit of angular velocity per unit of time; such as, degree per second per second (deg./sec. ${ }^{2}$ ), revolution per minute per second (rev./min./ sec.) radian per second per second (rad./sec.. $\left.{ }^{2}\right)$, etc.

\section{PROBLEMS}

288. What is the angular acceleration of a point on the rim of the flywheel having the motion described in Problem 286?

289. What is the angular acceleration of the train having the motion described in Problem 282? Ans. $\alpha=0.000555 \mathrm{rad} . / \mathrm{sec}^{2}$

290. A particle moves on a circular path according to the law $\theta=3 t^{2}+2 t$. What is the angular velocity and the angular acceleration of the particle at the end of 4 sec.?

121. Relation between Linear and Angular Accelerations.-In many problems in kinematics it is convenient to make use of the relation between the linear and angular accelerations of a point. This relation may be found as follows: In Fig. 287 let a point move on the circular path shown, $O$ being the center of the

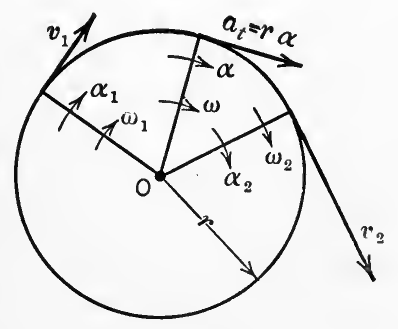

Fig. 287. 
path and $r$ the radius. If the magnitude of the velocity of the point changes (assumed to increase) there is, at any instant during the change, a tangential acceleration given by the equation,

$$
a_{\imath}=\frac{d v}{d t} .
$$

But,

$$
v=r \omega .
$$

Therefore $a_{t}=\frac{d(\omega r)}{d t}=r \frac{d \omega}{d t}$, since $r$ is constant. But $\frac{d \omega}{d t}$ is the angular acceleration, $\alpha$, of the point at the given instant. Hence,

$$
a_{t}=r \alpha .
$$

Therefore the tangential acceleration, ${ }^{1}$ at any instant, of a point moving on a circular path is equal to the angular acceleration of the point, at the same instant, about the center of the path

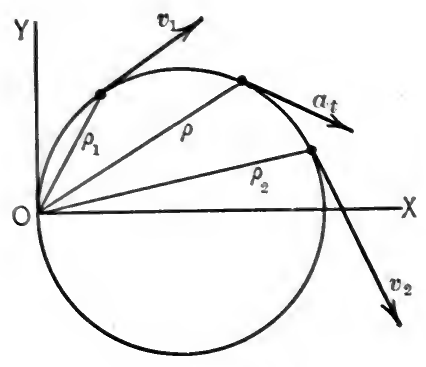

Fig. 288.

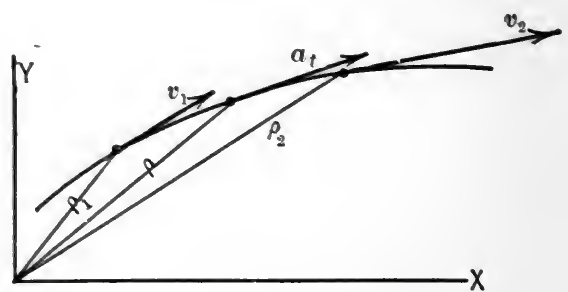

FIG. 289.

times the radius of the circle. If the particle does not move on a circular path, the equation is also true provided that $r$ is the radius of curvature of the path at the given position of the particle and that $\alpha$ is the angular acceleration of the particle with reference to the center of curvature.

If, however, the point moves on a path of any form and the pole is not taken at the center of curvature of the path at the given position of the particle (Figs. 288 and 299), then, although the equation $a_{t}=\frac{d v}{d t}$ is true, $a_{t}$ is not equal to $\rho \alpha$ since $v$ is not equal to $\rho \omega$ (Art. 115).

${ }^{1}$ See footnote for Art. 115. 
The normal acceleration, $r \omega^{2}$, of the particle (not shown in the above diagrams), unlike the tangential acceleration, is independent of the angular acceleration. It depends on the angular velocity at the instant, and not on the rate at which the angular velocity is changing at the instant.

\section{PROBLEMS}

291. As the drum (Fig. 290) turns, the weight $A$ is wound up with decreasing speed. If its speed decreases $20 \mathrm{ft}$. $/ \mathrm{sec}$. each second, what is the angular acceleration of a point on the rim of the drum?

292. A rod $3 \mathrm{ft}$. long is rotated in a horizontal plane about a vertical axis through one end of the rod so that the angular velocity increases uniformly from 10 to 40 r.p.m. in 3 sec. Find the tangential acceleration of the mid-point of the rod. Ans. $a_{t}=1.57 \mathrm{ft} . /$ sec. $^{2}$

122. Axial Components of Acceleration.-The component, in a given direction, of an acceleration of a point is the

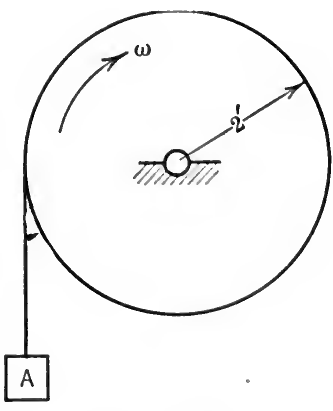

FIG. 290. rate of change, in the given direction, of the velocity of the point. In general, the rate of change, in the given direction, of the velocity of the point is due partly to a change in the magnitude and partly to a change in the direction

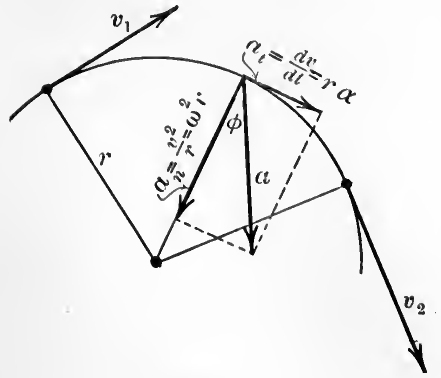

FIG. 291. of the velocity. It will be noted that the tangential and normal accelerations, $a_{t}$ and $a_{n}$, which are discussed in the preceding articles are components of an acceleration, one of . which, $a_{t}$, is the rate of change of the velocity due to a change in its magnitude only, and the other, $a_{n}$, is the rate of change of the velocity due to a change in its direction only. Thus, if a point moves on a curved path with increasing (or decreasing) speed (Fig. 291), both the magnitude and the direction of the velocity changes and the expressions for the total acceleration as found in the preceding articles are, 


$$
\begin{aligned}
a & =a_{t} \rightarrow a_{n} \\
& =\frac{d v}{d t}+\frac{v^{2}}{r}=r \alpha+r \omega^{2} \\
& =\sqrt{a_{l}^{2}+a_{n}^{2}}=r \sqrt{\omega^{4}+\alpha^{2}},
\end{aligned}
$$

and the direction of $a$ is expressed by the equation,

$$
\tan \phi=\frac{a_{t}}{a_{n}} .
$$

Acceleration, like any directed quantity, may be resolved into various sets of components. The axial components, in addition to the tangential and normal components, are frequently desired. The axial components $\left(a_{x}\right.$ and $a_{y}$ ) are parallel, respectively, to the $x$-and $y$-axes. Expressions for the axial components may be found as follows:

If the velocity of a point is resolved into components in arbi-

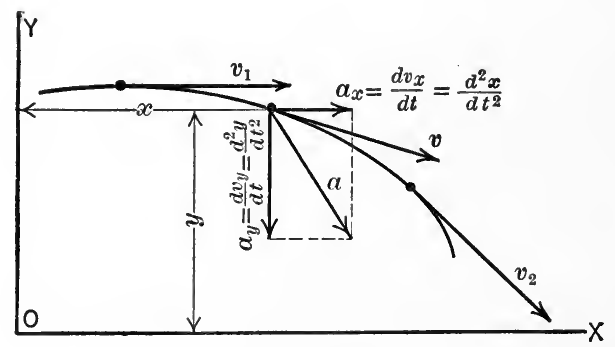

FIG. 292.

trarily selected directions, as, for example, parallel to the $x$ - and $y$ axes, then the acceleration of the point is the (vector) sum of the rates of change in these components. But since the directions of the velocity components are fixed, the components can change in magnitude only, and hence the acceleration in each of the selected directions is of the type $\frac{d v}{d t}$ (Theorem I, Art. 118). Therefore the axial components of the acceleration of a point (Fig. 292) are,

$$
\boldsymbol{a}_{x}=\frac{d v_{x}}{d t} \quad \text { and } \quad \boldsymbol{a}_{y}=\frac{d v_{y}}{d t}
$$


And since $v_{x}=\frac{d x}{d t}$ and $v_{y}=\frac{d y}{d t}$, the axial components may also be written,

$$
a_{x}=\frac{d^{2} x}{d t^{2}} \quad \text { and } \quad a_{y}=\frac{d^{2} y}{d t^{2}} .
$$

In order to determine the axial components from the above equations, $v_{x}$ (or $x$ ) and $v_{y}$ (or $y$ ) must be expressed in terms of $t$, unless $v_{x}$ and $v_{y}$ change uniformly in which case,

and,

$$
a_{x}=\frac{\Delta v_{x}}{\Delta t}=\frac{v^{\prime \prime}{ }_{x}-v_{x}^{\prime}}{t_{2}-t_{1}}
$$

$$
a_{y}=\frac{\Delta v_{y}}{\Delta t}=\frac{v^{\prime \prime}{ }_{y}-v^{\prime}{ }_{y}}{t_{2}-t_{1}}
$$

It is important to note that the velocity of a point in a given direction may be zero without making the acceleration in that direction zero. For example, a point moving on a circular path has no velocity component normal to the path, that is, $v_{t}$ is the total velocity $\left(v_{n}=0\right)$ but there is a normal acceleration (rate of change, in the normal direction, of the velocity) the magnitude of which as already shown, is $\frac{v^{2}}{r}$. Similarly, a ball thrown horizontally from a window has no vertical velocity just as it leaves the window, that is, $v_{y}=0$, but the acceleration of the ball at that instant is $a_{y}=32.2 \mathrm{ft}$. $/ \mathrm{sec}^{2} \quad$ In other words, $v_{y}$ is changing through its zero value at the rate of $32.2 \mathrm{ft}$./ sec. each second. Likewise, the velocity of the crosshead of a steam engine is zero at the end of the stroke, but its acceleration, $\frac{d v}{d t}$, has a large value as the velocity changes through its zero value.

\section{PROBLEMS}

293. The total acceleration of a point on the rim of a pulley, at a given instant, is $40 \mathrm{ft} . / \mathrm{sec}^{2}$ in a direction making an angle of $20^{\circ}$ with the radius to the point. If the radius of the pulley is $18 \mathrm{in}$. what is the angular velocity and the.angular acceleration of the point at the given instant?

Ans. $\omega=5.01 \mathrm{rad} . / \mathrm{sec}$.; $\alpha=9.12 \mathrm{rad} . / \mathrm{sec}^{2}$

294. A particle moves on the path $x y=4$, according to the law, $x=2 t^{2}$. (a) Find the $x$-and $y$-components of the velocity and of the acceleration at the end of 2 sec. (b) Determine the total acceleration by combining the components graphically. (c) Find the tangential and normal components graphically by resolving the total acceleration in the tangential and normal directions. 
123. Motion of a Projectile.-The equations developed in the preceding article will here be used in the analysis of the motion of a projectile. The actual motion of a projectile is influenced by a number of factors, such as the rotation of the projectile due to the rifling of the barrel, wind velocity, humidity, etc., which require modifications in the results found from the assumed ideal conditions. The motion of a projectile moving without rotation in a vacuum will here be considered. The motion may be studied by treating the components of the motion in the $x$ - and $y$-directions as follows:

Let a projectile be given an initial velocity $u$ in a direction

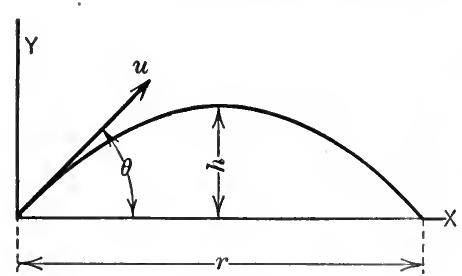

Fig. 293. making an angle $\theta$ with the horizontal and let the point of projection be taken as the origin as shown in Fig. 293. The acceleration of the projectile during its flight is constant and is directed vertically downward. This constant acceleration, denoted by $g$, is the acceleration due to gravity, since the projectile is a freely falling body, that is, no forces except the earth-pull (its weight) act on it. Hence,

$$
a_{x}=\frac{v_{x}-u_{x}}{t}=0 \quad \text { or } \quad v_{x}=u_{x}=u \cos \theta, \quad \text {. . . . . }
$$

and,

$$
a_{y}=\frac{v_{y}-u_{y}}{t}=-g \quad \text { or } \quad v_{\nu}=u_{\nu}-g t=u \sin \theta-g t, \quad .
$$

considering $v_{y}$ and $u_{y}$ positive when directed upward.

The horizontal distance, $x$, traveled in any time interval $t$ is,

$$
x=v_{x} \cdot t=u \cos \theta \cdot t,
$$

and the vertical distance, $y$, traveled in any time interval $t$ is the average velocity times the time interval. Hence,

$$
y=\frac{u \sin \theta+(u \sin \theta-g t)}{2} \cdot t=u \sin \theta \cdot t-\frac{1}{2} g t^{2} . \quad .
$$

Particular values found from the above equations are of special importance, namely, the range on a horizontal plane, the greatest height, and the time corresponding to each of these distances. 
Time of Flight.-Since $y$ equals zero when the projectile reaches the $x$-axis, the time of flight, $t_{r}$, is given by $t$ in the equation,

Therefore,

$$
y=u \sin \theta \cdot t-\frac{1}{2} g t^{2}=0 .
$$

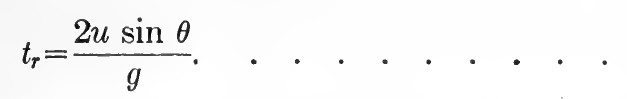

Range.-The range, $r$, equals the value of $x$ in equation (3) when $t=t_{r}$. Therefore,

$$
r=\frac{u^{2} \sin 2 \theta}{g} .
$$

Time to Reach Greatest Height.-When the projectile reaches its greatest height, $v_{\nu}=0$. Hence, the time, $t_{h}$, required for the projectile to reach its greatest height is given by $t$ in the equation,

Therefore,

$$
v_{\nu}=u \sin \theta-g t=0 .
$$

$$
t_{h}=\frac{u \sin \theta}{g} \text {. }
$$

Greatest Height.-The greatest height, $h$, will be given by $y$ in equation (4) when $t$ has the value $t_{h}$. Hence,

$$
h=\frac{1}{2} \frac{(u \sin \theta)^{2}}{g} .
$$

The equation of the path of the projectile (called the trajectory) may be obtained by eliminating $t$ from equations (3) and (4), which gives the following equation:

$$
y=x \tan \theta-\frac{g x^{2}}{2 u^{2} \cos ^{2} \theta} .
$$

Hence the trajectory is a portion of a parabola with its axis vertical.

\section{PROBLEMS}

295. A bullet is projected upward at an angle of $60^{\circ}$ with the horizontal with a velocity of $2000 \mathrm{ft}$./sec. Find the range and time of flight.

Ans. $r=108,000 \mathrm{ft}$.

296. A shot is fired from a gun on the top of a cliff $400 \mathrm{ft}$. high, with a velocity of $768 \mathrm{ft} . / \mathrm{sec}$., the angle of elevation of the gun being $30^{\circ}$. Find the range on a horizontal plane through the base of the cliff. Ans. $r=5543 \mathrm{yd}$. 
124. Uniformly Accelerated Rectilinear Motion.-Many examples of straight-line motion with constant acceleration occur in engineering practice, such as the motion of a freely falling body or of a train leaving a station under the action of a constant draw-bar pull. Further, from the preceding article it will be noted that one of the components of a curvilinear motion may be a uniformly accelerated rectilinear motion. The relations between the distance, time, velocity, and acceleration, for uniformly accelerated rectilinear motion may be deduced as follows:

By definition,

$$
\begin{aligned}
& a=\frac{\Delta v}{\Delta t}=\frac{v-u}{t}, \\
& v=u+a t,
\end{aligned}
$$

in which $u$ and $v$ are the initial and final velocities, respectively, corresponding to the time interval $\Delta t$ or simply $t$.

Since the velocity increases or decreases uniformly, the average velocity is $\frac{u+v}{2}$ and the distance, $s$, traveled in time $t$ is,

$$
\begin{aligned}
s & =\frac{u+v}{2} \cdot t \\
& =\frac{u+u+a t}{2} \cdot t \\
& =u t+\frac{1}{2} a t^{2} . \quad . \quad . \quad . \quad . \quad . . .
\end{aligned}
$$

By eliminating $t$ from equations (2) and (4), the following equation is obtained:

$$
v^{2}=u^{2}+2 a s
$$

Hence, for uniformly accelerated, rectilinear motion, the following equations express the relations between the distance, time, velocity, and acceleration,

$$
\begin{aligned}
& v=u+a t, \\
& s=u t+\frac{1}{2} a t^{2}, \\
& v^{2}=u^{2}+2 a s, \\
& s=\frac{u+v}{2} \cdot t .
\end{aligned}
$$


If the point starts from rest, $u$ in each of the above equations is zero, and the equations reduce to simpler forms. For freely falling bodies, $a$ is the acceleration due to the earthpull on the body and is usually denoted by $g$, its value being $32.2 \mathrm{ft}$. $/ \mathrm{sec}^{2}$ (approximately). Further, the above equations also apply to the motion of a point moving on a curved path if the speed changes uniformly and if the tangential acceleration $a_{b}$ of the point is used for $a$.

\section{PROBLEMS}

297. Deduce equations (6) and ( 7 ) by calculus methods, starting with the equations, $v=\frac{d s}{d t}$ and $a=\frac{d v}{d t}$.

298. Draw a speed-time graph for uniformly accelerated, rectilinear motion and deduce equations $(\overline{7})$ and $(9)$.

299. The brakes are set on a train running at $30 \mathrm{mi}$. per $\mathrm{hr}$., when $\frac{1}{2} \mathrm{mi}$. from a station. The train slows down uniformly, coming to rest at the station. Find the acceleration, and the time in stopping.

$$
\text { Ans. } a=0.366 \mathrm{ft} . / \mathrm{sec}^{2}{ }^{2} . \quad t=120 \mathrm{sec} .
$$

300. If the maximum allowable speed of an elevator is $800 \mathrm{ft}$. $/ \mathrm{min}$. and if it acquires this speed uniformly in a distance of $12 \mathrm{ft}$., what acceleration does it have?

301. A train in starting is uniformly accelerated and attains a speed of 60 $\mathrm{mi}$. $/ \mathrm{hr}$. in $5 \mathrm{~min}$. After running for a certain period of time at this speed, the brakes are applied and it stops at a uniform rate in $4 \mathrm{~min}$. If the total distance traveled is $10 \mathrm{mi}$. find the total time. Ans. $t=14.5 \mathrm{~min}$.

302. If the cam $A$ (Fig. 294) moves

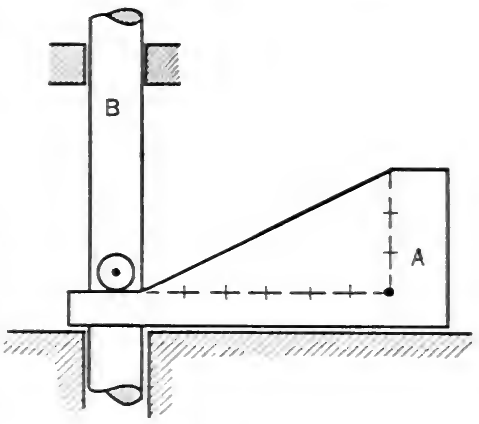

FIG. 294. to the left, changing its velocity uniformly 5 in. per sec. each second, what is the acceleration of the rod $B(a)$ before the pin comes in contact with the cam; (b) just as it comes in contact with the cam; (c) while it is in contact with the cam?

125. Uniformly Accelerated Circular Motion.-Many problems involving the motion of a point on a circular path with constant angular acceleration occur in engineering practice. The relation between the angular displacement, angular velocity, 
angular acceleration, and time may be deduced in a manner similar to that used in the preceding article.

By definition,

or,

$$
\begin{aligned}
& \alpha=\frac{\Delta \omega}{\Delta t}=\frac{\omega-\omega_{0}}{t}, \quad . \quad . \quad . \quad . \quad . \\
& \omega=\omega_{0}+\alpha t, \quad . \quad . \quad . \quad . \quad . . .
\end{aligned}
$$

in which $\omega_{0}$ and $\omega$ are the initial and final angular velocities, respectively, corresponding to the time interval $\Delta t$ or simply $t$.

The angular displacement $\Delta \theta$, or simply $\theta$, is the average angular velocity times the time interval. Hence,

$$
\begin{aligned}
\theta & =\frac{\omega_{0}+\omega}{2} \cdot t \quad . \quad . \quad . \quad . \\
& =\frac{\omega_{0}+\omega_{0}+\alpha t}{2} \cdot t \\
& =\omega_{0} t+\frac{1}{2} \alpha t^{2} . . .
\end{aligned}
$$

By eliminating $t$ from equations (2) and (4), the following equation is obtained,

$$
\omega^{2}=\omega_{0}^{2}+2 \alpha \theta . \quad . \quad . . \quad . \quad . \quad .
$$

To uniformly accelerated circular motion, therefore, the following equations apply:

$$
\begin{aligned}
& \omega=\omega_{0}+\alpha t, \quad . \quad . \quad . \quad . \quad . . \\
& \theta=\omega_{0} t+\frac{1}{2} \alpha t^{2}, \text {. . . . . . . . } \\
& \omega^{2}=\omega_{0}^{2}+2 \alpha \theta, \text {. . . . . . . . } \\
& \theta=\frac{\omega_{0}+\omega}{2} \cdot t . \quad . \quad . \quad . \quad . \quad .
\end{aligned}
$$

It will be noted that the above equations may be derived from the corresponding set of equations in Art. 124 by making use of the equations, $u=\omega_{0} r, v=\omega r, a_{t}=r \alpha$, and $s=r \theta$.

\section{PROBLEMS}

303. Derive equations (6) and (7) by calculus methods starting with the equations, $\omega=\frac{d \theta}{d t}$ and $\alpha=\frac{d \omega}{d t}$.

304. A wheel starting from rest turns so that a point on its rim has its angular velocity increased uniformly to 200 r.p.m. in 6 sec. After turning for a 
certain period of time at this speed a brake is applied and the wheel stops at a uniform rate in 5 sec. If the total number of revolutions is 3100 , find the total time.

126. Simple Harmonic Motion. - If the velocity of a point does not vary uniformly, the acceleration is not constant, and hence the equations of Art. 124 do not apply. One special case of rectilinear motion with variable acceleration is simple harmonic motion. A simple harmonic motion is defined as the motion of a point in a straight line such that the acceleration of the point is proportional to the distance, $x$, of the point from some fixed origin $O$, in the line and is directed toward $O$. Or expressed mathematically,

$$
a=\frac{d^{2} x}{d t^{2}}=-k x, \quad . \quad . \quad . \quad . \quad .
$$

where $k$ is a constant and the negative sign indicates that the sense of the acceleration is opposite to that of the displacement $x$ (Fig. 295), that is, $a$ is negative when $x$ is positive, and positive when $x$ is negative.

One example of a simple harmonic motion is the motion of a weight attached to the lower end of an elastic spring (the upper end being fixed) which is allowed to vibrate freely. The motion of the crosshead of a steam engine closely approximates a harmonic motion, the approximation becoming closer

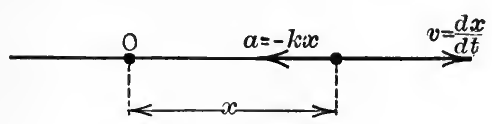

Fig. 295.

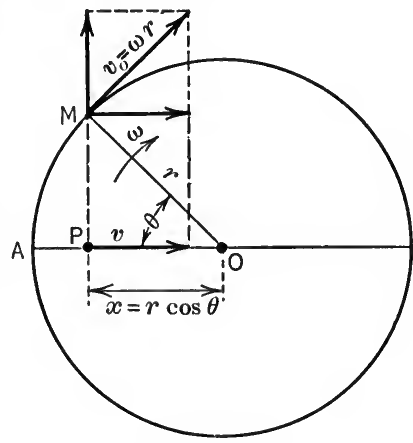

FIG. 296.

as the ratio of the length of the connecting rod to that of the crank increases. The motion of an oscillating pendulum also approximates closely a simple harmonic motion if the arc through which the pendulum swings is small. Further, if a point moves with constant speed in a circular path, the motion of the projection of the point on a diameter of the circle is a simple harmonic motion. This last statement may be proved as follows: In Fig. 
296 let $M$ be a point moving with constant speed, $v_{0}$, and angular velocity, $\omega$, along a circle of radius $r$. If $t$ is the time required for $M$ to move from $A$ to its given position, then,

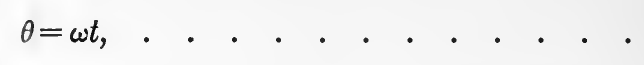

and

$$
x=r \cos \theta=r \cos \omega t \text {. . . . . . . . }
$$

Hence the velocity of $P$, the projection of $M$, is,

$$
\begin{aligned}
& v=\frac{d x}{d t}=-\omega r \sin \omega t=-\omega y, \ldots . . \\
& a=\frac{d^{2} x}{d t^{2}}=-\omega^{2} r \cos \omega t=-\omega^{2} x . \quad . \quad .
\end{aligned}
$$

Therefore, the motion of $P$ is harmonic and the constant $k$ in cquation (1) is here equal to $\omega^{2}$.

This method of generating a simple harmonic motion is a convenient one for studying certain features of the motion. From the definition it follows that a simple harmonic motion is a periodic motion. In the study of harmonic motion certain terms are used which are defined as follows: the amplitude is one-half the length of the path of the point, that is, in this example, the radius of the circle; the frequency is the number of complete oscillations of the point per unit of time, equal, in this example, to the number of revolutions per unit of time of the point $M$; the period is the time required for one complete oscillation, that is, the time required for $M$ to make one revolution. Hence the period $T$ is,

and the frequency $n$ is,

$$
T=\frac{2 \pi}{\omega} \quad \text {. . . . . . . . }
$$

$$
n=\frac{1}{T}=\frac{\omega}{2 \pi} . . . . . . .
$$

\section{ILLUSTRATIVE PROBLEM}

305. A point moves with a simple harmonic motion such that its speed is $90 \mathrm{in} . / \mathrm{sec}$. when it is $4 \mathrm{in}$. from the center of its path and $80 \mathrm{in} . / \mathrm{sec}$. when it is 6 in. from the center. Determine the period and the amplitude of the motion. Determine also the maximum velocity and the maximum acceleration of the point. 
Solution.-From equation (4) of Art. 126,

Hence,

$$
v=\omega y=\omega \sqrt{r^{2}-x^{2}}, \text {. . . . . . . . }
$$

and,

$$
\begin{aligned}
& 90=\omega \sqrt{r^{2}-16}, \\
& 80=\omega \sqrt{r^{2}-36} .
\end{aligned}
$$

Dividing (2) by (3),

$$
\frac{9}{8}=\frac{\sqrt{r^{2}-16}}{\sqrt{r^{2}-36}} \text {. }
$$

Squaring and transposing,

Hence,

That is,

Therefore,

$$
81\left(r^{2}-36\right)=64\left(r^{2}-16\right) .
$$

$$
17 r^{2}=1892
$$

$$
r^{2}=111.3
$$

$$
r=10.56 \text { in. }
$$

Substituting this value of $r$ in (2), we have,

$$
\omega=9.22 \mathrm{rad} . / \mathrm{sec} \text {. }
$$

From equation (6) of Art. 126,

$$
T=\frac{2 \pi}{\omega} .
$$

Hence,

$$
T=\frac{2 \pi}{9.22}=.68 \mathrm{sec}
$$

The velocity at any point may be found from equation (1). Since the velocity is a maximum when $x=0$, the maximum value is,

$$
\begin{aligned}
v & =\omega r \\
& =9.22 \times 10.56=97.5 \mathrm{in} . / \mathrm{sec} .
\end{aligned}
$$

The acceleration is a maximum when the displacement of the point is greatest. Hence, by using Equation (5) of Art. 126, the maximum value of the acceleration is found to be,

$$
\begin{aligned}
a & =\omega^{2} x \\
& =(9.22)^{2} \times 10.56=897 \mathrm{in} . / \mathrm{sec}^{2}{ }^{2}
\end{aligned}
$$

306. The drivers of a Mikado locomotive are $60 \mathrm{in}$. in diameter and the length of the crank is $\mathbf{1 5} \mathrm{in}$. If the speed of the locomotive is $30 \mathrm{mi}$. per hr., determine the maximum velocity and the maximum acceleration of the crosshead and piston relative to the engine frame, assuming that the connecting rod is so long that the motion of the crosshead is harmonic.

Ans. $v=22 \mathrm{ft} . /$ sec.; $a=387 \mathrm{ft} . /$ sec. $^{2}$ 
307. A point moves with a simple harmonic motion the amplitude of which is $10 \mathrm{in}$. If the period is 2 sec., determine the maximum velocity and maximum acceleration.

308. The maximum velocity of a point which has a simple harmonic motion is $10 \mathrm{ft}$. per sec. and the period is $\frac{1}{3}$ sec. Determine the amplitude of the motion and the maximum acceleration.

127. Non-uniformly Accelerated Motion.-One special case of non-uniformly accelerated motion is treated in the preceding article. Each case of this type of motion presents details peculiar to itself, but the fundamental equations expressing the relations between the displacement, velocity, acceleration, and time are the same for all cases. For rectilinear motion the equations are,

$$
\begin{aligned}
& v=\frac{d s}{d t}, \quad \text {. . . . . . . . . . } \\
& a=\frac{d v}{d t}=\frac{d^{2} s}{d t^{2}} . \quad . \quad . \quad . \quad . \quad .
\end{aligned}
$$

Eliminating $t$ from these two equations, we have

$$
a d s=v d v . \quad \text {. . . . . . . . . }
$$

By integrating equation (1), the following equations are obtained:

or.

and,

$$
\int_{s_{1}}^{s_{2}} d s=\int_{t_{1}}^{t_{1}} v d t
$$

$$
s_{2}-s_{1}=\Delta s=\int_{t_{1}}^{t_{2}} v d t, \ldots . \quad . \quad . \quad .
$$

or,

$$
\int_{t_{1}}^{t_{2}} d t=\int_{s_{1}}^{s_{2}} \frac{1}{v} d s
$$

$$
t_{2}-t_{1}=\Delta t=\int_{s_{1}}^{s_{2}} \frac{1}{v} d s . . . \quad . \quad . \quad .
$$

By integrating equation (2), the following equations are obtained:

or,

$$
\int_{v_{1}}^{v_{2}} d v=\int_{t_{1}}^{t_{2}} a d t
$$

$$
v_{2}-v_{1}=\Delta v=\int_{t_{1}}^{t_{2}} a d t . \quad . \quad . \quad . \quad .
$$


and,

$$
\int_{t_{1}}^{t_{2}} d t=\int_{v_{1}}^{v_{2}} \frac{1}{a} d v
$$

or,

$$
t_{2}-t_{1}=\Delta t=\int_{v_{1}}^{v_{2}} \frac{1}{a} d v . . . . . .
$$

The above equations may also be used in the case of curvilinear motion if the tangential component of the acceleration is used, since $a_{t}=\frac{d v}{d t}$. The equations may also be used if only a component, in a given direction, of the motion is considered, provided that $s, v$, and $a$ in the above equations be replaced by the components of displacement, velocity, and acceleration, respectively, in the given direction. Thus,

$$
\begin{aligned}
a_{x} & =\frac{d v_{x}}{d t}=\frac{d^{2} x}{d t^{2}}, \\
a_{x} d x & =v_{x} d v_{x}, \text { etc. }
\end{aligned}
$$

The relations between the displacement, velocity, acceleration, and the time, may, in general, be determined from the above equations by either of two methods; namely, by solving the equations by the methods of calculus, or by a graphical method through the use of distance-time, velocity-time, and acceleration-time graphs. The use of distance-time and speed-time graphs in finding the speed and acceleration, respectively, have been discussed in Arts. 113 and 119. The more convenient of the two methods for any given problem depends upon the character of the problem.

Calculus Method.-Equations (1) to (7) may be solved by the methods of calculus provided that certain relations between the variables are known. Thus, if $s$ is expressed as a function of $t$, the velocity $v$ may be obtained from equation (1). Likewise, if $v$ is expressed as a function of $s$, the time required for a given displacement may be obtained from equation (5), and so on.

\section{ILLUSTRATIVE PROBLEMS}

309. A point moves along a straight path according to the law, $v=3 t^{2}+4$, the units of distance and time being the foot and second, respectively. If $s=0$ when $t=0$, what is the value of $s$ when $t=10$ sec.? 
Solution.-

Hence,

$$
v=\frac{d s}{d t} \text { or } \quad s=\int_{12}^{t_{2}} v d t .
$$

$$
s=\int_{0}^{10}\left(3 t^{2}+4\right) d t=\left[t^{3}+4 t\right]_{0}^{10}=1040 \mathrm{ft} .
$$

310. A point moves along a curved path according to the law $a_{t}=10 t+5$. If $v=10 \mathrm{ft}$. $/ \mathrm{sec}$. when $t=4 \mathrm{sec}$., what is the value of $v$ when $t=7 \mathrm{sec}$.?

Solution. First Method:

Hence,

$$
a_{t}=\frac{d v}{d t}=10 t+5
$$

$$
\begin{aligned}
\Delta v & =\int_{t_{1}}^{t_{2}} a_{t} d t=\int_{4}^{7}(10 t+5) d t \\
& =\left[5 \iota^{2}+5 t\right]_{4}^{7}=180 \mathrm{ft} . / \mathrm{sec} .
\end{aligned}
$$

Hence, the gain in speed in the interval between the end of the fourth second and the end of the seventh second is $180 \mathrm{ft}$./sec. and, therefore, the speed at the end of the seventh second is,

$$
v_{7}=10+180=190 \mathrm{ft} . / \mathrm{sec} .
$$

Second Method.-Instead of using a definite integral as above, the problem may be solved by means of an indefinite integral as follows:

$$
\begin{aligned}
v & =\int a_{t} d t=\int(10 t+5) d t \\
& =5 t^{2}+5 t+C .
\end{aligned}
$$

The constant of integration may be determined by means of the initial condition that $v=10 \mathrm{ft}$. $/ \mathrm{sec}$. when $t=4 \mathrm{sec}$. Hence, using this condition, the above equation becomes,

$$
10=5 \times 4^{2}+5 \times 4+C .
$$

Therefore, $C=-90$ and the velocity at any time may be obtained from the equation,

$$
v=5 t^{2}+5 t-90 \text {. }
$$

If $t=7 \mathrm{sec}$., the corresponding value of $v$ is,

$$
v_{7}=5 \times 7^{2}+5 \times 7-90=190 \mathrm{ft} . / \mathrm{sec} .
$$

311. A point starts from rest at the vertex of the parabola $y^{2}=x$ and moves along the parabola according to the law $x=\frac{t^{2}}{4}, x$ and $y$ being in feet and $t$ in seconds. Find the magnitude of the velocity and of the acceleration of the point at the end of 2 sec. 
Solution.-The axial components of the velocity and of the acceleration may be found from the equations of Art. 122. Thus,

$$
\begin{aligned}
& v_{x}=\frac{d x}{d t}=\frac{d}{d t}\left(\frac{t^{2}}{4}\right)=\frac{t}{2}=1 \mathrm{ft} . / \mathrm{sec} . \text { when } t=2 \mathrm{sec} . \\
& v_{y}=\frac{d y}{d t}=\frac{d}{d t}\left(\frac{t}{2}\right)=\frac{1}{2} \mathrm{ft} . / \mathrm{sec} . \\
& a_{x}=\frac{d v_{x}}{d t}=\frac{d}{d t}\left(\frac{t}{2}\right)=\frac{1}{2} \mathrm{ft} . / \text { sec. }^{2} \\
& a_{y}=\frac{d v_{y}}{d t}=\frac{d}{d t}\left(\frac{1}{2}\right)=0 .
\end{aligned}
$$

Therefore,

$$
\begin{aligned}
v & =\sqrt{v_{x}^{2}+v_{y}^{2}} \\
& =\sqrt{(1)^{2}+\left(\frac{1}{2}\right)^{2}}=1.12 \mathrm{ft} . / \mathrm{sec} .
\end{aligned}
$$

and,

$$
\begin{aligned}
a & =\sqrt{a_{x^{2}+a_{y}^{2}}} \\
& =\sqrt{\left(\frac{1}{2}\right)^{2}+(0)^{2}}=0.5 \mathrm{ft} . / \text { sec. }^{2}
\end{aligned}
$$

312. The length of the crank, $O A$, of a steam engine (Fig. 297) is denoted by $r$ and the length of the connecting rod, $B A$, is denoted by $l$. If the crank

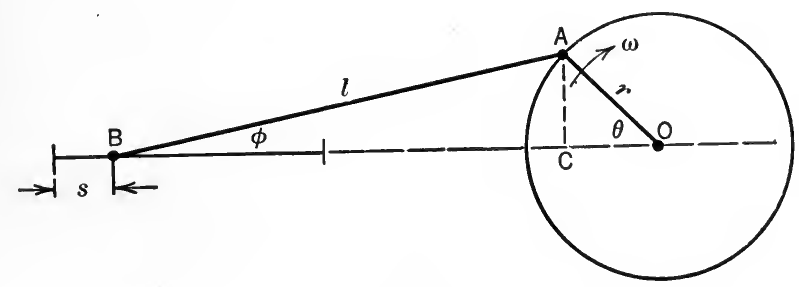

FIG. 297.

turns with constant angular velocity, $\omega$, determine the velocity and the acceleration of the crosshead, $B$, in terms of $r, l, \omega$, and the angle, $\theta$, which the crank makes with the horizontal.

Solution.-The displacement, $s$, of the crosshead from its extreme position may be determined in terms of $\theta$, and since $\theta=\omega t, s$ may also be expressed as a function of $t$, since $\omega$ is known. Thus,

But,

$$
s=l+r-l \cos \phi-r \cos \theta .
$$

and,

$$
A C=l \sin \phi=r \sin \theta,
$$

$$
l \cos \phi=\sqrt{l^{2}-l^{2} \sin ^{2} \phi}=\sqrt{l^{2}-r^{2} \sin ^{2} \theta} .
$$


By expanding the last expression and using only the first two terms of the expansion, since $\frac{r}{l}$ is generally small, the last equation may be written, with a close degree of approximation,

Therefore,

$$
l \cos \phi=l-\frac{r^{2}}{2 l} \sin ^{2} \theta .
$$

Hence,

$$
s=r-r \cos \theta+\frac{r^{2}}{2 l} \sin ^{2} \theta .
$$

and,

$$
\begin{aligned}
v & =\frac{d s}{d t}=r \sin \theta \frac{d \theta}{d l}+\frac{r^{2}}{l} \sin \theta \cos \theta \frac{d \theta}{d t} \\
& =r \omega\left(\sin \theta+\frac{r}{l} \sin \theta \cos \theta\right),
\end{aligned}
$$

$$
\begin{aligned}
a & =\frac{d v}{d l}=r \omega\left[\cos \theta+\frac{r}{l}\left(\cos ^{2} \theta-\sin ^{2} \theta\right)\right] \frac{d \theta}{d t} \\
& =r \omega^{2}\left(\cos \theta+\frac{r}{l} \cos 2 \theta\right) .
\end{aligned}
$$

Graphical Method.-The solution of equations (4) and (6) by the aid of speed-time and acceleration-time graphs will now be shown. The speed-time graph has already been defined (Art. 119). The acceleration-time graph is a curve, the coordinates of any point of which represent simultaneous values of the acceleration and the time. The acceleration used is either the total acceleration if the point has rectilinear motion, or the tangential acceleration if the point moves on a curved path.

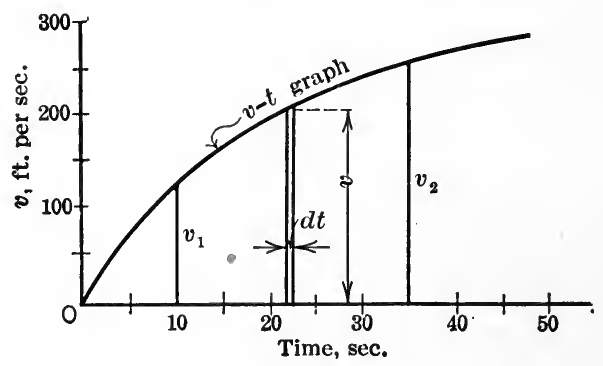

FIG. 298.

In Fig. 298 is represented a speed-time curve for a certain rectilinear motion. The area under the curve between the ordinates $v_{1}$ and $v_{2}$ is expressed by,

$$
\text { Area }=\int_{t_{1}}^{t_{2}} v d t
$$


But by equation (4), $\quad \Delta s=\int_{i_{2}}^{t_{2}} v d t$.

Therefore, the area under the speed-time curve represents, to some scale (depending on the scales used in plotting the graph), the distance traveled during the time interval $t_{2}-t_{1}$, corresponding to a change in the speed from $v_{1}$ to $v_{2}$.

\section{ILLUSTRATIVE PROBLEM}

313. The speed-time curve for the rectilinear motion of a certain point is shown in Fig. 298. The scales are: 1 in., vertically, equals $200 \mathrm{ft}$./sec., and $1 \mathrm{in}$., horizontally, equals $20 \mathrm{sec}$. If the area under the curve between $t=10$ and $t=35$ is 1.2 sq. in., how far does the point travel in the interval?

Solution. -1 sq. in. represents $200 \mathrm{ft} . /$ sec. $\times 20$ sec. $=4000 \mathrm{ft}$.

Hence,

the distance $=1.2 \times 4000=4800 \mathrm{ft}$.

Let the curve in Fig. 299 represent the acceleration-time graph for the rectilinear motion of a point. The area under the curve between the ordinates $a_{1}$ and $a_{2}$ is expressed by,

$$
\text { Area }=\int_{t_{1}}^{t_{2}} a d t
$$

But, by equation (6),

$$
\Delta v=\int_{t_{1}}^{t_{2}} a d t
$$

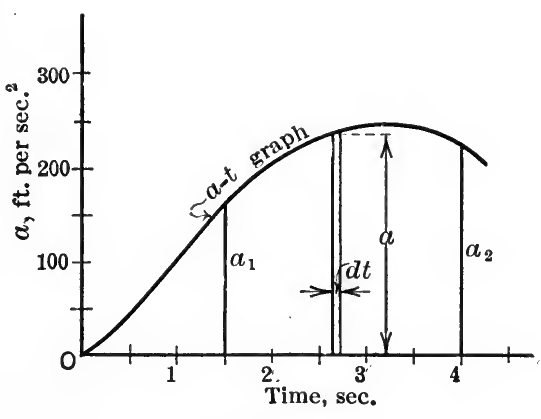

Fig. 299.

Therefore, the area under the acceleration-time curve represents, to some scale (depending on the scales used in plotting the graph), the change in speed during the time interval $t_{2}-t_{1}$.

\section{ILLUSTRATIVE PROBLEM}

314. The scales used for the acceleration-time curve in Fig. 299 are: $1 \mathrm{in.}=200 \mathrm{ft} . / \mathrm{sec}^{2}$ and $1 \mathrm{in} .=2 \mathrm{sec}$. If the area under the curve between the ordinates $t=1.5 \mathrm{sec}$. and $t=4 \mathrm{sec}$. is $1.3 \mathrm{sq}$. in., what is the speed at the end of $4 \mathrm{sec}$. if the speed at the end of $1.5 \mathrm{sec}$. is $20 \mathrm{ft}$./sec.?

Solution. -1 sq. in. represents $200 \mathrm{ft} . / \mathrm{sec}^{2} \times 2 \mathrm{sec} .=400 \mathrm{ft} . / \mathrm{sec}$. 
Hence,

Therefore,

$$
\Delta v=1.3 \times 400=520 \mathrm{ft} . / \mathrm{sec} .
$$$$
v_{4}=20+520=540 \mathrm{ft} . / \mathrm{sec} .
$$

\section{PROBLEMS}

315. A point moves on a circular path having a radius of $10 \mathrm{ft}$., according to the law, $v=20 t$. Determine the position of the moving point, with respect

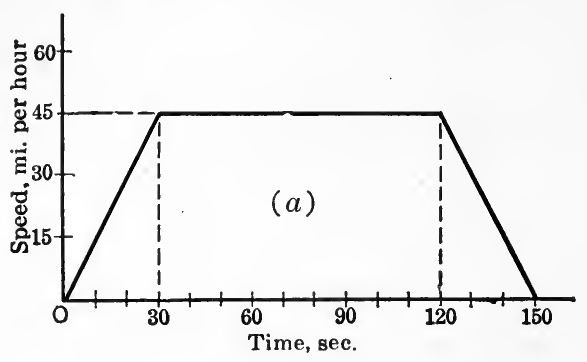
to its initial position, at the end of 5 sec.

316. A bellcrank operated by a cam (see Fig. 280) rotates so that its angular acceleration $\alpha$ follows the law, $\alpha=4 t^{2}+6$. If its initial angular velocity is 2 radians per sec., what is the angular velocity of the bellcrank at the end of 0.5 sec.?

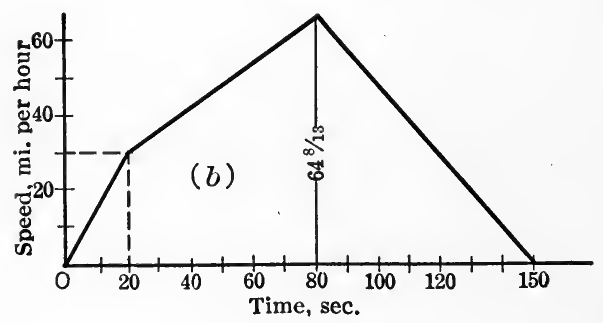

Ans, $\omega=5.17 \mathrm{rad} . / \mathrm{sec}$.

317. Two railway stations are connected by two s t r a i g t parallel tracks. Two trains $A$ and $B$ start from rest at one station and reach the second station in 150 seconds. The Fig. 300. speed-time curve for $A$ is shown in Fig. $300(a)$ and that for $B$ in Fig. $300(b)$. Draw the acceleration-time curve for each train.

318. What, in Fig. 300, represents the distance between the stations? From the diagrams find the distance in miles. Ans. 1.5 miles.

128. Relative Motion.-In the preceding articles, the motion of a particle is defined or described with reference to a point or a set of axes assumed to be fixed. All points and bodies, however, are in motion, but in most practical problems it is convenient to consider the earth to be fixed. Therefore a set of axes passing through any point on the earth will be regarded as a fixed reference frame. The motion of a particle, described with reference to a point on the earth, is called its absolute motion. The motion of a particle, described with reference to a point that is moving with respect to the earth, is called its relative motion. It will be observed that 
the absolute motion of a particle is its relative motion with respect to the earth.

In considering the motion of two moving particles, the motion of one particle relative to the other particle is often required. The relation between the absolute and relative motions of two particles $A$ and $B$ (assumed, for convenience, to be moving in a plane) may be stated by the following important theorem.

The absolute displacement, velocity, or acceleration of $A$ is the geometric or vector sum of the relative displacement, velocity, or acceleration, respectively, of $A$ with respect to $B$ and the absolute displacement, velocity, or acceleration, respectively, of $B$.

This theorem may be expressed in the form of equations as follows:

$$
\begin{array}{lll}
s_{A}=s_{\frac{A}{B}} \rightarrow s_{B} & \text { or } & s_{\bar{A}}=s_{A} \rightarrow s_{B} . \\
v_{A}=v_{\frac{A}{B}} \mapsto v_{B} & \text { or } & v_{\frac{A}{B}}=v_{A} \rightarrow v_{B} . \\
a_{A}=a_{\frac{A}{B}} \mapsto a_{B} & \text { or } & a_{\underline{A}}=a_{A} \rightarrow a_{B} .
\end{array}
$$

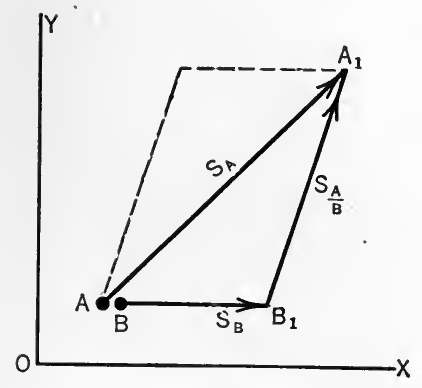

Fig. 301.

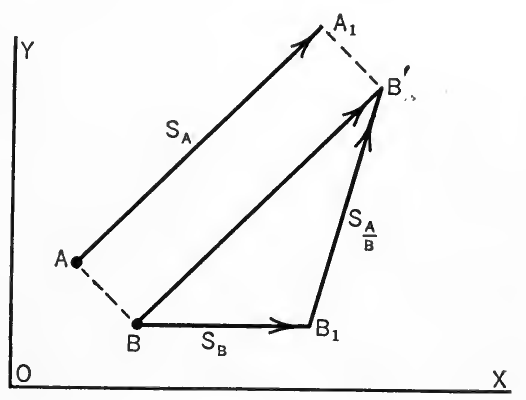

Fig. 302.

where $s_{A}$ denotes the absolute displacement of $A, s_{B}$ denotes the absolute displacement of $B$, and $s_{\frac{A}{B}}$ denotes the relative displacement of $A$ with respect to $B$, and similiarly for velocities and accelerations.

Relative Displacement.-As applying to displacements the above theorem is nearly self-evident. To illustrate, let $A$ and $B$ be two particles which occupy the same position with reference to a set of 
axes as shown in Fig. 301. Let the point $A$ be given a displacement $s_{A}=A A_{1}$ and the point $B$, a displacement $s_{B}=B B_{1}$. The point $A$ moves relative to the point $B$ a distance $B_{1} A_{1}$ and in the direction $B_{1} A_{1}$. Hence $B_{1} A_{1}$ is the displacement of $A$ relative to $B$ and it is evident that,

$$
s_{A}=s_{\bar{A}} \nrightarrow s_{B} \quad \text { or } \quad s_{\bar{A}}=s_{A} \rightarrow s_{B}
$$

If the points $A$ and $B$ do not occupy the same positions, the reasoning is changed but little, the final conclusion being the same as stated above as will be evident from a study of the diagram in Fig. 302.

\section{ILLUSTRATIVE PROBLEM}

319. The current in a river with parallel sides is $4 \mathrm{mi}$./ $\mathrm{hr}$. A motor boat starting from one side keeps headed perpendicular to the sides and moves at $6 \mathrm{mi}$./hr. If the river is one mile wide, what is the absolute displacement of the boat after reaching the other side?

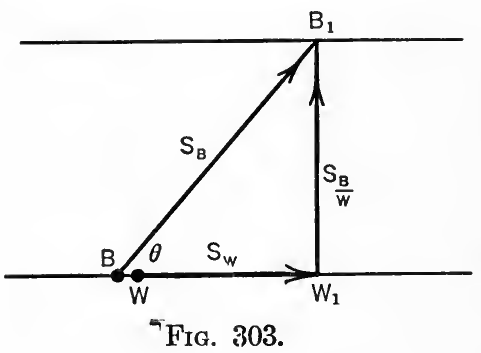

Solution.-Let $B$ (Fig. 303) represent the boat and $W$ the water that is in contact with the boat when the boat starts. Then,

$$
s_{B}=s_{W} \mapsto s_{\frac{B}{W}} .
$$

The displacement of the water while the boat is moving across is,

$$
s_{W}=W W_{1}=\frac{2}{3} \text { mile. }
$$

After the boat has reached the other side its displacement relative to the water (now at $W_{1}$ ) is,

$$
s_{\frac{B}{W}}=W_{1} B_{1}=1 \text { mile. }
$$

Therefore,

$$
s_{B}=1 \mapsto \frac{2}{3}=\sqrt{(1)^{2}+\left(\frac{2}{3}\right)^{2}}=1.2 \text { miles, }
$$

and,

$$
\tan \theta=\frac{s_{\frac{B}{W}}}{s_{W}}=\frac{3}{2} \quad \therefore \theta=56^{\circ} 20^{\prime} .
$$

Relative Velocity.- -The above theorem, as applying to the velocities of two moving particles, may be proved as follows: 
Let $A$ and $B$ (Fig. 304) be any two moving particles (assumed in a plane), let the absolute velocity, $v_{A}$, of $A$ be represented by $A A_{1}$, and let, $B B_{1}$ represent the absolute velocity, $v_{B}$, of $B$. Let a velocity equal to $v_{B}$ but reversed in direction be given to each point. The point $B$ will then be at rest and, hence, the resulting velocity of the point $A$ is the velocity of $A$ relative to $B$. Hence, it is evident from the diagram that,

$$
v_{A}=v_{\frac{A}{B}}+v_{B}
$$

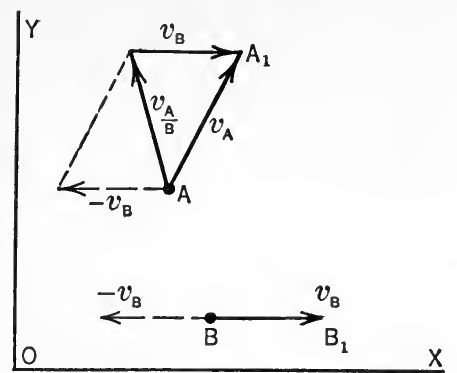

FIG. 304.

In this proof it is assumed that the additions of equal velocities to each particle does not influence the relative velocity of the two particles. This assumption is in accord with an important principle in kinematics, namely, that the relative motion of two particles is not affected by any motion that they have in common.

The above equation as already noted, may be written in the form

$$
v_{\frac{A}{B}}=v_{A} \rightarrow v_{B},
$$

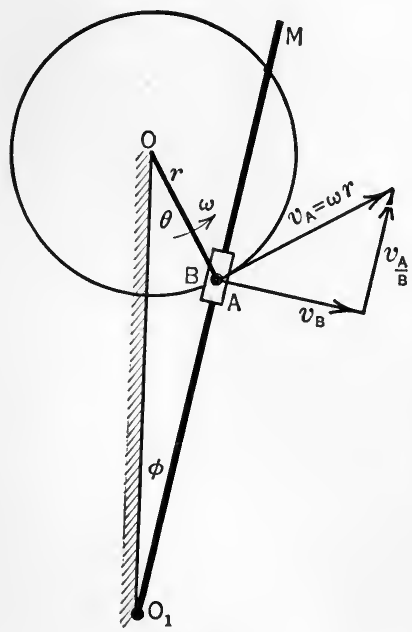

Fig. 305.

which states that the relative velocity of two points is the difference of their absolute velocities, as is shown in the diagram.

\section{ILLUSTRATIVE PROBLEM}

320. In the shaper mechanism shown in Fig. 305, let $A$ be the sliding block and let $B$ be the point on the rocker arm $O_{1} B M$ which is coincident with $A$ at the instant. The distance $O O_{1}$ is 20 in. and $\theta$ is $30^{\circ}$. If $\omega=20$ r.p.m. and $r=\frac{1}{2} \mathrm{ft}$., find $v_{B}$, the absolute velocity of $B$, and $v_{\frac{A}{B}}$, the relative velocity of $A$ with respect to $B$.

Solution.-Since the block $A$ moves on a circular arc at constant speed, its velocity is given in magnitude by the equation,

$$
v_{A}=\omega r=\frac{20 \times 2 \pi}{60} \times \frac{1}{2}=1.05 \mathrm{ft} . / \mathrm{sec} \text {. }
$$


and its direction is perpendicular to $r$, as shown in Fig. 305. The direction of the absolute velocity of $B$ is perpendicular to $O_{1} B M$ and its magnitude is unknown. Likewise, the direction of the velocity of $A$ relative to $B$ is known since it is along (parallel to) the rocker arm. By applying the equation,

$$
v_{A}=\frac{v_{A}}{B}+v_{B}
$$

the magnitudes of $\frac{v_{A}}{B}$ and $v_{B}$ are determined by the intersection of the lines that represent their directions. By scaling off the magnitudes, the following values are found,

$$
v_{\bar{A}}=0.70 \mathrm{ft} . / \mathrm{sec} . \quad \text { and } v_{B}=0.79 \mathrm{ft} . / \mathrm{sec} .
$$

\section{PROBLEMS}

321. Two trains $A$ and $B$ travel on parallel straight tracks. The speed of $A$ is $40 \mathrm{mi} . / \mathrm{hr}$. and that of $B$ is $50 \mathrm{mi} . / \mathrm{hr}$. in the same direction. What is the velocity of $A$ relative to $B$ ? Of $B$ relative to $A$ ?

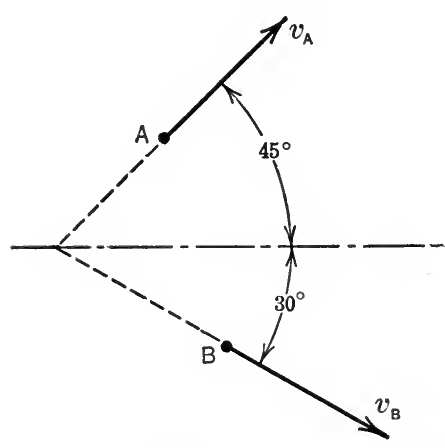

FIG. 306.

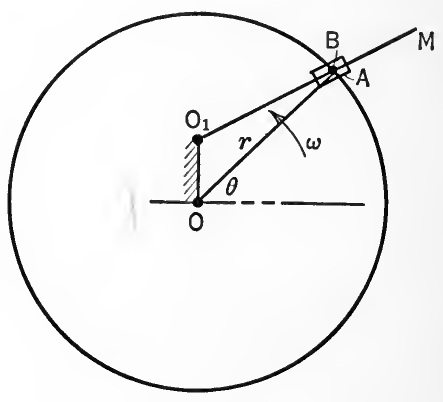

Fig. 307.

322. A train $A$ travels with a velucity $v_{A}=40 \mathrm{mi}$. $/ \mathrm{hr}$. and another train $B$ travels with a velocity $v_{B}=50 \mathrm{mi}$./hr. in the directions shown in Fig. 306 . What is the magnitude and the direction of the relative velocity of $B$ with respect to $A$ ? Ans. $55.4 \mathrm{mi} . / \mathrm{hr}$.

323. In Fig. 307, let $A$ be a block which revolves about $O$ at a constant angular velocity $\omega=30$ r.p.m. and let $B$ be the point on the arm $O_{1} B M$ coincident with $A$ at the instant. If $r=O A=9$ in., $O O_{1}=3$ in., and $\theta=45^{\circ}$, find the absolute velocity of $B$.

Relative Acceleration.-In order to prove the above theorem as it relates to accelerations, it will be assumed that the relative accel- 
eration of two particles is not changed if equal additional accelerations be given the two particles. This is analogous to the assumption made in the discussion of relative velocities. Hence, if equal velocities and accelerations be given to each of two particles the relative motion of one with respect to the other will not be changed. Consider, then, two particles $A$ and $B$ which move along paths as indicated in Fig. 308. Assume that a velocityequal and opposite to the velocity of $B\left(-v_{B}\right)$ is imposed on each particle (not shown at $A$ in Fig. 308). Assume also that accelerations equal to the acceleration of $B$ but of opposite sense are imposed on the two particles. This will not change the relative motion of $A$ with respect to $B$. Imposing a velocity of $-v_{B}$ and an acceleration

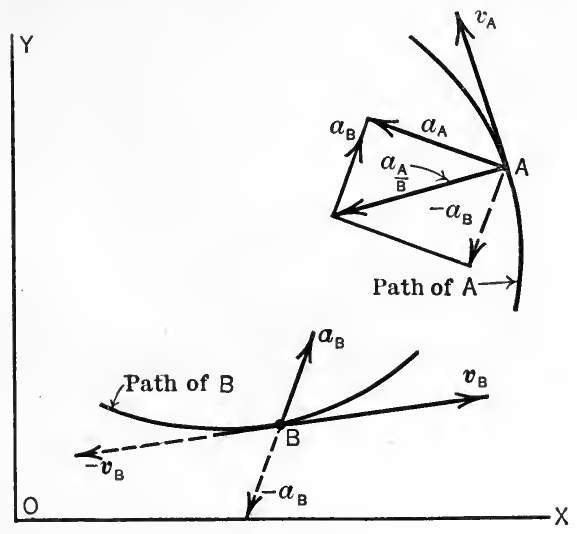

FIG. 308 of $-a_{B}$ on the particle $B$ will make its position fixed, and hence the acceleration of $A$ with respect to $B$ will be the same as the absolute acceleration of $A$. The acceleration of $A$, however, is now the vector sum of $a_{A}$ and $-a_{B}$ and hence,

$$
a_{A}=a_{\frac{A}{B}} \nrightarrow a_{B}
$$

or,

$$
a_{\frac{A}{B}}=a_{A} \rightarrow a_{B}
$$

Hence, the relative acceleration of one point with respect to a second point is the vector difference of the absolute accelerations of the two points.

\section{ILLUSTRATIVE PROBLEM}

324. Two friction disks (Fig. 309) rotate with constant angular velocities. Let $A$ and $B$ be points on the circumferences of the large and small disks, respectively. If $\omega_{1}=40$ r.p.m., $r_{1}=9 \mathrm{in}$., and $r_{2}=3 \mathrm{in}$., find the magnitude and the direction of the acceleration of $A$ relative to $B$. 


\section{Solution.-}

$$
\begin{aligned}
\omega_{1} r_{1} & =\omega_{2} r_{2}, \\
\omega_{2} & =\frac{\omega_{1} r_{1}}{r_{2}}=\frac{40 \times 9}{3}=120 \text { r.p.m., } \\
a_{A} & =\omega_{1}{ }^{2} r_{1}=\left(\frac{40 \times 2 \pi}{60}\right)^{2} \times \frac{9}{12}=13.3 \mathrm{ft} . / \text { sec. }^{2}, \\
a_{B} & =\omega^{2}{ }_{2} r_{2}=\left(\frac{120 \times 2 \pi}{60}\right)^{2} \times \frac{3}{12}=40 \mathrm{ft} . / \text { sec. }^{2} .
\end{aligned}
$$

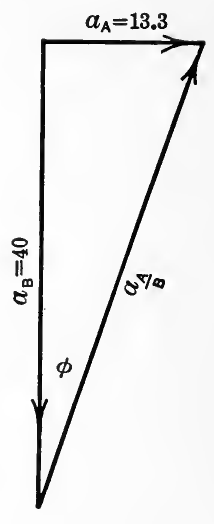

Therefore,

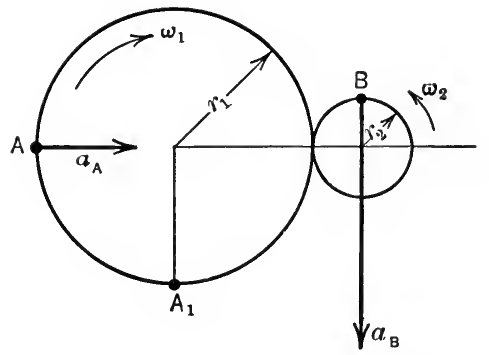

Fig. 309.

$$
\begin{aligned}
\underset{\frac{A}{B}}{a_{A}} & =a_{A} \rightarrow a_{B} \\
& =\sqrt{(13.3)^{2}+(40)^{2}}=42.2 \mathrm{ft} . / \text { sec. }^{2},
\end{aligned}
$$

and,

Therefore,

$$
\tan \phi=\frac{a_{A}}{a_{B}}=\frac{13.3}{40}=0.33 \text {. }
$$

$$
\phi=18^{\circ} 25^{\prime} \text {. }
$$

\section{PROBLEMS}

325. What is the relative acceleration of $A_{1}$ (Fig. 309) with respect to $B$, the data being the same as in the

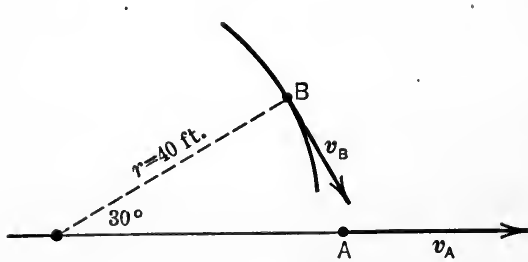

Fig. 310. preceding problem?

326. Two trains $A$ and $B$ travel in the same direction on parallel tracks; $A$ increases its speed uniformly $10 \mathrm{mi} . / \mathrm{hr} . / \mathrm{min}$. and $B$ decreases its speed uniformly $5 \mathrm{mi} . / \mathrm{hr} . / \mathrm{min}$. What is the relative acceleration of $A$ with respect to $B$ ? 
327. An automobile, $A$, is traveling on a straight road, increasing its speed at the rate of $300 \mathrm{ft} . / \mathrm{min} . / \mathrm{sec}$. when in the position shown (Fig. 310). At the same time another automobile, $B$, is traveling in a circular path increasing its speed at the rate of $5 \mathrm{ft} . / \mathrm{sec}^{2}$ Its speed when in the position shown is $n_{B}=12 \mathrm{mi}$. $/ \mathrm{hr}$. and the radius of the circular path is $40 \mathrm{ft}$. What is the relative acceleration of $A$ with respect to $B$ ?

Ans. $12.3 \mathrm{ft} . /$ sec. $^{2}$

129. Transverse and Radial Components of Acceleration.-In addition to the $x$-and $y$-components and the $n$ - and $t$-components of the acceleration of a particle (Art. 122), the transverse and radial ( $T$ and $R$ ) components are sometimes convenient to use. The transverse direction is perpendicular to the radius vector

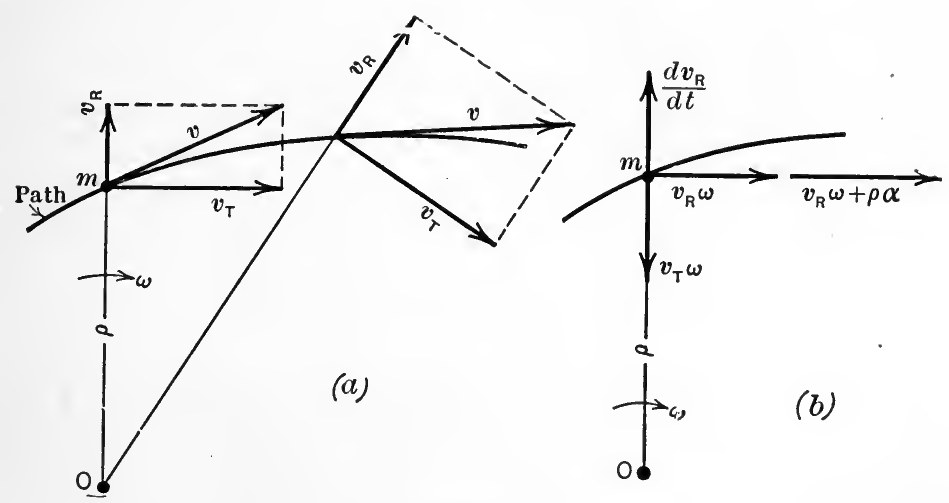

Fig. 311

drawn from any point taken as the center or pole, and the radial direction is along, or parallel to, the radius vector (see Fig. 311).

The $T$-and $R$-components may be derived by the application of Theorems I and II (Art. 118). In Fig. 311(a) let $m$ represent a particle moving on a fixed path. Let $O$ be any center or pole (not the instantaneous center of rotation, Art. 135). Then, the total velocity, $v$, of the particle may be replaced by the two components $v_{T}$ and $v_{R}$. Thus,

$$
v=v_{T} \nrightarrow v_{R}
$$

Fig. 311(a) indicates that in general at any instant

$v_{T}$ is changing in magnitude and also in direction, and that,

$v_{R}$ is changing in magnitude and also in direction.

Hence there will be four components of the acceleration: two of 
the type $\frac{d v}{d t}$; and two of the type $v \omega$, as follows (shown in Fig. $311 b)$ :

(1) $\frac{d v_{R}}{d t}=\frac{d}{d t}\left(\frac{d \rho}{d t}\right)=\frac{d^{2} \rho}{d t^{2}}$ parallel to $\rho$, being the rate of change of the magnitude of $v_{R}$.

(2) $v_{R} \omega$ perpendicular to $v_{R}$, being the rate of change of $v_{R}$ due to the change in its direction only.

(3) $\frac{d v_{T}}{d t}=\frac{d(\omega \rho)}{d t}=\omega \frac{d \rho}{d t}+\rho \frac{d \omega}{d t}=\omega v_{R}+\rho \alpha$ parallel to $v_{T}$, being the rate of change of the magnitude of $v_{T}$.

(4) $v_{T} \omega=\omega^{2} \rho=\frac{v_{T}^{2}}{\rho}$ perpendicular to $v_{T}$, being the rate of change of $v_{T}$ due to a change in its direction only.

The $T$ - and $R$-components of the total acceleration are, then (Fig. 2b),

$$
\begin{aligned}
& a_{T^{\prime}}=v_{R} \omega+\left(v_{R} \omega+\rho \alpha\right)=2 v_{R} \omega+\rho \alpha=2 \omega \frac{d \rho}{d t}+\rho \frac{d \omega}{d t}=\frac{1}{\rho} \frac{d}{d t}\left(\rho^{2} \omega\right), \\
& a_{R}=\frac{d v_{R}}{d t}-\omega v_{T}=\frac{d^{2} \rho}{d t^{2}}-\rho \omega^{2} .
\end{aligned}
$$

130. Coriolis' Law.- - It is convenient to consider the motion of a point of a body as being generated by a motion of the point along a path (line) as the path moves. Thus, in plane motion of a rigid body the motion of any point in the body may be considered as a combination of a motion along a path and a motion due to a translation of the path.

If a point moves along a path as the path is translated, the acceleration of the point is the vector sum of the acceleration relative to the path and the acceleration of the point of the path which is coincident with the point at the instant (or of any point of the path since the path is translated). This leads to the same expression for the acceleration of the point as was obtained in Art. 128 , as will be shown later.

If, however, a point moves along a path as the path rotates, the acceleration of the point is obtained as the vector sum of three component accelerations. The relation between these three component accelerations and the total acceleration is known as Coriolis' law, which may be derived by use of the theorems of Art. 118 as follows: 
In Fig. 312(a), let $m$ represent a particle (or bead) traveling along a path (curved wire) with the velocity $u$, and at the same time let the path rotate about $O$ with angular velocity $\omega$. The velocity of the particle will then have, at any instant, two components, $u$ and $w, u$ being the velocity along (relative to) the path, and $w$ the velocity of the point on the path which, at the instant, coincides with the particle.

It will be noted from Fig. 312(a) that, as the particle passes from one position to some other position, the velocity $u$ changes in magnitude and also in direction. Likewise $w$ changes in magni-

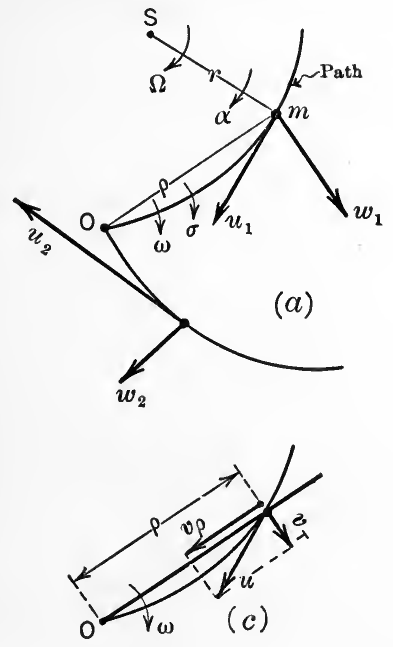

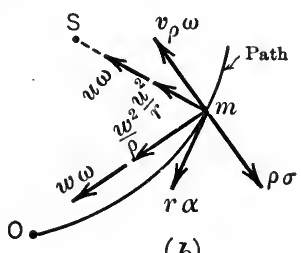

(b)

FIG. 312.

tude and also in direction. At any instant, therefore, the acceleration of the particle $m$ will have at least four components. It will be found convenient, however, to consider the change in both $u$ and $w$ to be made up of several parts, as follows:

The change in $u$ from $u_{1}$ to $u_{2}$ is made up of:

(1) A change in magnitude, due to an increasing (or decreasing) speed along the path.

(2) A change in direction, due to the curvature of the path.

(3) A change in direction, due to the turning of the path.

The change in $w$ from $w_{1}$ to $w_{2}$ is made up of:

(4) A change in magnitude, due to the rotation of the path with increasing (or decreasing) angular velocity 
(assuming $\rho$ to remain constant, that is, considering the particle to be fixed to the path).

(5) A change in direction, due to the rotation of the path (considering the particle fixed to the path).

(6) A change in magnitude, due to the change in length of $\rho$ (assuming $\omega$ to remain constant), caused by the movement along the path.

(7) A change in direction, due to the change in the direction of $\rho$, caused by the motion of the particle along the path (assuming the path fixed). The change in direction of $\rho$ and of $w$ are the same since they remain at right angles.

At any instant, therefore, the acceleration of $m$ will have seven components, unless (4) and (6) are combined, three being of the type $\frac{d v}{d t}$ and four of the type $v \omega$. These components (represented in Fig. 312b) are expressed as follows:

(1) $\frac{d u}{d t}=r \alpha$ parallel to $u$ and having the same sense as $u$ since $\Omega$, the angular velocity, and $\alpha$, the angular acceleration, of the particle $m$ with respect to the center of curvature, $S$, of the path were assumed to agree in sense as indicated in Fig. 312(a).

(2) $u \Omega=\Omega^{2} r=\frac{u^{2}}{r}$ perpendicular to $u$, towards $S$.

(3) $u \omega$ perpendicular to $u$ toward $S$.

(4) $\frac{d w}{d t}=\rho \sigma$ parallel to $w$ and having the same sense as $w$, since $\omega$, the angular velocity, and $\sigma$, the angular acceleration, of the path with respect to $O$ are assumed to agree in sense.

(5) $w \omega=\omega^{2} \rho=\frac{w^{2}}{\rho}$ perpendicular to $w$, toward 0 .

By referring again to Fig. 312(a), it will be noted that the velocity $u$ could also be produced by allowing the particle (bead) to slide along the straight line $\rho$ as the line $\rho$ is rotated about the center $O$ with the same angular velocity, $\omega$, as that of the path. The two (rnmponents of $u$ would then be $v_{T}=\omega \rho$ and $v_{\rho}=\frac{d \rho}{d t}$ (see Fig. 312c). 
It will be noted that these components are the radial and transverse components discussed in Art. 116. We are concerned at present only with the changes in $v_{T}$ due to this motion of the particle along the path, since the changes in $w$, not already considered in (4) and (5), are due to the motion of the point along the path and are the same as the changes in $v_{T}$. Now $v_{T}$ changes in magnitude and also in direction; these changes correspond to the change in length and in direction of $\rho$. The accelerations thus produced by reason of the change in $v_{T}$ (or $w$ ) are then;

(6) $\frac{d v_{T}}{d t}=\frac{d(\omega \rho)}{d t}=\omega \frac{d \rho}{d t}=v_{\rho} \omega$, parallel to $v_{T}$ (or $w$ ) and opposite to the sense of $w$ since $w$ is decreasing. And,

(7) $v_{T} \omega$ perpendicular to $v_{T}(\mathrm{o} \alpha w)$ toward $O$.

In (4) $\rho$ was considered constant and $\omega$ was assumed to change whereas in (6) $\omega$ was considered constant while $\rho$ was assumed to change. Of course (4) and (6) are parts of one expression, namely, $\frac{d(\omega \cdot \rho)}{d t}=\rho \frac{d \omega}{d t}+\omega \frac{d \rho}{d t}$, where $\omega$ and $\rho$ are considered to change at the same time. Separating the operation into two parts, however, makes clear the need for both $\omega$ and $\rho$ to vary.

Finally, the total acceleration is expressed in terms of the seven components as follows:

$$
a=r \alpha+\left\{\begin{array}{c}
u \Omega \\
\frac{u^{2}}{r} \\
\Omega^{2} r
\end{array}\right\} H u \omega+\rho \sigma+\left\{\begin{array}{c}
w \omega \\
\frac{w^{2}}{\rho} \\
\omega^{2} \rho
\end{array}\right\} H \omega v_{\rho} H \omega v_{T}
$$

These components may be grouped in several ways, the most useful grouping depending upon the particular problem under consideration. One such grouping leads to Coriolis' law. By combining the last two components into one term, we have $\omega\left(v_{\rho} \gg v_{T}\right)=u \omega$ since $v_{\rho} \rightarrow v_{T}=u$ (Fig. 312c). And since $u \omega$ represents an acceleration due to a change in the direction of $u$ it must be perpendicular to $u$ (Theorem II, Art. 118). This may be added to the third component, since the two will always have the same sense. Hence the third, sixth, and seventh components combine into $2 u \omega$ directed perpendicular to $u$ with a sense such that if it were applied as a force at the end of the vector $u$ it would cause the vector $u$ to turn in its actual direction of rotation. Further, let $a_{r}$ 
represent the vector sum of the first two components and let $a_{m}$ represent the vector sum of the fourth and fifth, that is, let

$$
a_{r}=r \alpha+\frac{u^{2}}{r} \text { and } \quad a_{m}=\rho \sigma+\frac{w^{2}}{\rho} \text {. }
$$

The total acceleration, then, may be expressed as follows,

$$
a=a_{r} \nrightarrow a_{m}+2 u \omega .
$$

This equation may be stated in words as follows: If a particle moves on a path as the path rotates, the acceleration of the particle is the geometric or vector sum of (1) the acceleration the particle would have if the path were fixed, (2) the acceleration the particle would have if it were fixed on the path as the path rotated, and (3) $2 u \omega$ called the compound supplementary acceleration. This statement is known as Coriolis' law.

From the above derivation, it is seen that $2 u \omega$ is due (1) to a part of the change of direction of $u$, (2) a part of the change of the direction of $w$, and (3) a part of the change of the magnitude of $w$, all of which would be absent if the path were translating instead of rotating. Thus, as noted at the beginning of this article, if the path is translated, the equation $a=a_{r} \rightarrow a_{m}$ expresses the same fact as does the equation of Art. 128 which involves accelerations.

\section{ILLUSTRATIVE PROBLEM}

328. The circular arc $A P B$ (Fig. $313 a$ ) represents the vane of a centrifugal pump, $P$ being a particle of water. Find the acceleration of the particle $P$
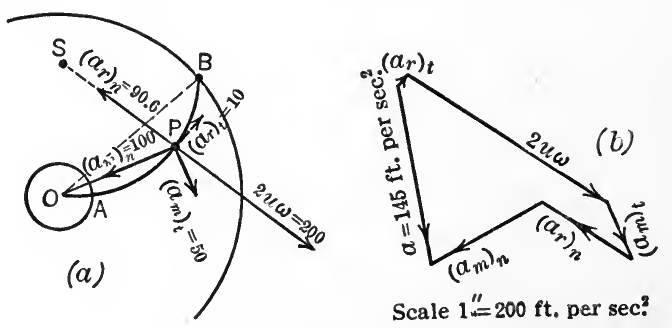

FIG. 313.

when it is $12 \mathrm{in}$. from $O$, the center of the shaft, if, at that instant, the angular velocity of the wheel is $10 \mathrm{rad}$. per sec. clockwise and its angular acceleration is $50 \mathrm{rad}$. per sec. ${ }^{2}$ clockwise. The tangential velocity of the particle along (relative to) the vane is $10 \mathrm{ft}$. per sec. and the tangential acceleration relative 
to the vane is $10 \mathrm{ft}$. per sec. ${ }^{2} \quad O B$ makes an angle of $45^{\circ}$ with the horizontal and is $18 \mathrm{in}$. long; $O A$ is $3 \mathrm{in}$; and $O P$ is $12 \mathrm{in}$. The radius of the arc $A P B$ is $13 \frac{1}{4} \mathrm{in}$.

Solution.-By Coriolis' law the total acceleration $a$ is,

$$
a=a_{r} \nrightarrow a_{m} \mapsto 2 u \omega,
$$

$a_{r}$ and $a_{m}$ are found most easily from their $t$ - and $n$-components, Hence,

$$
\begin{aligned}
& a=\left(a_{r}\right)_{t} \mapsto\left(a_{r}\right)_{n} \mapsto\left(a_{m}\right)_{t}+\left(a_{m}\right)_{n}+2 u \omega \\
& =10 \nrightarrow \frac{10^{2}}{\frac{13 \frac{1}{4}}{12}} \mapsto 50 \times \frac{12}{12} \mapsto 10^{2} \times \frac{12}{12} \mapsto 2 \times 10 \times 10 \\
& =10 \nrightarrow 90.56+50 \nrightarrow 100+200,
\end{aligned}
$$

each of the quantities being expressed in $\mathrm{ft}$. per sec. ${ }^{2}$ The five components are shown in their proper directions in Fig. 313(a) and the resultant acceleration $a$ as found from the acceleration polygon is shown in Fig. 313(b). By scaling the closing line of the polygon, $a$ is found to be $145 \mathrm{ft}$. per sec. ${ }^{2}$ in the direction shown in Fig. $313(b)$.

\section{PROBLEM}

329. Point $P$ (Fig. 314) moves on the rod $O M$ which rotates about the fixed point $O$. In the given position $O P=6 \mathrm{ft}$; the velocity along the rod is $5 \mathrm{ft}$. per sec. toward $M$ and is increasing at the rate of $5 \mathrm{ft}$. per sec. each second. The angular velocity of the rod is $2 \mathrm{rad}$. per sec. in a clockwise direction, and is decreasing at the rate of $1.5 \mathrm{rad}$. per sec. each sec. Find the acceleration of the point $P$. Notw.Use scales: $1 \mathrm{in} .=2 \mathrm{ft}$.; $1 \mathrm{in}$. $=5 \mathrm{ft}$. per sec.; $1 \mathrm{in} .=5 \mathrm{ft}$. per sec. $^{2}$ Use $8 \frac{1}{2}$ in. by 11 in. paper with the long edge as the bottom. Lay the diagram out on left part of sheet, placing point $O 1 \mathrm{in}$. from the left edge and $2 \mathrm{in}$. from the bottom edge. Show all component accelerations of $P$ to scale.

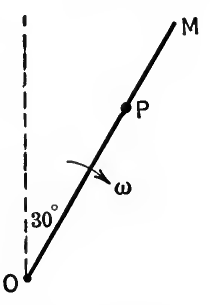

FIg. 314. Combine the components graphically, starting at a point $1 \frac{1}{4}$ in. from the top edge and $1 \mathrm{in}$. from the right edge. Combine the components in the order of $\left(a_{m}\right)_{n},\left(a_{m}\right)_{t}, a_{r}$ and $2 u \omega$.

Ans. $a_{P}=22.0 \mathrm{ft} . /$ sec. $^{2}$ vertically downwards. 


\section{CHAPTER VIII}

\section{MOTION OF RIGID BODIES}

131. Introduction.--In the preceding chapter the motion of a point or particle and the relation between the motions of two particles have been considered. In engineering problems in general, however, the motion of bodies, not particles, must be considered. In some problems, the dimensions of the body may be assumed to be negligible in comparison with its range of motion, with very small error, and hence, the methods and equations of the preceding chapter apply directly to the motion of the body. This assumption is involved in a number of problems in the preceding chapter, in which a body is assumed to be a particle. In general, however, the motion of bodies as met in engineering practice is such that the various points of a body have different motions.

The object of this chapter is to analyze certain common types of motion of rigid bodies so that the displacement, velocity, and acceleration, both linear and angular, may be found from the methods and equations developed in the preceding chapter. The motions considered are translation, rotation, and plane motion.

132. Translation.- Translation of a rigid body is a motion such that no straight line in the body changes direction, that is, each straight line remains parallel to its initial direction. Hence, at any instant, all points in the body move along parallel paths and have the same velocity and acceleration. If the translation is such as to move the particles on curved paths the motion is called curvilinear translation as, for example, the motion of the parallel rod of a locomotive. If the particles move on straightline paths the motion is called rectilinear translation. The body may have a uniformly or non-uniformly accelerated motion. Hence any point in a translating body may have any of the motions treated in the preceding chapter. Further, since all particles in the body have the same motion at any instant, the displacement, velocity, and acceleration of the body is described by the displacement, velocity, and acceleration of any particle of the body. 


\section{PROBLEM}

330. A locomotive is running on a straight track at a constant speed of $40 \mathrm{mi}$./hr. The diameter of the drivers is $6 \mathrm{ft}$. and the radius of the crankpin circle is $15 \mathrm{in}$. What is the magnitude and the direction of the velocity

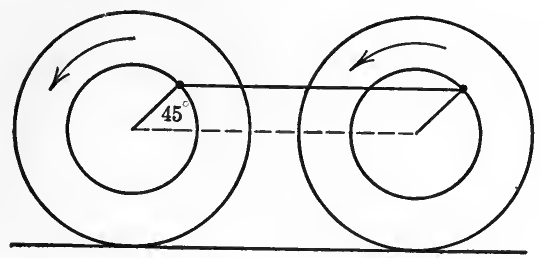

FIG. 315 .

and of the acceleration of the parallel rod, relative to the engine frame, when the rod is in the position shown in Fig. 315? What is the absolute velocity and the absolute acceleration for the same position?

133. Rotation.-Rotation of a rigid body is a motion such that one line in the body (or body extended) remains fixed in space while all points of the body describe circular paths having centers on the fixed line. The fixed line is called the axis of rotation and the plane in which the mass-center of the body moves is called the plane of motion. The point of intersection of the axis of rotation and the plane of motion is called the center of rotation. It will be noted that any line parallel to the plane of motion changes direction. The motion of a body having rotation cannot be defined or described by stating the linear displacement, velocity, and acceleration of any point in the body, as was the case for translation, since all points in the body do not have the same linear motion. However, the angular displacements, velocities, and acceleration, respectively, are the same for all particles in the body. Hence the motion of a rotating rigid body may be described by the angular motion of any point in the body. Thus all the equations in the preceding chapter dealing with the angular motion of a point moving on a circular path, in which the radius vector is the radius of the circle, apply to the motion of a rotating rigid body, as well as to each point in the body. The linear displacement, velocity, and acceleration of any point may also be found from the equations in the preceding chapter, that deal with the linear motion of a point moving on a circular path. 


\section{PROBLEMS}

331. A straight stick $4 \mathrm{ft}$. Iong rotates in a horizontal plane about a vertical axis through one end of the stick. Its angular velocity changes uniformly from 20 to 50 r.p.m. in 5 sec. What is the linear velocity of its mid-point at the end of 2 sec.?

332. The flywheel of a punching machine fluctuates from 100 to 80 r.p.m. at a uniform rate when a hole is punched. If the flywheel makes $1 \frac{1}{2}$ revolutions while this change of speed takes place, how long does it take to punch the hole?

Ans. $t=1$ sec.

333. The flywheel of a rolling-mill engine is $14 \mathrm{ft}$. in diameter. Just before the steel is fed in the rolls the speed of the flywheel is 90 r.p.m. As the steel enters the rolls the speed decreases uniformly during $\frac{1}{2}$ sec., before the governor can operate. If the angular acceleration (negative) of the flywheel is 20 r.p.m. per sec., what is the decrease in the speed of the flywheel, expressed in r.p.m.?

134. Plane Motion.-Plane motion of a rigid body is a motion such that each point in the body remains at a constant distance from a fixed plane. The motion of the connecting rod of a steam engine is an example of plane motion. The wheels of a locomotive when running on a straight track also have plane motion. A plane parallel to the fixed plane, containing the mass-center of the body, is called the plane of motion. It is evident that a rotation is always a special case of plane motion, whereas a translation may or may not be a plane motion.

In plane motion, in general, a straight line in the body lying in the plane of motion changes direction and, hence, the body rotates, but not about a fixed axis. The body, therefore, has angular motion, and its angular displacement, velocity, and acceleration are the same as that of any straight line in the body, in the plane of motion, since all such lines have the same angular motion if the body is rigid. The angular motion of the body, therefore, may be studied by means of the same equations that apply to the rotation of a rigid body about a fixed axis.

Rotation, however, is only one part of the motion of a rigid body having plane motion (except in a special case as noted in the next article). Plane motion of a rigid body may be resolved into two component motions, a rotation and a translation, according to the following theorem:

Plane motion of a rigid body, at any instant, is a combination of: (1) a pure rotation of the body, about an axis 
(perpendicular to the plane of motion) passing through any point $B$ in the body, with an angular velocity and acceleration the same as that which the body has at the instant; and, (2) a translation of the body which gives to each point the same linear velocity and acceleration that the point $B$ has at the instant.

The point $B$ is called the base point. It is evident that all points except the base point have two motions; a rotation about the base point, and a motion the same as that of the base point. From the analysis of the motion according to the above theorem, the displacement, velocity, and acceleration of any point, $A$, in the body may be found from the equations developed in Art. 128 of the preceding chapter. The equations are,

$$
\begin{aligned}
& s_{A}=s_{\frac{A}{B}} \mapsto s_{B}, \\
& v_{A}=\frac{v_{A}}{B} \rightarrow v_{B} \\
& a_{A}=a_{\frac{A}{B}} \rightarrow a_{B} .
\end{aligned}
$$

To arrive at the above theorem, consider the motion of the connecting rod of a steam engine (Fig. 316). Let $P$ denote the position of the crosshead and $Q$ that of the crank-pin, the crank being of length $O Q$. When the crank moves from position $O Q$

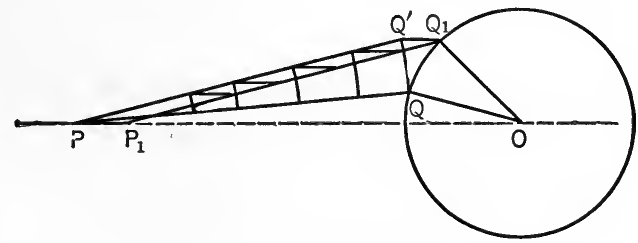

Fig. 316.

to position $O Q_{1}$ the connecting rod moves from position $P Q$ to position $P_{1} Q_{1}$. This change of position can be given the rod by first rotating the rod about $P$, until it becomes parallel to its new position, and then giving the rod a translation such that each point receives a displacement equal to $P P_{1}$ : By this combination of motions the point $P$ moves along its actual path but any other point does not travel in its actual path. The point $Q$, for 
example, moves along the path $Q Q^{\prime} Q_{1}$ instead of its actual circular path $Q Q_{1}$. However, as the change of position is made smaller and smaller, the path $Q Q^{\prime} Q_{1}$ approaches the circular path $Q Q_{1}$ and, in the limit, as the two motions are generated simultaneously, each point is made to move on its actual path by successive combinations of a proper rotation and a proper translation. The rotation, at any instant, must give the body its actual angular velocity and acceleration at the instant, since the translation does not influence the angular motion of the body. The translation must give all points of the body the same motion that the base point has at the instant, since the base point receives its total motion from the translation.

By the above method, the rod is given a rectilinear translation, since the point $P$ moves on a straight-line path. If, however, another base point is chosen as, for example, the point $Q$,

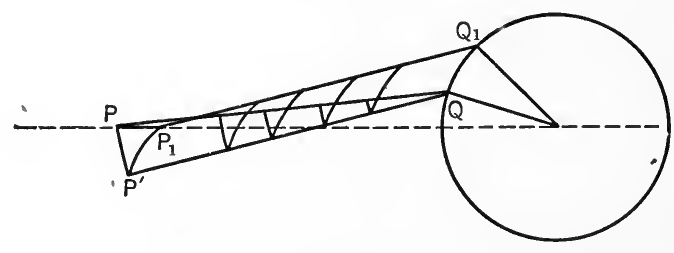

Fig. 317.

the change of position from $P Q$ to $P_{1} Q_{1}$ may then be made by: (1) a rotation about $Q$ (Fig. 317 ); and, (2) a curvilinear translation giving to each point the same motion that $Q$ has. By this combination of a rotation and a translation the point $P$ is made to take the path $P P^{\prime} P_{1}$ instead of its actual path $P P_{1}$, but, reasoning as above, if the change of position is made smaller and smaller the path $P P^{\prime} P_{1}$ approaches the path $P P_{1}$ and, in the limit, as the two motions are imposed simultaneously, each point in the body is given its exact motion at any instant. Likewise, any other point in the body may be selected as the base point. And, the plane motion of any other rigid body may be treated in like manner. Hence, a plane motion of a rigid body may be considered, at any instant, as a combination of a rotation of the body about any base point in the body, with an angular velocity and acceleration equal to the angular velocity and acceleration that the body has at the instant, and a translation of the body that 
gives to each point the same linear velocity and acceleration that the base point has at the instant.

In applying the equations,

$$
v_{P}=v_{\frac{P}{Q}} \mapsto v_{Q} \text { and } a_{P}=a_{\frac{P}{Q}} \nrightarrow a_{Q},
$$

to the motion of any two points in a rigid body having plane motion, the point $Q$ represents the base point. However, as stated above, the base point may be any point in the body. Further, the angular velocity (and acceleration) of the body is the same with reference to all base points in the body.

Since any two points in a rigid body remain a fixed distance apart, there can be no relative velocity of one point toward the other, that is, the relative velocity of either point with respect to the other is perpendicular to the line joining the two points. This is not true, however, of the relative acceleration, since it is made up of a tangential and a normal component, the normal component having a direction along the line joining the two points. The principles discussed in this article are involved in the solution of the following problem.

\section{ILLUSTRATIVE PROBLEM}

334. A 50 h.p. engine has a cylinder $10 \mathrm{in}$. in diameter and a stroke of 10 in. The engine runs at a constant speed of $\omega=300$ r.p.m. The ratio of the length of the connecting rod to that of the crank is 5 . Find the velocity and the acceleration of the crosshead when the crank angle is $30^{\circ}$.

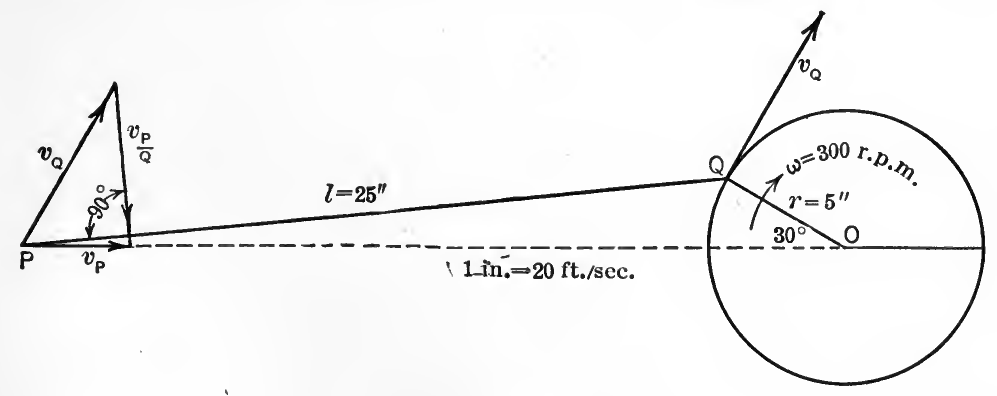

FIG. 318.

Solution.-Let $P$ be the crosshead and $Q$ the crank pin. Then in Fig. $318 P Q$ represents 25 in. and $O Q$ or $r$ represents 5 in., according to the scale used. The velocity of $P$ may be found from the equation,

$$
v_{P}=v_{P}+v_{Q} \text {. }
$$


There are six elements involved in the equation-three magnitudes and three directions, four of which must be found before the equation can be used to determine the other two. The direction of the velocity of $Q$ is perpendicular to $O Q$ and its magnitude is,

$$
v_{Q}=\omega r=\left(\frac{300 \times 2 \pi}{60}\right) \times \frac{5}{12}=13.1 \mathrm{ft} . / \mathrm{sec} .
$$

The direction of $v_{\frac{P}{Q}}$ is perpendicular to the line joining $P$ and $Q$ and its magnitude is unknown. The direction of $v_{P}$ is horizontal. Hence by laying off $v_{Q}$ to a convenient scale in the proper direction from $P$, and by drawing a line from the end of $v_{Q}$, perpendicular to the connecting rod, until it intersects a horizontal line through $P$, the magnitudes of $v_{P}$ and $v_{P}$ are determined by the intersection. By scaling off the values of $v_{\frac{P}{Q}}$ and $v_{P}$, the following results are found

$$
v_{\frac{P}{Q}}=11.4 \mathrm{ft} . / \mathrm{sec} \text {. and } v_{P}=7.8 \mathrm{ft} . / \mathrm{sec} .
$$

Thinking of the plane motion of the connecting rod as a combination of a rotation and a translation, $v_{\underline{P}}$ is the velocity which $P$ is given by the rotation of the rod about $Q$ and $v_{Q}$ is the velocity given to $P$ by the translation of the rod. The two velocities produce the resultant velocity $v_{P}$.

The acceleration of $P$ is given by the equation,

$$
a_{P}=\underset{\bar{Q}}{a_{P}} \mapsto a_{Q} \text {. }
$$

For convenience, $a_{P}$ will be replaced by its tangential and normal components.

Hence,

$$
\bar{Q}
$$

$$
a_{P}=\left(\frac{a_{P}}{Q}\right)_{\imath} \mapsto\left(\frac{a_{P}}{Q}\right)_{n} \mapsto a_{Q}
$$

Eight elements are involved in the equation-four magnitudes and four directions, six of which must be found before the graphical construction representing the equation can be completed. $a_{Q}$ is directed from $Q$ toward $O$ and its magnitude is,

$$
a_{Q}=\omega^{2} r=\left(\frac{300 \times 2 \pi}{60}\right)^{2} \times \frac{5}{12}=412 \mathrm{ft} . / \text { sec. }^{2}
$$

$a_{P}$ is known in direction, being parallel to $P O$ (horizontal), since $v_{P}$ changes in magnitude only. $\left(\frac{a_{P}}{\bar{Q}}\right)_{n}$ is directed from $P$ toward $Q$ and its magnitude is,

$$
\left(\frac{a_{P}}{Q}\right)_{n}=\frac{\left(\begin{array}{c}
v_{P} \\
\bar{Q}
\end{array}\right)^{2}}{P Q}=\frac{(11.4)^{2}}{\frac{25}{12}}=62.4 \mathrm{ft} . / \text { sec. }^{2}
$$


$\left(\frac{a_{P}}{Q}\right)_{t}$ has a direction perpendicular to $P Q$. The two unknown elements are, therefore, the mágnitudes of $a_{P}$ and $\left(\begin{array}{c}a_{P} \\ \bar{Q}\end{array}\right)_{i}$.

In Fig. 319, starting at $P$, the vectors are laid off to a convenient scale. Thus $a_{Q}$ and $\left(\frac{a_{P}}{Q}\right)_{n}$ (both of which are completely known) are drawn and then, fron the end of $\left(\begin{array}{c}a_{P} \\ \frac{Q}{Q}\end{array}\right)_{n}$, a line is drawn that represents the direction of

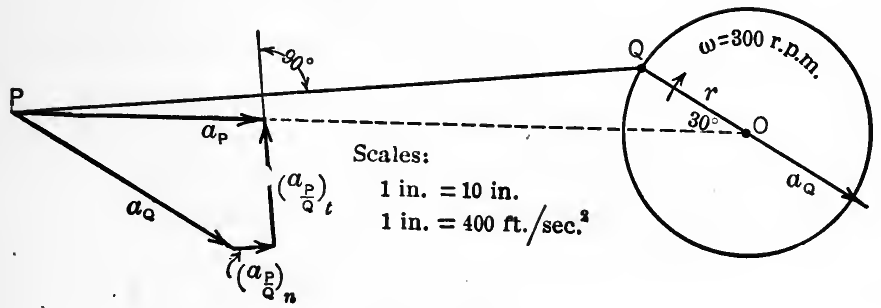

Fig. 319.

$\left(\frac{a_{P}}{Q}\right)_{i}$, that is, it is drawn perpendicular to $P Q$. The intersection of this line with the line $P O$ (which represents the direction of $a_{P}$ ) determines the lengths of the vectors that represent $\left(\begin{array}{c}a_{P} \\ \bar{Q}\end{array}\right)_{\ell}$ and $a_{P}$. By scaling off the values, the following results are obtained,

$$
\left(\frac{a_{P}}{Q}\right)_{t}=200 \mathrm{ft} . / \mathrm{sec}^{2} \text { and } a_{P}=404 \mathrm{ft} . / \text { sec. }^{2} .
$$

\section{PROBLEMS}

335. A wheel $4 \mathrm{ft}$. in diameter rolls, without slipping, on a horizontal track (Fig. 320). The velocity of its center, at a given instant, is $4 \mathrm{ft}$./sec. to the right and the acceleration of the center is 6 $\mathrm{ft} . / \mathrm{sec}^{2}$ in the same, direction. Find the vielocities and the accelerations of the points $A$ and $B$.

336. Find, by the graphical method, the velocity and the acceleration of the point $C$ of the four-link mechanism described in Problem 337 (Fig. 322). Use the following scales: $1 \mathrm{in} .=1 \mathrm{ft}$; 1 in. $=2$ ft./sec.; and $1 \mathrm{in} .=10 \mathrm{ft} . / \mathrm{sec}^{2}$

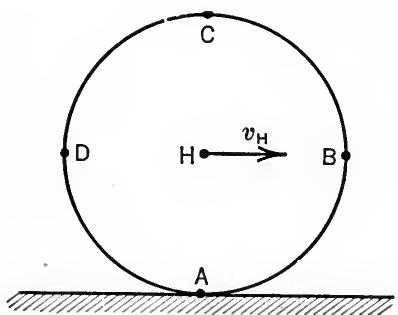

Fic. 320

Ans. $v_{c}=1.54 \mathrm{ft} . /$ sec.; $a_{c}=55.5 \mathrm{ft} . / \mathrm{sec}^{2}{ }^{2}$ 
135. Instantaneous Center.-It was shown in the previous article that a plane motion of a rigid body may be considered, at any instant, as a rotation, about an axis through any point in the body, combined with a translation. However, by choosing a particular axis in the body (or its extension), the motion of the body, at any instant, becomes one of rotation only, that is, no translation need be combined with the rotation to produce the actual motion of the body. This axis is called the instantaneous axis of rotation or the instantaneous axis of zero velocity. Its intersection with the plane of motion is called the instantaneous center of rotation.

To show a method of locating the instantaneous center, let $A$ and $B$ be any two points in the plane of motion of a rigid body having plane motion (Fig. 321). Let $v_{A}$ denote the velocity of $A$, and $v_{B}$, the velocity of $B$. From $A$ draw

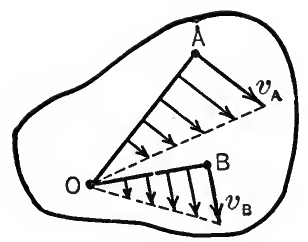

FIG. 321. a line perpendicular to $v_{A}$ and from $B$ draw a line perpendicular to $v_{B}$, the two lines intersecting in $O$. Any point in the body on the line $O A$, if not at rest, must have a velocity perpendicular to $O A$, since any two points in the body cannot move toward or away from each other if the body is rigid. Likewise, any point on the line $B O$, if not at rest, must move perpendicular to $B O$. Hence, the point of intersection of $A O$ and $B O$ must be at rest since it cannot, at the same instant, move in two directions. Therefore, the body, if not at rest, must rotate about the point $O$ at the instant.

If, then, $A$ and $O$ are two points in the rigid body having plane motion, $A$ being any point, and $O$ the instantaneous center, the equation,

$$
v_{A}=v_{\bar{A}}+v_{O}
$$

reduces to,

$$
v_{A}=v_{\bar{A}} \rightarrow 0=\omega \times O A,
$$

in which $\omega$ is the angular velocity of the body. Further, since $\omega$ is the same for all points in the body, it follows that the velocities of any two points $A$ and $B$ vary as their distances 
from the instantaneous center. Or, expressed in equational form,

$$
\frac{v_{A}}{v_{B}}=\frac{\omega \cdot O A}{\omega \cdot O B}=\frac{O A}{O B} \text {. }
$$

It should be noted that the instantaneous center is the center of zero velocity and not of zero acceleration. In the case of the motion of rotation of a rigid body about a fixed axis, which is a special case of plane motion, the axis of rotation is also the instantaneous axis and it has zero acceleration as well as zero velocity since it is at rest. In the general case of plane motion, however, the instantaneous center changes its position in the body and also in space. Although the velocity of the point in the body, coinciding with the instantaneous center at a given instant, is zero, the velocity is changing through its zero value and hence the point has an acceleration. Therefore in the equation,

$$
a_{A}=a_{\frac{A}{o}} \mapsto a_{o}
$$

in which $A$ is any point and $O$ is the instantaneous center, $a_{o}$ is not zero. Hence the actual acceleration, at any instant, of a point in a rigid body having plane motion cannot be found by considering the body to be rotating about a fixed axis through the instantaneous center, as may be done in determining the velocity of any point of the body.

\section{ILLUSTRATIVE PROBLEM}

337. The four-link mechanism, $A B C D$, shown in Fig. 322 has the following dimensions: $A B=6 \mathrm{in}$.; $B C=3 \mathrm{ft}$.; $D C=2 \mathrm{ft}$.; $A D=4 \mathrm{ft} . ; \theta=45^{\circ}$. Find the instantaneous center for the link $B C$. If the orank $A B$ rotates at a constant angular velocity $\omega=10 \mathrm{rad} . / \mathrm{sec}$., find the angular velocities, $\omega_{2}$, of the link $D C$ and, $\omega_{3}$, of the link $B C$. Also find the linear velocity, $v_{H}$, of $H$, the midpoint of $B C$.

Solution.-The instantaneous center for the link $B C$ is $O$, the point of intersection of the lines $A B$ and $D C$ extended. By scaling off the lengths of $O B, O C$, and $O H$, the following values are found:

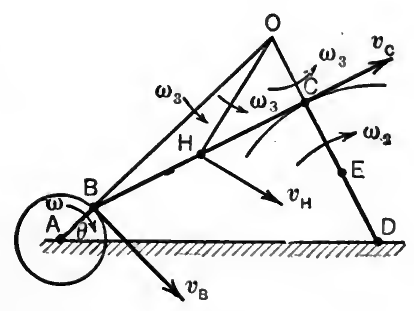

Fig. 322.

$$
O B=3.1 \mathrm{ft} . \quad O C=0.96 \mathrm{ft} . \quad O H=1.72 \mathrm{ft} .
$$


The velocity of $B$ is,

$$
v_{B}=\omega \times A B=10 \times \frac{\beta}{12}=5 \mathrm{ft} . / \mathrm{sec} .
$$

Therefore the angular velocity of $B$, considered as a point on $B C$, is,

$$
\omega_{3}=\frac{v_{B}}{O B}=\frac{5}{3.1}=1.61 \mathrm{rad} . / \mathrm{sec} .
$$

and the angular velocities of all points on $B C$ are the same. The linear velocity of $C$ is, therefore,

And,

Therefore,

$$
\begin{aligned}
& v_{C}=\omega_{3} \times O C=1.61 \times 0.96=1.54 \mathrm{ft} . / \mathrm{sec} . \\
& v_{H}=\omega_{3} \times O H=1.61 \times 1.72=2.77 \mathrm{ft} . / \mathrm{sec} .
\end{aligned}
$$

$$
\omega_{2}=\frac{v_{C}}{D C}=\frac{1.54}{2}=0.77 \mathrm{rad} . / \mathrm{sec} \text {. }
$$

\section{PROBLEMS}

338. What is the magnitude and the direction of the velocity of the point $E$ (Fig. 322) on link $D C$ midway between $D$ and $C$.

Ans. $v_{E}=0.77 \mathrm{ft} . /$ sec. perpendicular to $D C$.

339. Consider the linł $B C$ (Fig. 322) to be the triangular piece $B O C$ and let $P$ be a point on the triangular piece midway from $O$ to $H$. What is the magnitude and the direction of the velocity of the point $P$ ?

340. Locate the instantaneous center of the connecting rod of the steamengine mechanism described in Problem 334. Find the angular velocity of the connecting rod, the linear velocity of the crosshead, and the linear velocity of a point on the connecting rod midway from the crosshead to the crank-pin. Ans. $\omega_{2}=5.47 \mathrm{rad} . / \mathrm{sec}$.; $v_{P}=7.70 \mathrm{ft}$. $/ \mathrm{sec}$.; $v_{M}=9.12 \mathrm{ft} . / \mathrm{sec}$.

341. Find the instantaneous center of the wheel described in Problem 335. Find also the angu!ar velocity of the wheel and the magnitudes and directions of the velocities of the points $B, C$, and $D$. 


\section{PART III. KINETICS}

\section{CHAPTER IX \\ FORCE, MASS, AND ACCELERATION}

\section{§1. Preliminary Considerations. Kinetics of a Particle}

136. Introduction.-Kinetics is that branch of mechanics which treats of the laws in accordance with which the motion of physical bodies takes place.

A change in the state of motion of a body always occurs when an unbalanced force system acts on the body, the unbalanced part (resultant) of the force system being the cause of the change in the motion. Common experience teaches that the change of motion of the body. is influenced both by the characteristics (Art. 5) of the resultant of the forces acting on the body and by the nature of the body itself. For example, different force systems acting, in turn, on the same body do not produce the same change of motion of the body. Further, the same force system applied to different bodies, even if they are of the same size and form, does not produce the same change in the motion of all of the bodies.

Although experience suggests that relations exist between the force system acting on the body, the properties of the body, and the change in the motion of the body, it required the work of many eminent men and a period of several centuries before definite and complete fundamental relations between these three factors were finally established. These relations were formulated by Sir Isaac Newton (1642-1727) and are known as Newton's laws of motion.

Newton's fundamental laws, however, apply only to the motion of a particle under the action of a single force, whereas in engineering practice the motion of a body (system of particles) under the action of a system of forces must be considered. The force system acting on the body may produce any 
type of motion. Thus, in some cases a motion of translation is given to the body, in other cases the body is given a motion of rotation, and in still other cases the force system produces a plane motion of the body. These three types of motion, only, are considered in this chapter.

137. The General Kinetics Problem.-In each type of motion however, the general character of the kinetics problem is the same: A physical body is acted on by a force system that has a resultant, which causes a change in the motion of the body. In each problem it is required to deduce, by the use of Newton's laws, the equations expressing the definite relations between (1) the resultant of the force system, (2) the kinetic properties of the body, and (3) the change of motion of the body, so that the motion of a given body produced by a given force system may be determined, or the force system required to produce a given motion may be found. The equations which express these relations are called the equations of motion for the body.

These three elements or factors which are involved in the equations of motion of bodies may be considered briefly before stating Newton's laws and before deriving the equations which express the relations between these three factors. Change of motion is measured in terms of distance, time, velocity, and acceleration, relations between which, for various types of motion, have already been considered fully in Chapters VII and VIII. The characteristics of the resultant of a force system have also been considered in Chapter II, and need only be reviewed briefly at this point (see next article) to show their connection with the general problem in kinetics. The nature or property of the body which enables it to have an influence in determining its own motion, however, needs to be discussed at greater length (see Art. 139).

138. Characteristics of a Force System.-The only part of a force system that influences the motion of a body is the unbalanced part (resultant) of the force system. The forces which produce the $i$ ypes of motion considered in this chapter are coplanar forces or may be so regarded. ${ }^{1}$ The resultant of a coplanar force

${ }^{1}$ Although in most kinetics problems here considered, the motions are produced by coplanar force systems, the system of forces may be non-coplanar. It will be evident, however, after discussing the equations of motions for the three types of motion here considered, that non-coplanar forces that produce these motions may be replaced by equivalent coplanar force systems. 
system is either a force or a couple (Art. 28), although the resultant, when a force, may, for convenience, be considered as another force and a couple (Art. 18). If the resultant is a force, the characteristics of the resultant which influence the motion of the body on which the force system acts are (1) its magnitude, (2) the position of its action line in the body, and (3) its sense (Art. 5). If, however, the resultant is a couple, the characteristics of the resultant which influence the motion of the body on which the force system acts are (1) the moment of the couple, (2) the sense, and (3) the aspect or direction of the plane of the couple (Art. 16). These statements appeal directly to one's experience and may be verified by simple experiments which the reader may readily perform.

Since the equations of motion of a body must take account of all the factors influencing the motion, there must be a sufficient number of equations to determine the influence of all of the characteristics of the resultant of the force system as mentioned above. This may be done, for the types of motion considered in this chapter, by means of three equations. These three equations will contain the algebraic sum of the $x$-components of the forces acting on the body, the algebraic sum of the $y$-components, and the algebraic sum of the moments of the forces about some axis in the body. For, if the resultant of the force system acting on the body is a force, the algebraic sums of the $x$ - and $y$-components of the forces are needed to take account of the influence of the magnitude of the resultant force and of the direction of its action line, and the algebraic sum of the moments of the forces is needed to measure the influence of the position of the action line of the resultant force. And, if the resultant of the force system is a couple, the motion of the body is one of rotation as will be seen later (Art. 147), in which case, the algebraic sum of the moments of the forces is needed to measure the influence of the moment and sense of the couple. The aspect of the couple is not involved in the equations of motion since the plane of the couple always agrees with that of the plane of motion of the body.

139. Inertia and Mass.-The property of a body by virtue of which it offers resistance to any change in its motion, and thereby makes the body itself a factor in determining the motion which unbalanced forces impress upon it, is called inertia. All physical bodies are inert or possess inertia, but different bodies possess different amounts of inertia. That is, all bodies influence their 
own motion according to the same law but not to the same degree. ${ }^{1}$

Bodies possess many other properties such as hardness, gravitation, elasticity, strength, etc. In taking into account the influence which any one of these properties has on the behavior of the body, under the conditions which make the property a factor in the problem, a quantity (value of the property) is found which measures the particular property; that is, which indicates its relation to the other influencing factors and which, if possible, has a constant value for the body or for the material of the body. Thus, when the property of strength is involved in a problem, the constant values called " elastic limit" and "ultimate strength" are frequently used as a measure of the strength of the material. Similarly, the constant value called "modulus of elasticity" is used as a measure of the property of elasticity or stiffness of a material.

In the kinetics problem, that is, in a problem in which a change in the motion of a body occurs, the property of inertia of the body is involved. In taking account of the influence which the property of inertia has on the motion of the body, a quantity called the mass of the body is used. The two essential characteristics of mass are its direct relation to the other factors (forces and acceleration) which are involved in the kinetics problem and its constant value. Thus the mass of a body is a constant of the body which measures a kinetic property (inertia) of the body.

The term mass then, as used in mechanies, denotes the amount of inertia possessed by a body. Or, the mass of a body may be defined as the constant of the body which measures the influence which the body exerts in determining its own motion, when acted upon by an unbalanced force system. The amount of resistance offered by a body to a change in its motion (that is, the amount of inertia possessed by a body), must be determined experimentally. An experiment for determining the mass may be outlined as follows:

${ }^{1}$ The way in which bodies resist motion is analogous to the manner in which elastic materials, such as steel or timber, resist being stretched. Each material, within limits, resists according to the same law (stretch is proportional to stress), but some materials resist to a greater degree, that is, some materials are stiffer than others, which means that some materials require a greater force to produce a given stretch than do other materials, just as some bodies require a greater force to overcome their inertia at a definite rate than do other bodies. 
Let a body $A$ be acted upon by a single force $F_{1}$ causing a change in its motion such that the acceleration of the body is found to be $a_{1}$. In the same manner let the forces $F_{2}, F_{3}$, etc., act on the same body, in turn, causing accelerations which are found to be $a_{2}, a_{3}$, etc., respectively. The results of such an experiment show that,

$$
\frac{F_{1}}{a_{1}}=\frac{F_{2}}{a_{2}}=\frac{F_{3}}{a_{3}}=\text { etc. }=\text { a constant }=C_{1} \text { (say). }
$$

If any other body is acted upon in a similar manner ${ }^{1}$ by other forces $\left(F^{\prime}, F^{\prime \prime}, F^{\prime \prime \prime}\right.$, etc.), one at a time, such that the body is given the same series of accelerations $\left(a_{1}, a_{2}, a_{3}\right.$, etc.) as was given to the first body, it will be found that,

$$
\frac{F^{\prime}}{a_{1}}=\frac{F^{\prime \prime}}{a_{2}}=\frac{F^{\prime \prime \prime}}{a_{3}}=\text { etc. }=\text { a constant }=C_{2} \text { (say). }
$$

That is, the manner in which a body influences its own motion is by requiring that any force which is impressed on it shall bear a constant ratio to the acceleration which the force gives to the body. Further, it will be noted that the particular change in the motion of the body that is related directly to the forces acting on the body and the kinetic properties of the body is the acceleration of the body.

Although the ratio of the impressed force to the acceleration produced is constant for any given body it has different values for different bodies. That is, $C_{1}$ and $C_{2}$ as used above are, as a rule, not equal. Now $C_{1}$ and $C_{2}$ are measures of the inertia resistance offered by the particular bodies to a change in their motion since they measure the force required to overcome the inertia at a definite rate (acceleration). The constants $C_{1}$ and $C_{2}$, therefore, are proportional, respectively, to the masses of the two bodies. T'hat is, $C_{1}=k M_{1}$ and $C_{2}=k M_{2}$ in which $k$ is a constant and $M_{1}$ and $M_{2}$ are the masses of the bodies. If now the mass, $M_{2}$, of the second body is taken as the unit by which the mass of the first body is measured, then the ratio $\left(C_{1} / C_{2}\right)$ of the constants expresses the number of units of mass in the first body. The numerical value of this ratio, therefore, depends upon the choice of the value of $\mathrm{C}_{2}$, and hence, of the unit of mass.

${ }^{1}$ It is assumed in this experiment that a motion of rectilinear translation is given to the body in each case and hence the only cha-acteristic of the force that changes is its magnitude. 
Now, as shown above, $C_{2}$ is expressed by the equation $C_{2}=\frac{F}{a}$. Therefore, the unit of mass will depend upon the units in which $F$ and $a$ are expressed. The various units of force and acceleration, and the resulting units of mass are discussed in detail in Art. 141. Mass is considered at this point only to show its general connection with the other quantities (force and acceleration) involved in the kinetics problem and to show a method of determining it.

The experiment suggested above for determining the mass of a body by finding the ratio of an impressed force to the acceleration produced, is difficult and inconvenient to perform. Fortunately, a much simpler experiment serves the same purpose. Careful experiments have shown that the weights (earth-pulls) of bodies, when the weights $(W)$ are the only forces acting on the bodies, produce the same acceleration, $g$, of all the bodies at a given location on the earth's surface, the approximate value of $g$ for most localities being $32.2 \mathrm{ft}$. per sec. per sec. Hence, if instead of using the ratio of any force, $F$, to the corresponding acceleration, $a$, the ratio $\frac{W}{g}$ is used, the only experiment needed to determine the mass of a body is that of weighing the body on an ordinary spring balance since the value of $g$ is known. Therefore,

$$
\frac{F}{a}=\frac{W}{g} .
$$

But, as shown above, the ratio of the force $F$ to the acceleration, $a$, produced by the force is proportional to the mass $M$ of the body, that is, $\frac{F}{a}=k M$. Therefore,

$$
\frac{W}{g}=k M \text {. }
$$

And if units for $W, g$, and $M$ are so chosen that $k$ equals unity, as will be discussed in Art. 141, then the number of units of mass in a body may be found from its weight by the equation

$$
M=\frac{W}{g} .
$$


140. Newton's Laws.-Newton established his laws of motion from a study of the motion of planets whose orbits and speeds were then well known. Since the dimensions of a planet are negligible in comparison with the range of its motion, Newton's laws really apply directly only to a particle, that is, to a body, all points of which may be considered at any instant to have the same acceleration. In most cases of motion of bodies, however, the accelerations of different particles of the body are not the same. Thus, the accelerations of various parts of a rotating flywheel or of the connecting rod of a steam engine are quite different. The expression "acceleration of a body" is, therefore, indefinite and meaningless, except for a body having the motion of translation.

Newton's laws may be stated as follows:

First Law.-A particle remains at rest or continues to move uniformly in a straight line unless acted upon by an unbalanced force.

Second Law.-When a single (unbalanced) force acts on a particle the particle is accelerated; the direction of the acceleration is the same as that of the force and its magnitude is directly proportional to the force and inversely proportional to the mass of the particle.

Third Law.-There are mutual actions between any two particles of a system (body) such that the action of the one on the other is equal, collinear, and opposite to that of the other on the one.

1. The first law is a qualitative one. It states that a particle has inertia, that is, it resists having its motion changed. It statcs that a force must act on the particle if its motion (velocity) is changed either in direction or in magnitude, that is, if an acceleration is produced.

2. The second law is a quantitative one. It states what the magnitude and the direction of the force must be in order to produce a given acceleration of a given particle, and shows that although a particle cannot, of itself, change its state of motion it does nevertheless influence the change of motion caused by the force, by regulating or governing the manner in which the acceleration shall take place; namely, that it shall be always inversely proportional to the mass of the particle.

3. In the first two laws it is assumed that a single particle is acted upon by a single force. But the third law brings out the 
fact that a single force does not exist. One force requires the presence of another force. A push cannot be exerted unless there is a resisting force to develop the push; that is, forces always occur in pairs. A pair of forces, however, requires the presence of a pair of particles each of which acts on the other, and hence requires that each offers a resistance to the other. The resistance which the second particle offers to the first, in general, may arise in two ways: (1) by transmitting to the first particle the force (static reaction) which a third particle (or several particles) exerts on it when holding it in equilibrium, or (2) by virtue of its inertia as it allows the first particle to change its motion (kinetic reaction). Or, the resistance may be a combination of these causes.

In a moving system of particles, therefore, the motion of each particle is influenced by every other particle in the system. These actions and reactions which influence the motion of the particles of the body are, in general, impossible to determine. However, since they occur as collinear pairs (third law) the equations of motion for a system of particles (body) may be found without introducing them into the equations, for, in finding the sums of $x$ - and $y$-components and the sum of the moments of all the forces acting on all the particles, the mutual actions and reactions between the pairs of particles drop out of the expressions, since these sums are equal to zero. Hence, by use of Newton's third law, the second law, which applies directly only to a single particle under the action of a single force, may be used to extend the equations of motion to a system of particles under the action of a system of forces.

141. Mathematical Statement of Newton's Second Law. Units. -Newton's second law may be expressed mathematically by the equation

$$
F=k m a,
$$

in which $a$ is the acceleration of the particle of mass $m, F$ is the single force acting on the particle, and $k$ is a constant factor the value of which depends upon the units used to express the other quantities $(F, m$, and $a)$ in the equation. But in Art. 139 it was shown that $\frac{F}{a}=\frac{W}{g}$. Hence for any particle,

$$
\frac{F}{a}=\frac{W}{g}=k m
$$


It will be observed, therefore, that although the mass, $m$, of a particle is always proportional to $\frac{F}{a}$, the numerical value of $m$ (that is, the number of units of mass in the particle), is expressed by $\frac{F}{a}$ (or more conveniently by $\frac{W}{g}$ as discussed in Art. 139), only when units are chosen for the force $F$ and the acceleration $a$ such that $k$ becomes unity. It is obvious that such a system of units is desirable for, when such a system is chosen, Newton's second law may be written simply

$$
F=m a=\frac{W}{g} a .
$$

A system of units which gives a valua of unity to $k$ may be chosen as follows: In the equation $F=k m a$ four quantities are involved. If a particular value (unity) is assigned to $k$ then the units for only two of the other quantities may be chosen arbitrarily. In engineering, the unit of force and of acceleration are chosen and hence the unit of mass is derived from the units selected for force and acceleration. If the pound is chosen as the unit of force and the foot per sec. per sec. as the unit of acceleration, as is usual in engineering, it is evident that the body which has a unit of mass is one which, when acted on by a unit force (pound), will be given a unit acceleration (foot per second ${ }^{2}$ ). That is, if in the equation $F=k m a, k$ is made unity, $F$ is made unity (one pound), and $a$ is made unity (one foot per second ${ }^{2}$ ), then $m$ must be unity (one unit).

The body which is given an acceleration of $1 \mathrm{ft}$. per sec. per sec. by a 1-lb. force, that is, a body of unit mass, may be found by experiment. The direct method would be to allow a 1-lb. force to act on different bodies until one is found which is given an acceleration of $1 \mathrm{ft}$. per sec. per sec. However, experiments have been made which supply sufficient data from which the weight of a body of unit mass may be found. Thus, experiments have shown that the earth-pull on any body, if it is the only force acting, gives the body an acceleration of $g$, the value of which at most localities is $32.2 \mathrm{ft} . /$ sec. $^{2}$ (approximately). Hence, if the earth-pull on a body is $1 \mathrm{lb}$., that is, if the body weighs $1 \mathrm{lb}$., it will be given an acceleration of $32.2 \mathrm{ft} . / \mathrm{sec}^{2}$ and not $1 \mathrm{ft} . / \mathrm{sec}^{2}$ And, if a $1-\mathrm{lb}$. force acts on a free body which weighs more than $1 \mathrm{lb}$. the acceleration produced will decrease in proportion to the increase in weight (New- 
ton's second law). Therefore, a body weighing $32.2 \mathrm{lb}$. will be given an acceleration of $1 \mathrm{ft}$. per sec. per sec. by a 1-lb. force. Therefore, a body weighing $32.2 \mathrm{lb}$. is a body of unit mass. A body weighing $64.4 \mathrm{lb}$. has two units of mass, or, in general, a body weighing $W$ pounds has $\frac{W}{g}$ units of mass.

No name for the unit of mass as here used has gained general acceptance as has the name pound for the unit of force. The name slug (from sluggishness which suggests inertia) is used to some extent, particularly in England. The name geepound which suggests $32.2 \mathrm{lb}$. is also used to some extent, but, in general, the unit of mass here considered is called simply a unit of mass (sometimes the engineer's unit of mass as contrasted with that used by the physicist). If a special name is given to it, such as a slug or geepound, the tendency is to think of the unit of mass as an arbitrarily chosen unit like that of force and of acceleration and to forget that it is a derived unit; derived from the units of force and acceleration. Thus, the number of units of mass in a body when expressed by $\frac{W}{g}$, as discussed above, will be a certain number of $\frac{\text { lb. sec. }^{2}}{\mathrm{ft} .}$, for, according to the dimensional equation for mass,

$$
\text { mass }=\frac{\text { Force }}{\text { Acceleration }}=\frac{F}{\frac{L}{T^{2}}}=\frac{F^{\prime} T^{2}}{L} .
$$

Hence the mass of a body which weighs $966 \mathrm{lb}$. is

$$
\frac{W}{g}=\frac{966}{32.2}=30 \frac{\text { lb. sec. }^{2}}{\mathrm{ft} .} \text {. }
$$

Or, if each of the units of mass is called a slug or a geepound the mass of the body may be denoted as 30 slugs or 30 geepounds. A quantity expressed in the units $\frac{\mathrm{lb} \text {. sec. }}{2}$ is difficult to visualize or to interpret as a physical quantity, which accounts for the fact that mass as used by the engineer sometimes fails to appeal to common experience.

142. Other Systems of Units.-If the unit of force and unit of acceleration are chosen arbitrarily and the unit of mass derived therefrom, as was done in the preceding article, the system of units thus obtained is called a gravitational system. 
If the unit of mass and of acceleration are chosen arbitrarily and the unit of force derived therefrom, the system of units thus obtained is called an absolute system.

If, in either the gravitational or the absolute system of units, the units are so chosen that the value of $k$ in the equation $F=k m a$ becomes unity, the system is called a systematic or kinetic system.

If the kilogram is selected as the unit of force and the meter per sec. per sec. as the unit of acceleration instead of selecting the pound and the foot per sec. per sec. as was done in the preceding article, then the number of units of mass in the body will be, as before, expressed by $\frac{W}{g}$, but each unit of the mass will be expressed in terms of kilograms, meters, and seconds, instead of pounds, feet, and seconds.

A systematic absolute system of units commonly used by physicists in accurate scientific work and in engineering electrical measurements is a system in which the gram, centimeter, and second are used as the units of mass, length, and time, respectively. Thus, if the gram is chosen for the unit of mass and the centimeter per sec. per sec. for the unit of acceleration and if $k$ is to have a value of unity, then in the equation $F=k m a, F$ must be unity. Hence, the force that will give a mass of 1 gram an acceleration of $1 \mathrm{~cm}$. per sec. per sec. is the unit of force and is called a dyne. A dyne is $\frac{1}{981}$ of a gram. This fact may be shown as follows: The earth-pull on a body of unit mass (gram) produces an acceleration of $981 \mathrm{~cm}$. per sec. per sec. instead of $1 \mathrm{~cm}$. per sec. per sec. Hence, from Newton's law, it follows that a force of $\frac{1}{981}$ gram acting on the same body (unit mass) will produce an acceleration of $1 \mathrm{~cm}$. per sec. per sec. That is, $\frac{1}{981}$ gram is the unit of force in the absolute system of units.

If the pound is used for the unit of mass and the foot per sec. per sec. for the unit of acceleration, an absolute system of units is obtained in which the unit of force is called the poundal, corresponding to the dyne in the metric absolute system. The poundal is $\frac{1}{32.2}$ of a pound. This fact may be shown by reasoning in a way similar to that used above in showing that a dyne is $\frac{1}{981}$ of a gram.

143. Equations of Motion for a Particle.-Since Newton's laws apply directly only to a particle, they are limited to the action of a single force or of a force system which has a single force (not a 
couple) for a resultant, for the reason that the force system which acts on a particle is a concurrent system having as its resultant a single force acting through the particle (point of concurrence). Newton's laws, therefore, state the relation between (1) the resultant force acting on a particle, (2) the mass of the particle, and (3) the acceleration of the particle. As noted above, however, by means of the third law, this relation may be extended so as to apply to the motion of a system of particles under the action of a system of forces.

From Newton's second law the equation of motion of a particle, when systematic units are used, is,

$$
R=m a
$$

in which $R$ is the resultant of all the (concurrent) forces acting on the particle, $m$ is the mass of the particle, and $a$ is the acceleration of the particle. The action line of $R$ passes through the particle since the particle is the point of concurrence of the forces acting on it. Both $R$ and $a$ are directed quantities and their directions always agree; hence, both sides of the equation may be resolved into components. For convenience, three rectangular axes, $x, y$, and $z$, are chosen and, since $R_{x}=\Sigma F_{x}, R_{y}=\Sigma F_{y}$, etc., three equations of motion may be written as follows:

$$
\begin{aligned}
& \Sigma F_{x}=m a_{x} \\
& \Sigma F_{y}=m a_{y} \\
& \Sigma F_{z}=m a_{z}
\end{aligned}
$$

in which $\Sigma F_{x}, \Sigma F_{y}, \Sigma F_{z}$ are the algebraic sums, in the specified directions, of all the forces acting on the particle.

It is important to note that all of the forces acting on the particle are external to the particle. But, when the particle considered is one of a system of particles, some of the forces acting on it are exerted by the other (neighboring) particles of the system and are, therefore, internal forces when considering the motion of the whole system of particles.

NoтE.- In the problems which follow, the assumption is made that the bodies having the motions described may be considered to be particles without introducing serious errors in the analysis of the motion. The problems may be divided roughly into two classes; (1) the forces acting on the particle are given and the characteristics (distance, time, velocity, acceleration) of the resulting motion are required, and (2) the characteristics of the motion are 
given and the forces required to produce the motion are required. In solving the problems, therefore, the fact should be kept in mind that the kinematics equations of Chapter VII are to be used, when needed, in conjunction with the equations of motion of the particle, since the acceleration of the particle is involved both in the kinematics and the kinetics of the particle. In solving problems in kinetics it is important to follow a rather definite procedure the main steps in which may be outlined as follows:

1. Determine carefully what is given in the problem.

2. Determine carefully what is required in the problem. These two steps, as a rule, require a sketch with the known and unknown quantities either indicated on the sketch or briefly listed.

3. Draw a complete free-body diagram of the body (particle). That is, show the actions of all other bodies on the particle considered. This diagram is of particular importance in determining what shall be used for the left-hand member of each of the equations of motion for the particle.

4. Write the three equations of motion for the particle and select the three coordinate axes to be used in applying the equations. Frequently, by a proper choice of axes, only two or even one of the equations of motion will be sufficient for the solution.

5. Write any necessary additional equations such as kinematics equations, definitions, equations of equilibrium, etc., which apply to the particular problem, keeping in mind that there must be as many equations as there are unknown quantities.

6. Solve the equations, using the known quantities stated in the problem.

\section{ILLUSTRATIVE PROBLEMS}

342. A box weighing $16.1 \mathrm{lb}$. rests on the floor of an elevator. If the elevator starts up with an acceleration of $8 \mathrm{ft}$. per sec. ${ }^{2}$, what is the pressure on the floor of the elevator?

Solution.-The box is given the acceleration of $8 \mathrm{ft}$. per sec. per sec. by the upward pressure, $P$, of the elevator and the downward weight, $W$, as indicated in the freebody diagram (Fig. 323). Taking the $y$-axis as vertical, we need only one of the equations of motion, namely, $\Sigma F_{y}=m a_{y}$, for the solution of the problem. Hence using this equation we havo

$$
\begin{gathered}
P-W=\frac{W}{g} a_{y}, \\
P-16.1=\frac{16.1}{32.2} \times 8,
\end{gathered}
$$

Therefore,

$$
P=20.1 \mathrm{lb} .
$$

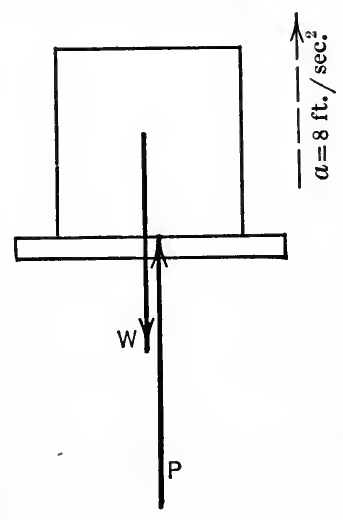

FIg. 323. 
343. A small body weighing $4 \mathrm{lb}$. is attached to one end of a string $5 \mathrm{ft}$. long and is made to revolve as a conical pendulum with a constant angular velocity, $\omega$, so that the string is inclined $30^{\circ}$ with the vertical as shown in Fig. 324. What is the tension, $T$, in the string and the linear velocity, $v$, of the body?

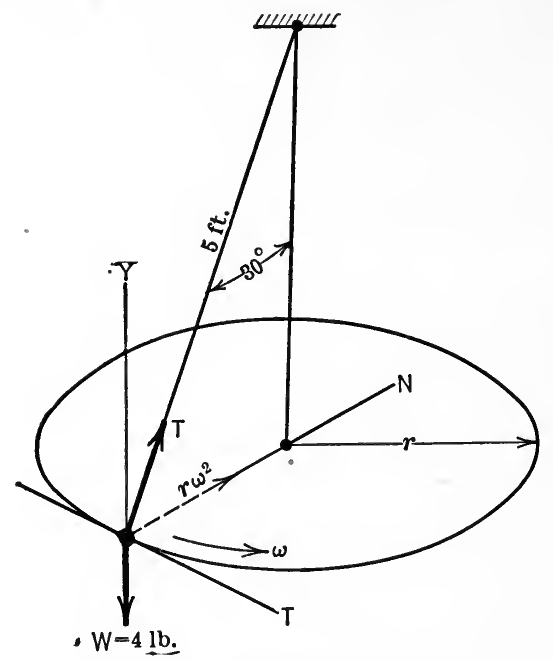

FIG. 324.

Solution.-The body moves on a circular path in a horizontal plane under the influence of two forces $T$ and $W$ as shown in the free-body diagram (Fig. 324). The acceleration of the body is $r \omega^{2}$, or $\frac{v^{2}}{r}$, toward the center of the circle. The equations of motion are:

$$
\begin{aligned}
& \Sigma F_{n}=m a_{n}=\frac{W}{g} r \omega^{2}=\frac{W}{g} \frac{v^{2}}{r}, \quad . \quad . \quad . \quad . \\
& \Sigma F_{t}=m a_{t}=\frac{W}{g} r \alpha=0, \quad \text { since } \alpha=0, \quad . \quad . \\
& \Sigma F_{y}=m a_{y}=0, \text { since } a_{y}=0 . \quad . \quad . \quad . \quad .
\end{aligned}
$$

From (1),

$$
T \cos 60^{\circ}=\frac{4}{32.2} \times \frac{v^{2}}{5 \sin 30^{\circ}} \text {. . . . . . . . . . }
$$

From (3),

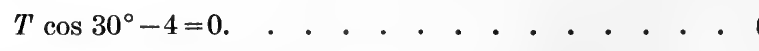

The value of $T$ may be found from (5). Thus,

$$
T=\frac{4}{\cos 30^{\circ}}=\frac{4}{.866}=4.62 \mathrm{lb}
$$


By substituting this value of $T$ in (4), the value of $v$ may be found. Thus,

Hence,

$4.62 \cos 60^{\circ}=\frac{4}{32.2} \times \frac{v^{2}}{5 \sin 30^{\circ}}$.

Therefore,

$$
v^{2}=\frac{4.62 \times 0.5 \times 32.2 \times 5 \times 0.5}{4}=46.3 .
$$

$$
v=6.8 \mathrm{ft} . / \mathrm{sec} \text {. }
$$

344. Boxes are sent from the street into the basement of a store by means of an inclined plane as shown in Fig. 325. The plane is $20 \mathrm{ft}$. long and makes an angle of $30^{\circ}$ with the floor. The boxes are given an initial velocity, $v_{0}$, of $10 \mathrm{ft}$. per sec. Assuming that the coefficient of friction for the box while on the incline is 0.4 , what is the velocity, $v$, of the box as it reaches the bottom of the incline and how many seconds does it take to reach the bottom?

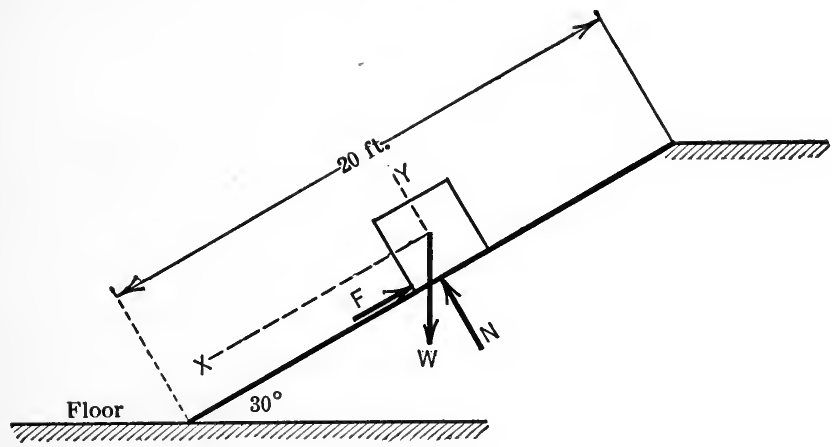

FIG. 325.

Solution.-The forces acting on the box during its motion down the incline are shown in Fig. 325. The $x$-axis is chosen in the direction of the total acceleration, hence $a_{x}=a$.

The equations of motion are,

$$
\begin{aligned}
& \Sigma F_{x}=m a_{x}=m a, \text {. . . . . . . . } \\
& \Sigma F_{y}=m a_{y}=0 \text {, since } a_{y}=0 \text {. . . . . }
\end{aligned}
$$

In addition to the equations of motion the following equations are needed:

$$
\begin{aligned}
& F=\mu N, \text {. . . . . . . . . . } \\
& v^{2}=v_{0}^{2}+2 a s, \quad \text {. . . . . . . . . . }
\end{aligned}
$$

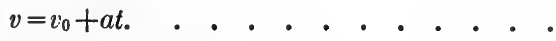

From (1),

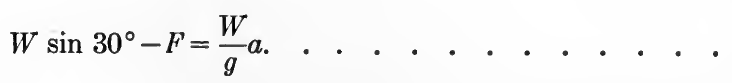

From (2),

From (3),

$$
N-W \cos 30^{\circ}=0 \text {. }
$$

$$
F=0.4 N \text {. }
$$


Substituting the value of $F$ from (8) and (7) in (6) we obtain,

Therefore,

$$
0.5 W-0.4 \times .866 W=\frac{W}{32.2} a .
$$

And from (4)

Therefore,

$$
a=4.95 \mathrm{ft} . / \text { sec. }^{2}
$$$$
v^{2}=(10)^{2}+2 \times 4.95 \times 20 .
$$

And from (5),

Therefore,

$$
v=17.3 \mathrm{ft} . / \mathrm{sec} .
$$

$$
\begin{gathered}
17.3=10+4.95 t . \\
t=1.47 \mathrm{sec} .
\end{gathered}
$$

\section{PROBLEMS}

345. A balloon weighing $400 \mathrm{lb}$. has a vertical acceleration of $2 \mathrm{ft}$. per sec. ${ }^{2}$ The horizontal wind pressure causes the balloon to travel in a direction making an angle of $30^{\circ}$ with the vertical. Find the horizontal component of the acceleration of the balloon and the horizontal wind pressure.

$$
\text { Ans. } 1.15 \mathrm{ft} . / \mathrm{sec}^{2} ; 14.35 \mathrm{lb} \text {. }
$$

346. The winding drum of a mine hoist (Fig. 326) is $15 \mathrm{ft}$. in diameter. A cage weighing 8 tons is raised by it. If the cage is rising at the rate of 60 $\mathrm{ft}$. per sec. when it is $104 \mathrm{ft}$. below the surface of the ground, and power is then shut off, what is the tension, $T$, in the cable while the cage is coming to the surface if it comes to rest just as it reaches the surface?

Ans. $7410 \mathrm{lb}$.

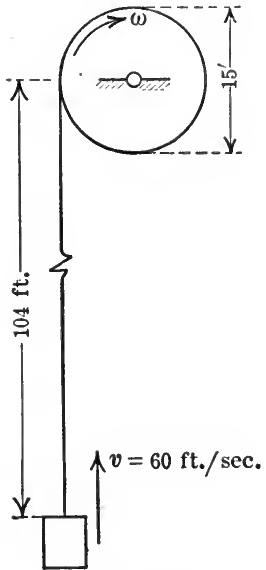

FIG. 326.

347. An engine weighing 64.4 tons travels round a curve having a constant radius of $1000 \mathrm{ft}$., at a constant speed of 45 miles per hour. Find the horizontal thrust on the rails.

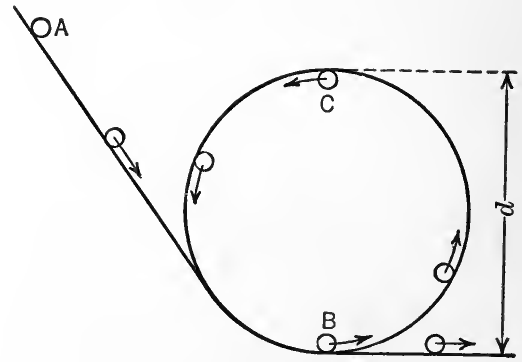

FIG. 327.

348. In "looping the loop" (Fig. 327), show that if friction is neglected, the minimum value of the velocity of the car when at $C$ is $\sqrt{\frac{g d}{2}}$ if the car does not leave the track. 
349. A small body weighing $12 \mathrm{lb}$. rests on an inclined surface (Fig. 328) which is revolved about a vertical axis with a constant angular velocity of 20 r.p.m. If the body is attached to the axis of rotation by a cord as shown in the figure, and if friction between the body and plane is neglected, find the tension, 7 ', in the cord.

350. A box is projected up an inclined plane, which makes an angle of $20^{\circ}$ with the horizontal, with an initial velocity of $2400 \mathrm{ft}$. per minute. If the coefficient of kinetic friction is 0.2 , how far up the plane does the box travel before coming to rest? Will the box remain at rest? If not, how long does it take it to reach the bottom of the incline?

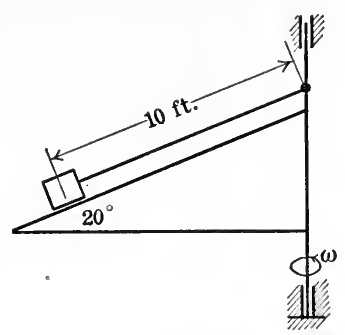

FIG. 328.

Ans. $s=46.9 \mathrm{ft}$.

\section{§ 2. Kinetics of Bodies}

144. Introduction. Methods of Analysis.-As stated in the preceding section, the general character of a problem in kinetics of bodies is the same whether the body has a motion of translation, of rotation, or of plane motion: A physical body is acted on by a force system that has a resultant which causes a change in the motion of the body. For each type of motion, relations are found between (1) the resultant of the external force system, (2) the properties of the body (mass, moment of inertia, etc.), and (3) the change in the motion of the body. For each of the types of motion of rigid bodies treated in this section, the equations which express the relations between the three factors or elements in the problem (equations of motion) are found by the same procedure or series of steps, as follows:

1. From the motion of the body, the acceleration, $a$, of any (and every) particle in the body is found, both in magnitude and in direction. This step involves the use of the equations of kinematics as developed in Chapters VII and VIII.

2. From the acceleration, $a$, of any particle and its mass, $m$, the force required to produce the acceleration is found, both in magnitude and in direction, by applying Newton's second law. This force, $R$, is expressed by the equation $R=m a$, its direction being the same as that of $a$. This force is called the effective force for the particle and is, of course, the resultant of the actual forces acting on the particle. Some (most) of the particles of a body are acted on by internal forces only (in addition to their weights), 
that is, by the neighboring particles of the body, and some of the particles are acted upon by both internal and external forces. The effective force for a particle, therefore, may be the resultant of internal forces only or of internal and external forces.

3. The magnitude and the direction of the effective force for each particle of the body having been determined, in terms of the mass and acceleration of the particle, the resultant of the effective forces for all the particles of the body is found completely by the same methods as were used in Chapter II for finding the resultant of a given system of forces. The effective forces for bodies having the motions considered in this section may be assumed to form a coplanar force system. ${ }^{1}$ Therefore, the resultant of the effective forces, in general, may be determined by writing three equations (Art. 30) involving the summations of the $x$-components of the effectives forces, of the $y$-components of the effective forces, and of the moments of the effective forces about an axis in the body.

4. The equations obtained in step 3 , which determine the resultant of the effective forces for the whole body, contain all the internal forces and all the external forces which act on all of the particles. But, in obtaining the summations of the $x$ - and of the $y$-components and of the moments of the effective forces, the internal forces drop out of the expressions since they occur in collinear pairs, the forces of each pair being equal and opposite (Newton's third law). Hence, the sum of their components in any direction and the sum of their moments about any axis are zero. Therefore,

The resultant of the effective forces for the particles of a

${ }^{1}$ In the discussion of translation, in this section, the motion will be restricted to a uniplanar translation. Further, the bodies considered in connection with the three types of motion discussed in this chapter will be assumed to be homogeneous and to have planes of symmetry which are the planes of motion of the bodies (Arts. 133 and 134) and hence, in discussing the kinetics of such a body having a motion of translation or rotation, or a plane motion, the mass of the body may be assumed to be concentrated in the plane of motion. Thus, the body may first be imagined to be made up of elementary rods perpendicular to the plane of symmetry and then each rod may be imagined to be squeezed or compressed without having its cross-sectional area changed, until the rod becomes an elemental disc lying in the plane of symmetry and having the same mass as does the elementary rod. Therefore, the effective forces may be considered to form a coplanar force system in the plane of motion of the body. If the body does not have a plane of symmetry, more than three equations are needed to define its motion completely. 
body is identical with the resultant of the external forces which act on the body. Or,

The resultant of the effective forces for all the particles of a body, if reversed and assumed to act on the body with the external forces, will hold the body in equilibrium.

This is a statement of D'Alembert's principle. It will be noted, therefore, that D'Alembert's principle makes it possible to reduce a problem in kinetics to an equivalent problem in statics by introducing a force (or forces) which may be found completely from the motion of the body by means of the first three steps outlined above.

\section{Translation}

145. Kinetics of a Translating Rigid Body.-If a rigid body is acted on by an unbalanced force system that causes a motion of translation, the resulting accelerations of all the particles of the body are the same, both in magnitude and in direction (Art. 132). And, whenever a particle of a body is accelerated, a resultant force must act on the particle. This force, as already noted, is called the effective force for the particle. The magnitude of the effective force is the product of the mass, $m$, of the particle and its acceleration, $a$, and its direction is the same as that of $a(R=m a$ from Newton's second law). Therefore, the effective forces for the particles of a translating rigid body form a parallel system and, since all of the forces have the same sense, their resultant must be a single force (never a couple), the direction of which is the same as that of each of the effective forces. And, as noted above, if the body is symmetrical with respect to a plane through the masscenter (plane of motion), the parallel forces may be treated as a coplanar parallel system (see footnote under Art. 144). Thus, let the rigid body represented in Fig. $329(a)$ be given a rectilinear translation along a smooth plane by the force $P$ which produces the acceleration, $a$, of each particle of the body. Let the body be made up of sixteen small cubes (representing the particles of the body) attached (glued) so that they move as a rigid system. The cubes may be made of different materials and hence their masses are not necessarily equal. For convenience, each cube will be thought of as a particle. The effective force for the first particle is $m_{1} a$, for the second particle, $m_{2} a$, etc. The sixteen effective forces form a coplanar parallel system as shown. 
Resultant of Effective Forces.-The resultant of these effective forces may be found in magnitude, action line, and sense by the method used in Art. 26 for finding the resultant of a sytem of parallel forces in a plane. Thus, the resultant of the effective forces is a force having a magnitude equal to the sum of the forces, its sense being the same as that of the common acceleration. Hence,

The magnitude of the resultant of the effective forces $=\Sigma m a$

$$
\begin{aligned}
& =a \Sigma m \\
& =M a
\end{aligned}
$$
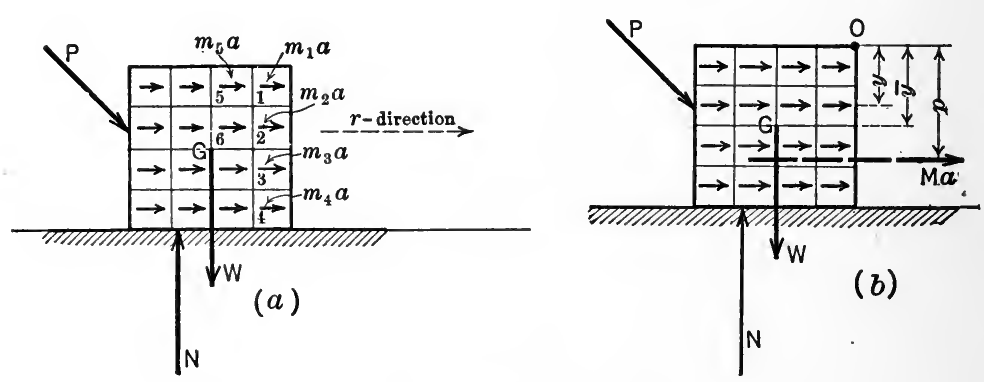

FIG. 329.

in which $M$ denotes the mass of the whole body, that is, the sum of the masses of the particles $(M=\Sigma m)$ and $a$ is the acceleration, which is common to all the particles and hence is a constant and may, therefore, be taken outside the summation sign.

The action line of the resultant of the effective forces is located by use of the principle of moments (Art. 25). Thus, in Fig. 329(b), the sum of the moments of the effective $(\mathrm{ma})$ forces about any point, $O$, must equal the moment of their resultant $(M a)$ about the same point. Hence, if $y$ denotes the moment-arm of the effective force for any particle with respect to $O$, and $p$ denotes the moment arm of the resultant $M a$ with respect to $O$, the principle of moments is expressed by the equation,

That is,

$$
\Sigma(m a \cdot y)=M a \cdot p
$$

Therefore,

$$
\Sigma(m y)=M p \text {, since } a \text { is constant. }
$$

$$
p=\frac{\Sigma(m y)}{M} .
$$


This equation shows, however, that the resultant of the effective forces passes through the mass-center (and not as shown in Fig. $329 b$ ) since the expression for $p$ is also the expression for the $y$-coordinate $(\bar{y})$ of the mass-center (Art. 85).

Relation between Effective Forces and External Forces.-The relation between the resultant of the effective forces and the resultant of the external forces which act on the body may now be found. The resultant of the effective forces is the resultant of all the forces acting on all the particles and hence includes all of the internal forces and all of the external forces. Therefore, if $F_{r}$ denotes the component of a force parallel to the direction of $a$, we may write,

$$
\left(\Sigma F_{r}\right)_{\text {External }}+\left(\Sigma F_{r}\right)_{\text {Internal }}=\Sigma(m a)=M a .
$$

However, in obtaining the summation of the $r$-components of all the effective forces, the sum of the $r$-components of the internal forces becomes zero since the internal forces occur in pairs of equal, opposite, and collinear forces (Newton's third law). Therefore, the resultant of the external forces is identical with the resultant of the effective forces (D'Alembert's principle). That is, it has the same magnitude, action line, and sense as the resultant of the effective forces. Hence, the magnitude of the resultant of the external forces $\left(\Sigma F_{r}\right)$ is given by the equation

$$
\Sigma F_{r}=M a \text {. }
$$

Thus, the resultant of the external forces $(P, W$, and $N)$ is represented completely by the force $M a$ in Fig. $330(a)$. Or, to express the same idea in other words, if an additional force were acting on the body with the external forces $P, W$, and $N$, the magnitude and the action line of the additional force being the same as the magnitude and the action line of the resultant $(M a)$ of the effective forces, but reversed in sense (opposite to $a$ ), as shown in Fig. $330(b)$, then the forces acting on the body would be in equilibrium. This additional (imaginary) force is sometimes called the reversed effective force for the body or the inertia force for the body. It will be noted that the normal pressure $N$ cannot be collinear with $W$ when the force $P$ has the action line as given in Fig. 330(b). 
The fact that the action line of the resultant of the external forces passes through the mass-center of the body may be expressed by stating that the sum of the moments of the external forces with respect to an axis through the mass-center is zero $(\Sigma \bar{T}=0)$.
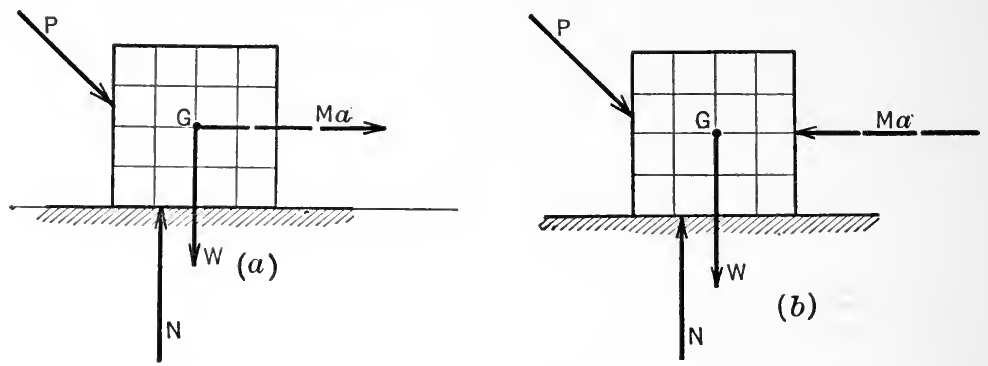

Fig. 330 .

Therefore, the equations which express the relations between the external forces, the kinetic property of the body (mass), and the change of motion (acceleration) for a translating rigid body are

$$
\left.\begin{array}{l}
\Sigma F_{r}=M a \\
\Sigma \bar{T}=0
\end{array}\right\} \cdot
$$

For convenience, the first of these two equations may be replaced by two equations which involve the components of the external forces and of the acceleration of the body, in the directions of two rectangular axes. Thus if $x$ and $y$ denote the rectangular axes, the equations of motion then become,

$$
\left.\begin{array}{l}
\Sigma F_{x}=M a_{x} \\
\Sigma F_{y}=M a_{y} \\
\Sigma \bar{T}=0
\end{array}\right\} \cdot . \quad \cdot \quad . \quad . \quad \cdot .
$$

If the $x$-axis is chosen in the direction of the acceleration, then $a_{x}$ is the total acceleration. The second equation then becomes $\Sigma F_{y}=0$ since $a_{y}$ is zero, that is, the body is in equilibrium as far as motion in a direction perpendicular to the direction of the acceleration is concerned, and equations (2) reduce to equations (1). However, the equation $\Sigma F_{y}=0$ may give an important relation 
between the forces in the solution of a particular problem. Further, it is sometimes convenient to select $x$ - and $y$-axes neither of which agree in direction with the acceleration. Equations (2) therefore, will, as a rule, be used and referred to as the equations of motion of a translating rigid body.

Note.-In analyzing and solving problems in kinetics of bodies, the same general procedure should be followed as was outlined in the note at the end of Art. 143.

\section{ILLUSTRATIVE PROBLEMS}

351. The dimensions of block $A$ (Fig. 331) are $3 \mathrm{ft}$. by $3 \mathrm{ft}$. by $5 \mathrm{ft}$. and the weight of the block is $1200 \mathrm{lb}$. The block rests on a carriage, $B$, which is given an acceleration $a$ in the direction shown. If the friction between the block and carriage is sufficient to prevent slipping, what is the maximum acceleration that the carriage can have without causing the block to tip over?

Solution.-The block has a motion of translation under the action of two forces, namely, the weight, $W$, and the reaction, $R$, of the carriage. For convenience the latter force, which acts at $O$ when the block is on the point of tipping, will be resolved into the normal pressure, $N$, and the frictional force, $F$, as indicated in the figure: The equations of motion for

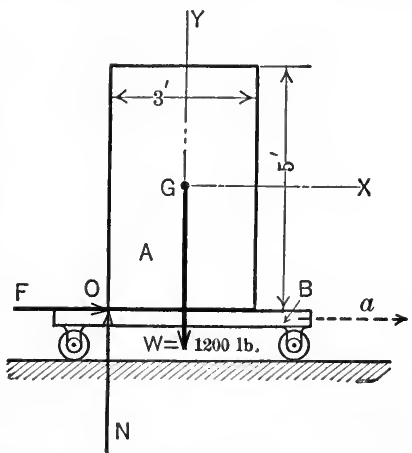

FIG. 331. the block are,

$$
\begin{aligned}
& \Sigma F_{x}=M a_{x}, \quad . \quad . \quad . \quad . \quad . \quad . . \\
& \Sigma F_{y}=M a_{y}, \quad . \quad . \quad . \quad . \quad . \quad . \\
& \Sigma \bar{T}=0 .
\end{aligned}
$$

Since the $x$-axis is chosen in the direction of the acceleration of the body, it follows that $a_{x}=a$ and $a_{y}=0$.

From (1),

$$
F=\frac{1200}{32.2} a \text {. . . . . . . . . }
$$

From (2),

From (3),

$$
N-1200=0, \quad \text {. . . . . . . . }
$$

$$
\frac{5}{2} F-\frac{3}{2} N=0, \quad \text {. . . . . . . . . . }
$$


By solving (5) and (6) for $F$ and substituting its value in (4), the values of $F$ and $a$ become,

$$
\begin{aligned}
F & =720 \mathrm{lb} ., \\
a & =19.32 \mathrm{ft} . / \text { sec. }^{2}
\end{aligned}
$$

Second Method. Inertia Force Method.-If the inertia force (reversed effective force) for the body is assumed to act on the body with the external forces, the body may be assumed to be in equilibrium (D'Alembert's principle) and hence the equations of equilibrium may be applied to the force system thus formed.

The inertia force for the translating block $A$ is,

$$
M a=\frac{1200}{32.2} a .
$$

Its direction is opposite to that of $a$ and its action line passes through the masscenter of the block. Therefore, the forces acting on the block as shown in

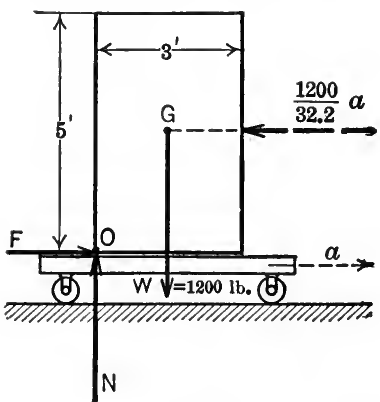

Fig. 332 .

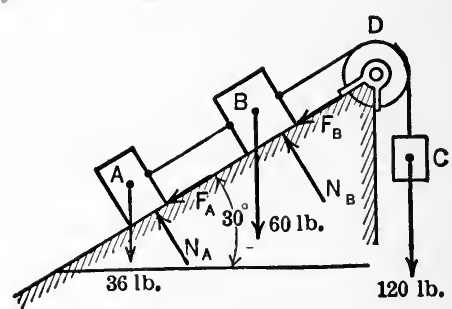

Fig. 333.

Fig. 332 will hold the block in equilibrium. The equations of equilibrium for the force system (Art. 50) are,

$$
\begin{aligned}
& \Sigma F_{x}=F-\frac{1200}{32.2} a=0, \\
& \Sigma F_{y}=N-1200=0, \\
& \Sigma M_{0}=\frac{1200}{32.2} a \times \frac{5}{2}-1200 \times \frac{3}{2}=0 .
\end{aligned}
$$

The solution of the equations leads to the same results as were found by the first method of solution.

It should be noted that in obtanning the moments of the forces in the above equilibrium equation $\left(\Sigma M_{0}=0\right)$, the moment-center $O$ may be taken as any point in the plane of the forces, whereas the moments of the forces in the third equation of motion $(\Sigma T=0)$ used in the first method of solution must be taken about the mass-center of the body.

352. Three bodies $A, B$, and $C$ are connected by two cords as shown in Fig. 333 so that all three bodies move with the same acceleration. The pulley, 
$D$, is assumed to be weightless and frictionless. Body $A$ weighs $36 \mathrm{lb}, B$ weighs $60 \mathrm{lb}$., and $C$ weighs $120 \mathrm{lb}$. The coefficient of kinetic friction for $A$ and the plane is $\frac{1}{4}$, and for $B$ and the plane it is $\frac{1}{5}$. Find (1) the acceleration of the bodies, (2) the tension, $T_{1}$, in the cord between $A$ and $B$, and (3) the tension, $T_{2}$, in the cord between $B$ and $C$.

Solution.-Each body has a motion of translation. Hence, the equations of motion for a translating body apply to each body; namely,

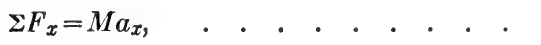

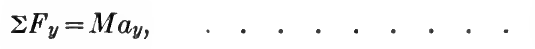

$$
\begin{aligned}
& \Sigma \bar{T}=0 \text {. }
\end{aligned}
$$

In addition, two equations involving the coefficients of friction for bodies $A$ and $B$ are needed, namely,

$$
\begin{aligned}
& F_{A}=\frac{1}{4} N_{A}, \quad \cdot \quad \cdot \quad \cdot \quad \cdot \quad \cdot \quad \cdot \quad \cdot \\
& F_{B}=\frac{1}{5} N_{B}
\end{aligned}
$$

Equation (3) is not needed in determining the quantities asked for. If the action lines of $N_{A}$ and $N_{B}$ were desired, however, all five equations would be needed.

A free-body diagram for body $A$ is shown in Fig. 334(a). Applying the equations of motion to $A$ we have,

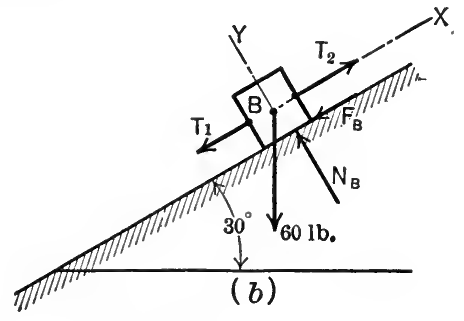

Fig. 334.

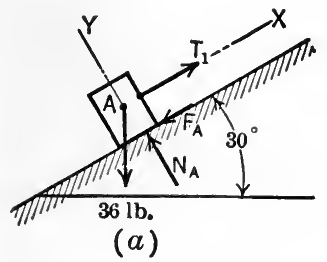

(a)

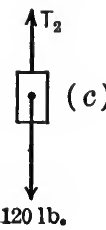

From (1),

$$
T_{1}-36 \sin 30^{\circ}-F_{A}=\frac{36}{32.2} a_{x} . \quad . \quad . \quad . \quad .
$$

From (2),

$$
N_{A}-36 \cos 30^{\circ}=0 \text {. }
$$

$$
F_{A}=\frac{1}{4} N_{A} \text {. }
$$

By solving for $F_{A}$ from (7) and (8) and substituting its value in (6), the following equation is obtained,

$$
T_{1}-36 \sin 30^{\circ}-\frac{1}{4} \times 36 \cos 30^{\circ}=\frac{36}{32.2} a_{x}
$$


In like manner, by applying the equations of motion to body $B$, the following equations are obtained (see Fig. $334(b)$ for frec-body diagram):

$$
\begin{aligned}
T_{2}-T_{1}-F_{B}-60 \sin 30^{\circ} & =\frac{60}{32.2} a_{x}, \\
N_{B}-60 \cos 30^{\circ} & =0, \\
F_{B} & =\frac{1}{5} N_{B} .
\end{aligned}
$$

From the last three equations we obtain,

$$
T_{2}-T_{1}-\frac{1}{5} \times 60 \cos 30^{\circ}-60 \sin 30^{\circ}=\frac{60}{32.2} a_{x} .
$$

For the body $C$ the equation of motion is (see Fig. $334 c$ ),

$$
120-T_{2}=\frac{120}{32.2} a_{x} \text {. . . . . . . . . }
$$

Equations I, II, and III contain three unknowns, $T_{1}, T_{2}$, and $a_{x}$ or $a$ (the $x$-axis is chosen in each case in the direction of the total acceleration, $a$ ). By solving the three equations for the three unknowns the following results are obtained;

$$
\begin{aligned}
a & =a_{x}=8.02 \mathrm{ft} . / \text { sec. }^{2}, \\
T_{1} & =34.76 \mathrm{lb} ., \\
T_{2} & =90.1 \mathrm{lb} .
\end{aligned}
$$

353. An elevator starts from rest and moves upwards, acquiring a velocity of $800 \mathrm{ft}$. per minute in a distance of $18 \mathrm{ft}$. If the acceleration is constant what is the pressure of a man on the floor of the elevator if the man weighs $161 \mathrm{lb} . ?$

Solution.-The man forms the body (assumed rigid), the motion of which is considered. Since the body has a motion of translation, the three equations

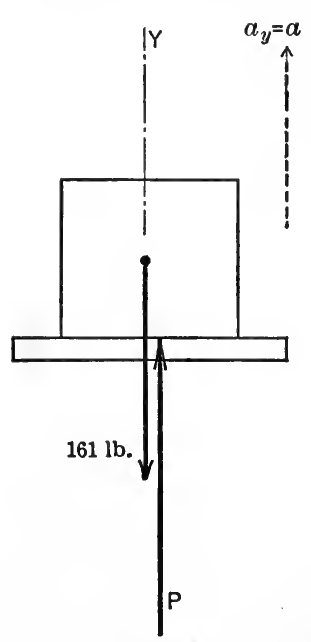

FIG. 335 . of motion for a translating rigid body apply, but since the forces acting on the body pass through the mass-center and since one of the axes is chosen in the direction of the acceleration, only one of the equations is needed; namely,

$$
\Sigma F_{y}=M a_{y} . \quad . \quad . \quad . \quad . \quad . \quad . \quad . \quad .
$$

In addition to the equation of motion one kinematics equation is needed; namely,

$$
v^{2}=v_{0}^{2}+2 a s . \quad \text {. . . . . . . . . }
$$

Fig. 335 shows the free-body diagram, the two forces being collinear.

From (1),

From (2),

$$
P-161=\frac{161}{32.2} a_{y}=\frac{161}{32.2} a .
$$

$$
\left(\frac{800}{60}\right)^{2}=0+2 a \times 18 .
$$


By solving the last two equations the following results are found,

$$
\begin{aligned}
& a=4.93 \mathrm{ft} . / \text { sec. }^{2}, \\
& P=185.7 \mathrm{lb} .
\end{aligned}
$$

In the above problems the bodies have rectilinear translation only. The parallel or side rod of a locomotive when running on a straight track has a motion of curvilinear translation with reference to the engine frame. Its motion is considered in the following problem.

354. The parallel rod of a locomotive (Fig. 336a) weighs $400 \mathrm{lb}$. The crank length, $r_{1}$, is $15 \mathrm{in}$. and the radius, $r_{2}$, of the drivers is $3 \mathrm{ft}$. If the speed of the engine is 50 miles per hour, what is the reaction of the pin at each end of the rod when the rod is in its lowest position?

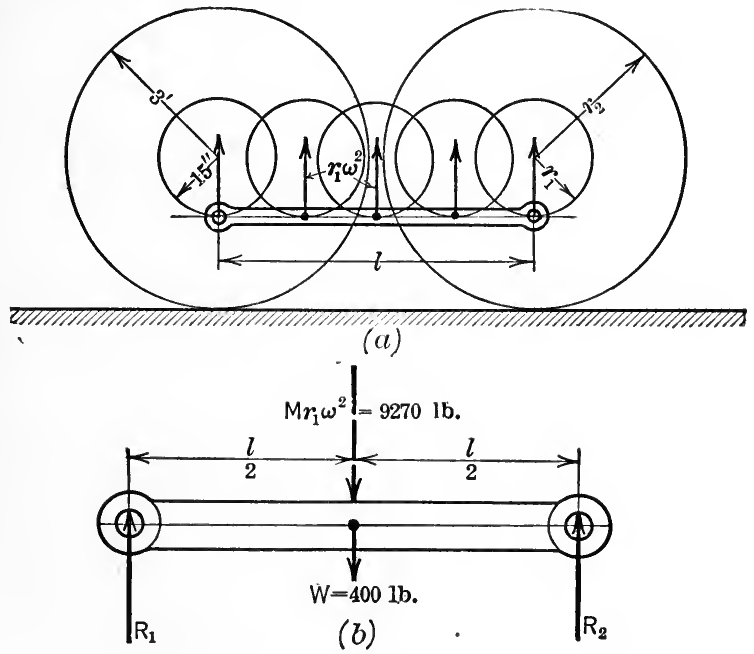

Fig. 336 .

Solution.-Each particle of the rod has the same acceleration at any instant. When the rod is in its lowest position, the acceleration of each particle with reference to the engine frame is directed vertically upwards, its value being $\omega^{2} r_{1}$. The angular velocity, $\omega$, is

$$
\omega=\frac{v}{r_{2}}=\frac{5280 \times 50}{60 \times 60} \times \frac{1}{3}=24.44 \mathrm{rad} . / \mathrm{sec} .
$$

The resultant of the effective forces acts through the mass-center and its magnitude is,

$$
M a=M \omega^{2} r_{1}=\frac{400}{32.2} \times(24.44)^{2} \times \frac{15}{12}=9270 \mathrm{lb} .
$$

If this resultant is reversed and assumed to act on the body with the external 
forces, as shown in Fig. 336(b), the forces will be in equilibrium (D'Alembert's. principle).

It will be observed that the forces form a parallel force system. The equations of equilibrium for a parallel force system (Art. 49) are,

$$
\begin{aligned}
& \Sigma F=0, \\
& \Sigma M=0 .
\end{aligned}
$$

Using (1),

Using (2),

$$
R_{1}+R_{2}-9270-400=0 \text {. }
$$

$$
R_{1} \times l-(9270+400) \times \frac{l}{2}=0 .
$$

Whence,

$$
R_{1}=R_{2}=4835 \mathrm{lb} .
$$

\section{PROBLEMS}

355. A man who is just strong enough to lift a 150-lb. weight when standing on the ground can lift a 200-lb. weight from the floor of an elevator when the elevator is going down with a certain acceleration. What is the acceleration? What weight can the man lift from the floor of the elevator when going up with the same acceleration? Ans. $a=8.05 \mathrm{ft} . / \mathrm{sec}^{2} ; W=120 \mathrm{lb}$.

356. A 3-ton cage descending a shaft with a speed of $9 \mathrm{yd}$. per sec. is brought to rest with a uniform acceleration in a distance of $18 \mathrm{ft}$. What is the tension in the cable while the cage is coming to rest?

357. The dimensions of body $A$ (Fig. 337) are $3 \mathrm{ft}$. by $2 \mathrm{ft}$. by $4 \mathrm{ft}$. and its weight is $1000 \mathrm{lb}$. Assuming that the body will not slip on the carriage, what is the maximum weight that $B$ may have without causing $A$ to tip over when the acceleration of the carriage is $8 \mathrm{ft}$. per sec. ${ }^{2}$ ? The pulley $D$ is assumed to be frictionless and weightless.

Ans. $201 \mathrm{lb}$.

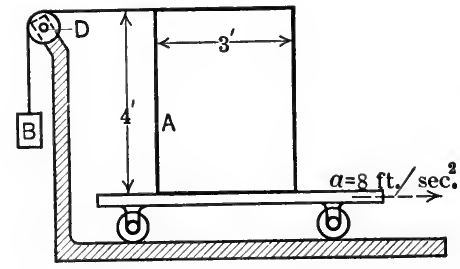

Fig. 337.

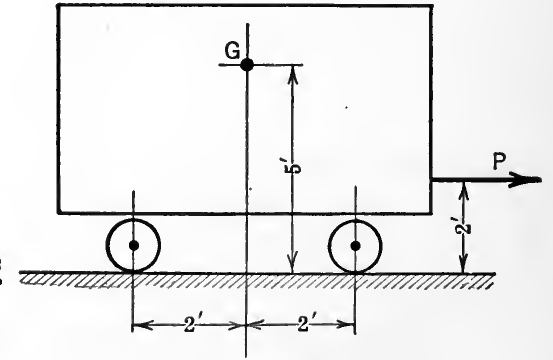

FIG. 338.

358. A man weighing $150 \mathrm{lb}$. leaves his room by way of a window which is $50 \mathrm{ft}$. above the ground. He has a rope that is long enough to reach to the ground but it can support a force only of $125 \mathrm{lb}$. What is the least velocity with which he can reach the ground? 
359. Two strings pass over a smooth drum. On one side of the drum the strings are attached to a $50-\mathrm{lb}$. weight; on the other side one string is attached to a 40-1b. weight and the other string to a $30-\mathrm{lb}$. weight. Find the acceleration of the weights and the tension in each of the strings during motion.

$$
\text { Ans. } T_{30}=25 \mathrm{lb} \text {; } T_{40}=33.3 \mathrm{lb} \text {; } a=5.37 \mathrm{ft} . / \text { sec. }^{2}
$$

360. Steam is shut off when a train running at a speed of 30 miles per hour reaches a 0.4 per cent down-grade. What will be the velocity of the train after $100 \mathrm{sec}$. if the train resistance is $10 \mathrm{lb}$. per ton?

361. A small car (Fig. 338) with its load weighs $800 \mathrm{lb}$. and the center of gravity, $G$, of the total weight is $5 \mathrm{ft}$. from the track. A force $P$ of $120 \mathrm{lb}$. is applied to the car as shown. Neglecting the friction on the track, find the acceleration of the car and the reactions of the track on each pair of wheels. What would be the reactions on the wheels if the force $P$ acted through the point $G$ ?

362. A door is hung on a track as shown in Fig. 339. The coefficient of friction for each of the shoes ( $A$ and $B$ ) and the track is $\frac{1}{4}$. The door weighs

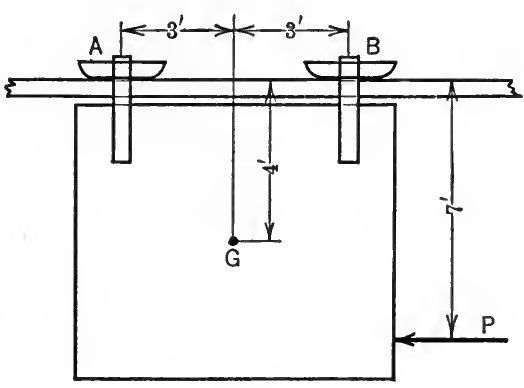

Fig. 339. $300 \mathrm{lb}$. What force $P$ is required to give the door an acceleration of $4 \mathrm{ft}$. per sec. ${ }^{2}$ ? Find the reactions of the shoes on the track. How far will the door travel in 2 sec.? Ans. $P=112 \mathrm{lb}$.

\section{Rotation}

146. Kinetics of a Rotating Rigid Body.-If a rigid body is acted on by an unbalanced force system that produces a motion of rotation of the body, the resulting linear accelerations of the various particles of the body are not the same, but vary directly as the distances of the particles from the axis of rotation (Art. 133). The angular velocities and accelerations of all the particles, however, are the same, at any instant, and the linear acceleration of any (and every) particle in the body may be expressed in terms of the common angular velocity, $\omega$, and the angular acceleration, $\alpha$. Thus, in Fig. 340 is represented a physical body acted on by the external forces $P, W$, and the reaction (not shown) of the axis at $O$, which cause the body to rotate with an angular acceleration, $\alpha$, about an axis through $O$. Each particle of the body moves on a circular path and the linear acceleration of any particle in the body 
has two components; a tangential component, $a_{t}$, and a normal component, $a_{n}$, (Art. 118) such that,

$a_{\imath}=r \alpha$, directed tangent to the path of the particle, and $a_{n}=r \omega^{2}=\frac{v^{2}}{r}$, directed normal to the path of the particle, towards the center of rotation.

in which $\omega$ and $\alpha$ are the angular velocity and angular acceleration, respectively, of the particle (and also of the body) at the instant, $v$ is the linear velocity of the particle at the instant, and $r$ is the distance of the particle from the axis of rotation. The acceleration components for several particles are shown in Fig. 340.

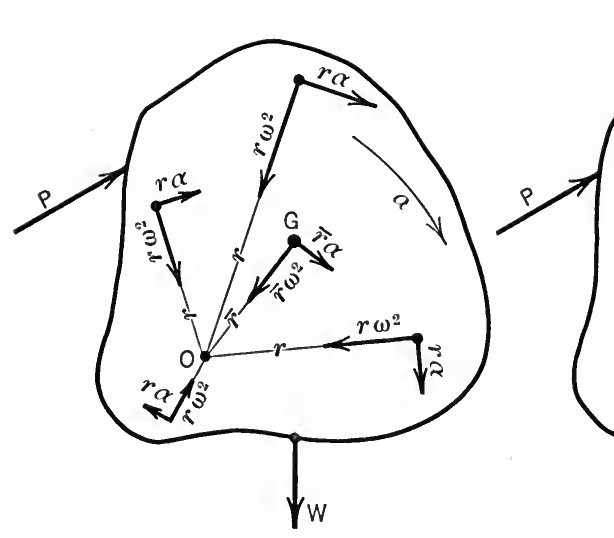

FIG. 340 .

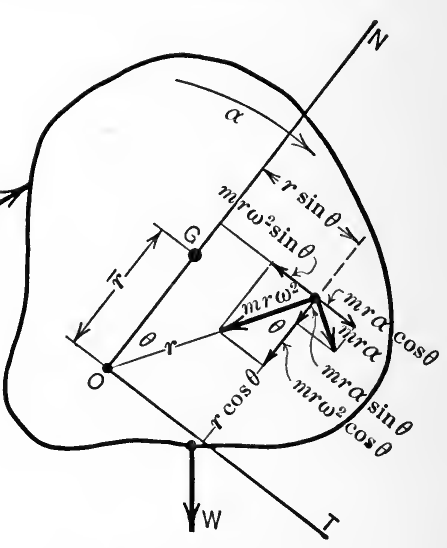

FIG. 341 .

From the components of the acceleration of any particle of the body, expressed above, the components of the resultant force (effective force) required to produce these acceleration components may now be found. Thus, if $m$ denotes the mass of the particle, then from Newton's second law,

$m a_{t}=m r \alpha$ is the tangential component of the effective force, and $m a_{n}=m r \omega^{2}$ is the normal component of the effective force, as shown in Fig. 341.

Resultant of the Effective Forces.-The resultant of the effective forces for the whole body may now be found. As already noted 
(see footnote under Art. 144), the effective forces form a coplanar system in the plane of motion and, as indicated in Fig. 341, the force system is non-concurrent. According to Art. 28, the resultant of such a coplanar system is either a force or a couple. In either case the resultant may be determined completely from the summation of the components of the forces along each of two rectangular axes and the summation of the moments of the forces about some point in the plane of the forces. Thus, let the two axes be the $T$ - and $N$-axes as shown in Fig. 341, where the $N$-axis is drawn through the center of rotation, $O$, and the mass-center of the body. Let each of the two components, $m r \alpha$ and $m r \omega^{2}$, of the effective force for any particle be resolved parallel to the $N$ - and $T$ axes respectively. Let $\theta$ be the angle (measured clockwise from the $N$-axis) between the radius, $r$, to the particle and the $N$-axis. Then, from Fig. 341, the component of the effective force parallel to the $\mathrm{N}$-axis is,

$$
-m r \omega^{2} \cos \theta-m r \alpha \sin \theta,
$$

and the component of the effective force parallel to the $T$-axis is,

\section{Hence,}

$$
m r \alpha \cos \theta-m r \omega^{2} \sin \theta .
$$

The algebraic sum of the components of the effective forces parallel to the $N$-axis $=\Sigma\left(-m r \omega^{2} \cos \theta-m r \alpha \sin \theta\right)$

$$
\begin{aligned}
& =-\omega^{2} \Sigma m r \cos \theta-\alpha \Sigma m r \sin \theta \\
& =-M \bar{r} \omega^{2}-0 .
\end{aligned}
$$

In the above equation $\Sigma m r \cos \theta$ represents the moment of the body with respect to the $T$-axis and hence may be replaced by $M \bar{r}$; and $\Sigma m r \sin \theta$ represents the moment of the body with respect to the $N$-axis and hence is zero since the $N$-axis passes through the mass-center of the body. Further, the negative sign indicates that $M \bar{r} \omega^{2}$ is directed from the mass-center towards the center of rotation. In like manner,

The algebraic sum of the components of the effective forces parallel to the $T$-axis $=\Sigma\left(m r \alpha \cos \theta-m r \omega^{2} \sin \theta\right)$

$$
\begin{aligned}
& =\alpha \Sigma m r \cos \theta-\omega^{2} \Sigma m r \sin \theta \\
& =M \bar{r} \alpha-0 .
\end{aligned}
$$


The moment of the effective force for any particle about the center of rotation, $O$, is $m r \alpha \cdot r$ since the normal component, $m r \omega^{2}$, of the effective force for each particle passes through the axis of rotation and has, therefore, no moment about the center $O$. Hence, taking moments about $O$ :

The algebraic sum of the moments of the effective forces with respect to the axis of rotation $=\Sigma(m r \alpha \cdot r)$

$$
\begin{aligned}
& =\alpha \Sigma m r^{2} \\
& =I_{0} \alpha,
\end{aligned}
$$

where $\mathrm{I}_{0}$ denotes the moment of inertia of the body with respect to the axis of rotation.

Since the sum of the components, in any direction, of the forces of a system is equal to the component of their resultant in the same direction, and since the sum of the moments of the forces about any point is equal to the moment of their resultant about the same point, the above results may be summarized as follows:

The component of the resultant of the effective forces parallel to the $N$-axis $=M \bar{r} \omega^{2}$.

The component of the resultant of the effective forces parallel to the $T$-axis $=M_{\bar{r}} \alpha$.

The moment of the resultant of the effective forces about the center of rotation $=I_{0} \alpha$.

If the resultant of the effective forces is a force, all of the above expressions are required to determine it completely. If, however, the resultant is a couple, the first two expressions reduce to zero, in which case $I_{0} \alpha$ expresses the moment of the resultant couple, as will be discussed in greater detail later in this article.

Relation between Effective Forces and External Forces.-The relation between the resultant of the effective forces for the whole body and the resultant of the external forces which act on the body may now be found. Since the effective force for a particle is the resultant of all the forces (both internal and external) which act on the particle, the effective forces for all the particles will include all of the internal forces (exerted by the particles among themselves) and all of the external forces. Hence, the summation of the $N$-components of the effective forces is the same as the summa- 
tion of the $N$-components of the internal forces plus the summation of the $N$-components of the external forces, and a similar statement applies to the $T$-components. Therefore, if $F$ denotes a force and $T$ the moment of a force, the relation between the above expressions and the forces acting on the particles is expressed by the following equations:

$$
\begin{aligned}
& \left(\Sigma F_{n}\right)_{\text {External }}+\left(\Sigma F_{n}\right)_{\text {Internal }}=M \bar{r} \omega^{2} \\
& \left(\Sigma F_{t}\right)_{\text {External }}+\left(\Sigma F_{t}\right)_{\text {Internal }}=M \bar{r} \alpha \\
& \left(\Sigma T_{0}\right)_{\text {External }}+\left(\Sigma T_{0}\right)_{\text {Internal }}=I_{0} \alpha .
\end{aligned}
$$

But the algebraic sum of the components, in any direction, of the internal forces and the algebraic sum of the moments, about the axis of rotation, of the internal forces are zero, since the internal forces occur in pairs of equal, collinear, and opposite forces (Newton's third law). Therefore, the resultant of the effective forces for the whole body and the resultant of the external forces, only, are identical (D'Alembert's principle).

Therefore, in the above equations, the expressions which involve the internal forces drop out since as just stated,

$$
\left(\Sigma F_{n}\right)_{\text {Internal }}=0,\left(\Sigma F_{t}\right)_{\text {Internal }}=0,\left(\Sigma T_{0}\right)_{\text {Internal }}=0 .
$$

Hence, if $F$ is now used to denote an external force only, and $T$ is used to denote the moment of an external force, the equations which express the relations between (1) the external forces, (2) the kinetic properties of the body, and (3) the change of motion (the equations of motion for a rotating rigid body) are,

$$
\left.\begin{array}{l}
\Sigma F_{n}=M \bar{r} \omega^{2} \\
\Sigma F_{t}=M \bar{r} \alpha \\
\Sigma T_{0}=I_{0} \alpha
\end{array}\right\}
$$

It will be noted that $\bar{r} \omega^{2}$ and $\bar{r} \alpha$ are the normal and tangential accelerations, respectively, of the mass-center of the body, and hence the first two of the above equations may be written $\Sigma F_{n}=M \bar{a}_{n}$ and $\Sigma F_{t}=M \bar{a}_{t}$.

Further, if the body rotates about an axis through the masscenter, that is, if the points $O$ and $G$ coincide, then the right- 
hand members of the first two equations are equal to zero since $\bar{r}$ is equal to zero, and therefore the algebraic sums of the components of the external forces in the two directions are equal to zero. Hence, if a rigid body rotates about an axis through its masscenter, the equation of motion for the body is,

$$
\Sigma \bar{T}=\bar{I} \alpha,
$$

in which $\Sigma \bar{T}$ is the algebraic sum of the moments of the external forces acting on the body with respect to an axis passing through the mass-center (axis of rotation) and $\bar{I}$ is the moment of inertia of the body with respect to the same axis.

\section{ILLUSTRATIVE PROBLEMS}

363. The rod $B C E$ (Fig. 342a) is made to oscillate by means of the crank $A D$ and link $D C$. The members are connected by smooth pins at $B, C$, and $D$. The rod $B C E$ has a constant cross-section and weighs $16.1 \mathrm{lb}$. In the position shown its angular velocity, $\omega$, is 60 r.p.m. and its angular acceleration, $\alpha$, is $40 \mathrm{rad} . / \mathrm{sec}^{2}$ (see Art. 134 for method of finding $\omega$ and $\alpha$ ). Find the pressure, $P$, of link $D C$ at $C$ and the reaction, $R$, of the pin at $B$.

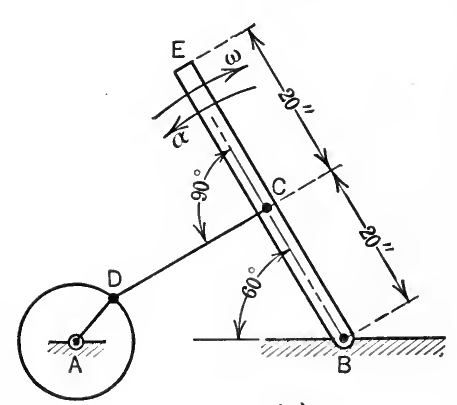

(a)

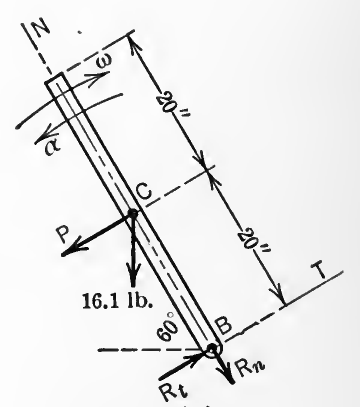

(b)

Fig. 342 .

Solution.-The rod rotates about the center $B$. The equations of motion for the rod are,

$$
\begin{aligned}
& \Sigma F_{n}=M \bar{r} \omega^{2}, \quad \ldots . . \quad . \quad . \quad . .
\end{aligned}
$$

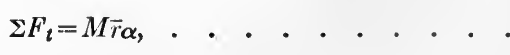

$$
\begin{aligned}
& \Sigma T_{B}=I_{B} \alpha
\end{aligned}
$$


Referring to the free-body diagram for the rod (Fig. 342b) we obtain:

From (1),

From (2),

$$
R_{n}+16.1 \cos 30^{\circ}=\frac{16.1}{32.2} \times \frac{20}{12} \times\left(\frac{60 \times 2 \pi}{60}\right)^{2} \cdot . . . .
$$

From (3),

$$
P-R_{t}+16.1 \sin 30^{\circ}=\frac{16.1}{32.2} \times \frac{20}{12} \times 40 . \quad \text {. . . . . . . }
$$

$$
\left(16.1 \cos 60^{\circ}+P\right) \frac{20}{12}=I_{B} \quad \alpha=\frac{1}{3} \times \frac{16.1}{32.2} \times\left(\frac{40}{12}\right)^{2} \times 40 . . .
$$

Solving (4) we obtain,

Solving (6) we obtain,

$$
\begin{gathered}
R_{n}=18.9 \mathrm{lb} . \\
P=36.4 \mathrm{lb} .
\end{gathered}
$$

Substituting the value of $P$ in (5) and solving for $R_{t}$ we obtain,

Therefore,

$$
R_{t}=11.1 \mathrm{lb} \text {. }
$$

$$
R=\sqrt{(18.9)^{2}+(11.1)^{2}}=21.9 \mathrm{lb} .
$$

364. In Fig. $343, C D$ represents a brake for regulating the descent of the suspended body, $A$. $B$ is the drum from which the cable attached to $A$ unwinds as $A$ descends. The radius, $r_{1}$, of the drum is $6 \mathrm{ft}$. The radius, $r_{2}$, of the brake wheel is $7 \mathrm{ft}$. The radius of gyration, $k_{0}$, of the rotating parts (drum and brake wheel) about the axis of rotation is $4 \mathrm{ft}$. The rotating parts weigh $2000 \mathrm{lb}$. and the body $A$ weighs $1000 \mathrm{lb}$. The coefficient of brake friction is $\frac{1}{4}$. If friction on the axle of the rotating parts is neglected, find the acceleration, $a$, of the body $A$, the tension, $P$, in the cable, and the horizontal and vertical components, $R_{1}$ and $R_{2}$, of the axle reaction, assuming the force at $C$ to be $100 \mathrm{lb}$. (Consider the cable to be flexible and neglect its weight.)

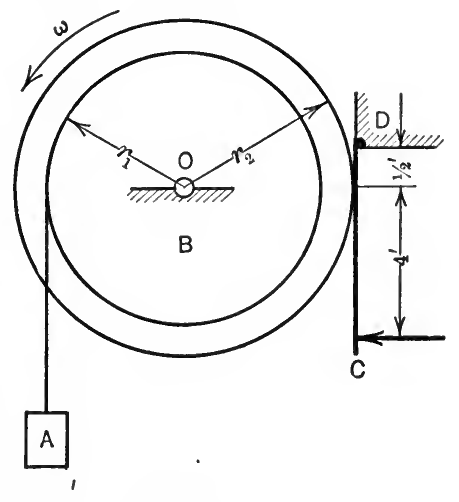

FIG. 343.

Solution.-Three bodies are to be considered, (1) the brake $C D$ which is in equilibrium, (2) the drum and the brake wheel which have a motion of rotation, and (3) body $A$ which has a motion of translation. The free-body diagram for each body is shown in Fig. 344. The brake is held in equilibrium by a non-concurrent force system in a plane for which the equations of equilibrium are:

$$
\begin{aligned}
& \Sigma F_{x}=0, \quad \cdot \quad . \quad . \quad \cdot \quad \cdot \quad \cdot \quad \cdot \quad \cdot \quad \cdot \\
& \Sigma F_{\nu}=0 \text {, . . . . . . . . . . } \\
& \Sigma M_{D}=0 \text {. . . . . . . . . . . }
\end{aligned}
$$


Equation (3) only is needed in this problem since all of the forces acting on the brake are not required.

The equations of motion for the drum and brake wheel are:

$$
\begin{aligned}
& \Sigma F_{n}=M \bar{r} \omega^{2}, \ldots \ldots \\
& \Sigma F_{t}=M_{\bar{r}} \alpha \\
& \Sigma T_{0}=I_{0} \alpha . \quad . \quad . . . . . . .
\end{aligned}
$$

In addition to these equations the defining equation of the coefficient of friction must be used, namely,

$$
F=\mu N \text {. }
$$
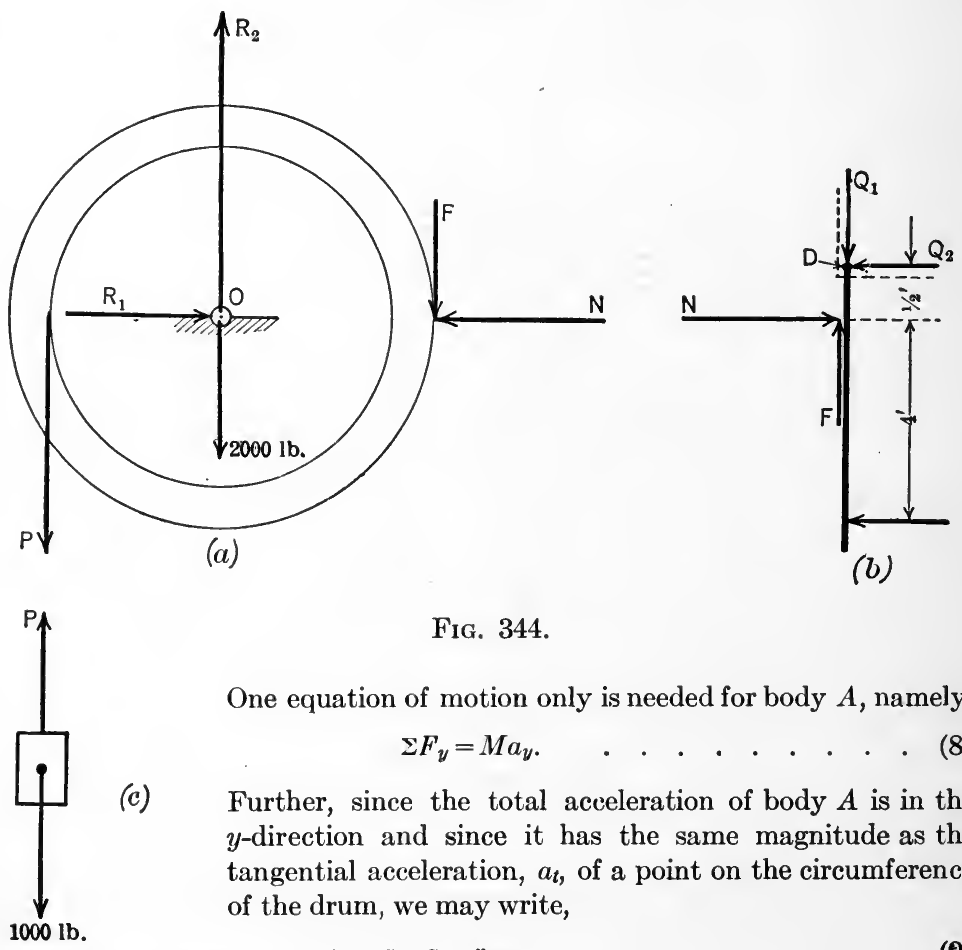

FIG. 344.

One equation of motion only is needed for body $A$, namely,

$$
\Sigma F_{y}=M a_{y} \text {. }
$$

(c) Further, since the total acceleration of body $A$ is in the $y$-direction and since it has the same magnitude as the tangential acceleration, $a_{t}$, of a point on the circumference of the drum, we may write,

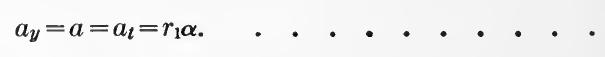

Applying the equations we have:

From (3),

Whence,

From (7),

$$
100 \times 4.5-0.5 N=0 .
$$

$$
N=900 \mathrm{lb} .
$$

$$
F=\frac{1}{4} \times 900=225 \mathrm{lb} \text {. }
$$


From (4),

Whence,

From (6),

$$
R_{1}-900=0 \text {, since } \bar{r}=0 \text {. }
$$

$$
R_{1}=900 \mathrm{lb} .
$$

From (8),

$$
6 P-225 \times 7=\frac{2000}{32.2} \times 4^{2} \times \alpha . . . \quad . \quad . \quad .
$$

$$
1000-P=\frac{1000}{32.2} \times a_{y} . \quad . \quad . \quad . \quad . \quad .
$$

Substituting $\frac{a}{r_{1}}$ from (9) for $\alpha$ in (10) and replacing $a_{y}$ in (11) by: $a$ from (9), we have,

$$
6 P-225 \times 7=\frac{2000}{32.2} \times 16 \times \frac{a}{16}
$$

and,

$$
1000-P=\frac{1000}{32.2} a \text {. }
$$

And, from (5),

$$
R_{2}-P-225-2000=0 \text {, since } \bar{r}=0 \text {. }
$$

These last three equations contain the three required quantities. The solution of the equations gives,

$$
\begin{aligned}
& a=12.54 \mathrm{ft} . / \mathrm{sec} . \\
& P=609 \mathrm{lb} . \\
& R_{2}=2834 \mathrm{lb} .
\end{aligned}
$$

365. Two spherical balls are connected by a light, slender, rigid rod and made to rotate in a horizontal plane about a vertical axis midway between the balls as shown in Fig. 345. Each sphere is $12 \mathrm{in}$. in diameter and weighs $64.4 \mathrm{lb}$. What turning moment must a couple have which gives the spheres an angular velocity of 30 r.p.m. in 4 sec., starting from rest? If one of the two forces of the couple is applied $9 \mathrm{in}$. from the axis of rotation and the other force is the reaction of the axis, what is the magnitude of each force?

Solution.--Since the two spheres

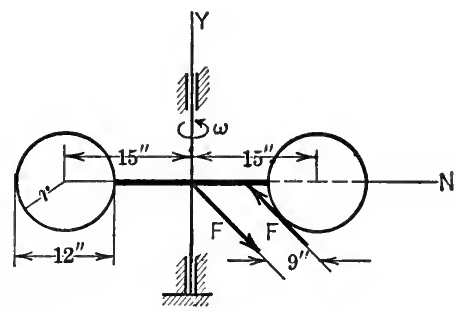

FIG. 345 . have a motion of rotation the equations of motion are,

$$
\begin{aligned}
& \Sigma F_{n}=M \bar{r} \omega^{2}=0, \text { since } \bar{r}=0 \\
& \Sigma F_{t}=M r \alpha=0, \\
& \Sigma T_{0}=I_{0} \alpha .
\end{aligned}
$$


Letting the moment of the couple be denoted by $C$ and the mass of each sphere by $M$ we have, from (3),

$$
\begin{aligned}
\Sigma T_{0} & =C=I_{0} \alpha \\
& =2\left(\bar{I}+M d^{2}\right) \alpha=2\left(\frac{2}{5} M r^{2}+M d^{2}\right) \alpha \\
& =2\left[\frac{2}{5} \times \frac{64.4}{32.2} \times\left(\frac{6}{12}\right)^{2}+\frac{64.4}{32.2} \times\left(\frac{15}{12}\right)^{2}\right] \alpha \\
& =6.65 \alpha .
\end{aligned}
$$

But, from definition,

$$
\alpha=\frac{\omega-\omega_{0}}{t}=\frac{30 \times 2 \pi}{60 \times 4}=0.785 \mathrm{rad} . / \text { sec. }^{2}
$$

Therefore,

And since,

then

Whence,

$$
C=6.65 \times 0.785=5.23 \mathrm{lb} . \mathrm{-ft} .
$$

$$
C=F \times \frac{9}{12},
$$

$$
F^{\prime} \times \frac{8}{12}=5.23
$$

$$
F=6.97 \mathrm{lb} \text {. }
$$

\section{PROBLEMS}

366. A solid sphere $15 \mathrm{in}$. in diameter revolves with an angular velocity of 500 r.p.m. about a fixed axis which passes through its center. What force acting tangent to its surface will stop the sphere in 5 sec. if friction on the axis is neglected? The weight of the sphere is $500 \mathrm{lb}$. Ans. $40.6 \mathrm{lb}$.

367. A weight of $30 \mathrm{lb}$. is suspended from a solid homogeneous cylinder by a weightless cord which is wrapped around the cylinder. The cylinder weighs $193.2 \mathrm{lb}$. and its radius is $18 \mathrm{in}$. Bearing friction is $18 \mathrm{lb}$. and the diameter of the shaft on which the cylinder rotates is $4 \mathrm{in}$. If the suspended weight has an initial velocity of $10 \mathrm{ft}$. per sec. downwards, what will be its velocity after it has moved $10 \mathrm{ft}$.? What time is required to move the $10 \mathrm{ft}$.?

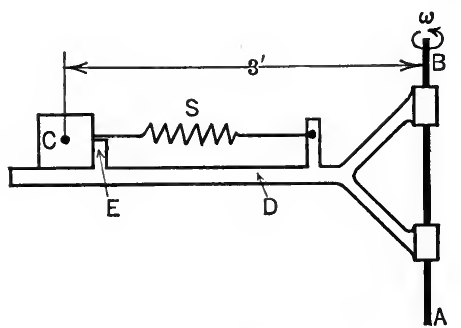

Fig. 346.

368. A body $C$ weighing $10 \mathrm{lb}$. rests upon a frame $D$ (Fig. 346) which rotates about a vertical axis $A B$. When the frame is not rotating, the tension in the spring, $S$, is $20 \mathrm{lb}$. If the angular velocity of the frame is 30 r.p.m. and the friction under $C$ is neglected, what is the pressure against the stop at $E$ ?

Ans. $10.8 \mathrm{lb}$.

369. If the rocker arm $B C E$ in Prob. 363 carries on its outer end $E$ a spherical ball which weighs $4 \mathrm{lb}$., find the values of $P, R_{t}$, and $R_{n}$, assuming that the ball is small enough to be considered as a particle.

370. A block $A$ (Fig. 347) weighs $257.6 \mathrm{lb}$. and rests on a plane inclined $30^{\circ}$ with the horizontal. A flexible rope attached to the block passes over the 
disc, $C$, at the top of the plane and is attached to a suspended body, $B$, which weighs $644 \mathrm{lb}$. $C$ weighs $322 \mathrm{lb}$. and is $20 \mathrm{in}$. in diameter. The axle is $4 \mathrm{in}$. in diameter and the axle friction is $10 \mathrm{lb}$. The coefficient of friction for body $A$ is 0.1 . Find $(a)$ the acceleration of $B,(b)$ the angular acceleration of the disc, (c) the pull of the rope on body $B$. Neglect the material cut from the dise for the shaft.

371. A constant turning moment of 240,000 in.-lb. turns a flywheel, the weight

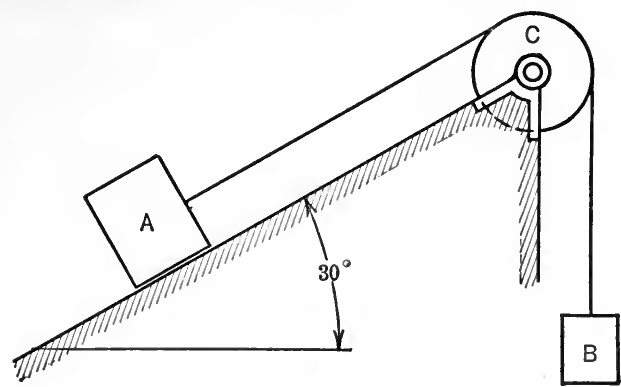

Fig. 347. of which is $3220 \mathrm{lb}$. The

diameter of the flywheel is $8 \mathrm{ft}$. Its angular velocity is changed from $40 \mathrm{rad}$. per sec. to $80 \mathrm{rad}$. per sec. in 20 revolutions. What is the radius of gyration of the flywkeel with respect to the axis of rotation? Neglect axle friction.

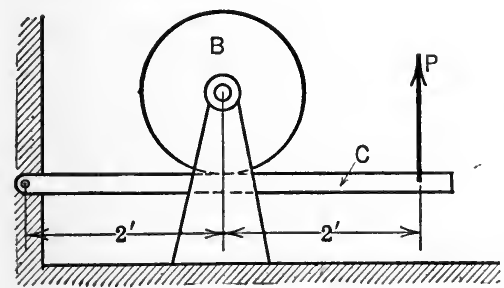

Fig. 348.

372. The drum $B$ (Fig. 348 ) is rotating with an angular velocity $\omega=120$ r.p.m. when the brake $C$ is applied. The drum is a solid cylinder and has a radius of $10 \mathrm{in.}$ Its weight is $2000 \mathrm{lb}$. If the coefficient of brake friction is 0.2 , what force, $P$, is required to reduce the angular velocity of the drum to 30 r.p.m. in 3 sec.? Neglect axle friction. Ans. $P=203 \mathrm{lb}$.

373. Two bodies, $A$ and $B$ (Fig. 349 ) rest on a frame, $E$, which rotates about an axis $C D$. $A$ weighs $8 \mathrm{lb}$. and $B$ weighs $24 \mathrm{lb}$. The tension in

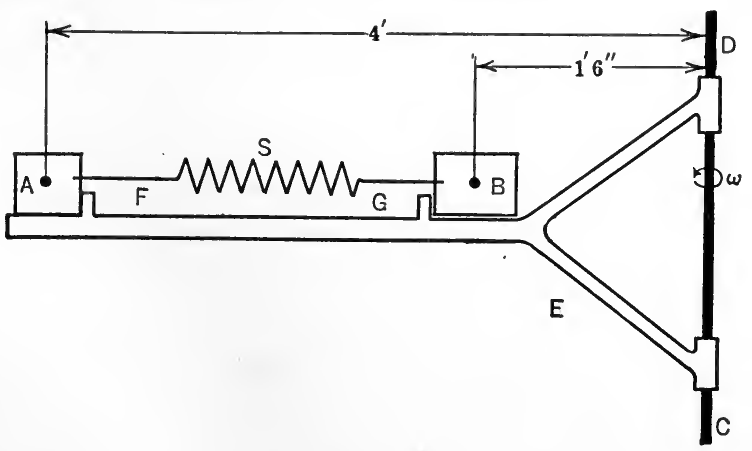

Fig. 349 . 
the spring, $S$, when the frame is not rotating is $30 \mathrm{lb}$. If the friction under bodies $A$ and $B$ and the mass of the spring are neglected, what are the pressures against the stops $F$ and $G$ when the frame revolves with an angular velocity of 30 r.p.m.?

374. In Prob. 365, what is the tension in the rod?

147. Second Method of Analysis. Inertia Forces.-In some problems dealing with the rotation of a rigid body under the action of an unbalanced force system, it is convenient to assume that the resultant of the effective forces is reversed and acts on the body with the external forces, thereby forming a force system that is in equilibrium (D'Alembert's principle), and thus reducing the kinetics problem of a rotating body to an equivalent statics problem. In the preceding articles it was shown that the resultant of the effective forces for a rotating body is either a force or a couple in the plane of motion of the body. The reversed resultant force (or resultant couple) is called the inertia force (or inertia couple) for the body. In using this method of analysis it is important, therefore, to determine under what conditions of the motion the resultant is a force and under what conditions it is a couple. Further, if the external forces are to be put in equilibrium by the addition of the anti-resultant of the effective forces, that is, by the inertia force for the body, the action line of the resultant force must be determined. The facts stated in the following proposition are of great importance in connection with the method of solution as outlined above.

Proposition.-If a rigid body rotates about a fixed axis through its mass-center, the resultant of the effective forces (and of the external forces also) is a couple the moment of which is equal to $\bar{I} \alpha$, the product of the moment of inertia of the body about the axis of rotation and the angular acceleration of the body. If the body rotates about an axis which does not pass through the mass center, the resultant of the effective forces is a single force having: a magnitude equal to the product of the mass of the body and the acceleration of the mass-center of the body $(M \bar{a})$; a direction the same as that of the mass-center; and an action line which does not pass through the mass-center of the body. For convenience, however, this single force may be considered to be equivalent to another force, having an action line which does pass through the mass-center, and a couple; the magnitude of the force 
is equal to $M \bar{a}$ and its direction is the same as that of $\bar{a}$; and the moment of the couple is equal to $\bar{I} \alpha$.

The facts stated in the above proposition, together with certain additional facts, are discussed and applied in the following pages, in connection with the use of inertia forces. It was found in the preceding article that the expressions $M \bar{r} \alpha$ and $M \bar{r} \omega^{2}$ represented the $T$ - and $N$-components, respectively, and that $I_{0} \alpha$ represented the moment, of the resultant of the effective force system for the rotating body. From these expressions, the resultant of the effective forces for various conditions of motion may be determined completely. Thus, it will be noted that if $\bar{r}$ is zero in these expressions, that is, if the distance from the axis of rotation to the masscenter of the body is zero, then each of the components, $M \bar{r} \alpha$ and $M \bar{r} \omega^{2}$, of the resultant of the effective forces is zero. Hence, the resultant is not a force. And, since the effective forces have a moment, the value of which is $I_{0} \alpha$, the resultant is a couple of moment $I_{0} \alpha$ or $\bar{I} \alpha$ since $I_{0}$ is denoted by $\bar{I}$ when the axis of rotation passes through the mass-center of the body. The sense of the resultant couple is, of course, the same as that of $\alpha$, the angular acceleration of the body. Further, since the resultant of the external forces that act on the body is identical with that of the effective forces for the body, the body may be considered to be in equilibrium if an additional couple having a magnitude equal to $\bar{I} \alpha$ and a sense opposite to that of $\alpha$ is assumed to act on the body with the external forces. This couple is often called the reversed effective couple or the inertia couple for the body.

If the body rotates about an axis which does not pass through its mass-center, as indicated in Fig. 350, then $\bar{r}$ in the above expressions is not zero. The resultant of the effective forces for the rotating body, then, is a single force since $M \bar{r} \alpha$ and $M \bar{r} \omega^{2}$ express the components of the resultant force, the moment of the resultant force (or of its two components) being expressed by $I_{0} \alpha$. The action line of each of the two components may now be located.

The action line of the normal component, $M \bar{r} \omega^{2}$, of the resultant force lies along the line connecting the center of rotation and the mass-center of the body, that is, along the $N$-axis as shown in Fig. 350. This fact may be proved as follows: $M \bar{r} \omega^{2}$ was obtained by resolving the components $m a_{t}$ and $m a_{n}$, of the effective force for each particle, parallel to the $N$-axis and determining the algebraic sum of these $N$-components (Art. 146). And, it was shown 
that the $m a_{n}$ components, only, have an influence in determining the magnitude $M \bar{r} \omega^{2}$ since the sum of the $m a_{t}$ components was found to be equal to zero. Now the $m a_{n}$ component of the effective force for each particle passes through the center of rotation. Therefore, the sum, $M \bar{r} \omega^{2}$, of their components parallel to the $N$-axis must lie along the $N$-axis.

The action line of the tangential component, $M \bar{r} \alpha$, of the resultant of the effective forces may be found from the principle of moments; namely, the moment of the resultant of the effective forces about the axis of rotation equals the sum of the moments of the effective forces about the same axis. The sum of the moments

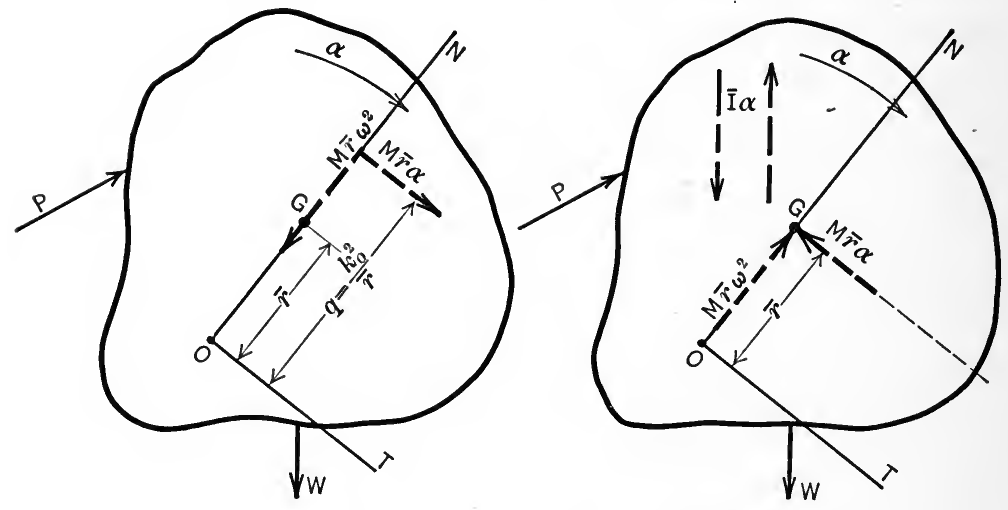

FIG. 350.

FIG. 351.

of the effective forces, as already shown, is $I_{0} \alpha$. Further, the moment of the resultant of the effective forces is the moment of its tangential component, $M \bar{r} \alpha$, only, since the normal component, $M_{\bar{r}} \omega^{2}$, passes through the center of rotation. Hence, the principle of moments is expressed by the equation,

$$
M \bar{r} \alpha \cdot q=I_{0} \alpha,
$$

in which $q$ is the moment arm of $M \vec{r} \alpha$ with respect to the center of rotation $O$, as shown in Fig. 350 . And, since $I_{0}=M k_{0}^{2}$, in which $k_{0}$ is the radius of gyration of the body with respect to the axis of rotation, we may write,

$$
\begin{gathered}
M \bar{r} \alpha \cdot q=M k_{0}^{2} \alpha \\
q=\frac{k_{0}^{2}}{\bar{r}}
\end{gathered}
$$

whence, 
Therefore, the action lines of the two components, $M \bar{r} \alpha$ and $M \bar{r} \omega^{2}$, of the resultant of the effective forces intersect in a point on the $N$-axis at a distance $\frac{k_{0}{ }^{2}}{\bar{r}}$ from the center of rotation, as shown in Fig. 350. And, since the resultant of the external forces is identical with the resultant of the effective forces, the body may be considered to be in equilibrium if the two forces $M \bar{r} \alpha$ and $M \bar{r} \omega^{2}$, having the proper action lines as determined above and shown in Fig. 350 , but reversed in sense, are assumed to act on the body with the external forces. The component $M \bar{r} \omega^{2}$ of the inertia force is sometimes called the centrifugal force.

However, instead of determining the resultant of the effective forces as a single force (or as components of a single force) as was done above, it is usually more convenient to find the resultant of the effective forces as an equivalent force and couple; the force having components equal to $M \bar{r} \alpha$ and $M \bar{r} \omega^{2}$ parallel, respectively, to the $T$-and $N$-axes, as before, but with action lines passing through the mass-center of the body. The fact that the components of this new force are the same in magnitude and sense as those of the single resultant force, follows from Art. 18, in which the resolution of a force into a force and a couple is discussed. The fact that the moment of the couple, $C$, is equal to $\bar{I} \alpha$ may be proved as follows: The moment of the effective forces about the axis of rotation is $I_{0} \alpha$ as already proved. And, if the resultant of the effective forces is a force having components $M \bar{r} \alpha$ and $M \bar{r} \omega^{2}$ acting through the mass-center, and a couple the moment of which is represented by $C$, as stated above, then, the principle of moments is expressed by the equation,

$$
M \bar{r} \alpha \cdot \bar{r}+C=I_{0} \alpha .
$$

But,

Hence,

$$
I_{0}=\bar{I}+M \bar{r}^{2} \text { (Art. 102). }
$$

$$
C=\bar{I} \alpha \text {. }
$$

Therefore, a rigid body (Fig. 351) rotating about a fixed axis under the action of an unbalanced force system $(P, W$, and the reaction of the axis at $O$ ) may be considered to be in equilibrium if an additional force and couple are assumed to act on the body with the external forces. The additional inertia force must act through the mass-center of the body; it must have components 
equal to $M \bar{r} \alpha$ and $M \bar{r} \omega^{2}$, the directions of which are parallel respectively to the $T$ - and $N$-axes; and the senses of the components must be opposite to those of the corresponding components of acceleration of the mass-center, as shown in Fig. 351. The additional inertia couple must have a moment equal to $\bar{I} \alpha$ and must have a sense of rotation opposite to that of the angular acceleration, $\alpha$, of the body, as shown in Fig. 351. The forces of the couple, of course, may be assumed to act anywhere in the plane of motion of the body, provided that the moment of the couple remains constant (Art. 27).

\section{ILLUSTRATIVE PROBLEMS}

375. A horizontal bar $B$ (Fig. 352, $a$ ) rotates with a constant angular velocity of 45 r.p.m. about a vertical axis $Y Y$. A slender $\operatorname{rod} C$, of constant

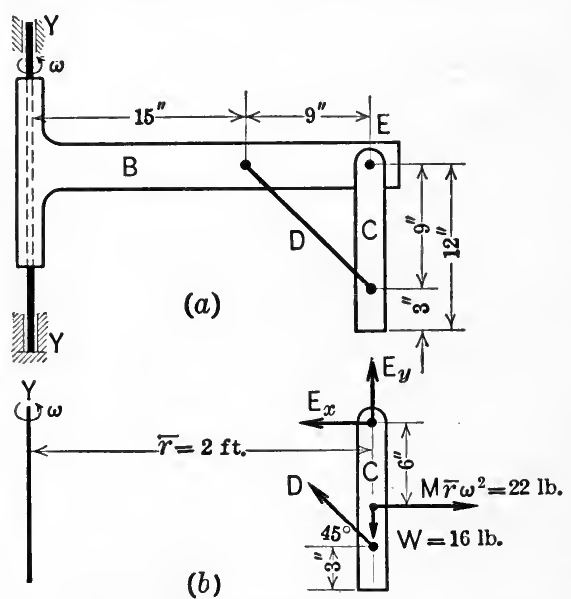

Fig. 352. cross-section, having a length of 12 in. and a weight of $16 \mathrm{lb}$. is attached to the rotating bar by means of a smooth pin at $E$, and is held in a vertical position by a weightless cord $D$. Find the tension in $D$ and the magnitude of the reaction of the pin at $E$ on the $\operatorname{rod} C$.

Solution.-A free-body diagram of the rod $C$ is shown in Fig. $352, b$. The rod has a motion of rotation about the vertical axis $Y Y$ under the influence of three forces $W, D$, and the pin pressure at $E$ (the components of the pin pressure being denoted by $E_{x}$ and $E_{y}$ ).

If the reversed resultant of the effective forces (inertia force) for the rod is assumed to act on the rod with $W, D, E_{x}$, and $E_{y}$, the rod may be considered to be in equilibrium. The resultant of the effective forces is $M \bar{r} \omega^{2}$, since $\alpha=0$ and hence $M \ddot{r} \alpha=0$.

$$
\begin{aligned}
M \vec{r} \omega^{2} & =\frac{16}{32.2} \times 2 \times\left(\frac{45 \times 2 \pi}{60}\right)^{2} \\
& =22.0 \mathrm{lb} .
\end{aligned}
$$

The action line of $M \bar{r} \omega^{2}$ passes through the mass-center of the rod. Thus, the forces $W, D, E_{x}, E_{y}$, and $M \bar{r} \omega^{2}$, as shown in the free-body diagram, would hold the rod in equilibrium. Using the equations of equilibrium, we have: 


$$
\begin{aligned}
& \Sigma F_{x}=22-E_{x}-D \cos 45^{\circ}=0 \\
& \Sigma F_{y}=E_{y}+D \cos 45^{\circ}-16=0 \\
& \Sigma M_{E}=22 \times 6-D \times 9 \cos 45^{\circ}=0
\end{aligned}
$$

The solution of these equations gives the following results:

$$
\begin{aligned}
& D=20.7 \mathrm{lb} . \\
& E_{x}=7.33 \mathrm{lb} ., \quad E_{y}=1.33 \mathrm{lb} ., \quad E=7.45 \mathrm{lb} .
\end{aligned}
$$

376. A bar $B, 2$ in. by 6 in. by $4 \mathrm{ft}$., is attached to a cylindrical disc, $A$, by means of two steel straps, $D$ and $E$, as shown in Fig. 353(a). The cylindrical disc is keyed to a shaft which causes the disc and the bar to rotate in a vertical plane. When the bar is in a horizontal position as shown, the angular velocity, $\omega$, of the shaft is 30 r.p.m. and it is decreasing, at the instant, at the rate of 120 r.p.m. per sec. The weight of the bar is $96 \mathrm{lb}$. The diameter of the disc is $18 \mathrm{in}$. and that of the shaft is $4 \mathrm{in}$. The straps, $D$ and $E$, are attached to the bar by means of smooth pins. Find $(a)$ the torque transmitted from the shaft to the bar $B,(b)$ the sum of the pin pressures in the horizontal direction, and $(c)$ the sum of the pin pressures in the vertical direction.

Solution.-The resultant of the effective forces may be considered to be a force having the components $M \bar{r} \omega^{2}$ and $M \bar{r} \alpha$ which act through the mass-center, and a couple, the moment of which is $\bar{I} \alpha$. If the effective forces be reversed as shown in Fig. 353(b) and assumed to act on the body with the external forces, the
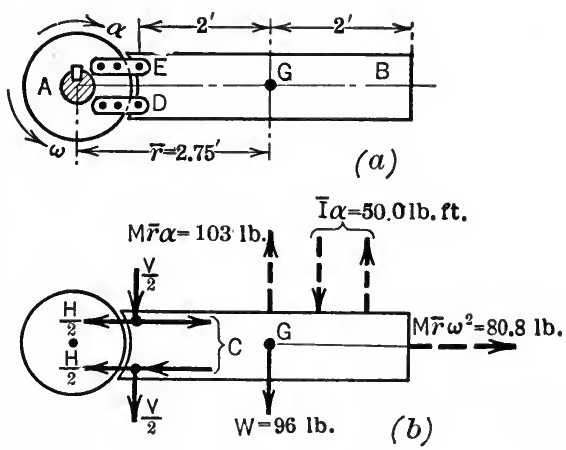

FIG. 353. body may be considered to be in equilibrium. Hence, the pins must exert a horizontal pressure $H\left(\frac{1}{2} H\right.$ for each pin) to balance the only horizontal force $M \bar{r} \omega^{2}\left(\Sigma F_{x}=0\right)$. They must also exert a vertical force $V\left(\frac{1}{2} V\right.$ for each pin) to balance $M \bar{r} \alpha$ and $W\left(\Sigma F_{y}=0\right)$. Finally, the pins must exert a couple $C$ whose moment about any point, $G$ say, balances the moments of the other forces about the point $\left(\Sigma M_{G}=0\right)$. The values of the reversed effective forces as shown in Fig. 353(b) are as follows:

$$
\begin{aligned}
M \bar{r} \omega^{2} & =\frac{96}{32.2} \times 2.75 \times\left(\frac{30 \times 2 \pi}{60}\right)^{2}=80.8 \mathrm{lb} . \\
M \bar{r} \alpha & =\frac{96}{32.2} \times 2.75 \times \frac{120 \times 2 \pi}{60}=103 \mathrm{lb} . \\
\bar{I} \alpha & =\frac{1}{12} M l^{2} \alpha=\frac{1}{12} \times \frac{96}{32.2} \times 4^{2} \times 4 \pi=50.0 \mathrm{lb} . \mathrm{ft} .
\end{aligned}
$$


Thus, the force system acting on the bar is a non-concurrent system in a plane, the equations of equilibrium for which are,

$$
\begin{array}{rlrl}
\Sigma F_{x} & =0, & H-80.8 & =0, \\
\Sigma F_{y} & =0, & 103-V-96=0, \\
\Sigma M_{G}=0, & -C+50.0+2 V=0 .
\end{array}
$$

Solving these equations we obtain,

$$
\begin{aligned}
& H=80.8 \mathrm{lb} ., \\
& V=7.0 \mathrm{lb} . \\
& C=64 \mathrm{lb} . \mathrm{ft} .
\end{aligned}
$$

The magnitude of each of the forces that form the couple, $C$, depends on the distance between the pins. If this distance is $3 \mathrm{in}$., then each force is $256 \mathrm{lb}$.

\section{PROBIEMS}

377. Solve Prob. 376 by introducing a single force as the reversed resultant of the effective forces instead of introducing a reversed force and couple.

378. A small body is placed on a rough horizontal disc which rotates about a vertical axis. If the distance of the body from the axis is $9 \mathrm{in}$. and the coefficient of friction between the body and disc is $\frac{2}{3}$, find (1) the greatest ang llar velocity and (2) the greatest angular acceleration the disc can have without causing the body to slide. Ans. $\omega=51$ r.p.m ; $\alpha=28.5$ rad. $/$ sec. $^{2}$

379. A flywheel used on a punching machine is $8 \mathrm{ft}$. in diameter and has a rim which weighs 1 ton. Each operation of punching a hole causes the speed

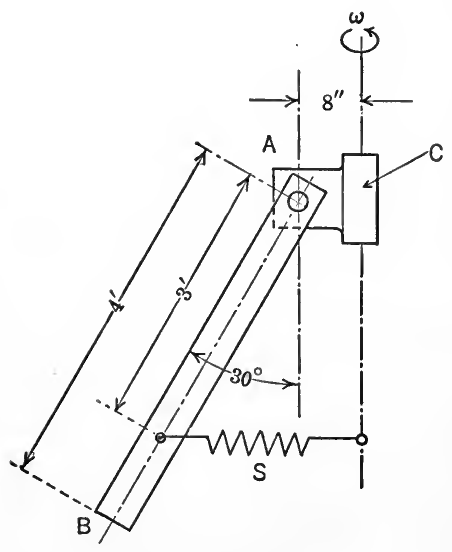

Fig. 354 . of the flywheel to decrease uniformly from 100 r.p.m. to 80 r.p.m. The flywheel has 6 spokes, each $3.5 \mathrm{ft}$. long. If the time of punching a hole is 0.5 sec., what moment is transmitted from the rim to the hub by each spoke. Assume that the thickness of the rim is small in comparison with the radius of the flywheel and neglect the weight of the hub and spokes.

Ans. 607 lb.-ft.

380. A uniform bar $A B$ and a member $C$, to which $A B$ is pinned at $A$ (Fig. $354)$, rotate, at a speed of 40 r.p.m. about a vertical axis. The weight of the bar is $40 \mathrm{lb}$. and its length is $4 \mathrm{ft}$. If the bar is connected to the axis by a spring $S$ (assumed horizontal), as shown 
in the figure, find the pull of the spring and the horizontal and vertical components of the pin pressure at $A$.

381. In Fig. $355, A$ represents a frame which revolves about a vertical axis $Y Y$ at a constant angular velocity $\omega=40$ r.p.m. A bar, $B$, is attached to the frame at $E$ by means of a smooth pin. At the end of $B$ a spherical ball, $C$, is fastened. $B$ weighs $20 \mathrm{lb}$. and is 16 in. long. $C$ weighs $8 \mathrm{lb}$. and is $4 \mathrm{in}$. in diameter. Find the reaction of the pin at $E$ and of the frame at $F$, on the bar.

148. Center of Percussion.The point $P$ (Fig. 356) on the $N$-axis, through which the resultant of the effective forces for a

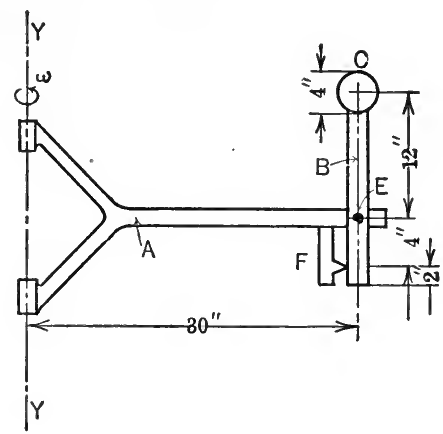

FIG. 355. rotating rigid body acts, is called the center of percussion of the body with respect to the given axis of rotation. Hence, the center

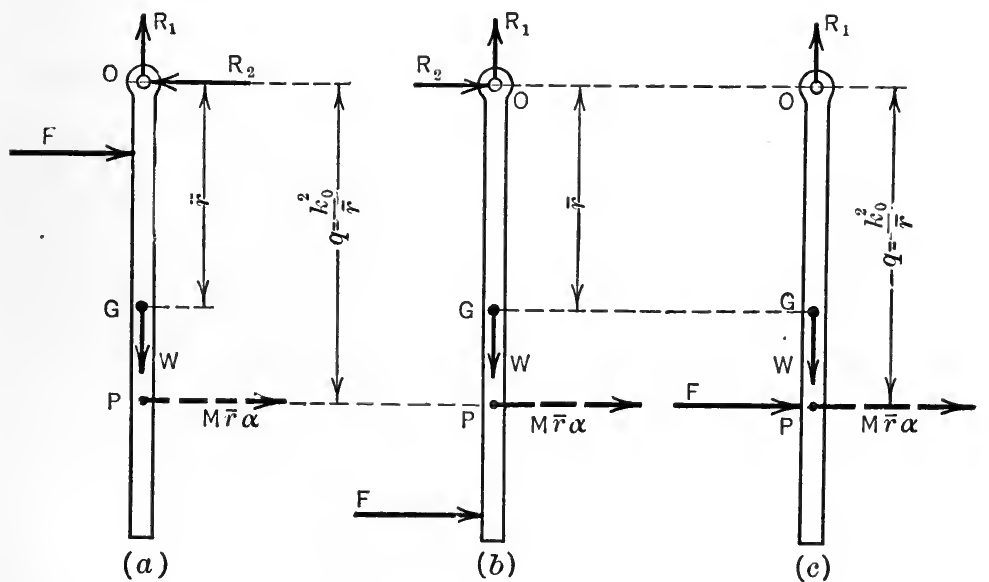

FIG. 356.

of percussion is a point on a line joining the center of rotation and the mass-center, at a distance $q$ from the center of rotation, such that,

$$
q=\frac{k_{0}^{2}}{\bar{r}}
$$

in which $k_{0}$ is the radius of gyration of the body about the axis 
of rotation and $\bar{r}$ is the distance from the axis of rotation to the mass-center of the body.

The physical significance of the center of percussion is suggested in the following illustration. Let a bar (Fig. 356) of weight $W$ be free to rotate about a horizontal axis when a horizontal force, $F$, is suddenly applied to it. If the force, $F$, is applied above the center of percussion, as shown in Fig. 356(a), the horizontal reaction, $R_{2}$, of the axis of rotation acts towards the left and becomes larger as the force $F$ is applied closer to the axis of rotation. If the bar is struck below the center of percussion, the reaction $R_{2}$ acts towards the right, as shown in Fig. 356(b). And if the bar is struck so that the center of percussion is on the action line of the force, as in Fig. 356(c), the horizontal reaction at $O$ is zero, since the action line at $F$ is collinear with the action line of the tangential component, $M \bar{r} \alpha$, of the resultant of the effective forces. It will be noted that the resultant of $F$ and $R_{2}$, in each case, is collinear with $M \bar{r} \alpha$, since the component of the resultant of the external forces in any direction is identical with the component of the resultant of the effective forces in the same direction, that is, if $M_{\bar{r}} \alpha$ were reversed and applied to the body as an external force, it would hold $F$ and $R_{2}$ in equilibrium.

An excellent illustration of the effect of varying the position of the force $F$ as above discussed is found in batting a baseball. If the ball strikes the bat at the center of percussion (about threefourths the length of the bat from the end, assuming the axis of rotation at the hands) no reaction perpendicular to the bat is experienced by the batter. If, however, the ball strikes the bat near the end or near the hands, the batter experiences a painful stinging of the hands as a result of the reaction perpendicular to the bat.

\section{ILLUSTRATIVE PROBLEM}

382. A slender rod $A B$ is caused to rotate about its lower end $A$ by the crank $C D$, the length of which is $1 \mathrm{ft}$., and the link $D E$ (Fig. 357a). The angular velocity, $\omega$, of the crank is very small at the given instant but its rate of change, $\alpha$, is $20 \mathrm{rad}$. per sec. ${ }^{2}$ The weight of the $\operatorname{rod} A B$ is $64.4 \mathrm{lb}$. and its length, $l$, is $5 \mathrm{ft}$. A small body $F$ which weighs $16.1 \mathrm{lb}$. is attached to the rod at a distance of $2 \mathrm{ft}$. from $A$. Body $F$ may be considered to be a particle. What is the distance, $d$, from $A$ at which the link $D E$ must be attached if no horizontal reaction occurs at $A$ ?

Solution.-The resultant of the effective forces for the rod is $M \bar{r} \alpha_{1}$ (where $\alpha_{1}$ is the angular acceleration of the rod) since $\omega_{1}$ is very small and hence 
$M \bar{r} \omega_{1}{ }^{2}$ is negligible. The tangential acceleration of $E$, as a point on $A B$, is $d \cdot \alpha_{1}$ and the tangential acceleration of $D$, as a point $C D$, is $\overline{C D} \cdot \alpha$. Since the accelerations of $D$ and $E$ along $E D$ are equal, we have (Art. 121),

$$
d \alpha_{1}=\alpha \text {, since } \overline{C D}=1 \mathrm{ft} \text {. }
$$

Hence,

$$
\alpha_{1}=\frac{\alpha}{d}=\frac{20}{d}
$$
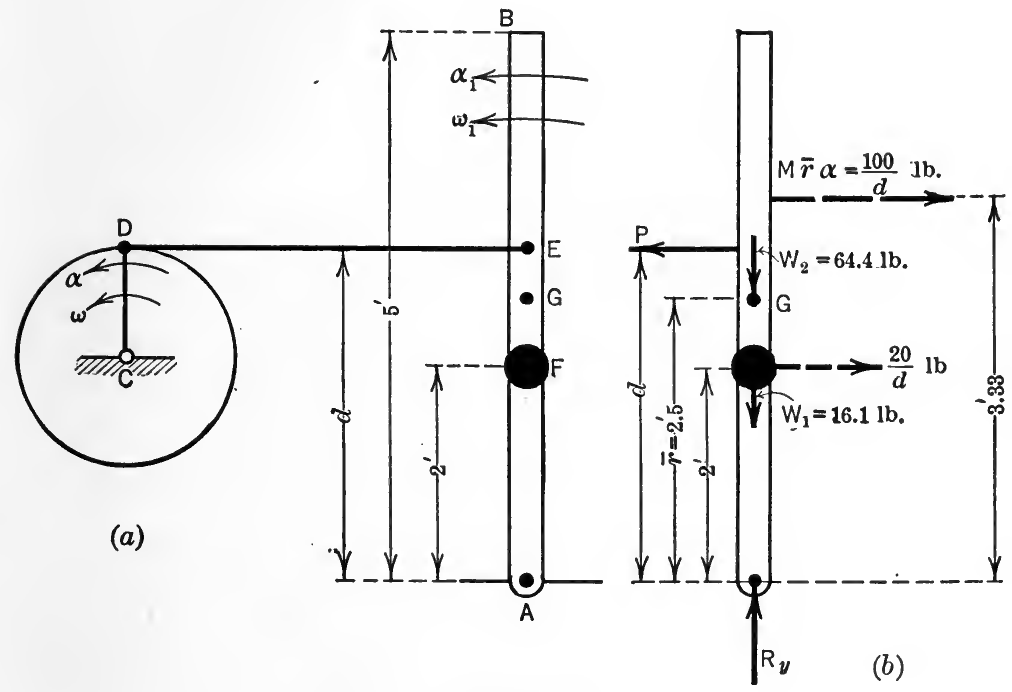

FIG. 357.

Therefore, the resultant of the effective forces for the rod is,

$$
M \bar{r} \alpha_{1}=\frac{64.4}{32.2} \times 2.5 \times \frac{20}{d}=\frac{100}{d} \mathrm{lb} .
$$

The distance of the action line of $M \bar{r} \alpha_{1}$ from $A$ is,

$$
q=\frac{k_{A}^{2}}{\bar{r}}=\frac{\frac{1}{3} l^{2}}{\frac{1}{2} l}=\frac{2}{3} l=3.33 \mathrm{ft} .
$$

The effective force for particle $F$ is,

$$
m \times 2 \alpha_{1}=\frac{16.1}{32} .2 \times 2 \times \frac{20}{d}=\frac{20}{d} \mathrm{lb} .,
$$

and its action line passes through the particle $F$. If these two forces, $\frac{100}{d} \mathrm{lb}$. and $\frac{20}{d} \mathrm{lb}$., are reversed and assumed to act on the rod with the forces $P, R_{\nu}$, $W_{1}$, and $W_{2}$, as shown in Fig. $357(b)$, the rod may be assumed to be in equilib- 
rium. The value of $d$ may be determined by using only two of the equilibrium equations. Thus,

$$
\begin{aligned}
\Sigma F_{x} & =\frac{100}{d}+\frac{20}{d}-P=0, \\
\Sigma M_{A} & =P d-\frac{100}{d} \times 3.33-\frac{20}{d} \times 2=0 .
\end{aligned}
$$

Solving the equations, we have,

$$
\begin{gathered}
P=38.6 \mathrm{lb} . \\
d=3.11 \mathrm{ft} .
\end{gathered}
$$

\section{PROBLEMS}

383. A uniform slender rod, $6 \mathrm{ft}$. long and weighing $20 \mathrm{lb}$., is suspended from a horizontal axis at one end and is acted on by a horizontal force of 20 lb. at its mid-point. Determine $(a)$ the resulting angular acceleration, $(b)$ the resulting linear acceleration of the mass-center, $(c)$ the horizontal reaction of the axis on the rod, and $(d)$ the distance from the axis at which the force must be applied so as to cause no horizontal reaction.

$$
\begin{aligned}
& \text { Ans. (a) } 8.05 \mathrm{rad} \text {. per sec. }{ }^{2} \text { (c) } 5 \mathrm{lb} \text {. }
\end{aligned}
$$

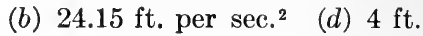

384. A door of constant cross-section is $3 \mathrm{ft}$. wide and weighs $32.2 \mathrm{lb}$. per $\mathrm{ft}$. of width. It swings on its hinges so that its outer edge has a speed of $8 \mathrm{ft}$. per sec. (a) Find the force applied perpendicularly to the door at the outer edge to bring it to rest in a distance of $1 \mathrm{ft}$. (b) What is the horizontal reaction of the hinges perpendicular to the door while the force is acting? (c) How far from the hinge line must the force be applied in order that the hinge reaction shall have no horizontal component perpendicular to the door?

\section{Plane Motion}

149. Kinetics of Plane Motion of a Rigid Body.-As shown in Art. 134, a plane motion of a rigid body may be considered, at any instant, as a combination of a rotation about an axis through any point, $O$, in the plane of motion of the body, and a translation of the body which gives to each particle the same velocity and acceleration that the point $O$ has at the instant. The motion of any particle of the body, therefore, may be resolved into two component motions, (1) a rotation about $O$ and (2) a motion identical with that of $O$. Hence, the acceleration of any particle has a normal component, $a_{n}=r \omega^{2}$, and a tangential component, $a_{t}=r \alpha$, due to the rotation of the body about $O$, and also an accelertion, $a_{0}$, the same as that of $O$, due to the translation of the body. 
Thus, in Fig. 358, let the diagram represent a body which has plane motion. The body is assumed to be symmetrical with respect to its plane of motion and hence may be considered to be concentrated in the plane of motion (see footnote under Art. 144). Let the angular velocity and angular acceleration of the body, at the given instant, be $\omega$ and $\alpha$, respectively ( $\omega$ and $\alpha$ are the same with respect to all axes perpendicular to the plane of motion and all particles have the same $\omega$ and $\alpha$ at a given instant, Art. 134). Let the motion be resolved into a rotation about an axis through any point $O$, giving to the body, of course, its actual angular

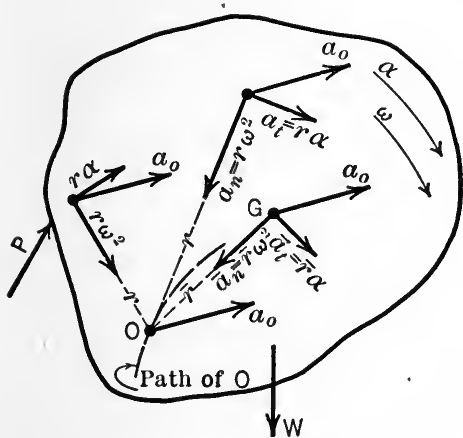

FIG. 358.

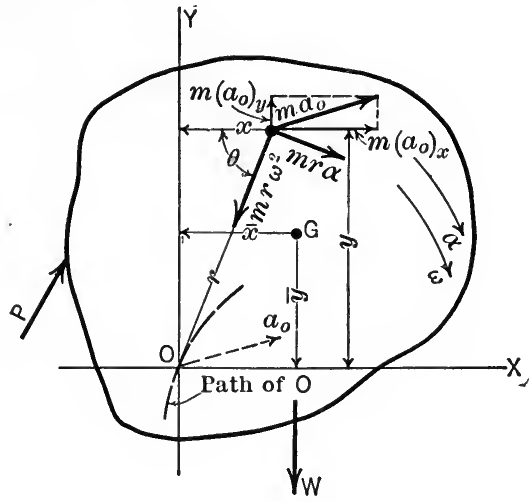

FIG. 359.

velocity and acceleration, and a translation which gives to each particle a motion the same as that of $O$. Let $a_{0}$ denote the acceleration of the point $O$. Then, the acceleration of any particle at a distance $r$ from $O$ (Fig. 358) has three components; $r \omega^{2}, r \alpha$, and $a_{0}$, the directions of which are shown for several particles in the figure.

After the components of the acceleration of any particle of the body have been found, the components of the resultant force (effective force) required to produce the component accelerations may be found from Newton's second law. Thus, as shown in Fig. 359, the components of the effective force for any particle of mass $m$ are $m a_{t}=m r \alpha$ in the direction of $a_{t}, m a_{n}=m r \omega^{2}$ in the direction of $a_{n}$, and $m a_{0}$ in the direction of $a_{0}$. For convenience, $m a_{0}$ will be resolved into its $x$ - and $y$-components, $m\left(a_{0}\right)_{x}$ and $m\left(a_{0}\right)_{y}$, as shown in Fig. 359. 
Resultant of Effective Forces.-The resultant of the effective forces for the whole body may now be found. The effective forces form a non-concurrent system in the plane of motion. The resultant of such a system of forces is either a force or a couple (Art. 28). It will be shown, however, that when the resultant is a force it may be resolved, for convenience, into another force which acts through the mass-center of the body and a couple.

The resultant of a non-concurrent force system in a plane may be determined completely from the algebraic sums of the $x$-components of the forces, of the $y$-components of the forces, and of the moments of the forces about an axis in the body through $O$ (Art. 30). Thus, referring to Fig. 359, and considering the effective forces for the whole body we may write:

The algebraic sum of the $x$-components of the effective forces

$$
\begin{aligned}
& =\Sigma m\left(a_{0}\right)_{x}+\Sigma m r \alpha \sin \theta-\Sigma m \omega^{2} r \cos \theta \\
& =\left(a_{0}\right)_{x} \Sigma m+\alpha \Sigma m y-\omega^{2} \Sigma m x \\
& =M\left(a_{0}\right)_{x}+M \bar{y} \alpha-M \bar{x} \omega^{2} .
\end{aligned}
$$

The algebraic sum of the $y$-components of the effective forces

$$
\begin{aligned}
& =\Sigma m\left(a_{0}\right)_{y}-\Sigma m r \alpha \cos \theta-\Sigma m \omega^{2} r \sin \theta \\
& =\left(a_{0}\right)_{y} \Sigma m-\alpha \Sigma m x-\omega^{2} \Sigma m y \\
& =M\left(a_{0}\right)_{y}-M \bar{x} \alpha-M \bar{y} \omega^{2} .
\end{aligned}
$$

The algebraic sum of the moments of the effective forces about $O$

$$
\begin{aligned}
& =\Sigma m r \alpha \cdot r+\Sigma m\left(a_{0}\right)_{x} y-\Sigma m\left(a_{0}\right)_{y} x \\
& =\alpha \Sigma m r^{2}+\left(a_{0}\right)_{x} \Sigma m y-\left(a_{0}\right)_{y} \Sigma m x \\
& =I_{0} \alpha+M \bar{y}\left(a_{0}\right)_{x}-M \bar{x}\left(a_{0}\right)_{y} .
\end{aligned}
$$

But the algebraic sum of the $x$-components of the effective torces is equal to the $x$-component of the resultant of the effective forces, and similarly for the $y$-components. Further, the algebraic sum of the moments of the effective forces with respect to $O$ is equal to the moment of the resultant of the effective forces with respect to $O$. Hence, summarizing,

The $x$-component of the resultant of the effective forces

$$
=M\left(a_{0}\right)_{x}+M \bar{y} \alpha-M \bar{x} \omega^{2} .
$$


The $y$-component of the resultant of the effective forces

$$
=M\left(a_{0}\right)_{y}-M \bar{x} \alpha-M \bar{y} \omega^{2} .
$$

The moment of the resultant of the effective forces about $O$

$$
=I_{0} \alpha+M \bar{y}\left(a_{0}\right)_{x}-M \bar{x}\left(a_{0}\right)_{y} .
$$

Relation between External and Effective Forces. - Since the effective force for a particle of a body is the resultant of all the forces acting on the particle, it is the resultant of both the internal and the external forces which act on the particle. Therefore, the component, in any direction, of the resultant of the effective forces for the whole body is the algebraic sum of the components, in the given direction, of all the internal forces and all the external forces which act on the particles. Likewise, the moment of the resultant of the effective forces about any axis perpendicular to the plane of motion is the algebraic sum of the moments of all the internal forces and all the external forces which act on the particles. Hence, if $F$ denotes a force and $T$ denotes the moment (torque) of a force, the above expressions are connected with the forces acting on the particles according to the following equations:

$$
\begin{aligned}
& \left(\Sigma F_{x}\right)_{\text {External }}+\left(\Sigma F_{x}\right)_{\text {Internal }}=M\left(a_{0}\right)_{x}+M \bar{y} \alpha-M \bar{x} \omega^{2} \\
& \left(\Sigma F_{y}\right)_{\text {External }}+\left(\Sigma F_{y}\right)_{\text {Internal }}=M\left(a_{0}\right)_{y}-M \bar{x} \alpha-M \bar{y} \omega^{2} \\
& \left(\Sigma T_{0}\right)_{\text {External }}+\left(\Sigma T_{0}\right)_{\text {Internal }}=I_{0} \alpha+M \bar{y}\left(a_{0}\right)_{x}-M \bar{x}\left(a_{0}\right)_{\lrcorner} .
\end{aligned}
$$

But, since the internal forces occur in pairs of equal, opposite, and collinear forces (Newton's third law), the algebraic sum of their components in any direction, and the algebraic sum of their moments about any axis perpendicular to the plane of motion, are zero (that is, the resultant of the external forces alone and the resultant of the effective forces for the body are identical). Hence, in the above equations, the terms which involve the internal forces may be omitted and therefore the equations expressing the relation between (1) the external forces acting on the body, (2) the kinetic properties of the body, and (3) the change in motion of the body are:

$$
\left.\begin{array}{l}
\Sigma F_{x}=M\left(a_{0}\right)_{x}+M \bar{y} \alpha-M \bar{x} \omega^{2} \\
\Sigma F_{y}=M\left(a_{0}\right)_{y}-M \bar{x} \alpha-M \bar{y} \omega^{2} \\
\Sigma T_{0}=I_{0} \alpha+M \bar{y}\left(a_{0}\right)_{x}-M \bar{x}\left(a_{0}\right)_{y}
\end{array}\right\} \cdot \cdot \cdot \cdot \cdot
$$


The equations of motion, however, may be written in a simpler form. As already noted, the center, $O$, about which the assumed rotation takes place and about which the moments of the forces are taken may be any point in the plane of motion of the body. Thus, if the mass-center is selected for the center about which moments are taken, that is, if $O$ coincides with $G$ (Fig. 359) then, in the above equations, $\bar{x}$ and $\bar{y}$ are zero; $a_{0}$ becomes $\bar{a} ; I_{0}$ becomes $\bar{I}$; and $\Sigma T_{0}$ becomes $\Sigma \bar{T}$. Hence the right-hand member of each of the above equations reduces to one term. Thus the equations of motion for a rigid body having plane motion may be written:

$$
\left.\begin{array}{l}
\Sigma F_{x}=\boldsymbol{M} \overline{\boldsymbol{a}}_{x} \\
\Sigma F_{y}=\boldsymbol{M} \overline{\boldsymbol{a}}_{y} \\
\Sigma \bar{T}=\overline{\boldsymbol{I}} \boldsymbol{\alpha}
\end{array}\right\} \cdot \cdot \cdot \cdot \cdot \cdot \cdot \cdot \cdot \cdot \cdot
$$

\section{ILLUSTRATIVE PROBLEMS}

385. A homogeneous cylinder which is $3 \mathrm{ft}$. in diameter and which weighs $805 \mathrm{lb}$. rolls down an inclined plane which makes an angle of $30^{\circ}$ with the

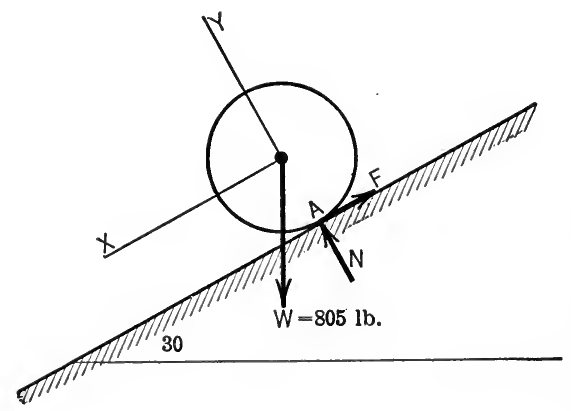

F1G. 360 . horizontal (Fig. 360). The mass-center of the cylinder has an initial velocity $v_{0}=50 \mathrm{ft}$. per sec. The plane is rough so that the cylinder rolls without slipping. Find (1) the acceleration of the mass-center, (2) the magnitude of the friction force, and (3) the velocity, $v$, of the mass-center at the end of $10 \mathrm{sec}$.

Solution.-The cylinder has plane motion under the action of three forces, $F, N$, and $W$, as shown in Fig. 360. Let the $x$ - and $y$-axes be chosen as shown in the figure. The equations of motion are,

From (1),

$$
\begin{aligned}
& \Sigma F_{x}=M \bar{a}_{x}, \quad \ldots \ldots \ldots \\
& \Sigma F_{y}=M a_{y}, \\
& \Sigma \bar{T}=\bar{I} \alpha .
\end{aligned}
$$

$$
805 \sin 30^{\circ}-F=\frac{805}{32.2} \bar{a}_{x}
$$


From (2),

From (3),

$$
-805 \cos 30^{\circ}+N=0 \text {, since } \bar{a}_{y}=0 \text {. . . . . . . }
$$

$$
\frac{3}{2} F=\frac{1}{2} \times \frac{805}{32.2}\left(\frac{3}{2}\right)^{2} \alpha .
$$

There are four unknown quantities involved in these three equations and hence another equation is needed. From the kinematics of the problem (Art. 121), we obtain the equation,

$$
\bar{a}_{x}=\bar{a}=r \alpha=\frac{3}{2} \alpha .
$$

By substituting the value of $\alpha$ from (7) in (6) and solving for $F$, we obtain the equation,

$$
F=\frac{25}{2} \bar{a}
$$

Substituting this value of $F$ in (4), we obtain,

Hence,

$$
\bar{a}_{x}=\bar{a}=10.73 \mathrm{ft} . / \text { sec. }^{2}
$$

$$
F=\frac{25}{2} \times 10.73=134.1 \mathrm{lb} \text {. }
$$

Since the mass-center moves with uniformly accelerated rectilinear motion, we may use the equation,

Hence,

$$
\begin{aligned}
\bar{v} & =\bar{v}_{0}+\bar{a} t, \\
\bar{v} & =50+10.73 \times 10 \\
& =157.3 \mathrm{ft} . / \text { sec. }
\end{aligned}
$$

386. The connecting rod of a steam engine has a length $l=6 \mathrm{ft}$. and a weight $W=220 \mathrm{lb}$. (Fig. 361). The length of the crank is $r=1 \mathrm{ft}$. and the

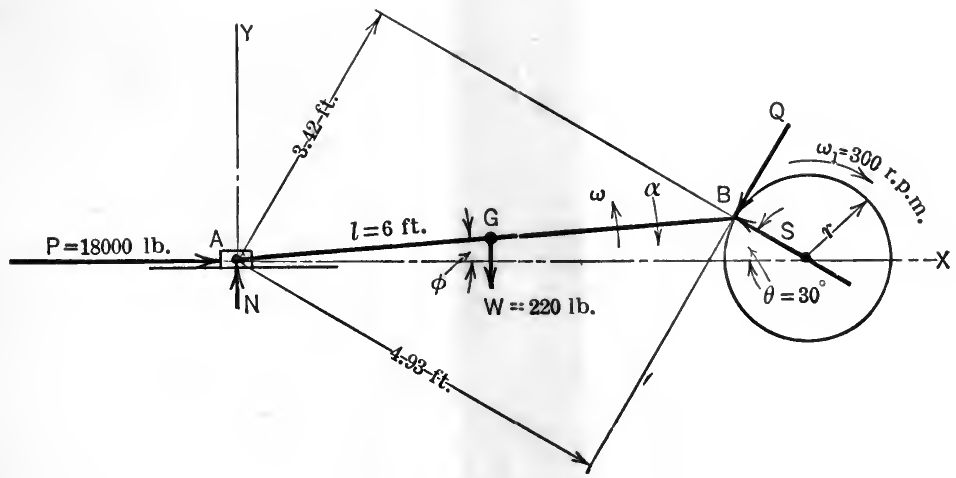

FIG. 361.

engine runs at a speed of $\omega_{1}=300$ r.p.m. The pressure $P$ on the crosshead, when $\theta=30^{\circ}$, is $18,000 \mathrm{lb}$. Assume that the cross-section of the connecting rod is constant. Find the tangential crankpin pressure, $Q$, the com- 
pression, $S$, in the crank arm, and the guide pressure, $N$, which is assumed to act vertically (friction neglected).

Solution.-The connecting rod has plane motion. Either equations (1) or equations (2) of Art. 149 may be used. Let equations (1) be chosen and let the crosshead end of the connecting rod be selected as the origin. Further, let the $x$ - and $y$-axes be chosen horizontal and vertical respectively. The equations of motion then are,

in which,

$$
\begin{aligned}
& \Sigma F_{x}=M\left(a_{A}\right)_{x}+M \bar{y} \alpha-M \bar{x} \omega^{2}, \quad . \quad . \quad . \quad . \quad . .
\end{aligned}
$$

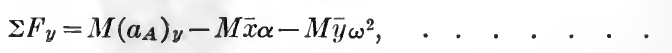

$$
\begin{aligned}
& \Sigma T_{A}=I_{A \alpha}+M \bar{y}\left(a_{A}\right)_{x}-M \bar{x}\left(a_{A}\right)_{y}, \quad \text {. . . . . . }
\end{aligned}
$$

$$
\left(a_{A}\right)_{y}=0,\left(a_{A}\right)_{x}=a_{A}, M=\frac{220}{32.2}=6.84 \text { slugs, } I_{A}=\frac{1}{3} M l^{2}=82 \text { slug-ft. }^{2}
$$

Further,

Hence,

Therefore,

$$
l \sin \phi=r \sin \theta_{i} \text { or } \sin \phi=\frac{1}{6} \sin 30^{\circ}=\frac{1}{12} \text {. }
$$

$$
\phi=4^{\circ} 47^{\prime} \text {. }
$$

$$
\begin{aligned}
& \sin \phi=.0833, \quad \bar{x}=3 \cos \phi=2.99 \mathrm{ft} ., \\
& \cos \phi=.9965, \quad \bar{y}=3 \sin \phi=0.25 \mathrm{ft} .
\end{aligned}
$$

$a_{A}, \omega$, and $\alpha$ may be found by use of the semi-graphical method discussed in

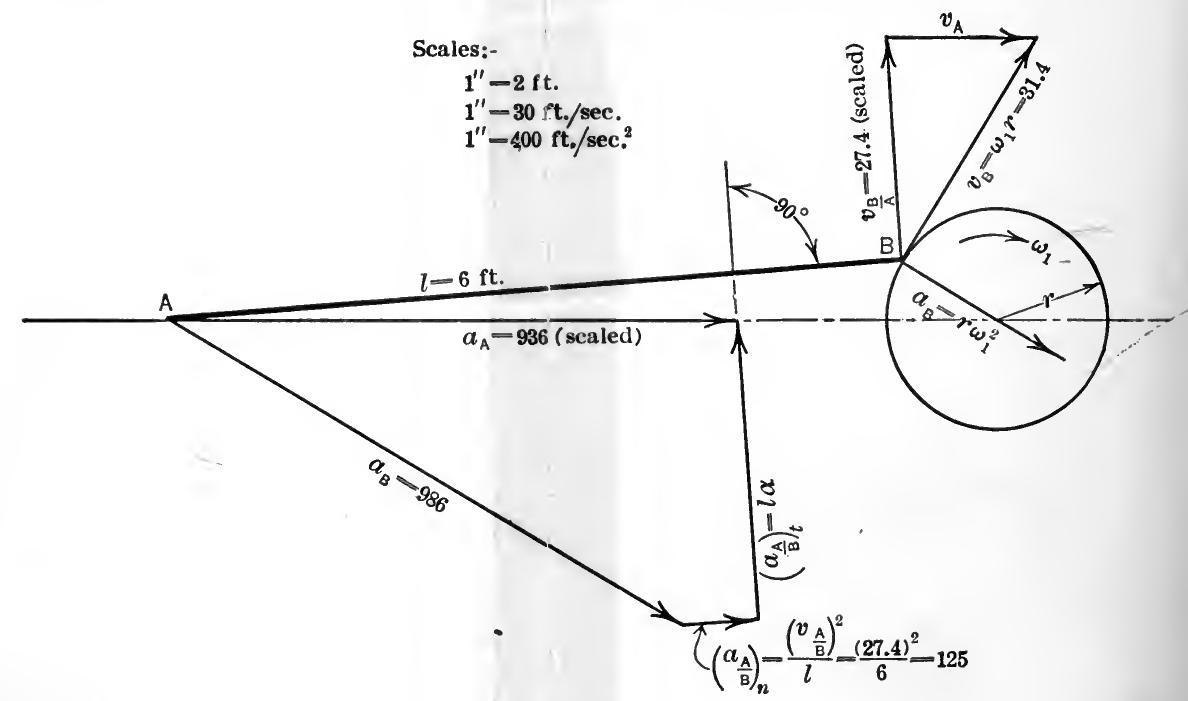

FIG. 362 .

Prob. (334). Thus, in Fig. 362, the following two equations, (4) and (5), are solved,

$$
v_{B}=v_{\frac{B}{A}} \mapsto v_{A},
$$




$$
\begin{aligned}
& a_{A}=a_{\frac{A}{15}}+a_{B} \\
& =\left(\frac{a_{A}}{B}\right)_{n} \mapsto\left(\frac{a_{A}}{B}\right)_{t} \mapsto a_{B} \\
& =\frac{\left(\begin{array}{c}
v_{A} \\
B
\end{array}\right)^{2}}{l}+\left(a_{\frac{A}{B}}\right)_{t} H \omega_{1}^{2} r=\frac{(27 \cdot 4)^{2}}{6}+\left(a_{\frac{A}{B}}\right)_{0} \nrightarrow\left(\frac{300 \times 2 \pi}{60}\right)^{2} .
\end{aligned}
$$

$v_{A}, v_{\frac{A}{B}}=-v_{\frac{B}{A}},\left(a_{\frac{A}{B}}\right)_{\iota}=-\left(a_{\frac{B}{A}}\right)_{\iota}$ and $a_{A}$ are found, by scaling off their

lengths, to have the following values:

$$
v_{A}=18 \mathrm{ft} . / \text { sec., } v_{\frac{B}{A}}=27.4 \mathrm{ft} . / \text { sec., }\left(a_{\frac{B}{A}}\right)_{\imath}=492 \mathrm{ft} . / \text { sec. }^{2}, a_{A}=936 \mathrm{ft} . / \text { sec. }^{2}
$$

But,

$$
v_{\frac{B}{A}}=\omega l, \text { and } \quad\left(\frac{a_{B}}{A}\right)_{t}=\alpha l .
$$

Hence,

$$
\omega=\frac{27.4}{6}=4.56 \mathrm{rad} . / \mathrm{sec} . \quad \text { and }, \quad \alpha=\frac{492}{6}=82 \mathrm{rad} . / \mathrm{sec} .^{2} .
$$

From (1),

$$
18,000-S \cos 30^{\circ}-Q \cos 60^{\circ}=6.84\left(936+0.25 \times 82-2.99 \times \overline{4.56}^{2}\right) .
$$

From (2),

$$
N+S \sin 30^{\circ}-Q \sin 60^{\circ}-220=6.84\left(0-2.99 \times 82-0.25 \times \overline{4.56}^{2}\right) .
$$

From (3),

$$
-3.42 S+4.93 Q+2.99 \times 220=82 \times 82+6.84 \times 0.25 \times 936 .
$$

Solving for the three unknown forces, we obtain the following values:

$$
Q=7950 \mathrm{lb} ., \quad S=9150 \mathrm{lb} ., \quad N=795 \mathrm{lb} .
$$

\section{PROBLEMS}

387. A homogeneous solid sphere rolls without slipping down a rough plane which is inclined at an angle $\theta$ with the horizontal. Show that the acceleration of the center of the sphere is $\frac{5}{7} g \sin \theta$, and that the ratio of the friction to the normal pressure must be not less than $\frac{2}{7} \tan \theta$ to prevent the sphere from slipping. 
388. A solid sphere having a radius of $8 \mathrm{in}$. and a weight of $161 \mathrm{lb}$. is made to roll up a rough inclined plane (Fig. 363) by means of a flexible cord, one end of which is attached to an axis through the center of the sphere. The cord passes over a smooth peg and has attached to its other end a suspended body $B$ which weighs $100 \mathrm{lb}$. Find the acceleration of the body $B$ and the tension in the cord.

Ans. $a=1.93 \mathrm{ft} . /$ sec. $^{2}$

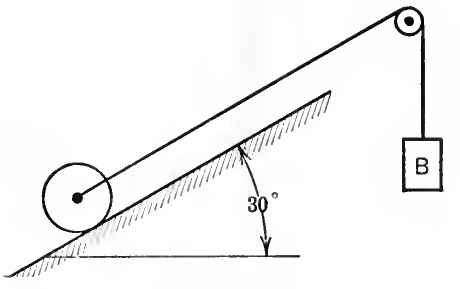

Fig. 363.

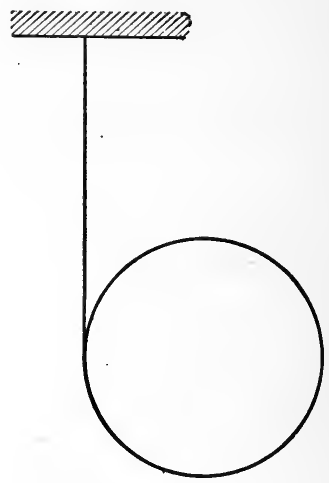

FıG. 364.

389. A homogeneous cylinder $1 \mathrm{ft}$. in diameter has a flexible cord wrapped around its central plane. One end of the cord is attached to a fixed plane as shown in Fig. 364. The cord is taut when the cylinder is allowed to fall. Find $(a)$ the acceleration of the mass-center, $(b)$ the angular acceleration of the cylinder, and $(c)$ the distance traveled by the mass-center in 10 sec.

396. Solve Prob. 386 by use of equations (2) of Art. 149.

391. A uniform rod $A B$ (Fig. 365) moves with its ends $B$ and $A$ in contact

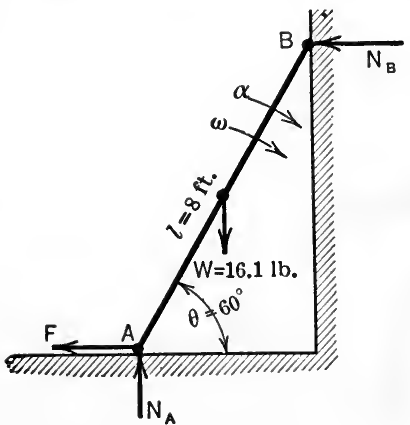

FIG. 365 . with smooth planes which are vertical and horizontal respectively. A variable horizontal force, $F$, is applied at the end $A$. The weight of the rod is $16.1 \mathrm{lb}$. and its length is $8 \mathrm{ft}$. If the value of $F$ for the position of the rod shown $\left(\theta=60^{\circ}\right)$ is such that the angular acceleration, $\alpha$, of the rod is $3 \mathrm{rad} . / \mathrm{sec}^{2}$, and the angular velocity, $\omega$, is $2 \mathrm{rad} . / \mathrm{sec}$., what are the values of $F, N_{A}$, and $N_{B}$ ?

$$
\text { Ans. } \begin{aligned}
& N_{A}=6.17 \mathrm{lb} . ; F=-.03 \mathrm{lb} . ; \\
& N_{B}=1.22 \mathrm{lb} .
\end{aligned}
$$

392. Two solid cylindrical discs are keyed to an axle as shown in Fig. 366. A string is wrapped around the axle in its central plane and a force, $P$, is exerted by the string in a directic 
parallel to the plane on which the discs roll and tangent to the under surface of the axle. Each disc weighs $20 \mathrm{lb}$. and is $2 \mathrm{ft}$. in diameter. The axle is $6 \mathrm{in}$. in diameter and weighs $40 \mathrm{lb}$. The magnitude of the force $P$ is $8 \mathrm{lb}$. W. Il the discs and axle roll forward or backward? Find the acceleration of the central axis of the discs and axle.

Ans. $a=1.91 \mathrm{ft} . /$ sec. $^{2}$

393. If the string in the preceding problem is wrapped around the axle in the opposite direction so that the force $P$ is tangent to the top of the axle, what is the acceleration of the central axis of the discs and axle?

394. A wheel has an eccentric weight attached near its rim so that its mass-center is not the geometric center of the wheel. If the wheel rolls on a horizontal track, will the wheel leave the track when its velocity attains some particular value?

150. Second Method of Analysis. Inertia Forces.--In some problems which deal with the plane motion of a rigid body under the action of an unbalanced force system, it is convenient to assume that the reversed resultant of the effective forces (inertia force for the body) acts on the body with the external forces, thereby forming a force system that is in equilibrium and thus reducing the kinetics problem to an equivalent statics problem. In order to use this method of analysis, the location of the action line of the resultant of the effective forces must be known, as well as the magnitude and sense of the resultant force.

Equations (1) of the preceding article state that the resultant of the effective forces for the body (and hence also of the external forces which act on the body) is a force having $x$ - and $y$-components equal to $M\left(a_{0}\right)_{x}+M \bar{y} \alpha-M \bar{x} \omega^{2}$ and $M\left(a_{0}\right)_{y}-M \bar{x} \alpha-M \bar{y} \omega^{2}$, respectively, as shown in Fig. 367 (a), and that the moment of the resultant force with respect to the axis through the origin $O$ is equal to $I_{0} \alpha+M \bar{y}\left(a_{0}\right)_{x}-M \bar{x}\left(a_{0}\right)_{y}$. Equations (2) state that the resultant of the effective forces for the same body is a force having $x$ - and $y$ components equal to $M \bar{a}_{x}$ and $M \bar{a}_{y}$, respectively, as shown in Fig. $367(b)$, and that the moment of the resultant force with respect to an axis through the mass-center of the body is $\bar{I} \alpha$. The resultant of the effective forces for any given body as obtained from each set 
of equations is, of course, the same force ${ }^{1}$ and its components may intersect at any point on its action line such as points $A$ and $A^{\prime}$ in Fig. 367(a) and (b).

The action line of the resultant force as located by its moment arm $q_{0}$ in Fig. 367(a) or $q$ in Fig. 367(b) may be found from the principle of moments by equating the moment of the resultant of

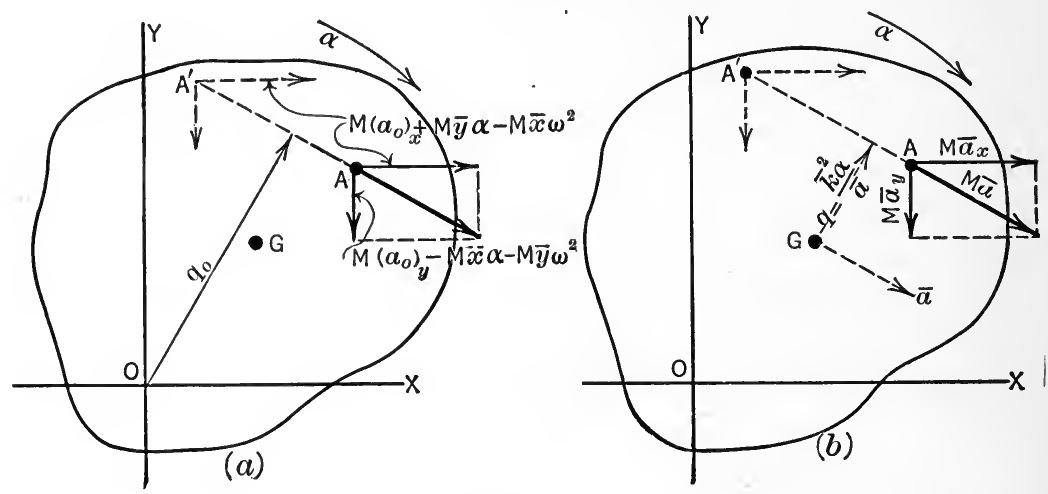

FIG. 367 .

the effective forces to the sum of the moments of the effective forces. The sum of the moments of the effective forces about the mass-center, as already found, is $\bar{I} \alpha$. And, the moment of the resultant force having the components $M \bar{a}_{x}$ and $M \bar{a}_{y}$ is $M \bar{a} \cdot q$ (Fig. 367b). Thus the principle of moments is expressed by the equation,

Therefore,

$$
M \bar{a} \cdot q=\bar{I} \alpha . \quad \text { But } \bar{I}=M \bar{k}^{2} .
$$

$$
q=\frac{\bar{k}^{2} \alpha}{\bar{a}}-
$$

${ }^{1}$ Thus we may write,

$$
\begin{aligned}
& M\left(a_{0}\right)_{x}+M \bar{y} \alpha-M \bar{x} \omega^{2}=M \bar{a}_{x}, \\
& M\left(a_{0}\right)_{y}-M \bar{x} \alpha-M \bar{y} \omega^{2}=M \bar{a}_{y} .
\end{aligned}
$$

Or, canceling the $M$ 's, we have the equations dealing with accelerations as follows,

$$
\begin{aligned}
& \left(a_{0}\right)_{x}+\bar{y} \alpha-\bar{x} \omega^{2}=\bar{a}_{x}, \\
& \left(a_{0}\right)_{y}-\bar{x} \alpha-\bar{y} \omega^{2}=\bar{a}_{y},
\end{aligned}
$$

which state that the acceleration of the mass-center is equal to the relative acceleration of the mass-center with respect to any axis, $O$, plus the acceleration of $O$; a proposition which is discussed in Art. 128. 
The value of $q_{0}$ (Fig. $367 a$ ) may be found in a similar manner. However, it is more convenient, in general, to resolve the single resultant force $M \bar{a}$, having an action line which is located at the distance $q=\frac{\bar{k}^{2} \alpha}{\bar{a}}$ from the mass-center, into another force equal and parallel to $M \bar{a}$, but with its line of action passing through the mass-center, and a couple, $C$, having a moment equal to $M \bar{a} \cdot q$ (Art. 18) as shown in Fig. 368(a). But, as shown above, $M \bar{a} \cdot q=\bar{I} \alpha$ and hence the moment of the couple is $\bar{I} \alpha$.

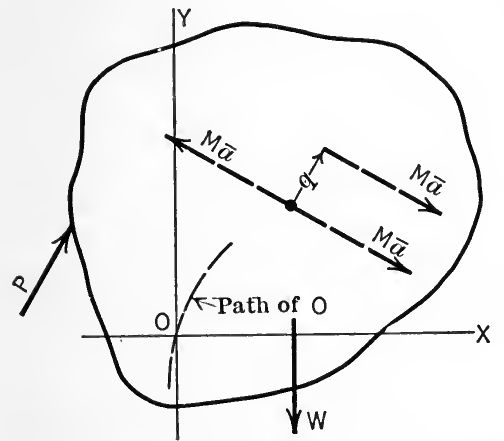

(a)

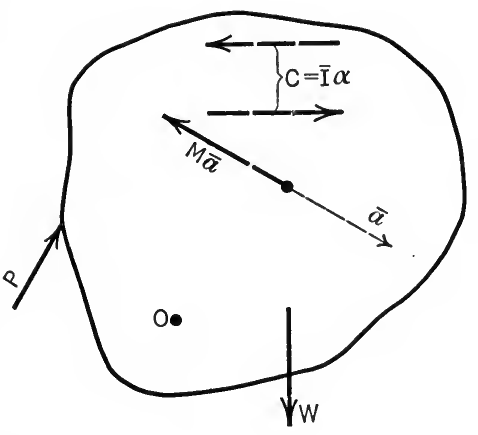

(b)

FIG. 368.

Therefore, the resultant of the effective forces for a rigid body having plane motion may be considered to be a force $M \bar{a}$ having an action line which passes through the mass-center of the body in the direction of the acceleration, $\bar{a}$, of the mass-center, and a couple having a moment equal to $\bar{I} \alpha$, the sense of the couple being the same as that of the angular acceleration, $\alpha$, of the body. The above facts together with D'Alembert's principle lead to a statement or proposition which is of great importance in the method of reducing the kinetics problem of a rigid body having plane motion to an equivalent problem in equilibrium by introducing the inertia force (or forces) for the body. The proposition may be stated as follows:

If a rigid body is acted upon by an unbalanced force system which gives to the body a plane motion, the body may be considered to be in equilibrium if an additional force and couple is assumed to act on the body with the external forces; the additional force has a magnitude equal to $M \bar{a}$, its line of action 
passes through the mass-center of the body, and its direction is opposite to that of $\bar{a}$; the couple has a moment equal to $\bar{I} \alpha$ and its direction of rotation is opposite to that of $\alpha$.

The forces of the couple are not necessarily equal to $M \bar{a}$ or parallel to $\bar{a}$ as shown in Fig. 368(a), that is, the couple may be rotated in the plane of motion and the magnitude of the forces of the couple may be changed provided the moment of the couple is always equal to $\bar{I} \alpha$ (Art. 27). Thus, if a force having a magnitude equal to $M \bar{a}$, and a couple having a moment equal to $M \bar{a} \cdot q$ (or $\bar{I} \alpha$ ), act on the body with the external forces $(P$ and $W$ ), as shown in Fig. 368(b), the force system (and the body) would be in equilibrium.

\section{ILLUSTRATIVE PROBLEMS}

395. At what height should the cushion on a billiard table be placed so that the billiard ball in rebounding from the cushion starts off without causing any friction on the table top?

Solution.-The billiard ball has plane motion and while in contact with the cushion it is acted upon by three forces; the cushion pressure $P$, the weight $W$, and the table pressure $N$, as shown in Fig. 369 .

If there is no horizontal friction force at $A$, then $P$ is the resultant of the external forces which act on the ball and it must be collinear with the

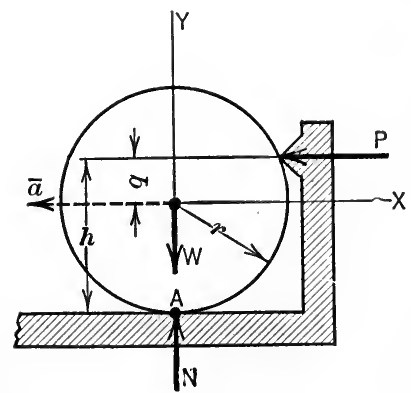

Fig. 369 . resultant of the effective forces. The resultant of the effective forces is a force of magnitude $M \bar{a}$, the distance of the action line from the mass-center being given by the equation,

$$
q=\frac{\bar{k}^{2} \alpha}{\bar{a}^{-}} .
$$

And since $\bar{a}=r \alpha$, we have

Hence,

$$
q=\frac{\bar{k}^{2}}{r}=\frac{\frac{2}{5} r^{2}}{r}=\frac{2}{5} r .
$$$$
h=\frac{7}{5} r \text {. }
$$

396. Solve Prob. 386 by introducing the reversed resultant of the effective forces (inertia force for the rod).

Solution.-The resultant of the effective forces is a force of magnitude $M \bar{u}$ acting through the mass-center of the rod in the direction of $\bar{a}$, and a couple of moment $\bar{I} \alpha$, as shown in Fig. $370(a)$. If $G$ denotes the mass-center, then 
$\bar{a}$ may be written $a_{G}$ for convenience. $a_{G}$ may be found from the graphic equation,

$$
\begin{aligned}
a_{G} & =a_{\frac{G}{B}} \mapsto a_{B}=\left(\frac{a_{G}}{\bar{B}}\right)_{n} \mapsto\left(\frac{a_{G}}{B}\right)_{t} \mapsto a_{B} \\
& =\frac{\left(v_{G}\right)^{2}}{\frac{1}{2} l}+\frac{l}{2} \alpha H \omega_{1}{ }^{2} r,
\end{aligned}
$$

in which $v_{\frac{G}{B}}=\frac{1}{2} v_{\frac{A}{B}}=13.7$ and $\alpha=82$. (See Prob. 386.) Hence,

$$
\begin{gathered}
a_{G} \quad \frac{(13.7)^{2}}{3}+3 \times 82+986 \\
=62.6+246+986 \\
=930 \mathrm{ft} . / \text { sec. }^{2}
\end{gathered}
$$

as scaled from the acceleration polygon of Fig. $370(a)$.
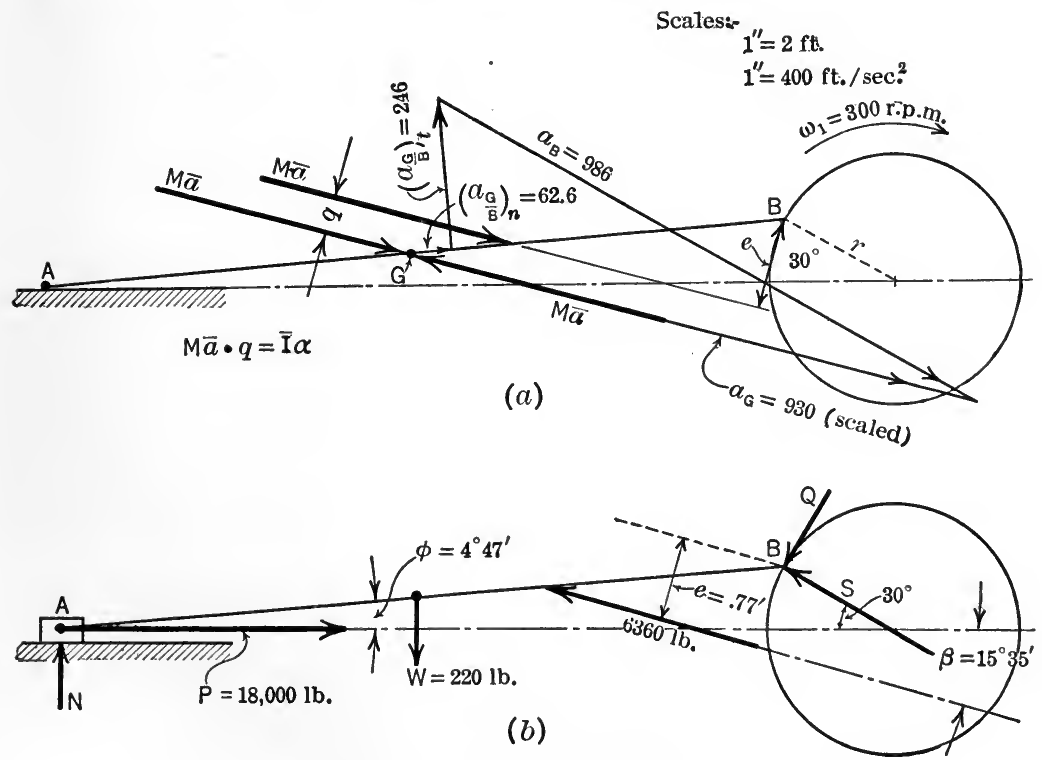

FIG. 370 .

Therefore the resultant of the effective forces is a force,

$$
M \bar{a}=\frac{220}{32.2} \times 930=6360 \mathrm{lb} .,
$$

and a couple,

$$
\bar{I} \alpha=\frac{1}{12} M l^{2} \alpha=\frac{1}{12} \times \frac{220}{32.2} \times 6^{2} \times 82=168.1 \mathrm{lb} . \mathrm{ft} .
$$

But,

$$
\overline{I \alpha}=M \bar{a} \cdot q \quad \text { or } \quad 168.1=6360 q . \quad \therefore q=0.264 \mathrm{ft} .
$$


Hence $q=.264 \mathrm{ft}$., and the inertia force for the rod is a single force of magnitude $6360 \mathrm{lb}$. as shown in Fig. $370(b)$. If the inertia force is assumed to act on the rod with the external forces, the rod may be considered to be in equilibrium. The three unknown forces may be found graphically by use of the force and funicular polygons or, the values of $\beta$ and $e$ may be scaled off and the equations of equilibrium used. The values of $\beta$ and $e$ are found to be $\beta=15^{\circ} 35^{\prime}$ and $e=0.77 \mathrm{ft}$.

The equations of equilibrium for the non-concurrent forces in a plane as shown in Fig. $370(b)$ are,

$$
\begin{gathered}
\Sigma F_{x}=0,18,000-6360 \cos \beta-S \cos 30^{\circ}-Q \cos 60^{\circ}=0, \ldots \\
\Sigma F_{y}=0, N-220+6360 \sin \beta+S \sin 30^{\circ}-Q \sin 60^{\circ}=0, \\
\Sigma M_{B}=0,18,000 l \sin \phi-N l \cos \phi+220 \times 2.99-6360 \times 0.77=0,
\end{gathered}
$$

in which $\sin \phi$ and $\cos \phi$ have the same values as were used in Prob. 386. Thus,

$\sin \phi=.0833, \cos \phi=.9965, \sin \beta=\sin 15^{\circ} 35^{\prime}=.268, \cos \beta=.963, l=6 \mathrm{ft}$. Sutstituting the known values in the above equations, we obtain the following equations:

$$
\begin{aligned}
.5 Q+.866 S-11,875 & =0 \\
.866 Q-.5 S-2282 & =0 \\
-5.98 N+4758 & =0
\end{aligned}
$$

the solution of which gives the following values for the three unknown forces:

$$
Q=7970 \mathrm{lb} . ; S=9100 \mathrm{lb} . ; \quad N=797 \mathrm{lb} \text {. }
$$

\section{PROBLEMS}

397. A solid homogeneous dise $3 \mathrm{ft}$. in diameter rolls on a straight horizontal track (Fig. 371). A small body, $B$, is attached to the disc at a distance

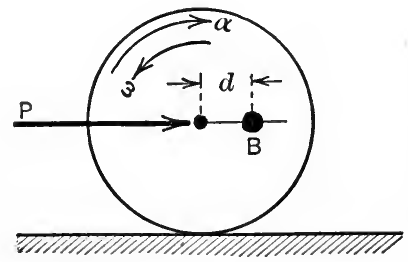

Fig. 371 . $d=0.75 \mathrm{ft}$. from the center of the disc. When in the position shown in Fig. 371 the angular velocity of the disc is 6 rad. per sec., and a force $P$ is retarding the angular velocity at the rate of $4 \mathrm{rad}$. per sec. each second. The disc weighs $60 \mathrm{lb}$. and $B$ weighs $8 \mathrm{lb}$. Find the inertia force for the disc and for the body $B$. Also find the value of $P$ and of the friction force. Ans. $P=7.91 \mathrm{lb}$.; $F=1.96 \mathrm{lb}$.

398. The resultant, $R$, of the forces acting on the connecting rod (Fig. $372)$ is $320 \mathrm{lb}$. and its action line is located as shown. If the connecting 
rod is $30 \mathrm{in}$. long and weighs $83 \mathrm{lb}$, what is the linear acceleration of the mass-center of the rod and the angular acceleration of the rod, assuming the rod to be of constant cross-section?

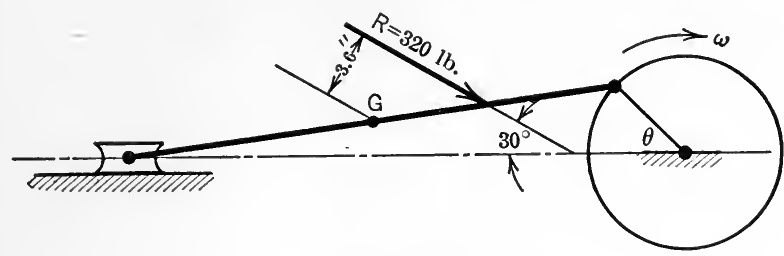

FIG. 372 .

151. Limitations of the Moment Equation $T=I \alpha$. - As discussed in Art. 134, a rigid body having plane motion may be given its actual motion, at any instant, by a combination of a rotation and a translation. And, it was shown that in giving the body the rotational motion, the axis about which the body is assumed to rotate may be any axis, $O$, in the body, perpendicular to the plane of motion, the corresponding translation of the body being defined by the motion which the arbitrarily chosen axis, $O$, has at the instant. It does not follow, however, that the resultant external force which is required to produce the translation is the product of the mass of the body and the translatory acceleration, $a_{0}$, which each particle receives, as is the case for a body for which the actual (or sole) motion is translation (Art. 145). Nor does it follow that the moment of the resultant of the external forces which is required to produce the rotational part of the motion is the product of the moment of inertia, $I_{0}$, of the body about the arbitrarily chosen axis and the angular acceleration, $\alpha$, of the body, as is the case for a body for which the actual (or sole) motion is rotation (Art. 146).

Equations (1) of Art. 149 show that the resultant force required to produce the translatory part of a plane motion is the product of the mass of the body and the translatory acceleration, only when the axis $O$ is chosen through the mass-center of the body, that is, when $\bar{x}$ and $\bar{y}$ in equations (1) are zero. The simple moment equation, $T_{0}=I_{0} \alpha$, also applies to a rigid body having a plane motion when the arbitrarily chosen axis, $O$, passes through the mass-center of the body, as was also shown in Art. 149. It also applies, however, for two other positions of the axis $O$, 
as stated under (3) and (4) below. Thus, in considering a plane motion of a rigid body under the action of an unbalanced force system, the moment of the resultant of the external forces about an axis in the body is equal to the product of the moment of inertia of the body with respect to the same axis and the angular acceleration of the body only when one (or more) of the following four conditions are satisfied, the first of which restricts the motion of the body to a special case of plane motion (rotation) and the other three restrict the choice of the axis in the body, about which the rotational part of the motion is assumed to take place:

1. If the body rotates about an axis which is fixed in space as well as in the body, then $a_{0}$, in equations (1) of Art. 149, is zero and hence the moment equation reduces to $T_{0}=I_{0} \alpha$. This restriction on the axis limits the motion to pure rotation, which is treated in Art. 146 and is, of course, a special case of plane motion.

2. If the arbitrarily chosen axis in the body, about which moments are taken, passes through the mass-center of the body, then $\bar{x}$ and $\bar{y}$ in equations (1) are zero and the moment equation reduces to $\bar{T}=\bar{I} \alpha$ as discussed above.

3. If the acceleration of the point $O$ (in the plane of motion) through which the moment axis is taken, is directed toward the mass-center of the body, then, the quantity $\left[M\left(a_{0}\right)_{x} \bar{y}\right.$ $\left.-M\left(a_{0}\right)_{y} \bar{x}\right]$ in the expression for the moment, in equations (1), is zero and hence the moment equation reduces to $T_{0}=I_{0} \alpha$. The quantity $\left[M\left(a_{0}\right)_{x} \bar{y}-M\left(a_{0}\right)_{y} \bar{x}\right]$ is the moment of the force $M a_{0}$ with respect to the mass-center of the body, and if $a_{0}$ passes through the mass-center, then $M a_{0}$ also passes through the mass-center and hence its moment with respect to the mass-center is zero.

4. If the arbitrarily chosen axis in the body, about which moments are taken, passes through the instantaneous center of zero acceleration (not the instantaneous center of zero velocity, Art. 135) then $a_{0}$ in equations (1) is zero and the moment equation reduces to $T_{0}=I_{0} \alpha$.

The location of the arbitrarily chosen axis as required under (3) and (4) above is of considerable importance in the study of certain problems in Dynamics of Machinery, a detailed discussion of which is beyond the scope of this book. 


\section{PROBLEM}

399. Solve Prob. 385 by use of the equations; $\Sigma F_{x}=M \bar{a}_{x}, \Sigma F_{y}=M \bar{a} \bar{a}_{y}$, and $\Sigma T_{A}=I_{A} \alpha$, in which moments are taken about an axis through $A$, the point of contact of the cylinder with the inclined plane. Does the moment equation $\Sigma T_{A}=I_{A} \alpha$ apply for this position of the moment axis because $A$ is the point about which the body is rotating at the instant (instantaneous center of zero velocity)? If not, which one of the four special cases stated above applies when the moment axis passes through $A$ ?

152. Principle of the Motion of the Mass-Center.-For each of the three types of motion of rigid bodies (translation, rotation, and plane motion) already discussed in this section, there are three equations of motion. The first two of each of these sets of equations ((2) of Art. 145; (1) of Art. 146; and (2) of Art. 149) may be written so as to apply to all three types of motion. The equations are,

$$
\boldsymbol{\Sigma} \boldsymbol{F}_{x}=\boldsymbol{M} \overline{\boldsymbol{a}}_{x} \text { and } \boldsymbol{\Sigma} \boldsymbol{F}_{y}=\boldsymbol{M} \overline{\boldsymbol{a}}_{y}
$$

in which $x$ and $y$ denote any two axes at right angles to each other. Thus, for the motion of translation of a rigid body, $\bar{a}_{x}$ and $\bar{a}_{y}$ are the same as the $a_{x}$ and $a_{y}$ of any other point in the body and hence, in equations (2) of Art. 145, $a_{x}=\bar{a}_{x}$ and $a_{y}=\bar{a}_{y}$. For the motion of rotation of a rigid body the $T$ - and $N$-axes were chosen and in the equations (1) of Art 146, $\bar{r} \alpha=\bar{a}_{t}$ and $\bar{r} \omega^{2}=\bar{a}_{n}$. For plane motion of a rigid body the first two of equations (2) of Art. 149 are already in the form written above. However, these two equations are not restricted to the motion of rigid bodies having the three types of motion treated in the preceding articles. They apply to any mass-system having any type of motion. They express mathematically a principle which is called the principle of the motion of the mass-center. The extension of the proof of this principle to any motion of any mass-system involves the same fundamental laws and the same methods as were used in the preceding articles in deriving the equations of motion for a rigid body having the three special types of motion. The principle of the motion of the mass-center may be stated in words as follows:

If an unbalanced external force system acts on a body (whether rigid or not), the resultant of the external force system, if a force, has a magnitude which is equal to the product of the mass of the body and the acceleration of the 
mass-center of the body, and the direction of the resultant force is the same as that of the acceleration of the mass-center. Likewise, the component, in any direction, of the resultant of the external forces is equal to the product of the mass of the body and the component of the acceleration of the mass-center in the given direction.

The statement of the principle as given above is in the form which makes it most directly applicable to the method of analysis which reduces the kinetics problem to an equivalent statics problem by introducing inertia forces. The principle may also be stated in the following form:

The algebraic sum of the components, in any direction, of the external forces which act on any mass-system is equal to the mass of the whole body times the component, in the given direction, of the acceleration of the mass-center of the mass system.

It will be noted that, according to the principle of the motion of the mass-center, the mass-system or body may be assumed to be reduced to a particle having a mass which is equal to the total mass of the body and which is located at, and moving with, the mass-center of the body. That is, the magnitude and direction of the resultant of the actual (non-concurrent) forces which act on the actual body are the same as the magnitude and direction of the resultant of the (concurrent) forces which would have to act on the mass if it were concentrated at, and moving with, the masscenter of the body.

It is important to note, however, that the action line of the resultant of the forces acting on a body, does not, in general, pass through the mass-center of the body whereas it would necessarily do so if the mass of the body were actually concentrated at the mass-center. Further, the principle cannot be used to determine the resultant of the forces acting on a body when the resultant is a couple. Nevertheless, the principle simplifies many problems and is of much importance in the study of kinetics. 


\section{ILLUSTRATIVE PROBLEM}

400. Two bodies $A$ and $B$ (Fig. 373) are connected by a light slender rod and revolve in a horizontal plane about an axle fixed in the top of a vertical post which supports the two bodies. Body $A$ weighs $4 \mathrm{lb}$. and $B$ weighs $12 \mathrm{lb}$. The bodies rotate with an angular velocity of 80 r.p.m. What horizontal force acts on the post tending to bend the post? The mass of the rod may be neglected.

Solution.-The mass-center of the two bodies is found by the principle of moments to be at a distance $\vec{r}=5 \mathrm{in}$.

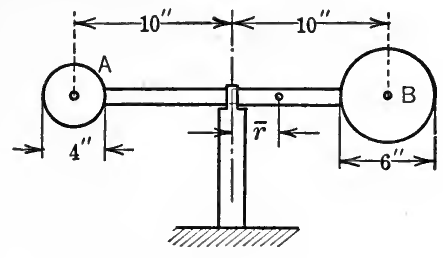

Fig. 373. from the axis of rotation. The acceleration of the mass-center is,

$$
\bar{a}=\bar{a}_{n}=\omega^{2} \bar{r}=\left(\frac{80 \times 2 \pi}{60}\right)^{2} \times \frac{5}{12}=29.2 \mathrm{ft} . / \text { sec. }^{2},
$$

directed toward the center of rotation. The resultant force acting on the two bodies is,

$$
M \bar{a}_{n}=\frac{4+12}{32.2} \times 29.2=14.5 \mathrm{lb} .
$$

This force is exerted on the two bodies by the post, and the two bodies exert an equal and opposite force on the post. (Check the answer by finding the force acting on each body and subtracting one force from the other.)

\section{PROBLEMS}

401. A solid wooden disc $10 \mathrm{ft}$. in diameter rotates in a horizontal plane about its geometric axis. Two small bodies each weighing $50 \mathrm{lb}$. are attached to the disc at a radius of $4 \mathrm{ft}$. from the axis of rotation so that the radii make an angle of $90^{\circ}$. If the disc rotates at 40 r.p.m. what is the resultant horizontal pull on the axis? Solve by two methods. Ans. $154 \mathrm{lb}$.

402. A flat-topped boat having a weight of $300 \mathrm{lb}$. and a length of $12 \mathrm{ft}$. is resting in still water. A man weighing $150 \mathrm{lb}$. stands at one end of the boat. The man starts to run with a speed increasing at the rate of $10 \mathrm{ft}$. per sec. each second. When he reaches the other end of the boat he jumps. Assuming that the water is a perfect (frictionless) fluid what is the acceleration of the mass-center of the boat and man (considered as one body) before the man starts to run? While he is running on the boat? After he jumps from the boat but before he strikes the water? What is the acceleration of the boat while the man is running on it?

403. Two bodies $A$ and $B$ (Fig. 374), each weighing $8 \mathrm{lb}$., are connected by a string. : Body $A$ is placed on a smooth table and the other body $B$ is sus- 
pended over the edge of the table. The two bodies start from rest and move two seconds before body $A$ reaches the table edge. What is the acceleration of the mass-center of the two bodies while $A$ is sliding on the table? What is the acceleration of the mass-center after $A$ leaves the table edge? Discuss the motion of the system after $A$ leaves the table.

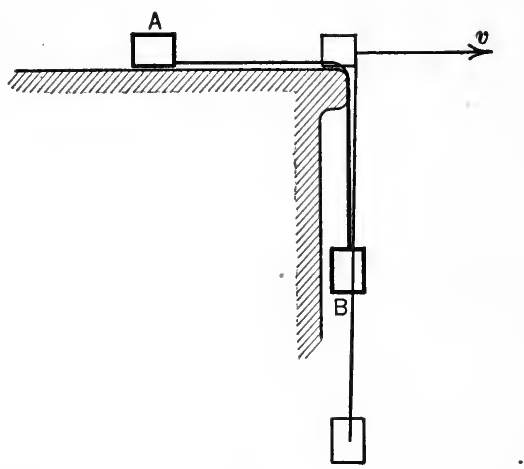

FIG. 374 .

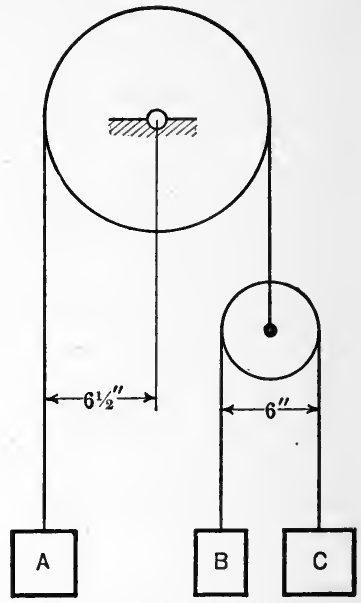

FIG. 375 .

404. The three bodies $A, B$, and $C$ (Fig. 375) weigh $6 \mathrm{lb}, 4 \mathrm{lb}$., and $10 \mathrm{lb}$., respectively. They are held in the position shown and then released simultaneously. The mass of the pulleys and of the strings is negligible. Locate the mass-center after the bodies have been moving 2 sec. Find the tensions in the strings. Ans. $\bar{y}=13.7 \mathrm{ft}$.; $T_{A}=7.87 \mathrm{lb}$.

\section{§3. Special Topics in Kinetics}

153. Introduction.-In this section are discussed several special problems or topics in kinetics which find direct application in engineering. The principles and equations of motion employed in their treatment are developed in the preceding sections of this chapter and all of the topics here considered could have been treated as special problems in the preceding sections. However, the topics are grouped and discussed in this section in order to give an opportunity to review the principles already developed and to emphasize their engineering applications.

154. Hoop Tension in Flywheels.-The stress developed in the rim of a flywheel or pulley, when revolving with a high 
angular velocity, is sometimes of considerable importance. Likewise the stress developed in a belt, due to the same cause, as it passes around a flywheel or pulley, must sometimes be considered. If the tension in the spokes of a flywheel is neglected, the tensile stress in the rim (often called hoop tension) corresponding to an angular velocity, $\omega$, may be found as follows: In Fig. 376 is represented one-half of the rim of a flywheel. As the wheel rotates, each half of the rim tends to separate from the other half and is prevented from doing so by the stresses $P, P$ which are developed in

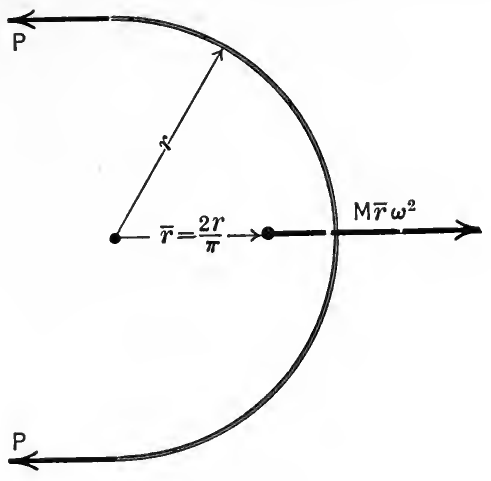

FIG. 376. the rim. The reversed effective force for the half of the rim is $M_{\bar{r}} \omega^{2}$ and it acts through the mass-center of the half-rim. And, since the reversed effective force (inertia force) is in equilibrium with the external forces $(P, P)$ which act on the half-rim, the following equation of equilibrium may be written,

$$
2 P=M \bar{r} \omega^{2}=\frac{W}{g} \bar{r} \omega^{2},
$$

in which $W$ is the weight of the half-rim. Now if the thickness of the rim is small in comparison with the mean radius $r$, the masscenter of the rim may be considered to coincide with the centroid of the semicircular arc, and hence $\bar{r}=\frac{2 r}{\pi}$ (Art. 82). Whence,

$$
P=\frac{1}{2} \frac{W}{g} \times \frac{2 r}{\pi} \omega^{2}=\frac{W r \omega^{2}}{g \pi} .
$$

The stress, $s$, per unit of area of the rim cross-section is

$$
s=\frac{P}{a},
$$

in which $a$ is the area of the cross-section. Therefore,

$$
s=\frac{W}{g} \times \frac{r}{\pi} \times \frac{\omega^{2}}{a}=\frac{\pi r a k}{g} \times \frac{r}{\pi} \times \frac{\omega^{2}}{a}=\frac{k r^{2} \omega^{2}}{g},
$$


in which $k$ is the weight of the material per unit volume. Or, since the velocity, $v$, of the mid-points of the rim is equal to $\omega r$, the expression for $s$ may be written in the form,

$$
s=\frac{k v^{2}}{g} .
$$

The units in which $s$ is expressed are lb. per sq. $\mathrm{ft}$. if $k$ is expressed in lb. per cu. ft., $r$ in ft., $g$ in ft. por sec. ${ }^{2}$, and $\omega$ in rad. per sec. It will be noted, therefore, that the intensity of stress, $s$, developed in the rim of a rotating wheel, if the rim is thin and the effect of the spokes is neglected, varies directly as the square of the linear speed of the rim.

\section{PROBLEMS}

405. A common rule limits the peripheral speed of cast-iron flywheels or pulleys to $6000 \mathrm{ft}$. per min. (Sometimes stated one mile per minute.) Calculate the tensile unit-stress in the rim corresponding to this speed, assuming that the effect of the spokes may be neglected. Ans. $970 \mathrm{lb}$. per sq. in.

406. Calculate the greatest number of revolutions per minute (r.p.m.) at which a thin cast-iron hoop can rotate without bursting. Assume that the maximum tensile strength of the cast iron is $20,000 \mathrm{lb}$. per square inch, that the material weighs $450 \mathrm{lb}$. per cubic foot: and that the radius is $2 \mathrm{ft}$.

$>$ 155. Superelevation of Railroad Track.-The wheels of a locomotive, electric car, etc., when traveling round a curve of radius, $r$, on a level track exert a horizontal thrust, $H$ (flange pressure), on the outer rail as shown in Fig. 377(a). The reversed effective force, $M r \omega^{2}$ or $M \frac{v^{2}}{r}$, if acting on the car with the four external forces $R_{1}, R_{2}, H$, and $W$, would hold the car in equilibrium. Hence $M r \omega^{2}$ and $H$ form a couple the moment of which, for a given car and radius of curvature, depends both upon the speed of the car and the height of the center of gravity, $G$, above the rails. In order to reduce the magnitude of the horizontal thrust and of the overturning couple, the outer rail is elevated above the inner rail a distance, $e$, which is called the superelevation.

It is desired to determine the superelevation required to reduce the flange pressure to zero for a given speed of the car and curvature of the track. Thus, in Fig. 377(b), the pressures of the rails are $R_{1}$ and $R_{2}$, the resultant of which is $R ; W$ is the weight of the 
car; and $r$ is the radius of the curve around which the car is traveling. Since the center of mass of the car travels in a horizontal plane, the reversed effective force $M r \omega^{2}$ is horizontal and its action line passes through the center of gravity, $G$, of the car, as shown. Since the reversed effective force is in equilibrium with the external forces, the three forces $W, M r \omega^{2}$, and $R$ form a concurrent system in equilibrium. Therefore, we may write,

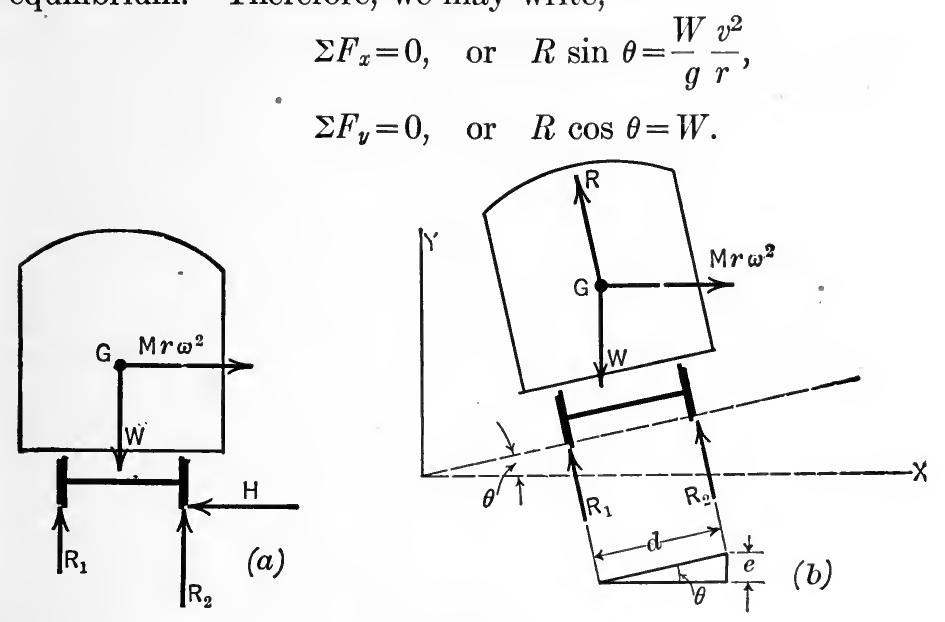

FIG. 377.

And, by dividing the first of these equations by the second, the resulting equation is,

$$
\tan \theta=\frac{v^{2}}{g r}
$$

Now for small angles the sine and the tangent of the angle are approximately the same. But from Fig. $377(b), \sin \theta=\frac{e}{d}$ in which $d$ is the distance between the action lines of the rail pressures (usually taken as $4.9 \mathrm{ft}$.). Therefore,

$$
\tan \theta=\frac{e}{d}=\frac{v^{2}}{g r}
$$

Hence, if $v$ is expressed in $\mathrm{ft}$. per sec., $g$ in $\mathrm{ft}$. per sec. ${ }^{2}$, and $d$ and $r$ in $\mathrm{ft}$., the superelevation (in $\mathrm{ft}$.) is found from the equation

$$
e=\frac{v^{2} d}{g r} \text {. }
$$

It will be noted that the value of $e$ which reduces the flange 
pressure to zero also reduces the overturning couple to zero regardless of the height of the center of gravity above the rails. However, if a car travels round a curve at a speed greater than that for which the flange pressure is zero, there will be, of course, an overturning couple and its magnitude will depend directly upon the height of the center of gravity of the car and its cargo above the rails. In order to indicate common values of the superelevation, the values used on one particular steam railroad are given in the following table:

\section{SUPERELEVATION OF OUTER RAIL IN INCHES}

\begin{tabular}{c|c|c|c|c}
\hline \multirow{2}{*}{$\begin{array}{c}\text { Degree * of } \\
\text { Curve. }\end{array}$} & \multicolumn{4}{|c}{ Speed in Miles per Hour. } \\
\cline { 2 - 4 } & 30 & 45 & 60 & 75 \\
\hline & & & & \\
1 & $\frac{3}{8}$ & $1 \frac{1}{8}$ & 2 & $3 \frac{1}{8}$ \\
2 & $\frac{7}{8}$ & $2 \frac{1}{8}$ & 4 & $6 \frac{1}{2}$ \\
3 & $1 \frac{1}{4}$ & $3 \frac{1}{8}$ & 6 & $9 \frac{3}{4}$ \\
4 & $1 \frac{5}{8}$ & $4 \frac{1}{4}$ & 8 & - \\
5 & 2 & $5 \frac{1}{4}$ & - & - \\
\hline
\end{tabular}

* A one-degree curve is a curve (circle) in which a $100-\mathrm{ft}$. chord is subtended by a central angle of one degree. In a two-degree curve a chord of $100 \mathrm{ft}$. is subtended by a central angle of two degrees, and so on.

\section{PROBLEMS}

407. The radius of a railroad curve is $1800 \mathrm{ft}$. What must be the superelevation of the outer rail in order to make the flange pressure zero when the speed of a car around the curve is 50 miles per hour? Ans. $e=5.46$ in.

408. Do the superelevations given in the above table reduce the flange pressure to zero for the speeds specified? Test several values.

\section{Pendulums}

156. Simple Circular Pendulum.-A simple circular pendulum consists of a single particle suspended by a weightless thread and allowed to swing or vibrate in the arc of a circle, in a vertical plane, under the influence of gravity and the pull of 
the string. Although these ideal conditions cannot be realized fully in any physical apparatus, the motion of a small body suspended by a light thread will be approximately that of the ideal simple pendulum.

It is desired to find the time or period of vibration of a simple circular pendulum. Let $C$ (Fig. 378) represent a small body which is suspended by a thread from the point $O$ and is allowed to swing as a simple pendulum in the arc $B B^{\prime}$ of radius $O C$ or $l$. By resolving forces in the direction of the tangent to the path we have,

$$
\begin{aligned}
\Sigma F_{t} & =m a_{t}, \\
\text { or, } & \\
-W \sin \theta & =\frac{W}{g} a_{t},
\end{aligned}
$$

whence,

$$
a_{t}=-g \sin \theta .
$$

And from Art. 127,

$$
v d v=a_{t} d s .
$$

Therefore,

But,

$$
v d v=-g \sin \theta d s .
$$

Therefore,

$$
d s=l d \theta
$$

$$
v d v=-\lg \sin \theta d \theta .
$$

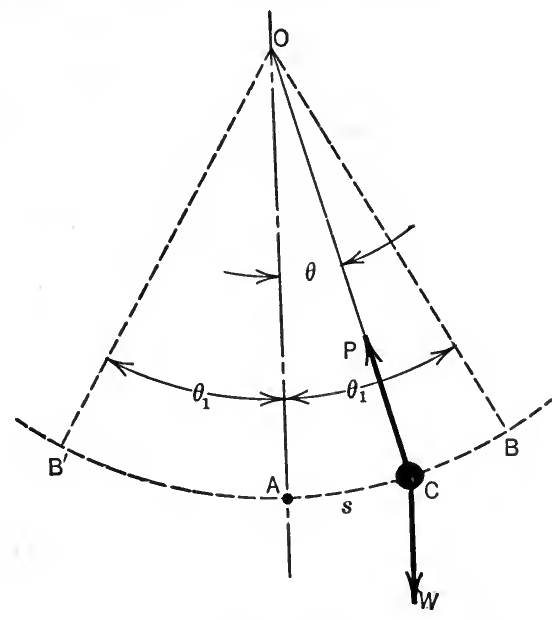

FIG. 378.

The integration of this equation, after expressing $v$ in terms of $t$ and $\theta$, leads to a complicated relation between $t$ and $\theta$. However, if the angle of vibration is small, a close approximation to the correct solution may be obtained by assuming that $\theta=\sin \theta$. The last equation then becomes

And by integrating,

$$
v d v=-\lg \theta d \theta .
$$

$$
v^{2}=-\lg \theta^{2}+C_{1} .
$$

To determine, $C_{1}$, the constant of integration, let $v=0$ when $\theta=\theta_{1}$. Using these values in the above equation, we have, $C_{1}=\lg \theta_{1}^{2}$.

Hence,

or,

$$
\begin{aligned}
v^{2} & =\lg \left(\theta_{1}^{2}-\theta^{2}\right), \\
v & =\sqrt{\lg } \sqrt{\theta_{1}^{2}-\theta^{2}} .
\end{aligned}
$$


The time, $t$, may now be introduced in the equation since $v=\frac{d s}{d t}$. And, if the particle is moving towards $A, s$ decreases as $t$ increases and hence $\frac{d s}{d t}$ is negative. Thus,

$$
v=\frac{d s}{d t}=\frac{l d \theta}{d t}=-\sqrt{l g} \sqrt{\theta_{1}^{2}-\theta^{2}},
$$

or,

$$
\frac{d \theta}{\sqrt{\theta_{1}^{2}-\theta^{2}}}=-\sqrt{\frac{g}{l}} d t
$$

And, by integrating,

$$
\sin ^{-1} \frac{\theta}{\theta_{1}}=-\sqrt{\frac{g}{l}} t+C_{2}
$$

If time is measured from the instant the body is at $B$, then $\theta=\theta_{1}$ when $t=0$. By substituting these values of $\theta$ and $t$ in the last equation, the value of $C_{2}$ is found to be $\sin ^{-1} 1$ or $\frac{\pi}{2}$. Thus, the last equation becomes,

$$
t=\sqrt{\frac{l}{g}}\left(\frac{\pi}{2}-\sin ^{-1} \frac{\theta}{\theta_{1}}\right)
$$

By making $\theta$ equal to zero in the last equation, the corresponding value for $t$, that is, the time required for the body to move from $B$ to $A$ is $\frac{\pi}{2} \sqrt{\frac{l}{g}}$. Since the body gains velocity during the displacement $B A$ at the same rate that it loses velocity in the displacement $A B^{\prime}$, the average velocities for the two displacements are equal. Therefore, the time required for a single oscillation ( $B$ to $\left.B^{\prime}\right)$ is $\pi \sqrt{\frac{l}{g}}$. The time or period, $P$, of a complete uscillation (from $B$ to $B^{\prime}$ and back to $B$ ) then is,

$$
P=2 \pi \sqrt{\frac{l}{g}}
$$

This equation shows that the period $P$ is independent of $\theta_{1}$, that is, of the amplitude of the oscillation. However, it must be remembered that the equation applies to oscillations of relatively small amplitudes.

If the amplitude of oscillation is not small enough to permit of 
the assumption that $\sin \theta=\theta$, then the time of a complete oscillation is expressed by the series,

$$
P=2 \pi \sqrt{\frac{l}{g}}\left[1+\left(\frac{1}{2}\right)^{2} k^{2}+\left(\frac{1 \cdot 3}{2 \cdot 4}\right)^{2} k^{4}+\left(\frac{1 \cdot 3 \cdot 5}{2 \cdot 4 \cdot 6}\right)^{2} k^{6}+\ldots . .\right]
$$

in which

$$
k=\sin \frac{\theta_{1}}{2} \text {. }
$$

\section{PROBLEMS}

409. A simple pendulum $4 \mathrm{ft}$. long swings through an angle of $60^{\circ}$ (that is, $\left.\theta_{1}=30^{\circ}\right)$. Find the period of oscillation, (1) by the approximate method and (2) by the exact method. Ans. (1) $P=2.21$ sec.; (2) $P=2.25$ sec.

410. Find the length of the simple pendulum which beats half-seconds, that is, one for which the period of a complete oscillation is one second.

411. What is the length of a clock pendulum which will make one beat per second. If the clock loses 5 sec. per hour, how much should the pendulum be shortened?

157. Compound Pendulum.-A physical body which oscillates or swings about a horizontal axis under the influence of gravity and the reaction of the supporting axis is called a compound or physical pendulum.

It is desired to find the time of oscillation of a compound pendulum. Let Fig. 379 represent a section of such a pendulum which rotates about an axis through $O$. From Art. 146 we have as one of the equations of motion for a rotating body,

Or,

$$
\Sigma T_{0}=I_{0} \alpha .
$$

$$
-W \vec{r} \sin \theta=I_{0} \alpha
$$

$$
=\frac{W}{g} k_{0}^{2} \alpha \text {. }
$$

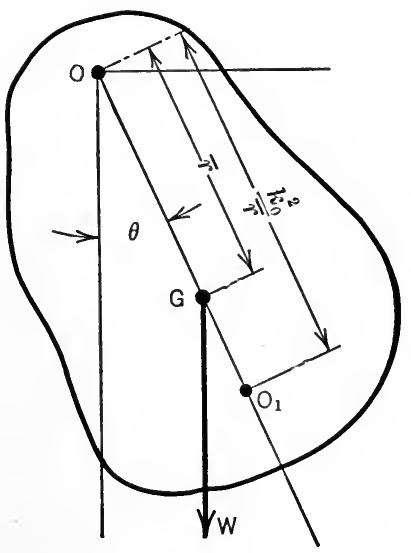

FIG. 379 .

Whence,

$$
\alpha=-\frac{g \bar{r}}{k_{0}^{2}} \sin \theta \text {. }
$$


Now, it was shown in the preceding article that the tangential acceleration of a simple pendulum of length $l$ is,

But,

$$
a_{t}=-g \sin \theta \text {. }
$$

Whence,

$$
a_{\imath}=l \alpha \quad \text { (Art. 121). }
$$

$$
\alpha=\frac{a_{t}}{l}=-\frac{g}{l} \sin \theta . \quad . \quad . \quad . \quad . \quad . \quad . \quad .
$$

By comparing equations (1) and (2) it will be noted that the angular motion of a simple pendulum may be made exactly the same as that of a compound pendulum (if the two start from the same position) by fixing the length $l$ of the simple pendulum such that,

$$
l=\frac{k_{0}^{2}}{\bar{r}} .
$$

A simple pendulum of this length is called an equivalent simple pendulum. The period, then, of a complete oscillation, of small amplitude, of a compound pendulum is,

$$
P=2 \pi \sqrt{\frac{k_{0}^{2}}{g \bar{r}}} .
$$

158. Center of Oscillation.-The point, $O_{1}$, in the compound pendulum (Fig. 379 ) at the distance $\frac{k_{0}^{2}}{\bar{r}}$ from the center of rotation is called the center of oscillation. That is, the center of oscillation is that point at which the whole mass of the compound pendulum may be concentrated without changing the period of vibration. It will be noted that the center of oscillation is also the center of percussion (see Art. 148).

Further, the center of oscillation may be made the center of rotation without changing the period of oscillation. That is, in a compound pendulum the centers of oscillations and suspension are interchangeable. This fact may be shown as follows: The distance $G O_{1}$ from the center of gravity of the compound pendulum to the center of oscillation (Fig. 379) is,

But, from Art. 102,

$$
G O_{1}=\frac{k_{0}^{2}}{\bar{r}}-\bar{r} \text {. }
$$

$$
k_{0}^{2}=\bar{k}^{2}+\bar{r}^{2}
$$


Whence,

$$
G O_{1}=\frac{\bar{k}^{2}}{\bar{r}}
$$

Now if $O_{1}$ is made the center of rotation, then the new center of oscillation, $O_{2}$, will be a distance $G O_{2}=\frac{\bar{k}^{2}}{\bar{r}}$ from the center of gravity. But $\bar{r}$ is now equal to $G O_{1}$; hence,

$$
G O_{2}=\frac{\bar{k}^{2}}{G O_{1}}=\frac{\bar{k}^{2}}{\frac{\overline{\bar{r}}}{\overline{\bar{r}}}}=\bar{r} .
$$

Therefore $O_{2}$ coincides with $O_{1}$ and hence the centers of suspension and oscillation are interchangeable.

\section{PROBLEMS}

412. A uniform slender rod $4 \mathrm{ft}$. long oscillates as a compound pendulum about a horizontal axis through one end of the rod, the rod being perpendicular to the axis. (a) Find the period of oscillation. (b) About what other point could the rod rotate and have the same period of oscillation.

Ans. (a) $P=1.80$ sec.; (b) $1.33 \mathrm{ft}$. from end of rod.

413. Find the length of a uniform slender bar having a period of oscillation of 1 sec. when allowed to swing as a compound pendulum about an axis through one end of the bar.

Ans. $l=1.22 \mathrm{ft}$.

414. A steel pendulum consists of a circular disc $10 \mathrm{in}$. in diameter and $1 \mathrm{in}$. thick, and a rectangular bar $30 \mathrm{in.}$ long, $3 \mathrm{in}$. wide, and 1 in. thick, as shown in Fig. 380. If the pendulum oscillates about a horizontal axis through $O$, what is the period of oscillation?

159. Torsion Pendulum.-A torsion pendulum consists of a body (Fig. 381) which is suspended

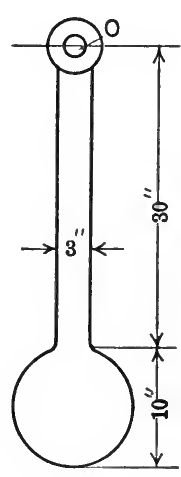

FIG. 380 . by a wire or slender rod and allowed to oscillate about the axis of the wire. The body is rigidly attached to the wire so that its center of gravity lies on the axis of the wire and the wire is rigidly attached at the support. Thus, if the disc of Fig. 381 is given an initial angular displacement, $\theta_{1}$, the wire is twisted and when the disc is released the wire exerts a turning moment on the disc, which starts it to oscillate.

The torque or turning moment, $T$, exerted by the wire on the disc is directly proportional to the angular displacement, provided 
that the elastic limit of the material is not exceeded. That is, $T=-k \theta$ in which $k$ is a constant; the minus sign indicates that when $\theta$ is positive $T$ is negative.

Since the disc has a motion of rotation,

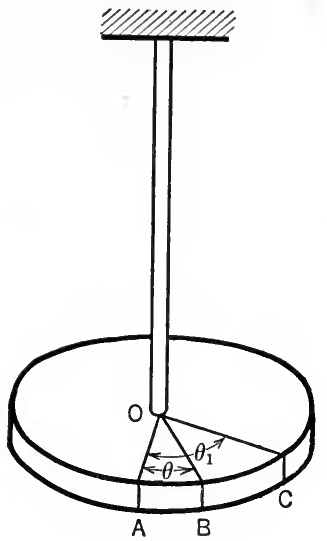

Fig. 381 . the equation of motion is,

Hence,

$$
\Sigma T_{0}=I_{0} \alpha .
$$

But,

$$
-k \theta=I_{0} \alpha \text {. }
$$

Therefore,

$$
\alpha=\frac{d^{2} \theta}{d t^{2}} .
$$

$$
\frac{d^{2} \theta}{d t^{2}}=-\frac{k}{I_{0}} \theta .
$$

The time of an oscillation of the pendulum may now be found by the integration of this equation. Multiplying each side of the equation by the factor $\frac{d \theta}{d t}$, we have, $\frac{d \theta}{d t} \frac{d^{2} \theta}{d t^{2}}=-\frac{k}{I_{0}} \theta \frac{d \theta}{d t}$.

Whence, by integrating,

Now, when

$$
\left(\frac{d \theta}{d t}\right)^{2}=-\frac{k}{I_{0}} \theta^{2}+C_{1}
$$

Therefore,

$$
\theta=\theta_{1}, \frac{d \theta}{d t}=0 \text {. }
$$

Hence,

$$
C_{1}=\frac{k}{I_{0}} \theta_{1}^{2} .
$$

$$
\left(\frac{d \theta}{d t}\right)^{2}=\frac{k}{I_{0}}\left(\theta_{1}^{2}-\theta^{2}\right) .
$$

By a method similar to that used in Art. 156, the detailed steps of which are left to the student, the time of a complete oscillation is found to be

$$
P=2 \pi \sqrt{\frac{I_{0} \theta}{T}},
$$

in which $I_{0}$ is the moment of inertia of the body (disc) with respect to the axis of the wire and $\theta$ is the angular displacement corre- 
sponding to which the wire exerts the torque $T$ on the disc. It will be observed that the period of an oscillation is independent of the initial displacement, that is, of the amplitude of the vibration, assuming that the elastic limit of the wire is not exceeded.

\section{PROBLEMS}

415. The disc of a torsional pendulum is turned through an angle of $10^{\circ}$ by a torque of $1 \mathrm{ft} .-\mathrm{lb}$. When released it is observed to make five complete oscillations per second. What is the moment of inertia of the disc? (In using the formula of Art. 159, the angle $\theta$ must be expressed in radians).

Ans. $I=.00582$ slug-ft. ${ }^{2}$

416. The disc of a torsional pendulum is $1 \mathrm{ft}$. in diameter and $2 \mathrm{in}$. thick. A torque of $1 \mathrm{ft}$.-lb. is required to turn the disc through $8^{\circ}$. Find the period of oscillation, assuming that the disc is made of cast iron, the weight of which is $450 \mathrm{lb}$. per cubic foot.

160. Experimental Determination of Moment of Inertia.As noted in Art. 106, the calculation of the moment of inertia of many bodies is a difficult process and frequently is impossible. The moment of inertia, however, may be found experimentally by allowing the body to oseillate as a compound pendulum and observing the period of oscillation or by allowing it to oscillate either as a torsion pendulum itself or with a given torsion pendulum to which it is attached.

I. By Means of a Compound Pendulum.-The period of a complete oscillation of a compound pendulum, from Art. 157, is

Or,

$$
P=2 \pi \sqrt{\frac{k_{0}^{2}}{g r}} \text {. }
$$

But,

$$
k_{0}^{2}=\frac{P^{2}}{4 \pi^{2}} g \bar{r} .
$$

Hence,

$$
I_{0}=M k_{0}^{2}=\frac{W}{g} k_{0}^{2} .
$$

$$
I_{0}=\frac{M P^{2}}{4 \pi^{2}} g \bar{r}=\frac{W P^{2} \dot{\bar{r}}}{4 \pi^{2}},
$$

in which $I_{0}$ is the moment of inertia of the body with respect to the axis of suspension Now $W$ may be found by weighing the body, $\bar{r}$ by balancing the body over a knife edge, and $P$ by observing the period_of_oscillation. 
After $I_{0}$ is found, the moment of inertia with respect to a parallel axis through the mass-center $(\bar{I})$ may be calculated from the equation,

$$
I_{0}=\bar{I}+M \bar{r}^{2} .
$$

II. By Means of the Torsional Pendulum.-In order to determine the moment of inertia of a body by means of a torsional pendulum, it will be convenient to replace the disc of the pendulum as described in Art. 159 by two discs rigidly connected as shown in Fig. 382. If $I_{0}$ denotes the moment of inertia of the two dises with respect to the axis of the wire, the time of a complete oscillation of the pendulum (denoted here for convenience by $P_{0}$ ) is, by Art. 159,

$$
P_{0}=2 \pi \sqrt{\frac{I_{0} \theta}{T}}=2 \pi \sqrt{\frac{\theta}{T}} \sqrt{I_{0}}=C \sqrt{I_{0}}, \quad . \quad . \quad . .
$$

in which $C$ is a constant. The value of $\frac{\theta}{T}$ is a constant of the wire,

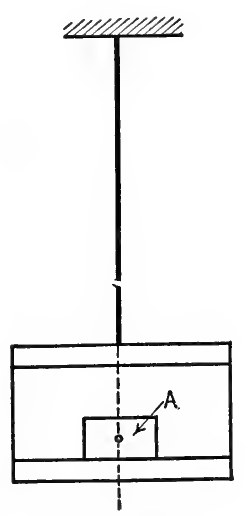

FIG. 382 . and depends on the material of the wire and its diameter. If this value is known (and it is readily found), $I_{0}$ may be obtained from the above equation by observing the period of oscillation of the pendulum. The method of determining the moment of inertia of a body $A$ is as follows: First allow the pendulum to oscillate and observe the period of oscillation, $P_{0}$. Next, place the body $A$ on the lower disc (Fig. 382) so that the center of gravity of $A$ is vertically below the wire. If the moment of inertia of $A$ with respect to the axis of the wire is denoted by $I_{A}$ and the period of oscillation of the pendulum (and $A$ ) is denoted by $P$, we have, by Art. 159,

$$
P=2 \pi \sqrt{\frac{\left(I_{0}+I_{A}\right) \theta}{T}}=C \sqrt{I_{0}+I_{A}} . \quad . \quad .
$$

From (1) and (2) the following equation is obtained:

$$
\frac{P_{0}}{P}=\frac{\sqrt{I_{0}}}{\sqrt{I_{0}+I_{A}}}
$$


That is,

Hence,

$$
I_{0}+I_{A}=\frac{P^{2}}{P_{0}^{2}} I_{0}
$$

$$
I_{A}=I_{0}\left(\frac{P^{2}}{P_{0}^{2}}-1\right)
$$

It may be noted that if the discs are of such form that their moment of inertia may be calculated it is not necessary to know the constant $\frac{\theta}{T}$ of the wire.:

\section{PROBLEMS}

417. The connecting rod of a steam engine weighs $300 \mathrm{lb}$. and the distance of the center of gravity from the crank-pin is found (by balancing) to be 50 in. When suspended from the crank-pin end and allowed to vibrate as a compound pendulum, it is found to make thirty complete oscillations in 75 seconds. Determine the moment of inertia of the rod with respect to the axis of the crank-pin and also with respect to a parallel axis through the center of gravity.

$$
\text { Ans. } I=198 \text { slug-ft. }{ }^{2} \quad \bar{I}=36.5 \text { slug-ft. }{ }^{2}
$$

418. The pendulum (Fig. 383) of a Charpy impact machine, which is used to determine the resistance of materials to impact, weighs $50.5 \mathrm{lb}$. and the distance of the center of gravity from the axis of rotation, as determined by balancing, is found to be $27.33 \mathrm{in}$. When allowed to vibrate about the axis of rotation, the pendulum is observed to make

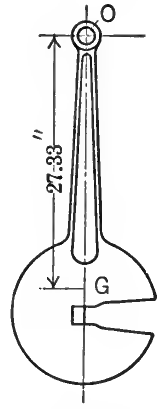

FIG. 383. 35 complete oscillations in 61 sec. Find the moment of inertia of the pendulum with respect to the axis of rotation.

\section{BALANCING}

161. Need for Balancing. - A moving part of a machine, as a rule, has either a reciprocating motion similar to that of the crosshead of a steam engine or a motion of rotation such as that of the crank-shaft of an automobile engine or the motor of a dynamo. In any case, if the moving parts are accelerated, forces must be supplied to produce the accelerations. If the moving parts are not balanced, the forces which act on the moving masses are transmitted to them from the stationary parts of the machine such as the bearings and the machine frame. And, in supplying these accelerating forces, serious trouble may arise such as, vibrations in automobiles, turbines, etc.; defective commutations in elec- 
trical machinery; heavy bearing pressures which cause undue wear; defective work with grinding dises, high-speed drilling machines, etc.; and defective lubrication. It is of great importance, therefore, to properly neutralize or balance these forces in various types of machines.

The moving parts of a machine may be (1) in static or standing balance or (2) in dynamic or running balance. Standing balance exists if the forces which act on the parts, when the parts are not running, are in equilibrium regardless of the positions in which the parts are placed. Dynamic balancing consists in distributing the moving masses or in introducing additional masses so that the inertia forces exerted by the masses of the moving system are in equilibrium amongst themselves, thereby making it unnecessary for the stationary parts of the machine to supply any of the accelerating forces. The complete balancing of a machine, however, is not always practicable or possible.

The method of balancing rotating masses, only, is here discussed. For methods of balancing reciprocating masses and for an excellent discussion of the whole subject of the balancing of engines see Dalby's "Balancing of Engines."

162. Balancing of Rotating Masses.-The inertia forces or kinetic loads due to unbalanced rotating masses may be treated, in general, (1) as a system of centrifugal forces or (2) as a system of centrifugal couples or (3) as a combination of the two.

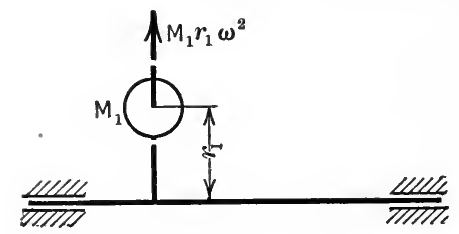

(a)

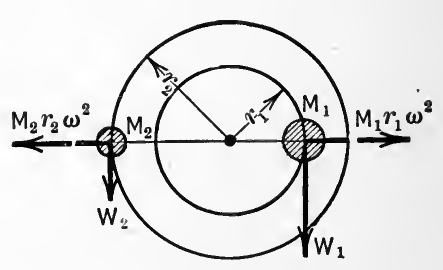

(b)

FIG. 384 .

A Single Rotating Mass. Centrifugal Force.-If a shaft (Fig. $384 a$ ) rotates at an angular velocity $\omega$ and carries a single mass $M_{1}$ the center of gravity of which is at the distance $r_{1}$ from the axis of rotation, the shaft will be subjected to a kinetic load (centrifugal force) of magnitude $M_{1} r_{1} \omega^{2}$. This kinetic load causes the shaft 
to exert forces on the bearings which in turn are transmitted to the machine frame. The reactions at the bearings may be eliminated by balancing the rotating mass. This may be done by the addition of a single mass $M_{2}$ diametrically opposite to $M_{1}$ (Fig. $384 b$ ), the center of gravity of $M_{2}$ being at a distance $r_{2}$ from the axis of rotation, such that,

$$
M_{1} r_{1} \omega^{2}=M_{2} r_{2} \omega^{2} \quad \text { or } \quad \frac{W_{1}}{g} r_{1} \omega^{2}=\frac{W_{2}}{g} r_{2} \omega^{2} .
$$

But, since $\frac{\omega^{2}}{g}$ is a common factor, the conditions for running or dynamic balance may be expressed by the equation

$$
W_{1} r_{1}=W_{2} r_{2} \text {. }
$$

Now, as is evident from Fig. 384(b), this equation expresses the condition for standing balance also. Thus, a shop method of obtaining approximate running balance with a rotating member, in which the material is substantially in a plane of rotation such as a disc, a pulley, or a flywheel, consists in drilling out material on the heavy side or adding material on the light side until standing balance is obtained.

Two Rotating Masses. Centrifugal Couple.-If a shaft carries two rotating masses $M_{1}$ and $M_{2}$ in different planes of rotation but in the same axial plane (Fig. 385) and, further, if the centrifugal forces $M_{1} r_{1} \omega^{2}$ and $M_{2} r_{2} \omega^{2}$ of the two masses are equal, then the shaft is subjected to an unbalanced centrifugal couple which is resisted by an equal couple exerted by the bearings. Or, if the two rotating masses are to be balanced, two additional masses, $M_{3}$ and $M_{4}$, must be introduced in the same axial plane (Fig. 385) such that the centrifugal couple

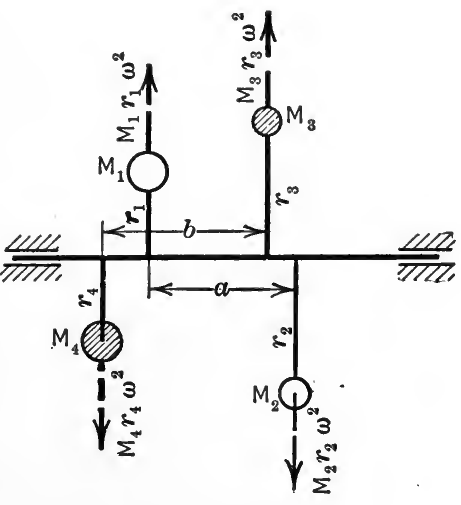

FIG. 385 . $M_{3} r_{3} \omega^{2} b$ or $M_{4} r_{4} \omega^{2} b$ which they set up equals the centrifugal couple of $M_{1}$ and $M_{2}$. Hence,

$$
M_{1} r_{1} \omega^{2} a=M_{3} r_{3} \omega^{2} b,
$$

Or,

$$
M_{2} r_{2} \omega^{2} a=M_{4} r_{4} \omega^{2} b \text {. }
$$


And, as before, omitting the common factor $\frac{\omega^{2}}{g}$ we may write,

$$
W_{1} r_{1} a=W_{3} r_{3} b \text {, etc. }
$$

It will be noted that the shaft when carrying only the two masses $M_{1}$ and $M_{2}$ is in standing balance but not in running balance.

163. Several Masses in a Single Plane of Rotation.-If several masses $M_{1}, M_{2}, M_{3}$, etc., lie in the same transverse plane (Fig. $386 a)$, the shaft is subjected to the centrifugal forces,

$$
M_{1} r_{1} \omega^{2}, M_{2} r_{2} \omega^{2}, M_{3} r_{3} \omega^{2} \text {, etc., }
$$

which form a concurrent system of forces.

The condition that such a force system shall balance is that the force polygon shall close. That is, the vectors representing the forces $M_{1} r_{1} \omega^{2}, M_{2} r_{2} \omega^{2}, M_{3} r_{3} \omega^{2}$, etc., if laid off in succession, each in
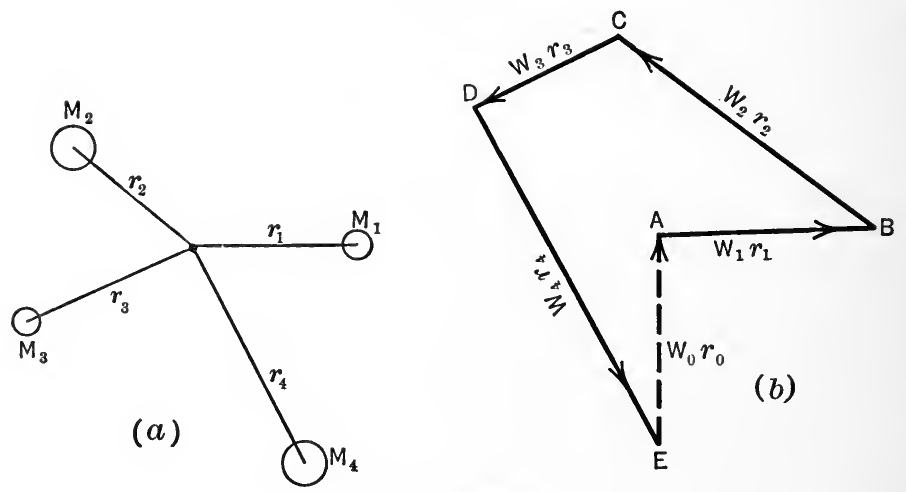

FIG. 386.

its proper direction, shall form a closed polygon. Or, since $M r \omega^{2}$ may be written $\frac{W}{g} r \omega^{2}$ and since $\frac{\omega^{2}}{g}$ is a factor common to the expression for each force, the products $W_{1} r_{1}, W_{2} r_{2}$, etc., may be used instead of the actual forces. Thus, let the four masses as shown in Fig. $386(a)$ be a system of masses which rotate in a transverse plane. Suppose that the products $W_{1} r_{1}, W_{2} r_{2}, W_{3} r_{3}$, and $W_{4} r_{4}$ when laid off as vectors (Fig. 386b) do not form a closed polygon. It is evident then that the four masses are not in running balance. In order to balance the system of masses, a mass, $M_{0}$, must be added at a distance, $r_{0}$, such that the product $W_{0} r_{0}$ is represented both in 
magnitude and direction by the closing side, $E A$, of the vector polygon. By assuming a convenient value for $r_{0}$, a value of $M_{0}$ may be found. The gap, $E A$, may be closed, however, by two or more vectors from which two or more masses may be found that will balance the system.

164. Masses in Different Transverse Planes.-In Fig. 387, let the masses $M_{1}$ and $M_{2}$ be connected with the shaft at $A$ and $B$, respectively, and through some point, $O$, of the shaft let a

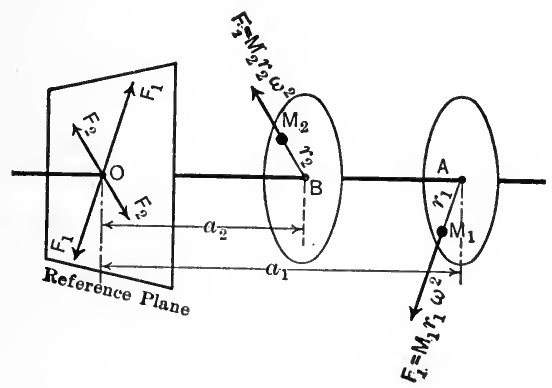

FIG. 387.

transverse plane, called a reference plane, be chosen. The mass $M_{1}$ exerts a kinetic load or centrifugal force, $F_{1}$, on the shaft such that

$$
F_{1}=M_{1} r_{1} \omega^{2}
$$

Now at $O$ introduce two equal and opposite forces each equal and parallel to $F_{1}$. The force $F_{1}$ at $A$ and the equal opposite force at $O$ form a couple, $C_{1}$, the moment of which is

$$
C_{1}=M_{1} r_{1} \omega^{2} a_{1}
$$

Thus the single force $F_{1}$ at $A$ is replaced by an equal parallel force at $O$ and the couple $C_{1}$. In like manner the single force $F_{2}=M_{2} r_{2} \omega^{2}$ at $B$ may be replaced by the equal parallel force $F_{2}$ in the reference plane and a couple $C_{2}=M_{2} r_{2} \omega^{2} a_{2}$.

Thus, the kinetic loads on a shaft exerted by a system of rotating masses may be reduced to a system of concurrent forces 
in an arbitrarily chosen reference plane and a system of couples which lie in different axial planes. The resultant of the system of concurrent forces, if not balanced, is a single force in the reference plane, and the resultant of the system of couples, if not balanced, is a single couple in some axial plane. Hence, in general, the system of kinetic loads, if not balanced, may be reduced to a single force and a couple. The magnitude of the couple will, of course, depend on the position chosen for the reference plane.

The conditions then which must be fulfilled to have a system of rotating masses in equilibrium are,

(1) The resultant of the system of concurrent forces must be zero. That is, the force polygon for the forces in the reference plane must close.

(2) The resultant of the system of couples must be zero. That is, the couple polygon must close.

These conditions may be satisfied by the addition of two rotating masses in different transverse planes. Thus, let the shaft (Fig. 388) carry an unbalanced system of rotating masses, $M_{1}, M_{2}$, and
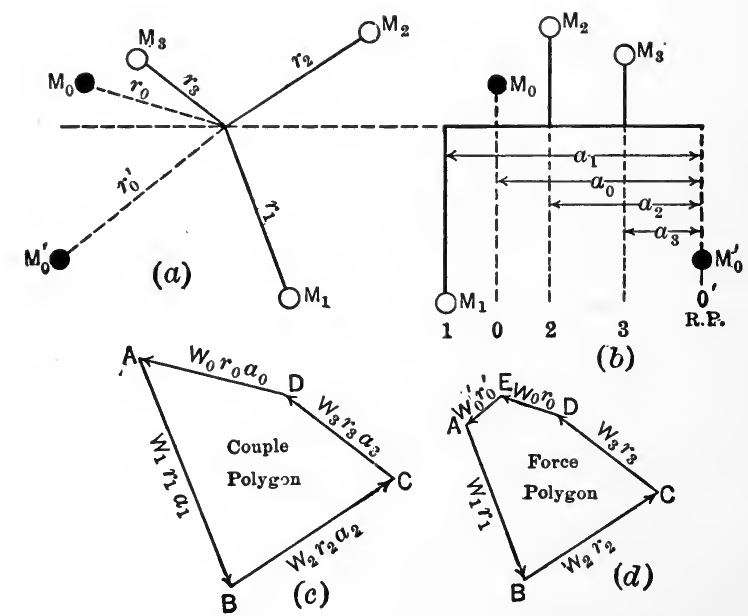

(b)

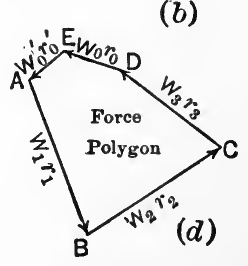

FIG. 388.

$M_{3}$, and let the two balancing masses be denoted by $M_{0}$ and $M^{\prime}{ }_{0}$. Let the transverse planes in which these balancing masses are to lie be chosen arbitrarily but let the plane of one of the masses be chosen 
as the reference plane. The plane of mass $M^{\prime}{ }_{0}$ will here be selected as the reference plane. Let $a_{0}, a_{1}, a_{2}$, and $a_{3}$ denote the respective distances of the masses $M_{0}, M_{1}, M_{2}$ and $M_{3}$ from the reference plane. The couples $W_{1} r_{1} a_{1}, W_{2} r_{2} a_{2}$, etc., may now be calculated (the common factor $\frac{\omega^{2}}{g}$ is omitted in each term for simplicity). The only unknown couple is $W_{0} r_{0} a_{0}$, since the couple $W^{\prime}{ }_{0} r^{\prime}{ }_{0} a_{0}^{\prime}$ is zero due to the fact that the reference plane was chosen as the plane of $M_{0}^{\prime}$, which makes $a_{0}^{\prime}$ equal to zero. By laying off the vectors that represent the known couples as the sides of a polygon, the closing side determines both the magnitude of the unknown couple $W_{0} r_{0} a_{0}$ and the axial plane in which it lies. By assigning any convenient value to the moment arm $a_{0}$, the product $W_{0} r_{0}$ may be found, and by assuming a convenient value for $r_{0}, W_{0}$ may be determined and placed in the plane indicated by the closing vector of the couple polygon. Thus, by the addition of the couple $W_{0} r_{0} a_{0}$ condition (2) is satisfied.

Condition (1) may now be satisfied as follows: The kinetic load due to the added mass $M_{0}$ is replaced by a force in the reference plane and the couple $M_{0} r_{0} a_{0}$ as was done for the kinetic loads due to the original masses. Now, if the products $W_{1} r_{1}, W_{2} r_{2}$, etc. (including $\left.W_{0} r_{0}\right)$ are laid off as the sides of a polygon, the closing side gives the magnitude and the direction of a product $W^{\prime}{ }_{0} r_{0}$ for a body $W^{\prime}{ }_{0}$ which must be added in the reference plane to balance the system of concurrent forces in the reference plane and thereby satisfy condition (1). By choosing a convenient value for $r_{0}^{\prime}$, the mass $M_{0}^{\prime}$ may be determined and the direction of the closing side of the polygon gives the direction of $M^{\prime}{ }_{0}$ from the axis of the shaft.

If the couple polygon is formed by drawing the couple vectors perpendicular to the planes of the couples, as explained in Art. 17, and then is turned through $90^{\circ}$, it will be the same as the polygon formed by drawing the couple vectors according to the following rule: Draw the couple vectors parallel to the respective crank directions; outwards for masses on one side of the reference plane; inwards towards the axis for masses on the opposite side of the reference plane.

The vectors of the force polygon are drawn, of course, from the axis outwards parallel to the radii to the masses. 


\section{ILLUSTRATIVE PROBLEM}

419. Three weights $W_{1}, W_{2}$, and $W_{3}$ (Fig. 389) which revolve in the planes 1,2 , and 3 are to be balanced by the addition of two weights. Plane 1 is chosen as the plane of one of the weights $\left(W_{0}\right)$ and the plane of the other weight $\left(W_{0}^{\prime}\right)$ will arbitrarily be taken $1.4 \mathrm{ft}$. to the right of plane 3 and will be selected as the reference plane. It is required to determine values of $W_{0}$ and $W^{\prime}{ }_{0}$ and the lengths and directions of the corresponding radii for kinetic balance. The accompanying table gives the values of the weights, the lengths and directions of the radii, and the distances from the reference plane.

\begin{tabular}{l|c|c|c|c|c|c}
\hline Plane. & $\begin{array}{c}\text { Weight } \\
W \text { (lb.) }\end{array}$ & $\begin{array}{c}\text { Radius } \\
\text { (in.) }\end{array}$ & Angle $\theta$. & $\begin{array}{c}\text { Distance } \\
\text { from R.P. } \\
a \text { (ft.) }\end{array}$ & $W r$ (lb.-in.) & $\begin{array}{c}W r a \\
\text { (lb.-in.-ft.) }\end{array}$ \\
\hline 1 & 2 & 9 & $270^{\circ}$ & 4.4 & 18 & 79.2 \\
2 & 3 & 8 & $30^{\circ}$ & 2.6 & 24 & 62.4 \\
3 & 3 & 6 & $150^{\circ}$ & 1.4 & 18 & 25.2 \\
0 & $(1.36)$ & $(8)$ & $\ldots \ldots$ & 4.4 & $(10.9)$ & $(48.0)$ \\
$0^{\prime}$ (R.P.) & $(2.28)$ & $(5)$ & $\ldots \ldots$ & 0 & $(11.4)$ &. \\
\hline
\end{tabular}

Solution.-From the given data, the values of the products $W r$ and $W r a$ for the known weights are calculated and entered in the last two columns of the above table. The couple polygon is then drawn as shown in Fig. 389(c). $A B$ is laid off in the direction of $W_{1}$ outwards from the shaft and its length represents to scale the value of the product $W_{1} r_{1} a_{1}$ which is 79.2. Next $B C$ is laid off in the direction of $W_{2}$ such that its length represents to the same scale $W_{2} r_{2} a_{2}(62.4)$. Similarly $C D$ is laid off to represent $W_{3} r_{3} a_{3}(25.2)$. The closing side $D A$ of the polygon represents the product $W_{0} r_{0} a_{0}$ due to the balancing weight $W_{0}$ in plane 1 ( or $O$ ). This product is found by measuring to be 48.0. Hence,

$$
W_{0} r_{0}=\frac{48.0}{4.4}=10.9 \mathrm{lb} \cdot-\text { in }
$$

This product is now entered in the column of the above table with the other $W r$-products. The addition of the weight $W_{0}$ in the plane 1 at the distance $r_{0}$ reduces the resultant couple to zero. There are left, however, the forces in the reference plane, including the force corresponding to the product $W_{0} r_{0}$ just found, and these forces will, in general, not be balanced. Now if the vectors corresponding to these $W r$-products are laid off in order as in Fig. $389(d)$, the closing vector $E A$ represents the product $W^{\prime}{ }_{0} r^{\prime}{ }_{0}$ which by measurement is found to be 11.4. If the values of $W_{0} r_{0}$ and $W^{\prime}{ }_{0} r^{\prime}{ }_{0}$ are divided by assumed values of $r_{0}$ and $r_{0}^{\prime}$ ( $8 \mathrm{in}$. and $5 \mathrm{in}$. respectively) we obtain the values, $W_{0}=1.36$ and $W_{0}^{\prime}=2.28$. Hence if weights of $1.36 \mathrm{lb}$. and $2.28 \mathrm{lb}$. are placed 
in planes $O$ and $O^{\prime}$ at radial distances 8 in. and 5 in., respectively, as indicated in Fig. 389, the system will be in kinetic balance.

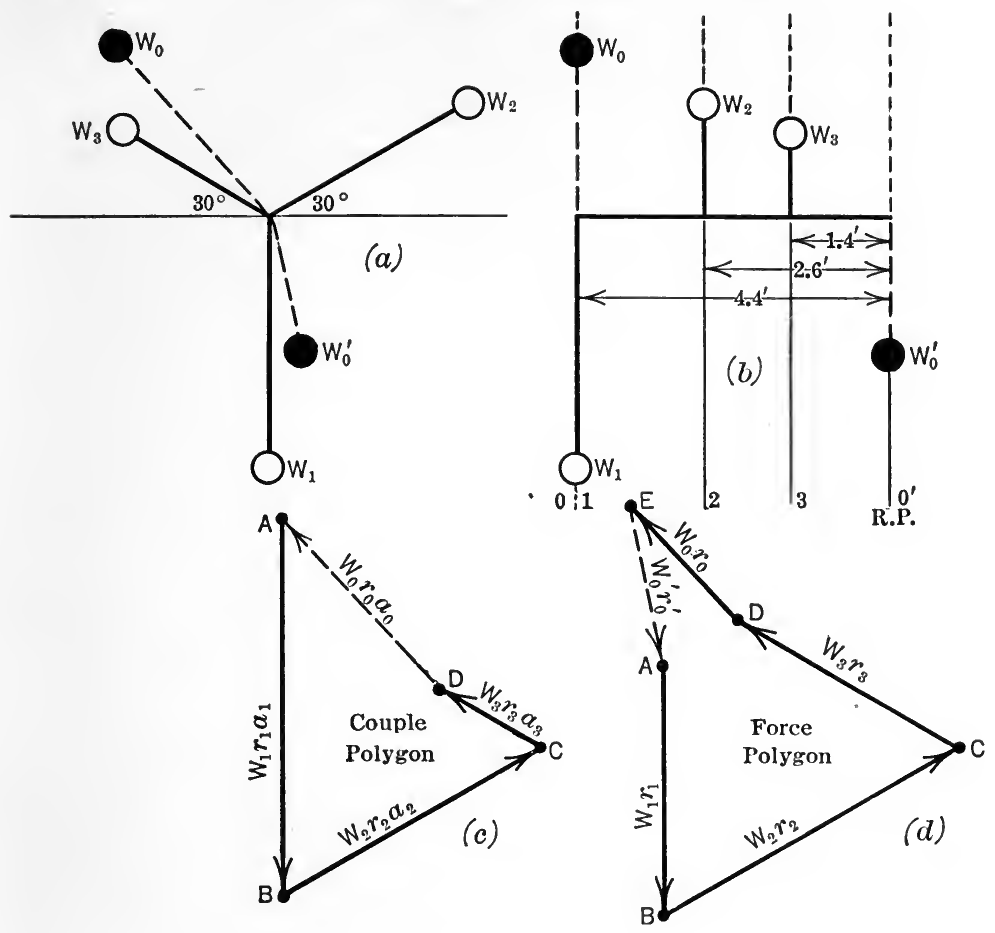

FIG. 389 .

\section{PROBLEMS}

420. Five bodies are attached to a disc which revolves with constant angular velocity. In the following table are given the weights of the bodies, the angles which the radii from the mass-centers to the axis of rotation make with the $x$-axis, and the lengths of the radii to the mass-centers. The angles, $\theta$, are measured counterclockwise from the $x$-axis.

\begin{tabular}{|r|r|r|}
\hline$W$ & $\theta$ & $r$ \\
\hline $5 \mathrm{lb}$. & $45^{\circ}$ & $18 \mathrm{in}$. \\
\hline $6 \mathrm{lb}$. & $120^{\circ}$ & $15 \mathrm{in}$. \\
$10 \mathrm{lb}$. & $150^{\circ}$ & $12 \mathrm{in.}$ \\
\hline $4 \mathrm{lb}$. & $240^{\circ}$ & $12 \mathrm{in}$. \\
$6 \mathrm{lb}$. & $315^{\circ}$ & $18 \mathrm{in}$. \\
\hline
\end{tabular}


Determine sufficient data for kinetic balance of the system of bodies:

(a) By the addition of a single weight placed $2 \mathrm{ft}$. from the axis of rotation.

(b) By the addition of two weights, one of $2 \mathrm{lb}$. placed on the $y$-axis, and the other of $2.25 \mathrm{lb}$. placed $2 \mathrm{ft}$. from the axis of rotation.

421. Four bodies are attached to a revolving shaft in different transverse planes. The weights and positions of the bodies are indicated in the following table, the reference plane being the transverse plane in which the mass-center of the 6-lb. body $\left(W_{3}\right)$ lies.

\begin{tabular}{|c|c|c|c|}
\hline$W$ & $\theta$ & $r$ & $a$ \\
\hline $5 \mathrm{lb}$. & $30^{\circ}$ & $2 \mathrm{ft}$. & $-1 \mathrm{ft}$. \\
$4 \mathrm{lb}$. & $45^{\circ}$ & $1 \frac{1}{2} \mathrm{ft.}$ & $2 \mathrm{ft}$. \\
$6 \mathrm{lb}$. & $150^{\circ}$ & $1 \mathrm{ft}$. & $0 \mathrm{ft}$. \\
$4 \mathrm{lb}$. & $240^{\circ}$ & $2 \mathrm{ft.}$ & $\frac{1}{2} \mathrm{ft}$. \\
\hline
\end{tabular}

The given masses are to be balanced by two masses, one mass to be placed in plane 4 at a radial distance of $1 \frac{1}{2} \mathrm{ft}$., and the other to be placed in the reference plane at a radial distance of $2 \mathrm{ft}$. Find the weights and values of $\theta$ for the two masses.

Ans. $W_{0}=2.93 \mathrm{lb}$.; $W^{\prime}{ }_{0}=4.85 \mathrm{lb}$.

422. The crank shaft of a gas engine carries two flywheels $A$ and $B$, the planes of revolution of which are $3.5 \mathrm{ft}$. apart. The plane of revolution of the crank is between the flywheels and $1 \mathrm{ft} .7 \mathrm{in}$. from the plane of $A$. The crank arms and pin are equivalent to a weight of $108 \mathrm{lb}$. at a radial distance of $10 \mathrm{in}$. from the crank shaft and in the plane of revolution of the crank. What weights placed at a radial distance of $2 \mathrm{ft}$., one in each flywheel, will balance the crank? Solve algebraically. Ans. $W_{A}=24.6 \mathrm{lb}$; $W_{B}=20.4 \mathrm{lb}$.

\section{The Gyroscope}

165. The Problem Defined.-Gyroscopic motion occurs whenever a body rotates about an axis in the body as the axis (and body) is turned about a second axis. Thus, the wheels of a locomotive when rounding a curve, or the screw propeller of a ship when the ship is pitching in a rough sea, are given gyroscopic motion. The forces that act on the body in giving it gyroscopic motion may be of considerable importance since under certain conditions they are very undesirable as, for example, the forces exerted on the propeller of an aeroplane when making a sharp turn. On the other hand the gyroscope is sometimes used to introduce desirable forces as, for example, in reducing the rolling of ships. 
The gyroscope here considered is a body symmetrical with respect to each of three rectangular axes. The body rotates or spins with constant angular velocity about one of the axes and at the same time turns about one of the other axes with constant angular velocity. Thus, in Fig. 390, let $A B C D$ represent a circular disc which is symmetrical with respect to the three axes $x, y$, and $z$. Let the disc rotate or spin with a high constant angular velocity $\omega$

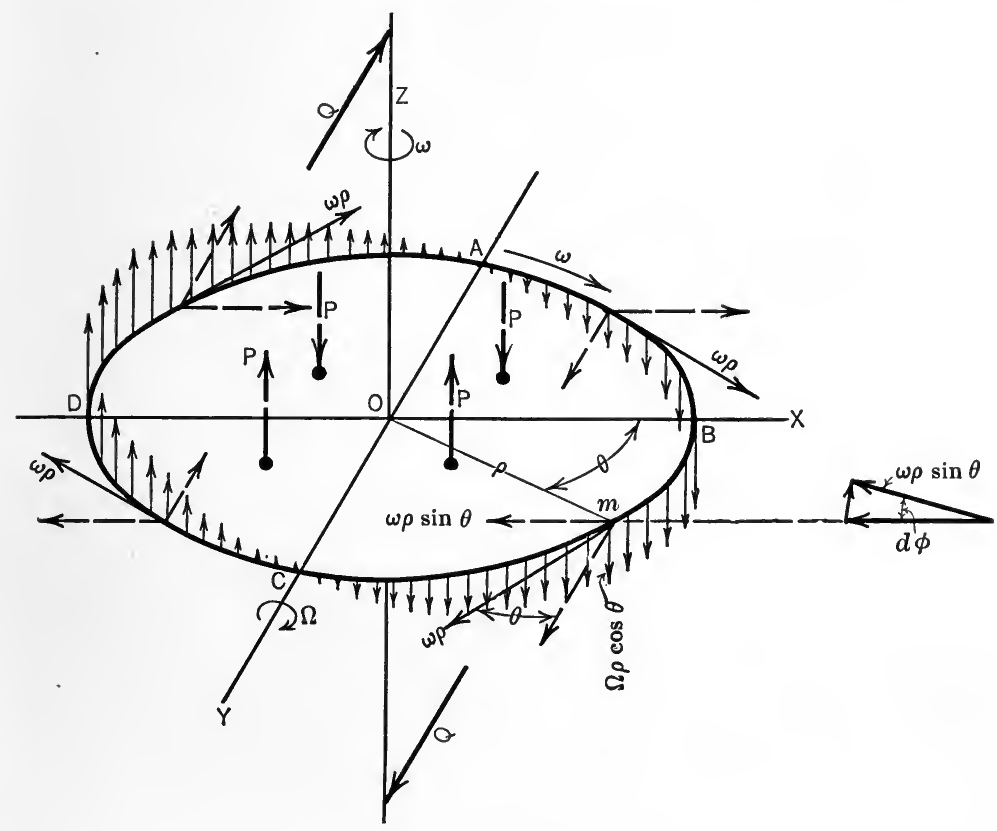

FIG. 390 .

about the $z$-axis and at the same time let the disc turn about the $y$-axis with the constant angular velocity $\Omega$. The problem which arises is that of determining the forces which must act on the disc or its axles (axle reactions) in order to maintain this gyroscopic motion.

166. Analysis of Forces in the Gyroscope.-Due to the two rotations imposed on the disc, a particle, $m$, at the distance $\rho$ (assumed at the circumference of the disc for convenience) has at any instant, two velocities: (1) a constant velocity $\omega \rho$ in the plane of the disc due to the rotation about the $z$-axis with angular 
velocity $\omega$, and (2) the velocity $\Omega \rho \cos \theta$ perpendicular to the disc (parallel to the $z$-axis) due to the rotation about the $y$-axis with angular velocity $\Omega$.

The changes that occur in each of these two velocities of any particle due to each of the rotations which are given to the disc will first be investigated, since the effective force for any particle must have components corresponding to the change in the magnitude and to the change in the direction of each of these two velocities (Theorems I and II, Art. 118, and Newton's second law, Art. 140). And, the effective forces for the particles must be found in order to determine the external forces required to maintain the gyroscopic motion. (Read Art. 144 for the general method of procedure, keeping in mind that gyroscopic motion is not uniplanar motion.) It will be found, however, that external forces come into action as the result of only two of the changes which occur in the two velocities; namely, the change in $\Omega \rho \cos \theta$ due to the rotation about the $z$-axis and the change in $\omega \rho$ due to the rotation about the $y$-axis.

Changes in the Velocities Due to Rotation about Z-axis

(1) Change in $\omega \rho$.- Since $\omega$ is constant, $\omega \rho$ changes in direction only. The resulting acceleration $\left(\omega^{2} \rho\right)$ and the corresponding effective force for the particle, is directed towards the center of rotation (towards the $z$-axis). And, since the body is symmetrical, these effective forces for all the particles form a balanced system and hence, according to D'Alembert's principle, no external forces act on the body by reason of this change in the velocity of the particles.

(2) Change in $\Omega \rho \cos \theta$.- - In one revolution of the disc about the $z$-axis, this component of the velocity changes, as follows: It gradually increases in magnitude downwards from zero at $A$ to a maximum at $B$; it then decreases gradually to zero at $C$; then increases in the opposite direction to a maximum at $D$; and finally decreases to zero again at $A$. The acceleration, $a_{1}$, at any instant then is (Theorem I, Art. 118),

$$
a_{1}=\frac{d(\Omega \rho \cos \theta)}{d t}=-\Omega \rho \sin \theta \frac{d \theta}{d t}=-\Omega \rho \sin \theta \omega=-\omega \Omega y,
$$

in which $y$ is the distance of the particle from the $x$-axis, and the 
minus sign indicates that the sense of the acceleration is opposite to that of the velocity for the position of the particle as shown in Fig. 390. Hence, for the position of the particle shown, the direction of $\omega \Omega y$ is upwards. The effective force, correspcnding to this acceleration, for any particle in the quadrants $O B C$ and $O C D$, is an upward force perpendicular to the plane of the disc. And, in the quadrants $O D A$ and $O A B$, it is a downward force. The resultant of the effective forces for the four quadrants, then, may be represented by the forces $P$ (Fig. 390). These forces form two couples which have moments with respect to only one of the rectangular axes; namely, the $x$-axis. Further, according to D'Alembert's principle, the effective forces require that external forces act on the disc such that the resultant of the external forces is equivalent to that of the effective forces. Hence, an external couple must act on the disc (or its axles) as indicated by the forces $Q$ (Fig. 390).

\section{Changes in Velocities Due to Rotation about Y-axis}

(3). Change in $\omega \rho$.- - Let the velocity $\omega \rho$ be resolved into two components; one parallel and one perpendicular, respectively, to the $y$-axis (Fig. 390). The component parallel to the $y$-axis undergoes no changes due to the rotation about the $y$-axis; but the component $\omega \rho \sin \theta$, which is perpendicular to the $y$-axis, changes in direction due to the rotation with angular velocity $\Omega$ about the $y$-axis. And, according to Theorem II of Art. 118, the acceleration $a_{2}$ corresponding to this change in velocity is the product of the magnitude of the velocity and its angular velocity of turning, that is,

$$
a_{2}=\omega \rho \sin \theta \cdot \Omega=\omega \Omega y,
$$

and it is directed upwards perpendicular to the plane of the disc. This acceleration, therefore, is equal to $a_{1}$ (see above) and has the same direction and sense as that of $a_{1}$ for all positions of the particle, as will be observed from a study of Fig. 390. Therefore, external forces which act on the disc as a consequence of the changes in $\omega \rho \sin \theta$ due to the rotation of the disc about the $y$-axis must constitute a couple exactly the same as that found under (2).

(4) Change in $\Omega \rho \cos \theta$. - This velocity changes in direction, only, due to the rotation about the $y$-axis with constant angular velocity $\Omega$. The resulting acceleration, $\Omega^{2} \times \rho \cos \theta$ or $\Omega^{2} x$, is 
directed towards the center, on the $y$-axis, about which the particle is rotating at the instant. The corresponding effective forces for the particles of the whole disc, therefore, form a parallel system in the plane of the disc. But, since the disc is symmetrical with respect to the $y$-axis, this effective force system is balanced and hence no external forces act on the disc as a consequence of the changes caused in this velocity by the rotation about the $y$-axis.

It will be noted, therefore, that if the disc is rotated about the $z$-axis and at the same time is turned about the $y$-axis, it will rotate about the $x$-axis unless an external couple acts on the disc to prevent the rotation. This external couple is called the gyroscopic couple.

A simple experiment for demonstrating the existence of the gyroscopic couple may be made by holding a bicycle wheel (dismounted from the frame) with one hand on either end of the projecting (horizontal) axle. If the wheel is spinning in the vertical plane about the axle which is held in the hands, any attempt to turn the axle (and hands) in the horizontal plane will cause the wheel (and hands) to turn about a horizontal axis perpendicular to the axis of the wheel unless the hands exert a couple to prevent this rotation.

167. The Moment of the Gyroscopic Couple.-In the preceding article it was shown that the only acceleration of a particle of the dise which requires the action of external forces on the disc is

$$
\begin{aligned}
a & =a_{1}+a_{2}=\omega \Omega y+\omega \Omega y \\
& =2 \omega \Omega y,
\end{aligned}
$$

and that it is directed perpendicular to the plane of the disc; upwards in the two quadrants $B C D$, and downwards in the two quadrants $D A B$.

The force required to produce this acceleration (effective force) of the particle of mass $m$ is,

$$
F=m a=m \times 2 \omega \Omega y,
$$

the direction of which agrees with that of $a$.

The moment of this effective force for the particle, about the $x$-axis is,

$$
F \times y=2 m \omega \Omega y^{2},
$$


and the sum of the moments of the effective forces for all the particles of the disc is,

\section{$\Sigma 2 m \omega \Omega y^{2}$.}

But since $\omega$ and $\Omega$ are constant this may be written,

$$
2 \omega \Omega \Sigma m y^{2}=2 I_{x} \omega \Omega
$$

in which $I_{x}$ is the moment of inertia of the disc with respect to the $x$-axis.

Now the sum of the moments of the effective forces is equal to the moment of their resultant, but the resultant of the effective forces is a couple, as shown in the preceding article. Further, this resultant couple is equal to the external or gyroscopic couple. Therefore, the moment, $C$, of the gyroscopic couple is

$$
C=2 I_{x} \omega \Omega
$$

But, since the disc is symmetrical with respect to the $x$ - and $y$-axes, $I_{x}$ is equal to $I_{y}$. Hence, by making use of the equation of Art. 89, $2 I_{x}$ may be replaced by the moment of inertia of the disc with respect to the axis of spin (z-axis). Therefore, the moment of the gyroscopic couple is,

$$
C=I \omega \Omega,
$$

in which $I$ is the moment of inertia of the dise with respect to the axis of spin.

The following conclusion then may be drawn: If a body is symmetrical with respect to each of two rectangular axes $(x$ and $y$ ) and rotates or spins with a constant angular velocity $\omega$ about a third axis perpendicular to each of the two axes (the $z$-axis or axis of spin), a couple having a moment about one of the two axes (the $x$-axis) is required to maintain an angular velocity, $\Omega$, about the other of the two axes (the $y$-axis); the moment of the couple is equal to the product of (1) the moment of inertia, $I$, of the body with respect to the axis of spin (z-axis), (2) the angular velocity, $\omega$, of spin, and (3) the angular velocity, $\Omega$, about the $y$-axis.

The angular velocity $\Omega$ which is maintained by the couple is called the velocity of precession and the corresponding axis (y-axis) is called the axis of precession. The axis about which the 
couple $I \omega \Omega$ tends to rotate the disc ( $x$-axis) is called the torque axis. Hence the dise when spinning about the $z$-axis with angular velocity $\omega$ is said to precess about the $y$-axis when acted on by a couple having a moment of $I \omega \Omega$ about the $x$-axis.

By referring to Fig. 390, it will be seen that the sense of rotation about the axis of precession ( $y$-axis) is in accordance with the folowing rule:

The sense of precession is such as to turn the axis of spin toward the torque axis, that is, the axis of spin tends to become coincident with the torque axis.

In the interpretation of this rule the torque axis must be regarded as that part or end of the $x$-axis which, considered as a vector drawn outward from the origin, represents the moment or torque of the couple $I \omega \Omega$, and the axis of spin must be regarded as that part or end of the $z$-axis which, considered as a vector drawn outward from the origin, represents the angular velocity $\omega$ about the $z$-axis.

Thus, if a disc of weight $W$ (Fig. 391) is given an angular velocity $\omega$ about the $z$-axis and

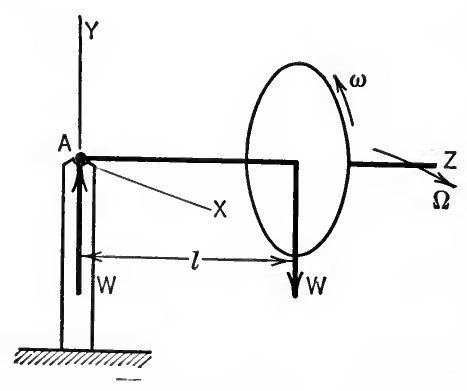

Fig. 391. then one end of the axis is placed on the vertical post at $A$, the couple having a moment $W l$ will cause the disc (and $z$-axis) to rotate with angular velocity $\Omega$ about the axis of the post ( $y$-axis) such that

Or,

$$
W l=I \omega \Omega .
$$$$
\Omega=\frac{W l}{\frac{W}{g} k^{2} \omega}=\frac{g l}{k^{2} \omega},
$$

in which $k$ is the radius of gyration of the disc with respect to the axis of spin (z-axis). Further, if the sense of $\omega$ be as represented in Fig. 391, the sense of precession about the $y$-axis will be clockwise as viewed from the positive end of the $y$-axis. For a further discussion of the gyroscopic couple see Art. 221. 


\section{ILLUSTRATIVE PROBLEM}

423. The flywheel of an engine on a ship weighs $6000 \mathrm{lb}$. and has a radius of gyration of $3.75 \mathrm{ft}$. It is mounted on a horizontal axle which is parallel to the longitudinal axis of the ship, and has a speed of 400 r.p.m. clockwise when viewed from the rear. Find the gyroscopic couple when the ship is turning to the left with an angular velocity of $0.1 \mathrm{rad}$. per sec. What are the axle reactions if the distance between the centers of bearings is $4 \mathrm{ft}$.?

Solution.-The moment of inertia of the flywheel about the axis of spin is,

And,

$$
I=\frac{6000}{32.2} \times(3.75)^{2}=2620 \text { slug-ft. }{ }^{2}
$$

$$
\omega=\frac{400 \times 2 \pi}{60}=41.9 \mathrm{rad} . / \mathrm{sec}
$$

Hence the gyroscopic couple is,

$$
I \omega \Omega=2620 \times 41.9 \times 0.1=10,980 \text { lb.-ft } .
$$

In accordance with the rule stated in Art. 167, the vector representing this couple is perpendicular to the axis of the ship and is directed towards the right. The forces constituting the gyroscopic couple are the axle reactions and hence the reaction at the forward bearing is downwards and that at the rear bearing is upwards. Since the distance between centers of bearings is $4 \mathrm{ft}$. the magnitude of each of these reactions is $10,980 \div 4=2745 \mathrm{lb}$. The effect of the gyroscopic motion, then, is to increase the reaction at the rear bearing and to decrease it at the forward bearing. The reaction at each bearing due to the weight of the flywheel is $3000 \mathrm{lb}$. Hence the resultant reaction at the rear bearing is $3000+2745=5745 \mathrm{lb}$. and that at the forward bearing is $3000-2745=255 \mathrm{lb}$.

\section{PROBLEMS}

424. A disc $4 \mathrm{ft}$. in diameter (Fig. 392) rolls on a circular track having a radius of $10 \mathrm{ft}$. The center of the disc has a velocity of $20 \mathrm{ft}$. per sec. The

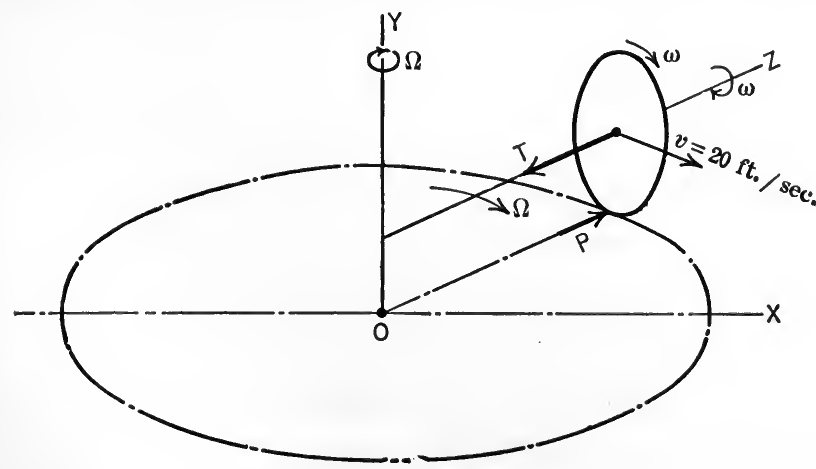

Fig. 392. 
disc is attached to the central axis $O Y$ by means of a rod which is collinear with the $z$-axis about which the disc turns. The disc has a flange similar to that on a car wheel. If the weight of the disc is $450 \mathrm{lb}$. find the tension, $T$, in the rod and the pressure, $P$, of the track against the flange of the wheel.

425. A circular dise is mounted on a horizontal axle which is free to rotate about a vertical axis as shown in Fig. 391, the distance from the center of the disc to the vertical axis being $2 \mathrm{ft}$. The radius of the disc is $6 \mathrm{in}$. and its weight is $10 \mathrm{lb}$. If the disc rotates about the horizontal axle with a speed of 300 r.p.m., with what velocity will it rotate about the vertical axis?

$$
\text { Ans. } \Omega=157 \text { r.p.m. }
$$

426. The propellor of an aeroplane rotates clockwise when viewed from the rear. If the aeroplane turns to the right when moving in a horizontal plane, what will be the effect of the gyroscopic couple?

427. The flywheel of an automobile engine is mounted on a horizontal axle parallel to the longitudinal axis of the automobile. The flywheel rotates counter-clockwise when viewed from the rear. What will be the effect of the gyroscopic couple on $(a)$ the axle reactions of the flywheel, $(b)$ the pressures of the wheels on the road?

\section{Governors}

168. The Action of Governors.-The governor of a steam engine, hydraulic turbine, gas engine, or other motor, automatically regulates the supply of the steam, water, gas, or other fluid, so as to keep the driving force exerted by the working fluid constantly adjusted to the resistance to be overcome. The governor partakes of the motion of the motor so that an increase in the speed of the motor due to a decrease in the load, causes a corresponding increase in the speed of the moving parts of the governor which, in turn, causes, by means of a suitable mechanism, either a decrease in the pressure of the fluid or a decrease in the quantity of fluid delivered to the motor.

The forces which cause the adjustment of the controlling valve and which are brought into play by the change of motion of the governor parts, depend in the main upon (1) the actual change in the speed of the moving parts or (2) the rate of change of the speed of the moving parts. Governors in which the governing action depends mainly upon the actual change of speed of the governor parts are called centrifugal governors and may be either pendulum or shaft governors. Governors in which the governing action depends mainly upon the rate of change of speed of the moving parts are called inertia governors and are shaft governors. 
A brief analysis of the forces brought into play by a change in the motion of each of these types of governors will here be made.

169. The Conical Pendulum.In Fig. 393( $a)$ a ball of mass $M$ and weight $W$ is held at the end of an arm $A B$ and the ball and arm are caused to rotate about an axle $A O$ with velocity $\omega$. The acceleration of the center of the ball is directed towards, $O$, the center of its circular path and its magnitude is $r \omega^{2}$. Hence, the force, $R$, required to produce this acceleration of the ball is,

$$
R=M r \dot{\omega}^{2}=\frac{W}{g} r \omega^{2} .
$$

Now, the accelerating (effective) force $R$ must be the resultant of

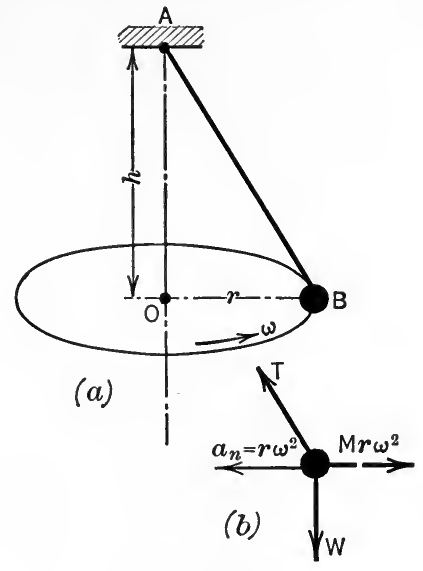

Fig. 393. the external forces which act on the ball. These external forces are the weight, $W$, and the tension, $T$, in the arm, as shown in Fig. $393(b)$. If a force which is equal but opposed to $R$ (the inertia force) is assumed to act with $W$ and $T$ as shown in Fig. 393(b), the three forces will be in equilibrium and hence the sum of their moments with respect to the point $A$ is equal to zero. Thus,

Therefore,

$$
W r=\frac{W}{g} r \omega^{2} \times h .
$$

$$
h=\frac{g}{\omega^{2}},
$$

in which $\omega$ is expressed in radians per second and $g$ is equal to 32.2 ft. per sec. ${ }^{2}$ If the number of revolutions per minute (r.p.m.) is denoted by $n$, then $\omega=\frac{2 \pi n}{60}$. Therefore, $h$, may be expressed (in inches) by the equation,

$$
h=\frac{35200}{n^{2}} \cdot \text {. . . . . . . . }
$$

This equation shows that the height $h$ of the cone depends only on the speed of rotation and not upon the weight of the ball nor the length of the arm $A B$. Now, in the pendulum governor, the governing action depends upon the manner in which $h$ varies with 
$\omega$. The accompanying table is obtained from equation (1). It will be noted that at low speeds a small change in speed of the ball

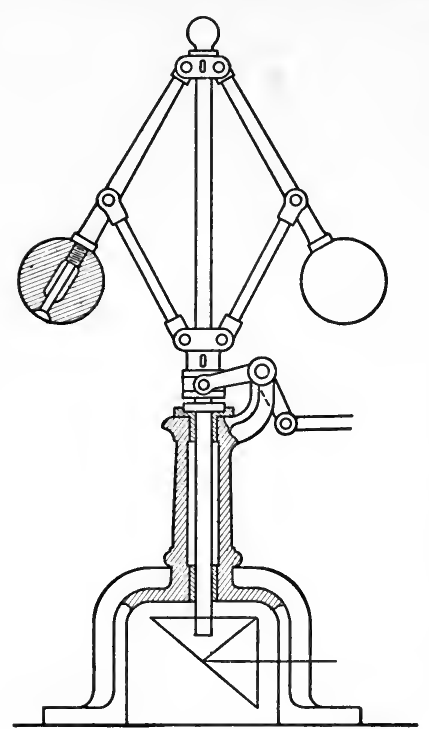

Fig. 394.

\begin{tabular}{|c|c|}
\hline$n$ (in r.p.m.) & $h$ (in inches). \\
\cline { 2 - 2 } 20 & 88.0 \\
40 & 22.0 \\
60 & 9.8 \\
80 & 5.5 \\
100 & 3.5 \\
150 & 1.56 \\
200 & .88 \\
300 & .39 \\
\hline
\end{tabular}

causes a large change in the height $h$ while at high speed a large change in speed causes only a small change in $h$. If the conical pendulum is used as a governor as in the simple Watt governor (Fig. 394) it may be made sensitive to small changes in speed

either by making $h$ large or by causing the governor to rotate slowly by gearing it down. However, a decrease in the speed of the governor results in a decrease in the energy available to overcome the resistances of the valve which is operated by the governor. In order to make the governor sensitive to small changes in speed without seriously reducing its available energy it may be loaded.

170. The Loaded Governor. -The height $h$ of the conical

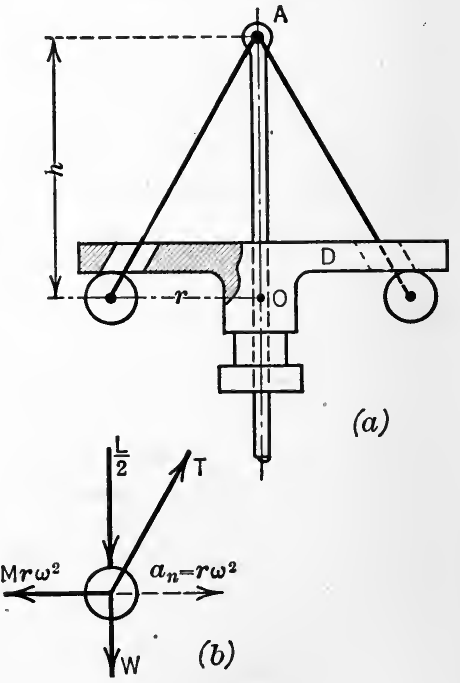

FIg. 395. pendulum may be increased by loading the balls as shown in Fig. $395(a)$. The disc, $D$, which rests on the balls (or cylinders) furnishes, by its weight, a vertical external force without influencing 
the inertia force of the ball or eylinder. If $L$ denotes the weight of the disc (load) then $\frac{L}{2}$ is the load on each ball and the external forces $W, \frac{L}{2}$, and $T$ which act on the ball form, with the reversed effective (inertia) force $M r \omega^{2}$, a system in equilibrium as shown in Fig. 395(b). The sum of their moments, therefore, about the point $A$ is equal to zero. Thus,

$$
\left(W+\frac{L}{2}\right) r=M r \omega^{2} \times h,
$$

and by expressing $\omega$ in revolutions per minute, $h$ is expressed in inches by the equation,

$$
h=\frac{W+\frac{L}{2}}{W} \times \frac{35200}{n^{2}} .
$$

This equation shows that the addition of the load to the balls of the conical pendulum increases the height $h$ in the ratio of $1+\frac{L}{2 W}$ to 1 . For example, if $W=5 \mathrm{lb}$. and $L=30 \mathrm{lb}$. then,

$$
1+\frac{L}{2 W}=1+\frac{30}{2 \times 5}=4 .
$$

Thus, the height $h$ of the simple conical pendulum corresponding to a speed of 200 r.p.m. is $0.88 \mathrm{in}$. and when loaded its height becomes $4 \times 0.88=3.52$ in.

For high rotative speeds, loading must be resorted to in order to increase the change of height for a given change in speed, that is, in order to increase the sensitiveness of the governor.

171. The Porter Governor.-In Fig. 396(a) is shown the Porter loaded governor, in which the load on the conical pendulum is suspended from additional links which are attached to the balls. With this arrangement the load is twice as effective as in the loaded governor discussed in the preceding article, since, for a given rise of the balls, the load rises twice the distance that the balls rise. If, therefore, $L$ denotes the load, the expression for $h$ (in inches) becomes,

$$
h=\frac{W+L}{W} \times \frac{35200}{n^{2}} .
$$


Thus, in the above example, for the Porter governor, the height becomes

$$
\frac{W+L}{W} \times .88=\frac{5+30}{5} \times 0.88=6.16 \text { in. }
$$

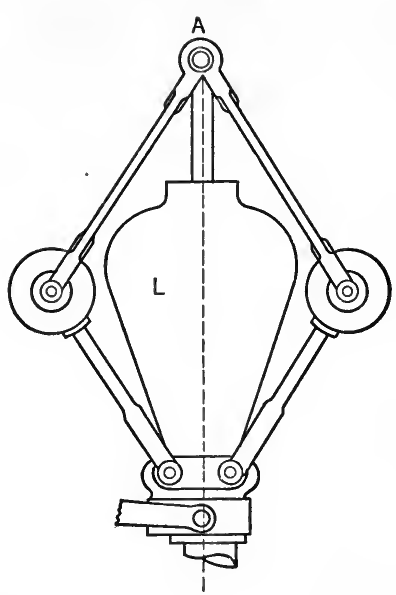

(a)

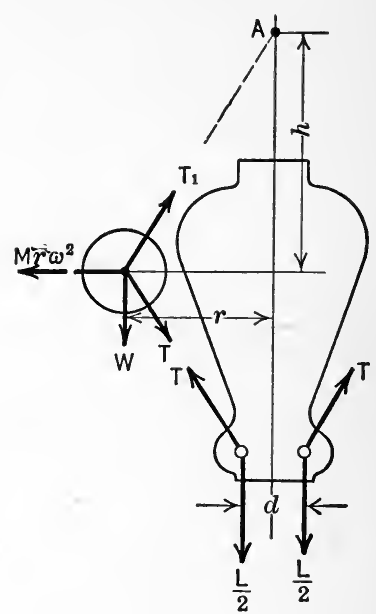

(b)

The student should derive the above equation for $h$ from the analysis of the forces in a manner similar to that used in the preceding two articles. Free-body diagrams

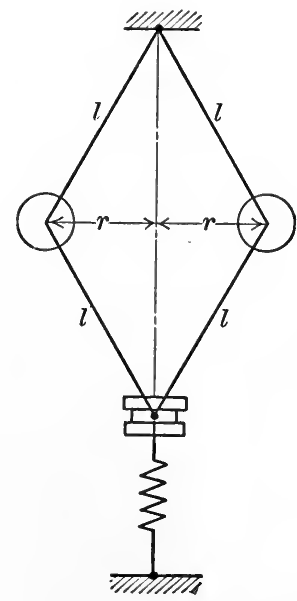

FIG. 397
FIG. 396. for the revolving ball and load are shown in Fig. 396(b).

\section{PROBLEM}

428. A spring-loaded governor is shown in Fig. 397. Let $W$ be the weight (lb.) of each ball; $r$ (ft.) the radius of the path of the balls; $l$ (ft.) the length of each of the four arms; $\omega$ the angular velocity in radians per second. When the radius, $r$, is zero, the tension in the spring is $T \mathrm{lb}$. and the force required to elongate the spring a unit length is $Q \mathrm{lb}$. Show that, if the weight of the balls be neglected,

$$
\omega^{2}=\frac{g\left[T+2 Q\left(l-\sqrt{l^{2}-r^{2}}\right)\right]}{W \sqrt{l^{2}-r^{2}}} .
$$

If $W=3 \mathrm{lb} ., l=1 \mathrm{ft}$., and the balls revolve at 26 
rad. per sec. when $r=3$ in., find the tension in the spring, assuming the modulus of the spring to be $58 \mathrm{lb}$. per in.

Ans. $T=16.8 \mathrm{lb}$.

172. The Centrifugal Shaft Governor.-In the pendulum governors considered above, the governing action is dependent upon the centrifugal force (inertia force) of a rotating mass and the same is true for some types of shaft governors. Thus, let a body of mass $M$ be pivoted to the arm of a flywheel as shown in Fig. 398. Let the distance of the center of the mass, $G$, from the shaft center, $S$, be denoted by $R$ and let the wheel rotate with an angular velocity $\omega$. The centrifugal or inertia force, then, of the mass $M$ is $M r \omega^{2}$. As long as $\omega$ remains constant this force has a definite value.

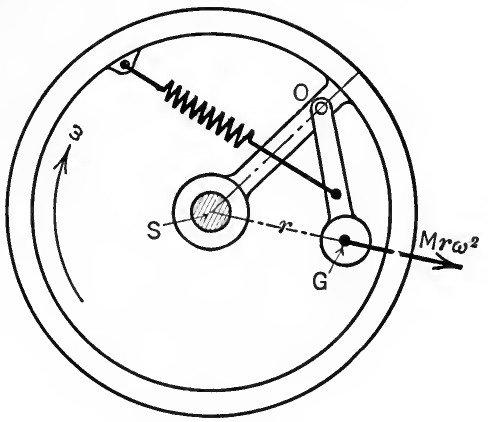

FIG. 398. To hold it in equilibrium a spring is employed. The moment of the spring tension about the point $O$ must therefore be equal to the moment of $M r \omega^{2}$ about the same point (the weight of the body is small in comparison with $M r \omega^{2}$ and hence its moment is neglected). If now the angular velocity of the wheel increases to $\omega^{\prime}$ due to a decrease in the load on the engine, assuming the value of $r$ to remain constant, the centrifugal force increases to $M r \omega^{2}$ and the excess force $\operatorname{Mr}\left(\omega^{2}-\omega^{2}\right)$ causes the mass to move outwards which in turn changes the time of cut off of the valve and adjusts the

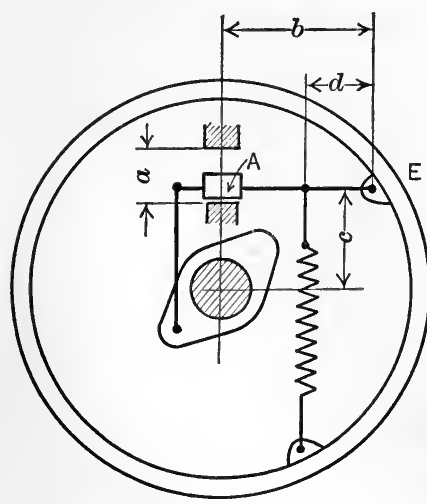

FIG. 399. amount of the working fluid delivered to the engine.

\section{PROBLEMS}

429. What must be the initial tension in the spring and the modulus of the spring so that the weight, $A$, in the governor shown in Fig. 399 will not leave the inner stop and pass to the outer stop until a speed of 210 r.p.m. is reached? Assume that the weight of $A$ is $16.1 \mathrm{lb} ., a=3 \mathrm{in}$., $b=24$ in., $c=8$ in., and $d=16$ in. If the spring should be placed farther from $E$ than 16 in., would $A$ reach the outer 
stop with the wheel revolving at the same speed that it had when $A$ left the inner stop? Ans. $T=242 \mathrm{lb}$; modulus $=45.4 \mathrm{lb}$. per in.

430. Given the arrangement as shown in the previous problem, with the following data: $a=3$ in., $b=20$ in., $c=9$ in., $d=12$ in., modulus of spring $=50$ in. If the mean speed, $n_{0}$, is 200 r.p.m., what must be the weight of $A$ for a coefficient of steadiness, $\frac{\left(n_{1}-n_{2}\right)}{n_{0}}$, of 0.01 , where $n_{1}$ and $n_{2}$ denote the maximum and minimum speeds respectively?

Ans. $W=14.85 \mathrm{lb}$.

173. The Inertia Shaft Governor.-As already noted, governors in which the regulating action depends upon a change in the centrifugal force, whether of the pendulum or of the shaft type, are

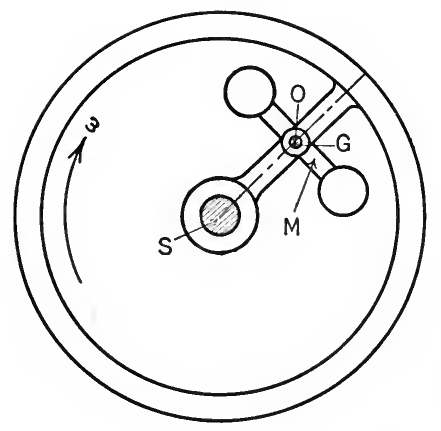

FIG. 400. called centrifugal governors. It is important to note that with centrifugal governors there must be an actual change of speed to give a governing action. In the inertia governor, however, the governing action is entirely different. Thus, in Fig. 400, let a mass $M$ be pivoted to the arm of a flywheel so that the pin, $O$, passes through the center of mass. The centrifugal force of the mass is balanced by the pin reaction at $O$ and hence the centrifugal force is not involved in the governing action.

In order to show how the forces which cause the governing action arise, let it be assumed first that the wheel of Fig. 400 is standing still and that the mass is turned on the pin, $O$, with an angular acceleration $\alpha$. To produce this acceleration a moment, $T$, is required having a magnitude,

$$
T=I_{0} \alpha
$$

in which $I_{0}$ is the moment of inertia of the mass with respect to the pin $O$. Now, if a change in speed of the wheel (and shaft) occurs, due to a change in the load on the motor, each particle in the mass $M$, by virtue of its inertia, will tend to maintain its linear velocity and hence will turn relative to the wheel about the axis $O$ and thereby cause the valve mechanism to exert forces on the mass, 
the moment of which about $O$ will be equal to $I_{0} \alpha$. In this type of governor, therefore, the governing action depends not upon the actual change of speed but upon the rate of change of angular speed, that is, upon the angular acceleration of the wheel or engine shaft. Shaft governors of this type are called inertia governors.

174. Comparison of the Two Types of Governors.-Centrifugal and inertia governors differ in two important particulars. Since, in the centrifugal type, the governing force depends upon the change in the speed there must be an increase (or decrease) in the speed of a definite amount before the governing force is sufficient to move the valve gear against the frictional forces. In the inertia governor, on the other hand, the governing force is proportional to the angular acceleration and, in general, the angular acceleration of the governing mass is greatest just at the beginning of the change of speed; hence, before the speed of the shaft has changed appreciably, the governor begins to act although the change in the centrifugal force is small. It follows then that the inertia governor acts more quickly and holds the speed within much smaller limits than does the centrifugal governor.

The centrifugal type of governor, however, has one essential property not possessed by the inertia type. The centrifugal force $M r \omega^{2}$ varies somewhat for different positions of the balls in Figs. 393, 394, 395, or 396, and of the body in Fig. 398, but for any fixed position it must have a fixed definite value which is determined by the weight of the balls (and load on the balls) in the pendulum governors and by the spring tension in the shaft governor. It follows that, with the governor in a definite position, the speed, $\omega$, of the shaft has a definite value and therefore the speed of the engine is fixed. Thus, if it is determined that the speed shall be 200 r.p.m. and the governor is properly adjusted, the speed cannot vary much from that speed as long as the governor is operative. The speed may sink to 195 r.p.m. under a heavy load or rise to 205 r.p.m. with a light load; but the centrifugal force imposes the average speed of 200 r.p.m., and the engine cannot be made to run at a different average speed without some adjustment of the governor, such as the addition of a weight or an increase in the spring tension.

175. Analysis of Forces in the Rite's Inertia Governor.In the shaft governor known as the Rite's inertia governor, a combination of the two governing actions discussed above is 
affected by means of a single heavy mass which is pivoted at a point at some distance from the shaft center but not at the center of the mass.

To gain a clear conception of the forces which are developed during the action of a shaft governor of the Rite's type, consider the motion of a heavy mass of any form (Fig. 401a) pivoted at a point $O$ which is at a distance $e$ from the shaft center $S$. The

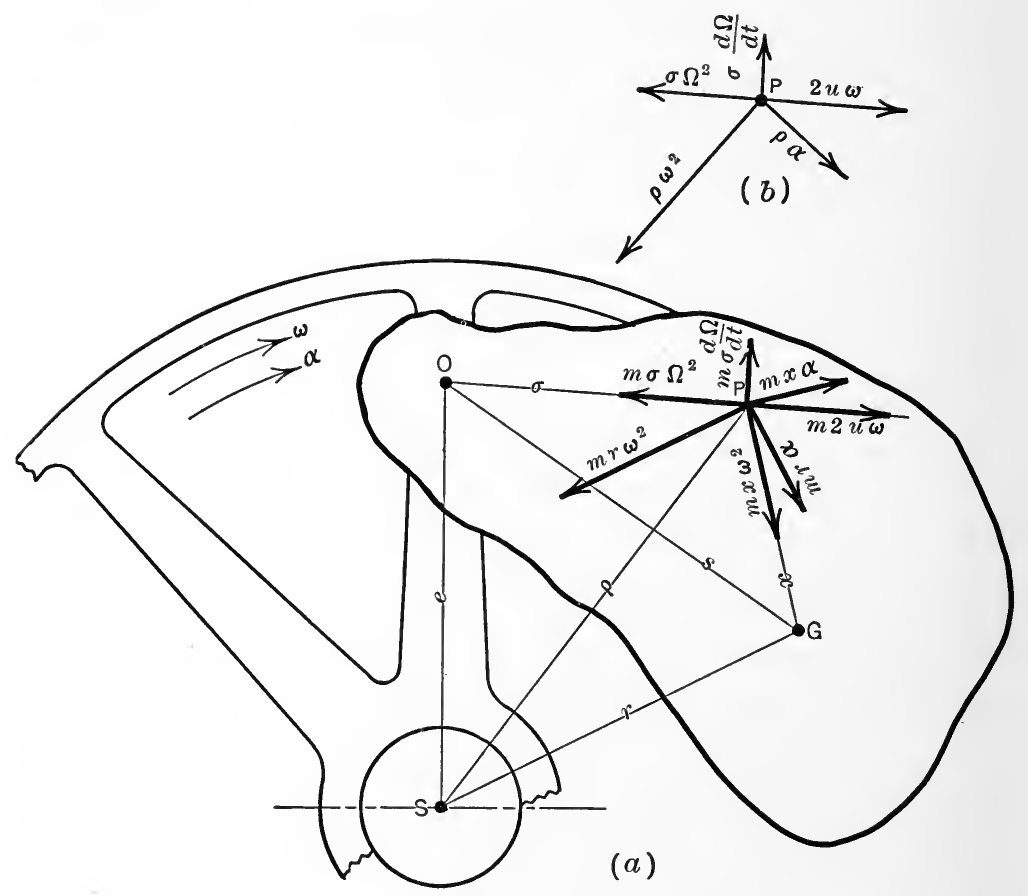

Frg. 401.

center of gravity, $G$, of the mass is at the fixed distance $s$ from the pivot $O$ and at the distance $r$ from the shaft center $S$; this latter distance may of course vary. Let any particle, $P$, of the mass be at the distances $\sigma$ and $\rho$ from $O$ and $S$, respectively. The procedure followed in the analysis of the forces in the Rite's governor is the same as that used in the analysis of the various kinetics problems in the preceding section. The main steps in the procedure are outlined in Art. 144. Let, 
$M=\frac{W}{g}=$ the mass of the body which is pivoted at $O$;

$\omega=$ the angular velocity of the wheel about the shaft center $S$;

$\Omega=$ the angular velocity of the mass $M$ about the pivot $O$;

$u=\sigma \Omega=$ the linear velocity of $P$ relative to $O$;

$\alpha=\frac{d \omega}{d t}=$ the angular acceleration of the wheel;

$\alpha^{\prime}=\frac{d \Omega}{d t}=$ the angular acceleration of the mass $M$ about $O$;

$I_{0}=$ the moment of inertia of the mass $M$ with respect to the pivot $O$, and

$\bar{I}=$ the moment of inertia of the mass $M$ with respect to the - center of gravity $G$.

Acceleration of Any Particle P.-According to Coriolis' law (Art. 130), the acceleration of the particle $P$ (Fig. 401b) has three components as follows:

(1) The acceleration that $P$ would have considering the wheel to be stationary and the mass to be rotating about $O$; this acceleration may itself be resolved into,

A radial component $\sigma \Omega^{2}$ along $P O$, and a tangential component $\sigma \frac{d \Omega}{d t}=\sigma \alpha^{\prime}$ perpendicular to $P O$;

(2) The acceleration of that point of the wheel which is coincident with $P$; this component may likewise be resolved into,

A radial component $\rho \omega^{2}$ along $P S$, and a tangential component $\rho \frac{d \omega}{d t}=\rho \alpha$ perpendicular to $P S$.

(3) The acceleration $2 u \omega$ along $O P$.

These components of the acceleration of the point $P$ are shown in Fig. 401(b). No attempt has been made to show the magnitudes of the accelerations by the lengths of the vectors.

It will be found convenient to replace the components $\rho \omega^{2}$ and $\rho \alpha$ by four other components, parallel and perpendicular, respectively, to the lines $S G$ and $P G$. These four components may be obtained by direct resolution or by considering the motion of the wheel to be replaced by a rotation and a translation (Art. 134). Thus, at any instant, the rotation of the wheel about its shaft, $S$, with angular velocity $\omega$ and angular acceleration $\alpha$ may be considered as a rotation with the same angular velocity and acceleration 
about a parallel axis in the wheel which passes through the point $G$, and a translation of the wheel in a direction perpendicular to $S G$. The components $\rho \omega^{2}$ and $\rho \alpha$ which, as found above, were due to the rotation of the wheel about its shaft $S$ will now be replaced by (1) the acceleration of the point $P$ due to the rotation of the wheel about $G$ and (2) the acceleration due to the translation, which is common to all points of the wheel. The accelerations of the point $P$ of the wheel (that is, the point on the wheel which is coincident with the point $P$ of the mass $M$ ), then, are,

and

$$
x \omega^{2} \text { along } P G
$$

$$
x \frac{d \omega}{d t}=x \alpha \text { perpendicular to } P G,
$$

which are due to the rotation of the wheel about $G$; and,

and

$$
r \omega^{2} \text { parallel to } S G \text {, }
$$

$r \alpha$ perpendicular to $S G$,

which are due to the (curvilinear) translation of the wheel.

Moment of Effective Forces about the Pin O.-The moment of the effective forces for the whole body with respect to the pin $O$ will now be found since the rotation of the mass about the pin $O$ is the cause of the movement of the valve mechanism. And, the moment of the effective forces must be found in order to determine the moment of the external forces which are brought into action as a result of this rotation and which are exerted on the valve mechanism. For, by D'Alembert's principle, the sum of the moments of the external forces must be equal to the sum of the moments of the effective forces.

The accelerating or effective force for the particle $P$ of mass $m$ must have a component in the direction of each of the acceleration components, the magnitude of each component of the force being the product of the mass $m$ of the particle and the acceleration component. These seven components of the effective force for the particle are shown in Fig. 401, acting at the particle $P$. The sum of the moments of these forces, for all the particles, about the pin $O$ may be found as follows:

(1) The components $m \sigma \Omega^{2}$ and $2 m u \omega$ are collinear and act through the pin $O$. The resultant of these components, therefore, 
for the whole body, is a force which acts through $O$. The sum of the moments of these components about the point $O$, then, is equal to zero and hence, these forces have no influence in the problem under discussion. Their only effect is to produce a pin pressure at $O$.

(2) Consider next the forces $m r \omega^{2}$. These forces arise from the translation given to the body. All of these forces, then, are parallel to $S G$ and all have the same sense. Therefore, their resultant is a single force (Art. 145) the magnitude of which is,

$$
\Sigma m r \omega^{2}=M r \omega^{2} .
$$

The line of action of this resultant force passes through $G$ (Art. 145), as shown in Fig. 402. The sum of the moments of the $m r \omega^{2}$

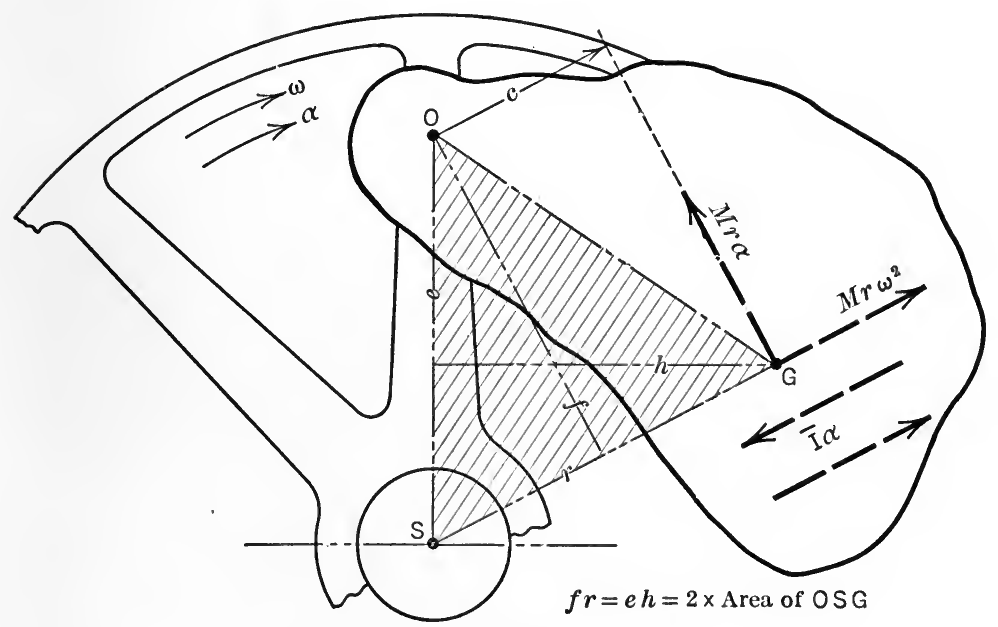

FIG. 402 .

forces about the pin $O$ (which, of course, is equal to the moment of their resultant), then, is (Fig. 402),

$$
M r \omega^{2} \times f .
$$

(3) Consider next the $m r \alpha$ forces. These forces also arise from the translatory motion which is given to the wheel (and mass $M)$. All of these forces then are perpendicular to $S G$ and have the 
same sense. Their resultant, therefore, is a single force (Art. 145) having a magnitude equal to

$$
\Sigma m r \alpha=M r \alpha .
$$

This resultant force also passes through $G$. The sum of the moments of the $m r \alpha$ forces with respect to the pin $O$, then, is (Fig. 402),

\section{$\operatorname{Mr} \alpha \times c$.}

(4) The moment of the $m x \omega^{2}$ forces will be found next. These forces are the normal forces due to the rotation of the wheel (and mass $M$ ) about an axis through $G$. They pass, then, through the axis of rotation and their resultant, therefore, is a single force having a magnitude equal to $M \bar{x} \omega^{2}$ which acts through $G$ (Art. 22). But, $G$ is the mass-center of the mass $M$ and hence, the quantity $M \bar{x} \omega^{2}$ is equal to zero since $\bar{x}$, the distance from the mass-center to the axis of rotation, is zero. Therefore, the sum of the moments of the $m x \omega^{2}$ forces about the pin $O$ is equal to zero.

(5) The $m x \alpha$ forces are the tangential forces which also arise due to the rotation of the wheel (and mass $M$ ) about the masscenter $G$. The resultant of these forces is a couple having a moment equal to $\bar{I} \alpha$ (Art. 146) in which $\bar{I}$ is the moment of inertia of the mass $M$ with respect to the axis through its mass-center. The sum of the moments of the $m x \alpha$ forces, then, with respect to the pin $O$ (or any other moment-center in the plane of motion, Art. 15) is equal to

\section{$\bar{I} \alpha$.}

(6) Lastly, the $m \sigma \alpha^{\prime}$ forces will be considered. These forces are the tangential forces which arise due to the rotation of the mass $M$ about the pin $O$. The sum of their moments, then, about $O$, the axis of rotation, is (Art. 146),

$$
I_{0} \alpha^{\prime}
$$

in which $I_{0}$ is the moment of inertia of the mass $M$ with respect to the pin $O$. This moment, however, for governors as constructed, is small compared with the other moments since $\alpha^{\prime}$ is small. Therefore, the moment $I_{0} \alpha^{\prime}$ will be neglected in the subsequent discussion. 
The Moment of the External Forces and the Governing Action.As noted above the moments of the external forces must be equal to the sum of the moments of the effective forces. Or, if the effective forces were reversed, external forces would have to act on the body such that they would hold the reversed effective (inertia) forces in equilibrium. Hence, external forces must act on the mass $M$ such that the moments of the external forces will hold in equilibrium the moment (Fig. 402),

$$
T_{c}=M r \omega^{2} f=M e h \omega^{2} \quad \text {. . . . . . . }
$$

of the centrifugal force, and the moment

$$
T_{i}=M r \alpha c+\bar{I} \alpha=\alpha(M r c+\bar{I}), \quad \text {. . . . . }
$$

which is called into action only when the angular acceleration, $\alpha$, exists.

It will be noted, therefore, that if the angular velocity, $\omega$, of the flywheel (and engine) remains constant, that is, if the angular acceleration, $\alpha$, of the flywheel is equal to zero, the moment $\left(M e h \omega^{2}\right)$ of the centrifugal force has a constant magnitude and must be equilibrated by the moment of the spring tension. Further, if $\omega$ is constant the only external moment acting on the mass $M$ is the moment of the spring tension since $\alpha$, and consequently $T_{i}$, is equal to zero. Thus, the moment $T_{i}$, unlike the moment $T_{c}$, is called into existence only when there is a change in speed of the flywheel and hence it does not require an opposing moment except that supplied by the resistance of the valve gear as the valve gear adjusts itself to the new speed.

To explain further the governing action in the Rite's governor, let it be assumed that the motor is running at a constant mean speed $\omega_{0}$; the moment $T_{c}\left(=M e h \omega^{2}\right)$ of the centrifugal force is then just balanced by the moment of the spring tension which may be denoted by $T_{s}$. Now, let the load on the motor be reduced; the excess of the effort over the resistance will cause the moving parts of the motor (flywheel, shaft, etc.) to rotate with an increased speed, $\omega_{1}$, and the moment $T_{c}$ will increase from $M e h \omega^{2}$ to $M e h \omega_{1}{ }^{2}$. The difference, $\operatorname{Meh}\left(\omega_{1}^{2}-\omega^{2}\right)$, is the excess of the moment of the centrifugal force over that of the spring tension and is available for producing a movement of the governor parts. However, if 
this difference alone is depended on for moving the valve mechanism, the speed $\omega_{1}$ must be considerably in excess of the mean speed $\omega_{0}$ before the governor and attached valve will move. On the other hand, the very instant the change in speed begins, the angular acceleration, $\alpha$, of the mass $M$ comes into existence and, with it, the unbalanced moment $T_{i}$ which is available for moving the valve gear. In the ideal governor, therefore, there is needed a moment, $T_{c}$, of a centrigufal force which is just sufficient to fix a mean speed $\omega_{0}$, and a moment, $T_{i}$, of considerable magnitude which arises from the rate of change of the angular velocity of the flywheel and which provides for the adjustment of the governor.

Distribution and Position of the Mass M.-An inspection of equation (2) shows that, with a given value of $r$, the magnitude of the moment $T_{i}$ depends on $M$ and $\bar{I}$. With a heavy flywheel, $\alpha$ is likely to be small; hence, to make $T_{i}$ large, either $M$ must be made large or, for a given $M, \bar{I}$ may be made large by constructing the swinging mass $M$ in two parts which are removed a considerable distance from the center of gravity, $G$, and are joined by a bar (see Figs. 404 and 405).

Further, the governing action of the swinging mass will depend largely on the relative position of the points $O, S$, and $G$. Thus, in Fig. 403, let a circle be drawn having a diameter equal to $O S$ or $e$ and let the sense of rotation of the shaft be assumed clockwise. As discussed above there are three moments ( $M r \alpha c, \bar{I} \alpha$, and $M e h\left(\omega_{1}^{2}-\omega_{0}^{2}\right)$ ) which are effective in producing the adjustment of the governor. Now these three moments may or may not have the same sense, depending on the location of the point $G$. If a counter-clockwise sense is denoted by + and a clockwise sense by -, the following table indicates the sense of each of the three moments about $O$ for a location of $G$ in each of the four regions, I, II, III, and IV indicated in Fig. 403.

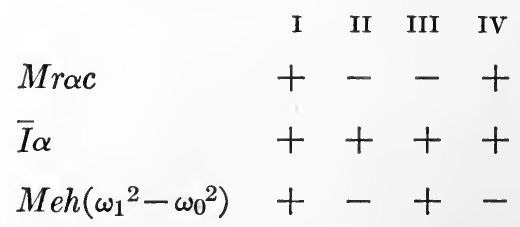

For the most powerful governing action, the mass-center, $G$, of the mass $M$, therefore, should be located in region I. If $G$ is 
located in region III, the moment $T_{i}$ becomes equal to $(\bar{I}-M r c) \alpha$ and this quantity may be positive or negative according as $\bar{I}$ is greater or less than $M r c$. With $G$ located in regions $I I$ and $I V$, that is, to the left of the vertical diameter, the moment of the centrifugal force and the moment $\bar{I} \alpha$ are opposite in sense. In a governor in which the whole mass is concentrated near to $G$ so that $\bar{I}$ is small, it is permissible to locate $G$ in region IV; with a governor of this type the engine may, therefore, rotate in either direction without changing the governor.

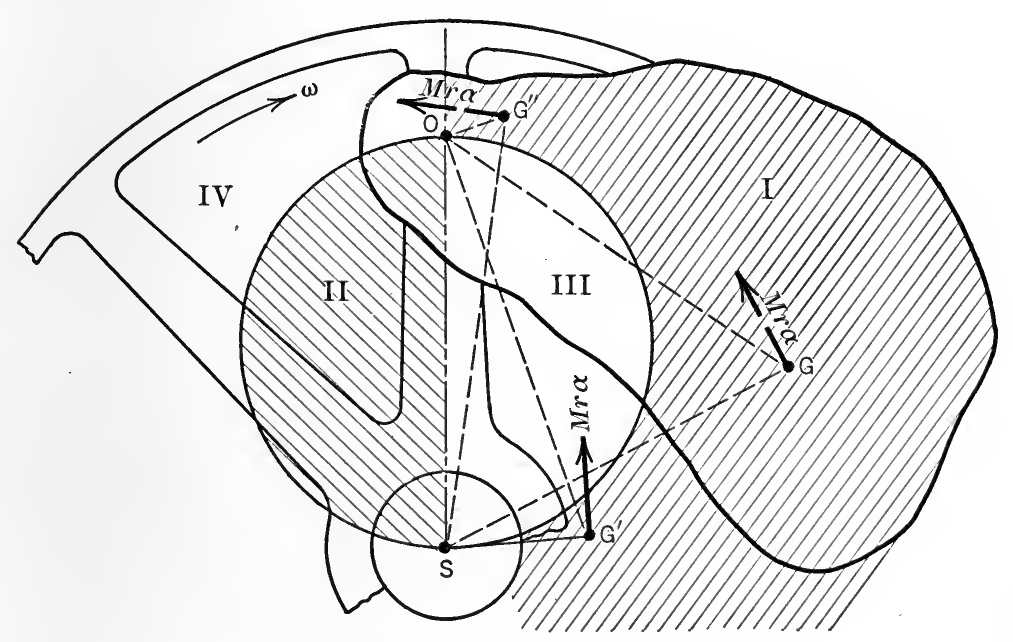

Fig. 403.

In modern governors of the Rite's type $\bar{I}$ is made very large and hence the moment $\bar{I} \alpha$ is very large compared with the moment $M r c \alpha$. Therefore, in these governors, the mass-center of $M$ may be located on the circle or even in region II if it is desirable to do so in order to decrease the centrifugal moment $M r c \alpha$ and, in consequence, the size of the spring required.

From Fig. 403, it will be noted that the moment of the centrifugal force $M r \omega^{2}$ is $2 M \omega^{2}$ times the area of the triangle $O S G$; hence, this moment is directly proportional to the area of the triangle $O S G$. From this fact, it appears that the size of the spring required varies directly as the area of the triangle $O S G$; and, to obtain as light a spring as possible, consistent with good regula- 
tion, the triangle $O S G$ should be made as small as possible. If $G$ is kept in region I, this object is accomplished by locating $G$ either near to $S$ at $G^{\prime}$, or near to $O$ at $G^{\prime \prime}$ (Fig. 403). In the governors shown in Fig. 404 and 405 it will be noted that $G$ is located near to $S$.

\section{ILLUSTRATIVE PROBLEM}

431. In Fig. 404 is shown a Rite's inertia governor designed for a 6-h.p. Nagle engine. Experience shows that from 0.5 to $8 \mathrm{ft}$.lb. of energy per

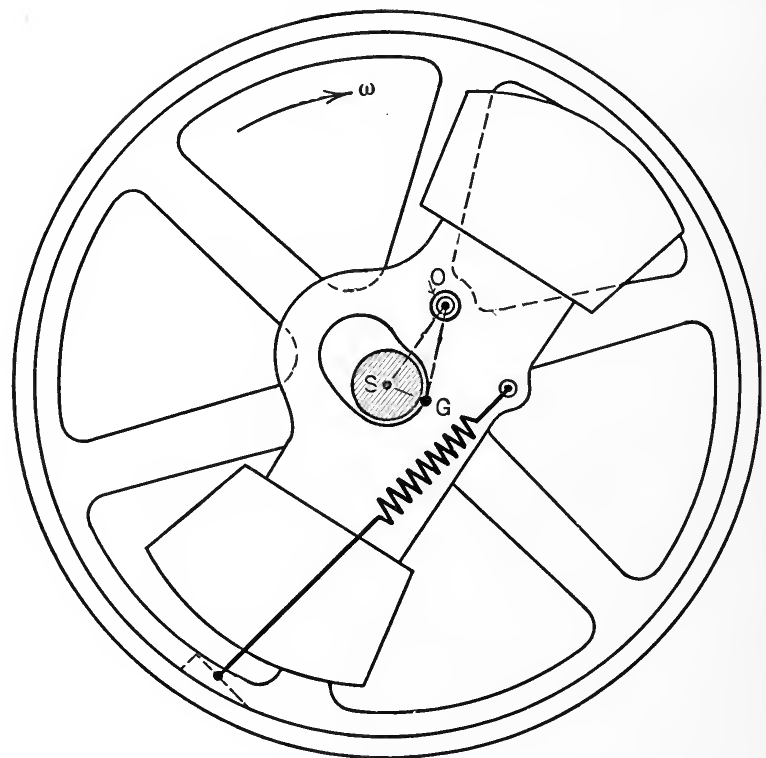

FIG. 404.

h.p. should be stored in the governor weight in moving through its arc (work done in stretching the spring). In the design, $5 \mathrm{ft}$.-lb. per h.p. was assumed. Find the inertia weight required and the modulus of the spring from the following data: $O S=e=3.4$ in.; $h=1.7$ in.; mean speed $=250$ r.p.m.; $l_{0}=9.4$ in. $=$ unstretched length of spring; $l_{1}=11$ in. $=$ minimum length of spring; $l_{2}=12.2$ in. = maximum length of spring; $p=3.8$ in. = moment arm of spring tension.

Solution.-Since the moment of the centrifugal force $\left(T_{c}\right)$ must equal the moment of the spring tension $\left(T_{s}\right)$ we have the equation,

That is,

$$
T_{c}=T_{s} .
$$

$$
\frac{W}{g} \dot{r} \omega^{2} f=T_{m} \times 3.8,
$$


or,

$$
\frac{W}{g} \omega^{2} h e=T_{m} \times 3.8(\text { since } r f=h e),
$$

where $T_{m}$ denotes the mean value of the spring tension. In order to determine the value of $T_{m}$, the work stored in the governor is equated to the work done in stretching the spring. Thus,

Hence,

$$
5 \times 6=T_{m} \times \frac{12.2-11.0}{12} \text {. }
$$

$$
T_{m}=300 \mathrm{lb} .
$$

Using this value of $T_{m}$ in the above equation, we have,

$$
\frac{W}{12 \times 32.2}\left(\frac{250 \times 2 \pi}{60}\right)^{2} \times 1.7 \times 3.4=300 \times 3.8 .
$$

From which,

$$
W=111 \mathrm{lb} .
$$

The length of the spring, when the governor is in its mean position is $\frac{1}{2}(12.2+$ $11.0)=11.6$. in. Hence the modulus of the spring is,

$$
\frac{300}{11.6-9.4}=\frac{300}{2.2}=136 \mathrm{lb} . \text { per in. }
$$

\section{PROBLEM}

432. In the Rite's governor shown in Fig. 405, the inertia weight is $111 \mathrm{lb}$. and the tension of the spring in mid-position is $500 \mathrm{lb}$. If $e=7 \mathrm{in}$., $h=2.6 \mathrm{in}$., $r=3$ in., and $p=8.2$ in.; find,

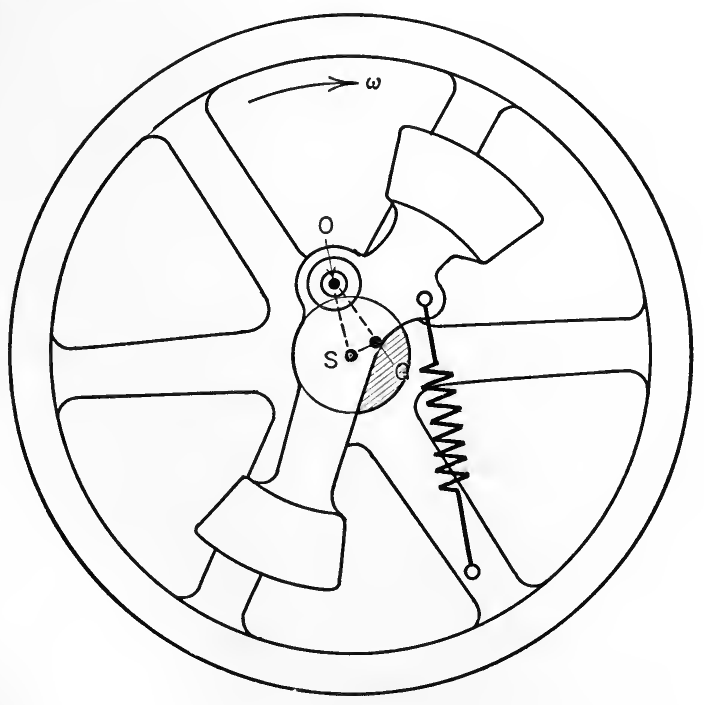

Fig. 405. 
(a) The normal speed of the engine, that is, the speed when the governor is in mid-position, and

(b) The power of the governor in ft.-lb. per h.p. if the engine is rated at 50 h.p., assuming that the spring stretches from $20 \mathrm{in}$. to $22 \mathrm{in.}$ when the governor moves through its whole range.

Ans. $\omega=268$ r.p.m.; 1.67 ft.-lb. per h.p. 


\section{CHAPTER $\mathrm{X}$}

\section{WORK AND ENERGY}

176. Introduction.- In the preceding chapter the relations between force, mass, and acceleration were developed from Newton's laws of motion and applied to the motion of bodies under the action of unbalanced forces. As already noted, the quantities involved directly in Newton's laws are force, mass, and acceleration. But, acceleration involves the quantities velocity, distance, and time. Now, in many problems in engineering, it is convenient to use certain other quantities, the more important of which are: work, power, energy, impulse, and momentum. The expression for each of these quantities is a combination of some of the six quantities (force, mass, acceleration, velocity, distance, and time) which are involved in Newton's laws of motion. Thus, force and distance combine to measure work; force, distance, and time combine to measure power; mass and velocity combine to measure momentum and some forms of energy; force and time combine to measure impulse, etc. Although the conceptions of these quantities are more or less a result of our experience with physical phenomena, the exact relations between them, as expressed in certain principles to be developed in the following pages, are based on the definite fundamental laws of Newton.

The present chapter is devoted to a discussion of the meaning and use of work, of energy, and of certain principles which involve these two quantities. Although no fundamental physical laws other than those of Newton are used in developing the principles of work and energy, nevertheless, the method of analysis which makes use of work and energy, possesses certain advantages over the method which makes use directly of force, mass, and acceleration, even in certain types of problems which involve only rigid bodies having rather simple types of motion such as translation, rotation, and plane motion. And, in dealing with non-rigid bodies having unordered motion, that is, motion in which the particles 
of the mass system (body) do not follow definite known paths, the principles of energy are of particular importance. In fact, the study of the behavior of non-rigid bodies in general, such as water, steam, gas, and air is largely based on the principles of energy and, hence, these principles play an important part in hydraulics, thermodynamics, meteorology, etc.

\section{$\S 1$. Work AND Power}

177. Work Defined.-A force does work on a body if the body on which the force acts is moved so that the displacement of the point of application of the force has a component in the direction of the force. The amount of work done by a force is the product of the force and the component of the displacement of its application point in the direction of the force. The work done by a force may also be expressed as the product of the component of the force in the direction of the displacement of its application point and the displacement. The component of the force in the direction of the displacement of its application point is often called the working component. And the component of the displacement in the direction of the force is called the effective displacement.

178. Algebraic Expressions for Work Done by a Force.-The mathematical expression for the work, $w$, done by a force, $F$, in a displacement, $s$, of its application point depends on the way the force varies during the displacement. Several important special cases are considered here.

I. The force is constant in magnitude and in direction and agrees in direction with the displacement as, for example, the force exerted in lifting a body vertically upward with a uniform acceler-

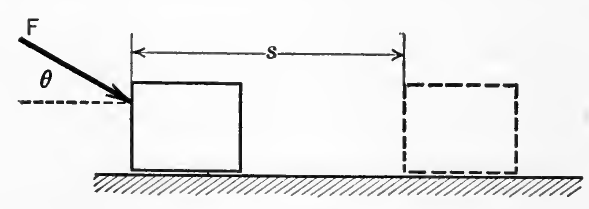

FIG. 406. ation. The amount of work done is,

$$
w=F \cdot s .
$$

II. The force is constant in magnitude and in direction but does not agree in direction with the displacement (Fig. 406). The amount of work done is,

$$
w=F \cos \theta \cdot s=F_{t} \cdot s
$$


in which $F \cos \theta$ is denoted by $F_{t}$ since $F \cos \theta$ is tangent to the path of the point of application.

III. The force varies in magnitude but not in direction and the direction agrees with that of the displacement as, for example, the force exerted in compressing a helical spring or the steam pressure against the piston of a steam engine after cut off. Thus, in compressing a spring, the force corresponding to any displacement, $s$, is $F$ (Fig. 407) and this force may be assumed to remain constant in an infinitesimal displacement, $d s$. Hence, according to case $I$, the work done by the force $F$ in the displacement $d s$ is $d w=F d s$ and, the total work done on the

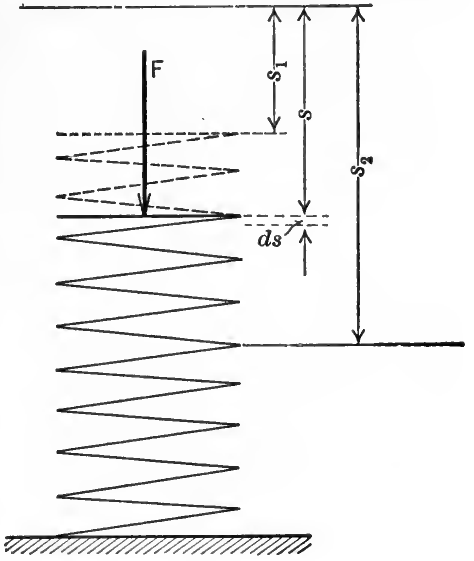

Fig. 407. spring, as $F$ varies from its initial to its final value, is

$$
w=\int_{s_{1}}^{s_{2}} F d s
$$

In order to evaluate the integral by the method of calculus, $F$ must be expressed in terms of $s$. That is, the manner in which $F$ varies with $s$ must be known.

$I V$. The force varies in magnitude and in direction as, for

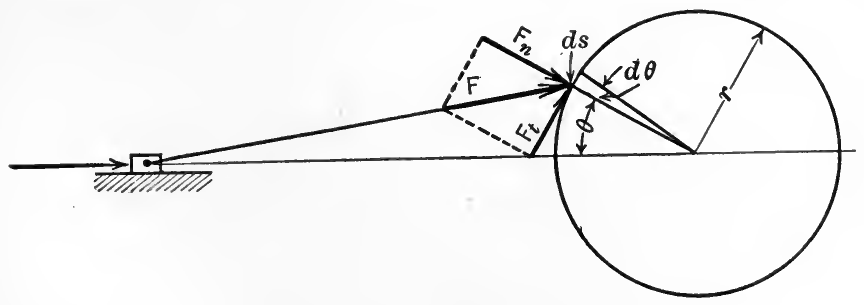

FIG. 408.

example, the pressure of the connecting rod on the crank-pin of an engine (Fig. 408). The expression for the work done by the force 
is found by the same method as was used in case $I I I$ except that the tangential component of the force must be used. Hence,

$$
w=\int_{s_{1}}^{s_{2}} F_{t} d s .
$$

This expression applies whether the displacement is along a circular path or not. But when the displacement takes place in a circular path the elemental displacement $d s$ is expressed by the equation $d s=r d \theta$. Whence,

$$
w=\int_{s_{1}}^{s_{2}} F_{t} d s=\int_{s_{1}}^{s_{2}} F_{t} r d \theta=\int_{\theta_{1}}^{\theta_{2}} T d \theta,
$$

in which $T$ is the torque or moment of the force about the center of the circular path. And if the torque remains constant during an angular displacement, $\theta=\theta_{2}-\theta_{1}$, then,

$$
w=T \int_{\theta_{1}}^{\theta_{2}} d \theta=T\left(\theta_{2}-\theta_{1}\right)=T \cdot \theta .
$$

Thus, in one revolution, the work done by the force $F$ having a moment $T$ is $w=T \cdot 2 \pi$. And if $n$ revolutions occur per unit of time, then the work done per unit of time is,

$$
w=T \cdot 2 \pi n \text {. }
$$

179. Work Done by a Couple.- Since the magnitude of the moment of a couple is the algebraic sum of the moments of the two forces which constitute the couple, and since the moment of a couple is the product of either force and the perpendicular distance between the action lines of the forces, it follows from the above discussion that the work done by a couple, having a moment $T$, in a displacement $d \theta$, is

$$
w=\int_{\theta_{1}}^{\theta_{2}} T d \theta
$$

when the moment of the couple varies. And the work done is,

$$
w=T \cdot \theta,
$$

when the moment of the couple is constant in the displacement. Hence, the work done by a couple having a constant moment is the product of the moment of the couple and the angular displacement of the couple. 
180. Work a Scalar Quantity. Sign and Units of Work.Work is a scalar quantity. Thus the work done by one force may be added (algebraically) to the work done by another force regardless of the directions of the forces or of the displacements of their points of application. And, the work done on one body of a system may be added (algebraically) to the work done on the other bodies of the system in order to obtain the total work done on the system, regardless of the manner in which the bodies move. The fact that work is a scalar quantity, frequently makes it convenient to use this quantity under conditions which make the use of vector quantities difficult or impossible.

It is convenient to regard the work done by a force as having sign. Work is positive when the working component of the force and the displacement of its application point agree in sense; and work is negative when the working component and the displacement are opposite in sense. Thus, a force which retards the motion of a body does negative work on the body.

The unit of work is the work done by a unit force acting through a unit distance and hence, depends on the units used for force and distance. Thus, the more common units for work in the gravitational (engineers') system of units are the foot-pound, inchpound, meter-kilogram, etc. No one-term names are given to the units of work in the gravitational system of units. The common units of work in the absolute system of units are the dyne-centimeter, which is called an erg, and the joule, which is $10^{7} \mathrm{ergs}$. For large units of work the horse-power-hour and the kilowatt-hour are used. For a definition of these units see Art. 184.

181. Graphical Representation and Calculation of Work.-In calculating the work done by a variable force, by the calculus method, the working component, $F_{t}$, of the force must be expressed in terms of the displacement $s$. If it is impossible to express $F_{t}$ in terms of $s$, or if, when possible, the expression for $F_{t}$ is complex and difficult to use, as in the case of the tangential effort on the crank-pin of a steam engine, the relation between $F_{t}$ and $s$ may be expressed graphically by means of a graph or curve, and the work done may be found from the graphical diagram as follows: If values of $F_{t}$ and $s$ are plotted on a pair of rectangular axes for all positions of the application point of the force $F$, the curve joining the plotted points is called a tangential-force-space $\left(F_{t}-s\right)$ curve (Fig. 409). In most problems, only a sufficient number of values of $F_{t}$ are 
plotted to make it possible to draw a reliable $F_{t}-s$ curve, values of $F_{t}$ being plotted more frequently when the value of $F_{t}$ is changing the more rapidly. The work done by a variable force $F$ as shown in the preceding article is $w=\int_{s_{1}}^{s_{2}} F_{t} d s$. But, $F_{t} d s$ represents an elemental part of the area (Fig. 409) between the $F_{t}-s$ curve and the $s$-axis. And, the total area under the $F_{t}-s$ curve between any two ordinates corresponding to abscissas $s_{1}$ and $s_{2}$ is,

$$
\text { area }=\int_{s_{1}}^{s_{2}} F_{t} d s .
$$

Therefore, the work done by a force in any displacement $s$ is represented by the area under the tangential-force-space curve between the ordinates $s_{1}$ and $s_{2}$. This diagram is called a work

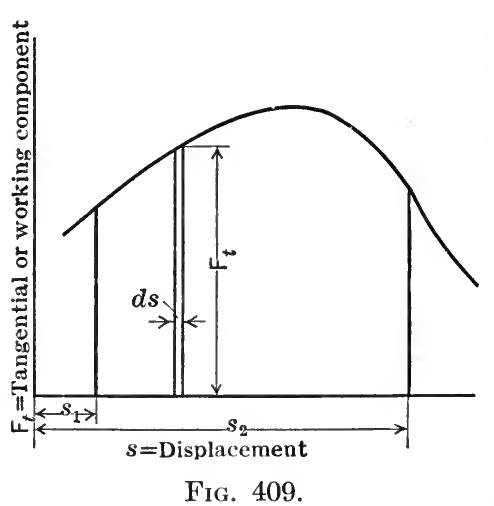
diagram. In determining the amount of work represented by the work diagram, the scales used in plotting the $F_{t}-s$ curve must be considered. Thus, if ordinates are plotted to a scale of $1 \mathrm{in} .=50 \mathrm{lb}$. and abscissas to the scale of $1 \mathrm{in} .=5 \mathrm{ft}$., then each square inch of area under the $F_{t}-s$ curve represents 250 ft.-lb. of work.

Since the area of a work diagram equals the product of the average ordinate and the base, the work done by a force equals the average value of the tangential (working) component of the force and the length of the path described by the application point.

The area of the work diagram may be found by means of a planimeter or by dividing the area into small strips and applying Simpson's rule. Or, in some cases, less exact methods may be employed in estimating the area.

\section{ILLUSTRATIVE PROBLEMS}

433. A helical spring (Fig. 410) having a modulus of $200 \mathrm{lb}$. per in. is compressed $s=4 \mathrm{in}$. by an axial load. How much work is done by the (variable) load in compressing the spring? 
Solution.-If $P_{y}$ denotes the force corresponding to any compression, $y$, of the spring, then from case III we have,

But,

$$
w=\int_{0}^{s} P_{\iota} d y .
$$

$$
P_{y}=200 y \text {. }
$$

Hence,

$$
\begin{aligned}
w & =\int_{0}^{s} 200 y d y \\
& =\frac{200 s^{2}}{2}=\frac{200(4)^{2}}{2}(\text { when } s=4) \\
& =1600 \text { in.-lb. }
\end{aligned}
$$

The expression

$w=\frac{200 s^{2}}{2}$ may be written,

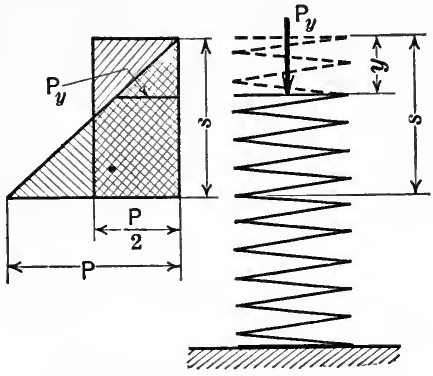

FIG. 410.

$w=\frac{200 s}{2} \times s=\frac{P}{2} \times s=$ area of triangular work diagram

$=$ average force times total displacement

$=$ area of rectangular diagram having the same area as the triangular diagram.

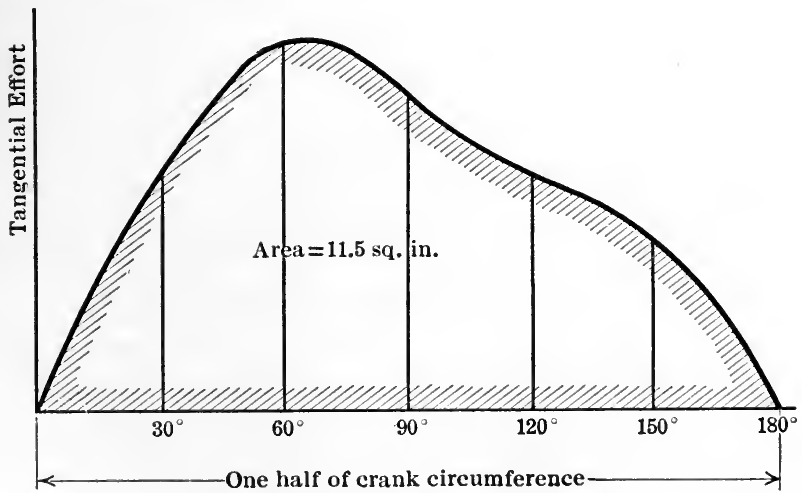

FIG. 411.

434. The tangential-effort diagram for a steam engine (similar to Fig. 411) is drawn to the following scales: 1 in. of ordinate $=24 \mathrm{lb}$. per sq. in. of piston area and $1 \mathrm{in}$. of abscissa $=\mathrm{a} 30^{\circ}$-arc of the crank-pin circle. The area under the curve is found to be $11.5 \mathrm{sq}$. in. Find the work done on the crankpin per square inch of the piston area per stroke (one-half revolution), if the crank length is $7.5 \mathrm{in}$. Also find the total work done per stroke, the diameter of the piston being $14 \mathrm{in}$. 
Solution.-A $30^{\circ}$-arc of the crank-pin circle $=3.92 \mathrm{in}$.

1 sq. in. of the work diagram $=24 \times 3.92=94.2$ in.-lb. Work done per stroke per square inch of piston $=94.2 \times 11.5$.

$$
\begin{aligned}
& =1083 \mathrm{in} .-\mathrm{lb} . \\
& =90.2 \mathrm{ft} .-\mathrm{lb} .
\end{aligned}
$$

Total work per stroke $=90.2 \times \frac{\pi \times(14)^{2}}{4}$

$$
=13,900 \mathrm{ft} .-\mathrm{lb} \text {. }
$$

435. In the design of punching machines (see Fig. 429) it is important to know how much work is done in

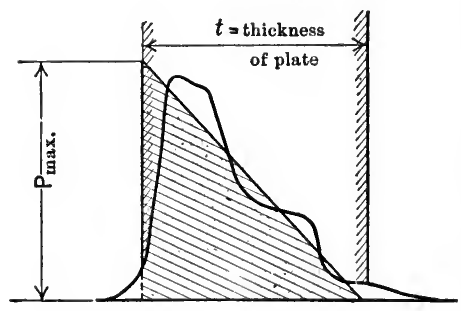

FIG. 412. punching a hole in a plate. Tests show that the work-diagram for stee! is approximately of the form shown by the heavy curved line in Fig. 412 . This diagram may be assumed, without serious error, to be equal to the triangular work-diagram in which the maximum pressure $P$ corresponds to a shearing strength in the steel plate of $60,000 \mathrm{lb}$. per sq. in. Find the work done in punching a $\frac{7}{8}$-in. hole in a $\frac{5}{8}$-in. steel plate.

Solution $-P_{\max }=$ shearing area $\times 60,000$

$$
\begin{aligned}
& =\pi d t \times 60,000 \\
& =\pi \times \frac{7}{8} \times \frac{5}{8} \times 60,000=103,000 \mathrm{lb} .
\end{aligned}
$$

The work done in punching the hole, assuming a triangular work diagram. is,

$$
\begin{aligned}
w & =\text { average pressure times thickness of plate } \\
& =\frac{P_{\text {max }}}{2} \times t \\
& =\frac{103,000}{2} \times \frac{5}{8} \\
& =32,200 \text { in-lb. }
\end{aligned}
$$

\section{PROBLEMS}

436. A box weighing $80 \mathrm{lb}$. is pulled up an inclined plane by a force, $P$, of $60 \mathrm{lb}$. as shown in Fig. 413. The coefficient of friction is $\frac{1}{4}$. Find the work done by each force acting on the box if it moves $20 \mathrm{ft}$. Find the total work done on the box.

Ans. $42.8 \mathrm{ft}$.-lb.

437. A rope which weighs $5 \mathrm{lb}$. per foot and which is $500 \mathrm{ft}$. long, is sus- 
pended by one end from a drum. How many foot-pounds of work must be done to wind up $200 \mathrm{ft}$. of the rope?

438. Water is pumped into an elevated tank from a reservoir at the rate of $10 \mathrm{cu} . \mathrm{ft}$. per second. How much work is done, per pound of water pumped, in raising the level of the water in the tank $12 \mathrm{ft}$. if the initial level is $40 \mathrm{ft}$. above the surface of the reservoir? Assume that the frictional resistance of the water in the pipe is equivalent to an additional lift of $4 \mathrm{ft}$. Ans. $50 \mathrm{ft}$.-lb.

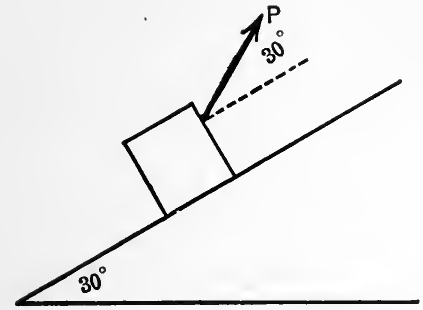

Fug. 413.

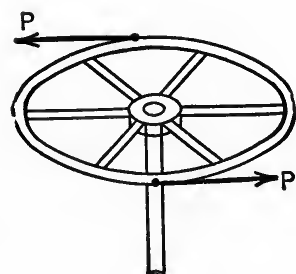

FIG. 414.

439. Two forces $P, P$ (Fig. 414) exert a constant turning moment (couple) on the hand-wheel of a large valve. The wheel is $18 \mathrm{in}$. in diameter. How much work is done in closing the valve if 8 revolutions of the hand-wheel are required and each force has a magnitude of $20 \mathrm{lb}$.

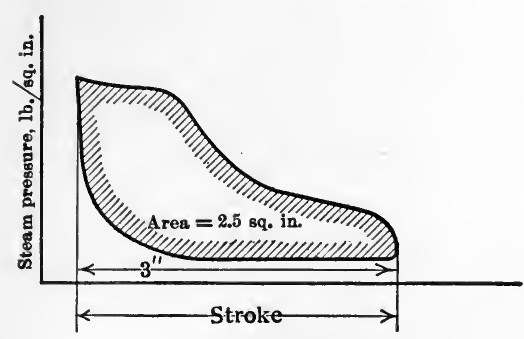

Fig. 415.

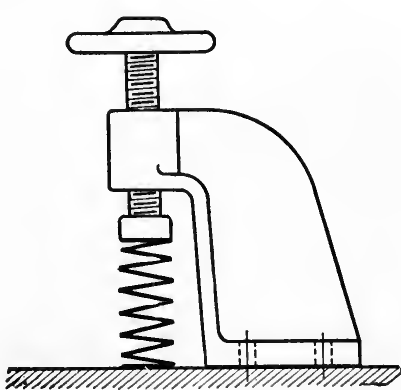

FIg. 416.

440. The steam indicator card (Fig. 415) is drawn to the following scales: 1 in. of ordinate $=100 \mathrm{lb}$. per square inch and $1 \mathrm{in}$. of abscissa $=5 \mathrm{in}$. of the stroke of the piston. The area of the indicator card is found to be $2.5 \mathrm{sq}$. in. and the length of the diagram is $3 \mathrm{in}$. (stroke $=15 \mathrm{in}$.). The diameter of the piston is $14 \mathrm{in.} \mathrm{Find} \mathrm{the} \mathrm{work} \mathrm{done} \mathrm{per} \mathrm{stroke} \mathrm{by} \mathrm{the} \mathrm{steam} \mathrm{on} \mathrm{the} \mathrm{piston.}$ What is the average steam pressure in lb. per sq. in. (mean effective pressure) which will do the same amount of work? Ans. $w=16,000 \mathrm{ft} .-\mathrm{lb}$. 
441. The screw of the bracket clamp (Fig. 416) moves vertically 1 in. when the hand-wheel is turned 4 revolutions. A helical spring having a modulus of $200 \mathrm{lb}$. per inch is compressed by turning the hand-wheel. The average frictional moment of the screw is $20 \mathrm{in} .-\mathrm{lb}$. What is the average turning moment applied to the hand-wheel in compressing the spring 3 in.?

182. Work Done on a Body by a Force System.--So far, the work done on a body by a single force has been considered. In general, however, a body is acted on by a force system, and, in order to find the work done on the body in any displacement, the work done by the whole force system must be found. In many cases the simplest and most direct method of obtaining the work done on the body is by finding the algebraic sum of the works done by the forces of the system. In certain cases, however, it is convenient to find the work done on a body by a force system from the resultant of the force system. In this connection the following propositions are important:

(a) The work done by a pair of equal opposite and collinear forces in any displacement of their application points is zero, provided that the dist nce between the points of application of the forces remains constant. Although the resultant of two such forces is always zero the work done, in general, is not zero. Thus, if one end of a helical spring is attached to a fixed body and a force is applied gradually to the other end, the applied force and the reaction of the fixed body are equal but the work done on the spring is not zero. It will be noted, therefore, that although the resultant of the internal forces in any body (whether rigid or not) is zero since they occur in pairs of equal, opposite, and collinear forces (Newton's third law), the work done by the internal forces of a body is not, in general, zero except for rigid bodies.

(b) The work done on a body by a concurrent force system is equal to the work done by the resultant force of the system. It is convenient, as a rule, to assume the point of application of the resultant force to be the point of concurrence of the forces.

(c) The work done on a rigid body, in any angular displacement, $\theta$, by a system of couples in a plane is equal to the work done by the resultant couple, $\Sigma T$. Thus, from Art. 179, the work done is

$$
w=\int_{\theta_{1}}^{\theta_{2}} \Sigma T \cdot d \theta
$$

Or, if the resultant couple remains constant in the displacement, then

$$
w=\Sigma T \cdot \theta
$$

(d) The work done by the earth-pull (weight) on a body (whether rigid or not) in any displacement equals the product of the weight of the body and the 
vertical displacement of the mass-center of the body irrespective of its lateral displacement. The work done by the earth-pull is positive if the center of gravity of the body is lowered and negative if the center of gravity is raised. It will be noted that the earth-pull on a system of particles (body) constitutes a special parallel force system and that the weight of the body is the resultant of the system.

In determining the work done by a force system which gives a motion of translation, rotation, or plane motion to a rigid body there are certain special cases of these motions for which it is especially convenient to use the resultant in finding the work done on the body. A brief discussion of these special cases follows.

By the method of Art. 18, any coplanar system of forces may be resolved into a system of concurrent forces acting at any chosen point in the plane and a system of couples in the plane. The resultant of the concurrent forces is a force (Art. 22); and the resultant of the system of couples is a couple (Art. 27). Hence the work done on a rigid body by the resultant of such a force system is the sum of the works done by the resultant force and couple. For convenience, the mass-center of the body will be chosen for the point of concurrence of the concurrent force system for the reason that the resultant force always agrees in direction with the acceleration of the mass-center (Art. 152). Further, the resultant force is equal to the algebraic sum of the components of the forces in the direction of the acceleration of the mass-center of the body. It will be denoted, therefore, by $\Sigma F \bar{a}$, and the working component of the resultant will be tangent to the path of the mass-center and will be denoted by $\Sigma F_{a_{t}}$ The resultant couple is the algebraic sum of the moments of the forces about the mass-center and will be denoted by $\Sigma \bar{T}$. The work done on a rigid body by a coplanar force system, therefore, is

$$
w=\int_{s_{1}}^{s_{2}} \Sigma F_{\bar{a}} d \bar{s}+\int_{\theta_{1}}^{\theta_{2}} \Sigma \bar{T} d \theta, \ldots . . .
$$

in which $\bar{s}$ denotes the distance moved by the mass-center along its path and $\theta$ denotes the angle through which the body turns. For certain important special cases of the motion of rigid bodies, this expression reduces to simpler forms as follows.

I. Uniformly Accelerated Rectilinear Translation. $-\Sigma \bar{T}$ is zero, $\Sigma F_{\bar{a}}$ is constant and acts in the direction of $\bar{s}$. But $\bar{a}$ and $\bar{s}$ are 
the same as the $a$ and $s$ of every other point in the body. Hence equation (1) reduces to

$$
w=\Sigma F_{\bar{a}} \cdot \bar{s}=\Sigma F_{a} \cdot s \quad . \quad . \quad . \quad .
$$

II. Uniformly Accelerated Rotation about an Axis through the Mass-center. $-\Sigma F_{\bar{a}}$ is zero and $\Sigma \bar{T}$ remains constant. Hence,

$$
w=\Sigma \bar{T} \cdot \theta \text {. }
$$

III. Plane Motion in which the Mass-center has Uniformly Accelerated Rectilinear Motion and the Body has Uniformly Accelerated Angular Motion. $-\Sigma F_{\bar{a}}$ is constant and acts in the direction of $\tilde{s}$, and $\Sigma \bar{T}$ is constant. Hence,

$$
w=\Sigma F_{\bar{a}} \cdot \dot{s}+\Sigma \bar{T} \cdot \theta .
$$

\section{ILLUSTRATIVE PROBLEM}

442. A solid cylinder of radius $r \mathrm{ft}$. and of weight $W \mathrm{lb}$. rolls down an inclined plane without slipping. Find the work done on the cylinder while rolling

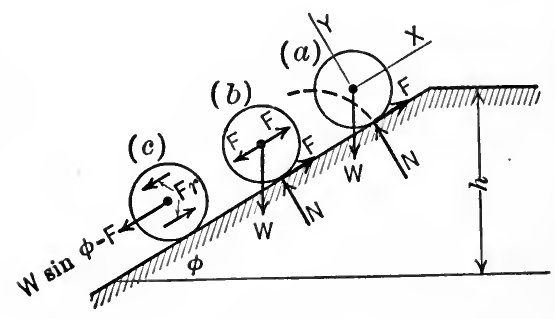

Fig. 417. down the plane if the plane is $s \mathrm{ft}$. long and makes an angle of $\phi$ degrees with the horizontal (Fig. 417).

Solution.-The forces acting on the cylinder as shown in Fig. $417(a)$ are the weight $W$, the normal pressure $N$, and the friction $F$. By introducing two equal and opposite forces, $F F$, at the mass-center (Fig. 417b), and then resolving $W$ into $x$ - and $y$-components, the original three forces may be resolved into a force, $W \sin \phi-F$, and a couple, $F r$, as shown in Fig. $417(c)$. From equation (4) the work done, then, is,

$$
w=(W \sin \phi-F) \bar{s}+F r \cdot \theta .
$$

But the displacement, $\bar{s}$, of the mass-center equals the length, $s$, of the plane. And, $s=r \theta$ in which $\theta$ is the angular displacement of the cylinder. That is,

Whence,

$$
\bar{s}=s=r \theta .
$$

$$
\begin{aligned}
w & =W \sin \phi \cdot s-F s+F s \\
& =W \sin \phi \cdot s \mathrm{ft} .-\mathrm{lb} .
\end{aligned}
$$

Thus, it will be noted that $F$ does no work, for, if there is no slipping, the point of application of $F$ moves always perpendicular to $F$. Likewise $N$ does no work since its point of application has no displacement in the direction of the 
force. Therefore, the work done on the body is the work done by $W$. But, the work done by $W$ is $W h$ (Art. 182), and from the diagram it will be noted that $h=s \sin \phi$. Hence the work done on the body is $w=W s \sin \phi$ which agrees with the result found above.

183. Power Defined.-The term power as used in mechanics is defined as the rate of doing work. The use or function of many machines depends upon the rate at which they do work as well as upon the amount of work performed. Thus, some machines such as electric generators, steam engines, etc., are rated in terms of the power they are able to develop.

If the rate of doing work is constant, the power, $P$, developed may be defined by the expression,

$$
P=\frac{w}{t}
$$

in which $w$ is the work done in time $t$. If the rate of doing work varies, the power at any instant may be defined by the expression,

$$
P=\frac{d w}{d t}
$$

184. Units of Power.-Power, like work, is a scalar quantity. The unit of power may be any unit of work per unit of time. Thus, in the gravitational system of units, the foot-pound per second (ft.-lb. per sec.) and kilogram-meter per second are common units, whereas in the absolute system, the dyne-centimeter per second (erg per sec.) or joule per second are in common use.

In many problems in engineering, however, it is more convenient to use a larger unit of power than those mentioned above. In the gravitational system of units these larger units are the British or American horse-power (h.p.) and the force de cheval or Continental horse-power. They are defined as follows:

One British or American horse-power $=550 \mathrm{ft}$.-lb. per sec .

$$
=33,000 \mathrm{ft} .-1 \mathrm{~b} \text {. per min. }
$$

One Continental horse-power $=75$ kilogram-meters per sec. $=4500$ kilogram-meters per min.

And in the absolute system, the larger units are the watt and kilowatt which are defined as follows:

One watt $=10^{7}$ ergs per sec.

One kilowatt $=1000$ watts. 
The watt and kilowatt are used extensively in electrical engineering. They may be converted into British horse-power by means of the relations

\section{One horse-power $=746$ watts}

One kilowatt $=1.34$ horse-power.

And, for approximate computations, it is convenient to use 1 horse-power $=\frac{3}{4}$ kilowatt or 1 kilowatt $=\frac{4}{3}$ horse-power.

For expressing very large quantities of work, the units used are the horse-power-hour (h.p.-hr.) and the kilowatt-hour (kw.-hr.). A horse-power-hour is the work done in one hour at a constant rate of one horse-power. Thus,

One horse-power-hour $=33,000 \times 60=1,980,000 \mathrm{ft}$.-lb. Similarly, one kilowatt-hour $=1.34 \times 1,980,000=2,650,000 \mathrm{ft}$.-lb.

185. Special Equations for Power.-If a force, $F$, remains constant in a given displacement of its application point and acts in the direction of the displacement as, for example, the draw-bar pull of a locomotive, the work done in one unit of time is $F v$, in which $v$ is the velocity of the application point, that is, the distance moved through in one unit of time. Hence, if $F$ is expressed in pounds and $v$ in feet per second, the horse-power developed by the force (or the body exerting the force) is,

$$
\text { h. } p .=\frac{F v}{550} .
$$

If the velocity varies, the above equation expresses the horse-power at the instant when the velocity is $v$. If the force does not act in the direction of the displacement of its application point, the working component may be used in the above equation. And, if the force agrees in direction with the displacement but varies in magnitude as, for example, the pressure of the steam against the piston of a steam engine or the tangential effort against the crankpin, then the value of $F$ (or $F_{t}$ ) at any instant may be used to obtain the power at that instant. However, the average power during a given cycle (or many cycles) of operations is generally more useful than the instantaneous power. Thus, in the case of a steam engine the average horse-power is expressed by,

$$
\text { h.p }=\frac{2 \text { Plan }}{33000}
$$


in which $P$ is the mean effective pressure (lb. per sq. in.), $a$ is the piston area (sq. in.), $l$ is the length of stroke (ft.), and $n$ is the number of revolutions per minute (r.p.m.). For, $P a$ is the average force (lb.) which acts through a distance $l \cdot 2 n$ (ft.) per minute, the number of strokes per minute being $2 n$ in a double-acting engine, and hence the work (ft.-lb.) done per minute (power) is $\mathrm{Pa} \cdot l \cdot 2 n$ and the horse-power is as given above.

If a couple having a constant moment, $T$, acts through a given angular displacement of $\theta$ radians, the work done is $T \cdot \theta$ (Art. 179). And, if the couple turns through $\omega$ radians per unit of time, the work done per unit of time is $T \omega$. But, $\omega$ is the angular velocity of turning. Hence, if the moment of the couple is expressed in pound-feet and $\omega$ in radians per second, the horse-power developed by the couple is

$$
h . p .=\frac{T \omega}{550} .
$$

If the angular velocity at which the couple is turning is not constant, the above equation expresses the horse-power at the instant at which the velocity is $\omega$. But, in most cases the average horse-power during a given cycle of operations is of more use than the instantaneous value.

\section{PROBLEMS}

443. A locomotive exerts a constant draw-bar pull of $35,000 \mathrm{lb}$. while increasing the speed of a train from 30 to 45 miles per hour. What horsepower does the engine develop (a) at the beginning of the period; $(b)$ at the end of the period? What is the average horse-power during the period?

444. A man in turning the crank on the winch of a crane was found to exert the forces shown in Fig. 418 at the positions indicated. Plot (freehand) carefully a tangential-effort diagram, the crank (radius) being 15 in. long. Estimate from the diagram the mean tangential effort and calculate the mean horse-power developed by the man assuming that he turns the crank at a constant speed of 40 r.p.m. Ans. 0.55 h.p.

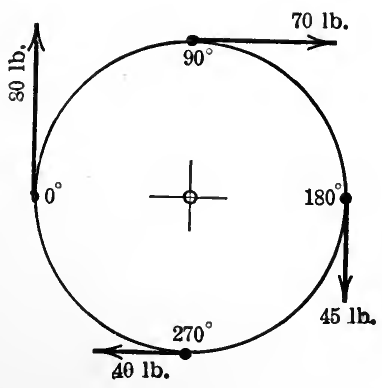

Fig. 418.

445. Two pulleys are keyed to the same shaft $10 \mathrm{ft}$. apart. One pulley is driven by a belt from an engine. The other pulley is belted to, and drives a machine. If the first (driving) pulley receives 3 h.p. from its belt what torque 
is transmitted to the shaft (and driven pulley), assuming that the shaft rotates at a constant speed of 150 r.p.m.

Ans. $105 \mathrm{lb} . \mathrm{ft}$.

446. What indicated horse-power will the engine referred to in Prob. 440 develop if it operates at a constant speed of 250 r.p.m. and is double acting?

447. A generator develops 500 k.w. and delivers 450 k.w. to a machine shop. A price of 4 cents per kw.-hr. is paid. Does the machine shop pay for power or for work? What is the cost to the machine shop per day of 8 hours?

448. A certain machine requires $5 \mathrm{~h}$.p. for its operation. If the machine is in use $6 \mathrm{hr}$. per day how many foot-pounds of work are delivered to the machine in one day?

449. An accumulator loaded to a pressure of $750 \mathrm{lb}$. per square inch is used in emergencies to supply the lubricant to a step bearing of a vertical steam turbine. If the ram is $10 \mathrm{in}$. in diameter and has a stroke of $15 \mathrm{ft}$., what horsepower does it deliver if one hour is required to complete the stroke?

\section{§ 2. ENERGY}

186. Energy Defined.-The energy of a body is the capacity of the body for doing work. Work may be considered to be done by forces, as in the preceding section, or, since forces are exerted by bodies, work may also be considered to be done by the bodies which exert the forces, the work being done by virtue of the energy which the bodies possess. A body may have the capacity to do work (possess energy) due to a variety of conditions or states of the body. Thus, energy may be classified as mechanical energy, heat or thermal energy, chemical energy, electrical energy, etc., depending on the state or condition of the body by virtue of which it is capable of doing work. Our knowledge of all the conditions which render bodies capable of doing work is far from complete, but experience shows that any of the forms of energy may, under the proper conditions, be transformed into any of the other forms.

Mechanical energy is of particular importance in connection with the kinetics of bodies and is therefore considered at some length in the following pages. The other forms of energy are discussed briefly in Art. 191. Mechanical energy is divided into potential energy, or energy of position or configuration, and kinetic energy or energy of motion.

From the definition of energy it follows that energy, like work, is a scalar quantity. Thus, the energy of any mass-system is the algebraic sum of the energies of the various particles of the system regardless of the directions of motion of the particles. 
The units of energy are the same as the units of work discussed in the preceding section.

187. Potential Energy. - The potential energy of a body is the capacity of the body for doing work due to its position or configuration. Thus, the water above a mill dam posesses potential energy which may be used in driving a water wheel; a compressed spring and the compressed steam in a boiler are capable of doing work by virtue of the relative positions (configuration) of their particles.

The potential energy of a body may be defined quantitatively as the amount of work which a body is capable of doing against forces, in passing from the given position or configuration to some standard position or configuration, assuming that no other change in the state or condition of the body takes place. This definition, however, does not lead to a definite quantity for the potential energy of the body for a given configuration, unless the work done by the body (mass-system) depends only on the initial and final configuration of the mass-system and not at all on the paths described by the parts of the system while coming to the standard state. Mass-systems for which this condition is fulfilled are called conservative mass-systems, and the force system which acts on such a mass-system while its potential state changes is called a conservative force system.

The potential energy of conservative systems, only, will be considered herein since, in most kinetics problems which involve non-conservative systems the kinetic energy of the system is of greater importance in the solution of the problems. The most common case of a non-conservative system is that in which the mass-system does work against frictional forces, such as sliding and journal friction and the friction of the particles developed in deforming an inelastic body. Conservative mass-systems occur frequently in engineering problems. In fact any rigid body under the action of a force system in which friction does not occur (or may be considered negligible) is a conservative system, provided, of course, that no change in the state or condition of the body except that of configuration takes place. A common example of a conservative system is that of the earth and an elevated body (whether rigid or not). The work done on the body in any displacement is equal to the earth-pull (weight) of the body times the vertical displacement of the center of gravity of the body (Art. 
182), regardless of the intermediate positions occupied by the body in moving from one position to another position. Another example is that of an elastic body, for, if the body is elastic the energy possessed by the body when in a given strained condition, that is, for a given configuration of its particles, is the same regardless of the relative displacements of the particles which occurred while being put in the given strained condition. The standard configuration may be arbitrarily chosen, but, for convenience, it is so chosen that the potential energy of the body is positive. Thus, in the case of the earth and an elevated body the earth is considered fixed and the standard configuration occurs when the body is in contact with the earth. A discussion of the mathematical test for a conservative system is beyond the scope of this book.

188. Kinetic Energy.-The kinetic energy of a body is its capacity for doing work due to its motion. Thus, by virtue of its kinetic energy, a body is capable of doing work against forces which change its motion. For example, a jet of water does work on a tangential water-wheel; a steam forging hammer does work on the material which is deformed by the hammer; the rotating flywheel on a punching machine does work in punching the hole in the metal plate, etc.

The kinetic energy of a body at any instant may be defined quantitatively as the amount of work that the body is capable of doing against forces which destroy its motion, that is, which bring it to a state of rest. The expression for the kinetic energy of a body (mass-system) should, therefore, contain a quantity (velocity) which is a measure of the motion of the body and also a quantity (mass, moment of inertia, etc.) which is a measure of the (kinetic) property of the body that has an influence in governing its change of motion. The "velocity of a mass-system," however, is, in general, an indefinite and meaningless phrase since the velocities of the various parts of a system are not the same. Hence, an expression for the kinetic energy of a particle is first obtained and, since energy is a scalar quantity, the kinetic energy of a system of particles (mass-system) is the algebraic sum of the kinetic energies of the particles. However, the expression for the kinetic energy of a particle is of considerable importance in itself since in many problems a physical body may be regarded as a particle without introducing serious errors.

189. Kinetic Energy of a Particle.-In Fig. 419, let $P$ be a par- 
ticle of mass $m$ in a body (assumed rigid for convenience only) which moves so that $P$ travels from position $P^{\prime}$ to $P^{\prime \prime}$, along the path shown, while its velocity decreases from $v$ at $P^{\prime}$ to zero at $P^{\prime \prime}$ due to the forces against which the particle does work. The work done on the particle by the forces (which form a concurrent system) is equal to the work done by their resultant $R$. Or, $w=\int_{s_{1}}^{s_{2}} R_{t} d s$.

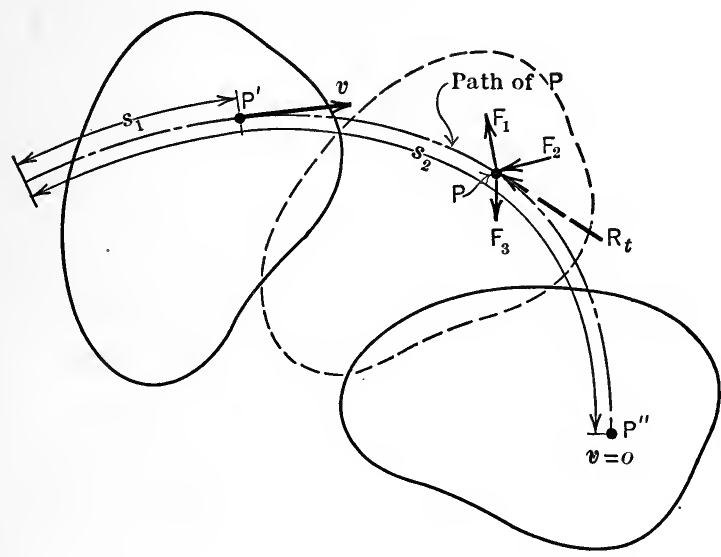

FIG. 419.

But, by definition, the kinetic energy, $E_{k}$, of the particle is the work which the particle does against the forces. Hence, the defining equation for the kinetic energy of a particle is,

$$
E_{\mathbf{k}}=-w=-\int_{s_{1}}^{s_{2}} R_{t} d s .
$$

This expression may be transformed so that $E_{\mathrm{k}}$ is expressed in terms of $m$ and $v$ by means of the following relations,

Thus,

$$
R_{t}=m a_{t}, \quad a_{t}=\frac{d v}{d t}, \quad \text { and } \quad \frac{d s}{d t}=v .
$$

$$
\begin{aligned}
E_{k} & =-\int_{s_{1}}^{s_{2}} R_{t} d s \\
& =-\int_{s_{1}}^{s_{2}} m a_{t} d s=-\int_{s_{1}}^{s_{2}} m \frac{d v}{d t} d s \\
& =-\int_{s_{1}}^{s_{2}} m \frac{d s}{d t} d v=-\int_{v}^{0} m v d v \\
& =\frac{1}{2} m v^{2} .
\end{aligned}
$$


Therefore, the kinetic energy of a particle of mass $m$ having a velocity $v$ is equal to $\frac{1}{2} m v^{2}$. That is,

$$
E_{\mathrm{k}}=\frac{1}{2} m v^{2}
$$

\section{PROBLEMS}

450. By making use of the equations of Art. 124, prove that the kinetic energy of a particle having uniformly accelerated rectilinear motion is $\frac{1}{2} m v^{2}$.

451. The German long-range gun which shelled Paris from a distance of approximately 76 miles was $118 \mathrm{ft}$. long. The muzzle velocity of the projectile was not far from $5000 \mathrm{ft}$. per sec. The diameter of the projectile was $8.15 \mathrm{in}$. and its weight was $264 \mathrm{lb}$. It attained a height of about 24 miles, was in flight about $3 \mathrm{~min}$., and reached Paris with a velocity of about $2300 \mathrm{ft}$. per sec. (For a description of the gun see Journal A. S. M. E., Feb., 1920.) Neglecting the energy due to the rotation of the projectile, calculate the kinetic energy of the projectile as it left the gun, and also its energy at the end of its flight. Find the loss of kinetic energy per second during the flight.

190. Kinetic Energy of a Body.-Since energy is a scalar quantity the kinetic energy of a body (whether rigid or not) is the algebraic sum of the kinetic energies of its particles. Hence, for any mass-system,

$$
E_{k}=\Sigma \frac{1}{2} m v^{2} .
$$

It is convenient, however, to express the kinetic - energy of a rigid body in terms of the mass (or some other kinetic property such as moment of inertia) of the whole body and the velocity of some particular point in the body (as, for example, the masscenter) or the angular velocity of the whole body. Thus, for rigid bodies having the special motions of translation, rotation, and plane motion the expressions for the kinetic energy are found as follows:

1. Translation of a Rigid Body.-All parts of the body have the same velocity at any instant whether the motion is rectilinear translation or curvilinear translation (Art. 132), that is, $v$ is constant. Hence,

$$
E_{\mathrm{k}}=\Sigma \frac{1}{2} m v^{2}=\frac{1}{2} v^{2} \Sigma m
$$

But, $\Sigma m$ is the mass of the body and may be denoted by $M$. Therefore,

$$
E_{\mathrm{k}}=\frac{1}{2} M v^{2}
$$


II. Rotation of a Rigid Body. - The angular velocities of all particles are the same at any instant, that is, the angular velocity $\omega$ of any particle is the angular velocity of the body. The linear velocity, $v$, of any particle, $P$, of mass $m$, at a distance, $r$, from the axis of rotation, $O$, (Fig. 420) is equal to $r \omega$. Hence, the kinetic energy of the body is

$$
E_{\mathrm{t}}=\Sigma \frac{1}{2} m v^{2}=\frac{1}{2} \Sigma m(\omega r)^{2}=\frac{1}{2} \omega^{2} \Sigma m r^{2} .
$$

But, $\Sigma m r^{2}$ is the moment of inertia of the body with respect to the axis from which $r$ is measured, that is, the axis of rotation, and is denoted by $I_{0}$. Thus, $I_{0}=\Sigma m r^{2}$.

Therefore,

$$
E_{\mathrm{k}}=\frac{1}{2} I_{0} \omega^{2} .
$$

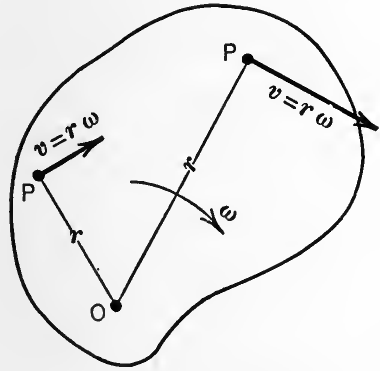

Fig. 420 .

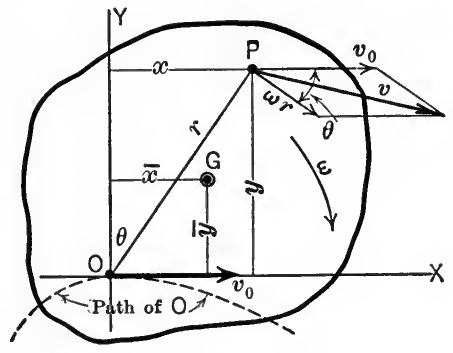

FIG. 421.

III. Plane Motion of a Rigid Body.-The angular velocities of all particles are the same at any instant, that is, the angular velocity $\omega$ of any particle is the angular velocity of the body. Further, as shown in Art. 134, the motion of the body at any instant may be considered to be a combination of a rotation about an axis through any point, $O$, in the plane of motion and a translation defined by the motion of $O$. Hence, the velocity, $v$, of any particle $P$ of mass $m$ (Fig. 421) is the resultant of the velocity, $\omega r$, which $P$ is given by the rotation about $O$, and the velocity, $v_{0}$, which is given to all particles by the translation. And, since the body is rigid the velocity $\omega r$ has a direction perpendicular to $r$. Thus,

$$
v^{2}=(\omega r)^{2}+v_{0}^{2}+2 v_{0} \omega r \cos \theta .
$$

In Fig. 421, let $O$ be the origin and, for simplicity, let the $x$-axis have the same direction as $v_{0}$. The kinetic energy of the body may 
then be found in terms of the mass of the whole body, the angular velocity of the body, and the linear velocity of one point (in this case the point $O$ ) in the body as follows:

$$
\begin{aligned}
E_{k} & =\Sigma \frac{1}{2} m v^{2}=\frac{1}{2} \Sigma m\left(\overline{\omega r}^{2}+v_{0}^{2}+2 v_{0} \omega r \cos \theta\right) \\
& =\frac{1}{2} \Sigma m \omega^{2} r^{2}+\frac{1}{2} \Sigma m v_{0}^{2}+\Sigma m v_{0} \omega r \cos \theta \\
& =\frac{1}{2} \omega^{2} \Sigma m r^{2}+\frac{1}{2} v_{0}^{2} \Sigma m+\omega v_{0} \Sigma m r \cos \theta .
\end{aligned}
$$

But, $\Sigma m r^{2}$ is the moment of inertia of the body with respect to the axis through $O$ from which $r$ is measured. Thus, $\Sigma m r^{2}=I_{0}$. Further, $r \cos \theta=y$, whence, $\Sigma m r \cos \theta=\Sigma m y=M \bar{y}$ in which $M$ is the mass of the body. Therefore,

$$
E_{k}=\frac{1}{2} I_{0} \omega^{2}+\frac{1}{2} M v_{0}^{2}+M \bar{y} \omega v_{0} .
$$

Now since the point $O$ is any point in the plane of motion it may be chosen at the mass-center. That is, the motion of the body may be resolved into a rotation about an axis through the mass-center and a translation defined by the motion of the mass-center. If the point $O$ is made the mass-center, then, $\overline{\bar{y}}=0, I_{0}$ becomes $\bar{I}$, and $v_{0}$ becomes $\bar{v}$. Hence, the kinetic energy is given by the expression,

$$
E_{\mathrm{k}}=\frac{1}{2} \bar{I} \omega^{2}+\frac{1}{2} M \bar{v}^{2} .
$$

It is important to note that, although plane motion of a rigid body may be resolved at any instant, into a rotation about an axis through any point in the plane of motion and a translation defined by the motion of that point, it does not follow that the kinetic

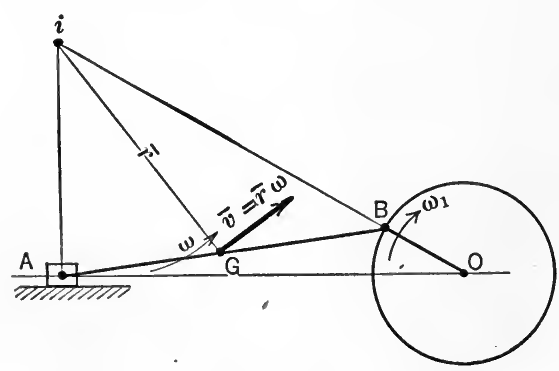

FIG. 422 . energy of the body at the given instant is the kinetic energy due to the rotation plus the kinetic energy due to the translation, unless the assumed rotation is about an axis through the mass-center of the body and the translation is defined by the motion of the mass-center.

Alternative Method.-Plane motion of a rigid body may be considered to be pure rotation about the instantaneous axis of 
rotation (Art. 135). The kinetic energy of a body, as for example, the connecting rod represented in Fig. 422, therefore, is,

$$
E_{\mathbf{k}}=\frac{1}{2} I_{i} \omega^{2}
$$

in which $I_{i}$ is the moment of inertia of the body with respect to the instantaneous axis. But, by the parallel axis theorem (Art. 102), $I_{i}=\bar{I}+M \bar{r}^{2}$. Therefore,

$$
E_{\mathrm{k}}=\frac{1}{2}\left(\bar{I}+M \bar{r}^{2}\right) \omega^{2}=\frac{1}{2} \bar{I} \omega^{2}+\frac{1}{2} M \bar{v}^{2} .
$$

\section{PROBLEMS}

452. A body $B$ weighing $60 \mathrm{lb}$. is attached by a flexible string to a spring $S$ (Fig. 423). The pulley over which the string passes is weightless and frictionless. The spring has a modulus of $40 \mathrm{lb}$. per inch. If a force $P$ of $50 \mathrm{lb}$. is gradually applied to $B$, how much is the potential energy of the spring changed? Of the spring and body $B$ considered as one system?

453. A slender rod, similar to the spoke of a flywheel, is $3 \mathrm{ft}$. long and rotates about an axis through one end at a constant speed of 120 r.p.m. The rod weighs $50 \mathrm{lb}$. Find the kinetic energy which the rod possesses.

Ans: $E_{k}=368 \mathrm{ft} .-1 \mathrm{~b}$.

454. Two spherical bodies each weighing $20 \mathrm{lb}$. are connected by a slender rod and revolve at 90 r.p.m. in a horizontal plane about a vertical axis located midway between the two bodies. The center of each ball is

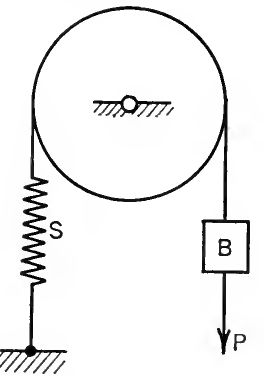

Fig. 423. $10 \mathrm{in}$. from the axis. The diameter of each ball is $4 \mathrm{in}$. The weight of the rod is $6 \mathrm{lb}$. Find the kinetic energy of the system. Ans. $E_{K}=40.5 \mathrm{ft} .-\mathrm{lb}$.

455. The winding drum of a mine hoist is $14 \mathrm{ft}$. in diameter, its radius of gyration is $6 \mathrm{ft}$., and its weight is 7 tons. A cage weighing 6 tons is raised by it. When the cage is rising at the rate of $40 \mathrm{ft}$. per sec. what is the kinetic energy of the system? Ans. $E_{k}=554,000 \mathrm{ft}$.-lb.

456. The cast iron flywheel (Fig. 424) is used on a punching machine in the forge shop at the University of Illinois. If the flywheel is rotating at a speed of 225 r.p.m. when the punch starts to punch a hole and has its speed reduced 20 per cent in punching the hole, how much energy does the flywheel give up; (a) neglecting hub and spoke, (b) including hub and spokes, assuming the spokes to have a constant mean cross-section and to run from the hub to the rim?

457. Find the kinetic energy of the rocker arm $A D$ (Fig. 425) from the following data: $O B=10 \mathrm{in}$.; $O A=3 \mathrm{ft}$.; angular velocity of crank $O B=50$ r.p.m.; $\theta=30^{\circ}$; length of $\operatorname{rod} A D=4.5 \mathrm{ft}$.; and the weight of the rod is $22.7 \mathrm{lb}$. 
458. Find the kinetic energy of the connecting rod, the motion of which is described in Prob. 386.

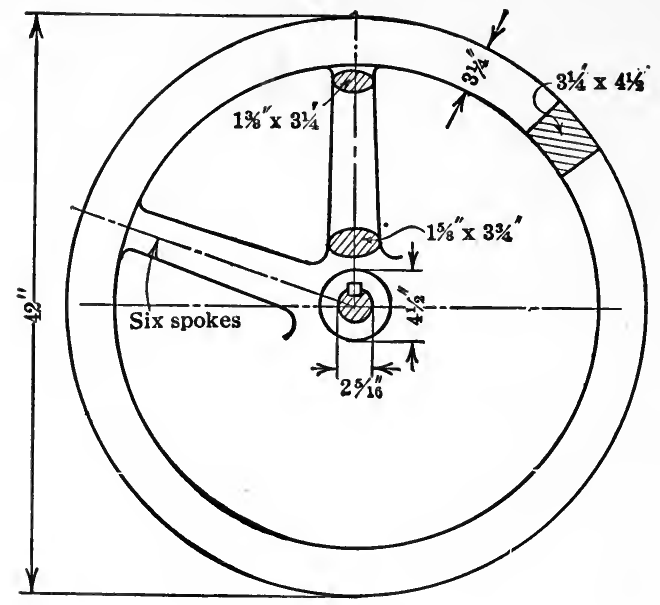

Fig. 424.

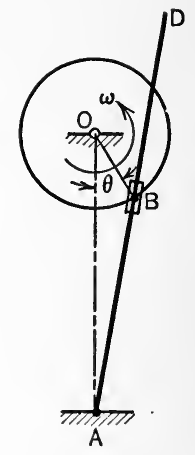

Fig. 425.

191. Non-mechanical Energy.-Experience shows that some bodies are capable of doing work (possess energy) by virtue of certain states or conditions of their parts, the nature of which is not definitely enough known to make it possible to determine their energy by the methods used in the preceding articles. Energy which cannot be determined directly as potential or kinetic energy is called non-mechanical or non-dynamic energy. Thus, heat or thermal energy, chemical energy, and electrical energy are forms of non-mechanical energy.

A body is capable of doing work by reason of its heated state or condition since by giving up its heat it may do work, under favorable conditions, as in the case of steam in the cylinder of a steam engine. Energy possessed by a body by virtue of its heated state is called heat or thermal energy.

Certain bodies are capable of doing work by reason of their chemical state or condition. Thus, hydrogen and oxygen, under favorable conditions, combine and give evidence of considerable energy by transferring heat to surrounding bodies. Likewise, carbon (coal) and oxygen combine and produce heat which as noted above may in turn do work. Energy possessed by bodies due to the state of their chemical elements is called chemical energy. 
Some bodies are capable of doing work by virtue of their electrical state or condition. Thus, a copper wire on an armature moving in a field of force may develop electric current which in turn may do work in driving a motor. Or, a charged storage battery may do work as its electrical condition changes, etc. Energy which arises out of the electrical conditions of bodies is called electrical energy.

Any one of these, so-called, special forms of energy may be converted, under favorable conditions, into mechanical energy, and there is considerable evidence to indicate that all energy is mechanical energy. Thus, according to this view, the heat energy of a body could be determined as kinetic energy if the motions of the individual particles were known. And, certain forms of chemical and electrical energy could be determined as potential energy if the molecular forces were definitely known. Therefore, the energy possessed by bodies by virtue of special states of their molecular structure are considered as non-mechanical forms of energy, not because these special forms are necessarily different from mechanical energy, but because the energy cannot be determined directly as mechanical energy, and, therefore, has to be transformed into mechanical energy and then measured. Thus, one unit of heat energy, the British thermal unit (B.t.u.), has a definite mechanical equivalent which carefully made experiments have shown to be

\section{B.t.u. $=778$ ft.-lb.}

However, the lack of knowledge of the molecular structure and conditions by virtue of which bodies possess energy does not prevent the application of certain principles of energy to such conditions. In fact, the outstanding feature concerning energy is that certain general principles of energy, such as principles of the conservation of energy and of degradation of energy, etc., are the basis upon which our knowledge of the behavior of non-rigid bodies, in general, is built and thus they furnish a method of approach to problems for which the principles of force, mass, and acceleration are inadequate. They are of special importance, therefore, in the study of hydraulics, thermodynamics, electrodynamics, physical chemistry, etc.

In the following section certain principles concerning mechanical energy are developed and applied to the motion of bodies 
(mainly rigid) in which the motion of the particles are definitely known. And even though the principles of force, mass, and acceleration may be used for many of the problems considered, nevertheless, it will be noted that even for rigid bodies the principles of work and energy are of great importance in many kinetics problems as met in engineering practice.

\section{§3. Principles of Work and Energy}

192. Preliminary.-As stated in Art. 137, in order to deal with the main problem in kinetics, a relation is found between the forces acting on the body, the kinetic properties (mass, moment of inertia, etc.) of the body by virtue of which it influences its own motion, and the change of motion (involving acceleration, velocity, distance, etc.) of the body. This may be done by determining the relation between the work done by the forces acting on the body and the kinetic energy of the body, since work and kinetic energy involve quantities in terms of which the three factors in the kinetics problem as mentioned above are expressed. And since, in general, the motion of all particles of a body are not the same, the principle of work and kinetic energy will be developed for a particle first and then extended to the motion of a body. However, as already noted, in many problems the body may be regarded as a particle without introducing serious errors.

193. Principle of Work and Kinetic Energy.-I. For a Particle.-In Fig. 426 let $A^{\prime}$

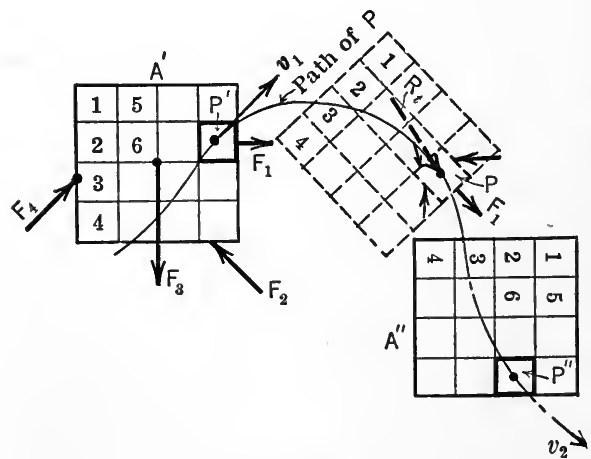

Fig. 426. and $A^{\prime \prime}$ be two positions of a body, the motion of which changes due to the unbalanced forces $\left(F_{1}, F_{2}\right.$, $F_{3}$, and $F_{4}$ ) which act on it. The body is assumed, for convenience, to be composed of small cubes of different materials rigidly attached (glued together), each cube being regarded as a particle of the body. Let $P$ be one of the particles which describes the path shown in the figure as the particle moves from $P^{\prime}$ to $P^{\prime \prime}$ while its velocity changes from $v_{1}$ to $v_{2}$ due 
to the unbalanced (concurrent) forces which act on it. Let the resultant of the forces acting on $P$ be denoted by $R$ and its component tangent to the path of $P$ be denoted by $R_{t}$. It will be noted that some of the particles (cubes) have their velocities changed (are accelerated) by forces exerted only by other particles of the body (internal forces) whereas other particles are acted on both by internal and by external forces.

The work done by the forces acting on the particle as it moves along its path from $P^{\prime}$ to $P^{\prime \prime}$ is $w=\int_{s_{1}}^{s_{2}} R_{t} d s$ but, as in Art. 189, $R_{t} d s$ may be expressed in terms of the mass, $m$, and velocity, $v$, of the particle by means of the relations,

$$
R_{t}=m a_{t}, \quad a_{t}=\frac{d v}{d t}, \quad \text { and } \quad v=\frac{d s}{d t} .
$$

The work done on the particle $=\int_{s_{1}}^{s_{2}} R_{t} d s$

$$
\begin{aligned}
& =\int_{s_{1}}^{s_{2}} m a_{t} d s=\int_{s_{1}}^{s_{2}} m \frac{d v}{d t} d s \\
& =\int_{v_{1}}^{v_{2}} m \frac{d s}{d t} d v \\
& =\int_{v_{1}}^{v_{2}} m v d v \\
& =\frac{1}{2} m v_{2}^{2}-\frac{1}{2} m v_{1}^{2} \\
& =\text { change in kinetic energy of the } \\
& \quad \text { particle. }
\end{aligned}
$$

Therefore, the work done by the forces acting on a particle of a body (whether rigid or not) during any displacement is equal to the change in the kinetic energy of the particle in the same displacement. Or, expressed in the form of an equation,

$$
w=\Delta E_{\mathrm{k}}=\frac{1}{2} m v_{2}^{2}-\frac{1}{2} m v_{1}^{2} .
$$

II. For a System of Particles.-The principle of work and kinetic energy for a system of particles (body) may now be derived. Since work and energy are scalar quantities, the work done on a body is the algebraic sum of the works done on all the particles. And, the change in the kinetic energy of the body is the algebraic sum of the changes in the kinetic energies of all the particles. 
But, the work done by all the forces acting on all the particles equals the work done by the external forces which act on the body plus the work done by the internal forces of the body. Thus, by writing the above equation for each particle and adding both sides of the equations, the resulting equation is,

$$
w_{e}+w_{i}=\frac{1}{2} \Sigma m v_{2}^{2}-\frac{1}{2} \Sigma m v_{1}^{2}=\Delta E_{k} .
$$

That is, the work done on a system of particles (whether rigid or not) by all of the external and internal forces in any displacement of the system is equal to the change in the kinetic energy of the system in the same displacement.

III. For a Rigid Body.-The principle may now be expressed for the special case of a rigid body, for, as pointed out in Art. 182, the internal forces in any mass-system occur in pairs of equal opposite and collinear forces whether the body is rigid or not, but the work done by these forces is zero, in general, only if the application points of each pair of forces remain a fixed distance apart, which is the case in a rigid body. Hence, for a rigid body, $w_{t}=0$. Therefore,

$$
w_{e}=\Delta \mathbf{E}_{k} .
$$

That is, the work done by the external forces acting on a rigid body in any displacement is equal to the change in the kinetic energy of the body in the same displacement.

Although an absolutely rigid body does not exist in nature, the work done by the external forces in causing the relative displacements of the part.c.es in physical bodies is usually negligible in comparison with the work done by the forces in causing the displacement of the body as a whole.

194. Application of Principle to Special Cases of Motion of Rigid Bodies.-By making use of the expressions developed in Art. 182 and 190, the principle of work and kinetic energy may be expressed for important special cases of motion of rigid bodies as follows:

I. For a Rigid Body Having Uniformly Accelerated Rectilinear Translation.-

$$
\Sigma F_{a} \cdot s=\frac{1}{2} M\left(v_{2}^{2}-v_{1}^{2}\right) .
$$

II. For a Rigid Body Rotating about an Axis through the Masscenter with Constant Angular Acceleration.-

$$
\Sigma \bar{T} \cdot \theta=\frac{1}{2} \bar{I}\left(\omega_{2}^{2}-\omega_{1}^{2}\right)
$$


III. For a Rigid Body Having Plane Motion Such that the Angular Acceleration is Constant and the Mass-center has Uniformly Accelerated Rectilinear Motion.-

$$
\Sigma F_{\bar{a}} \cdot s+\Sigma \bar{T} \cdot \theta=\frac{1}{2} M\left(\bar{v}_{2}^{2}-\bar{v}_{1}^{2}\right)+\frac{1}{2} \bar{I}\left(\omega_{2}^{2}-\omega_{1}^{2}\right) .
$$

As noted in Art. 182 the work done by the forces acting on any body may be found by taking the algebraic sum of the works done by all the forces. And in more general cases of motion than those stated above, it will be, as a rule, more convenient to find the work done in this way rather than from the resultant of the forces.

\section{ILLUSTRATIVE PROBLEMS}

459. An engine capable of exerting a maximum draw-bar pull of 51,000 lb. is used on a certain railroad having small grades to draw freight trains having a maximum weight of 2000 tons, the average weight of a freight car with its cargo being about 45 tons. The train resistance per ton of weight varies with the car weight and with the speed. If an average value of $8 \mathrm{lb}$. per ton is used and the engine pulls a 2000-ton train

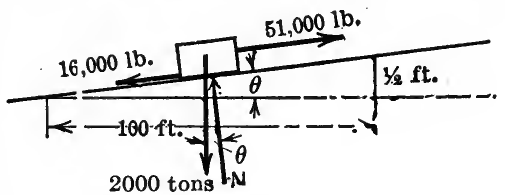

Fig. 427. while going up a $\frac{1}{2}$ per cent grade, how far will the train travel while its velocity is increasing from 15 to $30 \mathrm{mi}$. per hour? How long will it take?

Solution.-The forces acting on the train are shown in the free-body diagram (Fig. 427). The angle $\theta$ (exaggerated in the diagram) for a $\frac{1}{2}$ per cent grade is so small that $\tan \theta$ may be considered to be equal to $\sin \theta$. Hence, $\sin \theta=\frac{1}{200}$.

$$
\begin{gathered}
w_{e}=\Delta E_{k}, \\
\Sigma F_{a} \cdot s=\frac{1}{2} M\left(v_{2}{ }^{2}-v_{1}{ }^{2}\right) \\
\left(51,000-16,000-\frac{2000 \times 2000}{200}\right) s=\frac{1}{2} \frac{2000 \times 2000}{32.2}\left(\overline{44}^{2}-\overline{22}^{2}\right) \\
15,000 s=62,200 \times 1452, \\
s=6020 \mathrm{ft} .
\end{gathered}
$$

Since,

we have,

$$
s=\frac{v_{1}+v_{2}}{2} \times t
$$

Whence,

$$
6020=\frac{22+44}{2} \times t .
$$

$$
\begin{aligned}
t & =182.5 \mathrm{sec} . \\
& =3.04 \mathrm{~min} .
\end{aligned}
$$


460. In the friction-board type of drop hammer used in the production of drop forgings, the ram (Fig. 428) is attached to the lower end of a friction board which is lifted by friction driving rolls and these in turn are driven by spur gears. The rolls are pressed against the board by revolving the eccentric bearings and they may be released quickly to let the ram drop. The following

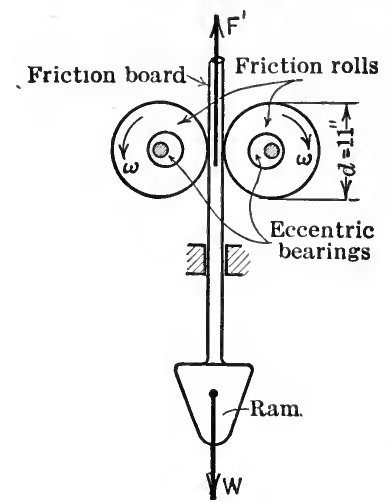

FIG. 428 . data apply to one particular hammer:

Weight of ram, $W=1000 \mathrm{lb}$.

Angular velocity of rolls, $\omega=130$ r.p.m.

Diameter of rolls, $d=11$ in.

Normal pressure against friction board, $N=6000 \mathrm{lb}$.

Coefficient of friction, $\mu=0.3$.

Total height to which ram is raised, $h=$ $4.5 \mathrm{ft}$.

Find the distance the ram travels $(a)$ while being accelerated, that is, while its velocity is being brought up to the peripheral speed of the friction rolls; $(b)$ while the ram is coming to rest after the rolls are released; $(c)$ while the ram is moving with constant velocity, after the acceleration ceases and before the rolls are released. Also find the time (seconds) required to travel each of these distances and the time to complete a cycle (total time). Assume that the friction rolls do not slip on the friction board. Although there is some slipping just as the rolls are pressed against the board, this assumption will not introduce serious errors in the problem.

Solution.-The friction force, $F^{\prime}$, during the acceleration period is,

$$
F^{\prime}=\mu N=0.3 \times 6000=1800 \mathrm{lb} .
$$

The peripheral speed of the rolls is,

$$
v=\omega r=\frac{130 \times 2 \pi}{60} \times \frac{5.5}{12}=6.25 \mathrm{ft} . / \mathrm{sec} .
$$

During the acceleration period, the forces acting on the ram are $F^{\prime}$ and $W$, as shown in Fig. 428. Applying the principle of work and energy, we have,

$$
\begin{aligned}
w_{\epsilon} & =\Delta E_{k}, \\
\left(F^{\prime}-W\right) s_{1} & =\frac{1}{2} M\left(v^{2}-0\right), \\
(1800-1000) s_{1} & =\frac{1}{2} \frac{1000}{32.2} \times(6.25)^{2} . \\
800 s_{1} & =606 . \\
s_{1} & =0.758 \mathrm{ft} .
\end{aligned}
$$

Hence, 
After the rolls are released, the ram is acted upon only by the earth-pull(weight) while it gives up its kinetic energy. Thus,

$$
\begin{gathered}
w_{e}=\Delta E_{k}, \\
-W \cdot s_{3}=0-\frac{1}{2} M v^{2}, \\
1000 \times s_{3}=\frac{1}{2} \frac{1000}{32.2} \times(6.25)^{2} .
\end{gathered}
$$

Therefore,

$$
s_{3}=0.606 \mathrm{ft} \text {. }
$$

Hence,

$$
\begin{aligned}
s_{2} & =h-\left(s_{1}+s_{3}\right) \\
& =4.5-(0.758+0.606) \\
& =3.14 \mathrm{ft} .
\end{aligned}
$$

Since the distance traveled in each period is the average speed times the time, we have,

Therefore,

$$
\begin{aligned}
s_{1} & =\frac{v}{2} \times t_{1}, \\
0.758 & =\frac{6.25}{2} \times t_{1} .
\end{aligned}
$$

Likewise,

$$
t_{1}=0.243 \text { sec. }=\text { duration of acceleration period. }
$$

$$
0.606=\frac{6.25}{2} \times t_{3} \text {. }
$$

Whence,

Also,

$$
t_{3}=0.194 \text { sec. }=\text { duration of decelerating period. }
$$

whence,

Further,

$$
3.14=6.25 \times t_{2} \text {, }
$$

$t_{2}=0.503$ sec. $=$ duration of constant velocity period.

$$
t_{4}=\sqrt{\frac{2 h}{g}}=\sqrt{\frac{2 \times 4.5}{32.2}},
$$

Hence,

$$
=0.528 \text { sec. }=\text { time of falling. }
$$

$$
\begin{aligned}
t & =t_{1}+t_{2}+t_{3}+t_{4} \\
& =1.468 \text { sec. }=\text { time of complete cycle. }
\end{aligned}
$$

461. The punching and shearing machine shown in Fig. 429 has a capacity for punching a $2 \frac{1}{2}$-in. hole in a $\frac{1}{2}$-in. steel plate. The pinion shaft (and flywheel) is driven at 220 r.p.m. from a counter shaft by means of a belt drive to the tight pulley on the pinion shaft. Each operation of punching a hole (punching cycle) causes a fluctuaton (decrease) in the speed of the flywheel. The "coefficient of speed fluctuation" is 0.8 , that is, the speed of the flywheel decreases 20 per cent in each punching cycle. If the work done in punching the hole is all supplied by the flywheel, what moment of inertia should the flywheel have? What is the moment of inertia of the flywheel on the machine 
as actually designed (as shown in Fig. 429), neglecting the material in the hub and spokes? If the difference in the belt tensions, $T_{2}-T_{1}$, is $286 \mathrm{lb}$. and the pinion shaft turns through an angle of $230^{\circ}$ while the hole is being punched, how much work is done on the shaft (and flywheel) by the beit while the hole is being punched? The diameter of the pulley is $22 \mathrm{in}$.

Solution.-The maximum value of the force $P$ required to punch the hole,

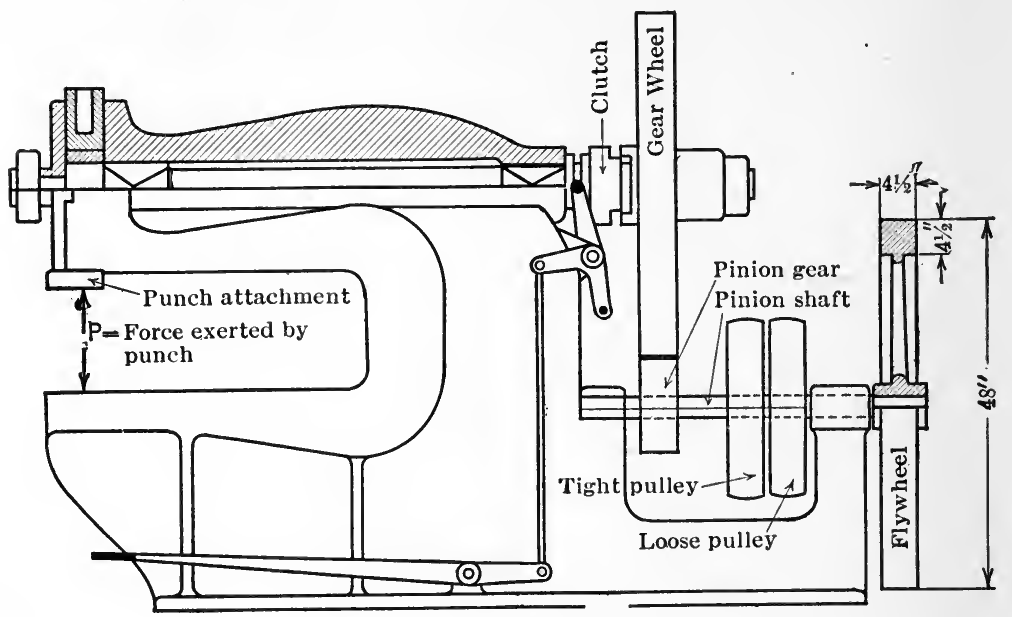

FIG. 429.

using $60,000 \mathrm{lb}$. per square inch for the ultimate shearing strength of the material of the plate, is

$$
\begin{aligned}
P & =\pi d \times t \times 60,000 \\
& =\pi \times \frac{5}{2} \times \frac{1}{2} \times 60,000 \\
& =235,500 \mathrm{lb} .
\end{aligned}
$$

The work done in punching the hole, assuming a triangular work diagram (see Prob. 435), is,

$$
\begin{aligned}
w & =\frac{P}{2} \times \frac{1}{2}=\frac{235,500}{4}=58,875 \text { in.-lb. } \\
& =4900 \mathrm{ft} .-\mathrm{lb} . \text { which is supplied by the flywheel. }
\end{aligned}
$$

For the flywheel we have then,

Whence,

$$
\begin{aligned}
w_{e} & =\Delta E_{k} \\
4900 & =\frac{1}{2} \bar{I}\left(\omega_{2}{ }^{2}-\omega_{1}{ }^{2}\right) \\
& =\frac{1}{2} \bar{I}\left[\left(\frac{220 \times 2 \pi}{60}\right)^{2}-\left(0.8 \times \frac{220 \times 2 \pi}{60}\right)^{2}\right]=95.5 \bar{I} .
\end{aligned}
$$

$\bar{I}=51.3$ slug-ft. ${ }^{2}$ 
Hence, the moment of inertia of the flywheel should be 51.3 slug-ft. ${ }^{2}$ in order that the speed be decreased not more than 20 per cent. Assuming the flywheel to be made of cast-iron which weighs $450 \mathrm{lb}$. per cubic foot, the moment of inertia of the flywheel as actually designed, neglecting hub and spokes; is

$$
\begin{aligned}
\bar{I} & =\frac{1}{2} M\left(r_{2}{ }^{2}+r_{1}{ }^{2}\right) \\
& =\frac{1}{2} \times \frac{\pi}{32.2}\left[2^{2}-\left(\frac{19.5}{12}\right)^{2}\right] \times \frac{4.5}{12} \times 450 \times\left[2^{2}+\left(\frac{19.5}{12}\right)^{2}\right] \\
& =\frac{1}{2} \frac{\pi}{32.2} \times 1.36 \times 0.375 \times 450 \times 6.64 \\
& =74.3 \text { slug-ft. }{ }^{2} .
\end{aligned}
$$

The work done on the flywheel while the hole is being punched is,

$$
\begin{aligned}
w & =\Sigma T \cdot \theta \\
& =286 \times \frac{11}{12} \times 230 \times \frac{\pi}{180} \\
& =1050 \mathrm{ft} .-\mathrm{lb} .
\end{aligned}
$$

462. A solid homogeneous cylinder rolls up an inclined plane without slipping (Fig. 430). The weight of the cylinder is $120 \mathrm{lb}$. and its diameter is $3 \mathrm{ft}$. The angle, $\phi$, of inclination of the plane is $15^{\circ}$. If the velocity of the center of the cylinder is $20 \mathrm{ft}$. per sec. just as it comes in contact with the incline, how far up the plane will the cylinder roll?

Solution.-The forces acting on the cylinder while rolling up the plane are shown in Fig.

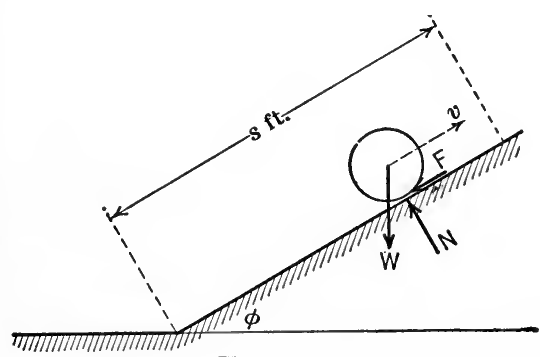

Frg. 430. 430. And, the work done by the forces, as was shown in Prob. 442, is $W \sin \phi \cdot s$. Thus,

$$
w_{e}=\Delta E_{k},
$$$$
-W \sin \phi \cdot s=\frac{1}{2}\left(M \bar{v}_{2}^{2}+\vec{I} \omega_{2}^{2}\right)-\frac{1}{2}\left(M \bar{v}_{1}^{2}+\widetilde{I} \omega_{1}^{2}\right) .
$$

Or,

$$
W \sin \phi \cdot s=\frac{1}{2}\left(M \bar{v}_{1}{ }^{2}+\bar{I} \omega_{1}^{2}\right) \text {, since } \bar{v}_{2} \text { and } \omega_{2} \text { are zero. }
$$

Also

Hence,

$$
\bar{v}=\bar{r} \omega=1.5 \omega .
$$

Whence,

$$
120 \times 0.2588 \times s=\frac{1}{2} \frac{120}{32.2}(20)^{2}+\frac{1}{2}\left(\frac{1}{2} \frac{120}{32.2} \times \overline{1.5}^{2}\right)\left(\frac{20}{1.5}\right)^{2} .
$$

$$
s=\frac{746+372}{31.1}=36 \mathrm{ft} \text {. }
$$




\section{PROBLEMS}

463. A body weighing $80 \mathrm{lb}$. is projected along a rough horizontal plane with a velocity of $8 \mathrm{ft}$. per sec. It comes to rest in a distance of $10 \mathrm{ft}$. Find the coefficient of kinetic friction.

Ans. $\mu=0.099$.

464. An automobile which weighs $W \mathrm{lb}$. is moving at the rate of 30 miles per hour when it comes to the foot of a hill. Power is then shut off. The slope of the hill is $1 \mathrm{ft}$. in $50 \mathrm{ft}$. How far will the machine coast up the hill if the total frictional resistance (parallel to the road) is $0.08 \mathrm{~W}$ ?

$$
\text { Ans. } s=301 \mathrm{ft} \text {. }
$$

465. A sphere rotates about an axis through its center. Its speed is increased from $600 \mathrm{rad}$. per min. to $90 \mathrm{rad}$. per sec. while it turns through 10 revolutions. If the moment of inertia of the sphere with respect to the axis of rotation is $20 \mathrm{lb}$. sec. ${ }^{2} \mathrm{ft}$. (or slug-ft. ${ }^{2}$ ), find the moment of the couple acting on the sphere.

466. The winding drum of a mine hoist is $15 \mathrm{ft}$. in diameter, its radius of gyration is $6 \mathrm{ft}$., and its weight is 8 tons. A cage weighing 6 tons is raised by it. If the cage is rising at the rate of $60 \mathrm{ft}$. per sec., at what level should the power be shut off in order that the cage may stop at the surface without braking? Neglect friction.

Ans. $103.5 \mathrm{ft}$.

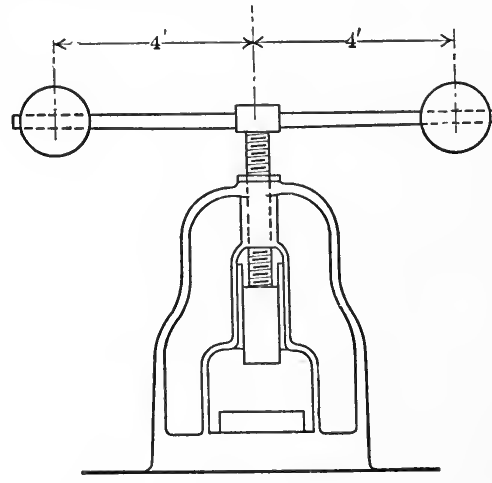

Fig. 431.

467. The hand-operated screw press shown in Fig. 431 is used for embossing, lettering dies, punching thin plates, etc. The diameter of the screw is $2 \frac{1}{2}$ in. The screw has triple threads with a pitch of $2 \frac{1}{2}$ in. Each ball weighs $100 \mathrm{lb}$. and the diameter of each ball is 9 in. If the balls are revolved at 60 r.p.m., what is the maximum size (diameter) of hole that can be punched in a $\frac{1}{4}$-in. steel plate, assuming the shearing strength of the steel to be $60,000 \mathrm{lb}$. per square inch and the efficiency of the screw to be 15 per cent. Also assume the work diagram for punching the hole to be triangular. (See Probs. 435 and 461.) Ans. $d=0.60$ in.

468. A shearing machine has 3 h.p. delivered to it by the belt. Every two seconds an operation occurs which requires seven-eighths of all the energy supplied during the two seconds; the other one-eighth of the energy is required to overcome the friction of the machine. During each operation the speed of the flywheel decreases from 120 to 80 r.p.m. Assuming that the work done in shearing is done by the flywheel, what should be the 
moment of inertia of the flywheel? If the weight of the flywheel is $400 \mathrm{lb}$. what is its radius of gyration?

469. A solid cylinder weighing $500 \mathrm{lb}$. is rolled up an inclined plane by means of a descending weight $B$ (Fig. 432). The diameter of the cylinder is $4 \mathrm{ft}$. The pulley over which the rope runs is assumed to be frictionless and weightless. Body $B$ weighs $300 \mathrm{lb}$. If the cylinder starts from rest at the bottom of the incline, with what velocity will its center reach the top?

470. A solid disc $18 \mathrm{in}$. in diameter is mounted on a shaft 4 in. in diameter (Fig. 433). The shaft rolls, without slipping, on two inclined tracks. The disc and shaft weigh $120 \mathrm{lb}$. The angle of inclination, $\phi$, is $15^{\circ}$ and the incline

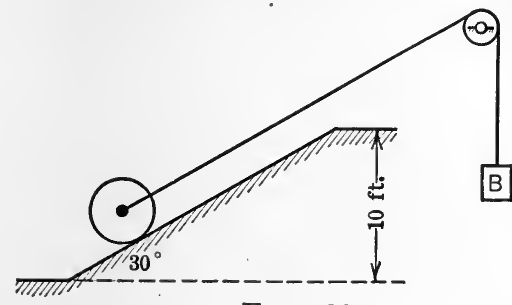

FIg. 432.

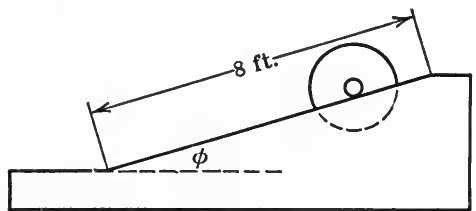

Fig. 433.

is $8 \mathrm{ft}$. long. If the disc starts from rest at the top, what will be the velocity of its center at the bottom of the incline?

Ans. $v=3.46 \mathrm{ft} . / \mathrm{sec}$.

195. Conservation of Energy.-One of the greatest achievements of the nineteenth century was the recognition and statement of the principle of the conservation of energy. Like Newton's laws of motion it is an inductive generalization from observation of, and experience with, physical phenomena. The principle states that in any change of the state or condition of an isolated material system the total amount of energy of the system remains constant. By an isolated system is meant one on which no bodies external to the system have any effect on the system. Hence, an isolated system neither gives nor receives energy. Thus, the distribution of energy within the isolated system may be altered and the various forms of energy changed into other forms but the total amount of energy remains constant. Or, as sometimes stated, energy may be transformed or transferred but cannot be created or destroyed. As noted in Art. 191, the principle of 'conservation of energy is of particular importance in the study of material systems which possess non-mechanical energy, although it is also of much value in the study of mechanical energy. Although an isolated system does not exist in nature, certain systems approach 
closely thereto, as for example, the earth and a falling body, provided that the action (and reaction) between the earth and body is large compared with the resistance of the air and the attractions of other bodies on the falling body. Again, although external forces act on a system of bodies, the work done by the forces may be zero (or negligible) as in the case of a simple swinging pendulum and the earth in which the pull of the string on the bob is always normal to the displacement of its application point and the effect of the air is negligible.

196. Relation between Potential and Kinetic Energy for Conservative Systems.-For non-rigid mass-systems it was found (Art. 193) that,

$$
w_{e}+w_{i}=\Delta E_{k},
$$

but $w_{i}$, which represents the work done by the internal forces in any displacement of the system, is equal (but opposite in sign) to the change in potential energy of the mass-system for the given displacement, ${ }^{*}$ that is,

Therefore,

$$
w_{i}=-\Delta E_{p}
$$

$$
w_{e}=\Delta E_{\mathrm{z}}+\Delta E_{p} .
$$

Now if the work done by the external forces which act on a masssystem is zero, then, $w_{e}=0$ and hence,

$$
\Delta E_{\mathrm{k}}+\Delta E_{p}=0 .
$$

Or,

$$
\Delta E_{\mathrm{k}}=-\Delta E_{p} .
$$

* This fact may be shown as follows: By definition the change of potential energy of a body is the work which the body does against forces in passing from one configuration to another configuration, providing no other change in the state of the body (such as a change in velocity) takes place. Then from the equation,

we obtain,

$$
w_{e}+w_{i}=\Delta E_{k},
$$

or,

$$
w_{e}+w_{i}=0 \text {, }
$$

$$
w_{i}=-w_{e},
$$

since $\Delta E_{k}$ is zero if no change of the velocity state occurs. But, by definition $-w_{e}$ is the potential energy of the system in its initial configuration. As explained in Art. 187, $w_{e}$ is a definite quantity only when the forces are conservative. 
That is, in any displacement of a conservative mass-system (or one on which the external forces do no work), the gain (or loss) in the kinetic energy of the system is equal to the loss (or gain) in the potential energy of the system.

If the system is not conservative, the transformation of the energy within the system from potential to kinetic energy (or vice versa) is accompanied by an increase in other forms of energy such as heat energy. And, although the total energy remains constant, the mechanical (potential and kinetic) energy does not remain constant. The relations between potential and kinetic energy for conservative systems, only, are considered herein.

\section{ILLUSTRATIVE PROBLEMS}

471. A body which weighs $50 \mathrm{lb}$. falls from a height, $h$, of $50 \mathrm{in}$. upon a helical spring (Fig. 434), the modulus of which is $800 \mathrm{lb}$. per inch. What is the maximum shortening, $s$, of the spring?

Solution.- - Little error will be introduced by assuming the spring and body to be perfectly elastic; by neglecting the air resistance on the falling body; and by neglecting the inertia of the spring. If this is done, the earth, the body, and the spring, may be considered to be a conservative system in which the earth is assumed to be fixed. Thus the mechanical energy of the system remains constant and hence,

$$
\Delta E_{p}=-\Delta E_{k} .
$$

But $\Delta E_{k}=0$, since the kinetic energy of the system is zero at the beginning and at the end of the displacement. And the change in the potential energy of the system is,

$$
\Delta E_{p}=-W(h+s)+\frac{800 \times s}{2} \times s=0 .
$$

Or,

$$
W(h+s)=\frac{800 s^{2}}{2},
$$

in which $h$ and $s$ are expressed in inches. This equation states that the loss of potential energy of the body is equal to the gain of potential energy of the spring. Solving the equation, we have,

$$
50(50+s)=400 s^{2}
$$

Or,

Whence,

$$
8 s^{2}-s-50=0 \text {. }
$$

$$
s=2.56 \text { in. }
$$

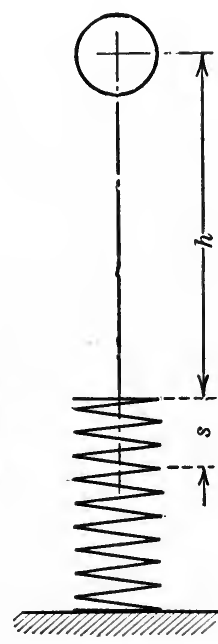

FIG. 434.

472. A simple pendulum (Fig. 435) consists of a cord having a length, $r$, of $4 \mathrm{ft}$. and a bob (assumed to be a particle) having a weight, $W$, of $6 \mathrm{lb}$. The 
pendulum is displaced an angle, $\theta$, of $60^{\circ}$. What will be the velocity, $v$, of the bob when in its lowest position, if air resistance is neglected?

Solution.-The tension $T$ in the string does no work. Thus, the earth and bob may be treated as a conservative system. Hence,

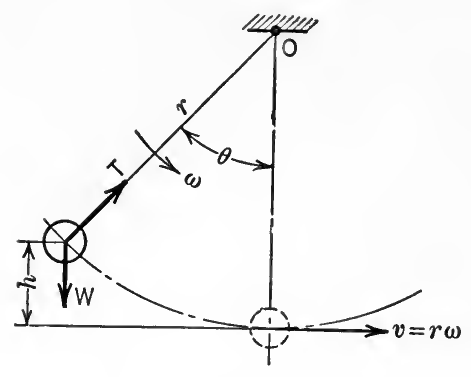

Fig. 435.

$$
\begin{aligned}
\Delta E_{p} & =-\Delta E_{k}, \\
W h=\frac{1}{2} m v^{2} & =\frac{1}{2} \frac{W}{g} v^{2}, \\
6\left(4-4 \cos 60^{\circ}\right) & =\frac{1}{2} \frac{6}{32.2} v^{2}, \\
v & =11.36 \mathrm{ft} . / \mathrm{sec} .
\end{aligned}
$$
whence,

473. Solve Prob. 462 by use of the principle expressed by the equation,

$$
\Delta E_{k}+\Delta E_{p}=0 \text {. }
$$

Solution.-As shown in Prob. 442, the only force which does work is the earth-pull. Hence, the earth and the cylinder form a conservative system Thus,

$$
\begin{aligned}
\Delta E_{p} & =-\Delta E_{k}, \\
W h & =\left(\frac{1}{2} M \bar{v}^{2}+\frac{1}{2} \bar{I} \omega^{2}\right), \\
W s \sin \phi & =\left(\frac{1}{2} M \bar{v}^{2}+\frac{1}{2} \bar{I} \omega^{2}\right) .
\end{aligned}
$$

This is the same equation that was obtained and solved in Prob. 462.

Substituting the given values in the above equation as was done in Prob. 462 , the value of $s$ is found to be $36 \mathrm{ft}$.

Note.-All three of the above problems may be solved by the principle of work and kinetic energy. It is important, however, to note the relation between potential and kinetic energy for conservative systems.

\section{§4. Efficiency. Dissipation of Energy}

197. Efficiency Defined.-The efficiency of a machine, as for example, a steam engine, an electric motor, a chain hoist, a jack screw, etc., is the ratio of the energy output of the machine in a given period of time to the energy input in the same period, provided that no energy is stored in the machine which becomes available at a later period. By input is meant the amount of energy received by the machine, a portion of which is transformed or transmitted into the work or energy for which the machine is designed. The work done or energy delivered by the machine is called the output. Thus, denoting efficiency by $e$, we have,

$$
e=\frac{\text { energy output }}{\text { energy input }} \text { or } e=\frac{\text { power output }}{\text { power input }} \text {. }
$$


As already noted, in the transformation and transference of energy (which is the main function of many machines), some of the energy always takes the form of a lower grade of energy (heat energy) and thereby becomes unavailable for the particular process for which the machine is used. The amount of energy which thus miscarries or leaks out in the process is spoken of by various names, such as lost energy (or lost work), energy leak, dissipated energy, etc. Since dissipation of energy occurs with every physical process, the output is always less than the input and, therefore, the efficiency is always less than unity.

The efficiency as defined above is the over-all efficiency of the machine. Certain parts of the machine, however, may have their individual efficiencies; and the over-all efficiency is the product of the efficiencies of the several elements of the machine.

198. Dissipation of Energy.-The work done against friction is the most frequent cause of dissipation of energy in machines. Energy dissipated in doing work against frictional forces is transformed into heat energy. Electrical resistance in connection with electrical machinery also causes a loss of available energy by developing heat. The work done against friction is, in some machines, a necessary evil to be reduced to a minimum as in the case of prime movers, bearings, teeth of gears, etc.; whereas, in other machines or machine elements, the main object of the machine is to dissipate all the energy received by the machine, as in the case of friction brakes and absorption dynamometers.

Two typical examples involving dissipation of energy in machines are here considered: (1) a bearing in which the loss of energy is made as small as practicable by lubrication and (2) a simple Prony brake or absorption dynamometer, the object of which is not only to absorb all the energy delivered to it but also to measure that energy (or power). Another class of dynamometers measures the power delivered by a machine without absorbing (dissipating) the energy. Dynamometers of this class are called transmission dynamometers.

199. Work Lost Due to Friction in Bearings.-The dissipation of energy in bearings of machines of various kinds reduces the useful energy transmitted or transformed by the machine and is, therefore, an important factor in the design and operation of such machines. For example, in textile machinery, which involves a 
large number of small parts moving at relatively high velocities. a large part of the load is due to friction.

Various types of bearings are used to meet the different needs. Thus, for shafts subjected to a considerable end thrust, as, for example, a screw-propellor shaft or the shaft of a centrifugal pump, a collar thrust bearing having several collars may be used, whereas for slow speeds, such as occur in certain types of rotary cranes, the end thrust due to the load and weights of the moving parts is usually carried by an ordinary flat pivot or step bearing.

Fig. 436 represents an ordinary flat pivot in which the work (and power) lost in friction is to be found. Certain assumptions

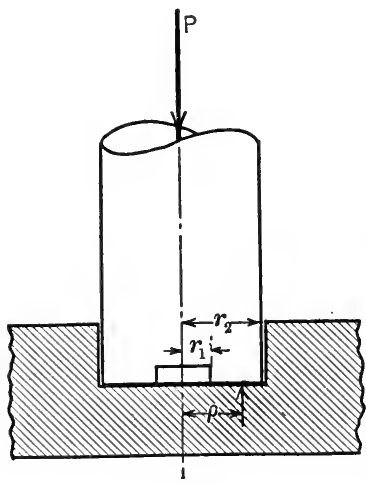

FIg. 436. must be made. It will be assumed that the coefficient of friction for the rubbing surfaces is constant over the whole area of contact and that the normal wear at any point (wear normal to the rubbing surfaces) is proportional to the work of friction. Thus, according to this assumption, the pressure after wear has occurred will not be uniform but will be a maximum at the center where no wear occurs and will decrease towards the edge, since the relative velocity of the rubbing surfaces is zero at the center and increases towards the edge. It will, therefore, be well to cut out the material near the center of the bearing to avoid crushing of the material. Let $p$ be the intensity of pressure on any elementary area $2 \pi \rho d \rho$, at a distance $\rho$ from the axis of the shaft. The total pressure $P$, then, is

$$
P=2 \pi \int p \rho d \rho .
$$

And from the assumption made above, the normal wear, $n$, is

in which $k$ is a constant. Therefore,

$$
n=k p \rho \quad \text { or } \quad p=\frac{n}{k \rho},
$$

$$
\begin{aligned}
P & =2 \pi \int_{r_{1}}^{r_{2}} \frac{n}{k} d \rho \\
& =\frac{2 \pi n}{k}\left(r_{2}-r_{1}\right) .
\end{aligned}
$$


The frictional force at any distance $\rho$ from the axis is,

$$
\begin{aligned}
F^{\prime} & =\mu p d A \\
& =\mu p 2 \pi \rho d \rho \\
& =\mu\left(\frac{n}{k \rho}\right) 2 \pi \rho d \rho \\
& =\mu \frac{2 \pi n}{k} d \rho .
\end{aligned}
$$

And the frictional moment $T_{f}$ for the whole area is,

$$
\begin{aligned}
T_{f} & =\frac{\mu 2 \pi n}{k} \int_{r_{1}}^{r_{2}} \rho d \rho \\
& =\frac{\mu \pi n}{k}\left(r_{2}{ }^{2}-r_{1}^{2}\right) . \quad . \quad . \quad . \quad . .
\end{aligned}
$$

By eliminating $n$ and $k$ from (1) and (2), the following equation is obtained,

$$
T_{f}=\frac{\mu P}{2}\left(r_{2}+r_{1}\right) . \quad \text {. . . . . . . }
$$

And, if $\omega$ is the angular velocity of the shaft in radians per second, the work lost per second, $w_{f}$, is,

$$
w_{f}=T_{f} \omega=\frac{\mu P \omega}{2}\left(r_{2}+r_{1}\right) \quad . \quad . \quad .
$$

If $P$ is expressed in pounds and $r_{1}$ and $r_{2}$ in feet, then $w_{f}$ is expressed in foot-pounds per second and the horse-power lost in friction is,

$$
\text { (h.p. })_{f}=\frac{\mu P \omega}{550} \times \frac{r_{2}+r_{1}}{2} . . . \quad . \quad . \quad .
$$

In order to increase the amount of rubbing surface and thereby diminish the intensity of pressure, a collar bearing with two or more collars may be used. Equations (3), (4), and (5) also apply to such bearings. It will be observed that the coefficient of friction is an important factor in the problem of reducing the dissipation of energy due to friction and this fact suggests the great importance of the choice of bearing materials and of lubricants.

Various other types of bearings may be treated in a manner similar to that used above. 
200. A Simple Dynamometer. Prony Brake.-A simple dynamometer, commonly called a Prony brake, is shown in Fig. 437. $A$ is a flanged pulley keyed to a rotating shaft. The power transmitted by the shaft is absorbed and measured by the brake. $B, B$ are bearing blocks against which the pulley develops a frictional resistance, the magnitude of which is varied by adjusting the nuts $C, C . \quad D$ is the frame or beam with its end $E$ resting on the platform of a weighing scale. When the pulley is running, the beam develops an additional pressure on the platform, due to the friction of the pulley on the bearing blocks. The work lost

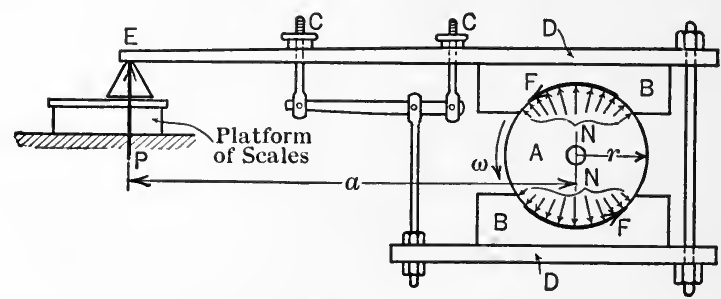

FIG. 437.

in friction and the power developed by the shaft may be found as follows:

It will be assumed that the scales are adjusted to read zero when the beam rests on the platform and the pulley is not running. Thus, the scale reading is a measure of the pressure, $P$, at $E$ due to the friction developed on the bearing blocks when the pulley is running. Since the brake frame is in equilibrium under the action of the forces, $N, P$, and $F$ ( $F$ denotes the total frictional force on the two blocks), the sum of the moments of $P$ and $F$ about the axis of the shaft must equal zero. Or,

$$
F r=P a .
$$

And the work done by the frictional moment $F r$ in one second is,

$$
\begin{aligned}
w_{f} & =F r \omega \\
& =P a \omega,
\end{aligned}
$$

in which $\omega$ is expressed in radians per second. And, since $\omega=\frac{2 \pi n}{60}$ 
where $n$ is the number of revolutions per minute (r.p.m.) of the shaft and pulley, the expression for $w_{f}$ becomes,

$$
\begin{aligned}
w_{f} & =\frac{2 \pi P a n}{60} \\
& =\frac{\pi P a n}{30} .
\end{aligned}
$$

If $P$ is expressed in pounds and $a$ in feet, then $w_{f}$ will be expressed in foot-pounds and the horse-power developed by the frictional moment (and hence by the shaft) is,

$$
\begin{aligned}
\text { h.p } & =\frac{\pi \text { Pan }}{30 \times 550} \\
& =\frac{\pi P a n}{16,500} .
\end{aligned}
$$

This expression may be simplified if the dynamometer is constructed so that $a$ has a special value. It should be remembered that the scale reading should not be used for the value of $P$ unless the scales are adjusted to read zero when the pulley is not running.

\section{PROBLEMS}

474. What effort, $P$, is required to raise a load, $Q$, of $200 \mathrm{lb}$. by means of the differential chain hoist shown in Fig. 438 if $e=30$ per cent, $r_{2}=8$ in., and $r_{1}=4$ in.? Ans. $P=167 \mathrm{lb}$.

475. In a test of a jackscrew (see Fig. 169) with a screw 1.5 in. in diameter and a pitch of $\frac{1}{3} \mathrm{in}$., it was found that a pull of $72 \mathrm{lb}$. at the end of a 15-in. lever was required to raise a load of $2400 \mathrm{lb}$. when no lubricant was used and a pull of $63 \mathrm{lb}$. when a hard oil lubricant was used. What is the efficiency of the jack for each case?

Ans. $e=11.8 \% ; e=13.5$ per cent.

476. The band brake described in Prob. 165 allows a certain load to lower at a constant speed such that the brake sheaves rotate at 120 r.p.m., in a counter-clockwise direction. What horse-power is absorbed by the brake?

477. The dynamometer shown in Fig. 439 was devised by Lord Kelvin in connection with the laying of the Atlantic cable for braking the cable drum as the cable was laid out. If it is used in the test of a steam engine, find the horse-power absorbed, using the following data: $W=400 \mathrm{lb}$; ; speed of engine = 150 r.p.m.; $r=4 \mathrm{ft}$.; reading of spring balance is $85 \mathrm{lb}$. Ans. 36 h.p. 
478. Derive an expression for the work done by friction in the spherical pivot shown in Fig. 179, assuming that normal wear is proportional to the

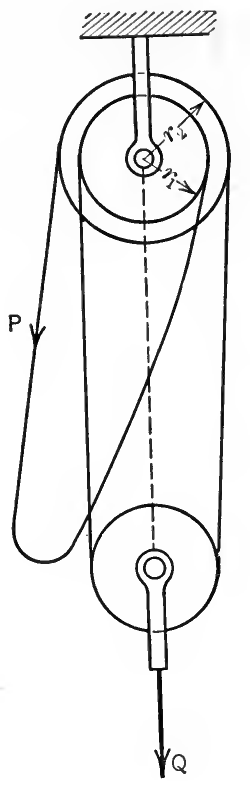

FIg. 438.

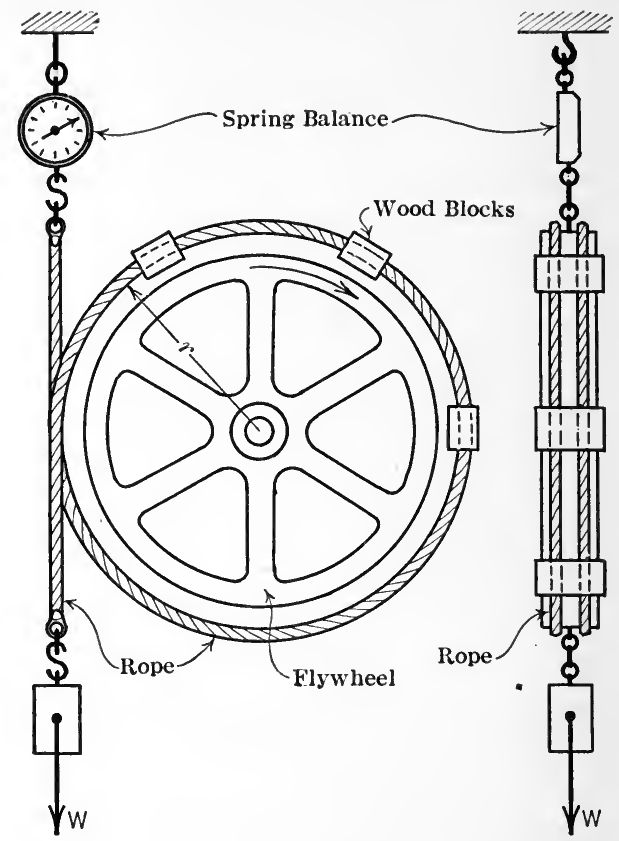

FIG. 439.

work of friction and that the coefficient of friction is the same for all parts of the rubbing surfaces.

$A n s$. Work per revolution $=2 \pi \mu W r \frac{\sin ^{2} \alpha}{\alpha+\sin \alpha \cos \alpha \text {. }}$ 


\section{CHAPTER XI}

\section{IMPULSE AND MOMENTUM}

201. Preliminary.--In Art. 176, the statement was made that impulse is a quantity which involves force and time, and that momentum is a quantity which involves mass and velocity. And the fact was noted that the use of these quantities in the analysis of the motion of bodies requires no fundamental laws in addition to Newton's laws of motion. However, methods which make use of impulse and momentum offer advantages, in certain types of problems, over the methods of work and energy (Chap. $\mathrm{X})$ and of force and acceleration (Chap. IX).

In determining the effect of forces on the motion of bodies, thus far, by the methods of force and acceleration and of work and energy, it has been assumed that the forces have acted on rigid bodies during a definite (comparatively large) interval of time, and when the forces were not constant the manner in which they varied during the period was assumed to be known. To such conditions the methods of impulse and momentum also apply, and in many cases offer a simpler method of solution than the methods previously discussed. Forces sometimes act, however, for a very short (indefinite) interval of time during which neither the value of the force at any instant nor its law of variation is known. These forces may, nevertheless, produce very appreciable changes in the motion (velocity) of the body. Such forces are called impulsive forces. The bodies upon which impulsive forces act deform under the excessive pressures produced and hence, in determining the motions of bodies under the influence of impulsive forces, the bodies cannot always be assumed to be rigid, as in the preceding chapters, without introducing appreciable errors. As examples of impulsive forces the following may be mentioned: the force exerted on a projectile due to the explosion of the powder; the action of one billiard ball on another; the force exerted by the ram of a pile driver on the pile; the pressure between two 
railway cars when making a flying coupling; the action of a steam jet on the blades of a high-speed steam turbine; the pressure exerted by the water in a pipe line on a valve which is closed suddenly. The principles of impulse and momentum are of special value when considering the motion of bodies under the action of impulsive forces.

The purpose of the present chapter is to make clear the conception or meaning of impulse and of momentum, to develop certain principles which express relations between these quantities, and to apply these principles to problems in kinetics.

\section{$\S 1$. ImpUlse}

202. Impulse and Impact Defined. Units.-The impulse of a constant force is defined as the product of the magnitude of the force and the time interval during which the force acts. Thus, if $Q$ denotes the impulse of a force $F$, the impulse of the force is defined by the equation,

$$
Q=F \cdot \Delta t,
$$

provided that the force remains constant during the time interval $\Delta t$. If the force varies in magnitude but not in direction, the impulse for an indefinitely short period of time $d t$, is $F \cdot d t$ and, for a time interval $\Delta t=t_{2}-t_{1}$, the impulse is,

$$
Q=\int_{t_{1}}^{t_{2}} F d t .
$$

In order to evaluate this integral by the method of calculus, $F$ must be expressed in terms of $t$. The impulse of a force which acts during a very short (indefinite) interval of time (impulsive force) is also given by the above expression but, as noted in the preceding article, the impulsive force cannot be expressed in terms of $t$, since its law of variation is not known and hence, the impulse cannot be determined directly but is found in terms of the change of momentum as is discussed in the subsequent pages.

The impulse of an impulsive force is sometimes called an impact, that is, an impact is a sudden impulse. The term impact, however, is also frequently used as descriptive of the act of collision of bodies. In some problems it is convenient to estimate the time interval of the impulsive force and to express the impact as 
the product of an average value of the force and an assumed or estimated time interval $\Delta t=t_{2}-t_{1}$. Thus,

$$
Q=\int_{t 1}^{t_{2}} F d t=F_{a v} \cdot \Delta t
$$

in which $F_{a v}$ denotes the average value of the impulsive force which is assumed to act during the time interval $\Delta t$. The impulse of a force frequently is called linear impulse in contrast with the moment of the impulse which is called angular impulse. (See Art. 204.)

Units.-The unit of an impulse is a combination of a unit of force and of time and hence, is a compound unit. The unit of impulse has no special name. In the gravitational or engineer's system of units, if the pound is selected as the unit of force and the second for the unit of time, then the unit of impulse is the poundsecond (lb.-sec.) or, if the kilogram is selected for the unit of force and the second for the unit of time, the unit of impulse is the kilogram-second (kg.-sec.).

203. Components of Linear Impulse.-The impulse of a force, like the force itself, is a directed or vector quantity, the sense and action line of the impulse being the same as that of the force. An impulse of a force, therefore, may be resolved into components and may have a moment with respect to a point or a line. The component, in any direction, of the impulse of a constant force is the product of the component of the force in the given direction and the time interval $\Delta t$ during which the force acts. That is,

$$
Q_{x}=F_{x} \cdot \Delta t ; \quad Q_{y}=F_{y} \cdot \Delta t, \text { etc. }
$$

And, if the force varies in magnitude during the time interval $\Delta t=t_{2}-t_{1}$, the component of the impulse is,

$$
Q_{x}=\int_{t_{1}}^{t_{2}} F_{x} d t ; \quad Q_{y}=\int_{t_{1}}^{t_{2}} F_{y} d t, \text { etc. }
$$

Linear Impulse of a Force System.-The linear impulse, in any direction, of a force system is the algebraic sum of the components, in the given direction, of the impulses of the forces of the system. 
Thus, for a force system in which the forces are constant, the linear impulse, $Q_{x}$, of the force system in any direction $x$ is,

$$
Q_{x}=\Sigma F_{x} \cdot \Delta t
$$

Or, if the forces of the system vary during the interval, then,

$$
Q_{x}=\Sigma \int_{t_{1}}^{t_{2}} F_{x} d t
$$

204. Moment of Impulse. Angular Impulse.-The moment of the impulse of a constant force about any point or axis is the product of the moment, $T$, of the force about the given point or axis, and the time interval, $\Delta t$, during which the force acts. Thus, by letting $L_{0}$ denote the moment of the impulse of a constant force with respect to an axis $O$, the moment of the impulse of the force is defined by the equation,

$$
L_{0}=T_{0} \cdot \Delta t
$$

And, if the force varies in magnitude during the time interval, $\Delta t=t_{2}-t_{1}$, the moment of the impulse is expressed by the equation,

$$
L_{0}=\int_{t_{1}}^{t_{2}} T_{0} d t
$$

in which $T_{0}$ is the moment of the force with respect to the axis $O$. The moment of the impulse of a force is also called the angular impulse of the force.

Angular Impulse of a Force System.-The angular impulse of a force system about any axis is the algebraic sum of the angular impulses of the forces of the system about the given axis. Thus, if the forces of the system are constant, the angular impulse, $L_{0}$, of the system about the axis $O$ is,

$$
L_{0}=\Sigma T_{0} \Delta t
$$

And, if the forces vary, the angular impulse of the force system about the axis $U$, for the time interval $\Delta t=t_{2}-t_{1}$, is expressed by the equation,

$$
L_{0}=\Sigma \int_{t_{1}}^{t_{2}} T_{0} d t .
$$


It should be noted that the linear (and angular) impulse of a constant force, or of a variable force for which the law of variation is known, is not as important a conception in the analysis of the motion of a body as is the impulse of an impulsive force. However, as already noted, even for constant forces which act during a comparatively long time interval, the methods of impulse and momentum may possess advantages over other methods.

\section{PROBLEMS}

479. A body weighing $40 \mathrm{lb}$. slides down an inclined plane in 4 sec. The plane makes an angle of $60^{\circ}$ with the horizontal. If the coefficient of friction is 0.2 find the component of the linear impulse of the force system acting on the body parallel to the plane.

$$
\text { Ans. } Q_{x}=123 \mathrm{lb} \text {-sec. }
$$

480. The linear impulse of the total pressure of the steam on the piston of a steam engine is $4200 \mathrm{lb}$.-sec. The diameter of the cylinder is $14 \mathrm{in}$. and the engine runs at 200 r.p.m. Find the average (time average) pressure (in lb. per sq. in.) of the steam against the piston during one stroke.

481. A train having a weight of 2000 tons travels up a $\frac{1}{2}$ per cent grade. The draw-bar pull of the engine is $50,000 \mathrm{lb}$. and the train resistance is $8 \mathrm{lb}$. per ton of weight. If it takes $2 \mathrm{~min}$. to travel up the grade, find the linear impulse of the force system acting on the train for the 2-min. interval.

$$
\text { Ans. 1,680,000 lb.-sec. }
$$

482. A constant frictional moment of $200 \mathrm{lb}$. $\mathrm{ft}$. is applied to a rotating drum by means of a band brake (see Fig. 185). If the moment decreases the angular velocity of the drum uniformly from 90 r.p.m. to 10 r.p.m. while the drum makes 30 revolutions, find the angular impulse of the band brake on the drum.

Ans. 7200 ib.-ft.-sec.

\section{§2. Momentum}

205. Momentum of a Particle Defined. Units. - The momentum of a particle of a moving body, at any instant, is defined as the product of the mass of the particle and its velocity at the instant. Momentum, like velocity, is a directed or vector quantity. Furthermore, like force, momentum is represented by a localized vector, that is, it has a definite position line. Thus, the direction of the momentum of a particle is the same as that of the velocity of the particle and its position line passes through the particle. Thus, if $U$ denotes the momentum of a particle of mass $m$ and velocity $v$, the magnitude of the momentum of the particle is defined by the equation,

$$
U=m v .
$$


The momentum of a particle frequently is called linear momentum in contrast with the moment of momentum of the particle which is called angular momentum.

Units. - The unit of momentum is a combination of a unit of mass and a unit of time and hence, is a compound unit. The unit of momentum has no special name. In the gravitational or engineer's system of units, the unit of mass is a derived unit as explained in Art. 141; derived from the units of force, length, and time and hence, the unit of momentum is also a derived unit. Thus, if the pound, foot, and second are chosen for the units of force, length, and time, respectively, the unit of momentum is expressed by,

1 unit of mass $\times 1$ unit of velocity $=\frac{1 \mathrm{lb} . \times 1 \text { sec. }^{2}}{1 \mathrm{ft} .} \times \frac{1 \mathrm{ft} .}{1 \mathrm{sec} .}$

$$
=1 \text { lb.-sec. }
$$

It will be observed, therefore, that momentum is expressed in the same fundamental units as is impulse.

206. Components of Momentum. Moment of Momentum.Since the momentum of a particle is a vector quantity it may be resolved into components and, like any localized vector, it has a

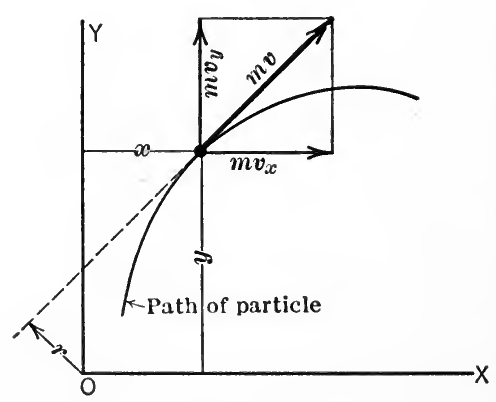

Fig. 440 . moment with respect to any point, the moment being defined as the product of the magnitude of the momentum and the perpendicular distance from the position line to the point or moment-center. If, then, $U_{x}$ and $U_{y}$ denote the components of the momentum of a particle of mass $m$ and of velocity $v$ (Fig. 440) and, if $H_{0}$ denotes the moment of momentum of the particle with respect to the point $O$, the components of the momentum of the particle and the moment of momentum of the particle are expressed by the equations,

and

$$
U_{x}=(m v)_{x}=m v_{x} ; \quad U_{y}=(m v)_{y}=m v_{y},
$$

$$
H_{0}=m v \cdot r ; \quad \text { or, } \quad H_{0}=m v_{x} \cdot y-m v_{y} \cdot x .
$$


The latter expression for $H_{0}$ expresses the fact that the moment of the momentum of a particle equals the algebraic sum of the moments of the components of the momentum of the particle.

207. Linear Momentum of a Body.- The linear momentum, in any direction, of any body (mass-system) is the algebraic sum of the components in the given direction of the momentums of the particles of the body. In general the momentums of the particles of a body are not the same either in magnitude or in direction. It will now be shown, however, that the linear momentum of the whole body may be found from the mass of the whole body and the velocity of the mass-center of the body. Thus, in Fig. 441 are shown three particles $m^{\prime}, m^{\prime \prime}$, and $m^{\prime \prime \prime}$ of a system assumed, for convenience only, to move in a plane. At a

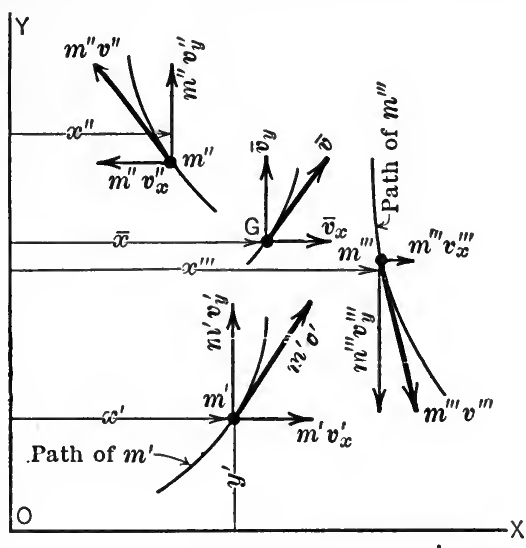

FIG. 441. given instant the particles and their mass-center are in the positions shown. The direction of the momentum of each particle is tangent to the path of the particle and the velocity, $\bar{v}$, of the masscenter is tangent to the path which the mass-center describes. The component, in the $x$-direction, of the linear momentum of the mass-system is,

$$
\begin{aligned}
U_{x} & =m^{\prime} v_{x}{ }^{\prime}+m^{\prime \prime} v_{x}{ }^{\prime \prime}+m^{\prime \prime \prime} v_{x}^{\prime \prime \prime}+\ldots \\
& =\Sigma\left(m v_{x}\right) .
\end{aligned}
$$

But, $\Sigma\left(m v_{x}\right)$ may be shown to be equal to $\bar{v}_{x} \Sigma m$ or $M \bar{v}_{x}$ as follows: The $x$-coordinate, $\bar{x}$, of the mass-center is defined by the principle of moments (Art. 80) by the equation,

$$
m^{\prime} x^{\prime}+m^{\prime \prime} x^{\prime \prime}+m^{\prime \prime \prime} x^{\prime \prime \prime}+\ldots=\bar{x} \Sigma m=M \bar{x} .
$$

By differentiating both sides of the equation, the following equation is obtained,

$$
m^{\prime} \frac{d x^{\prime}}{d t}+m^{\prime \prime} \frac{d x^{\prime \prime}}{d t}+m^{\prime \prime \prime} \frac{d x^{\prime \prime \prime}}{d t}+\ldots=M \frac{d \bar{x}}{d t} .
$$


But, $\frac{d x^{\prime}}{d t}$ is the $x$-component of the velocity of $m^{\prime}$ and hence,

Similarly,

$$
\frac{d x^{\prime}}{d t}=v_{x}^{\prime}
$$

and

$$
\frac{d x^{\prime \prime}}{d t}=v_{x}^{\prime \prime}, \text { etc., }
$$

$$
\frac{d x}{d t}=\bar{v}_{x}
$$

Thus, the above equation becomes,

Similarly,

$$
m^{\prime} v_{x}{ }^{\prime}+m^{\prime \prime} v_{x}^{\prime \prime}+m^{\prime \prime \prime} v_{x}^{\prime \prime \prime}+\ldots=M \bar{v}_{x}
$$

Whence,

$$
m^{\prime} v_{y}^{\prime}+m^{\prime \prime} v_{y}^{\prime \prime}+m^{\prime \prime \prime} v_{y}^{\prime \prime \prime}+\ldots=M \bar{v}_{y} .
$$

$$
U_{x}=M v_{x} ; \quad U_{y}=M v_{y} ; \text { and } U=M v \text {. }
$$

That is, the linear momentum of any moving mass-system is the product of the mass of the whole system and the velocity of the masscenter of the system and its direction agrees with that of the velocity of the mass-center.

208. Angular Momentum of a Rotating Rigid Body.-Let

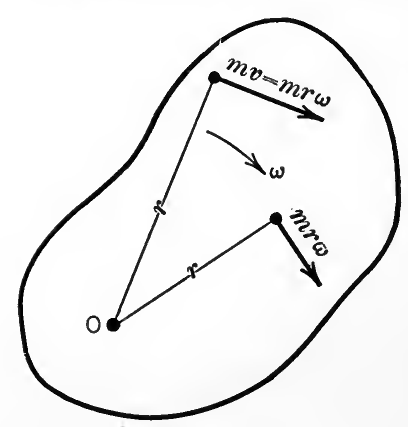

FIG. 442. Fig. 442 represent a rigid body rotating about a fixed axis through $O$ with an angular velocity $\omega$. The linear velocity of any particle of the body at a distance $r$ from the axis of rotation is $r \omega$ (Art. 115) and the linear momentum of the particle of mass $m$ is $m v$ or $m r \omega$. Therefore, the moment of momentum for one particle about the axis of rotation is,

$$
m v \cdot r=m r \omega \cdot r=m r^{2} \omega,
$$

and the algebraic sum of the moments of the momentums of all the particles about the same axis is,

whence,

$$
H_{0}=\Sigma m r^{2} \omega=\omega \Sigma m r^{2},
$$

$$
H_{0}=I_{0} \omega \text {. }
$$


in which $I_{0}$ is the moment of inertia of the body about the axis of rotation.

Therefore, the angular momentum of a rotating rigid body about the axis of rotation is equal to the product of the moment of inertia of the body with respect to the axis of rotation and the angular velocity of the body.

209. Angular Momentum of a Rigid Body Having Plane Motion.-Since a plane motion of a rigid body may be considered as a combination of a rotation and a translation (Art. 134), the velocity of any particle $P$ (Fig. 443) is the resultant of a velocity $r \omega$ due to the rotation of the body with angular velocity $\omega$ about an axis $O$ and, the velocity $v_{0}$ due to the translation which gives to each particle the velocity $v_{0}$ of the point $O$. Therefore, the components of the momentum of the particle $P$ are

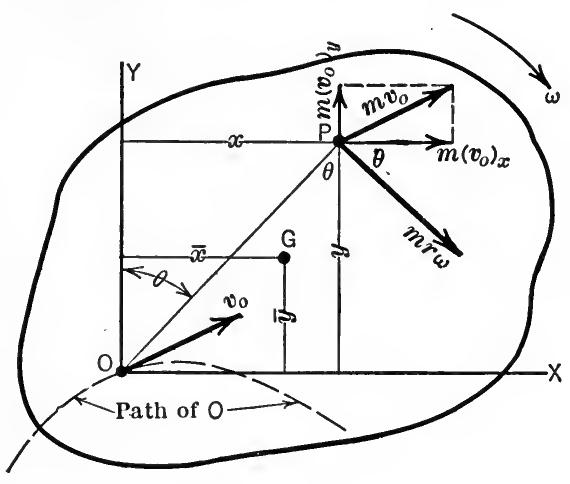

Fig. 443. $m r \omega$ and $m v_{0}$ as shown in Fig. 443. And, if $m\left(v_{0}\right)_{x}$ and $m\left(v_{0}\right)_{y}$ denote the $x$-and $y$-components of the momentum $m v_{0}$, then the angular momentum of the particle with respect to the axis $O$ is,

$$
m r \omega \cdot r+m\left(v_{0}\right)_{x} \cdot y-m\left(v_{0}\right)_{y} \cdot x,
$$

and the angular momentum, $H_{0}$, for the whole body with respect to the axis $O$ is,

$$
\begin{aligned}
H_{0} & =\Sigma m r^{2} \omega+\Sigma m\left(v_{0}\right)_{x} y-\Sigma m\left(v_{0}\right)_{y} x \\
& =\omega \Sigma m r^{2}+\left(v_{0}\right)_{x} \Sigma m y-\left(v_{0}\right)_{y} \Sigma m x \\
& =\boldsymbol{I}_{0} \boldsymbol{\omega}+\left(\boldsymbol{v}_{0}\right)_{x} \boldsymbol{M} \bar{y}-\left(v_{0}\right)_{y} \boldsymbol{M} \overline{\boldsymbol{x}} . .
\end{aligned}
$$

Now, as already noted, a plane motion of a rigid body may be considered as a rotation about an axis through any point in the plane of motion and a translation, etc. Hence, if the point $O$ is taken as the mass-center of the body, then $H_{0}$ becomes $\bar{H}$ and the above expression reduces to

$$
\bar{H}=\bar{I} \omega, \quad \text {. . . . . . . . . }
$$


since $\bar{y}$ and $\bar{x}$ are then equal to zero and $I_{0}$ becomes $\bar{I}$. Therefore, in general, the angular momentum of a rigid body having plane motion, with respect to an axis in the body, is not equal to the product of the moment of inertia of the body with respect to the given axis and the angular velocity of the body. But, for an axis passing through the mass-center of the body, the angular momentum is equal to the product of the moment of inertia of the body with respect to the axis through the mass-center and the angular velocity of the body.

It may be noted also that if the axis about which the angular momentum of the body is taken is the instantaneous axis of rotation, equation (1) becomes $H_{0}=I_{0} \omega$ since $\left(v_{0}\right)_{x}$ and $\left(v_{0}\right)_{y}$ are equal to zero. That is, the angular momentum of a rigid body having plane motion, about the instantaneous axis of rotation, is equal to the product of the moment of inertia of the body with respect to the instantaneous axis of rotation and the angular velocity of the body.

\section{PRORLEMS}

483. A small body (particle) weighing $8 \mathrm{lb}$. is attached to one end of a string and is made to revolve as a conical pendulum (see Fig. 324). If the body revolves at a distance of $15 \mathrm{in}$. from the axis with an angular velocity of 90 r.p.m., find $(a)$ the linear momentum of the body, $(b)$ the angular momentum of the body with respect to the axis of rotation.

$$
\text { Ans. (a) } U=2.93 \mathrm{lb} \text {.-sec.; (b) } H=3.66 \mathrm{lb} \text {.-ft.-sec. }
$$

484. A slender rod rotates in a horizontal plane about a vertical axis through one end of the rod. If the rod is $3 \mathrm{ft}$. long, weighs $4 \mathrm{lb}$. per foot, and rotates at 120 r.p.m., find its angular momentum about the axis of rotation. Ans. $H_{0}=14.05 \mathrm{lb} . \mathrm{ft}$. -sec.

485. Find the angular momentum, about the axis of rotation, of the flywheel shown on the punching machine in Fig. 429, when the flywheel is rotating at 225 r.p.m. Use the value of $I$ found in Prob. 461.

486. Find the angular momentum, about the axis of rotation, of the two spheres shown in Fig. 431, when the spheres are revolving at 120 r.p.m. Each sphere weighs $100 \mathrm{lb}$. and is $9 \mathrm{in}$. in diameter. Ans. $H_{0}=1250 \mathrm{lb}$.-ft.-sec.

487. A solid cylinder weighing $128.8 \mathrm{lb}$. rolls, without slipping, down an inclined plane. The linear velocity of the mass-center of the cylinder at a given instant is $30 \mathrm{ft}$. per sec. The diameter of the cylinder is $18 \mathrm{in}$. Find the angular momentum of the cylinder $(a)$ about an axis through the masscenter perpendicular to the plane of motion, $(b)$ about the instantaneous axis of rotation. (Ans.) (a) $\bar{H}=45 \mathrm{lb}$.-ft.-sec.; $(b) H_{i}=135 \mathrm{lb}$.-ft.-sec. 
488. The connecting rod shown in Fig. 422 is $30 \mathrm{in}$. long. It has a constant cross-section and weighs $52 \mathrm{lb}$. The crank length $O B$ is 5 in. and $\omega_{1}$ is 300 r.p.m. The distance from $i$ to $B$ is found to be 34.68 in. and $\bar{r}$ is found to be $23.75 \mathrm{in}$. Find the angular momentum of the connecting rod $(a)$ about an axis through the mass-center and $(b)$ about the instantaneous axis of rotation,

\section{§3. Principles of Impulse and Momentum}

210. Preliminary.-In order to determine the effect of a force system on the motion of a body, that is, in order to treat the usual problem in kinetics, by means of the quantities impulse and momentum, the relations which exist between the impulse of the force system and the momentum of the body on which the force system acts must be established. These relations are expressed by means of two principles, namely, (1) the principle of linear impulse and linear momentum and (2) the principle of angular impulse and angular momentum.

211. Principle of Linear Impulse and Linear Momentum.It was stated in Art. 152 that the algebraic sum of the components in a given direction of the external forces acting on any body (whether rigid or not) is equal to the mass of the body times the component of the acceleration of the mass-center of the body in the given direction. That is, if $x$ denotes any direction,

$$
\Sigma F_{x}=M \bar{a}_{x}
$$

Now if the forces acting on the body remain constant, then $\bar{a}_{x}$ will be constant, that is, the mass-center of the body will be uniformly accelerated. Hence, according to Art. 124,

$$
\bar{a}_{x}=\frac{\bar{v}_{x}^{\prime \prime}-\bar{v}_{x}^{\prime}}{\Delta t}
$$

in which $\bar{v}_{x}^{\prime \prime}$ and $\bar{v}_{x}^{\prime}$ are the $x$-components of the velocity of the mass-center of the body at the end and beginning, respectively, of the time interval $\Delta t$. Therefore,

$$
\Sigma F_{x} \cdot \Delta t=M \bar{v}_{x}^{\prime \prime}-M \bar{v}_{x}^{\prime} .
$$

Now the left-hand member of this equation is the $x$-component of the linear impulse of the external force system which acts on the body (Art. 203) and the right-hand member is the change in the 
momentum of the body in the $x$-direction (Art. 207). The above equation is a mathematical statement of the principle of linear impulse and linear momentum for any body when acted upon by constant forces. The principle applies, however, to the motion of any body under the action of any force system. Thus, in general, $\bar{a}_{x}=\frac{d \bar{v}_{x}}{d t}$ and hence, equation (1) may be written,

or, since $M$ is constant,

$$
\Sigma F_{x}=M \frac{d \bar{v}_{x}}{d t}
$$

$$
\Sigma F_{x}=\frac{d}{d t}\left(M \bar{v}_{x}\right)
$$

And, by integrating this equation, the following equation which expresses the principle of linear impulse and linear momentum is obtained,

Or,

$$
\int_{t_{1}}^{t_{2}} \Sigma F_{x} d t=\int_{\bar{v}_{x}^{\prime}}^{\bar{v}_{x}^{\prime \prime}} d\left(M \bar{v}_{x}\right)
$$

$$
\Sigma \int_{t_{1}}^{t_{2}} F_{x} d t=M \bar{v}_{x}^{\prime \prime}-M \bar{v}_{x}^{\prime},
$$

in which, as before, $\bar{v}_{x}^{\prime \prime}$ and $\bar{v}_{x}^{\prime}$ are the $x$-components of the velocity of the mass-center of the body at the end and at the beginning, respectively, of the time interval $t_{2}-t_{1}$. The principle of linear impulse and linear momentum then, as expressed in equations (2) and (4), may be stated in words as follows: The algebraic sum of the components, in any direction, of the impulses of the external forces acting on a body during any time interval is equal to the change in the linear momentum of the body in the same direction during the same interval of time, or, stated in the form of an equation,

$$
Q_{x}=\Delta U_{x}
$$

in which $x$ represents any direction. The particular forms in which $Q_{x}$ and $U_{x}$ are expressed depend upon the type of force system, the type of motion, and the kind of body (see Art. 214 for expressions which apply to rigid bodies having particular types of motion).

It should be noted also that equation (3) expresses an important principle which may be stated in words as follows: The algebraic sum of the components, in any direction, of the external forces 
acting on any body is equal to the rate of change of the linear momentum of the body in the same direction.

212. Principle of Angular Impulse and Angular Momentum.It was shown in Art. 146 that if a rigid body rotates about a fixed axis, the algebraic sum of the moments of the external forces about the axis of rotation is equal to the product of the moment of inertia of the body about the axis of rotation and the angular acceleration of the body. That is,

$$
\Sigma T_{0}=I_{0} \alpha \text {. }
$$

Now, if the forces acting on the body remain constant, then $\alpha$ will be constant, that is, the body will rotate with uniformly accelerated motion and hence, from Art. 125,

Therefore,

$$
\alpha=\frac{\omega_{2}-\omega_{1}}{\Delta t} \text {. }
$$

$$
\Sigma T_{0} \cdot \Delta t=I_{0} \omega_{2}-I_{0} \omega_{1}
$$

Now the left-hand member of this equation is the moment of the impulse (angular impulse), about the axis of rotation, of the force system acting on the body, and the right-hand member is the change in the moment of momentum (angular momentum) of the body with respect to the axis of rotation.

If the forces acting on the body are not constant, then $\alpha$ will not be constant, its value at any instant being $\frac{d \omega}{d t}$ (Art. 120). Hence, in general,

$$
\Sigma T_{0}=I_{0} \frac{d \omega}{d t}
$$

and since $I_{0}$ is constant, this equation may be written in the form,

$$
\Sigma T_{0}=\frac{d}{d t}\left(I_{0} \omega\right) . \quad . \quad . \quad . \quad . \quad . \quad .
$$

Now by integrating this equation, the following equation, which expresses the principle of angular impulse and angular momentum for a rigid body rotating about a fixed axis, is obtained,

Or,

$$
\int_{t_{1}}^{t_{2}} \Sigma T_{0} d t=\int_{\omega_{1}}^{\omega_{2}} d\left(I_{0} \omega\right)
$$

$$
\Sigma \int_{l_{1}}^{l_{2}} T_{0} d t=I_{0} \omega_{2}-I_{0} \omega_{1} \text {. . . . . . }
$$


The principle, however, applies to any mass-system having any type of motion. The principle may be stated in words as follows: The algebraic sum of the angular impulses, about any axis, of the external forces acting on any body (mass-system) for any period of time is equal to the change in the angular momentum of the body about the same axis during the same interval of time. Or, expressed in terms of the symbols which already have been defined, the principle is expressed by the equation,

$$
L_{0}=\Delta H_{0}
$$

The particular forms in which $L_{0}$ and $H_{0}$ are expressed depend on the type of force system, the type of motion, and the kind of body. (See Art. 214 for expressions which apply to a rigid body having particular types of motion under the action of constant forces.)

It should be noted also that equation (3) expresses an important principle which may be stated in words as follows: The algebraic sum of the moments of the forces acting on a rotating rigid body about the axis of rotation is equal to the rate of change of the angular momentum of the body about the same axis. The principle, however, is not restricted to the motion of a rigid body rotating about a fixed axis. It applies to any body having any type of motion. It should be noted, however, that, in general, the angular momentum of a body is not equal to $I \omega$.

213. Method of Analysis of the Motion of a Body by Means of Impulse and Momentum.- - It was noted in Art. 144 that, in the analysis of the motion of any body under the action of an unbalanced force system, relations must be found which involve (1) the forces acting on the body, (2) the kinetic properties of the body, and (3) the kinematic properties of the motion of the body (linear and angular velocity or acceleration, etc.).

Now these three factors are involved in the principles of impulse and momentum. And, as noted in Art. 138, in the analysis of the motion of any mass-system in a plane, three equations are needed. Two of these equations are obtained by expressing the principle of linear impulse and linear momentum with reference to any two rectangular axes in the plane of motion, and the third equation is obtained by expressing the principle of angular impulse and angular momentum with reference to an axis perpendicular to the plane of motion. Thus, in terms 
of the symbols already defined, the three equations may be written as follows:

$$
\begin{aligned}
& Q_{x}=\Delta U_{x}, \\
& Q_{y}=\Delta U_{y}, \\
& L_{0}=\Delta H_{0} .
\end{aligned}
$$

The particular forms of the expressions for the above quantities depends upon the type of forces (whether constant or variable, etc.), the kind of body (whether rigid, etc.), and the type of motion (whether translation, rotation, plane motion, etc.). The particular equations for a rigid body having a motion of translation, of rotation, and of plane motion, under the action of constant forces, are given in the next article.

214. Application of the Principles of Impulse and Momentum to Special Types of Motion of Rigid Bodies.-The relation between the impulse of an unbalanced force system which acts on a body and the momentum of the body, as expressed in a general form by the three equations in the preceding article, may be expressed in a more detailed form for the special motions of translation, rotation, and plane motion of a rigid body as follows:

I. Translation of a Rigid Body under the Action of Constant Forces. - The velocities of all particles of the body are the same (Art. 132). Therefore, the linear momentum of the body is equal to $M v$ and since its position line passes through the masscenter of the body, the angular momentum of the body about an axis through the mass-center is equal to zero. Therefore (using symbols which have been defined in the preceding articles), the three equations of Art. 213 become,

$$
\begin{aligned}
& \Sigma F_{x} \cdot \Delta t=\Delta(M v)_{x}=M\left(v_{x}^{\prime \prime}-v_{x}{ }^{\prime}\right), \\
& \Sigma F_{y} \cdot \Delta t=\Delta(M v)_{y}=M\left(v_{y}^{\prime \prime}-v_{y}{ }^{\prime}\right), \\
& \Sigma \bar{T} \cdot \Delta t=0 .
\end{aligned}
$$

II. Rotation of a Rigid Body under the Action of Constant Forces.-The linear momentum of the body is $M \bar{v}$ (Art. 207) and the angular momentum about the axis of rotation is $I_{0} \omega$ (Art. 208). Therefore (using symbols which have been 
, defined in the preceding articles) the equations of Art. 213 become,

$$
\begin{aligned}
& \Sigma F_{x} \cdot \Delta t=\Delta(M \bar{v})_{x}=M\left(\overline{\boldsymbol{v}}_{x}{ }^{\prime \prime}-\overline{\boldsymbol{v}}_{x}{ }^{\prime}\right), \\
& \Sigma F_{y} \cdot \Delta t=\Delta(M \bar{v})_{\nu}=M\left(\overline{\boldsymbol{v}}_{y}{ }^{\prime \prime}-\overline{\boldsymbol{v}}_{y}{ }^{\prime}\right), \\
& \Sigma T_{0} \cdot \Delta t=\Delta\left(I_{o} \omega\right)=I_{o}\left(\omega_{2}-\omega_{1}\right) .
\end{aligned}
$$

III. Plane Motion of a Rigid Body under the Action of Constant Forces.-The linear momentum of the body is $M \hat{v}$ (Art. 207) and the angular momentum of the body about an axis through the mass-center is $\bar{I} \omega$ (Art. 209). Therefore (making use of symbols which have been defined in the preceding articles), the equations of Art. 213 become,

$$
\begin{aligned}
\Sigma F_{x} \cdot \Delta t & =\Delta(\boldsymbol{M} \bar{v})_{x}=M\left(\bar{v}_{x}{ }^{\prime \prime}-\bar{v}_{x}{ }^{\prime}\right), \\
\Sigma F_{y} \cdot \Delta t & =\Delta(M \bar{v})_{y}=M\left(\bar{v}_{y}{ }^{\prime \prime}-\bar{v}_{y}{ }^{\prime}\right), \\
\Sigma \bar{T} \cdot \Delta t & =\Delta(\bar{I} \omega)=\bar{I}\left(\omega_{2}-\omega_{1}\right) .
\end{aligned}
$$

It should be noted that each of the three sets of equations above may be readily transformed into the set of equations which was derived in Chapter IX for the corresponding motion. However, under certain conditions, the above equations are more convenient to use in the analysis and solution of problems than are the equations of Chapter IX. Furthermore, these equations lead to an important special principle called the principle of the conservation of momentum (Art. 215).

\section{ILLUSTRATIVE PROBLEMS}

489. A cylindrical jet of water $1 \frac{1}{2} \mathrm{in}$. in diameter impinges on a fixed blade which is inclined at an angle of $30^{\circ}$ with the direction of the jet as shown in

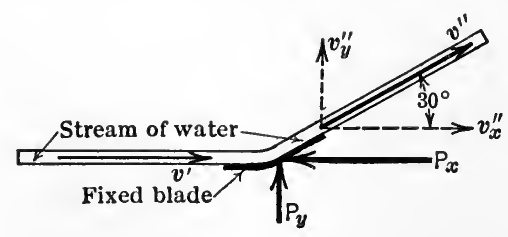

FIg. 444.

Fig. 444. The velocity of the jet is $25 \mathrm{ft}$. per sec. Find the horizontal and vertical components of the pressure of the water on the blade (or blade on the water). Assume that the magnitude of the velocity of the jet is not changed by the action of the blade. Also assume that the only force acting on the water while it is in contact with the blade is the pressure of the blade.

Solution.-Let $P_{x}$ and $P_{y}$ be the unknown horizontal and vertical pressures 
exerted by the blade on the water, these pressures being the cause of the change in the momentum of the water.

The principle of impulse and momentum states that,

$$
\begin{aligned}
& \Sigma F_{x} \cdot \Delta t=M\left(v_{x}^{\prime \prime}-v_{x}^{\prime}\right), \text {. . . . . . } \\
& \Sigma F_{y} \cdot \Delta t=M\left(v_{y}{ }^{\prime \prime}-v_{y}{ }^{\prime}\right) . \quad . \quad . \quad . \quad . \quad . .
\end{aligned}
$$

Let $\Delta t$ be taken as any convenient time interval ( 1 sec. say). Then $M$ is the mass of the water upon which the blade acts in the same time interval.

Taking the direction of the velocity of the impinging water as positive and the weight of water as $62.5 \mathrm{lb}$. per cubic foot, we have,

From (1)

Whence,

$$
P_{x} \cdot 1=\frac{\pi(1.5)^{2} \times 25 \times 62.5}{4 \times 144 \times 32.2}\left(25-25 \cos 30^{\circ}\right) .
$$

$$
P_{x}=0.594(25-21.65)
$$$$
=1.99 \mathrm{lb} \text {. }
$$

From (2),

Whence,

$$
P_{y} \cdot 1=0.594\left(25 \sin 30^{\circ}-0\right) \text {. }
$$

$$
P_{y}=7.43 \mathrm{lb} .
$$

490. A flywheel weighing $1288 \mathrm{lb}$. is keyed to a shaft 4 in. in diameter. The shaft transmits a turning moment of $1200 \mathrm{in.-lb.} \mathrm{to} \mathrm{the} \mathrm{flywheel,} \mathrm{thereby}$ increasing its angular velocity from $600 \mathrm{rad}$. per min. to $50 \mathrm{rad}$. per sec. in 10 sec. Find the radius of gyration of the flywheel.

Solution.-The flywheel is a rotating rigid body. Only one of the three equations which apply to a rotating rigid body is needed in this particular problem; namely,

Hence,

$$
\begin{aligned}
\Sigma T_{0} \cdot \Delta t & =I_{0}\left(\omega_{2}-\omega_{1}\right) \\
& =M k_{0}{ }^{2}\left(\omega_{2}-\omega_{1}\right) .
\end{aligned}
$$

Whence,

and,

$$
\frac{1200}{12} \times 10=\frac{1288}{32.2} k_{0}{ }^{2}\left(50-\frac{600}{60}\right) \text {. }
$$

$$
\begin{aligned}
k_{0}{ }^{2} & =0.625, \\
k_{0} & =0.79 \mathrm{ft} .
\end{aligned}
$$

\section{PROBLEMS}

491. A jet of water $2 \mathrm{in}$. in diameter has a velocity of $30 \mathrm{ft}$. per sec. in a horizontal direction. If the jet impingas normally against a fixed vertical plane, what is the pressure of the water on the plane? If the plane is moving in the direction of the jet with a velocity of $10 \mathrm{ft}$. per sec., what is the pressure on the plane?

Ans. $P=38.1 \mathrm{lb}$; $P=16.9 \mathrm{lb}$.

492. A $5 \frac{1}{2}$-oz. baseball moving horizontally with a velocity of $150 \mathrm{ft}$. per sec. is struck by a bat and is deflected $135^{\circ}$ from its original direction as 
indicated in Fig. 445. If the speed of the ball as it leaves the bat is $130 \mathrm{ft}$. per sec., compute the horizontal and vertical components of the impulse of

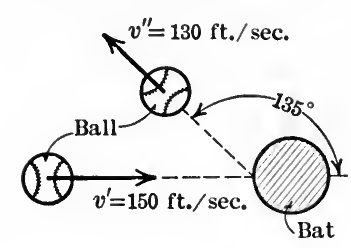

FIG. 445 . the bat on the ball. Assuming that the time of contact is $\frac{1}{50}$ sec., determine the average value of the force during the impact.

493. The table of a planing machine together with the material bolted on it weighs 5 tons. Find the time required to change its velocity from $20 \mathrm{ft}$. per min. (cutting stroke) to $40 \mathrm{ft}$. per min. in the opposite direction (return stroke) if the average force of the pinion on the rack while the velocity is being changed is $200 \mathrm{lb}$. Ans. $t=1.55 \mathrm{sec}$.

494. The rotating parts of a horizontal-shaft turbine weigh 20 tons and have a radius of gyration of $2 \mathrm{ft}$. It takes $10 \mathrm{~min}$. for the turbine to come to rest from a speed of 55 r.p.m. under the influence of journal friction alone. The shaft is 12 in. in diameter. What is the average coefficient of friction?

495. A sphere having a weight of $64.4 \mathrm{lb}$. and a diameter of $30 \mathrm{in}$. rolls without slipping down a plane inclined $30^{\circ}$ with the horizontal. What will be the velocity of its center at the end of 5 sec. if the initial velocity of its center is $30 \mathrm{ft}$. per sec.?

Ans. $v=87.5 \mathrm{ft} . / \mathrm{sec}$.

496. A body slides down a plane irclined $45^{\circ}$ with the horizontal. If the coefficient of kinetic friction is 0.2 , how many seconds will it take for the velocity of the body to change from $10 \mathrm{ft}$. per sec. to $30 \mathrm{ft}$. per sec.?

$$
\text { Ans. } t=1.09 \mathrm{sec} \text {. }
$$

497. A certain machine gun fires 350 bullets per minute. If each bullet weighs $1 \mathrm{oz}$. and the muzzle velocity of the bullets is $2200 \mathrm{ft}$. per sec., what is the average reaction of the gun against its support? Neglect the reaction due to the discharged gases.

498. In the relief valve shown in Fig. 446 the discharge area is assumed to be equal to the circumference of the pipe times the lift of the valve. The rate of discharge of the water is $2 \mathrm{cu}$. ft. per sec. The diameter, $d$, of the pipe is $6 \mathrm{in}$. The "lift" is $0.25 \mathrm{in}$. The pressure $p$ in the pipe is $30 \mathrm{lb}$. per sq. in. Find the force exerted by the spring on the valve. Hint: The force causing the change in the horizontal component of the momentum of the water from $M v_{1}$ to $M v_{2} \cos 45^{\circ}$ is the difference between the pressure

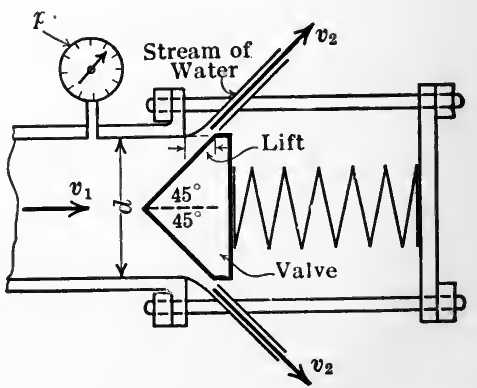

FIG. 446. on a cross-section of the water in the pipe and the force exerted by the spring. 
215. Conservation of Momentum.-I. Linear Momentum.As already noted, the principle of linear impulse and linear momentum for the motion of any mass-system under the action of an unbalanced external force system is expressed by the equation.

$$
\begin{aligned}
Q_{x} & =\Delta U_{x} \\
& =\Delta\left(M \bar{v}_{x}\right),
\end{aligned}
$$

in which $x$ represents any direction. Now if the resultant of the forces which act on the body has no component in the $x$-direction, then the impulse, $Q_{x}$, of the force system in the $x$-direction will be zero and hence, $\Delta\left(M \bar{v}_{x}\right)$ will be equal to zero. Thus,

$$
\Delta\left(M \bar{v}_{x}\right)=0 \text { or } \quad M \bar{v}_{x}=a \text { constant } .
$$

That is, if the resultant of the external forces which act on a body has no component in a given direction, then the linear momentum of the body in the given direction remains constant. This statement expresses the principle of the conservation of linear momentum.

II. Angular Momentum.-As already noted, the principle of angular impulse and angular momentum for the motion of any body under the action of an unbalanced external force system is expressed by the equation,

$$
L_{0}=\Delta H_{0}
$$

Now if the external forces which act on the body have no resultant moment about a given axis, $O$, then the angular impulse, $L_{0}$, of the forces about the same axis will be zero and hence $\Delta H_{0}$ will be equal to zero. Thus,

$$
\Delta H_{0}=0 \text { or } H_{0}=a \text { constant } .
$$

That is, if the external forces which act on a body have no resultant moment about an axis, then the angular momentum of the body with respect to that axis remains constant. This statement expresses the principle of the conservation of angular momentum.

It was shown in Arts. 208 and 209 that the angular momentum, $H_{0}$, of a body about an axis, $O$, is expressed by $I_{0} \omega$ if one of the following conditions is satisfied:. (a) The body is rigid and rotates about a fixed axis, the $\mathrm{O}$-axis being taken as the axis of rotation; (b) the body is rigid and has a plane motion and the $O$-axis passes through the mass-center of the body. Further, $I_{0} \omega$ also expresses 
the angular momentum of a non-rigid mass-system about a fixed axis of rotation provided that all parts of the mass-system have the same angular velocity. Thus, if a rod rotates about a fixed axis as bodies slide radially outwards (or inwards) along the rod, the mass-system is not rigid but the angular momentum of the system about the axis, $O$, of rotation is $I_{0} \omega$.

Therefore, the principle of conservation of angular momentum, when the above conditions are satisfied, may be expressed as follows:

$$
I_{0} \omega=\text { a constant. }
$$

Thus, if $I_{0}$ decreases $\omega$ must increase and vice versa. For example, a gymnast who leaves the swinging trapeze at the top of a circus tent with a relatively small angular velocity $\omega$ (his body being extended) may increase his angular velocity and make several complete turns in mid-air as he descends in a vertical plane by " doubling up." His moment of inertia is thereby decreased and his angular velocity is increased a sufficient amount to keep $I_{0} \omega$ constant since no external torque acts on him while he is descending.

\section{ILLUSTRATIVE PROBLEMS}

499. The weight of the parts of a 3 -in. field gun (Fig. 447) which move during recoil is $950 \mathrm{lb}$. The weight of the projectile is $15 \mathrm{lb}$. and that of the

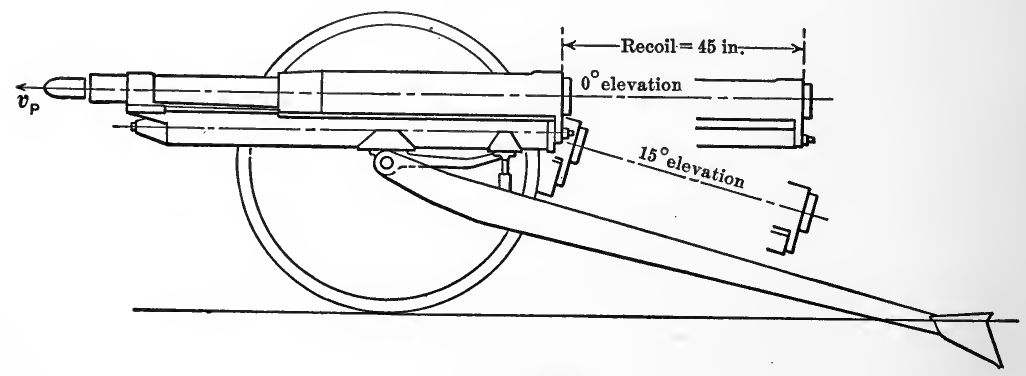

FIg. 447.

powder charge is $1.5 \mathrm{lb}$. The muzzle velocity is $1700 \mathrm{ft}$. per sec. Determine the velocity of free recoil at the time the projectile reaches the muzzle (end of barrel) assuming that the projectile leaves the gun with a horizontal velocity.

Solution.-Three bodies are to be considered; the projectile, the powder charge, and the recoiling parts of the gun. Since the recoil is free, there are no horizontal external forces acting on these three bodies while the projectile is reaching the muzzle of the gun and hence, the linear momentum of the system 
remains constant. That is, the momentum of the projectile plus the momentum of the gases is equal to the momentum of the recoiling parts. Thus,

$$
M_{p} v_{p}+M_{g} \bar{v}_{g}=M_{r} v_{r}
$$

The gases (and unburned powder) form a non-rigid body and hence the velocity, $\bar{v}_{g}$, of the mass-center must be used. It is usually assumed that $\bar{v}_{g}$ is one-half of the velocity of the projectile. Thus, using weights instead of masses since they are proportional, we have,

Hence,

$$
15 \times 1700+1.5 \times \frac{1700}{2}=950 v_{r} .
$$

$$
\begin{aligned}
v_{r} & =\frac{25,500+1275}{950} \\
& =28.1 \mathrm{ft} . / \mathrm{sec} .
\end{aligned}
$$

The velocity of free recoil as the projectile reaches the muzzle of the gun is about 0.7 of the maximum velocity of free recoil. The bore is filled with gases for a short interval after the projectile leaves the gun and these gases continue to exert pressure on the breech and thus to increase the velocity of recoil.

500. 'Two spheres (Fig. 448) are mounted on a light rod on which the spheres may slide without friction. The rod and spheres rotate about the vertical central axis. A string is attached to each sphere and runs over pulleys so that the pull of each string is directed along the rod. Each sphere weighs $8 \mathrm{lb}$. and is $2 \frac{1}{2}$ in. in diameter. When the distance of the center oi each sphere from the axis of rotation is $2 \mathrm{ft}$., the angular velocity of the rod is 60 r.p.m. If the spheres are pulled a distance of 6 in. along the rod

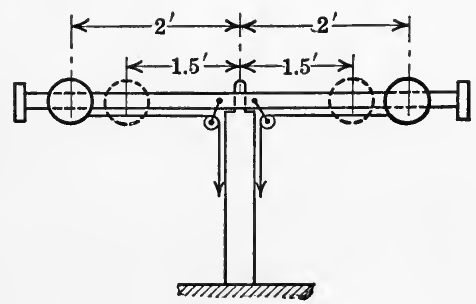

Fig. 448. toward the axis of rotation what will be the angular velocity of the rod?

Solution.-Since the external forces acting on the spheres have no moment about the axis of rotation, the angular momentum with respect to the axis of rotation remains constant. That is, the angular momentum of the spheres before they are pulled in is equal to their angular momentum after they are pulled in. Whence,

$$
I_{1} \omega_{1}=I_{2} \omega_{2}
$$

Little error will be introduced by considering the spheres to be particles and by neglecting the mass of the rod. Thus,

$$
2 M \times(2)^{2} \times \frac{60 \times 2 \pi}{60}=2 M \times(1.5)^{2} \omega_{2} .
$$


Hence,

$$
\begin{aligned}
\omega_{2} & =\frac{4 \times 2 \pi}{2.25}=11.15 \mathrm{rad} . / \mathrm{sec} . \\
& =106.6 \mathrm{r} . \mathrm{p} . \mathrm{m} .
\end{aligned}
$$

Thus, it will be noted that as the moment of inertia of the spheres decreases, their angular velocity must increase. And since the moment of inertia decreases as the square of the distance from the axis of rotation, a relatively small inward movement of the spheres causes a relatively large increase in the angular velocity.

If the pulls of the strings are released when the spheres are at any distance,

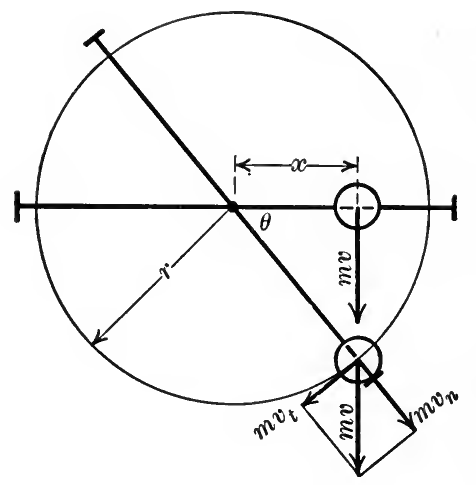

Fig. 449 . $x$ (Fig. 449), from the axis of rotation, then no force in the horizontal plane will be acting on either sphere, and hence each sphere will continue with constant speed in a straight line until it strikes the stop at the end of the rod as indicated in Fig. 449. That is, the linear momentum of each sphere remains constant until the stop is hit and then changes from $m v$ to $m v_{t}$. The angular momentum of the sphere, however, is the same after the sphere strikes the stop as it was before the sphere struck the stop since no moment is introduced by the pressure of the stop. Thus,

$$
m v \cdot x=m v_{t} \cdot r .
$$

\section{PROBLEMS}

501. If the pulls of the strings on the two spheres of Prob. 500 are released when each sphere is $2 \mathrm{ft}$. from the axis of rotation and the angular velocity of the rod is 90 r.p.m., what will be the linear velocity of each sphere after hitting the stop, assuming the rod to be $6 \mathrm{ft}$. long? What is the impulse of the sphere against the stop?

502. A 2-oz. bullet moving with a velocity of $2000 \mathrm{ft}$. per sec. strikes, centrally, a block of wood which is moving on a smooth horizontal plane in the same direction as the bullet with a velocity of $20 \mathrm{ft}$. per sec. If the block of wood in which the bullet embeds itself weighs $16.8 \mathrm{lb}$., what is the resulting velocity of the block and bullet?

$$
\text { Ans. } v=34.6 \mathrm{ft} . / \mathrm{sec} \text {. }
$$

503. Two similar pulleys are running loose on a shaft. One has an angular velocity of 10 r.p.m. and the other a velocity of 30 r.p.m. in the opposite direction. They are suddenly coupled together by means of a friction clutch. What will be the angular velocity of the pulleys after the clutch has ceased to slip? What proportion of the kinetic energy is lost?

Ans. 10 r.p.m.; 80 per cent. 
504. A 4.7 in. howitzer field gun rests on a wooden platform. Recoil is checked by heavy ropes attached to stakes driven into the ground in front of the gun. Assuming that the slack in the ropes allows the velocity of free recoil to be developed, find the velocity of recoil from the following data: Weight of gun, $7000 \mathrm{lb}$; weight of projectile, $63 \mathrm{lb}$.; weight of powder charge, $6 \mathrm{lb}$; muzzle velocity, $1500 \mathrm{ft}$. per sec.

216. Impact.-The equations of Art. 213 which express the principles of impulse and momentum apply to the motion of bodies whether the bodies move under the action of impulsive forces or of forces which act during a finite time interval. In fact, as stated in Art. 201, the principles of impulse and momentum are particularly well adapted to the solution of kinetics problems which involve sudden impulses (see Art. 201 for various illustrations).

The effect of impulsive forces on the motion of a body, in most problems, is so large in comparison with the effect of the other forces which act on the body that the effect of the other forces on the motion of the body, while the impact lasts, may be neglected. The only details of the change in the motion of a body that can be determined, when the change in the motion is caused by impulsive forces, are the initial and final velocities of the body. For, the distance traveled during the impact is indefinitely small; the time interval is also indefinitely small and hence, the acceleration produced is indefinitely large since the change in velocity is a finite quantity. Thus, the distance, time, and acceleration are indeterminate. There is, however, a definite (appreciable) change in the velocity although, as just noted, the manner in which the velocity changes during the period of the impact is unknown and only the initial and final values of the velocity can be determined. Therefore, the momentum of the body at the beginning and at the end of the impact period are definite quantities and, since these quantities are involved in the principles of impulse and momentum, problems which involve impulsive forces yield to this method of solution although the impulse of the impulsive forces is used and not the forces themselves.

Definitions.- It is convenient to classify or define some of the ways in which impact of bodies may occur.

Direct and Oblique Impact.-If two bodies collide and the velocity of each is directed normal to the striking surfaces, the impact is said to be direct. When this condition is not fulfilled the impact is called oblique. 
Central and Eccentric Impact.-If two bodies collide in such a way that the action line of the pressures exerted by the bodies on each other is directed along the line connecting the mass-centers of the two bodies, the impact is called central. If the action line of the pressures is not so directed the impact is called eccentric.

Impact may occur, of course, with two translating bodies; with two rotating bodies; with two bodies having plane motion; or, with two bodies, one having any one of these three types of motion and the other having either one of the remaining two types of motion. Two of these combinations of motion are treated in the subsequent articles.

The period of impact may be divided into two parts: the time of deformation during which the impulsive force is increasing to its maximum value as the two bodies deform, and (2) the time of restitution during which the bodies are separating and partially recovering from the deformation. If the two bodies were perfectly elastic, the period of deformation would be equal to the period of restitution and, the velocity of separation would be equal to the velocity of approach. But, all bodies are more or less inelastic and hence, the velocity of separation is always somewhat less than the velocity of approach.

Coefficient of Restitution.-For direct central impact of two bodies, the ratio of the relative velocity of separation to the relative velocity of approach is defined as the coefficient of restitution. Thus, if the velocities before impact are denoted by $v_{1}$ and $v_{2}$ and after impact by $v_{1}{ }^{\prime}$ and $v_{2}{ }^{\prime}$ and if $e$ denotes the coefficient of restitution, the value of $e$ is defined by the following equation,

$$
e=-\frac{v_{2}{ }^{\prime}-v_{1}{ }^{\prime}}{v_{2}-v_{1}} \text { or } \quad v_{2}^{\prime}-v_{1}^{\prime}=-e\left(v_{2}-v_{1}\right) \text {. }
$$

Experiments show that the value of the coefficient of restitution for two spheres in central direct impact depends only on the materials of the two spheres. It is generally assumed that the value of $e$ as found for two spheres of any two materials is the same for other bodies of the same materials whether the impact is central and direct or not. But if the impact is not central and direct the components of the velocities normal to the impact surfaces must be used in equation (1) instead of the total velocities.

Notation.-In dealing with the problem of the impact of two 
bodies as discussed in the following articles, the notation as indicated below will be used.

$M_{1}=$ the mass of one body.

$M_{2}=$ the mass of the second body.

$v_{1}\left(\right.$ or $\left.\omega_{1}\right)=$ the linear (or angular) velocity of the impact surface of $M_{1}$ before impact occurs.

$v_{2}\left(\right.$ or $\left.\omega_{2}\right)=$ the linear (or angular) velocity of the impact surface of $M_{2}$ before impact occurs.

$v_{1}^{\prime}\left(\right.$ or $\left.\omega_{1}^{\prime}\right)=$ the linear (or angular) velocity of the impact surface of $M_{1}$ after impact.

$v_{2}^{\prime}\left(\right.$ or $\left.\omega_{2}^{\prime}\right)=$ the linear (or angular) velocity of the impact surface of $M_{2}$ after impact.

$v($ or $\omega)=$ the linear (or angular) velocity of the impact surfaces at the end of the deformation period.

$t_{d}=$ time of deformation.

$t_{r}=$ time of restitution.

$t=t_{d}+t_{r}=$ total time of contact.

$\int_{0}^{t_{d}} P_{d} d t=$ impulse or impact for the time of deformation.

$\int_{t_{d}}^{t} P_{r} d t=$ impulse or impact for the time of restitution.

$\left(P_{d}\right)_{a v}=$ the average force for the time of deformation. That is,

$$
\int_{0}^{t_{d}} P_{d} d t=\left(P_{d}\right)_{a v} \cdot t_{d}
$$

$\left(P_{r}\right)_{a v}=$ the average force for the time of restitution. That is,

$$
\int_{t_{d}}^{t} P_{r} d t=\left(P_{r}\right)_{u v} \cdot t_{r} .
$$

217. Impact of Two Translating Bodies.-Direct Central Impact.-In Fig. 450 are represented two translating bodies which collide with direct central impact. It is

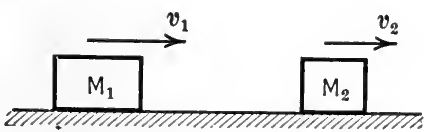

FIG. 450. assumed that the values of

$$
M_{1}, M_{2}, v_{1}, v_{2} \text {, and } e \text {, }
$$


are known, and it is required to find the values of

$$
v, v_{1}{ }^{\prime}, v_{2}{ }^{\prime}, \int_{0}^{t_{d}} P_{d} d t \text { or }\left(P_{d}\right)_{a v} \cdot t_{d} \text {, and } \int_{t_{d}}^{t} P_{r} d t \text { or }\left(P_{r}\right)_{a v} \cdot t_{r} \text {. }
$$

Evidently five equations must be found from which the five unknown quantities may be determined. The five equations may be found as follows: From the principle of conservation of linear momentum equations (1) and (2) below are obtained. Thus,

$$
\begin{gathered}
M_{1} v_{1}+M_{2} v_{2}=\left(M_{1}+M_{2}\right) v \text { for the period } t_{d} . . . \\
\left(M_{1}+M_{2}\right) v=M_{1} v_{1}{ }^{\prime}+M_{2} v_{2}{ }^{\prime} \text { for the period } t_{r} .
\end{gathered}
$$

And from Art. 216,

$$
v_{1}^{\prime}-v_{2}{ }^{\prime}=-e\left(v_{1}-v_{2}\right)
$$

From these three equations the values of $v, v_{1}{ }^{\prime}$, and $v_{2}{ }^{\prime}$ may be found. The impulses for the periods $t_{d}$ and $t_{r}$ may now be found by applying the principle of linear impulse and momentum for the periods $t_{d}$ and $t_{r}$, which leads to the following equations,

$$
\begin{aligned}
& \int_{0}^{t_{d}} P_{d} d t=M_{1}\left(v-v_{1}\right)=M_{2}\left(v-v_{2}\right), \ldots . . \\
& \int_{t_{d}}^{t} P_{r} d t=M_{1}\left(v_{1}^{\prime}-v\right)=M_{2}\left(v_{2}^{\prime}-v\right) . \quad . \quad . \quad . \quad .
\end{aligned}
$$

\section{PROBLEMS}

505. A freight car weighing 40 tons and traveling at a speed of $20 \mathrm{mi}$. per hour on a straight track overtakes another car weighing 30 tons and traveling on the same track in the same direction at a speed of $15 \mathrm{mi}$. per hour. If the value of $e$ is 0.2 , find the velocity of each car after impact and the impulse of each car on the other both for the time of deformation and for the time of restitution.

$$
\begin{aligned}
\text { Ans. } v_{1}{ }^{\prime}=17.4 \mathrm{mi} . / \mathrm{hr} . ; v_{2}{ }^{\prime}=18.4 \mathrm{mi} . / \mathrm{hr} . ; & \int_{0}^{t_{d}} P_{d} d t=7820 \mathrm{lb} .-\mathrm{sec} . ; \\
& \int_{t_{d}}^{t} P_{r} d t=1560 \mathrm{lb} \text {.-sec. }
\end{aligned}
$$

506. A body weighing $50 \mathrm{lb}$. moving to the right collides with a 30-lb. body moving to the left. The speed of each body is $15 \mathrm{ft}$. per sec. The impact of the two bodies is direct and central. If the coefficient of restitution is 0.6 , find $(a)$ the velocity of each body after impact, $(b)$ the velocity 
of each body at the end of the deformation period, $(c)$ the impulse or impact for the deformation period, and $(d)$ the impulse for the period of restitution. Ans. $v_{1}{ }^{\prime}=-3 \mathrm{ft} . / \mathrm{sec}$.; $v_{2}{ }^{\prime}=15 \mathrm{ft} . / \mathrm{sec}$.; $v=3.75 \mathrm{ft} . / \mathrm{sec}$.

218. Impact of a Translating Body with a Rotating Body.-In Fig. 451 let $M_{2}$ be the mass of a body which is rotating about a fixed axis through $O$ with angular velocity $\omega_{2}$ when a small translating body of mass $M_{1}$ having a velocity $v_{1}$ collides with it at a distance $h$ from the axis of rotation. This is an example of direct eccentric impact in which the known quantities are,

$$
b, h, M_{1}, M_{2}, I_{2}, v_{1}, \omega_{2}, \bar{v}_{2}=\omega_{2} b \text {, and } e
$$

where $I_{2}$ denotes the moment of inertia of

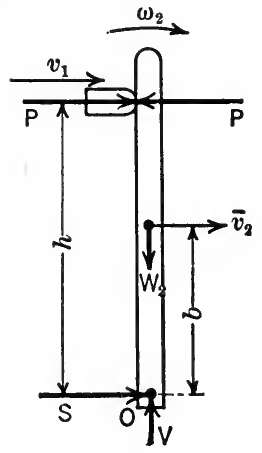

Fig. 451. $M_{2}$ about the axis of rotation. And the quantities to be found are,

$$
v, \omega, v_{1}{ }^{\prime}, v_{2}{ }^{\prime}, \int_{0}^{t_{d}} P_{d} d t, \int_{t_{d}}^{t} P_{r} d t, \int_{0}^{i_{d}} S_{d} d t \text {, and } \int_{t_{d}}^{t} S_{r} d t
$$

The necessary equations from which to solve for the eight unknown quantities may be obtained as follows: From the principle of conservation of angular momentum the equations (1) and (2) below are obtained. Thus,

and,

$$
M_{1} v_{1} h+I_{2} \omega_{2}=M_{1} v h+I_{2} \omega, \text { for the period } t_{d}, \quad .
$$

$$
M_{1} v h+I_{2} \omega=M_{1} v_{1}{ }^{\prime} h+I_{2} \omega_{2}{ }^{\prime} \text {, for the period } t_{r} . .
$$

And from Art. 216,

$$
v_{1}^{\prime}-v_{2}^{\prime}=-e\left(v_{1}-v_{2}\right) . \quad \text {. . . . . . . . }
$$

And from Art. 115,

$$
v=\omega h \text {. }
$$

From these four equations the quantities $v, \omega, v_{1}{ }^{\prime}$, and $v_{2}{ }^{\prime}$ may be determined. Then from the principle of linear impulse and momentum for the mass $M_{1}$ the values of the impulse of the pres- 
sure $P$ for the periods $t_{d}$ and $t_{r}$ may be found from the following equations,

$$
\begin{aligned}
& \int_{0}^{t_{d}} P_{d} d t=M_{1}\left(v-v_{1}\right) \text { for the period } t_{d}, \ldots \\
& \int_{t_{d}}^{t} P_{r} d t=M_{1}\left(v_{1}{ }^{\prime}-v\right) \text { for the period } t_{r} . \\
& .
\end{aligned}
$$

In a similar way by applying the principle of linear impulse and momentum to the mass $M_{2}$, the values of the impulse of the reaction $S$ for the periods $t_{d}$ and $t_{r}$ may be found from the following equations,

$$
\begin{aligned}
& \int_{0}^{t_{d}} P_{d} d t+\int_{0}^{t_{d}} S_{d} d t=M_{2}\left(\bar{v}-\bar{v}_{2}\right) \text { for the period } t_{d}, \ldots . \\
& \int_{t_{d}}^{t} P_{\tau} d t+\int_{t_{d}}^{t} S_{r} d t=M_{2}\left(\bar{v}_{2}{ }^{\prime}-\bar{v}\right) \text { for the period } t_{r} . \quad . \quad .
\end{aligned}
$$

The kinetics problems involving the impact of two rotating bodies, of a translating body with a body having plane motion, or of other combinations of motions may be solved by methods similar to those used in the last two articles.

\section{PROBLEM}

507. The following data apply to the bodies represented in Fig. 451. The weight of the translating body is $W_{1}=6 \mathrm{lb}$. and $v_{1}=40 \mathrm{ft}$. per sec. The rotating body is a slender rod $6 \mathrm{ft}$. long which is rotating at 4 rad.per sec. when impact occurs. The weight of the rod is $15 \mathrm{lb}$., $h=5 \mathrm{ft}$., and $e=0.8$. Find $v, v_{1}^{\prime}, v_{2}^{\prime}, \int_{0}^{t_{d}} P_{d} d t$ and $\int_{0}^{t_{d}} S_{d} d t$. Assuming that the deformation period is $\frac{1}{10}$ sec., what is the average value of $S$ during the deformation?

$$
\text { Ans. } v_{1}^{\prime}=20.4 \mathrm{ft} . / \text { sec.; } v_{2}{ }^{\prime}=36.4 \mathrm{ft} . / \text { sec.; } S_{a v}=5.1 \mathrm{lb} \text {. }
$$

219. Loss of Kinetic Energy in Direct Central Impact.-The linear momentum of two bodies before impact is the same as the momentum after impact but the kinetic energy of the two bodies after impact is less than the kinetic energy before impact since a part of the energy is dissipated during impact in doing work in compressing the two bodies which are more or less inelastic. If the value of the coefficient of restitution for two bodies were unity, that is if the two bodies were perfectly elastic, no energy would be dissipated during impact since the work done in the 
deformation period would equal the work recovered during the period of restitution. The amount of energy lost in impact is the difference between the energy of the two bodies before impact and the energy after impact. That is, the loss of kinetic energy, $E_{L}$, is given by the expression,

$$
E_{L}=\left(\frac{1}{2} M_{1} v_{1}^{2}+\frac{1}{2} M_{2} v_{2}^{2}\right)-\left(\frac{1}{2} M_{1} v_{1}{ }^{2}+\frac{1}{2} M_{2} v_{2}{ }^{2}\right) . . .
$$

The expression for $E_{L}$, however, is more convenient for use if the velocities $\left(v_{1}^{\prime}\right.$ and $\left.v_{2}^{\prime}\right)$ of the two bodies after impact are eliminated. This may be done as follows: From the principle of conservation of linear momentum the following equation is found,

$$
M_{1} v_{1}+M_{2} v_{2}=M_{1} v_{1}{ }^{\prime}+M_{2} v_{2}{ }^{\prime} . \quad \text {. . . . }
$$

And from definition,

$$
v_{1}^{\prime}-v_{2}^{\prime}=-e\left(v_{1}-v_{2}\right)
$$

By solving equations (2) and (3), the values of $v_{1}{ }^{\prime}$ and $v_{2}{ }^{\prime}$ are found to be,

and,

$$
v_{1}^{\prime}=v_{1}-(1+e) \frac{M_{2}}{M_{1}+M_{2}}\left(v_{1}-v_{2}\right),
$$

$$
v_{2}^{\prime}=v_{2}-(1+e) \frac{M_{1}}{M_{1}+M_{2}}\left(v_{2}-v_{1}\right) .
$$

By substituting these values of $v_{1}{ }^{\prime}$ and $v_{2}{ }^{\prime}$ in equation (1) the following expression for $E_{L}$ results,

$$
E_{L}=\frac{1}{2}\left(1-e^{2}\right) \frac{M_{1} M_{2}}{M_{1}+M_{2}}\left(v_{1}-v_{2}\right)^{2} .
$$

It will be noted that for two perfectly elastic bodies $(e=1)$ the loss of energy in impact is zero and for two plastic bodies $(e=0)$ the loss of energy is $E_{L}=\frac{1}{2} \frac{M_{1} M_{2}}{M_{1}+M_{2}}\left(v_{1}-v_{2}\right)^{2}$.

The loss of energy in direct eccentric impact and in other cases of impact may be found by a similar method.

220. Angular Momentum a Vector Quantity.-In the preceding articles of this chapter the magnitude, only, of the angular momentum of a body has been assumed to change. However, in expressing the principle of angular impulse and angular momentum for a body which moves so that its plane of motion changes in direction (such as the propeller of an aeroplane when making a 
turn), the angular momentum of the body must be considered as a quantity having direction as well as magnitude, that is, it must be considered to be a vector quantity. The angular momentum of a body may be represented by a vector drawn (1) perpendicular to the plane of motion of the body to indicate the direction of the plane of motion, (2) of such a length that it represents, to some scale, the magnitude of the angular momentum, and (3) with the sense of rotation indicated by an arrow which points in the direction along the vector in which a right-handed screw would advance if given the same sense of rotation as that of the body. Thus, if a disc (Fig. 452) rotates with angular velocity $\omega_{1}$ about the axis $O Z$, its angular momentum $\left(H_{1}=I \omega_{1}\right)$ about the axis of rotation is

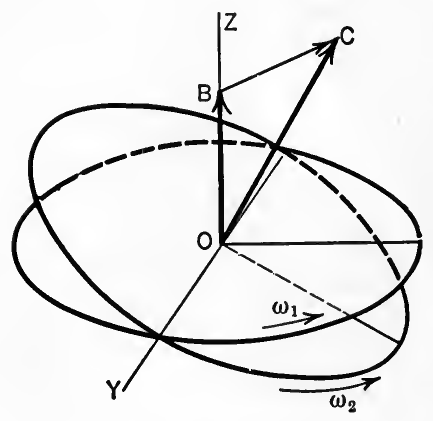

Fig. 452 . represented completely by the vector $O B$. It will be noted that the vector representing the angular momentum may change in length only, in direction only, or both in length and in direction. Thus, in Fig. 452, if the disc is rotated about the axis $O Y$ and at the same time the angular velocity about the $O Z$ axis is increased to $\omega_{2}$, the vector representing the angular momentum $\left(H_{2}=I \omega_{2}\right)$ of the disc is $O C$. Furthermore, the change in the angular momentum of a body is represented completely by the change in the angular momentum vector. Thus, in Fig. 452 the change in the angular momentum of the disc is represented by the vector $B C$.

221. The Gyroscopic Couple.-A disc which rolls round a curved track (Fig. 453a) revolves simultaneously about two rectangular axes and hence it has gyroscopic motion (Art. 165). Gyroscopic motion of a body will here be analyzed briefly by considering the changes in the angular momentum of the body. (See Arts. 166 and 167 for another method of analysis.)

In Fig. 453(a) is represented a disc or wheel which rotates with a constant angular velocity $\omega$ about its axis (axle) $O D$ as it moves round the curved track with a constant angular velocity $\Omega$. It will be observed that the angular velocity of the disc about an axis through $D$ perpendicular to the paper is also equal to $\Omega$. At a 
given instant, the disc is in the position $A_{1} B_{1}$ and its angular momentum, $H_{1}$, is equal to $I \omega$. After an interval of time $d t$ the disc is in the position $A_{2} B_{2}$ and its angular momentum is $H_{2}$, the magnitude of which is also equal to $I \omega$. That is, the angular momentum of the disc $(I \omega)$ has changed in direction, only, during the time interval $d t$.

The change in the angular momentum of the disc from $H_{1}$ to $H_{2}$ is represented by the vector $E F$ (Fig. 453b) which connects the ends of the vectors $H_{1}$ and $H_{2}$. Now, since the angle $d \theta$ is small

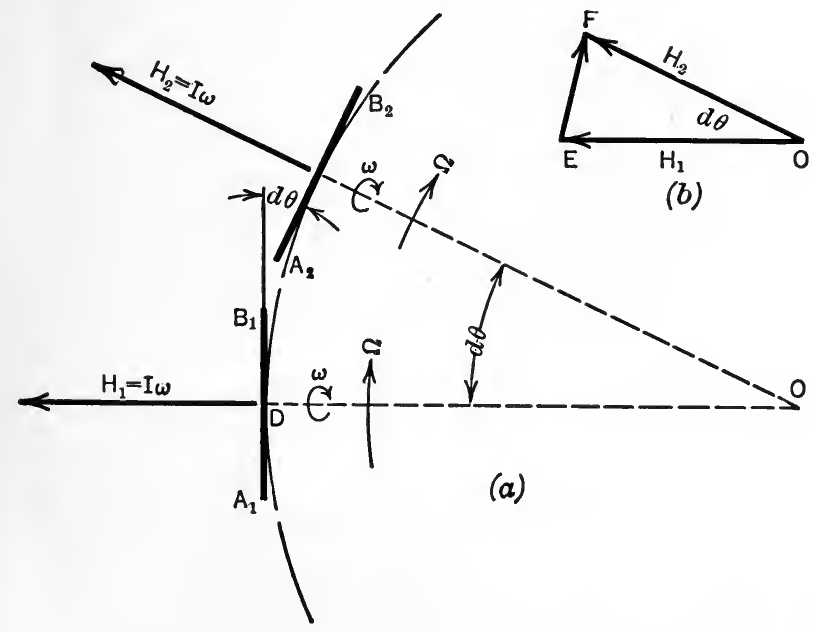

FIg. 453.

(greatly exaggerated in Fig. 453) the length of $D E$, that is, the magnitude of the change in the angular momentum, is

$$
\begin{aligned}
D E=H_{1} d \theta & =H_{2} d \theta \\
& =I \omega d \theta,
\end{aligned}
$$

and the limiting direction of the vector $E F$ as $d \theta$ becomes indefinitely small is perpendicular to $H_{1}$. The rate of change of the angular momentum, then, is

$$
\frac{I \omega d \theta}{d t}=I \omega \Omega
$$

and the direction of the vector which represents this rate of change of the angular momentum is also perpendicular to $H_{1}$. Now a 
torque or couple is always required to produce a change in the angular momentum of a body; the moment of the couple is equal to the rate of change of the angular momentum of the body (Art. 212); the plane in which it acts is perpendicular to the vector which represents the rate of change of the angular momentum; and the sense of rotation of the couple is such that it would cause a right-handed screw to progress (in the direction of the arrow) along the vector which represents the rate of change of the angular momentum.

Therefore, a couple $C$ must act on the disc in a plane perpendicular to the plane of the disc and to the plane of the paper, with a clockwise sense of rotation (as viewed from behind), the magnitude of the couple being

$$
C=I \omega \Omega .
$$

It is evident, therefore, that the disc would turn over counterclockwise (outward) unless a couple having a moment equal to $I \omega \Omega$ acted to prevent the turning. This couple is called the gyroscopic couple.

As noted in Art. 165, the forces of the gyroscopic couple frequently cause considerable pressure on the axle of the rotating body as, for example, in the case of the rotor of an electric locomotive when rounding a curve or of the propeller of an aeroplane when making a turn, etc.

If a body (Fig. 454) rotates or spins about the $z$-axis and a couple $W l$ having a moment about the $x$-axis is applied to the body, the body will rotate about the $y$-axis with an angular velocity $\Omega$ unless a couple having a moment about the $y$-axis acts on the body to prevent the rotation about the $y$-axis. The angular velocity $\Omega$ is called the velocity of precession. It is necessary that the body shall precess in order to develop a resistance to the couple $W l$ and hence prevent the disc from falling. This fact may be shown by following the changes which occur in the angular momentum about the $z$-axis. Thus, when the couple $W l$ (Fig. 454) first acts, the $z$-axis is turned through an angle $d \theta$ thereby causing a change $A B$ in the angular momentum of the disc. This change requires a couple in the horizontal plane with a clockwise sense as viewed from below. But, since there are no bodies to develop or supply this couple, the disc turns (precesses) in the horizontal plane and thus the necessary couple is developed from the inertia of the 
disc. As soon as precession starts, however, that is, as soon as the $z$-axis has turned through the angle $d \phi$, the change $A C$ in the angular momentum is produced. This change requires a couple in the vertical plane (clockwise as viewed by the reader) to prevent the disc and axle from rotating counter-clockwise in the vertical plane. This couple is supplied by the two forces $W$ having a moment $W l$. Hence, if the body is allowed to precess about the

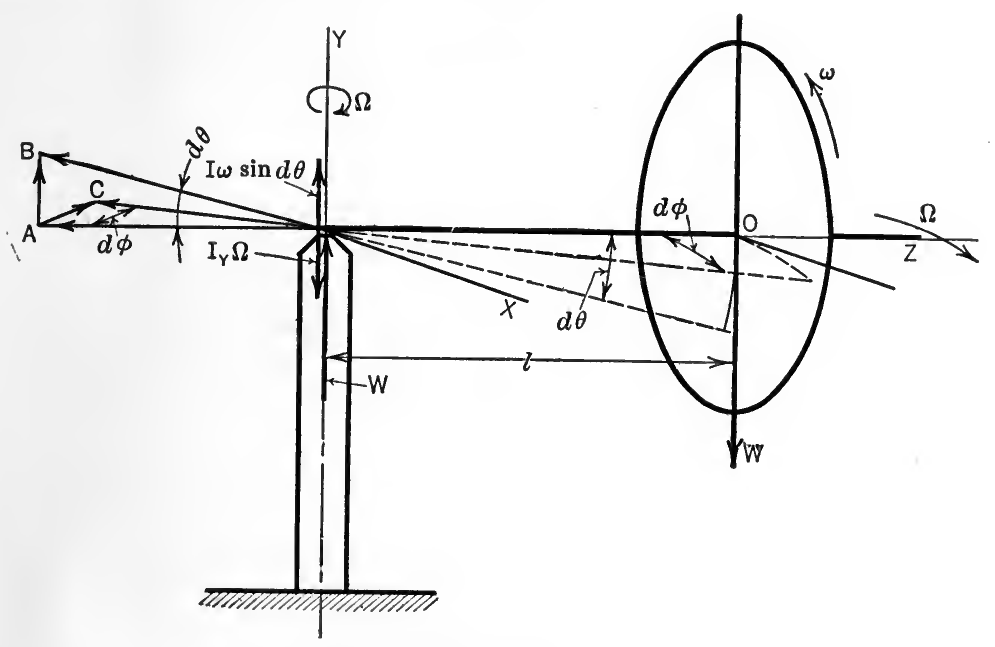

Fig. 454.

$y$-axis with angular velocity $\Omega$, the external couple $W l$ is resisted by the gyroscopic couple, that is,

$$
W l=I \omega \Omega=\frac{W}{g} k^{2} \omega \Omega,
$$

from which the velocity of precession is found to be,

$$
\Omega=\frac{l g}{\omega k^{2}}
$$

in which $k$ is the radius of gyration of the dise with respect to the $z$-axis.

If precession is prevented, a couple $(C=I \omega \Omega)$ must be set up in the horizontal plane, $\Omega$ here being the angular velocity, at any instant, produced by $W l$, which will be the same whether the disc rotates about the $z$-axis or not, since no resistance is offered to the 
external couple unless precession is allowed. This explains why a heavy rotating flywheel or armature on board a ship, with its axis horizontal and athwartship, will offer no more resistance to the rolling of the ship than when it is not rotating. The bearing of the axles, however, must exert a large couple $C=I \omega \Omega$ in a horizontal plane which tends to "nose" the ship around. $\Omega$ here represents the angular velocity of roll.

If an external couple is applied in a horizontal plane to increase or hurry the precession, the disc and axle (Fig. 454a) will rise since $I \omega \Omega>W l$. This principle is employed in the Brennan mono-rail car, the precession being hurried by the rolling of the axle of the revolving flywheels, extended, on a shelf attached to the side of the car. This principle is also used in the "active type" of gyroscope for stabilizing ships. In this case the precession is hurried by means of a precession engine which acts after the ship has rolled a very small amount thus producing a gyroscopic righting-couple sufficient to extinguish the roll. Since the roll is checked in its incipiency, only a small amount of work is done. The stresses produced in the hull of the ship are also small for the same reason, and hence, the weight and displacement of the active type of gyroscope likewise may be small. For those interested in the various ways in which gyroscopic motion may arise and in the progress which has been made in the application of the gyroscope to the stabilizing of ships, mono-rail cars, and aeroplanes; to the guiding of torpedoes; to compasses; and to the Griffin grinding mill, the following references are appended:

1. Journal of the Franklin Institute, May, 1913.

2. American Machinist, August 7 and 14, 1913.

3. Iron Age, December 1, 8, 15, and 22, 1910.

4. Scientific American Supplement, January 26, 1907.

5. Popular Science Monthly, July, 1909.

6. Scientific American, December 18, 1915.

7. Journal American Society of Mechanical Engineers, November, 1920.

Note.-For problems on the gyroscope see Art. 167. 


\section{INDEX}

The numbers refer to pages

Acceleration

- angular, 242

- components, 236, 245, 269

- linear, 235

- of gravity, 5

- relative, 266

- tangential and normal, 236

- uniform circular, 251

- - rectilinear, 250

Angle of repose, 133

Angular momentum, 454, 456, 457

- velocity, 229

Anti-resultant, 8

Balancing, 369

— of rotating masses, 370

Bearings, friction in, 145, 148, 443

Bow's notation, 7, 95

Cables, 102, 106

Catenary, 106

Center of gravity, defined, 173

- - - by experiment, 178

— - parallel force system, 173

- — oscillation, 364

- - percussion, 333, 364

- - suspension, 364

Centrifugal couple, 371

— force, 329, 370

Centroid, defined, 160

- by integration, 163

- graphical method, 177

- of composite figures and bodies, 168

Coefficient of friction, 128

- - restitution, 472

— - rolling resistance, 153

— - speed fluctuation, 435
Composition, defined, 8

- of couples, 60, 62

- - forces, 28, 29, 34, 48, 53, 56, 65

Compound pendulum, 363, 367

Conservation of energy, 439

- - momentum, 467

Conservative system, 421, 440

Coriolis' law, 270

Couple, characteristics of, 20

- definition of, 19

- gyroscopic, 382, 478

- moment of, 19

- resolution of, 64

- transformations of, 22, 44

- vector representation of, 20

D'Alembert's principle, 305, 307, 326, 347

Dimensional equations, 25

Displacement

- angular, 224

- linear, 222

- relative, 263

Dynamics, 2

Dynamometer, 446

Dyne, 297

Effective force, 303

Efficiency, 442

Energy, 420

- conservation of, 439

- dissipation of, 443

- kinetic, 422

- loss of, in impact, 476

- non-mechanical, 428

- of a body, 424

- - particle, 422

- potential, 421 
Equations of motion, defined, 288

- for a particle, 298

- - plane motion, 339, 340

- rotation, 319

- - translation, 308

Equilibrant, 8

Equilibrium, 8

- algebraic conditions of, 70

- diagrams, 69

- equations of, $69,72,73,78,82,110$ 113,114

- graphical conditions of, 70

— of collinear forces, 72

- concurrent forces in a plane, 73

- - - - space, 110

- - parallel forces in a plane, 78

- - - - space, 112

- - non - concurrent, non - parallel forces in a plane, 82

- - non - concurrent, non - parallel forces in space, 114

- - three forces, 75

Erg, 409, 417

First moment, 160

Flexible cables, 102, 106

Flywheel, stresses in, 356

Force, 1

- characteristics of, 4

- component of, 8

- conception of, 1, 3

- effective, 303

- external effect of, 4

— inertia, 307, 326, 345

- measure of, 4

- moment of, 14

- polygon, 31, 36, 49

- resolution of, 11, 22

- units of, 4

- vector representation of, 6

Forces, classification of, 7

- composition of, 8

- impulsive, 449

- inertia, 326, 345

- resultant of a system of, 8,28 ,

- system of, 7

- triangle of, 9
Friction, defined, 127

— belt, 155

- circle, 145

- coefficient of, 128

- cone of, 129

- journal, 145

- laws of, 134

- limiting, 128

- of inclined planes, 135

- - screws, 142

- - wedges, 138

- pivot, 148

- rolling, 153

- work lost due to, 443

Funicular polygon, 37

Geepound, 296

Governors, 386

- centrifugal shaft, 391

- inertia shaft, 392

- loaded, 388

- Porter, 389

- Rite's inertia, 393

Guldinus, theorem of, 171

Gyroscope, 378

- analysis of forces in, 379

- precession, axis of, 383

- - velocity of, 383,480

Gyroscopic couple, 382, 478

Harmonic motion, 253

Hoop tension, 356

Horsepower, 417

- -hour, 418

Impact, 450, 471

- loss of energy in, 476

Impulse, 450

- angular, 451, 452

- components of, 451

- linear, 451

- moment of, 451, 452

Inertia, defined, 289

- forces, 307, 326, 345

- moment of, 180, 205

— product of, 194 
Instantaneous center,

- of acceleration, 352

- - velocity, 284

Inclined plane, friction of, 135

Joule, 409

Journal friction, 145

Kilowatt, 417

Kilowatt-hour, 418

Kinematics, 2, 221

Kinetic energy, 420

- lost in impact, 476

- of a body having plane motion, 426

- - - - rotation, 425

- - - - - translation, 424

- - - particle, 422

Kinetics, 2, 287, 303

Lami's theorem, 74

Loss of energy, in bearings, 434

— in impact, 476

Mass, 289

Mass-center, 175

- motion of, 353

Moment-arm, 14, 19

Moment-center, 14, 87

Moment equation, limitations of, 351

Moment of inertia, 180, 205

—— - about inclined axes, 198

_ — - perpendicular planes, 208

— _ — by experiment, 220,367

_ — - composite areas, 190

— - - - - bodies, 218

_ - - graphical methods, 202

- - - integration, 184, 209

- - parallel axis theorem, 183, 207

- - - polar, 181

- - principal axes, 199

Momentum, 453

— angular, 454, 456, 457

- a vector quantity, 453,477

- components of, 454

- conservation of, 467

- linear, 454, 455

— moment of, $454,456,457$
Motion, curvilinear, 222

- gyroscopic, 378, 478

- Newton's laws of, 293

- non-uniformly accelerated, 256

— of a projectile, 248

- - mass-center of body, 353, 414

— — rigid bodies, 276

- rectilinear, 222

- relative, 262

- simple harmonic, 253

- uniformly accelerated curvilinear, 251

- uniformly accelerated rectilinear, 250

Motion graphs

- acceleration-time, 261

- distance-time, 228

- speed-time, 241, 260

Newton's laws of motion, 293

Pappus, theorem of, 171

Parabolic cable, 102

Parallelogram law, 8

Particle, 221

- equations of motion for, 297

Pendulum, compound, 363, 367

- conical, 300, 387

- simple, 360

- torsion, 365, 368

Period of oscillation, $362,364,366$

Pivots, friction of, 148

- loss of energy in, 443

Plane motion, 278, 336

Polar moment of inertia, 181

Pole of force polygon, 36

Poundal, 297

Power, 417

- special equations for, 418

Principal axis, 199

Principle of impulse and momentum, 459,461

- - moments, $16,38,49,57,65$

- - transmissibility, 8

- - work and energy, 430

Product of inertia, 194

- parallel axis theorem for, 196 
Projectile, motion of, 248

Prony brake, 446

Radius of gyration, 182, 207

Rays of force polygon, 36

Relative motion, 262

Resolution, 8

- of a force, 11

— - couples, 64

- directions of, 87

Resultant, 8

Resultants of force systems, 28

- - collinear forces, 28

- - concurrent forces in a plane, 29

- - - - space, 53

- - non - concurrent, non - parallel forces in a plane, 48

- - - - - space, 65

_- parallel forces in a plane, 34

- _ - - - space, 56

Reversed effective force for a body, $307,326,345$

Rigid body, 3

Rolling resistance, 153

Rotation, 277, 315

Scalar quantity, 6

Screws, 142

Second moment, 180

Sensitiveness of governors, 389

Slug, 296

Space diagram, 6

Speed, 226

Statically indeterminate force systems, 69
Statics, 2

Stresses in trusses, 88

Strings of funicular polygon, 37

Tangential-effort diagram, 411

Theorems of Pappus and Guldinus, 171

Translation, 276, 305

Triangle law, 9

Trusses, analysis of, 88

- graphical, 95

- method of joints, 89

- - sections, 90

Units, 294

- absolute system of, 297

- gravitational system of, 297

— systematic system of, 297

Varignon's Theorem, 16

Vector, 6

- diagram, 6

- quantity, 6

Velocity, angular, 229

- components of, 231

- linear, 226

- relative, 264

Work and energy, principles of, 430

Work, 406

- done in punching hole in plate, 412

- - by a force system, 414

- expressions for, 406, 408

- graphical representation of, 409

— units of, 409 



JAN 101982

FE'B 3998

THIS BOOK IS DUE ON THE LAST DATE STAMIPED BELOW

AN INITIAL FING OF 25 CFNTS WILL BE ASSESSED FOR FAILURE TO RETURN THIS BOOK ON THE DATE DUE. THE PENALTY WILL INCREASE TO 50 CENTS ON THE FOURTH DAY AND TO \$1.00 ON THE SEVENTH DAY OVERDUE.

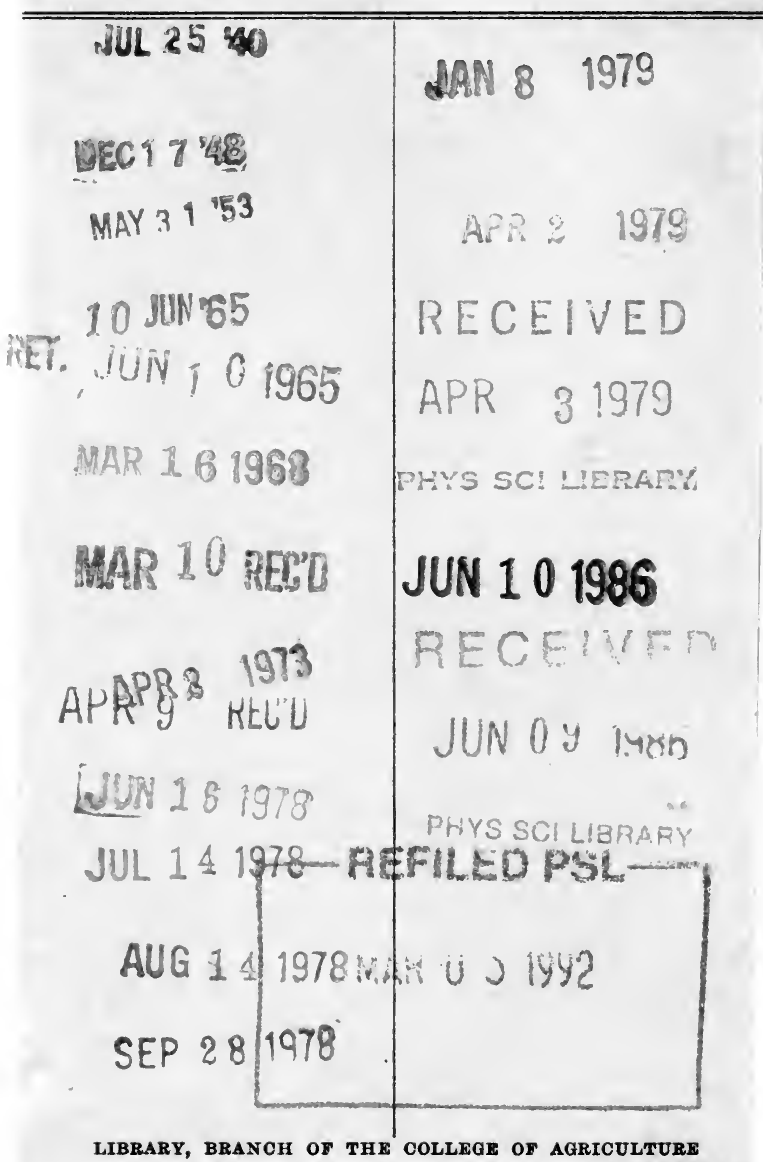

$5 m-9,35$ (s) 


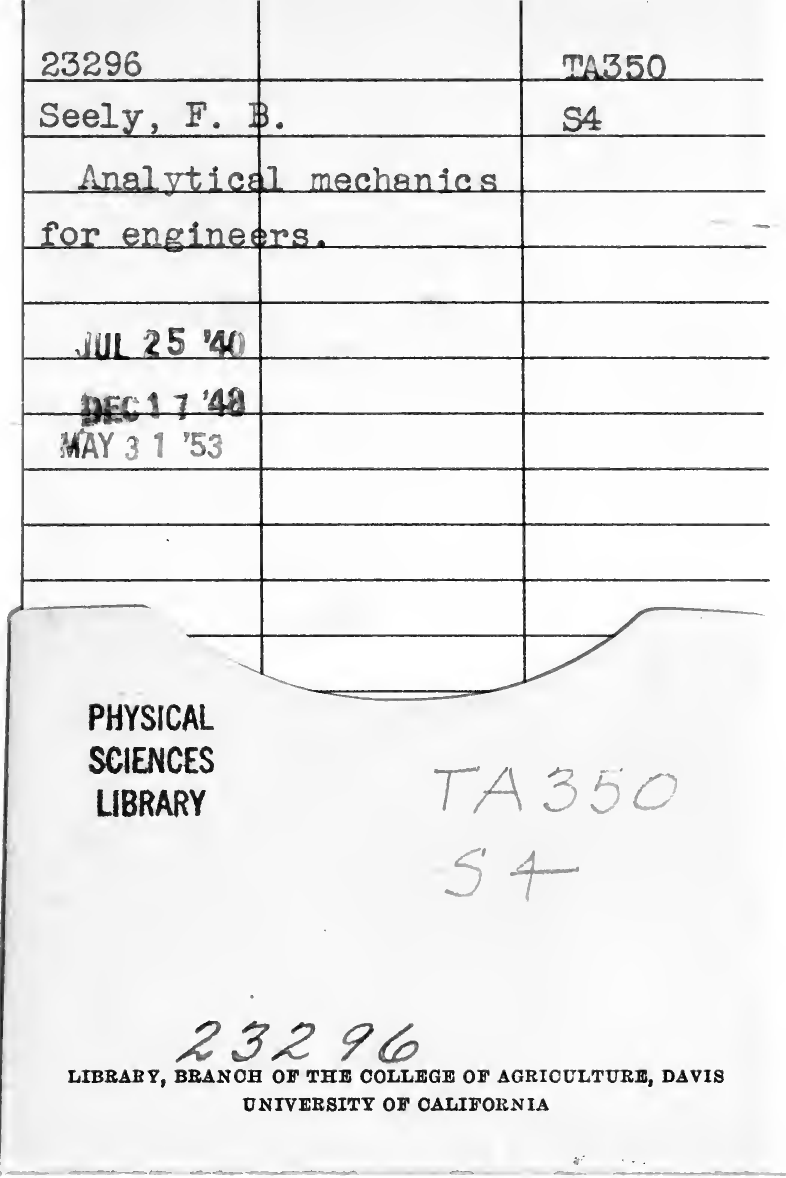


